\title{
TRANSIENT SMALL WIND TURBINE TOWER STRUCTURAL ANALYSIS WITH COUPLED ROTOR DYNAMIC INTERACTION
}

\author{
A Thesis \\ presented to \\ the Faculty of California Polytechnic State University, \\ San Luis Obispo
}

\author{
In Partial Fulfillment \\ of the Requirements for the Degree \\ Master of Science in Mechanical Engineering
}

by

George Robert Katsanis

May 2013 
(C) 2013

George Robert Katsanis

ALL RIGHTS RESERVED 
TITLE:

AUTHOR:

DATE SUBMITTED:

COMMITTEE CHAIR:

COMMITTEE MEMBER:

COMMITTEE MEMBER:
Transient Small Wind Turbine Tower Structural

Analysis with Coupled Rotor Dynamic Interaction

George Robert Katsanis

May 2013

Dr. Patrick Lemieux,

Professor of Mechanical Engineering

Dr. Peter Schuster,

Professor of Mechanical Engineering

Dr. Joseph Mello, Professor of Mechanical Engineering 


\section{ABSTRACT \\ Transient Small Wind Turbine Tower Structural Analysis with Coupled Rotor Dynamic Interaction \\ George Robert Katsanis}

Structural dynamics is at the center of wind turbine tower design - excessive vibrations can be caused by a wide range of environmental and mechanical sources and can lead to reduced component life due to fatigue, noise, and impaired public perception of system integrity. Furthermore, periodic turbulent wind conditions can cause system resonance resulting in significantly increased structural loads. Structural vibration issues may become exacerbated in small wind applications where the analytical and experimental resources for system verification and optimization are scarce. This study combines several structural analysis techniques and packages them into a novel and integrated form that can be readily used by the small wind community/designer to gain insight into tower/rotor dynamic interaction, system modal characteristics, and to optimize the design for reduced tower loads and cost. The finite element method is used to model the tower structure and can accommodate various configurations including fixed monopole towers, guy-wire supported towers, and gin-pole and strut supported towers. The turbine rotor is modeled using the Equivalent Hinge-Offset blade model and coupled to the tower structure through the use of Lagrange's Equations. Standard IEC Aeroelastic load cases are evaluated and transient solutions developed using the Modal Superposition Method and Runge-Kutta 4th order numerical integration. Validation is performed through comparisons to theoretical closed form solutions, physical laboratory test results, and peer studies. Finally a case study is performed by using the tool to simulate the Cal Poly Wind Power Research Center Wind Turbine and Tower System. Included in the case study is an optimization for hypothetical guy-wire placement to minimize tower stresses and maximize the tower's natural frequency.

Keywords: wind, turbine, tower, rotor, structural, structure, dynamic, vibration, optimization, IEC, stress, transient, coupling 


\section{ACKNOWLEDGMENTS}

I would like to acknowledge Dr. Patrick Lemieux for his guidance, mentorship, and patience over the course of several years as I worked to complete my thesis. Through my work with Dr. Lemieux I have learned the importance of technical rigor which has, and will no doubt continue to, serve me well.

I would also like to acknowledge my wife who has helped me with her encouragement and patience to complete this project. I love you!

I would also like to acknowledge my Mother and Father. I will be forever grateful for your love, support, and for the example that you have set for me.

I would like to dedicate this project to my grandfather, Dr. Robert Edgerley, whose work in aviation, toxicology, and the Apollo program inspired my interest in technical matters as a child. I will never forget the affirmations he encouraged me to repeat as a young child - I love math, I love math, I love math... 


\section{Table of Contents}

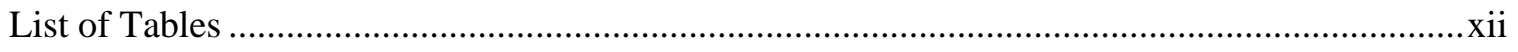

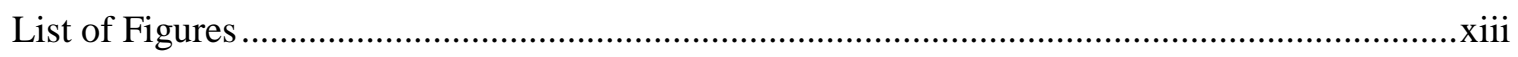

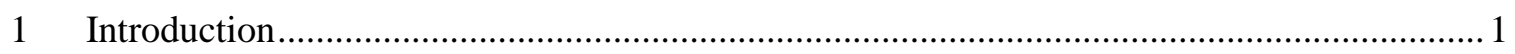

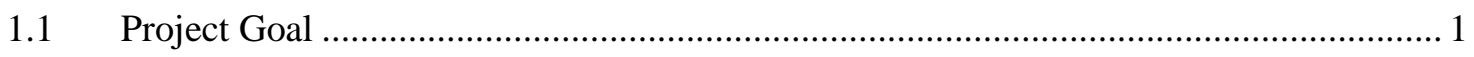

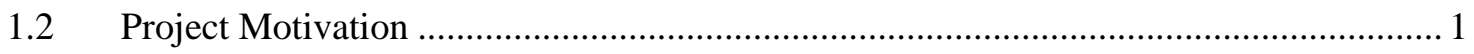

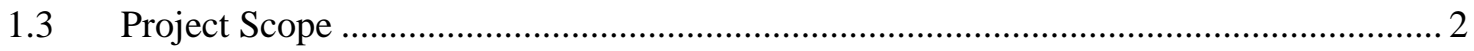

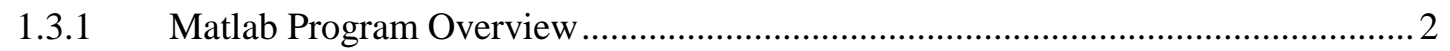

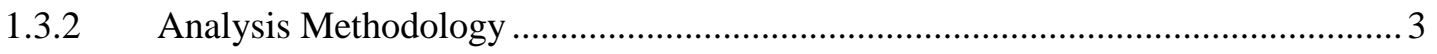

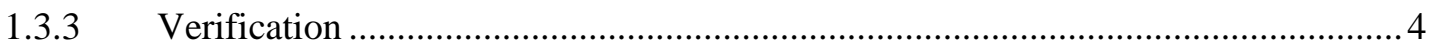

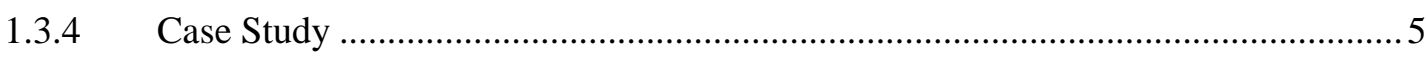

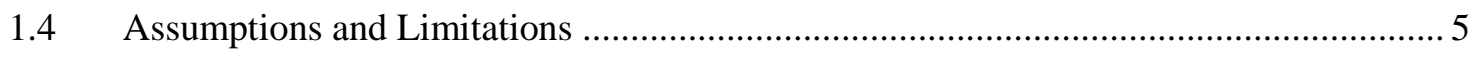

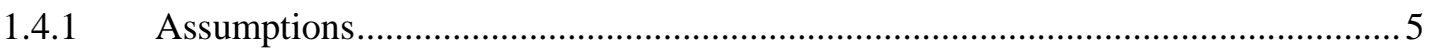

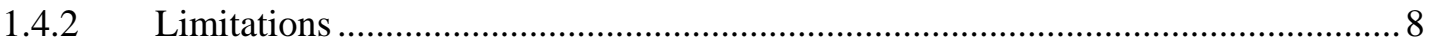

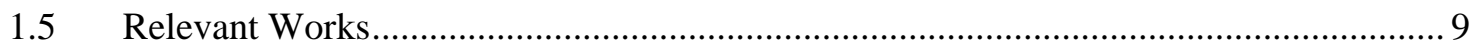

1.6 Wind Power History and Outlook ...................................................................... 10

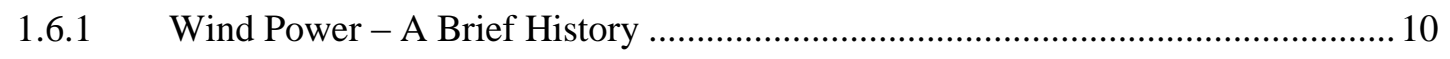

1.6.2 Wind Power - Current State, Economic Viability, and Future Outlook ................ 11

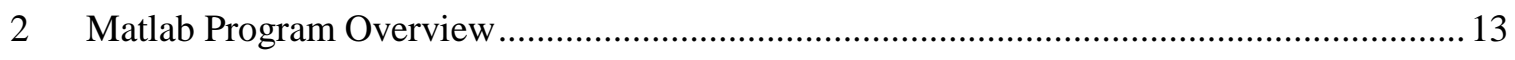

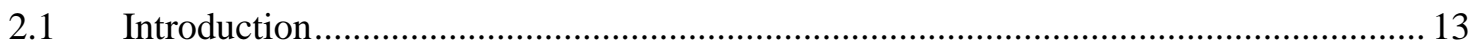

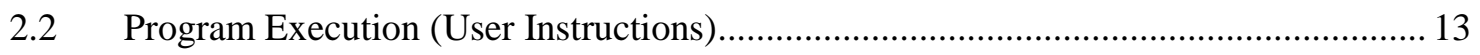




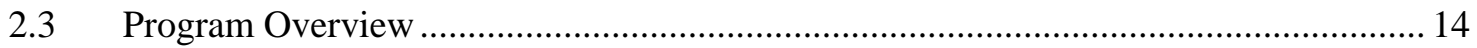

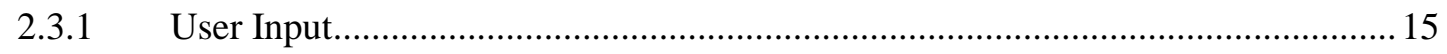

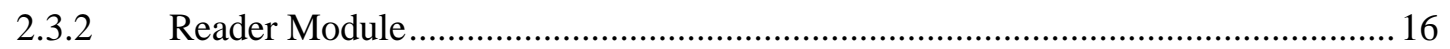

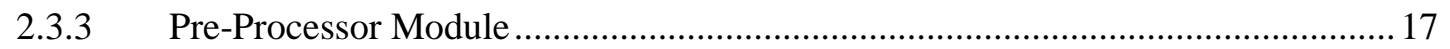

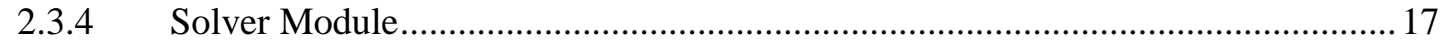

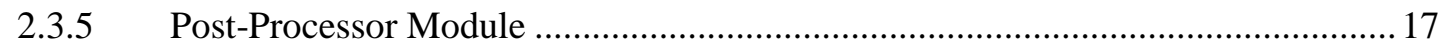

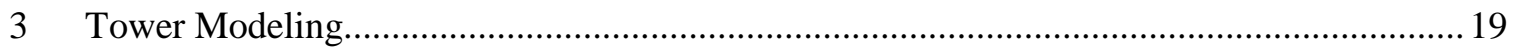

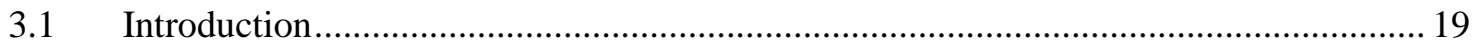

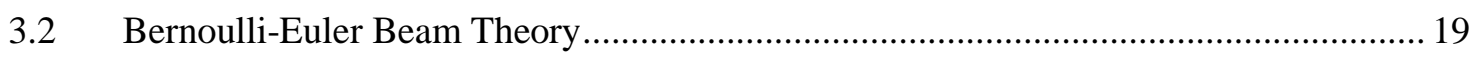

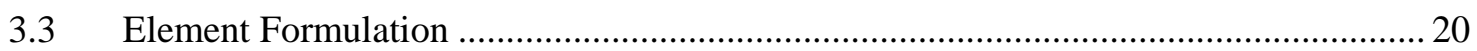

3.3.1 Method for Determining Finite Element Matrix Coefficients .............................20

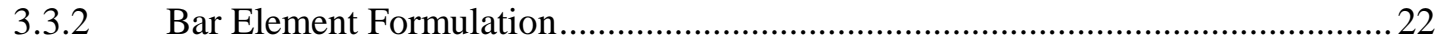

3.3.3 Simple Plane Beam Element Formulation …........................................................ 23

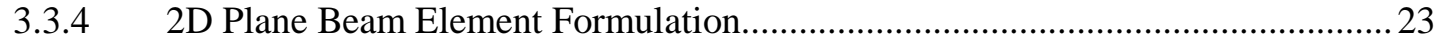

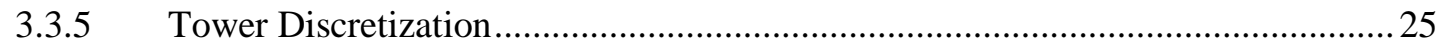

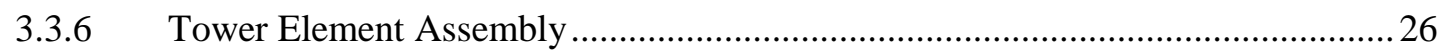

3.3.7 Boundary Conditions and Equation Elimination ............................................... 30

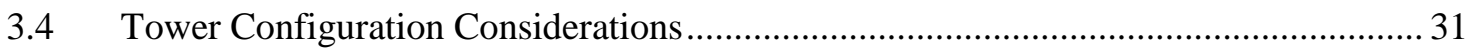

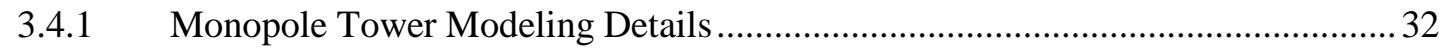

3.4.2 Guy-Wire Supported Tower Modeling Details .................................................... 32

3.4.3 Ginpole and Strut Supported Tower Modeling Details........................................ 34 


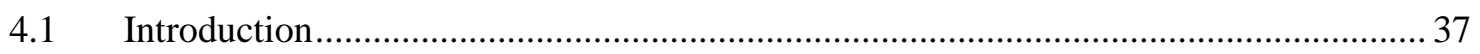

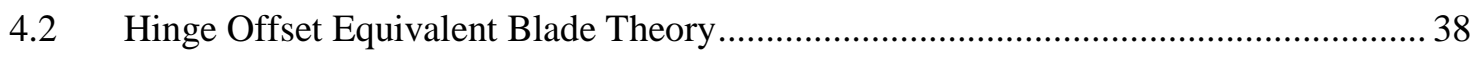

4.2.1 Assumptions of the Hinge Offset Equivalent Blade Theory ...................................39

4.2.2 Considered Blade Motions ............................................................................. 40

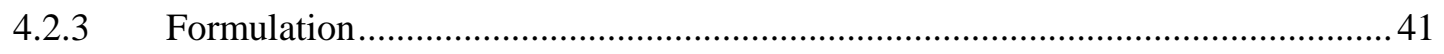

4.3 Rotor Tower Coupling - Simplified Rotor Model ................................................. 50

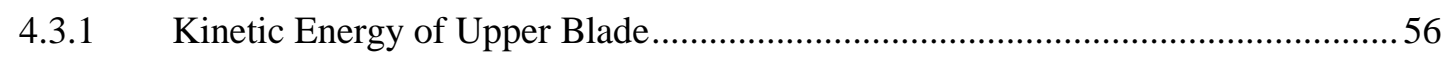

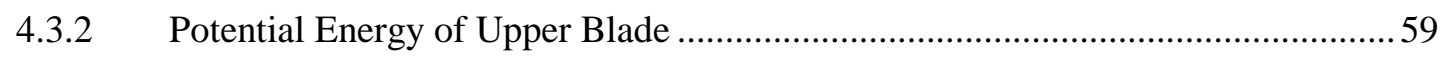

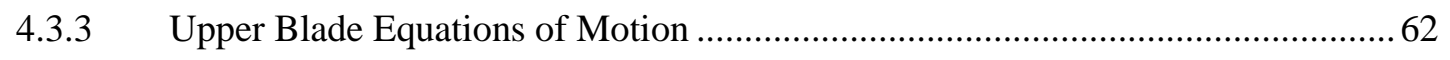

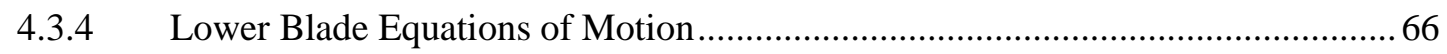

4.3.5 Final Equations of Motion for Simplified Rotor Model ..................................... 68

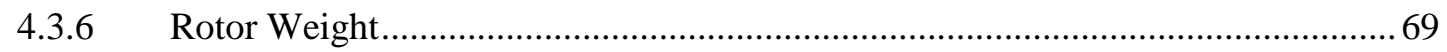

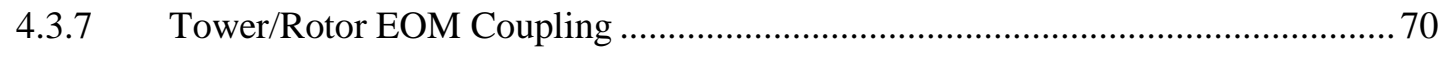

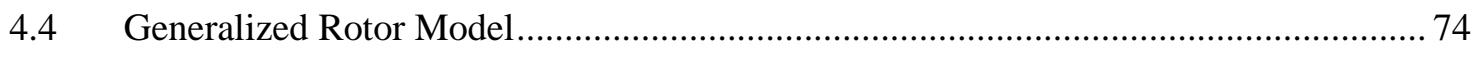

4.4.1 Generalized Rotor Model Azimuth Conventions.............................................. 75

4.4.2 Generalized Rotor Model Coordinate System …............................................... 77

4.4.3 Generalized Rotor Blade Kinetic Energy ...................................................... 80

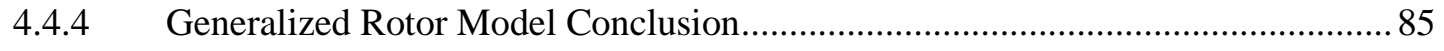

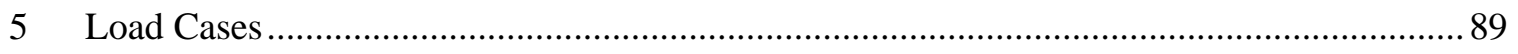

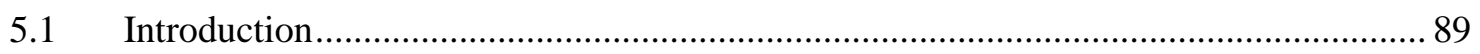




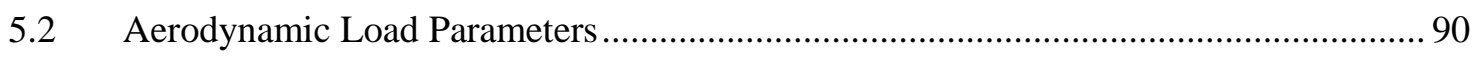

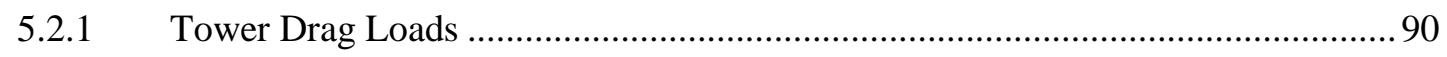

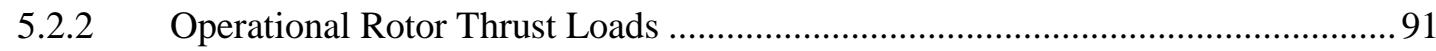

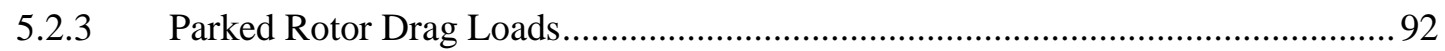

5.2.4 Rotor Blade Hinge Bending Moments .............................................................. 92

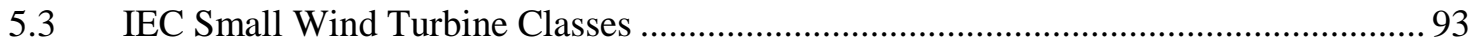

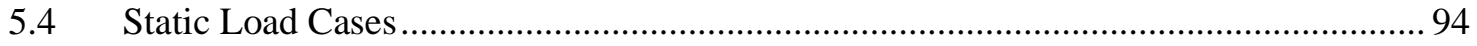

5.4.1 Static Load Case A - Tip Load ........................................................................ 94

5.4.2 Static Load Case B - 50 Year Extreme Wind Model (IEC DLC 5.1) ...................95

5.4.3 Static Load Case C - 1 Year Extreme Wind Model (IEC DLC 6.1) .....................96

5.4.4 Static Load Case D - Nominal Operation............................................................96

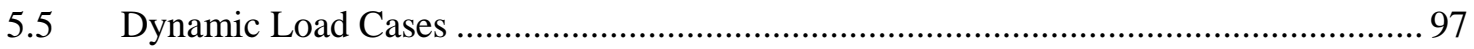

5.5.1 Dynamic Load Case E - Extreme Operating Gust (IEC DLC 1.3)........................97

5.5.2 Dynamic Load Case F - Extreme Coherent Gust (IEC DLC 1.5) .........................98

5.5.3 Dynamic Load Case G - Normal Turbulence Model (IEC DLC 2.2) ................... 99

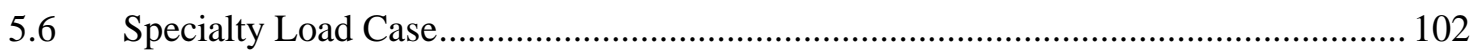

5.6.1 Specialty Load Case H - Rotor Thrust Swept Sine .......................................... 102

5.6.2 Specialty Load Case I - Tower Vortex Shedding ............................................ 105

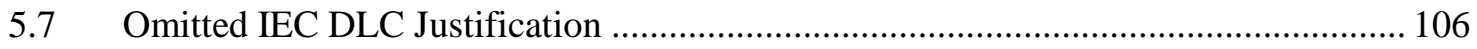

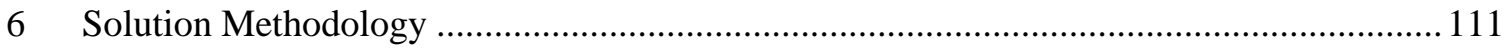

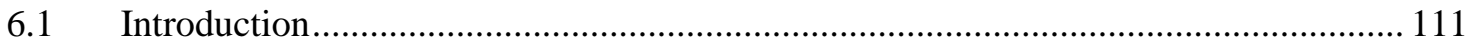




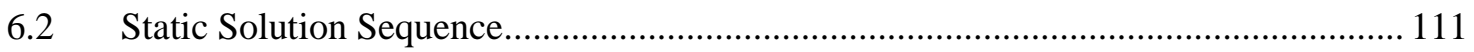

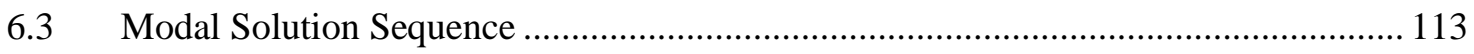

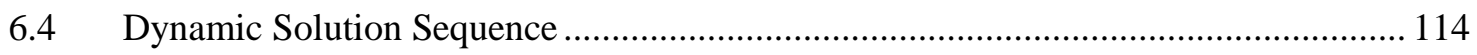

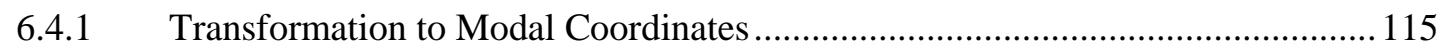

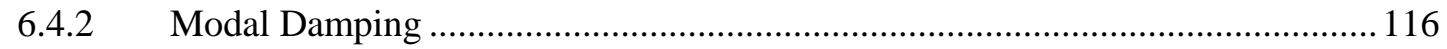

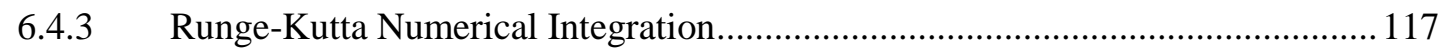

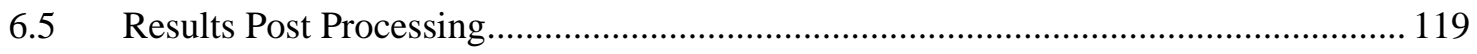

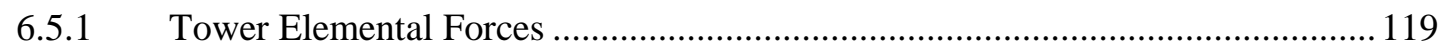

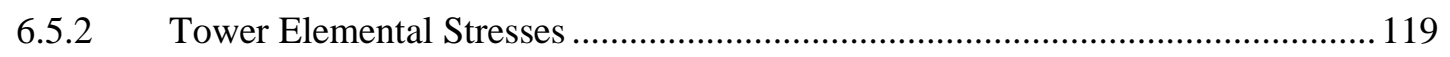

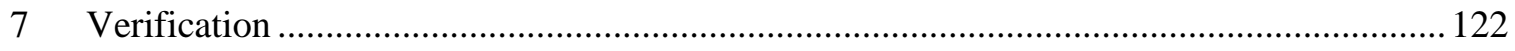

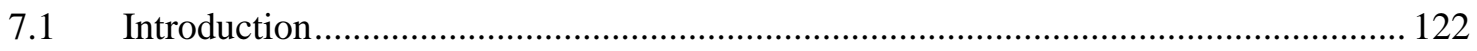

7.2 Tower - Comparisons to Theoretical Models ......................................................... 122

7.3 Tower - Physical Tower Testing and Model Correlation ......................................... 125

7.4 Tower - Case Study Comparison to Peer Work ................................................... 128

7.4.1 Case Study Comparison to Peer Work - Static Loading …............................... 129

7.4.2 Case Study Comparison to Peer Work - Modal Evaluation .................................. 130

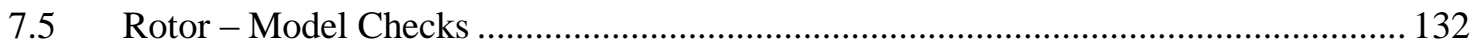

7.6 Dynamic - Validation of Runge-Kutta Numerical Integration................................. 133

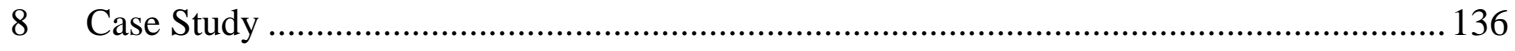

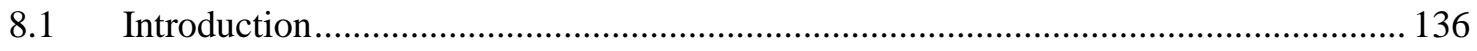

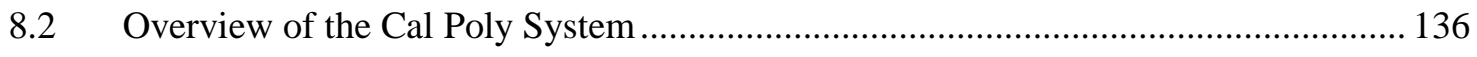




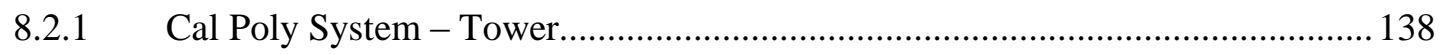

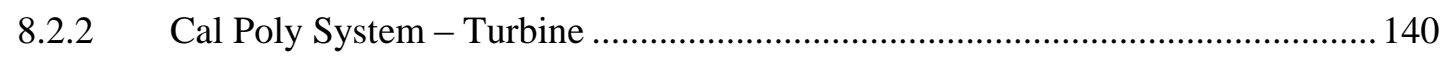

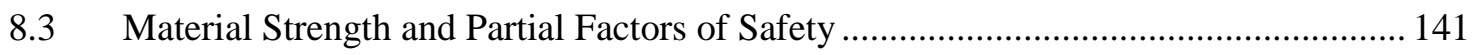

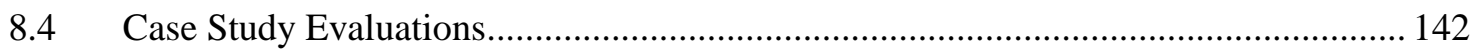

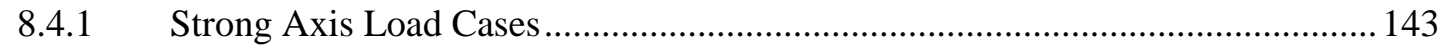

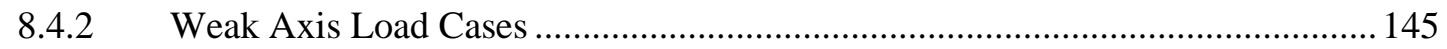

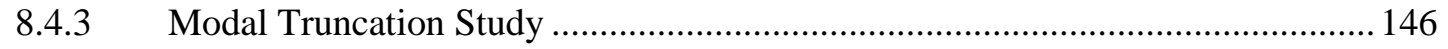

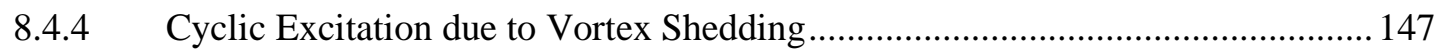

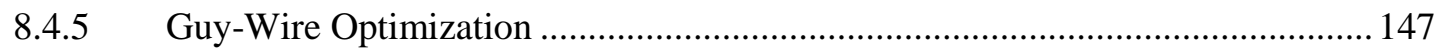

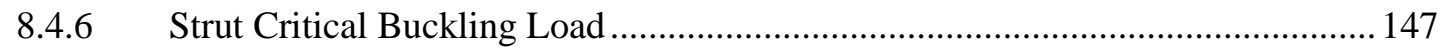

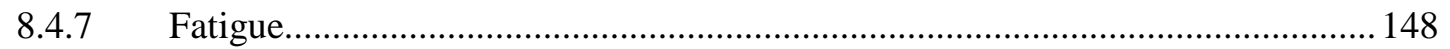

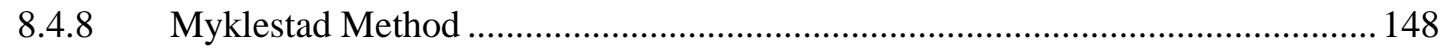

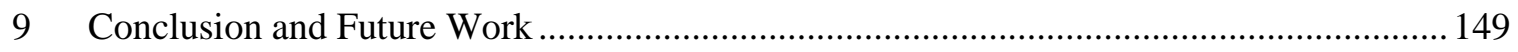

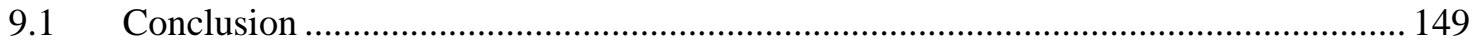

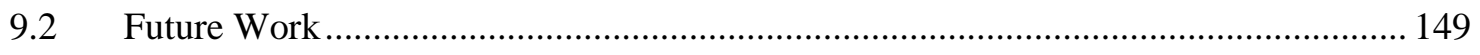

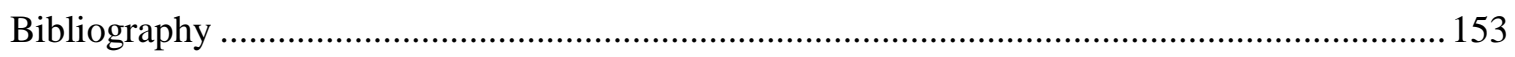

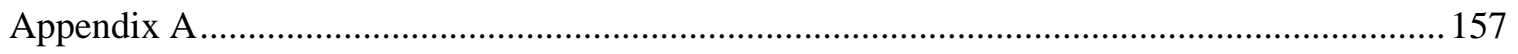

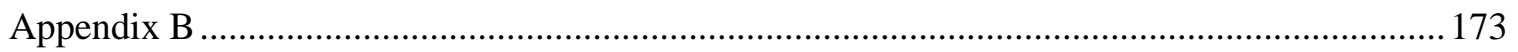

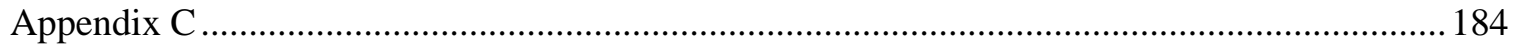

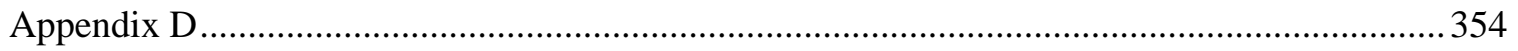




\section{List of Tables}

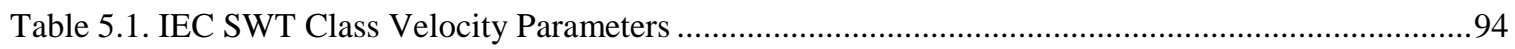

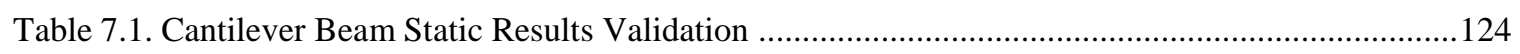

Table 7.2. Cantilever Beam Modal Convergence Study ………………………......................................125

Table 7.3. Comparison of Katsanis and Gwon Static 2100 lbf Static Thrust Load Results ........................129

Table 7.4. Comparison of Katsanis and Gwon Modal Frequency Results ...................................................131

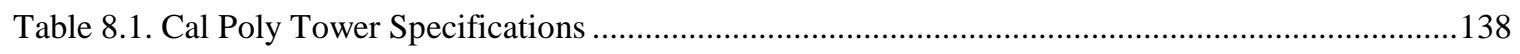

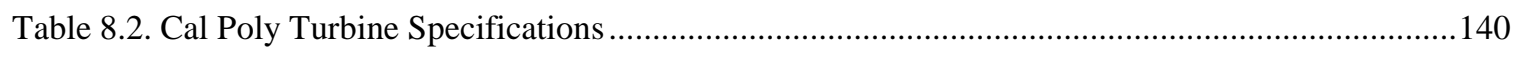

Table 8.3. Cal Poly Tower Material Strength and Factors of Safety ........................................................142 


\section{List of Figures}

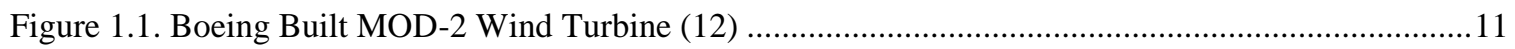

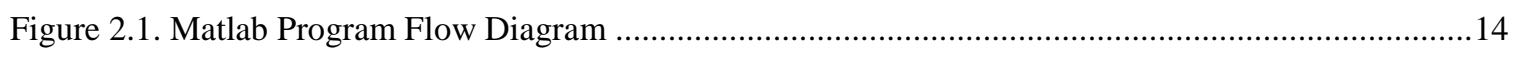

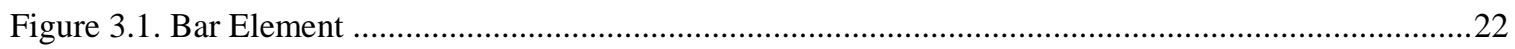

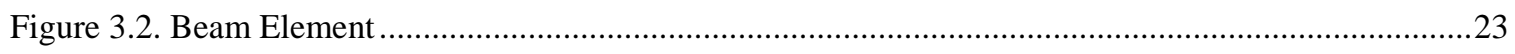

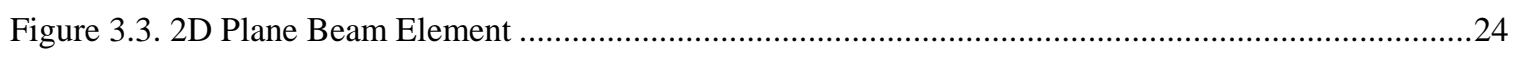

Figure 3.4. 2D Plane Beam Element rotated by $\lambda=90^{\circ}$ into vertical orientation .........................................2

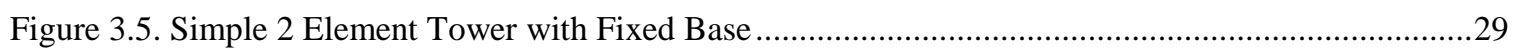

Figure 3.6. Tower Configurations, a) Monopole Tower, b) Guy-Wire Supported Tower, c) Ginpole and

Strut Supported Tower (Cal Poly Wind Turbine Configuration)........................................................ 31

Figure 3.7. Tower Coordinates for Ginpole and Strut Supported Tower a) Strong Axis $(\xi=0), b)$,

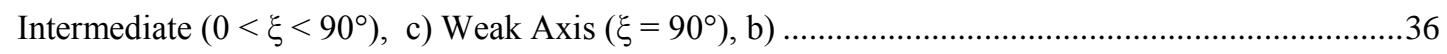

Figure 4.1. A wind turbine blade with complex geometry and the corresponding hinge equivalent blade

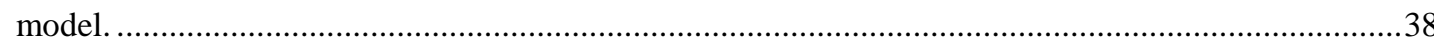

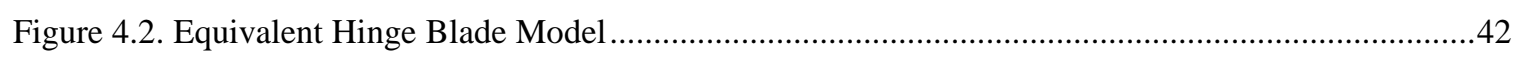

Figure 4.3. Hinge equivalent rotor blade with centrifugal force and dimensions. Distance R is the total

length of the blade and distance $r$ is the distance from the hinge to the differential mass, dm............44

Figure 4.4. The Simplified Rotor Model configuration.......................................................................... 51

Figure 4.5. Schematic of the tower, hub, and rotor blades used for the Simplified Rotor Model. Degrees of

freedom of tower tip and blade hinges appear in blue. $($ Note: wrt $=$ with respect to $)$...........................52

Figure 4.6. The coordinate systems of the upper blade used in the Simplified Rotor Model........................55

Figure 4.7. The coordinate systems of the lower blade used in the Simplified Rotor Model.......................66

Figure 4.8. Simplified Rotor Model coupled with the Simplified Tower Model. ..........................................71

Figure 4.9. Example Blade and rotor azimuth angles used for the Generalized Rotor Model ......................76

Figure 4.10. Baseline Blade Model for the i'th blade in the Generalize Rotor Model. Generalized

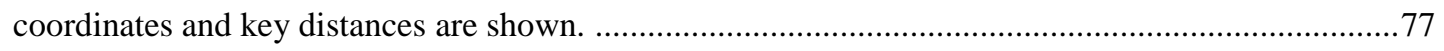

Figure 4.11. Unit vectors associated with the Baseline Blade Model. ......................................................79

Figure 4.12. Mass centered coordinate systems for the Baseline Blade Model...........................................80 
Figure 4.13. Section A from the Generalize Rotor Model - Baseline Blade Model................................84

Figure 5.1. EWM50, EWM1, and "Nominal Operation” 15MPH Wind Profiles ....................................95

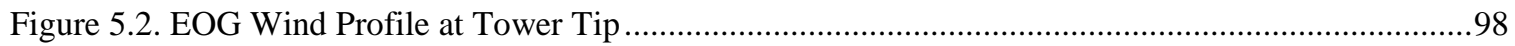

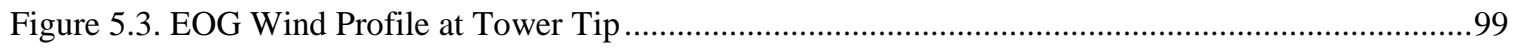

Figure 5.4. NTM Wind Profile at Tower Tip ............................................................................ 102

Figure 5.5. Swept Sine Rotor Thrust Load and Frequency ........................................................104

Figure 6.1. Tower Cross Section Stress Theta Location .................................................................121

Figure 7.1. Cantilever Beam used for Verification Study ….....................................................122

Figure 7.2. Cantilever Beam Modal and Static Results Comparison ..............................................124

Figure 7.3. Guy-Wire Supported Tower Experiment Configuration ...............................................125

Figure 7.4. Guy-Wire Supported Tower Experiment Dimensions .......................................................126

Figure 7.5. Guy-Wire Supported Tower Experiment Modal Tap Test ............................................127

Figure 7.6. Guy-Wire Supported Tower Experiment Modal Results ................................................127

Figure 7.7. Matlab Modal Simulation of Experimental Configuration ................................................. 128

Figure 7.8. Matlab Modal Simulation of Cal Poly Wind Turbine Tower.............................................131

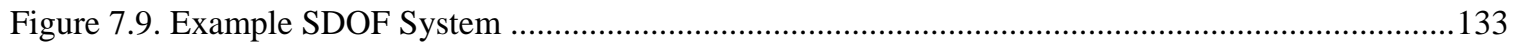

Figure 7.10. SDOF Theoretical vs Runge-Kutta Convergence Study ............................................... 135

Figure 8.1. The Cal Poly System - Coordinates....................................................................... 137

Figure 8.2. The Cal Poly System - Select Dimensions ............................................................139 


\section{Introduction}

\subsection{Project Goal}

The goal of this project is to simplify the structural analysis of small wind turbine towers by providing an integrated, high-level development tool that is based on proven engineering methods for both rotor and tower aeroelastic simulation, but does not require the in-depth training that other tools require.

\subsection{Project Motivation}

Small wind turbines are critical to the wind power industry because they allow full utilization of the diffuse resource. In order to make small wind viable, small local companies must be capable of effectively developing the enabling technologies. A major barrier for many startup companies is tower design because of the lack of resources. Wind turbine towers are one of the most critical components of a wind turbine system. Unlike many other components, a tower failure necessarily results in a catastrophic failure of the whole system.

Utility scale wind turbine structures undergo exhaustive analysis and optimization with some of the most robust and advanced techniques available. Small wind turbines intended for private use are a much different story. Novice designers of small wind turbines may lack an understanding of the complex environmental loading placed on the towers as well as critical structural issues such as modal coupling of the tower/rotor subsystems. In addition many small companies will lack the proper technical resources, such as structural modeling programs.

Free simulation codes are available through the National Wind Technology Center (NWTC), such as YawDyn and, FAST, but are not tailored to the specific requirements of tower analysis or are complex and require an experienced technical user. Furthermore these tools are not well 
integrated and require the user to work with several different software packages to develop a model and execute a simulation.

This work addresses these needs by introducing a highly integrated tool that can quickly and accurately verify the structural integrity of a small wind turbine tower. Furthermore the tool allows the designer to perform system optimization studies and explore system dynamic characteristics during the design phase. The method is practical for small startup companies which are common in the small wind turbine industry. The tool is implemented in Matlab, which is ubiquitous in technical workplaces, and as such is inexpensive to implement (assuming the user has a Matlab license). The tool was developed with the novice designer in mind, shelters the user from the underlying theory, and requires a limited set of well defined user inputs.

\subsection{Project Scope}

Sections 1.3.1 through 1.3.4 summarize the scope of this work. The organization of these sections mirrors that of the remainder of this report (Chapters 2-8). Note that Chapters 3, 4, 5, and 6 are all focused on methodology. Subsequently, the methods implemented by this work are verified in Chapter 7. Also note that results are reserved for Chapter 8, the case study chapter, and are not discussed elsewhere in this report.

\subsubsection{Matlab Program Overview}

A Matlab program has been created that allows the user to simulate coupled rotor / tower response to environmental (wind) conditions. The tool accommodates various tower configurations including a simple monopole tower, guy-wire supported tower, as well as towers supported by a gin-pole and strut. The tool can simulate towers which support two or three bladed horizontal axis wind turbines and can also be configured to simulate towers that support an arbitrary tip mass as well as bare towers. User inputs to the programmed are structured through the use of a user input Microsoft Excel spreadsheet which includes detailed definitions of each input parameter. Various solution sequences can be selected by the user and include static, modal, 
and dynamic simulations. The tool includes International Electrotechnical Commission (IEC) aeroelastic load cases as well as several customizable load cases. A digital copy of the Matlab tool has been submitted with this report. Chapter 2 includes a detailed overview of the Matlab tool as well as user instructions.

\subsubsection{Analysis Methodology}

\subsubsection{Analysis Methodology - Tower}

The methods implemented by the Matlab tool are all based on proven engineering simulation techniques. The finite element method is used to create a mathematical model of the tower using 2D Plane Beam elements which feature six degrees of freedom (DOF) per element. The 2D Plane Beam element is based on Euler-Bernoulli Beam Theory which is well documented (1) and has been widely used for over a century. Chapter 3 includes an in-depth overview of the tower modeling methods implemented by this work.

\subsubsection{Analysis Methodology - Rotor}

The rotor model used by this work is based on the Hinge Offset Equivalent Blade Theory (2) and features a single DOF for each blade that allows flapwise deflection of the blade. The rotor model is a simple first order model and cannot support detailed analysis of the turbine components but is effective at simulating coupled tower / rotor interaction loads and determining the influence of rotor parameters, such as blade natural frequency, on tower response. The details of the rotor model are discussed in Chapter 4.

\subsubsection{Analysis Methodology - Load Cases}

The Matlab program allows the user to select from several static (does not vary as a function of time) and dynamic (varies as a function of time) load cases. The load cases include several standard IEC-61400-2 Aeroelastic Design Load Cases (DLC) (3). Conservative simplifications have been made (primarily related to assumed system fault conditions) such that the selected IEC 
load cased bound the omitted IEC load cases. Several custom load cases have been included that can be easily modified by the user to simulate nominal operating conditions. A customizable swept sine dynamic load case has also been included which allows the user to gain additional insight into tower / rotor interaction and system dynamic characteristics. The load cases are fully detailed in Chapter 5.

\subsubsection{Analysis Methodology - Solution Methods}

The Matlab program utilizes several solution sequences which are automatically selected based on load case. A static solution sequence based on Hooke's Law (4) and Gaussian Elimination (5) is used to solve all static load cases. A modal solution sequence, which uses built-in Matlab Eigenvector / Eigenvalue solution sequences, is utilized to solve for system normal modes. Finally a dynamic solution sequence, utilizing the Truncated Mode Superposition Method (1) with modal damping, is used to solve for all dynamic load cases including the swept sine load cases. The theoretical foundations for each solution sequence are detailed in Chapter 6.

\subsubsection{Verification}

Several independent methods have been utilized to verify the accuracy of the Matlab program included in this work. The Matlab program was used to model a simple cantilever beam (i.e., monopole tower with fixed base) and beam deflections due to static load, as well as normal modes, were compared to the theoretical solution given by Euler-Bernoulli Beam Theory. A physical modal tap test was performed on a small (46 inch tall) guy-wire supported tower in a laboratory setting and results were compared to the Matlab tool. The Case Study static and modal results, see Section 7.4, were compared to peer results (6). Various rotor model checkouts were performed to ensure the simulated rotor characteristics were consistent with user inputs. Finally the Runge-Kutta dynamic solution sequence was compared to theoretical results for a simple model. The steps taken to verify the program are detailed further in Chapter 7. 


\subsubsection{Case Study}

The methods established by this work are demonstrated through the use of a case study. The subject of the case study is the Cal Poly Wind Power Research Center wind turbine and tower system. The system features a $70 \mathrm{ft}$ tapered tubular tower that is erected, and supported by, a ginpole and strut configuration. The tower supports a $3 \mathrm{~kW}$ horizontal-axis wind turbine with a $12 \mathrm{ft}$ rotor diameter. The system is evaluated for all load cases detailed in Chapter 5 and the overall maximum tower deflection was found to be 69.45 inches and the overall minimum Margin of Safety was 0.25 . The case study includes repeated use of the swept sine load case to explore tower / rotor coupled dynamic characteristics and evaluate the influence of various parameters, including rotor blade flapwise natural frequency, on tower response. A detailed discussion of the case study is captured in Chapter 8 . Note that all case study result tables, figures, and data are organized in Appendix C.

\subsection{Assumptions and Limitations}

\subsubsection{Assumptions}

The following is a summary of this work's assumptions. Each assumption includes a crossreference for additional information.

1. Tower Modeling Assumptions

a. Tower shear deformation is assumed to be negligible and is ignored. This assumption is appropriate for tall sender towers (tower height $>>$ tower diameter). See Section 3.2 for further details.

b. Tower torsion is assumed to be negligible and is ignored which is an appropriate assumption for passively controlled furling wind turbines which do not transfer any torsional moment to the tower. Furthermore, the load cases in this work all feature homogeneous wind profiles across the swept rotor area and as such do not 
generate any yaw torsion. See Section 3.3.4 for further details of the degrees of freedom that are considered.

c. Tower deflection is only allowed in a $2 \mathrm{D}$ plane (the plane defined by the wind direction, which is the $\mathrm{X}$-Axis, and the vertical direction, which is the Y-Axis) and out of plane loads and deflections are assumed to be negligible. See Section 3.3.4 for further details.

i. As discussed in Section 3.4.3 (item iv.b) the model can be rerun multiple times with the wind direction (X-Axis) in different orientations relative to the tower to determine tower loads and deflections for towers with asymmetric geometry, such as the Cal Poly configuration used for the case study.

d. Tower base boundary conditions must be set, by the user, to be either fixed or pinned. The ground soil stiffness and damping, and interaction with the tower, cannot be directly modeled. For gin-pole and strut supported towers, as well as guy-wire supported towers, the user may bound the tower / ground interaction by running the model with both pinned and fixed tower boundary conditions. For monopole towers the model must be run with a fixed tower base boundary condition. See Section 3.4 for more information.

e. For guy-wire supported towers the guys-wires are assumed to remain in a state of tension (i.e. do not go slack due to environmental loads). If the model returns a negative tension load for a guy-wire (i.e., guy-wire compression) the user should increase the pre-tensioning of the guy-wires and rerun the model. The Matlab program outputs guy-wire tension to the "Guy-Wire_Tension" tab of the MS Excel output file for each load case. See Section 3.4.2 for more information.

f. For gin-pole and strut supported towers the gin-pole and strut are assumed to be 2-force members (i.e., not capable of supporting bending moments). In addition, 
the gin-pole and strut are each modeled with a single 2D Plane Beam Element and as such bending mode shapes of the gin-pole and strut are not included in the simulation. See Sections 3.4.3 and 7.4.1 for further details.

\section{Rotor Modeling Assumptions}

a. The rotor orientation (X-Axis) is assumed to be parallel to the wind direction. Furthermore, it is assumed that no loads are developed orthogonal to the wind direction and that the turbine hub does not impart any torsion onto the tower. The turbine hub / tower interaction load components considered in this work are axial force, shear force, and bending moment about the Z-Axis. See Section 4.4 for further information.

b. The Hinge Offset Equivalent Blade Theory (2) is used to model the blades. There are various assumptions associated with this theory and are summarized in Section 4.2.1. The implementation in this work further simplifies the Hinge Offset Equivalent Blade Theory by limiting blade deformation to the flapwise direction only (into and out of the wind), see Section 4.2.2 for further details.

c. The solution sequence used in this work is a linear solution sequence. Therefore system mass and stiffness properties are not adjusted as a function of time during transient simulations. Nonlinear effects, such as the rigid body rotation of the rotor during operation are not considered. The analyst can perform a simulation with the rotor modeled in any user defined azimuth position and can include the influence of turbine operation on blade natural frequency caused by centrifugal stiffening. See Section 4.4 for further details.

\section{Load Case Assumptions}

a. Only some of the IEC Aeroelastic Design Load Cases have been included in this work. Conservative assumptions, primarily related to assumed system fault conditions, have been applied such that the load produced by the included cases 
bound the loads produced by the omitted cases. See Section 5.7 for further details.

b. The user must enter in various aerodynamic parameters for the program to determine the system loads caused by the wind. These parameters can be set to whatever the analyst thinks is appropriate. For the purpose of the Case Study (see Chapter 8) the aerodynamic parameters were assigned the values listed below. See Section 5.2 for an explanation of these parameters and the rationale behind the selected values.

i. Tower Aerodynamic Drag Coefficient: $\mathrm{C}_{\mathrm{d}}=1.3$

ii. Operational Rotor Thrust Coefficient: $\mathrm{C}_{\mathrm{T}}=0.5$

iii. Parked Rotor Drag Coefficient: $\mathrm{C}_{\mathrm{f}}=1.5$

c. The force acting on each rotor blade, induced by the wind, is assumed to have a force resultant that is located $2 / 3$ of the way down the blade away from the blade root. See Section 5.2.4 for further details.

d. Air density is based on the U.S Standard Atmosphere (7) at sea level and at 59 F.

\subsubsection{Limitations}

This tool can only be used to model monopole towers, guy-wire supported towers, and gin-pole and strut supported towers. The tower cross-section must be circular. Furthermore this tool can only model horizontal wind turbines with two or three blades.

The rotor model used in this work is a first order highly simplified model that is only intended to determine how rotor / tower interaction influences tower loads. Simulations from this model will return blade flapwise bending angles as well as hub forces but these results should NOT be used for turbine component design or analysis as they are approximate. 
The tower model used in this work only includes primary structure (i.e., the tower itself and if applicable guy-wires or a gin-pole and strut). Secondary structural elements (such as mounting flanges at tower section joints, guy-wire and strut clevis joints, tower base bearings and housings, brackets, etc...) are NOT included in this model. Furthermore, stress concentration factors caused by geometric discontinuities are not included. As such the loads produced by this tool should be used as only the first step in the structural verification process. Detailed stress analysis of all mechanical joints (welded, bonded, fastened, pinned, etc...) as well as all secondary structure should be performed by the analysis / designer. An example of such detailed / component level analysis is included in Gwon's work (6).

\subsection{Relevant Works}

As noted in Section 1.3.4, the case study presented in Chapter 8 is based on the Cal Poly Wind Power Research Center wind turbine and tower system. The Cal Poly Wind Power Research Center has sponsored several other student projects relevant to this work including the following:

1. Christopher Nosti studied the performance and fatigue life of small wind turbine blades in 2009 (8). Nosti's implementation of the Hinge Offset Equivalent Blade Theory and the Mykelstad Method inspired the blade modeling approach implemented in this work.

2. Bryan Edwards designed the composite rotor blades used by the Cal Poly Wind Power Research Center wind turbine as well as the manufacturing process required to create the blades in 2009 (9). The blade cross-sectional airfoil properties, as well as stiffness test results, documented by Edwards were used to develop the rotor blade model used in the case study of this work.

3. Tae-gyun (Tom) Gwon studied the same Cal Poly Wind Power Research Center wind turbine tower configuration that was used for the case study in this work. Gwon used Abaqus (a commercial finite element analysis program) to evaluate the tower's deflection due to a 2,100 lbf tip load (same load case as Load Case A in this work) as well as the 
tower modal frequencies and shapes. The results from Gwon's work were compared to the results from this work; see Chapter 7 for more details. In general, result agreement was good.

\subsection{Wind Power History and Outlook}

\subsubsection{Wind Power - A Brief History}

Harnessing the wind's power is certainly not a new idea. Mesopotamians used the wind to power sailboats as far back as 5,000 years ago (10). The origins of fixed on-shore wind power stretch as far back as $900 \mathrm{AD}$ when the Persians constructed vertical axis drag windmills (11). In the middle ages wind power entered main stream use with four bladed horizontal axis wind mills common across Europe. These European wind mills were used for a multitude of mechanical tasks including pumping water, milling grain, and even for sawing wood (11). During the industrial revolution (late $18^{\text {th }}$ through early $19^{\text {th }}$ century) wind power lost its relevance as it became displaced by emerging power production technologies, such as coal fired steam engines, that offered on-demand power production that could be collocated with energy users (11).

Shortly after the advent of practical electrical generators in the late $19^{\text {th }}$ century efforts began to use wind power to generate electricity. Notable early wind turbines used for electricity production include 20 - $35 \mathrm{~kW}$ turbines designed by Poul La Cour from 1891 through 1918 in Denmark, the large 1.25MW Smith-Putnam wind turbine built in the late 1930s in Vermont, and small 1-3kW wind turbines were built by Marcellus Jacobs for residential in the 1920s (11). Although modern wind turbines can trace their origins to the noted examples, wind power in the early $20^{\text {th }}$ century was not cost competitive with competing technologies and was relegated to research projects and niche usage.

The second half of the $20^{\text {th }}$ century ushered in a revival in wind power interest. In the 1960 s and 1970s the environmental movement took hold as the public became aware of the impact 
industrialization, and in particular the usage of fossil fuel, was having on the environment.

Despite the shifting public consciousness it was not until the oil crises of the mid-1970s, caused in part by the OAPEC oil embargo, that a concerted effort was made to invest in wind power (11). At this time the US Department of Energy (DOE) funded a sequence of utility scale wind turbine research projects. In 1973 Boeing received a NASA / DOE contract to build a series of $2.5 \mathrm{MW}$ MOD-2 two bladed wind turbines, shown in Figure 1.1, which enter production in the early 1980s (12).

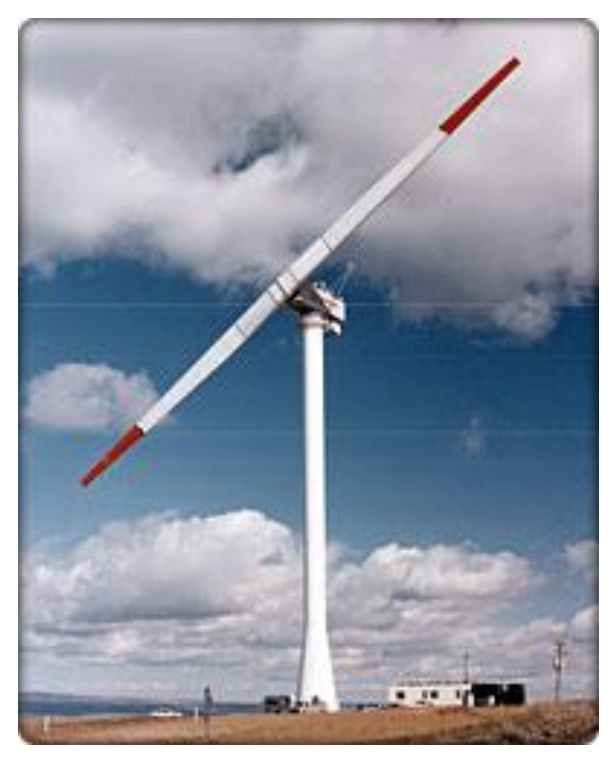

Figure 1.1. Boeing Built MOD-2 Wind Turbine (12)

In the late 1970s maturation of wind turbine technologies, regulatory changes allowing for wind power to be connected to the electrical grid, and federal investment tax credits lead to the economic viability of utility scale wind power production (11). Additional incentives provided by the state of California allowed it to lead the way with wind farm developments in Altamont Pass, San Gorgonio Pass, and Tehachipi.

\subsubsection{Wind Power - Current State, Economic Viability, and Future Outlook}

While improved designs have reduced the cost of wind power production the industry remains sensitive to government tax incentives. A pull back in the industry occurred in 1980 when the 
Reagan administration ended the federal tax credits (11). Through the early $21^{\text {st }}$ century the Production Tax Credit (PTC) was a major factor in the rapid expansion of wind power capacity in the US but also led to a boom and bust cycle in the industry due to PTC expiration concerns (13).

In February 2013 US Wind Production was 13.9 Trillion Watt-Hours, a year-over-year increase of $24.4 \%$, and accounted for $4.5 \%$ of US energy production (14). Meanwhile total year-over-year power production across all sources actually went down by $0.2 \%$. Some critics argue that the strong 2012 growth in wind power capacity was caused by a rush to complete projects prior to the expiration of the PTC, which was set to expire at the end of 2012, and that this may dampen growth in 2013 (13). Ultimately the PTC, which provides 2.2 cents per kilowatt-hour of wind power, was extended as a part of the "Fiscal Cliff" negotiations (15).

The outlook for wind power remains strong with the DOE setting a goal in 2008 to obtain $20 \%$ of US electrical power from wind energy by 2030 (16). While federal incentives can help to achieve this goal the outlook for the wind industry depends mainly on its ability to achieve cost reductions (17). The good news is that the industry expects the levelized cost of wind power energy to drop by $20-30 \%$ through 2030 , helping the DOE meet its production goal, though these estimates include significant uncertainty (17). Cost reductions are expected to be lead by technological advances that allow for wind turbine performance / reliability improvements as well as innovations in manufacturing and logistics (17). Pertinent to this work, improved tower designs are expected to reduce the cost of accessing the stronger, less turbulent, wind conditions that are high above the ground (17). 


\section{Matlab Program Overview}

\subsection{Introduction}

As discussed in Chapter 1 the primary product produced by this work is a Matlab program. The program can be used to evaluate coupled tower / rotor response to environmental wind loads, perform system optimization and trade studies, and demonstrate compliance of primary structure to IEC Aeroelastic Load Cases (3) . As discussed in Chapter 1, the program is capable of evaluating multiple tower and turbine configurations. This chapter offers a top level overview of the Matlab program with a focus on user instructions, program inputs and outputs. The theoretical foundation of the program and the methods implemented by the program are detailed in Chapters $3,4,5$, and 6 .

\subsection{Program Execution (User Instructions)}

The following steps must be used to execute the program:

1. Download the digital Matlab Script files accompanying this work.

2. Open Matlab and set the working directory to the folder containing the Run_Tower_Analysis.m script file (note this folder should also have a "utilities" subdirectory which contains various supporting scripts and functions called by Run_Tower_Analysis.m)

3. Complete the User Input spreadsheet (use the digital template provided with this work, snapshots of which are shown in Appendix D). The user inputs are discussed further in Section 2.3.1.

4. Type "Run_Tower_Analysis" into the Matlab Command Window and press enter

5. Follow the on-screen prompts:

a. Select the user input file

b. Select an output directory 
6. The program will begin running. Various status messages are outputted to the Command Window throughout execution. At the completion of each load case simulation plots will appear briefly on the screen and will then be automatically closed.

7. After the program has completed execution navigate to the output directory. The output directory will contain a sub-folder for each load case. Within each load case sub-folder the user will find result plots as well as Excel files with tabulated data.

\subsection{Program Overview}

The Matlab program is organized into several modules that each serve a distinct purpose. Each module, along with a brief summary of the module's function, is detailed in the flow diagram shown in Figure 2.1.

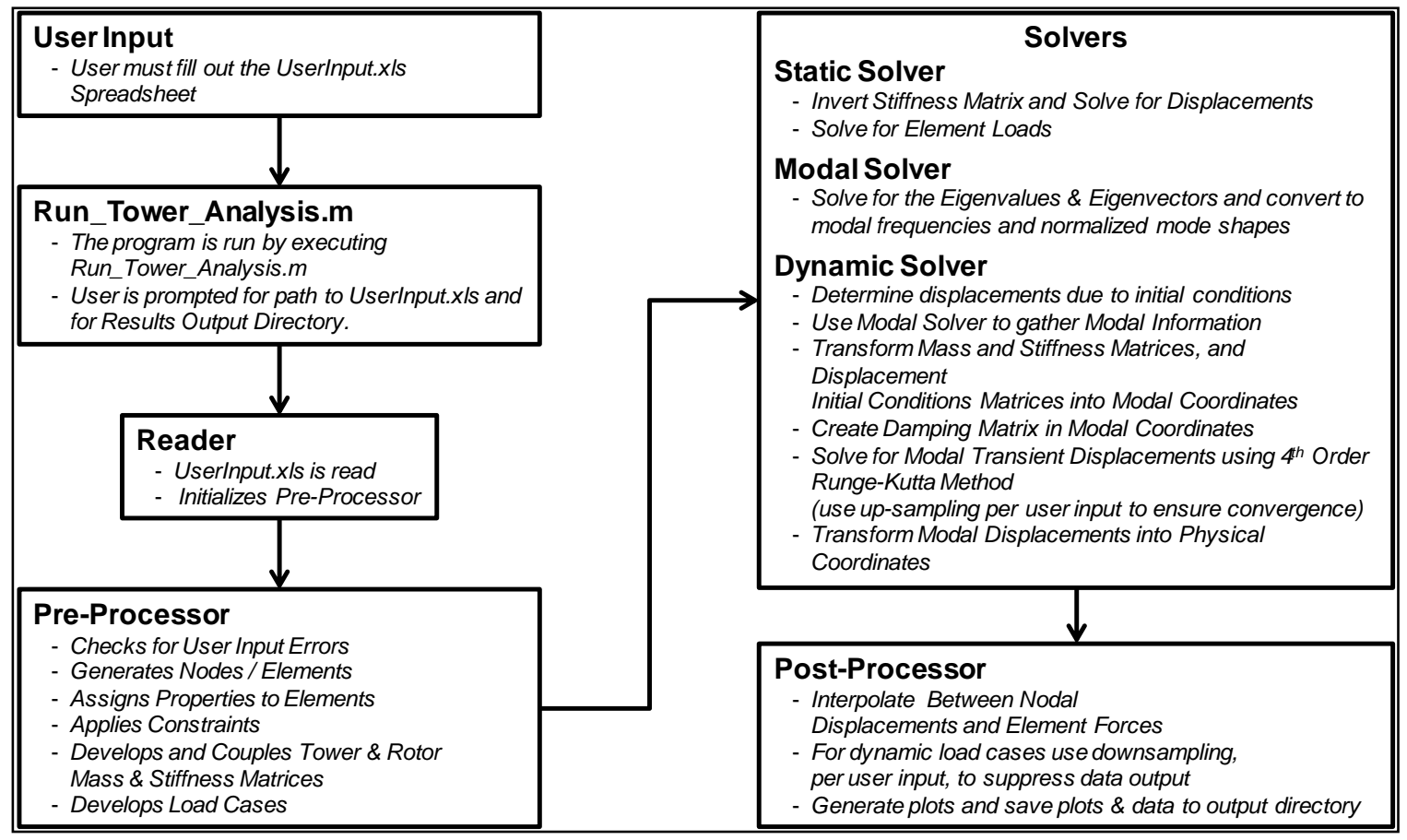

Figure 2.1. Matlab Program Flow Diagram 


\subsubsection{User Input}

There are many parameters that the user must provide to fully define the system being evaluated.

The user inputs are structured through the use of a Microsoft Excel User Input spreadsheet; a template of which is included electronically with this work and is shown in Appendix D. Note that the Appendix D template features the user inputs which correspond with the Case Study (Strong-Axis) discussed further in Chapter 8.

The User Input spreadsheet organizes input into four categories (reflected by the four tabs in the workbook) as listed below. The list below features a brief description of notable user inputs within each category. All user input parameters, along with allowable inputs and units, are defined in detail within the User Input spreadsheet template appearing in Appendix D.

\section{Tower Inputs}
a. Tower Geometry
b. Tower Materials
c. Number of Elements per Tower Section (i.e., "mesh seeding")
d. Tower Boundary Conditions (base support)
e. Auxiliary Support

i. Gin-Pole and Strut Parameters (if applicable)

ii. Guy-Wire Parameters (if applicable)

2. Rotor Inputs

a. User may select to run the analysis with or without a rotor.

i. If a rotor is included in the analysis the user enters parameters for the number of blades (2 or 3), blade weight, blade radius, blade flapwise natural frequency (for both rotating and non-rotating conditions), rated rotational speed, operating rotor speed, hub mass, hub offsets from the tower tip, and turbine projected area. 
ii. If a rotor is not including in the analysis any value entered for hub mass is included as a rigid point mass offset from the tower tip per user inputs.

3. Load Case Inputs

a. User selects IEC (3) Small Wind Turbine Class (1 through 4, see Chapter 5 for further details)

b. The user selects which load cases, all discussed in detail in Chapter 5, to run. At least one load cases must be selected (otherwise no results will be generated). If all load cases are selected the analysis may take several minutes to complete (depending on computer performance).

c. Some load cases (including the Static Tip Load, the Static Nominal Operation, and the Swept Sine load cases) include additional numeric inputs to define load magnitudes, and in the case of the Swept Sine load case, to define starting / ending frequencies and logarithmic sweep rate.

4. Analysis Parameters

a. Dynamic Analysis Parameters

i. User selects the number of modes (n) to include in the Mode Superposition Method, the system modal damping (separate values may be selected for each of the first 10 modes), the time step, Runge-Kutta up-sampling ratio, and results output down-sampling ratio.

b. Aerodynamic Analysis Parameters

i. User enters the tower drag coefficient, the operational rotor thrust coefficient, and the parked rotor drag coefficient.

\subsubsection{Reader Module}

The reader module prompts the user for the user input file and for the output directory. The reader module also reads in the user input file. The reader module is fully contained within the Run_Tower_Analysis.m file. The reader module ends by calling up the Pre-Processor Module. 


\subsubsection{Pre-Processor Module}

The Pre-Processor Module starts off by performing some basic error checking of the user inputs. Nodes and elements are then created and properties are assigned to the elements. Mass and stiffness matrices are then generated for both the tower and the rotor as detailed in Chapters 3 and 4, respectively. The tower and rotor matrices are then combined into the system level mass and stiffness matrices as detailed in Chapter 4. The tower base (and if applicable guy-wire or gin-pole and strut) boundary condition constraints are applied through the use of equation elimination as detailed in Chapter 3. Load cases are developed for each user selected load case, as detailed in Chapter 5. The pre-processor ends by selecting the appropriate solver sequence for each load case.

\subsubsection{Solver Module}

The Solver Module actually consists of three independent modules for static, modal, and dynamic solution sequences. The three solver modules are all similar in that their core function is to solve for system displacements. After solving for system displacements the static and dynamic solver modules then determine forces acting on each tower element (the modal solver does not include this feature).

A brief description of each solver module is included in Figure 2.1. The theory and methodology implemented by each solver module is detailed in Chapter 6 .

\subsubsection{Post-Processor Module}

As is the case with the solver module there are independent modules for post-processing of static, modal, and dynamic data.

The static post-processor module interpolates between nodal displacements by creating five displacement data points between each tower node. The displacement interpolation utilizes the 2D Plane Beam Element shape function, discussed further in Chapter 3, and helps to produce smooth displacement plots. Based on the element forces (produced by the Solution Module) the 
static post-processor then calculates shear force, bending moment, axial force, shear stress, bending stress, axial stress, and von Mises stress acting on each tower node. When applicable, axial force acting on the gin-pole and strut, or on guy-wires, is also determined. The static solver then proceeds with creating displacement and stress plots for the tower and saving them to the user selected output directory. Results are also saved in an Excel file to the output directory.

The modal post-processor includes similar functionality as the static post-processor (except nodal forces / stresses are not determined).

The dynamic solver creates several transient plots to display the wind speed at the tower tip, tower tip deflection, maximum tower von Mises stress and when applicable blade flapwise hinge rotation, gin-pole and strut tension, and guy-wire tension, all as a function of time. The dynamic solver then determines the moments in time when maximum tower von Mises stress occurs and when maximum tower displacement occurs (which are often coincident). The dynamic solver then executes the static solver for these moments in time based on the instantaneous tower displacement and load state (effectively taking two snapshots of tower displacement and loads). 


\section{Tower Modeling}

\subsection{Introduction}

The finite element modeling of the wind turbine tower forms the foundation of this work and the corresponding Matlab tool. The tower modeling approach employs Bernoulli-Euler beam theory and can accommodate any of the following tower configurations:

a) Monopole Towers with Fixed Base

b) Guy-Wires Supported Towers with Fixed or Pinned Base

c) Ginpole and Strut Supported Tower (Cal Poly Wind Turbine Configuration)

This chapter will offer a brief overview of beam theory, element formulation, and tailoring for the three tower configurations listed above.

\subsection{Bernoulli-Euler Beam Theory}

The finite element formulation used in this work is based on Bernoulli-Euler Beam Theory. Bernoulli-Euler Beam Theory is based on a set of simplifying assumptions that allow for a relatively simple differential equation of motion for beam bending. The assumptions are documented in detail in (1) and a brief summary of the more consequential assumptions are listed below:

1. Neutral Axis: There is an axis, running longitudinally down the length of the beam, which does not experience any strain. The neutral axis separates portions of the beam that experience a state of tension from those in compression when under the influence of moment induced bending.

2. Plane Sections Remain Plane: Plane sections of the un-deformed beam which are orthogonal to the neutral axis remain plane, and orthogonal to the neutral axis, as the beam deforms due to loading. 
The key implication of assumption \#2 above is that shear deformation is ignored (plane sections do not remain plane when shear deformation is considered). Wind turbine towers are considered slender beams (length >> width) and their deformation is dominated by bending and shear deformation is negligible. The inclusion of shear deformation in beam formulation is typically only considered when dealing with short stubby beams (length $\approx$ width). Timoshenko Beam Theory includes the influence of shear deformation and is typically used by commercial finite element codes, for example the PBEAM card in NASTRAN defaults to Timoshenko Beam Theory (18). When compared with Timoshenko Beam Theory, Bernoulli-Euler Beam Theory predicts a slightly stiffer beam and slightly higher modal frequencies because shear deformation is not allowed. Again, for wind turbine towers Bernoulli-Euler Beam Theory is appropriate (11) as shear deformation is negligible.

\subsection{Element Formulation}

The formulation used for the tower elements in this work is referred to as a 2D Plane Beam Element (4). Each end (node) of the 2D Plane Beam Element features three degrees of freedom and has axial, shear, and moment bearing capacity. The 2D Beam Element is a combination of the Bar Element (capable of axial load only) and a Simple Plane Beam Element (capable of shear and bending only). In the following sections a brief overview of the process for determining stiffness and mass matrix coefficients will be discuss, the formulation for the Bar Element and Simple Plane Beam Element will be detailed, and finally the 2D Plane Beam Element formulation will be documented.

\subsubsection{Method for Determining Finite Element Matrix Coefficients}

The process for determining the stiffness and mass matrix coefficients of bar and beam finite elements is well documented in many texts including (1) and (4). It would not be value added to detail the full finite element derivation here but rather the goal is to summarize the process and document the final formulation. 
The method for deriving finite element formulation is an extension of the Assumed Modes Method and Lagrange's Equations. The steps of the method are as follows:

1. Shape Functions:

a. Establish a set of admissible shape functions (a linearly independent set of functions that can satisfy the prescribed boundary conditions, see 1.c) that, together, can be used to interpolate the deformed shape of the finite element. The shape functions are assumed modes whose generalized coordinates are simply the displacements / rotations at the element ends (i.e., the element degrees of freedom).

b. The shape functions for the Bar Element are first order polynomials while the shape functions for the Simple Plane Beam Element are third order polynomials.

The polynomial order is established by solving the differential equations of motion for bars / beams loaded only at their ends.

c. To finalize the shape functions a sequence of boundary conditions are used in which a single elemental degree of freedom is set to a unit value and all others are set to zero. The number of boundary condition sets equals the element's number of degrees of freedom and each degree of freedom is modeled with a unit value in a single boundary condition set. Each boundary condition set is associated with a unique shape function. An example set of boundary conditions for one of the Simple Plane Beam Element shape functions is:

i. Translation at Node $1=1$

ii. Rotation at Node $1=0$

iii. Rotation at Node $2=0$

iv. Translation at Node $2=0$

2. Express the element's kinetic and potential energy as a function of the shape functions. 
3. Using the kinetic and potential energy expressions apply Lagrange's equations to determine the equations of motion and sort the coefficients of the generalized coordinates, and time derivatives of the generalized coordinates, into stiffness and mass matrices.

Note that a similar method, also involving Lagrange's equations, is used to determine the stiffness and mass matrices of the rotor model. In the case of the rotor model the full derivation is documented as the approach is unique to this work.

\subsubsection{Bar Element Formulation}

Consider a Bar Element, oriented in the X-Axis, as shown in Figure 3.1. The Bar Element is only capable of accommodating axial load. Using the method summarized in Section 3.3.1 it can be shown (1) that the stiffness $\left(\mathrm{K}_{\mathrm{Bar}}\right)$ and mass $\left(\mathrm{M}_{\mathrm{Bar}}\right)$ matrices of the Bar Element are as shown in Eq. 1 and Eq. 2. Note that this work uses the bar element to simulate guy-wires, ginpoles, and struts.

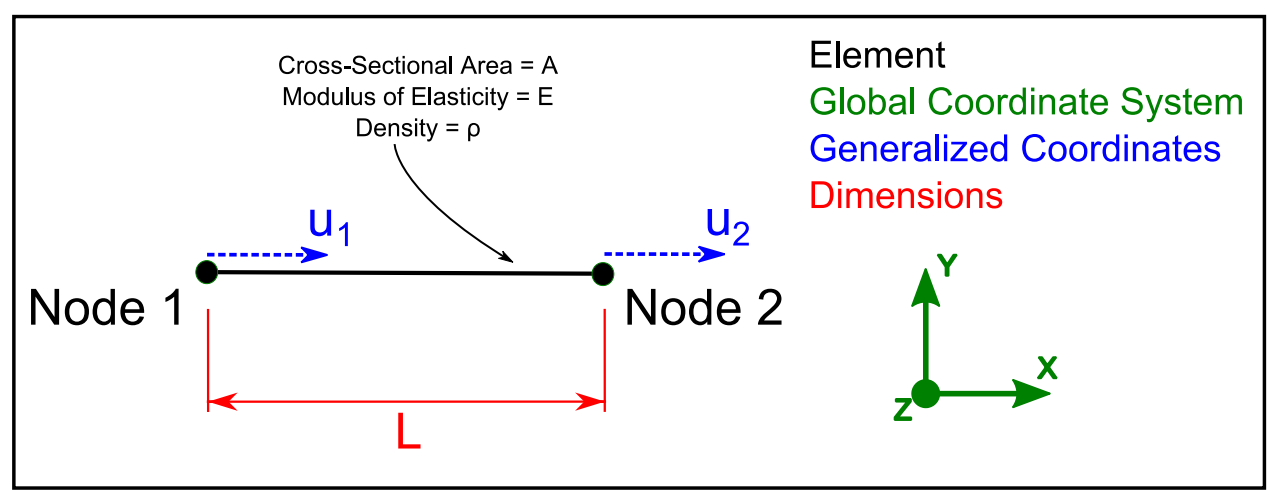

Figure 3.1. Bar Element

$$
\begin{gathered}
\boldsymbol{K}_{\text {Bar }}=\frac{A E}{L}\left[\begin{array}{cc}
1 & -1 \\
-1 & 1
\end{array}\right] \\
\boldsymbol{M}_{\text {Bar }}=\frac{\rho A L}{6}\left[\begin{array}{ll}
2 & 1 \\
1 & 2
\end{array}\right]
\end{gathered}
$$




\subsubsection{Simple Plane Beam Element Formulation}

Consider a Simple Plane Beam Element, oriented in the X-Axis, as shown in Figure 3.2. The Simple Beam Element is only capable of accommodating shear loads and bending moments.

Using the method summarized in Section 3.3.1 it can be shown (1) that the stiffness $\left(\mathrm{K}_{\text {SimpleBeam }}\right)$ and mass $\left(\mathrm{M}_{\text {SimpleBeam }}\right)$ matrices of the Beam Element are as shown in Eq. 3 and Eq. 4.

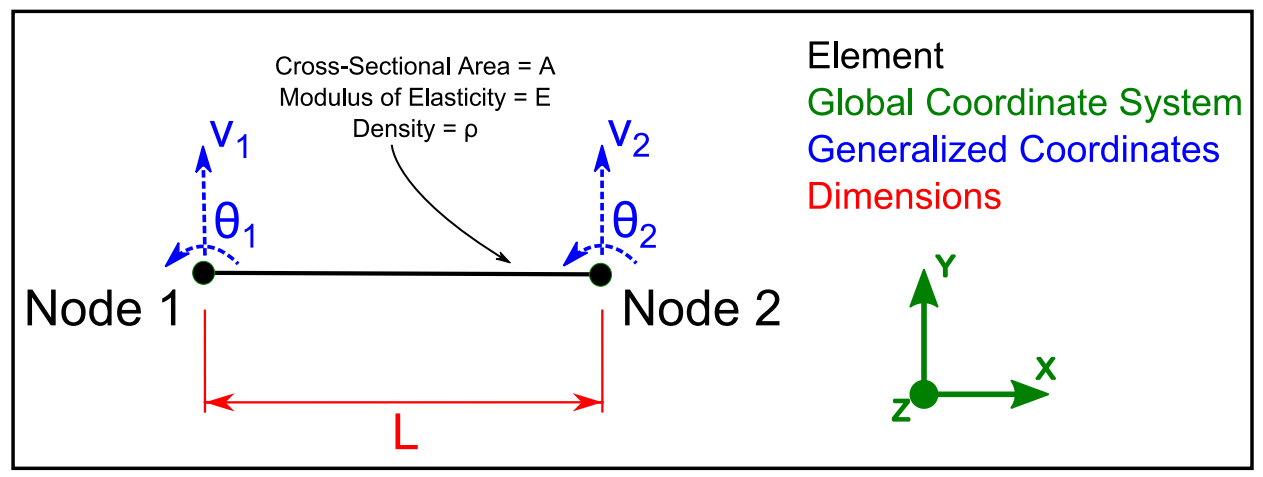

Figure 3.2. Beam Element

$$
\begin{gathered}
K_{\text {SimpleBeam }}=\frac{E I}{L^{3}}\left[\begin{array}{cccc}
12 & 6 L & -12 & 6 L \\
6 L & 4 L^{2} & -6 L & 2 L^{2} \\
-12 & -6 L & 12 & -6 L \\
6 L & 2 L^{2} & -6 L & 4 L^{2}
\end{array}\right] \\
M_{\text {SimpleBeam }}=\frac{\rho A L}{420}\left[\begin{array}{cccc}
156 & 22 L & 54 & -13 L \\
22 L & 4 L^{2} & 13 L & -3 L^{2} \\
54 & 13 L & 156 & -22 L \\
-13 L & -3 L^{2} & -22 L & 4 L^{2}
\end{array}\right]
\end{gathered}
$$

Eq. 4

\subsubsection{D Plane Beam Element Formulation}

By combining the mass and stiffness matrices for the Bar Element and the Simple Beam Element we arrive at the formulation for the 2D Plane Beam Element, shown in Figure 3.3. The 2D Plane Beam Element is used as the finite element for the tower structure in this work. The 2D Plane Beam Element is capable of accommodating axial load, shear load, and bending moments. The stiffness $\left(\mathrm{K}_{2 \mathrm{DBeam}}\right)$ and mass $\left(\mathrm{M}_{2 \mathrm{DBeam}}\right)$ matrices of the 2D Plane Beam Element are as shown in Equations Eq. 5 and Eq. 6. The stiffness and mass matrices can be used to form the Equation of 
Motion of the 2D Plane Beam Element as shown in Eq. 7. Note that Eq. 7 does not include damping terms; damping will be accounted for later and applied to the whole tower/rotor system through modal damping.

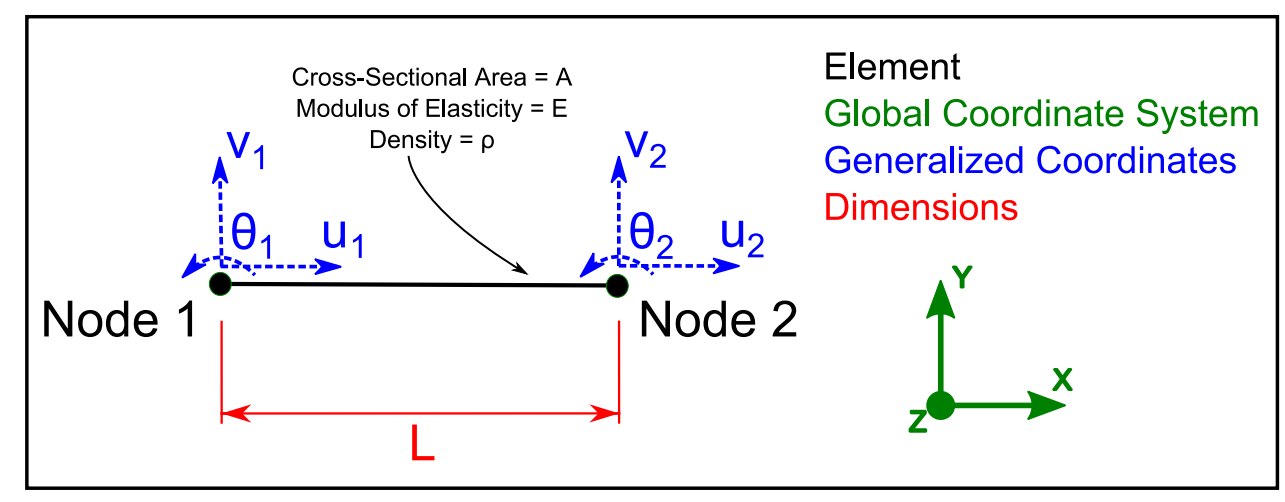

Figure 3.3. 2D Plane Beam Element

$$
\begin{aligned}
& \boldsymbol{K}_{2 D \_B e a m}=\left[\begin{array}{cccccc}
\frac{A E}{L} & 0 & 0 & -\frac{A E}{L} & 0 & 0 \\
0 & \frac{12 E I}{L^{3}} & \frac{6 E I}{L^{2}} & 0 & -\frac{12 E I}{L^{3}} & \frac{6 E I}{L^{2}} \\
0 & \frac{6 E I}{L^{2}} & \frac{4 E I}{L} & 0 & -\frac{6 E I}{L^{2}} & \frac{2 E I}{L} \\
-\frac{A E}{L} & 0 & 0 & \frac{A E}{L} & 0 & 0 \\
0 & -\frac{12 E I}{L^{3}} & -\frac{6 E I}{L^{2}} & 0 & \frac{12 E I}{L^{3}} & -\frac{6 E I}{L^{2}} \\
0 & \frac{6 E I}{L^{2}} & \frac{2 E I}{L} & 0 & -\frac{6 E I}{L^{2}} & \frac{4 E I}{L}
\end{array}\right] \\
& \boldsymbol{M}_{2 D_{\text {B_Beam }}}=\frac{\rho A L}{420}\left[\begin{array}{cccccc}
140 & 0 & 0 & 70 & 0 & 0 \\
0 & 156 & 22 L & 0 & 54 & -13 L \\
0 & 22 L & 4 L^{2} & 0 & 13 L & -3 L^{2} \\
70 & 0 & 0 & 140 & 0 & 0 \\
0 & 54 & 13 L & 0 & 156 & -22 L \\
0 & -13 L & -3 L^{2} & 0 & -22 L & 4 L^{2}
\end{array}\right] \\
& \boldsymbol{M}_{2 D \_B e a m}\left\{\begin{array}{l}
\ddot{u}_{1} \\
\ddot{v}_{1} \\
\ddot{\theta}_{1} \\
\ddot{u}_{2} \\
\ddot{v}_{2} \\
\ddot{\theta}_{2}
\end{array}\right\}+\boldsymbol{K}_{2 D \_B e a m}\left\{\begin{array}{l}
u_{1} \\
v_{1} \\
\theta_{1} \\
u_{2} \\
v_{2} \\
\theta_{2}
\end{array}\right\}=\boldsymbol{F}\left(t, u_{1}, v_{1}, \theta_{1}, u_{2}, v_{2}, \theta_{2}\right)
\end{aligned}
$$




\subsubsection{Tower Discretization}

The 2D Plane Beam Element formulation captured in Eq. 5 through Eq. 7 is for only one element. Any practical tower model will consist of many elements. The Cal Poly Wind Turbine case study, discussed further in Chapter 8, utilized a total of 37 2D Plane Beam Elements in the model of the tower. Appendix C-9 includes a modal convergence study which compares a 37 element model to models containing fewer elements as well as models containing more elements. A 37 element model offers excellent accuracy (modal convergence accuracy up to the $4^{\text {th }}$ mode shape is within $0.25 \%$ Error). A higher fidelity model would have a negligible accuracy benefit with a significant computational penalty.

If an analyst was only interested in simulating a constant cross-section monopole tower under the influence of a static rotor thrust load then a single 2D Plane Beam Element could be used to model the tower. Multiple tower elements are required to account for the following:

1. Tower with Variable Cross-Section:

a. Each element features a constant cross-section so many elements are required to smoothly interpolate the stiffness and mass properties of a tower with a variable cross-section.

2. Distributed Tower Loads, which can be caused by:

a. Aerodynamic drag loads acting on the tower.

b. Inertial loading, which is distributed by nature and influences modal analysis and dynamic transient analysis simulations.

3. Modal Analysis:

a. Modal analysis is limited by element count since the number of system modes will equal the number of unconstrained system degrees-of-freedom. 


\section{Tower Support Interfaces:}

a. Locations where the tower is supported by guy-wires, ginpoles, or struts there must be a element node to attach the supporting member to.

For the reasons listed above it is recommended that a minimum of 30 elements (where each element corresponds to a complete 2D Plane Beam Element) be used to model the tower with a minimum of 3 elements between tower support interfaces. As with any finite element analysis the analyst should incrementally increase mesh density (i.e., element count) until model convergence is achieved. Unnecessarily high mesh density will result in long processing times.

\subsubsection{Tower Element Assembly}

Stiffness and mass matrices are developed for each tower element and then assembled into the total tower stiffness and mass matrices using the following steps:

- Step 1: Stiffness and mass matrices for each element are transformed from their local elemental coordinate systems into the global tower coordinate system.

- Step 2: Individual stiffness and mass matrices are assembled into total tower stiffness and mass matrices by populating an $\mathrm{n} x \mathrm{n}$ matrix, where $\mathrm{n}$ is the number of DOFs in the system, where term $(i, j)$ relates displacements of DOF $i$ to forces acting on DOF $j$, and where term $(i, j)$ in the system matrix is the summation of all elemental matrix $(i, j)$ terms.

To illustrate step 1, consider that the 2D Plane Beam element show in Figure 3.3 is in a horizontal configuration and must be rotated $90^{\circ}$ about the + Z-Axis resulting in the vertical orientation, shown in Figure 3.4, necessary to model the tower. A transformation matrix, $\mathbf{T}$, must be constructed and used to transform the stiffness and mass matrices from their local coordinate systems to the global coordinate system. The general transformation matrix for an arbitrary rotation of $\lambda$ about the $+Z$-Axis, as shown in Figure 3.4, is detailed in Eq. 8 (4). The transformation matrix is applied as shown in Eq. 9 and Eq. 10 to transform the stiffness and mass 
matrices from their local coordinate systems to the global coordinate systems (4). The resulting transformed stiffness and mass matrices are indicated with a $\left(^{\wedge}\right)$ accent.

$$
\begin{gathered}
\boldsymbol{T}=\left[\begin{array}{cccccc}
\cos (\lambda) & \sin (\lambda) & 0 & 0 & 0 & 0 \\
-\sin (\lambda) & \cos (\lambda) & 0 & 0 & 0 & 0 \\
0 & 0 & 1 & 0 & 0 & 0 \\
0 & 0 & 0 & \cos (\lambda) & \sin (\lambda) & 0 \\
0 & 0 & 0 & -\sin (\lambda) & \cos (\lambda) & 0 \\
0 & 0 & 0 & 0 & 0 & 1
\end{array}\right] \\
\widehat{\boldsymbol{K}}_{2 D_{-} \text {Beam }}=[\boldsymbol{T}]^{T}\left[\boldsymbol{K}_{2 D_{-} \text {Beam }}\right][\boldsymbol{T}] \\
\widehat{\boldsymbol{M}}_{2 \text { D_Beam }_{-}}=[\boldsymbol{T}]^{T}\left[\boldsymbol{M}_{2 D_{-} \text {Beam }}\right][\boldsymbol{T}]
\end{gathered}
$$

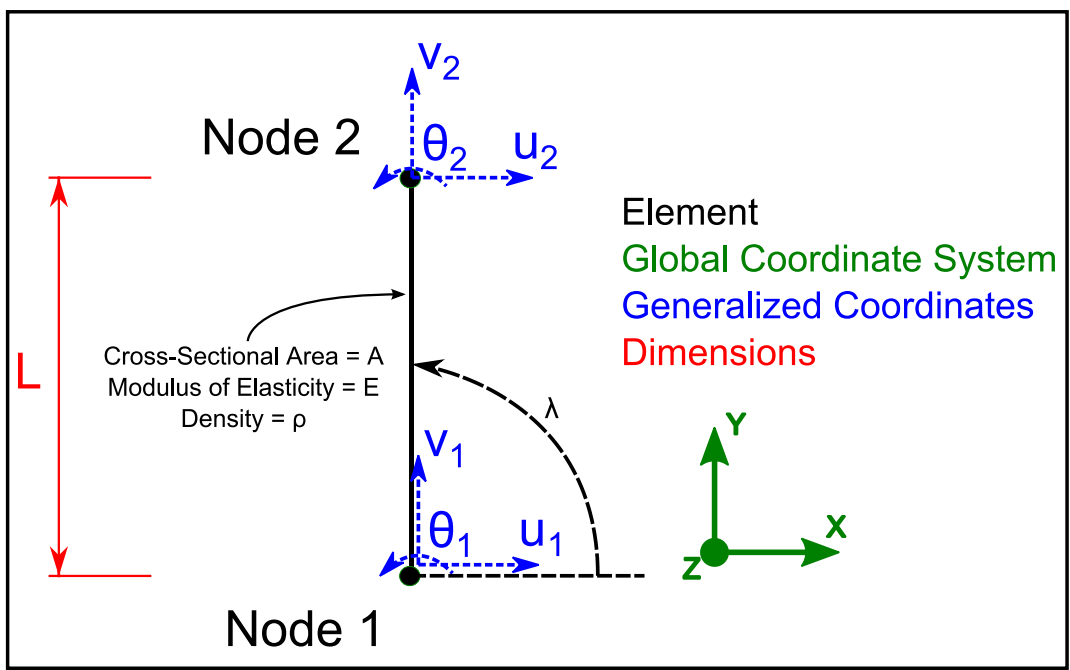

Figure 3.4. $2 D$ Plane Beam Element rotated by $\lambda=90^{\circ}$ into vertical orientation

The Eq. 8 transformation matrix reduces to Eq. 11 for the special case of $\lambda=90^{\circ}$ shown in Figure 3.4 and the resulting transformed stiffness matrix is as shown in Eq. 12 (transformed mass matrix is omitted for brevity) (4).

$$
\boldsymbol{T}_{90^{\circ}}=\left[\begin{array}{cccccc}
0 & 1 & 0 & 0 & 0 & 0 \\
-1 & 0 & 0 & 0 & 0 & 0 \\
0 & 0 & 1 & 0 & 0 & 0 \\
0 & 0 & 0 & 0 & 1 & 0 \\
0 & 0 & 0 & -1 & 0 & 0 \\
0 & 0 & 0 & 0 & 0 & 1
\end{array}\right]
$$




$$
\widehat{\boldsymbol{K}}_{2 D \_B e a m}=\left[\begin{array}{cccccc}
\frac{12 E I}{L^{3}} & 0 & -\frac{6 E I}{L^{2}} & -\frac{12 E I}{L^{3}} & 0 & -\frac{6 E I}{L^{2}} \\
0 & \frac{A E}{L} & 0 & 0 & -\frac{A E}{L} & 0 \\
-\frac{6 E I}{L^{2}} & 0 & \frac{4 E I}{L} & \frac{6 E I}{L^{2}} & 0 & \frac{2 E I}{L} \\
-\frac{12 E I}{L^{3}} & 0 & \frac{6 E I}{L^{2}} & \frac{12 E I}{L^{3}} & 0 & \frac{6 E I}{L^{2}} \\
0 & -\frac{A E}{L} & 0 & 0 & \frac{A E}{L} & 0 \\
-\frac{6 E I}{L^{2}} & 0 & \frac{2 E I}{L} & \frac{6 E I}{L^{2}} & 0 & \frac{4 E I}{L}
\end{array}\right]
$$

To illustrate step 2, the assembly of elemental matrices into the system matrices, consider the simple two element tower configuration shown in Figure 3.5. For this example we will assign the same properties (L, A, E, $\rho$ ) to both Element 1 and 2. Thus the stiffness matrix, in global coordinates, for both elements would be as shown in Eq. 12. As described in step 2, to assemble the two stiffness matrices, terms corresponding to the same degrees-of-freedom are added. The resulting system stiffness matrix for the simple 2 element tower configuration shown in Figure 3.5 is detailed in Eq. 13. The system mass matrix could be found in a similar manner. 


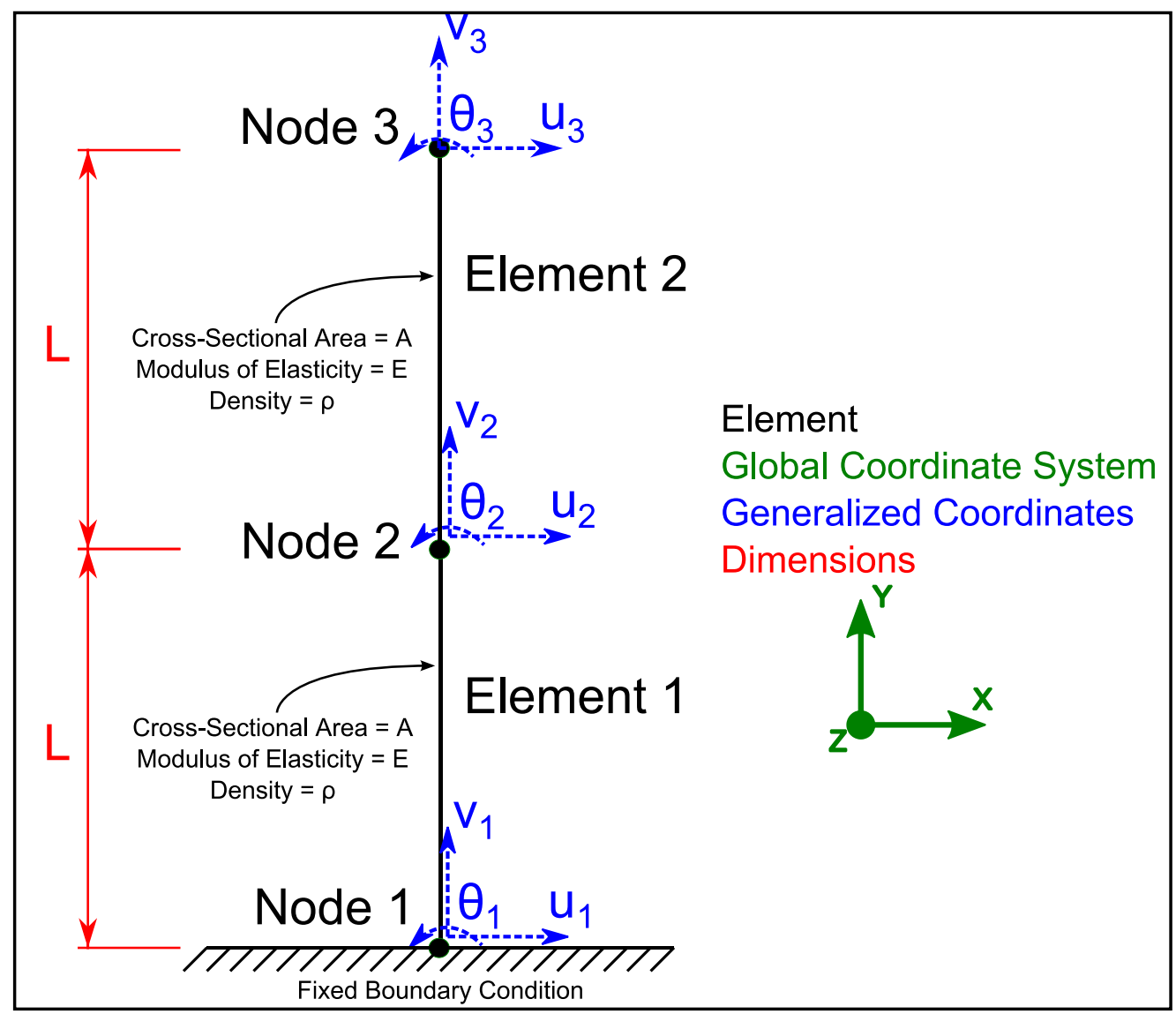

Figure 3.5. Simple 2 Element Tower with Fixed Base

$$
\boldsymbol{K}_{\text {SimpleTower }}=\left[\begin{array}{ccccccccc}
\frac{12 E I}{L^{3}} & 0 & -\frac{6 E I}{L^{2}} & -\frac{12 E I}{L^{3}} & 0 & -\frac{6 E I}{L^{2}} & 0 & 0 & 0 \\
0 & \frac{A E}{L} & 0 & 0 & -\frac{A E}{L} & 0 & 0 & 0 & 0 \\
-\frac{6 E I}{L^{2}} & 0 & \frac{4 E I}{L} & \frac{6 E I}{L^{2}} & 0 & \frac{2 E I}{L} & 0 & 0 & 0 \\
-\frac{12 E I}{L^{3}} & 0 & \frac{6 E I}{L^{2}} & \frac{24 E I}{L^{3}} & 0 & 0 & -\frac{12 E I}{L^{3}} & 0 & -\frac{6 E I}{L^{2}} \\
0 & -\frac{A E}{L} & 0 & 0 & \frac{2 A E}{L} & 0 & 0 & -\frac{A E}{L} & 0 \\
-\frac{6 E I}{L^{2}} & 0 & \frac{2 E I}{L} & 0 & 0 & \frac{8 E I}{L} & \frac{6 E I}{L^{2}} & 0 & \frac{2 E I}{L} \\
0 & 0 & 0 & -\frac{12 E I}{L^{3}} & 0 & \frac{6 E I}{L^{2}} & \frac{12 E I}{L^{3}} & 0 & \frac{6 E I}{L^{2}} \\
0 & 0 & 0 & 0 & -\frac{A E}{L} & 0 & 0 & \frac{A E}{L} & 0 \\
0 & 0 & 0 & -\frac{6 E I}{L^{2}} & 0 & \frac{2 E I}{L} & \frac{6 E I}{L^{2}} & 0 & \frac{4 E I}{L}
\end{array}\right]
$$

Eq. 13 


\subsubsection{Boundary Conditions and Equation Elimination}

It is necessary to have a set of boundary conditions that eliminate rigid body motion of the model; otherwise the stiffness matrix will be singular. By definition, a singular stiffness matrix cannot be inverted thus preventing static analysis. In the case of dynamic analysis the solution sequence, see Section 6.4, can be executed even when a model is under-constrained but, depending on excitation, rigid body motion may result.

Note that in Figure 3.5 the base of the tower, represented by Node 1, features a fully fixed boundary condition. In Eq. 13 all nine (9) degrees-of-freedom in the simple tower model, shown in Figure 3.5, are included in the stiffness matrix. Boundary conditions are applied by eliminating the constrained degrees-of-freedom from the system model. Rows 1 through 3 , as well as columns 1 through 3, of $\mathrm{K}_{\text {SimpleTower }}$ in Eq. 13, are associated with the constrained degrees-offreedom $\mathrm{u}_{1}, \mathrm{v}_{1}$, and $\theta_{1}$ and need to be eliminated to impose the boundary condition. Eliminating the noted rows and columns results in the updated stiffness matrix $\mathrm{K}_{\text {SimpleTowerCon }}$ of Eq. 14 (where Con indicates Constrained). The constrained mass matrix $\mathbf{M}_{\text {SimpleTowerCon }}$ can be found in a similar manner. The resulting system Equations of Motion are detailed in Eq. 15.

$$
\begin{gathered}
\boldsymbol{K}_{\text {SimpleTowercon }}=\left[\begin{array}{cccccc}
\frac{24 E I}{L^{3}} & 0 & 0 & -\frac{12 E I}{L^{3}} & 0 & -\frac{6 E I}{L^{2}} \\
0 & \frac{2 A E}{L} & 0 & 0 & -\frac{A E}{L} & 0 \\
0 & 0 & \frac{8 E I}{L} & \frac{6 E I}{L^{2}} & 0 & \frac{2 E I}{L} \\
-\frac{12 E I}{L^{3}} & 0 & \frac{6 E I}{L^{2}} & \frac{12 E I}{L^{3}} & 0 & \frac{6 E I}{L^{2}} \\
0 & -\frac{A E}{L} & 0 & 0 & \frac{A E}{L} & 0 \\
-\frac{6 E I}{L^{2}} & 0 & \frac{2 E I}{L} & \frac{6 E I}{L^{2}} & 0 & \frac{4 E I}{L}
\end{array}\right] \quad \text { Eq. } 14 \\
\boldsymbol{M}_{\text {SimpleTowerCon }}\left\{\begin{array}{l}
\ddot{u}_{2} \\
\ddot{v}_{2} \\
\ddot{\theta}_{2} \\
\ddot{u}_{3} \\
\ddot{v}_{3} \\
\ddot{\theta}_{3}
\end{array}\right\}+K_{\text {SimpleTowerCon }}\left\{\begin{array}{l}
u_{2} \\
v_{2} \\
\theta_{2} \\
u_{3} \\
v_{3} \\
\theta_{3}
\end{array}\right\}=\boldsymbol{F}\left(t, u_{2}, v_{2}, \theta_{2}, u_{3}, v_{3}, \theta_{3}\right) \text { Eq. } 15
\end{gathered}
$$




\subsection{Tower Configuration Considerations}

The preceding sections of this chapter introduced the general tower modeling methods and element formulation. As mentioned in Section 3.1 the Matlab tool accompanying this work can accommodate the three distinct tower configurations shown in Figure 3.6. Special considerations are required for each of the three tower configurations. The special considerations include element type, boundary conditions, and tower coordinate systems.

Note that the coordinate system in Figure 3.6 is the rotor coordinate system and is attached to the rotor such that the $\mathrm{X}$-Axis always points downwind, the Z-Axis always points up, and the Y-Axis follows the right-hand rule (i.e., $\mathrm{Y}=\mathrm{Z} \times \mathrm{X}$ ).

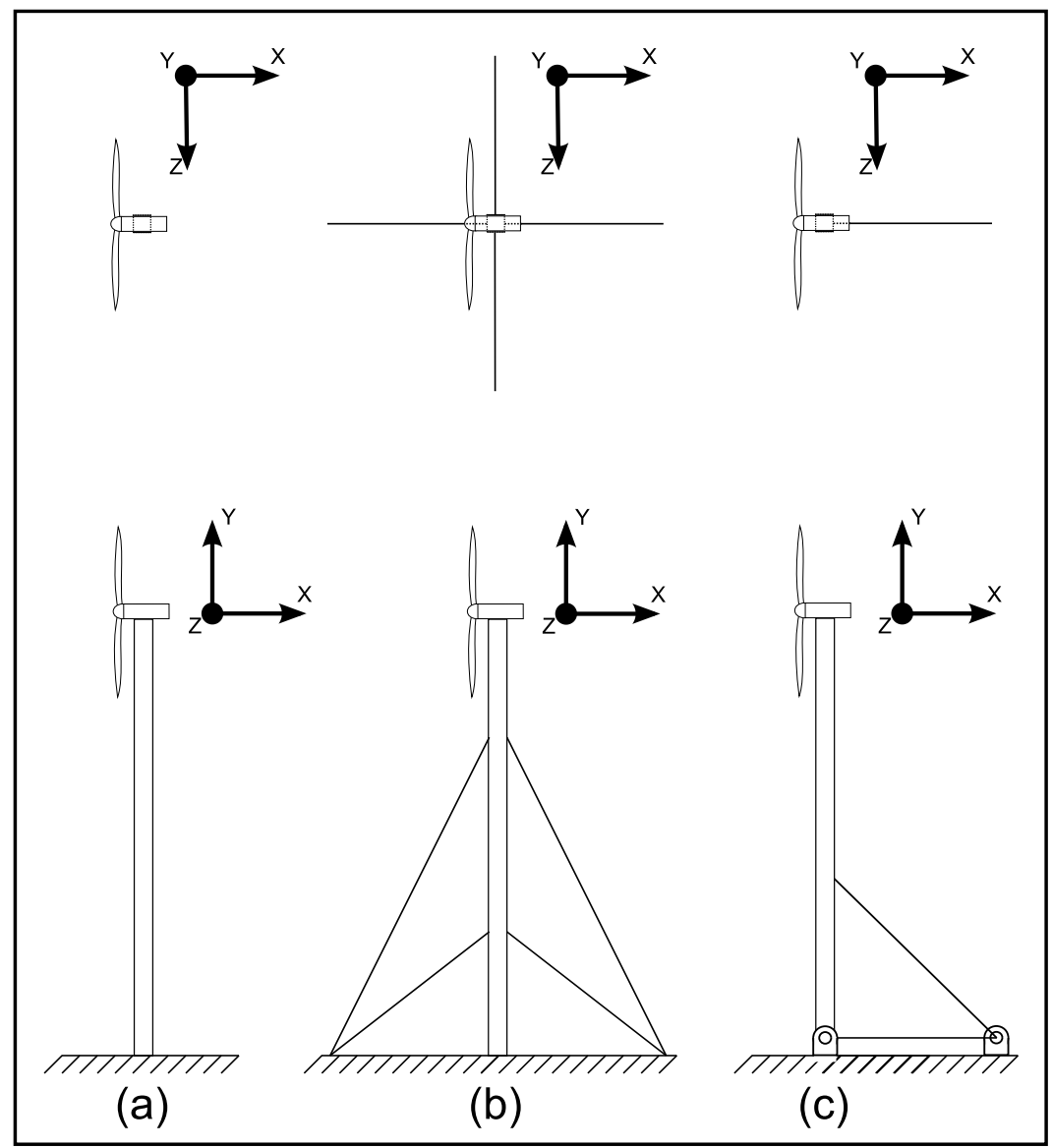

Figure 3.6. Tower Configurations, a) Monopole Tower, b) Guy-Wire Supported Tower,

c) Ginpole and Strut Supported Tower (Cal Poly Wind Turbine Configuration) 


\subsubsection{Monopole Tower Modeling Details}

Monopole tower modeling special considerations are as follows:

i. Element Type:

a. 2D Plane Elements are used for the tower

ii. Boundary Condition:

a. A fully fixed boundary condition is imposed on the tower base (i.e., translations and rotations are constrained).

iii. Tower Coordinate System:

a. No special tower coordinate system is required since the tower features rotational symmetry. The results of the tower analysis are in the rotor coordinate system.

\subsubsection{Guy-Wire Supported Tower Modeling Details}

Guy-wire supported tower modeling special considerations are as follows:

i. Guy-Wire Configuration

a. The Matlab tool can evaluate towers supported by up to 10 levels of guy-wires where each level consists of a total of four guy-wires with $90^{\circ}$ clocking. A four guy-wire support configuration is common for small wind turbine towers as it allows for tower erection using the gin-pole method.

Note: The tool is not currently configured to perform direct simulation of towers supported by a three guy-wire configuration with $120^{\circ}$ clocking. However, the tool may be used to perform an indirect simulation of a tower supported by a three guy-wire configuration by the use of conversion factors. The approach is based on the observation, which can be demonstrated through the use of Castigliano's Theorem (19), that the stiffness provided by a three guy-wire support is $75 \%$ of that provided by an equivalent four guy-wire support. Furthermore the maximum guy-wire tensile load for a three guy- 
wire support is $1 \frac{1}{3}$ times that of an equivalent four guy-wire support when acted upon by the same force profile. As such, a tower supported by a three guy-wire configuration may be indirectly simulated by multiplying the guy-wire Young's Modulus user input by a factor of 0.75 and subsequently multiplying the resulting maximum guy-wire live tensile load (the tensile load in excess of the pre-tension) by a factor of $1 \frac{1}{3}$.

ii. Element Type:

a. 2D Plane Elements are used for the tower.

b. The guy-wires are each modeled using a single bar element capable of supporting only axial loads. As discussed further in Chapter 6 a preload is applied to the guy-wires. Since this work performs a linear elastic analysis (i.e., it cannot handle the non-linear effects of slack guy-wire) the preload must be sufficient to maintain positive tension on all guywires for all load cases (this is also good operating practice).

iii. Boundary Condition:

a. The analyst may select a fixed or pinned boundary condition for the tower base.

i. Note that a pinned boundary condition constrains only node translations but not rotations.

b. The guy-wire interface to the ground is modeled as a pinned boundary condition.

c. Each guy-wire interface to the tower is modeled as pinned.

iv. Tower Coordinate System:

a. The tower coordinate system is the same as the rotor coordinate system with tower oriented such that two guy-wires sets are in the XY-Plane and the other two guy-wire sets are in the YZ-Plane, as shown in Figure 3.6.

i. For wind loading in the X-Axis two guy-wires will be actively loaded while the other two are not. 
ii. As shown in Appendix C-10 the effective stiffness of the four guy-wire configuration with $90^{\circ}$ clocking, as shown in Figure 3.6, is the same regardless of loading orientation in the YZ-Plane. Note that this is also true for a three guy-wire configuration.

iii. It follows from item iv.a.i that the simulated configuration will produce guy-wire loads that will bound loads produced by YZ-Plane loading in any orientation.

iv. It follows from item iv.a.ii that the simulated configuration will produce tower displacements, and loads, that are representative of YZ-Plane loading in any orientation.

\subsubsection{Ginpole and Strut Supported Tower Modeling Details}

Ginpole and Strut supported tower modeling special considerations are as follows:

i. Ginpole and Strut Configuration

a. The Matlab tool can evaluate towers supported by a single ginpole and strut set.

ii. Element Type:

a. 2D Plane Elements are used for the tower.

b. The ginpole and strut are each modeled using a single bar element capable of supporting only axial loads. Section 7.4.2 discusses and justifies the omission of ginpole and strut moment baring capacity.

iii. Boundary Condition:

a. The analyst may select a fixed or pinned boundary condition for the tower base.

b. The ginpole and strut interface to the ground is modeled as a pinned boundary condition.

c. The ginpole and strut interface to the tower is modeled as pinned. 
iv. Tower Coordinate System:

a. The system stiffness for a tower supported by a ginpole and strut configuration is asymmetric. Thus a tower specific coordinate system must be generated $\left(\mathrm{X}_{\mathrm{T}} \mathrm{Y}_{\mathrm{T}} \mathrm{Z}_{\mathrm{T}}\right)$ where the $\mathrm{Y}_{\mathrm{T}}$-Axis is aligned with the rotor $\mathrm{Y}$-Axis (i.e., upward) and the angle between the tower $\mathrm{X}_{\mathrm{T}}$-Axis and the rotor $\mathrm{X}$-Axis is defined as angle $\xi$ (yaw angle with respect to the Strong-Axis), as shown in Figure 3.7. The tower analysis should be performed for the following orientations:

i. Strong Axis: The Strong Axis is defined as $\xi=0$ and is shown in Figure 3.7a. This orientation is referred to as the Strong Axis because the strut provides its maximum stiffening effect to the tower in this orientation.

ii. Weak Axis: The Weak Axis is defined as $\xi=90^{\circ}$ and is shown in Figure 3.7c. This orientation is referred to as the Weak Axis because the strut provides no stiffening effect to the tower in this orientation and all wind induced load is reacted out at the tower's base.

b. Intermediate Axes: Intermediate Axes are any orientation between the Strong Axis and the Weak Axis (i.e., $0<\xi<90^{\circ}$ ). The strut provides some limited stiffening effect to the tower in the Intermediate Axes. An independent Matlab simulation is not conducted for the Intermediate Axes but rather the analyst can deduce the tower deflections and loads based on scaled load superposition using Eq. 16 where "Result" is any deflection or load parameter of interest. For example, as discussed in Chapter 8, the maximum tower tip deflection due to Lad Case A (2100 lbf thrust load) is 41.77 in for the Strong Axis and 69.45 in for the Weak Axis, for an Intermediate Axis of $\xi=45^{\circ}$ the tower tip deflection would be 57.31 in.

$$
\text { Result }_{\text {Intermediate }}=\sqrt{\left(\cos (\xi) \cdot \text { Result }_{\text {Strong }}\right)^{2}+\left(\sin (\xi) \cdot \text { Result }_{\text {Weak }}\right)^{2}} \quad \text { Eq. } 16
$$



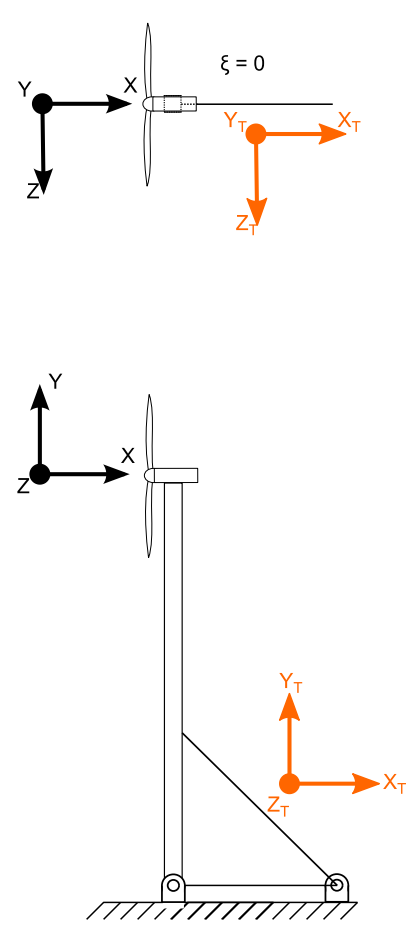

(a)
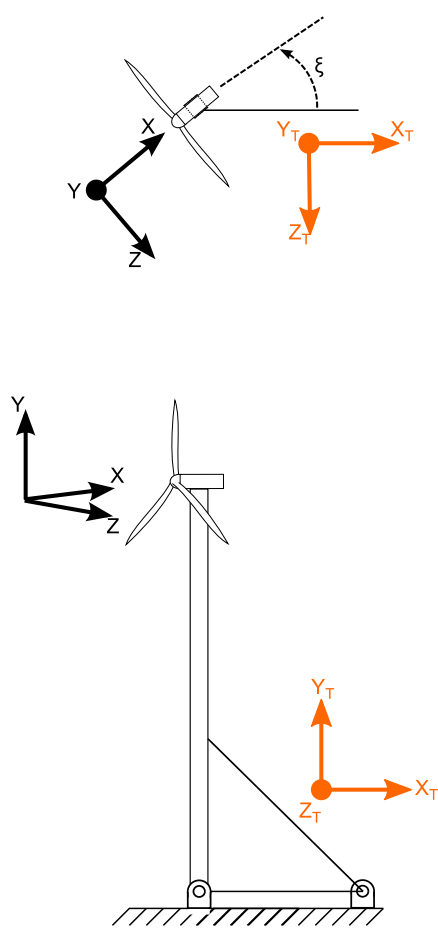

(b)
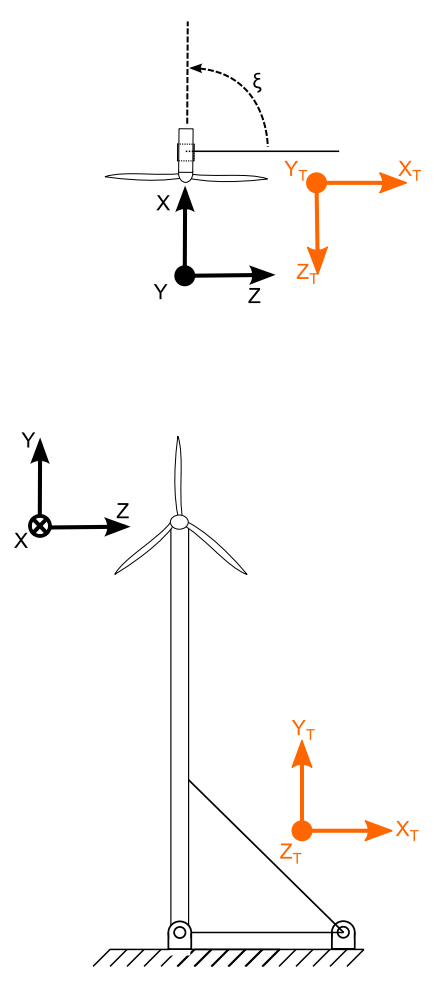

(c)

X-Axis Points Donwind for all Configurations

Figure 3.7. Tower Coordinates for Ginpole and Strut Supported Tower

a) Strong Axis $(\xi=0)$, b), Intermediate $\left(0<\xi<90^{\circ}\right)$,

c) Weak Axis $\left.\left(\xi=90^{\circ}\right), b\right)$ 


\section{Rotor Modeling}

\subsection{Introduction}

A wind turbine tower sees many complex loads, many of which are a result of the dynamic interaction between the tower and the rotor. It is conventional wisdom to design the tower and rotor such that the lowest natural frequencies, typically the first 3-4, of the subsystems do not overlap. If the tower and rotor exhibit natural frequencies which are sufficiently spaced it is common to model the rotor as simply a point mass when analyzing the tower (20). Although it can often be shown that this approximation is appropriate for a given system, it would be overly constraining to limit a tool, intended to assist the designer, to this approximation. Modern wind turbines often feature rotor passing frequencies and rotor flapping modes that approach tower modes so understanding this interaction is paramount to the designer (11). One of the goals of the present work is to provide a tool which is flexible enough to be effective in a variety of designs, namely designs in which a natural frequency of the tower approaches the fundamental frequencies of the rotor. In order to be effective in these situations, a first order approximation of the rotor's modal characteristics must be developed and coupled in with the tower mode. The present work uses the hinge offset equivalent blade theory as presented by Eggleston (2) as the foundation of the rotor model.

This chapter will focus on the theory of the hinge offset equivalent blade and the method used to couple the rotor model to the tower. We will begin with an overview of the hinge offset equivalent blade theory covered by Eggleston. Then, through the use of a 2 bladed Simplified Rotor Model, the process for developing the blade Equations of Motion (EOM) and coupling the blade models to the tower model will be presented in detail. This process will then be generalized and applied to the development of the Generalized Rotor Model. The resulting rotor model 
effectively couples the EOM for a two or three bladed rotor with the EOM for the tower. The final model allows the designer to evaluate the system at any user defined rotor speed.

\subsection{Hinge Offset Equivalent Blade Theory}

The basic premise of the hinge offset equivalent blade theory, sometimes referred to as the linearized hinge spring blade rotor model (11), is to reduce a blade with complex geometries and with infinite natural frequencies to a blade modeled with a constant cross section and only one Degree of Freedom (DOF).

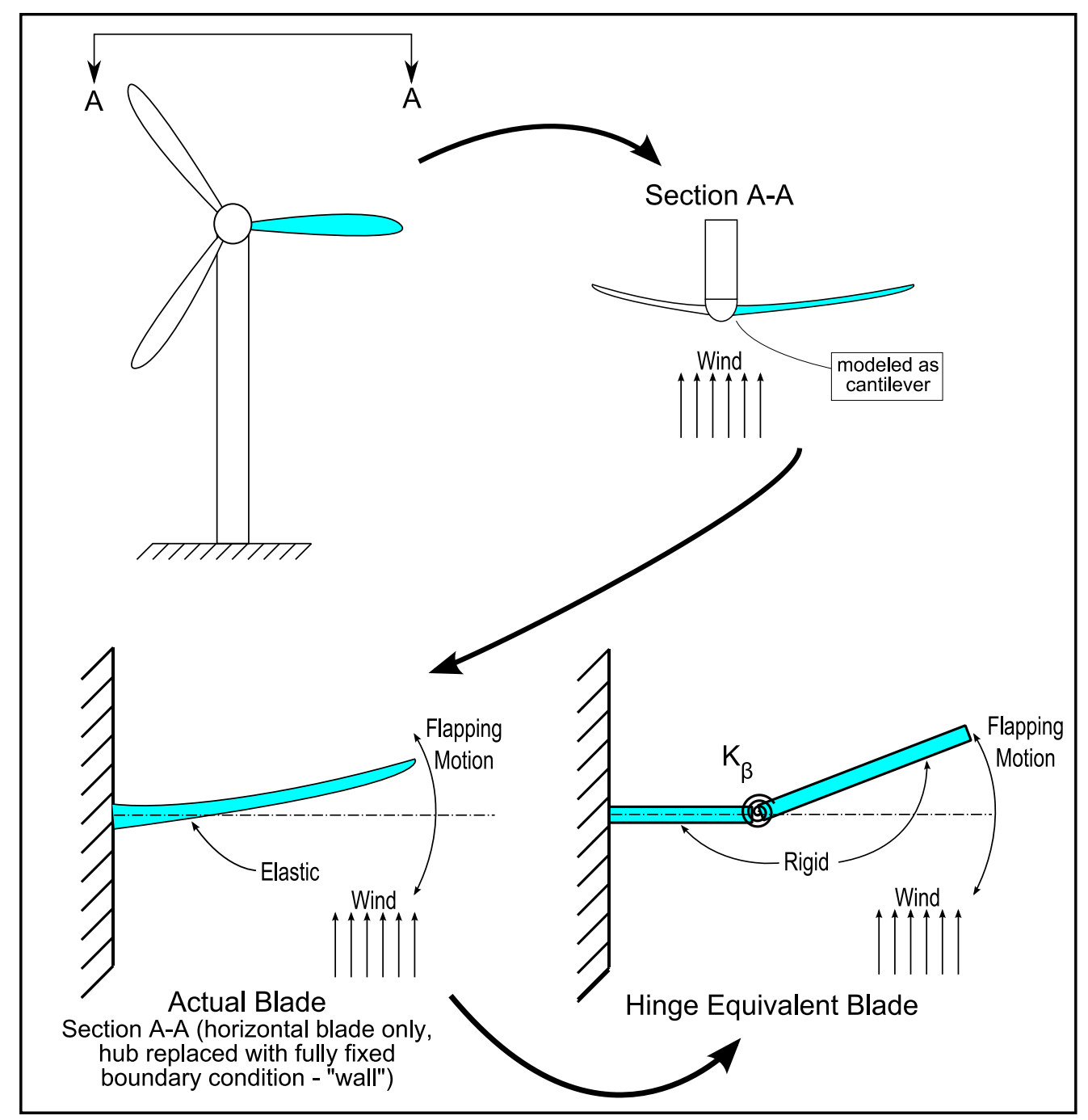

Figure 4.1. A wind turbine blade with complex geometry and the corresponding hinge equivalent blade model. 
Although this model may appear to be oversimplified, it has been used with great success in the past for the evaluation of helicopter blades (2). A certain level of uncertainty must be accepted with any type of analysis and this is especially true when considering the structural dynamics of wind turbine blades. Aside from their highly irregular geometry and nonisotropic composite materials, the aerodynamic loading that wind turbine blades are exposed to is by nature, unsteady, nonlinear, and aperiodic (2). It is commonly suggested that given the uncertainties involved with approximating the loading it would be unreasonable to allocate a large amount of resources to the rotor's structural model and that a first order approximation of the structural characteristics is acceptable. As such, the hinge offset blade model was chosen for the present work because of its proven record, prevalence in literature, and because its relatively simple formulation lends itself well to gaining an understanding of the underlining physical principles which influence the rotor dynamics. Additionally, the solutions of the model are algebraic and closed form. The simplified formulation greatly eases the process of coupling the mathematical rotor terms with the tower terms.

\subsubsection{Assumptions of the Hinge Offset Equivalent Blade Theory}

There are several assumptions involved with the hinge offset blade model, these assumptions are clearly laid out in Manwell's et al. (11) summary of Eggleston's work and are as follows:

- The blade's cross section, material, and mass properties are uniform from root to tip

- The blade, with the exception of the hinge, is rigid

- The blade hinge may be offset from the root

- The blade interface to the hinge is modeled as cantilever

- The rotor's angular velocity is constant, i.e., no angular acceleration 


\subsubsection{Considered Blade Motions}

The hinge offset blade model allows for up to three blade degrees of freedom. The directions in which the degrees of freedom allow for blade motions are as follows:

\section{Flapwise \\ 2. Lead-lag \\ 3. Torsion}

The present work only considers the flapwise direction; blade motion parallel to the axis of rotation as well as the primary wind direction. A horizontal wind turbine typically features a blade geometry which results in maximum blade flexibility in the flapwise direction, thus the fundamental mode of the blade will typically be dominated by deflection in the flapwise direction. Furthermore, it is the excitation of the fundamental flapwise blade mode that will create hub loads which are parallel to the rotor thrust force. This combination of hub loads, along with tower drag, will result in highest tower stresses. The present work is focused on tower analysis, and therefore, it is appropriate to limit the rotor modeling to the rotor characteristics most influential to tower design and analysis.

Motion in the lead-lag direction lies in the plane of rotation and is defined as a perturbation of the blade's motion from its steady state angular velocity and limited to the direction of the angular velocity. This motion will influence the torque applied to the drive train and the generator. Vibration in the lead-lag direction will produce tower loads, but unlike flapwise vibration, these loads will produce tip moments which will NOT combine with trust loads to produce critical tower load cases (as the overturning tower tip moment caused by lead-lag blade motion is orthogonal to the overturning moment caused by rotor trust loads). Lead-lag blade vibration is typically considered when sizing drive train components. 
Finally, the torsional DOF pertains to the blade twisting about its longitudinal axis, the axis connecting the blade's root and tip. This motion will not produce significant hub loads and is typically only considered in detail when sizing the components for the pitch control system used on many utility scale wind turbines (11).

\subsubsection{Formulation}

The hinge equivalent blade models the wind turbine blade, which likely features a variable cross section and non homogeneous material properties, as a straight and rigid cantilevered beam with a constant cross-section and constant material and mass properties. The flexibility of the blade is modeled with a single spring hinge, which in the case of the present work, only allows for flexion in the flapwise direction. The hinge is offset from the axis of rotation, this along with a variable spring and moments of inertia allow the model to account for centrifugal stiffening. Centrifugal stiffening occurs when a beam rotates about some axis and the resulting centrifugal forces stiffen the blade, this causes the natural frequency of the blade to increase.

We will now take a detailed look at the formulation of the hinged equivalent blade by summarizing the related work done by Eggleston (2). Many of the following equations are borrowed directly from Eggleston's work and are presented for completeness and the convenience of the reader.

The first step will be to relate the mass properties of the hinge equivalent blade model to the mass and geometric properties of the actual blade. The total radius, $\mathrm{R}$, of the hinged blade and the actual blade will be the same, as well as the total mass, $\mathrm{M}_{\mathrm{b}}$. The hinged blade has homogeneous mass distribution from root to tip. 


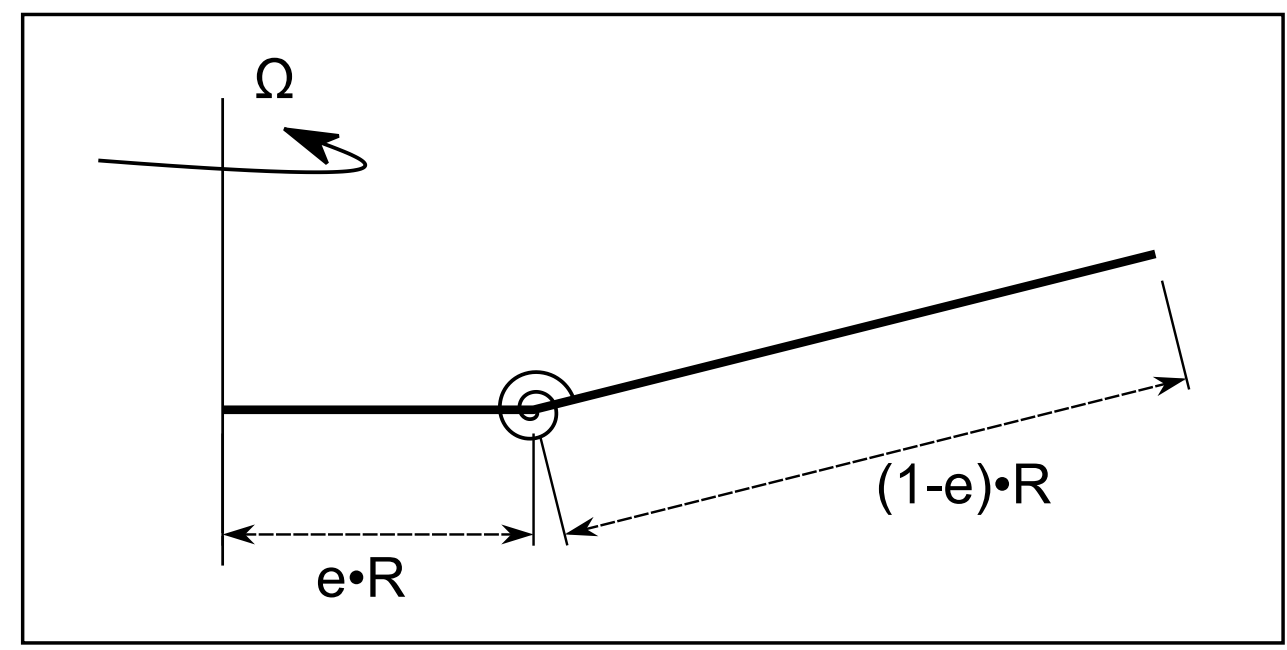

Figure 4.2. Equivalent Hinge Blade Model

To determine the mass moment of inertia of the hinged portion of the blade, about the hinge, we integrate starting at the hinge and continuing through the tip.

$$
I_{b}=\int_{0}^{R(1-e)} r^{2} d m
$$

Again, the mass distribution is constant so a simple relationship can be made to relate the differential mass unit to the differential spacial unit.

$$
d m=\frac{M_{b}}{R} d r
$$

Substituting Eq. 18 into Eq. 17 allows for integration over a spacial differential.

$$
\begin{gathered}
I_{b}=\frac{M_{b}}{R} \int_{0}^{R(1-e)} r^{2} d r \\
I_{b}=\frac{M_{b} R^{2}}{3}(1-e)^{3}
\end{gathered}
$$


The torsional spring at the hinge is assigned a constant stiffness value of $K_{\beta}$. Note that thus far the system is a 1 DOF system with a natural frequency of:

$$
\omega_{N R}=\sqrt{\frac{K_{\beta}}{I_{b}}}
$$

The natural frequency in Eq. 21 is given the subscript NR to emphasize that this is the natural frequency of the blade for its Non Rotating state. No centrifugal stiffening has yet been accounted for.

With the basic dynamic characteristics of the non-rotating hinged blade solved for, we now switch our attention to the rotating case. We will assume that the rotational speed of the rotor is a constant, $\Omega$. The centrifugal forces acting on the hinged portion of the blade create a stiffening effect on the blade, i.e., the forces act to straighten the blade. The effect of these forces will be determined by performing an energy balance. As a result of centrifugal stiffening we will find that the rotating natural frequency, $\omega_{\mathrm{R}}$, will be higher than the non-rotating natural frequency and will be of the following form:

$$
\omega_{R}^{2}=\omega_{N R}^{2}+\alpha \Omega^{2}
$$

Where $\sqrt{\alpha \Omega^{2}}$ is the natural frequency of the hinged blade when the only restoring force is the centrifugal force caused by the rotor rotation, $\Omega$.

To continue we must consider the potential and kinetic energy of a differential mass located on the hinged portion of the blade. In Figure $4.3 \mathrm{dm}$ is located a total distance of $e R+r \cdot \cos \beta \rightarrow \mathrm{r}^{\prime}$ from the rotational axis, about which the blade rotates at an angular velocity of $\Omega$. The mass, $\mathrm{dm}$, is acted upon by a centrifugal force of magnitude: $d m \cdot \Omega^{2} \cdot \mathrm{r}^{\prime}$. 


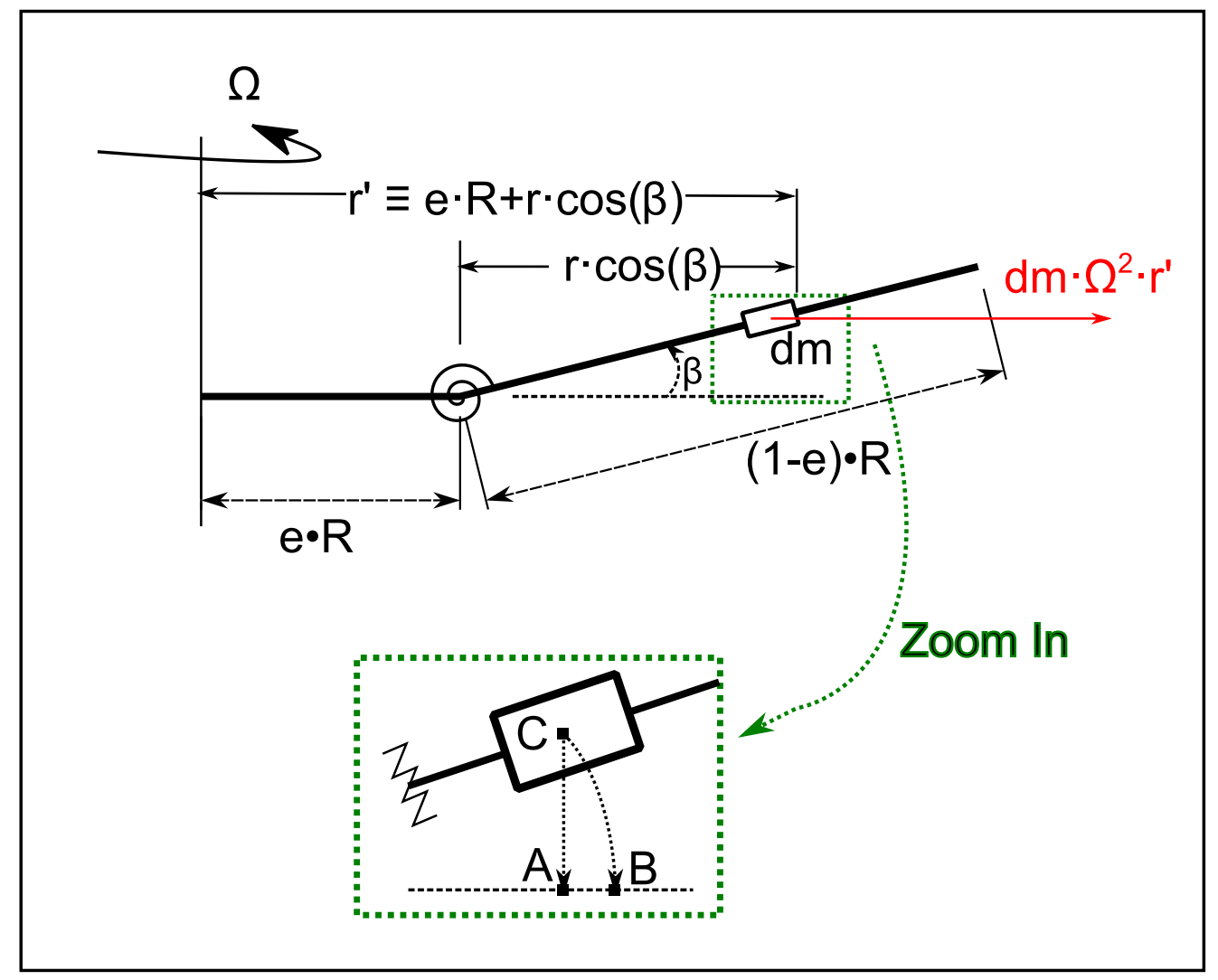

Figure 4.3. Hinge equivalent rotor blade with centrifugal force and dimensions. Distance $R$ is the total length of the blade and distance $r$ is the distance from the hinge to the differential mass, dm.

As the blade oscillates, the differential mass moves from $\mathrm{C}$ to $\mathrm{B}$, its horizontal position varies between $\mathrm{A}$ and $\mathrm{B}$, and the vertical position varies between $\mathrm{C}$ and $\mathrm{A}$. The change in energy state during oscillation is fundamental to the derivation of the hinge equivalent rotor blade model and is discussed below.

An energy balance is used to determine the effects of centrifugal stiffening and the $\alpha$ value in Eq.

22. The energy balance will only consider energies associated with $\mathrm{dm}$, which are:

- The potential energy due to the centrifugal force action on dm (this force attempts to straighten the blade).

- The kinetic energy resulting from oscillator movement driven by the centrifugal force. 
The following energies will be omitted:

- The potential energy due to the spring hinge strain (the influence of the spring hinge is captured in the $\omega_{N R}^{2}$ term of Eq. 22).

- The kinetic energy due to the rotation of the blade about the hub (this omission is discussed further in Section 4.4.3).

When the hinged portion of the blade is at its maximum deflection in its oscillatory path, the horizontal position of $\mathrm{dm}$ is at A. At this location $\mathrm{dm}$ only has potential energy due to the centrifugal force. As the position of dm approaches B its velocity increases as the potential energy transforms into kinetic energy. For now we ignore any damping effects and assume that no energy is dissipated from the system. Thus, due to conservation of energy, the energy of $\mathrm{dm}$ is the same when its horizontal position is at $\mathrm{A}$ or $\mathrm{B}$, or any position in between. By equating the two energy states we are able to solve for $\alpha$ in Eq. 22 .

Through simple geometry we find the distance $\mathrm{AB}$ is:

$$
A B=r-r \cdot \cos (\beta)
$$

The $\cos (\beta)$ term in Eq. 23 may be approximated by the first two terms of its Taylor Series expansion $1-\beta^{2} / 2$. Substituting this into Eq. 23 gives.

$$
A B=r\left(\frac{\beta^{2}}{2}\right)
$$

Potential energy may be simply expressed as the product of a force and the distance the force acts over. It follows that the potential energy, $\triangle \mathrm{PE}$, of mass $\mathrm{dm}$ at location $\mathrm{A}$ is:

$$
\Delta P E=\left(\Omega^{2} r^{\prime} d m\right) \cdot r\left(\frac{\beta^{2}}{2}\right)
$$


Integrating Eq. 25, as follows, from the location of the hinge to the tip, a distance of $R(1-e)$, results in the potential energy of the hinged potion of the blade when at its peak of oscillation.

$$
P E=\int_{0}^{R(1-e)} \Omega^{2} \mathrm{r}^{\prime} \cdot \mathrm{r}\left(\frac{\beta^{2}}{2}\right) \mathrm{dm}
$$

The relationship from Eq. 18 is used to transform the above integration into integration across spacial coordinates as follows.

$$
P E=\frac{M_{b}}{R} \int_{0}^{R(1-e)} \Omega^{2} \mathrm{r}^{\prime} \cdot \mathrm{r}\left(\frac{\beta^{2}}{2}\right) \mathrm{dr}
$$

Now r' is written in its expanded form as seen in Figure 4.3.

$$
P E=\frac{M_{b}}{R} \int_{0}^{R(1-e)} \Omega^{2}(\mathrm{eR}+\mathrm{rcos}(\beta)) \cdot \mathrm{r}\left(\frac{\beta^{2}}{2}\right) \mathrm{dr}
$$

Executing the integration and simplifying Eq. 28 results in the following expression for the Potential Energy of the hinged portion of the blade when at its peak of oscillation, location $\mathrm{C}$ in Figure 4.3. Note the use of Eq. 20 in the following equation.

$$
P E=\frac{1}{4} \Omega^{2} \beta^{2} \mathrm{M}_{\mathrm{b}} \mathrm{R}^{2} \mathrm{e}(1-\mathrm{e})^{2}+\frac{1}{2} \Omega^{2} \beta^{2} \mathrm{I}_{\mathrm{b}}
$$

We now proceed to determine the Kinetic Energy of the differential mass, dm, when it is at position B. The Kinetic Energy of the mass at any point in time is:

$$
\Delta K E=\frac{1}{2} d m \cdot V_{d m}^{2}
$$


Where $V_{d m}$ in Eq. 30 is the instantaneous velocity of mass $\mathrm{dm}$ as it passes through point B. Recall that the centrifugal force acts as a restoring force and allows for the oscillation of the hinged portion of the blade. Let the natural frequency of the hinged portion of the blade, with the centrifugal force acting as the only restoring force, be a constant: $\omega_{c f}$. The velocity of dm, $V_{d m}$, as it passes through point $\mathrm{B}$ is $\mathrm{AC} \omega_{c f}$. The magnitude of this velocity may not be intuitive and warrants further explanation. Recall that the position of a simple 1 DOF oscillating system is frequently expressed as $u(t)=u_{o} \sin \left(\omega_{c f} t\right),(1)$. Where $\mathrm{u}$ is some spacial coordinate system, $\mathrm{u}_{\mathrm{o}}$ is the amplitude of oscillation, and $\omega$ is the natural frequency. To find the velocity of this simplified example we take the time derivative of the position as follows:

$v(t)=\frac{d u(t)}{d t}=u_{o} \omega_{c f} \cdot \cos \left(\omega_{c f} t\right)$. Here we can clearly see that the maximum velocity is the amplitude $\left(u_{o}\right)$ times the natural frequency $\omega_{c f}$. In the hinged blade model the amplitude in ydirection as shown in Figure 4.3 is AC. Therefore, as with the preceding simplified example, the velocity of $\mathrm{dm}$ at position $\mathrm{B}$ is the amplitude times the frequency: $A C \omega_{c f}$. Substituting this velocity term into Eq. 30 gives:

$$
\Delta K E=\frac{1}{2} d m \cdot A C^{2} \omega_{c f}^{2}
$$

The amplitude is assumed to be small so AC may be approximated as $\beta r$ and Eq. 31 may be rewritten as:

$$
\Delta K E=\frac{1}{2} d m \cdot \beta^{2} \mathrm{r}^{2} \omega_{c f}{ }^{2}
$$

By integrating Eq. 32 from the hinge to the tip, the Kinetic Energy of the hinged potion of the blade when it is aligned with the centrifugal force is obtained as follows. 


$$
K E=\frac{1}{2} \int_{0}^{\mathrm{R}(1-\mathrm{e})} \beta^{2} \mathrm{r}^{2} \omega_{c f}^{2} d m
$$

Within the integration argument, distance $r$ is the only non-constant and by substituting in Eq. 17 the Kinetic Energy maybe written as:

$$
K E=\frac{1}{2} \beta^{2} \omega_{c f}^{2} I_{b}
$$

Now the important step of equating the Kinetic and Potential Energies is done. The goal here is to determine the natural frequency of the hinged equivalent blade model when the only restoring force is the centrifugal force. We proceed by equating the results of Eq. 29 and Eq. 34 .

$$
\frac{1}{2} \beta^{2} \omega_{c f}{ }^{2} \mathrm{I}_{\mathrm{b}}=\frac{1}{4} \Omega^{2} \beta^{2} \mathrm{M}_{\mathrm{b}} \mathrm{R}^{2} \mathrm{e}(1-\mathrm{e})^{2}+\frac{1}{2} \Omega^{2} \beta^{2} \mathrm{I}_{\mathrm{b}}
$$

Simplifying Eq. 35 along with Eq. 20 gives:

$$
\omega_{c f}^{2}=\Omega^{2}\left(1+\frac{3}{2}\left(\frac{e}{1-e}\right)\right)
$$

The result of Eq. 36 is equal to the $\alpha$ term in Eq. 22. Therefore the natural frequency of the rotating hinged blade maybe expressed as:

$$
\omega_{R}^{2}=\omega_{N R}^{2}+\Omega^{2}\left[1+\frac{3}{2}\left(\frac{e}{1-e}\right)\right]
$$

Eq. 37 corresponds to Eq. 7.9 in Eggleston's work and it is this insight that allows the hinged equivalent blade model to effectively model rotating blades (2). For the present work the above relationship will be rewritten as follows so that the value for e may be found. 


$$
e=\frac{\omega_{R}^{2}-\omega_{N R}^{2}-\Omega^{2}}{\omega_{R}^{2}-\omega_{N R}^{2}+\frac{1}{2} \Omega^{2}}
$$

Eq. 38 allows one to solve for e based on the non-rotating natural frequency, the rotating natural frequency, and the rotor frequency. It is important to note that Eq. 38 requires the engineer to know the blade rotating and non-rotating natural frequencies. The blade rotating and non-rotating natural frequencies can be obtained from experimentation or by analytically solving for the frequencies using the Myklestad-Prohl table analysis, a detailed description of which is offered in Rao's work (21). It is also important to note that because the rotating natural frequency, $\omega_{R}$, is solved for at only one rotor frequency, $\Omega$, the hinged model is only completely accurate at the rotor frequencies of zero and $\Omega$. Eggleston points out that despite this weakness the mode shapes, and in particular the first mode, change very little over the operational range and thus the model is effective for practical use over any rotor frequency between zero and $\Omega$. With this in mind it is practical to choose to evaluate the rotating natural frequency at the maximum rotor frequency expected, which under normal operation is typically the frequency at which the turbine is rated. Thus for the present work $\Omega$ will be considered the turbine rotor speed at its rated power output, $\Omega_{\text {Rated }}$. The hinge equivalent blade model is then effective for any rotor speed between parked and rated speed.

The final key is to modify Eq. 21 into a more useful form as follows:

$$
K_{\beta}=\omega_{N R}^{2} \cdot I_{b}
$$

Eq. 39 allows for the torsional spring located at the hinge in the hinge equivalent model to be determined based on known values.

In summary the preceding derivation sets the premise for the hinge equivalent blade model which allow the designer to model a non-homogeneous wind turbine blade with a very simple analytical 
model effective for dynamic simulation. The model is fully defined by equating the real and modeled blade radii and mass ( $\mathrm{R}$ and $\mathrm{M}_{\mathrm{b}}$ respectively) and through the following three equations: Eq. 20, Eq. 38, and Eq. 39. The model is accurate at all normal operating rotor speeds and simulates the first flapwise bending mode of the blade while compensating for centrifugal stiffening.

\subsection{Rotor Tower Coupling - Simplified Rotor Model}

With the basics of the hinge equivalent blade theory established the focus is now transitioned to the method and theory of analytically coupling the rotor and tower subsystems. The final rotor model used in this work allows for a two or three bladed rotor and can model the rotor in any rotational position by calculating the azimuth angle for each blade. The ability of the rotor model to account for an arbitrary blade azimuth angle adds a considerable amount of complexity to the model's formulation. Therefore the focus of the subsequent sections will be to demonstrate the analytical method of coupling the structural model of a blade with the structural model of the tower for a simplified case. By focusing on this simpler case, the complex coordinate transformations required for the general case will not burden the reader. After the simpler case is fully developed the coordinate transformations required for the general case will be stated as well as the results of the general case. For the interested reader the full derivation of the general case is

presented in Appendices A and B. The process, theories, and concepts used to develop the simplified model are identical to the ones used for the more complex general case.

The simplified configuration, referred to in this work as the Simplified Rotor Model, will be one in which a two blade wind turbine is parked with one blade pointing straight up and the other straight down. This configuration can be seen in Figure 4.4. As is shown in Figure 4.4, the base of each blade is offset from the tower tip due to the hub, drive train, generator, bedplate and nacelle (all of which is loosely referred to as the hub in this work). The spacial offset of the hub, as well as its mass properties, are included as a part of the rotor model (see green lines in Figure 4.4). As 
discussed in Sections 4.3.1 through 4.3.4 the kinetic and potential energies of the hub are split evenly between the upper and lower blade model.

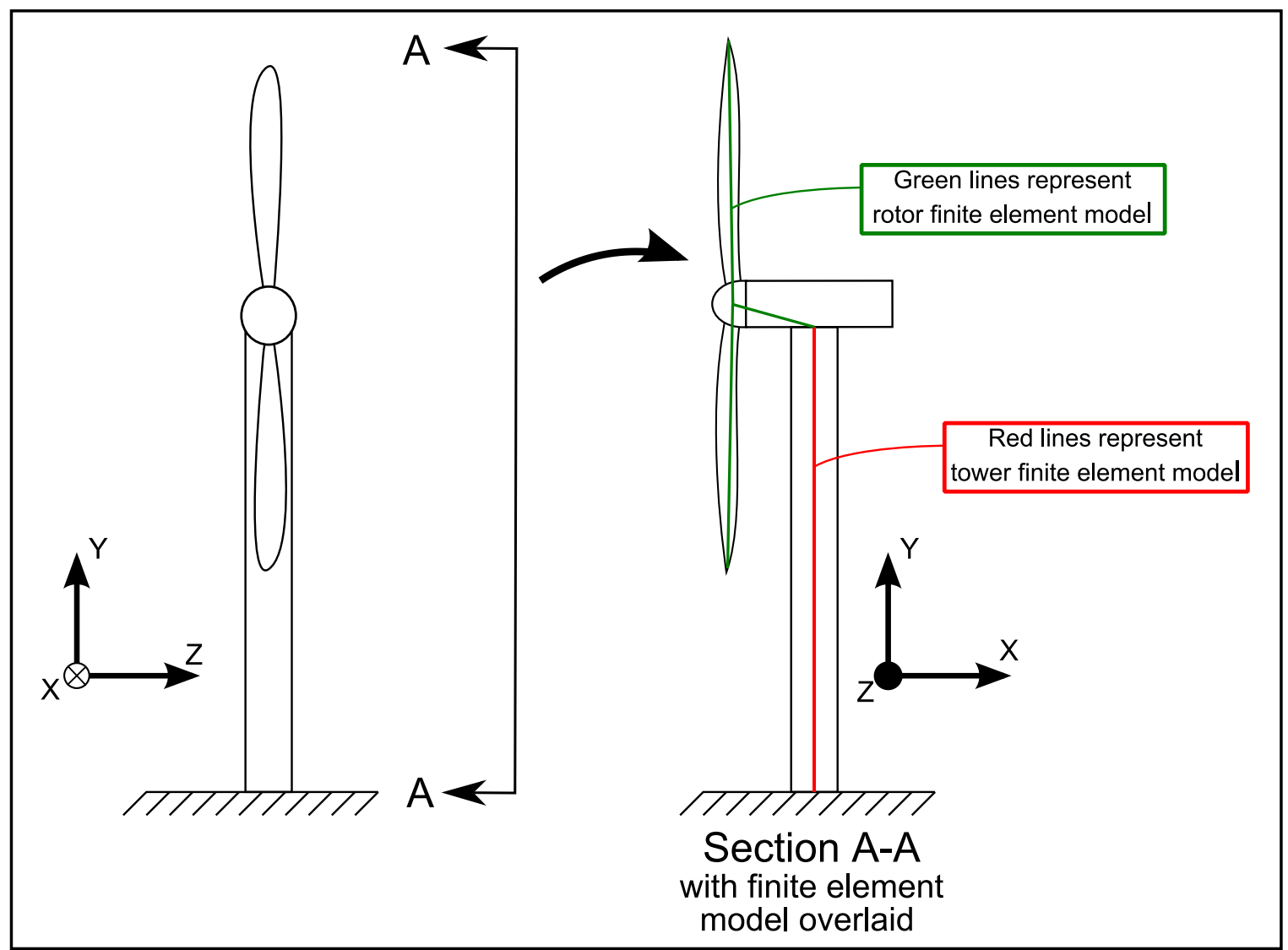

Figure 4.4. The Simplified Rotor Model configuration.

The blades will be modeled using the formulations developed in the Hinged Offset Equivalent Blade Theory section. Section 4.2 developed the physical parameters which describe the analytical blade model; theses parameters were developed using energy methods to ensure that the dynamic characteristics of the model accurately resemble the actual blade. The focus in this section is on the following three items:

- Develop EOM for the upper and lower blade of the Simplified Rotor Model

- Combine the upper and lower blade EOM into the rotor EOM 
- Integrate the rotor EOM with the tower EOM, a method commonly referred to dynamic coupling.

By coupling the rotor and tower EOM, the DOF which describe the location of the hub in the EOM for each blade will be mathematically equated to the DOF which describe the tower tip in the tower EOM. By coupling the two subsystems the movement of one will influence the movement of the other, just as is the case in actual wind turbines.

Figure 4.5 displays the major components of the two bladed wind turbine used in the Simplified Rotor Model. Note that the blades have been given an exaggerated deflection to emphasize the use of the Hinged Equivalent Blade model in this work.

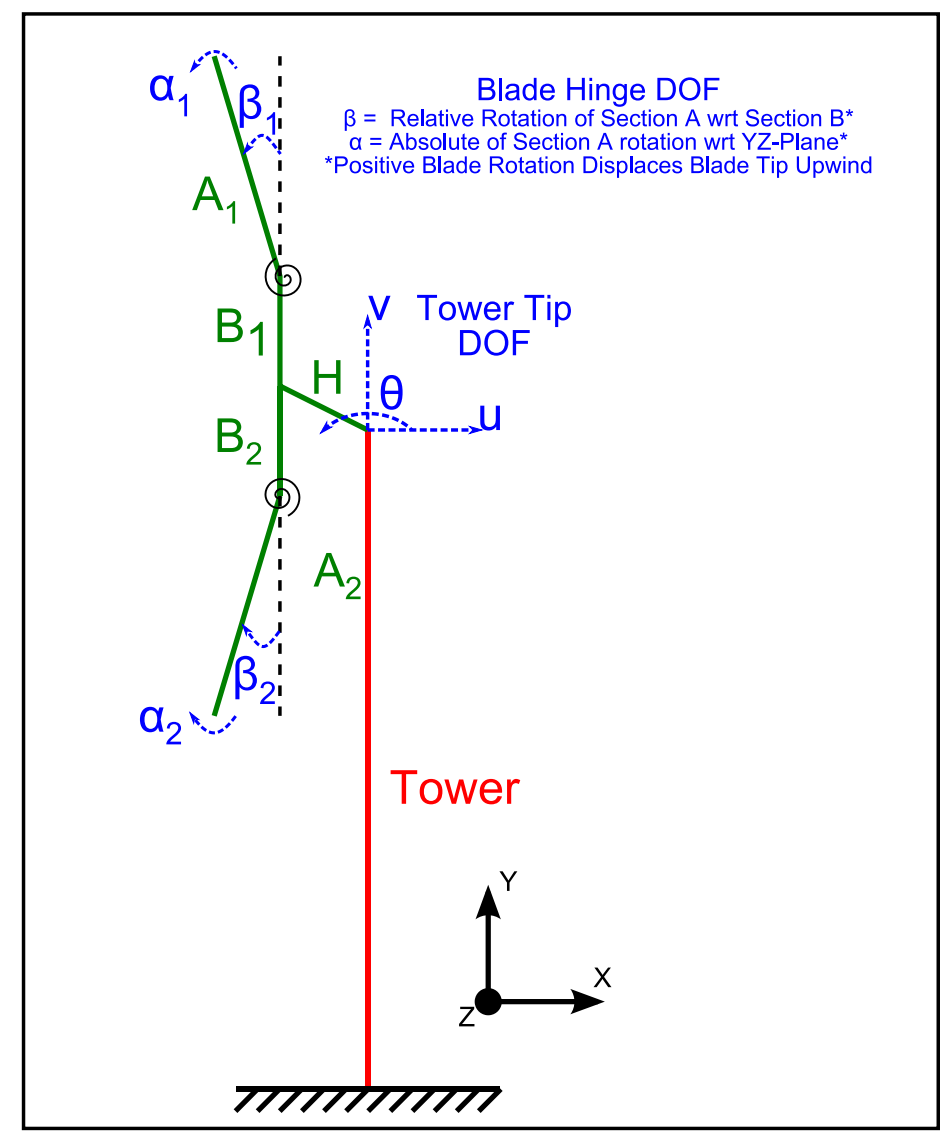

Figure 4.5. Schematic of the tower, hub, and rotor blades used for the Simplified Rotor Model. Degrees of freedom of tower tip and blade hinges appear in blue.

(Note: wrt $=$ with respect to $)$ 
In Figure 4.5 the symbols have the following definitions:

- Components
$\circ \quad \mathrm{A}_{1} \quad$ Hinged portion of upper blade
$\circ \quad \mathrm{A}_{2} \quad$ Hinged portion of lower blade
$\circ \quad B_{1} \quad$ Base portion of upper blade
$\circ \quad \mathrm{B}_{2} \quad$ Base portion of lower blade
$\circ \mathrm{H} \quad \mathrm{Hub}$

- Degrees of Freedom

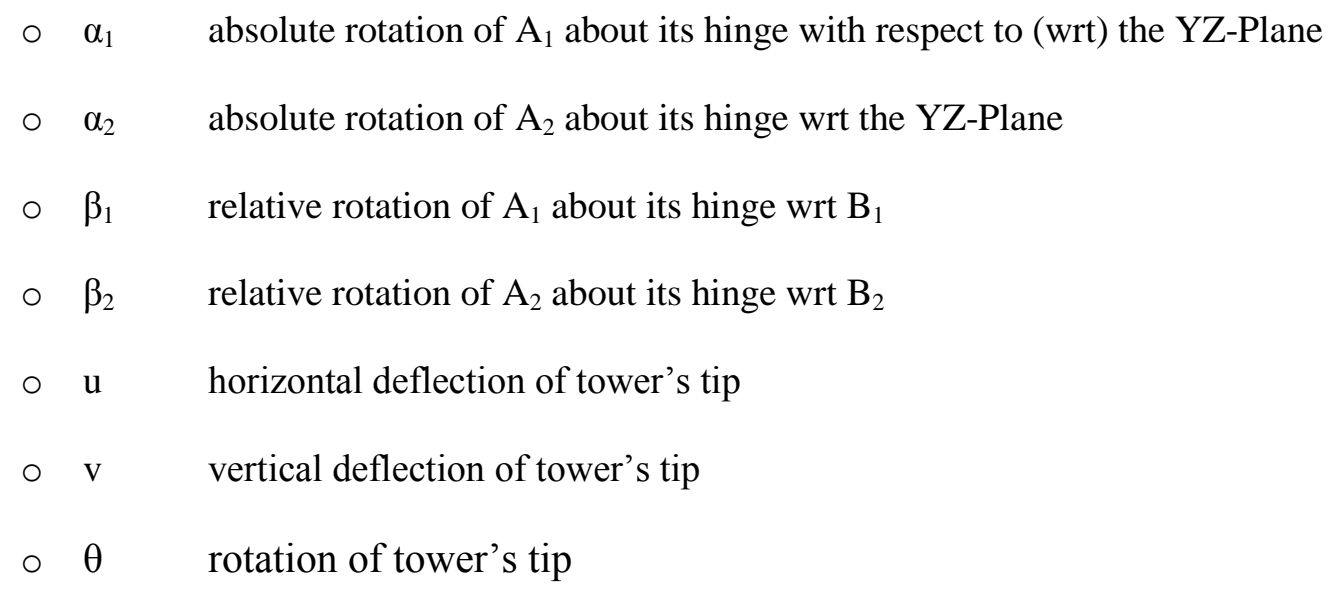

All of the coordinates displayed in Figure 4.5 are shown in their positive orientations. Note that positive blade tip rotation about the blade hinge results in an upwind displacement of the blade tip for both the upper and lower blade. The same polarity for rotations will also apply to the generalized rotor model discussed in Section 4.4.

The structural model used in this work accounts for the mass properties of the hub, but the hub will be assumed to be a rigid body. This structural approximation of the hub is reasonable given the relative stiffness of the hub when compared to the tower and rotor blades. Modeling of hub/nacelle/bedplate as a rigid member is very common in literature, see references (22), (23), (24), (25), and (26). For computational simplicity the hub will not be considered as an 
independent subsystem, rather its influence will be factored into the model for each blade. The method for combining blade/hub influences will be detailed in the Section 4.3.1.

The goal now becomes to develop EOM for each blade as a function of the tower's tip displacements $(\mathrm{u}, \mathrm{v}$, and $\theta$ ) and the absolute rotation of the hinged portion of the blade with respect to the YZ-Plane $\left(\alpha_{1}\right.$ and $\left.\alpha_{2}\right)$. Note that developing the EOM as a function of relative rotation of the hinged portion of the blade about the hinge $\left(\beta_{1}\right.$ and $\left.\beta_{2}\right)$ may at first seem more intuitive but that approach would not allow for proper coupling of the tower and rotor mass and stiffness matrices (hinge bending moment loads would not properly transfer through the blade and to the tower). Post processing of results data, using Eq. 40 and Eq. 41, may be used to determine relative blade hinge rotation.

$$
\begin{aligned}
& \beta_{1}=\alpha_{1}-\theta \\
& \beta_{2}=\alpha_{2}+\theta
\end{aligned}
$$

The method used is Lagrange's Method. Lagrange's method is a highly effective method for developing the EOM of mechanical and structural systems (1). Lagrange's method uses scalar quantities, kinetic and potential energy, instead of vector quantities, force and acceleration, to develop the EOM. Lagrange's method expresses the system energies based on generalized coordinates. Generalized coordinates are a coordinate set that is linearly independent, consistent with the system constraints, and sufficient to describe any configuration of the system. This approach is particularity useful for systems in which the generalized coordinates are not aligned with each other, as is the case for the generalized rotor/tower coupling formulation used in this work which allows for arbitrary azimuth angles.

The first step of Lagrange's Method is to express the potential and kinetic energies of a system in terms of the system's generalized coordinates. The potential and kinetic energies of each blade's 
components, $A_{1}$ and $B_{1}$ in the case of the upper blade, are most easily expressed about their mass centers. But because the mass centers are not the location of the generalized coordinates it is necessary to develop a series of coordinate transformations to switch between a mass centered coordinate system and the generalized coordinates. Figure 4.6 displays the mass centered coordinates for the upper blade, the generalized coordinates, and the spacial distances between the coordinate systems. Figure 4.6 will become the basis of the coordinate transformations.
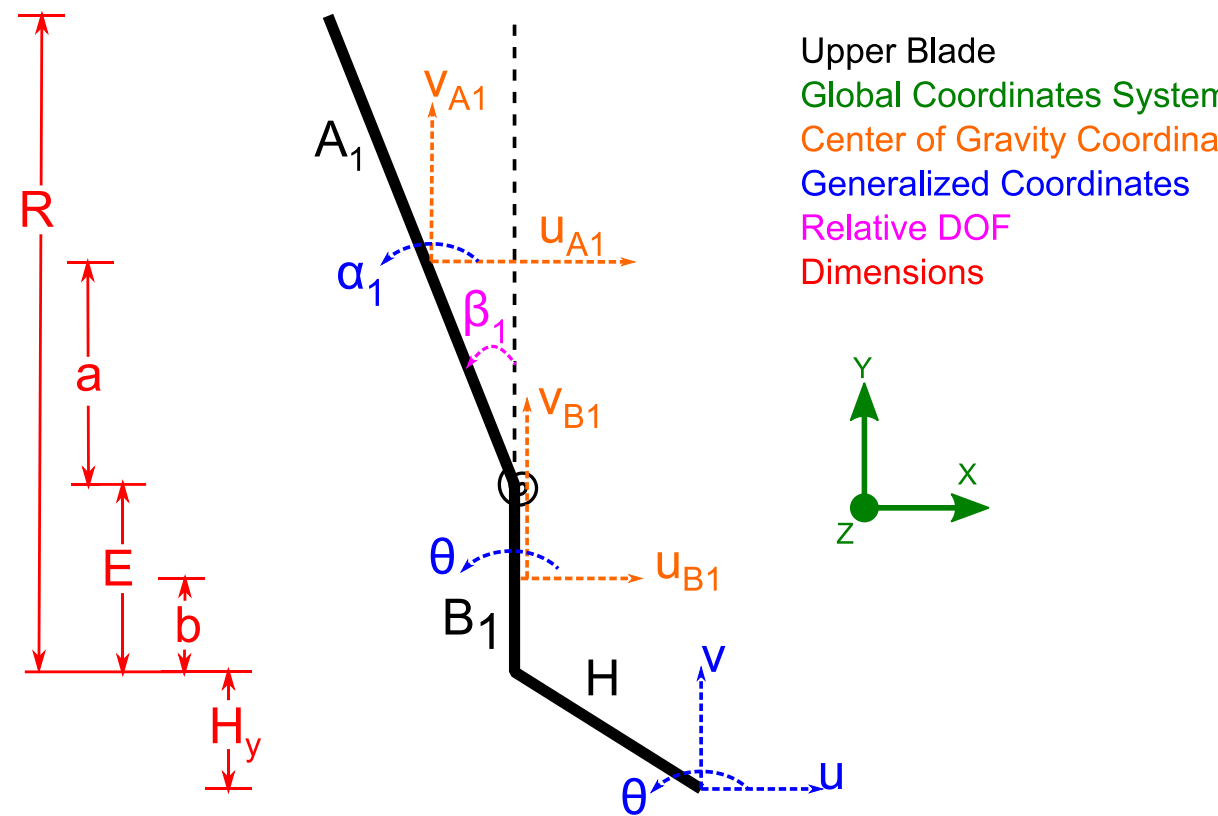

Center of Gravity Coordinates Systems Generalized Coordinates Relative DOF

Dimensions

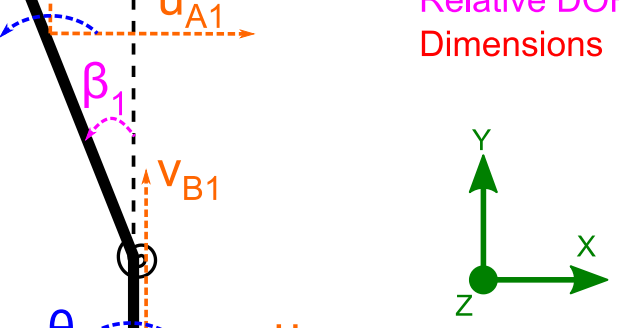

Figure 4.6. The coordinate systems of the upper blade used in the Simplified Rotor Model.

In Figure 4.6 dimension " $a$ " is the distance from the blade's hinge to the A1 coordinate system. Dimension " $\mathrm{b}$ " is the distance from the hub/blade root junction to the $\mathrm{B} 1$ coordinate system. The $\mathrm{A} 1$ and $\mathrm{B} 1$ coordinate systems have their origins located at the mass centers of Sections $\mathrm{A}_{1}$ and $\mathrm{B}_{1}$, respectively. 


\subsubsection{Kinetic Energy of Upper Blade}

The focus is to now develop an expression for the kinetic energy of the upper blade as a function of the generalized coordinates. By stating that the kinetic energy will be expressed as a function of the generalized coordinates, it is more specifically implied that the kinetic energy will be a function of the first time derivative of the four degrees of freedom which are each defined by a unique generalized coordinate.

It will be necessary to develop the kinetic energy expressions for the hub, the base of the blade, and the hinged portion of the blade.

\subsubsection{Kinetic Energy of Upper Blade - Hub}

We will begin with the simplest portion - the hub. The hub is represented in Figure 4.6 by the rigid member $\mathrm{H}$. In this work the hub is considered to be rigid and moves directly with the tip of the tower. Thus its location, velocities, and accelerations, are fully expressed through the tower tip degrees of freedom $u, v$, and $\theta$ - as shown in Figure 4.6. The hub has a mass of $\mathrm{M}_{\mathrm{H}}$ and mass moment of inertial of $\mathrm{I}_{\mathrm{H}}$ about the tower tip. The kinetic energy of the hub, $\mathrm{T}_{\mathrm{H}}$, may be expressed as:

$$
T_{H}=\frac{M_{H} \cdot\left(\dot{u}^{2}+\dot{v}^{2}\right)+I_{H} \cdot\left(\dot{\theta}^{2}\right)}{2}
$$

Eq. 42 expresses the entire kinetic energy of the hub. As mentioned in the previous section, it is computationally simpler to treat the hub as an extension of each blade as opposed to a distinct sub-system. Therefore the final expression for the upper blade's kinetic energy will factor in $1 / 2$ of $\mathrm{T}_{\mathrm{H}}$; the kinetic energy expression for the lower blade will factor in the other $1 / 2$. If a three bladed system is considered each blade would factor in $1 / 3$ of the kinetic energy of the hub, and so on. The same method is used for the hub's potential energy terms. 
The above expression for the kinetic energy of the hub, $\mathrm{T}_{\mathrm{H}}$, is fully expressed in terms of generalized coordinates and is already in a form which is very useful. Note that Eq. 42 displays the generalized coordinates accented with a dot; this signifies the first derivative in time (which is velocity). Similar expressions for the blade's kinetic energy must be developed, but a coordinate transformation will be required to arrive at a function in terms of generalized coordinates.

\subsubsection{Kinetic Energy of Upper Blade - Blade Base}

The kinetic energy of the blade base, B1, will first be expressed as a function of the coordinate system located at its center of gravity. The blade base has a mass of $\mathrm{M}_{\mathrm{B}}$ and a mass moment of inertia of $\mathrm{I}_{\mathrm{B}}$ about its center of gravity. The kinetic energy of the hub, $\mathrm{T}_{\mathrm{B} 1}$, may be expressed as:

$$
T_{B 1}=\frac{M_{B} \cdot\left(\dot{u}_{B 1}^{2}+\dot{v}_{B 1}^{2}\right)+I_{B} \cdot\left(\dot{\theta}^{2}\right)}{2}
$$

In Eq. 43 we see the $T_{B 1}$ is expressed as a function of one generalized coordinate $(\theta)$ and two local coordinates $\left(\mathrm{u}_{\mathrm{B} 1}\right.$ and $\left.\mathrm{v}_{\mathrm{B} 1}\right)$. The translational degrees of freedom used in Eq. 43 , $\mathrm{u}_{\mathrm{B} 1}$ and $\mathrm{v}_{\mathrm{B} 1}$, must be related to the generalized coordinates. The necessary coordinate transformations are as follows:

$$
\begin{gathered}
u_{B 1}=u-\left(\frac{E}{2}+H_{y}\right) \cdot \theta \\
v_{B 1}=v-H_{x} \cdot \theta
\end{gathered}
$$

These coordinate transformations both assume small rotations of $\theta$, namely that $\sin (\theta)=\theta$. Eq. 44 and Eq. 45 may be plugged into Eq. 43 such that $T_{B 1}$ is a function of only the generalized coordinates. The resulting expression for $\mathrm{T}_{\mathrm{B} 1}$ is as follows: 


$$
T_{B 1}=\frac{M_{B}}{2} \cdot\left[\left(\dot{u}-\left(\frac{E}{2}+H_{y}\right) \cdot \dot{\theta}\right)^{2}+\left(\dot{v}-H_{x} \cdot \dot{\theta}\right)^{2}\right]+\frac{I_{B}}{2} \cdot \dot{\theta}^{2}
$$

\subsubsection{Kinetic Energy of Upper Blade - Blade Tip}

A very similar process is now performed to determine an expression for the kinetic energy of the hinged portion of the upper blade. The kinetic energy of the hinged portion of the blade, A1, will first be expressed as a function of the coordinate system located at its center of gravity. A1 has a mass of $\mathrm{M}_{\mathrm{A}}$ and a mass moment of inertia of $\mathrm{I}_{\mathrm{A}}$ about its center of gravity. The kinetic energy of the hinged portion of the blade, $\mathrm{T}_{\mathrm{A} 1}$, may be expressed as:

$$
T_{A 1}=\frac{M_{A} \cdot\left(\dot{u}_{A 1}^{2}+\dot{v}_{A 1}^{2}\right)+I_{A} \cdot\left(\dot{\alpha}_{1}\right)^{2}}{2}
$$

The translational degrees of freedom used in Eq. $47, \mathrm{u}_{\mathrm{A} 1}$ and $\mathrm{v}_{\mathrm{A} 1}$, must be related to the generalized coordinates. The necessary coordinate transformations are as follows:

$$
\begin{gathered}
u_{A 1}=u-a \cdot\left(\alpha_{1}\right)-\left(E+H_{y}\right) \cdot \theta \\
v_{A 1}=v-H_{x} \cdot \theta
\end{gathered}
$$

Eq. 48 and Eq. 49 may be plugged into Eq. 47 such that $\mathrm{T}_{\mathrm{A} 1}$ is a function of only the generalized coordinates. The resulting expression for $\mathrm{T}_{\mathrm{A} 1}$ is as follows:

$$
T_{A 1}=\frac{M_{A}}{2} \cdot\left[\left(\dot{u}-a \cdot\left(\dot{\alpha}_{1}\right)-\left(E+H_{y}\right) \cdot \dot{\theta}\right)^{2}+\left(\dot{v}-H_{x} \cdot \dot{\theta}\right)^{2}\right]+\frac{I_{A}}{2} \cdot\left(\dot{\alpha}_{1}\right)^{2}
$$




\subsubsection{Kinetic Energy of Upper Blade - Total}

The kinetic energy, $\mathrm{T}_{1}$, for the whole upper blade along with the hub may be found by adding Eq. 46, Eq. 50, and 1 1/2 of Eq. 42.

$$
\begin{gathered}
T_{1}=T_{A 1}+T_{B 1}+\frac{1}{2} T_{H} \\
T_{1}=\frac{M_{A}}{2} \cdot\left[\left(\dot{u}-a \cdot\left(\dot{\alpha}_{1}\right)-\left(E+H_{y}\right) \cdot \dot{\theta}\right)^{2}+\left(\dot{v}-H_{x} \cdot \dot{\theta}\right)^{2}\right]+\frac{I_{A}}{2} \cdot\left(\dot{\alpha}_{1}\right)^{2} \\
+\frac{M_{B}}{2} \cdot\left[\left(\dot{u}-\left(\frac{E}{2}+H_{y}\right) \cdot \dot{\theta}\right)^{2}+\left(\dot{v}-H_{x} \cdot \dot{\theta}\right)^{2}\right]+\frac{I_{B}}{2} \cdot \dot{\theta}^{2} \\
+\frac{M_{H} \cdot\left(\dot{u}^{2}+\dot{v}^{2}\right)+I_{H} \cdot\left(\dot{\theta}^{2}\right)}{4}
\end{gathered}
$$

\subsubsection{Potential Energy of Upper Blade}

Having established the kinetic energy expression for the upper blade the focus is now placed on developing the potential energy expression; a similar method will be used. As with the kinetic energy, it will be necessary to develop the potential energy expressions for the hub, the base of the blade, and the hinged portion of the blade.

\subsubsection{Potential Energy of the Upper Blade - Hub}

The first component considered will be the hub. The hub's potential energy changes as a function of its vertical displacement.

$$
V_{H}=M_{H} \cdot v \cdot g
$$

Where $\mathrm{g}$ is the gravitational acceleration constant. Consistent with the method described in the kinetic energy section, the final expression for the upper blade's potential energy will factor in $1 / 2$ of $\mathrm{V}_{\mathrm{H}}$. 


\subsubsection{Potential Energy of the Upper Blade - Blade Base}

The potential energy of the blade base, B1, will first be expressed as a function of the coordinate system located at its center of gravity.

$$
V_{B 1}=M_{B} \cdot v_{B 1} \cdot g
$$

Using the coordinate transformation appearing in Eq. 45, the expression for $\mathrm{V}_{\mathrm{B} 1}$ is easily written as a function of generalized coordinates as follows:

$$
V_{B 1}=M_{B} \cdot g \cdot\left(v-H_{x} \cdot \theta\right)
$$

\subsubsection{Potential Energy of the Upper Blade - Blade Tip}

The potential energy of the upper blade's hinged portion, $\mathrm{V}_{\mathrm{A} 1}$, is substantially more complex than the other components. There are three distinct contributions to $\mathrm{V}_{\mathrm{A} 1}$ :

- the vertical displacement of section $\mathrm{A} 1\left[\mathrm{~V}_{\mathrm{A} 1 \mathrm{v}}\right]$

- the rotation of section $\mathrm{A} 1$, relative to the tower tip, about the hinge and the resulting spring loading $\left[\mathrm{V}_{\mathrm{A} 1 \mathrm{~s}}\right]$

- the rotation of section A1 about the hinge and the corresponding restoring force resulting from centrifugal stiffening $\left[\mathrm{V}_{\mathrm{Alcf}}\right]$

Each of the three contributions to $\mathrm{V}_{\mathrm{A} 1}$ will be discussed in detail, beginning with $\mathrm{V}_{\mathrm{Alv}}$. The change in potential energy caused by the vertical displacement of section B1 is first expressed as a function of the coordinate system located at its center of gravity.

$$
V_{A 1 v}=M_{A} \cdot v_{A 1} \cdot g
$$


Using the coordinate transformation appearing in Eq. 49, the expression for $\mathrm{V}_{\mathrm{B} 1}$ is easily written as a function of generalized coordinates as follows:

$$
V_{A 1 v}=M_{A} \cdot g \cdot\left(v-H_{x} \cdot \theta\right)
$$

The change in potential energy caused by the displacement of the spring hinge, $\mathrm{V}_{\mathrm{Al}}$, may be expressed as:

$$
V_{A 1 s}=\frac{1}{2} K_{\beta} \cdot \beta_{1}^{2}=\frac{1}{2} K_{\beta} \cdot\left(\alpha_{1}-\theta_{1}\right)^{2}=\frac{K_{\beta}}{2} \cdot \alpha_{1}^{2}+\frac{K_{\beta}}{2} \cdot \theta_{1}^{2}-K_{\beta} \cdot \alpha_{1} \cdot \theta_{1}
$$

Where $K_{\beta}$ is the spring constant of the hinge as described in Eq. 39. Next, the potential energy, $\mathrm{V}_{\mathrm{Alcf}}$, related to the restoring force resulting from centrifugal stiffening must be accounted for. The potential energy resulting from centrifugal stiffening was derived in Section 4.2.3, and appeared in final form in Eq. 29. A few substitutions must be made to Eq. 29 for the form to accurately represent the model of Figure 4.6.

- Absolute rotation of the hinged portion of the blade $\left(\alpha_{1}\right.$ and $\left.\alpha_{2}\right)$ will be used instead of relative rotation $(\beta)$

- The hinge offset, originally e, is now $\frac{E}{R}$

- The blade mass, originally $\mathrm{M}_{\mathrm{b}}$, will now be stated in terms of the mass of section A where $M_{A}=M_{b}\left(1-\frac{E}{R}\right)$.

- The flapping moment of inertia, $\mathrm{I}_{\mathrm{b}}$, of section A was originally centered about the blade's hinge. Now the section A flapping moment of inertia, $\mathrm{I}_{\mathrm{A}}$, will be centered about the section A center of gravity. Note that: $I_{b}=4 \cdot I_{A}$. 
With these substitutions Eq. 29 can be written as:

$$
V_{A 1 c f}=\frac{1}{4} \Omega^{2} \cdot\left(\alpha_{1}-\theta\right)^{2} \cdot \mathrm{M}_{\mathrm{A}} \cdot \mathrm{R} \cdot \mathrm{E} \cdot\left(1-\frac{\mathrm{E}}{\mathrm{R}}\right)+2 \Omega^{2} \cdot\left(\alpha_{1}-\theta\right)^{2} \mathrm{I}_{\mathrm{A}} \quad \text { Eq. } 59
$$

The potential energy of section $\mathrm{A}_{1}$, may be found by summing the three contributions, as follows.

$$
\begin{gathered}
V_{A 1}=V_{A 1 v}+V_{A 1 s}+V_{A 1 c f} \\
V_{A 1}=M_{A} \cdot g \cdot\left(v-H_{x} \cdot \theta\right)+\frac{K_{\beta}}{2} \cdot \alpha_{1}{ }^{2}+\frac{K_{\beta}}{2} \cdot \theta_{1}{ }^{2}-K_{\beta} \cdot \alpha_{1} \cdot \theta_{1} \\
+\frac{1}{4} \Omega^{2} \cdot\left(\alpha_{1}-\theta\right)^{2} \cdot \mathrm{M}_{\mathrm{A}} \cdot \mathrm{R} \cdot \mathrm{E} \cdot\left(1-\frac{\mathrm{E}}{\mathrm{R}}\right)+2 \Omega^{2} \cdot\left(\alpha_{1}-\theta\right)^{2} \mathrm{I}_{\mathrm{A}}
\end{gathered}
$$

Eq. 61

\subsubsection{Potential Energy of the Upper Blade - Total}

The total potential energy of the upper blade may be found by summing the contributions from section $A_{1}$, section $B_{1}$, and the hub.

$$
\begin{gathered}
V_{1}=V_{A 1}+V_{B 1}+\frac{1}{2} V_{H} \\
V_{1}=M_{A} \cdot g \cdot\left(v-H_{x} \cdot \theta\right)+\frac{K_{\beta}}{2} \cdot \alpha_{1}{ }^{2}+\frac{K_{\beta}}{2} \cdot \theta_{1}{ }^{2}-K_{\beta} \cdot \alpha_{1} \cdot \theta_{1}+ \\
\frac{1}{4} \Omega^{2} \cdot\left(\alpha_{1}-\theta\right)^{2} \cdot \mathrm{M}_{\mathrm{A}} \cdot \mathrm{R} \cdot \mathrm{E} \cdot\left(1-\frac{\mathrm{E}}{\mathrm{R}}\right)+2 \Omega^{2} \cdot\left(\alpha_{1}-\theta\right)^{2} \mathrm{I}_{\mathrm{A}}+M_{B} \cdot g \cdot\left(v-H_{x} \cdot \theta\right) \\
+\frac{1}{2} M_{H} \cdot v \cdot g
\end{gathered}
$$

\subsubsection{Upper Blade Equations of Motion}

With the kinetic energy and the potential energy now determined for the upper blade in terms of the blade's generalized coordinates, the blade's EOM may be found by utilizing Lagrange's Equations. Lagrange's Equations are as follows (1): 


$$
\frac{d}{d t} \frac{\partial T}{\partial \dot{q}_{i}}-\frac{\partial T}{\partial q_{i}}+\frac{\partial V}{\partial q_{i}}=p_{i}(t)
$$

In Eq. $64 \mathrm{~T}$ and $\mathrm{V}$ are kinetic and potential energy, respectively, $\mathrm{t}$ is time, $\mathrm{q}_{\mathrm{i}}$ denotes the $\mathrm{i}$ 'th generalized DOF. The $\mathrm{p}_{\mathrm{i}}(\mathrm{t})$ term denotes generalized forces which account for nonconservative forces which are caused by damping. At this stage in the analysis damping is considered negligible and thus the $\mathrm{p}_{\mathrm{i}}(\mathrm{t})$ terms vanish. Note that damping will later be accounted for and applied to the whole tower/rotor system through modal damping. The $\frac{\partial T}{\partial q_{i}}$ terms would only be nonzero if the kinetic energy was a function of the system's position. The kinetic energy of the upper blade, Eq. 52, is only a function of the system's generalized velocities and therefore the $\frac{\partial T}{\partial q_{i}}$ terms vanish. Simplifying Eq. 64 by only showing the nonzero terms gives:

$$
\frac{d}{d t} \frac{\partial T}{\partial \dot{q}_{i}}+\frac{\partial V}{\partial q_{i}}=p_{i}(t)
$$

Eq. 65 may be used to develop the EOM for the upper blade by using the kinetic energy, T, developed in Eq. 52 and the potential energy, V, developed in Eq. 63.

The generalized coordinates, $\mathrm{q}_{\mathrm{i}}$, for $\mathrm{i}=1$ through 4 , are: $\mathrm{u}, \mathrm{v}, \theta$, and $\alpha_{1}$. The final EOM will be placed into the following matrix form.

$$
\boldsymbol{M}\left\{\begin{array}{c}
\ddot{u} \\
\ddot{v} \\
\ddot{\theta} \\
\ddot{\alpha}_{1}
\end{array}\right\}+\boldsymbol{K}\left\{\begin{array}{c}
u \\
v \\
\vartheta \\
\alpha_{1}
\end{array}\right\}=0
$$

In Eq. $66 \mathbf{M}$ and $\mathbf{K}$ are $4 \times 4$ mass and stiffness coefficient matrices. 
Applying the appropriate derivatives seen in Eq. 65 to the kinetic energy (Eq. 52) results in the following expressions:

$$
\begin{aligned}
& \frac{d}{d t} \frac{\partial T}{\partial \dot{u}}=\left(\frac{M_{H}}{2}+M_{A}+M_{B}\right) \cdot \ddot{u}+\left[-M_{B} \cdot\left(\frac{E}{2}+H_{y}\right)-M_{A} \cdot\left(E+H_{y}\right)\right] \\
& \cdot \ddot{\theta} \quad+\left(-M_{A} \cdot a\right) \cdot \ddot{\alpha}_{1} \\
& \frac{d}{d t} \frac{\partial T}{\partial \dot{v}}=\left(\frac{M_{H}}{2}+M_{A}+M_{B}\right) \cdot \ddot{v}-H_{x}\left(M_{A}+M_{B}\right) \cdot \ddot{\theta} \\
& \frac{d}{d t} \frac{\partial T}{\partial \dot{\theta}}=\left[-M_{B} \cdot\left(\frac{E}{2}+H_{y}\right)-M_{A} \cdot\left(E+H_{y}\right)\right] \cdot \ddot{u}+-H_{x}\left(M_{A}+M_{B}\right) \cdot \ddot{v} \\
& +\left\{\frac{I_{H}}{2}+I_{A}+M_{A}\left[H_{x}{ }^{2}+\left(E+H_{y}\right)^{2}\right]+M_{B}\left(H_{x}{ }^{2}+\left(\frac{E}{2}+H_{y}\right)^{2}\right)\right\} \cdot \ddot{\theta} \\
& +\left[M_{A} \cdot a \cdot\left(E+H_{y}\right)\right] \cdot \ddot{\alpha_{1}} \\
& \frac{d}{d t} \frac{\partial T}{\partial \dot{\alpha}}=\left(-M_{A} \cdot a\right) \cdot \ddot{u}+\left[M_{A} \cdot a \cdot\left(E+H_{y}\right)\right] \cdot \ddot{\theta}+\left(M_{A} \cdot a^{2}+I_{A}\right) \cdot \ddot{\alpha}_{1}
\end{aligned}
$$

Applying the appropriate derivatives seen in Eq. 65 to the potential energy results in the following expressions:

$$
\begin{gathered}
\frac{\partial V}{\partial u}=0 \\
\frac{\partial V}{\partial v}=g \cdot\left(\frac{1}{2} M_{H}+M_{A}+M_{B}\right) \\
\frac{\partial V}{\partial \theta}=K_{e q} \cdot \theta-K_{e q} \cdot \alpha_{1}-H_{x} g \cdot\left(M_{A}+M_{B}\right) \\
\frac{\partial V}{\partial \beta}=-K_{e q} \cdot \theta+K_{e q} \cdot \alpha_{1}
\end{gathered}
$$

In equations Eq. 73 and Eq. $74 K_{e q}$ signifies the equivalent blade hinge stiffness which is a combination of the hinge spring and centrifugal force stiffness. $K_{e q}$ is defined in below.

$$
K_{e q}=K_{\beta}+4 \cdot I_{A} \cdot \Omega^{2}+\frac{1}{2} \Omega^{2} \cdot \mathrm{M}_{\mathrm{A}} \cdot \mathrm{R} \cdot \mathrm{E} \cdot\left(1-\frac{\mathrm{E}}{\mathrm{R}}\right)
$$


The results of Eq. 67 through Eq. 74 can be placed into the matrix form seen in Eq. 66. In doing so the upper blade mass matrix, $\mathbf{M}_{1}$, and stiffness matrix, $\mathbf{K}_{\mathbf{1}}$, are determined (the "1" subscript signifies the top blade). The mass matrix for the upper blade is:

$\boldsymbol{M}_{\mathbf{1}}=\left[\begin{array}{cccc}\left(\frac{M_{H}}{2}+M_{A}+M_{B}\right) & 0 & \left(-M_{B} \cdot\left(\frac{E}{2}+H_{y}\right)-M_{A} \cdot\left(E+H_{y}\right)\right) & \left(-M_{A} \cdot a\right) \\ 0 & \left(\frac{M_{H}}{2}+M_{A}+M_{B}\right) & \left(-H_{x}\left(M_{A}+M_{B}\right)\right) & 0 \\ \left(-M_{B} \cdot\left(\frac{E}{2}+H_{y}\right)-M_{A} \cdot\left(E+H_{y}\right)\right) & \left(-H_{x}\left(M_{A}+M_{B}\right)\right) & \left(\frac{I_{H}}{2}+I_{B}+M_{A}\left[H_{x}{ }^{2}+\left(E+H_{y}\right)^{2}\right]+M_{B}\left(H_{x}{ }^{2}+\left(\frac{E}{2}+H_{y}\right)^{2}\right)\right) & \left(M_{A} \cdot a \cdot\left(E+H_{y}\right)\right) \\ \left(-M_{A} \cdot a\right) & 0 & \left(M_{A} \cdot a \cdot\left(E+H_{y}\right)\right) & \left(M_{A} \cdot a^{2}+I_{A}\right)\end{array}\right]$ Eq. 76

The stiffness matrix for the upper blade is, where $K_{e q}$ is defined in Eq. 75:

$$
\boldsymbol{K}_{\mathbf{1}}=\left[\begin{array}{cccc}
0 & 0 & 0 & 0 \\
0 & 0 & 0 & 0 \\
0 & 0 & K_{e q} & -K_{e q} \\
0 & 0 & -K_{e q} & K_{e q}
\end{array}\right]
$$

Equations Eq. 66, Eq. 76, and Eq. 77 are combined to create the final EOM for the upper blade. The result is:

$$
\boldsymbol{M}_{\mathbf{1}}\left\{\begin{array}{c}
\ddot{u} \\
\ddot{v} \\
\ddot{\theta} \\
\ddot{\alpha_{1}}
\end{array}\right\}+\boldsymbol{K}_{\mathbf{1}}\left\{\begin{array}{c}
u \\
v \\
\vartheta \\
\alpha_{1}
\end{array}\right\}=0
$$




\subsubsection{Lower Blade Equations of Motion}

The method used to develop the EOM for the upper blade is also applied to the lower blade. The lower blade is shown schematically in Figure 4.7. As discussed in section 4.3.1.1, the hub, section "H", is included in both the upper and lower blade models (the kinetic and potential energy terms for the hub are split evenly between the upper and lower blade EOM).

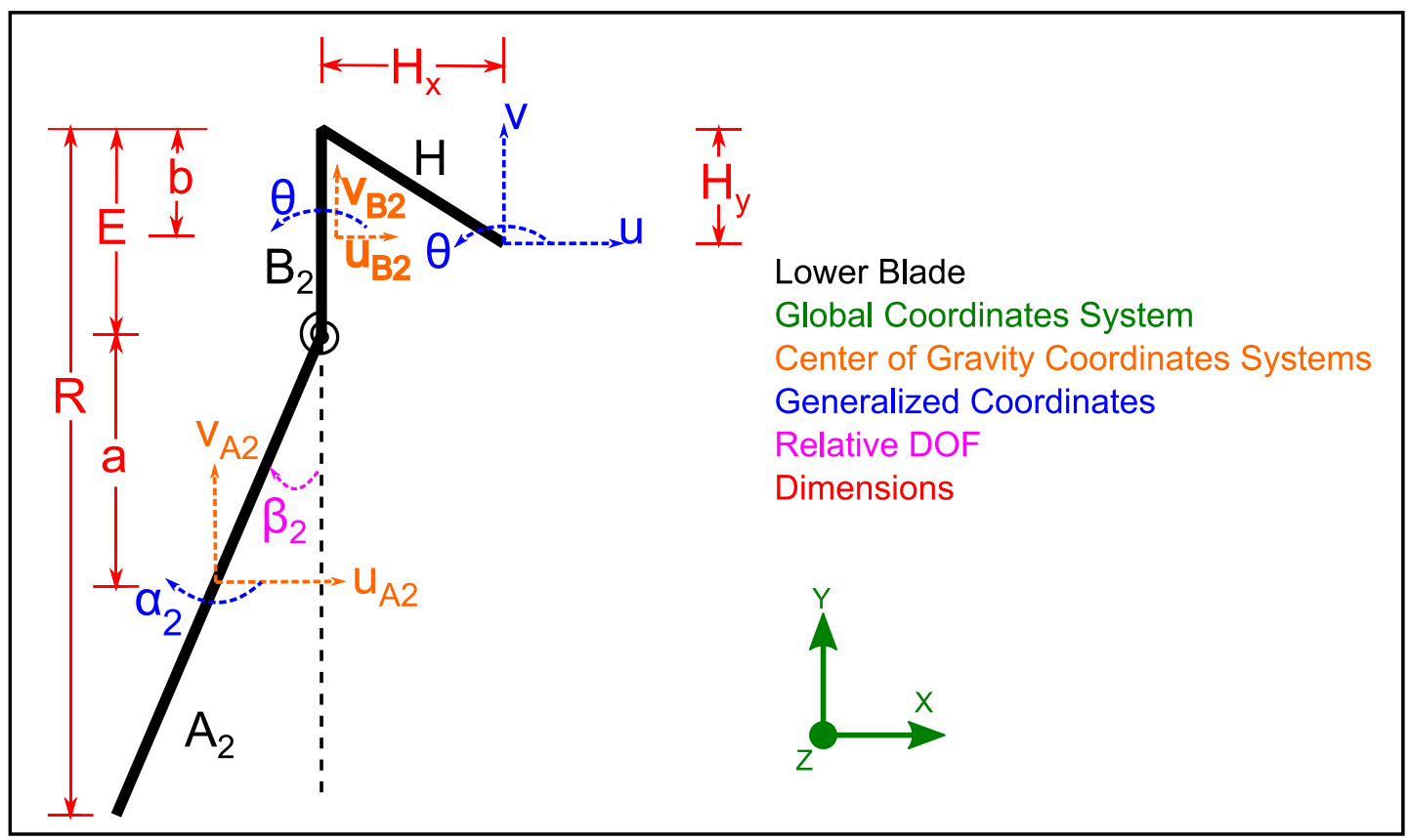

Figure 4.7. The coordinate systems of the lower blade used in the Simplified Rotor Model.

The hinged portion of the blade, A2, and the root of the blade, B2, both have coordinate systems at their respective centers of gravity. The hub, $\mathrm{H}$, moves with the coordinate system located at the tower's tip.

As was done for the upper blade, coordinate transformations must be made to relate the local coordinate systems to the generalized coordinates, kinetic and potential energy expressions must be developed, and finally Lagrange's Equations are used to put develop the EOM. The resulting EOM appear on the next page. 
The lower blade mass matrix, $\mathbf{M}_{2}$, where the subscript 2 signifies the lower blade, is as follows:

$\boldsymbol{M}_{2}=\left[\begin{array}{cccc}\left(\frac{M_{H}}{2}+M_{A}+M_{B}\right) & 0 & \left(M_{B} \cdot\left(\frac{E}{2}-H_{y}\right)+M_{A} \cdot\left(E-H_{y}\right)\right) & \left(-M_{A} \cdot a\right) \\ 0 & \left(\frac{M_{H}}{2}+M_{A}+M_{B}\right) & \left(-H_{x}\left(M_{A}+M_{B}\right)\right) & 0 \\ \left(M_{B} \cdot\left(\frac{E}{2}-H_{y}\right)+M_{A} \cdot\left(E-H_{y}\right)\right) & \left(-H_{x}\left(M_{A}+M_{B}\right)\right) & \left(\frac{I_{H}}{2}+I_{B}+M_{A}\left[H_{x}^{2}+\left(-E+H_{y}\right)^{2}\right]+M_{B}\left(H_{x}^{2}+\left(-\frac{E}{2}+H_{y}\right)^{2}\right)\right) & \left(M_{A} \cdot a \cdot\left(-E+H_{y}\right)\right) \\ \left(-M_{A} \cdot a\right) & 0 & \left(M_{A} \cdot a \cdot\left(-E+H_{y}\right)\right) & \left(M_{A} \cdot a^{2}+I_{A}\right)\end{array}\right]$

The lower blade stiffness matrix, $\mathbf{K}_{\mathbf{2}}$, is as follows, where $K_{e q}$ is defined in Eq. 75:

$$
\boldsymbol{K}_{2}=\left[\begin{array}{cccc}
0 & 0 & 0 & 0 \\
0 & 0 & 0 & 0 \\
0 & 0 & K_{e q} & K_{e q} \\
0 & 0 & K_{e q} & K_{e q}
\end{array}\right]
$$

Equations Eq. 66, Eq. 79, and Eq. 80 are combined to create the final EOM for the lower blade. The result is:

$$
\boldsymbol{M}_{2}\left\{\begin{array}{c}
\ddot{u} \\
\ddot{v} \\
\ddot{\theta} \\
\ddot{\alpha_{2}}
\end{array}\right\}+\boldsymbol{K}_{2}\left\{\begin{array}{c}
u \\
v \\
\vartheta \\
\alpha_{2}
\end{array}\right\}=0
$$




\subsubsection{Final Equations of Motion for Simplified Rotor Model}

The final rotor EOM for the Simplified Rotor Model is developed by combining the influence of the upper and lower blades. The final

EOM will be a function of all of the degrees of freedom which are required to express the state of the two blades, $u, v, \vartheta, \beta_{1}$, and $\beta_{2}$. The mass and stiffness matrices of the Simplified Rotor Model, $\mathbf{M}_{\text {SIMP }}$ and $\mathbf{K}_{\text {SIMP }}$, are determined by adding the terms for corresponding degrees of freedom from the upper and lower blade EOM.

$$
M_{S I M P}=M_{1}+M_{2}
$$

$$
\begin{aligned}
& M_{\text {SIMP }} \\
& =\left[\begin{array}{ccccc}
\left(M_{H}+2 M_{A}+2 M_{B}\right) & 0 & \left(-2 H_{y}\left(M_{A}+M_{B}\right)\right) & \left(-M_{A} \cdot a\right) & \left(-M_{A} \cdot a\right) \\
0 & \left(M_{H}+2 M_{A}+2 M_{B}\right) & \left(-2 H_{x}\left(M_{A}+M_{B}\right)\right) & 0 & 0 \\
\left(-2 H_{y}\left(M_{A}+M_{B}\right)\right) & \left(-2 H_{x}\left(M_{A}+M_{B}\right)\right) & (\theta \theta) & \left(I_{A}+M_{A} \cdot a \cdot\left(E+H_{y}+a\right)\right) & \left(I_{A}+M_{A} \cdot a \cdot\left(E-H_{y}+a\right)\right) \\
\left(-M_{A} \cdot a\right) & 0 & \left(I_{A}+M_{A} \cdot a \cdot\left(E+H_{y}+a\right)\right) & \left(M_{A} \cdot a^{2}+I_{A}\right) & 0 \\
\left(M_{A} \cdot a\right) & 0 & \left(I_{A}+M_{A} \cdot a \cdot\left(E-H_{y}+a\right)\right) & 0 & \left(M_{A} \cdot a^{2}+I_{A}\right)
\end{array}\right] \text { Eq.83 }
\end{aligned}
$$

In Eq. 83 center term, $(\theta \theta)$, is omitted and recorded below for compactness.

$$
(\theta \theta)=\left(I_{H}+2 I_{B}+M_{A}\left[2 H_{x}^{2}+\left(E+H_{y}\right)^{2}+\left(-E+H_{y}\right)^{2}\right]+M_{B}\left(2 H_{x}^{2}+\left(\frac{E}{2}+H_{y}\right)^{2}+\left(-\frac{E}{2}+H_{y}\right)^{2}\right)\right) \quad \text { Eq. } 84
$$


The system stiffness matrix is found in a similar manner as follows, where $K_{e q}$ is defined in Eq.

75:

$$
\begin{gathered}
\boldsymbol{K}_{\text {SIMP }}=\boldsymbol{K}_{\mathbf{1}}+\boldsymbol{K}_{\mathbf{2}} \\
\boldsymbol{K}_{\text {SIMP }}=\left[\begin{array}{ccccc}
\mathbf{0} & \mathbf{0} & \mathbf{0} & \mathbf{0} & \mathbf{0} \\
\mathbf{0} & \mathbf{0} & \mathbf{0} & \mathbf{0} & \mathbf{0} \\
\mathbf{0} & \mathbf{0} & \mathbf{2} K_{e q} & -K_{e q} & K_{e q} \\
\mathbf{0} & \mathbf{0} & -K_{e q} & K_{e q} & \mathbf{0} \\
\mathbf{0} & \mathbf{0} & K_{e q} & \mathbf{0} & K_{e q}
\end{array}\right]
\end{gathered}
$$

Equations Eq. 83, Eq. 84, Eq. 86, and Eq. 87 are combined to create the final EOM for the Simplified Rotor Model. The result is:

$$
\boldsymbol{M}_{\text {SIMP }}\left\{\begin{array}{c}
\ddot{u} \\
\ddot{v} \\
\ddot{\theta} \\
\ddot{\alpha_{1}} \\
\ddot{\alpha_{2}}
\end{array}\right\}+\boldsymbol{K}_{\text {SIMP }}\left\{\begin{array}{c}
u \\
v \\
\vartheta \\
\alpha_{1} \\
\alpha_{2}
\end{array}\right\}=\boldsymbol{F}\left(t, u, v, \theta, \alpha_{1}, \alpha_{2}\right)
$$

where $\mathrm{F}$ is a forcing function created by environmental and gravity loads. Eq. 87 represents the complete 5 DOF EOM required to capture the dynamic behavior of the simplified rotor system. The first three degrees of freedom in Eq. 87; $u, v, \theta$ are the same exact degrees of freedom used to describe the state of the tower's tip. With these three common degrees of freedom the tower and rotor models are easily coupled by combining the EOM for the two subsystems such that the terms with common degrees of freedom are added.

\subsubsection{Rotor Weight}

In Section 4.3.5 it was noted that Eq. 87 includes the forcing function $\boldsymbol{F}\left(t, u, v, \theta, \alpha_{1}, \alpha_{2}\right)$ term. The forcing function is a combination of aerodynamic loads and steady state weight loads, the environmental load cases will be described in detail in Chapter 5. 
Terms associated with the system's weights were originally captured in the potential energy expressions. As any portion of the system raises in height its potential energy increases. The relationship of potential energy to the generalized coordinates is a first order (linear) relationship (e.g. $V=m g h)$. When Lagrange's Equations are applied the potential energy terms are differentiated with respect to the generalized coordinates. In the case of the noted weight terms, the result of the differentiation is no longer related to any of the generalized coordinates; it is simply a weight (e.g.

$\left.\frac{d}{d h}(m g h)=m g\right)$. As a result the terms are not captured in either the mass matrix $(\mathbf{M})$ or the stiffness matrix (K). The weight terms must be captured as steady state terms within the forcing function, $\boldsymbol{F}\left(t, u, v, \theta, \beta_{1}, \beta_{2}\right)$.

In this work the weight of the rotor is applied directly to the tower tip. The rotor weight manifests itself as a downward axial load on the tower. The blades, hub, drive train, generator, bedplate and nacelle are assumed to be balanced on the tower tip and that there is no tip moment induced by gravity loads. The total rotor weight for the Simplified Rotor Model applied to the tower tip is found by adding Eq. 72 (weight terms for the upper blade model) to the weight terms for the lower blade model (which are the same as Eq. 72$)$. The resulting axial force applied to the tower tip $\left(F_{R W S I M P}\right)$ [Rotor Weight Simplified Rotor Model] is:

$$
F_{R W S I M P}=g \cdot\left(M_{H}+2 M_{A}+2 M_{B}\right)
$$

\subsubsection{Tower/Rotor EOM Coupling}

To illustrate the actual coupling of the tower and rotor subsystems, and their corresponding EOM, it is easiest to discuss a highly simplified example. Let us consider a very simple cantilevered tower model with three nodes, referred to as the Simplified Tower Model in this work. The bottom node is fully constrained to the ground $\left(u_{1}, v_{1}\right.$, and $\left.\theta_{1}=0\right)$. Following the tower modeling conventions laid out in Chapter 3 , the mass matrix would be a $6 \times 6$ matrix corresponding to the 6 active degrees of freedom $\left(u_{2}, v_{2}, \theta_{2}, u_{3}, v_{3}\right.$, and $\left.\theta_{3}\right)$. Now let us 
consider that attached to the tip of the tower is a rotor meeting the configuration of the Simplified Rotor Model displayed in Figure 4.5. Such a wind turbine configuration is displayed schematically in Figure 4.8 with the degrees of freedom shown.

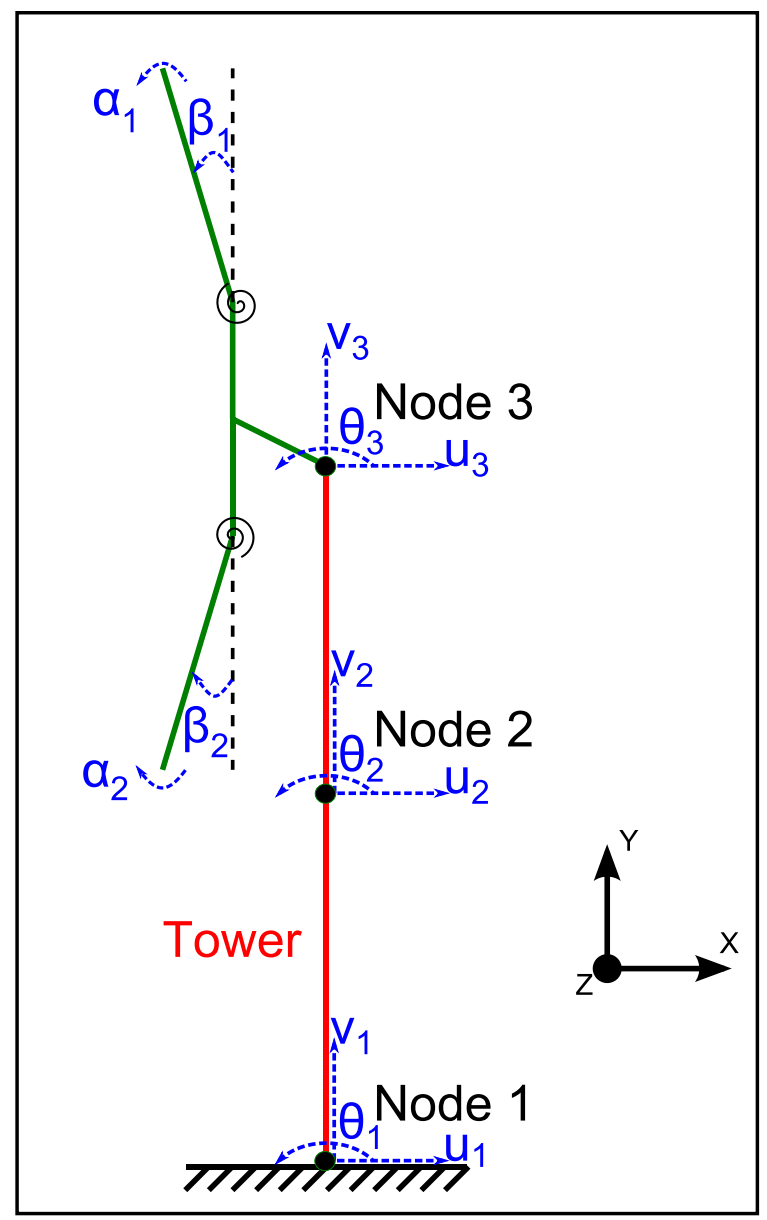

Figure 4.8. Simplified Rotor Model coupled with the Simplified Tower Model.

The mass matrix for the tower takes on the form of Eq. 89, which displays the matrix terms in indicial notation. The TM notation denotes Tower Mass and the subscripts indicate the term's corresponding degrees of freedom $\left(\mathrm{TM}_{\text {row,column }}\right)$. 


$$
\boldsymbol{T} \boldsymbol{M}=\left[\begin{array}{llllll}
T M_{u_{2}, u_{2}} & T M_{u_{2}, v_{2}} & T M_{u_{2}, \theta_{2}} & T M_{u_{2}, u_{3}} & T M_{u_{2}, v_{3}} & T M_{u_{2}, \theta_{3}} \\
T M_{v_{2}, u_{2}} & T M_{v_{2}, v_{2}} & T M_{v_{2}, \theta_{2}} & T M_{v_{2}, u_{3}} & T M_{v_{2}, v_{3}} & T M_{v_{2}, \theta_{3}} \\
T M_{\theta_{2}, u_{2}} & T M_{\theta_{2}, v_{2}} & T M_{\theta_{2}, \theta_{2}} & T M_{\theta_{2}, u_{3}} & T M_{\theta_{2}, v_{3}} & T M_{\theta_{2}, \theta_{3}} \\
T M_{u_{3}, u_{2}} & T M_{u_{3}, v_{2}} & T M_{u_{3}, \theta_{2}} & T M_{u_{3}, u_{3}} & T M_{u_{3}, v_{3}} & T M_{u_{3}, \theta_{3}} \\
T M_{v_{3}, u_{2}} & T M_{v_{3}, v_{2}} & T M_{v_{3}, \theta_{2}} & T M_{v_{3}, u_{3}} & T M_{v_{3}, v_{3}} & T M_{v_{3}, \theta_{3}} \\
T M_{\theta_{3}, u_{2}} & T M_{\theta_{3}, v_{2}} & T M_{\theta_{3}, \theta_{2}} & T M_{\theta_{3}, u_{3}} & T M_{\theta_{3}, v_{3}} & T M_{\theta_{3}, \theta_{3}}
\end{array}\right] \text { Eq. } 89
$$

Note that Eq. 89 does not display the terms associated with node 1 because the node 1 degrees of freedom are fully constrained and are thus inactive. The mass matrix for the rotor is a $5 \times 5$ matrix, corresponding to the two rotor blade hinge DOF and the three tower tip DOF. As with the tower's mass matrix, the rotor's mass matrix is displayed in indicial notation. The term RM indicates Rotor Mass and the subscripts indicate the term's corresponding degrees of freedom $\left(\mathrm{RM}_{\text {row,column }}\right)$.

$$
\boldsymbol{R} \boldsymbol{M}=\left[\begin{array}{lllll}
R M_{u_{3}, u_{3}} & R M_{u_{3}, v_{3}} & R M_{u_{3}, \theta_{3}} & R M_{u_{3}, \beta_{1}} & R M_{u_{3}, \beta_{2}} \\
R M_{v_{3}, u_{3}} & R M_{v_{3}, v_{3}} & R M_{v_{3}, \theta_{3}} & R M_{v_{3}, \beta_{1}} & R M_{v_{3}, \beta_{2}} \\
R M_{\theta_{3}, u_{3}} & R M_{\theta_{3}, v_{3}} & R M_{\theta_{3}, \theta_{3}} & R M_{\theta_{3}, \beta_{1}} & R M_{\theta_{3}, \beta_{2}} \\
R M_{\beta_{1}, u_{3}} & R M_{\beta_{1}, v_{3}} & R M_{\beta_{1}, \theta_{3}} & R M_{\beta_{1}, \beta_{1}} & R M_{\beta_{1}, \beta_{2}} \\
R M_{\beta_{2}, u_{3}} & R M_{\beta_{2}, v_{3}} & R M_{\beta_{2}, \theta_{3}} & R M_{\beta_{2}, \beta_{1}} & R M_{\beta_{2}, \beta_{2}}
\end{array}\right]
$$

The mass matrix for the coupled tower/rotor system is an $8 \times 8$ matrix, reflecting all eight of the active degrees of freedom of the system. Coupling TM and RM into SM, the System Mass matrix, is done by adding the terms with corresponding degrees of freedom. Terms that only appear in $\mathbf{T M}$ or $\mathbf{R M}$, such as $T M_{u_{2}, u_{2}}$ are simply carried over. Terms corresponding to a DOF combination that does not appear in either $\mathbf{T M}$ or $\mathbf{R M}$, such as $u_{2}, \alpha_{1}$, are simply set to zero. 
The final fully coupled, system level mass matrix, SM, is as follows:

$$
\boldsymbol{S} \boldsymbol{M}=\left[\begin{array}{cccccccc}
T M_{u_{2}, u_{2}} & T M_{u_{2}, v_{2}} & T M_{u_{2}, \theta_{2}} & T M_{u_{2}, u_{3}} & T M_{u_{2}, v_{3}} & T M_{u_{2}, \theta_{3}} & 0 \\
T M_{v_{2}, u_{2}} & T M_{v_{2}, v_{2}} & T M_{v_{2}, \theta_{2}} & T M_{v_{2}, u_{3}} & T M_{v_{2}, v_{3}} & T M_{v_{2}, \theta_{3}} & 0 \\
T M_{\theta_{2}, u_{2}} & T M_{\theta_{2}, v_{2}} & T M_{\theta_{2}, \theta_{2}} & T M_{\theta_{2}, u_{3}} & T M_{\theta_{2}, v_{3}} & T M_{\theta_{2}, \theta_{3}} & 0 \\
T M_{u_{3}, u_{2}} & T M_{u_{3}, v_{2}} & T M_{u_{3}, \theta_{2}} & T M_{u_{3}, u_{3}}+R M_{u_{3}, u_{3}} & T M_{u_{3}, v_{3}}+R M_{u_{3}, v_{3}} & T M_{u_{3}, \theta_{3}}+R M_{u_{3}, \theta_{3}} & R M_{u_{3}, \beta_{1}} & R M_{u_{3}, \beta_{2}} \\
T M_{v_{3}, u_{2}} & T M_{v_{3}, v_{2}} & T M_{v_{3}, \theta_{2}} & T M_{v_{3}, u_{3}}+R M_{v_{3}, u_{3}} & T M_{v_{3}, v_{3}}+R M_{v_{3}, v_{3}} & T M_{v_{3}, \theta_{3}}+R M_{v_{3}, \theta_{3}} & R M_{v_{3}, \beta_{1}} & R M_{v_{3}, \beta_{2}} \\
T M_{\theta_{3}, u_{2}} & T M_{\theta_{3}, v_{2}} & T M_{\theta_{3}, \theta_{2}} & T M_{\theta_{3}, u_{3}}+R M_{\theta_{3}, u_{3}} & T M_{\theta_{3}, v_{3}}+R M_{\theta_{3}, v_{3}} & T M_{\theta_{3}, \theta_{3}}+R M_{\theta_{3}, \theta_{3}} & R M_{\theta_{3}, \beta_{1}} & R M_{\theta_{3}, \beta_{2}} \\
0 & 0 & 0 & R M_{\beta_{1}, u_{3}} & R M_{\beta_{1}, v_{3}} & R M_{\beta_{1}, \theta_{3}} & R M_{\beta_{1}, \beta_{1}} & R M_{\beta_{1}, \beta_{2}} \\
0 & 0 & 0 & R M_{\beta_{2}, u_{3}} & R M_{\beta_{2}, v_{3}} & R M_{\beta_{2}, \theta_{3}} & R M_{\beta_{2}, \beta_{1}} & R M_{\beta_{2}, \beta_{2}}
\end{array}\right]
$$

The exact same method can be used to find the system level stiffness matrix, SK. With SM and SK fully defined the fully coupled, system level, EOM becomes:

$$
\boldsymbol{S M}\left\{\begin{array}{c}
\ddot{u}_{2} \\
\ddot{v}_{2} \\
\ddot{\theta}_{2} \\
\ddot{u}_{3} \\
\ddot{v}_{3} \\
\ddot{\vartheta}_{3} \\
\ddot{\alpha}_{1} \\
\ddot{\alpha}_{2}
\end{array}\right\}+\boldsymbol{S} \boldsymbol{K}\left\{\begin{array}{l}
u_{2} \\
v_{2} \\
\theta_{2} \\
u_{3} \\
v_{3} \\
\vartheta_{3} \\
\alpha_{1} \\
\alpha_{2}
\end{array}\right\}=\boldsymbol{F}\left(t, u_{2}, v_{2}, \theta_{2}, u_{3}, v_{3}, \theta_{3}, \alpha_{1}, \alpha_{2}\right)
$$

Eq. 92 represents the culmination of the Rotor Tower Coupling section. In summary the EOM for the upper blade and lower blade of a simplified rotor system were developed using the Lagrangian method. The individual blade EOMs were then combined into an overall EOM for the Simplified Rotor Model. Finally the rotor EOM was combined with the EOM for a simple tower model. The resulting EOM, represented by Eq. 92, are for the fully coupled rotor/tower model displayed in Figure 4.8 .

A practical tower model would feature more than three nodes so the tower mass and stiffness matrices would be larger than those in this simplified example. Nevertheless the method for coupling the tower and rotor EOM would be identical. 


\subsection{Generalized Rotor Model}

In Section 4.2 the basic principles of the hinge equivalent blade model were discussed and the important equations were derived. In Section 4.3 the EOM were developed for the Simplified Rotor Model and the method for coupling the rotor model with the Simplified Tower Model was illustrated. The simplified rotor model, displayed in Figure 4.5, has some major restrictions.

- The rotor model is limited to two blades.

- The rotor must be parked with the blades collinear with the tower (i.e., one blade points straight up, the other straight down)

Clearly, the restrictions associated with the simplified blade model render it impractical for most useful applications. For these reasons, a generalized model must be developed; this model will be referred to as the Generalized Rotor Model in this work. The Simplified Rotor Model was used for illustrating many important concepts in the Rotor Tower Coupling section because the formulas associated the Generalized Rotor Model are quite cumbersome. The Generalized Rotor Model uses the exact same individual blade model utilized by the Simplified Rotor Model and developed in Section 4.2.3. The approach for developing the Generalized Rotor Model kinetic and potential energy expressions for each blade are identical to methods used for the Simplified Rotor Model. Furthermore the application of Lagrange's Equations to develop the EOM for the Generalized Rotor Model are identical to the Simplified Rotor Model. Finally the method for coupling the Generalized Rotor Model EOM with the tower EOM is the same as the method illustrated in Section 4.3.6.

Despite the similarities between the Simplified and Generalized Rotor Models there are two very important differences. The Generalized Rotor Model:

- Allows for the rotor to be modeled in any azimuth position

- Allows for a 2 or 3 bladed rotor model 
The remainder of the Generalized Rotor Model section will discuss only the three key modeling differences which separate if from the Simplified Rotor Model, these differences are as follows:

- Conventions for tracking the azimuth location of each blade in the Generalized Rotor Model.

- See Section 4.4.1

- The 3D coordinate system used to describe each blade in the Generalized Rotor Model.

○ See Section 4.4.2

- Updated kinetic energy expression which accounts for blade azimuth location

- See Section 4.4.3

In the interest of brevity the full derivation of the Generalized Rotor Model will not be presented in this chapter as it was for the Simplified Rotor Model. The detailed generalized rotor model derivation was done in MathCAD and a print out of the MathCAD analysis is included in Appendix A (three bladed Generalized Rotor Model).

Section 4.4 will conclude by listing the expressions for each term of the Mass and Stiffness matrix for a three bladed Generalized Rotor Model. The terms of the two bladed Generalized Rotor Model can be found in Appendix B.

\subsubsection{Generalized Rotor Model Azimuth Conventions}

The Generalized Rotor Model allows for the rotor to be modeled with the blades in any rotational orientation based on the user defined rotor azimuth angle $\left(\Psi_{\mathrm{r}}\right)$. A positive $\Psi_{\mathrm{r}}$ value represents a rotor rotation in the counterclockwise direction (from the perspective of an upwind observer looking downwind toward the rotor). Each blade is associated with a relative blade azimuth angle $\Psi_{\mathrm{b}}$ which is the angular distance between each blade and the reference blade. For a three bladed rotor, blade 1, 2, and 3 would be associated with blade azimuth angles $\Psi_{1 \mathrm{~b}}, \Psi_{2 \mathrm{~b}}$, and $\Psi_{3 \mathrm{~b}}$ respectively. As with the rotor azimuth angle the blade $\Psi_{\mathrm{b}}$ values are positive in the counterclockwise direction. The reference blade has a $\Psi_{\mathrm{b}}$ of zero, thus the position of the reference blade is the same as the $\Psi_{\mathrm{r}}$ value. For simplicity blade 1 will always be treated as the 
reference blade, thus $\Psi_{1 \mathrm{~b}}$ is always equal to zero. A $\Psi_{\mathrm{r}}$ angle of zero corresponds with the reference blade, blade 1, positioned in the vertical upward position as shown in Figure 4.9a.

For a typical three bladed wind turbine with equally spaced blades the $\Psi_{2 \mathrm{~b}}$ and $\Psi_{3 \mathrm{~b}}$ values would be set to $\frac{2 \pi}{3}$ and $\frac{4 \pi}{3}$ respectively. The current angular position of the i'th blade, $\Psi_{\mathrm{ic}}$ (c for current) becomes:

$$
\Psi_{\mathrm{ic}}=\Psi_{\mathrm{r}}+\Psi_{\mathrm{ib}}
$$

For example, if the user enters a $\Psi_{\mathrm{r}}$ value of $\frac{3 \pi}{2}$ the resulting $\Psi_{1 \mathrm{c}}, \Psi_{2 \mathrm{c}}$, and $\Psi_{3 \mathrm{c}}$ angles would be $\frac{3 \pi}{2}, 2 \frac{\pi}{6}$, and $2 \frac{5 \pi}{6}$ respectively. This configuration is shown in Figure $4.9 \mathrm{~b}$.

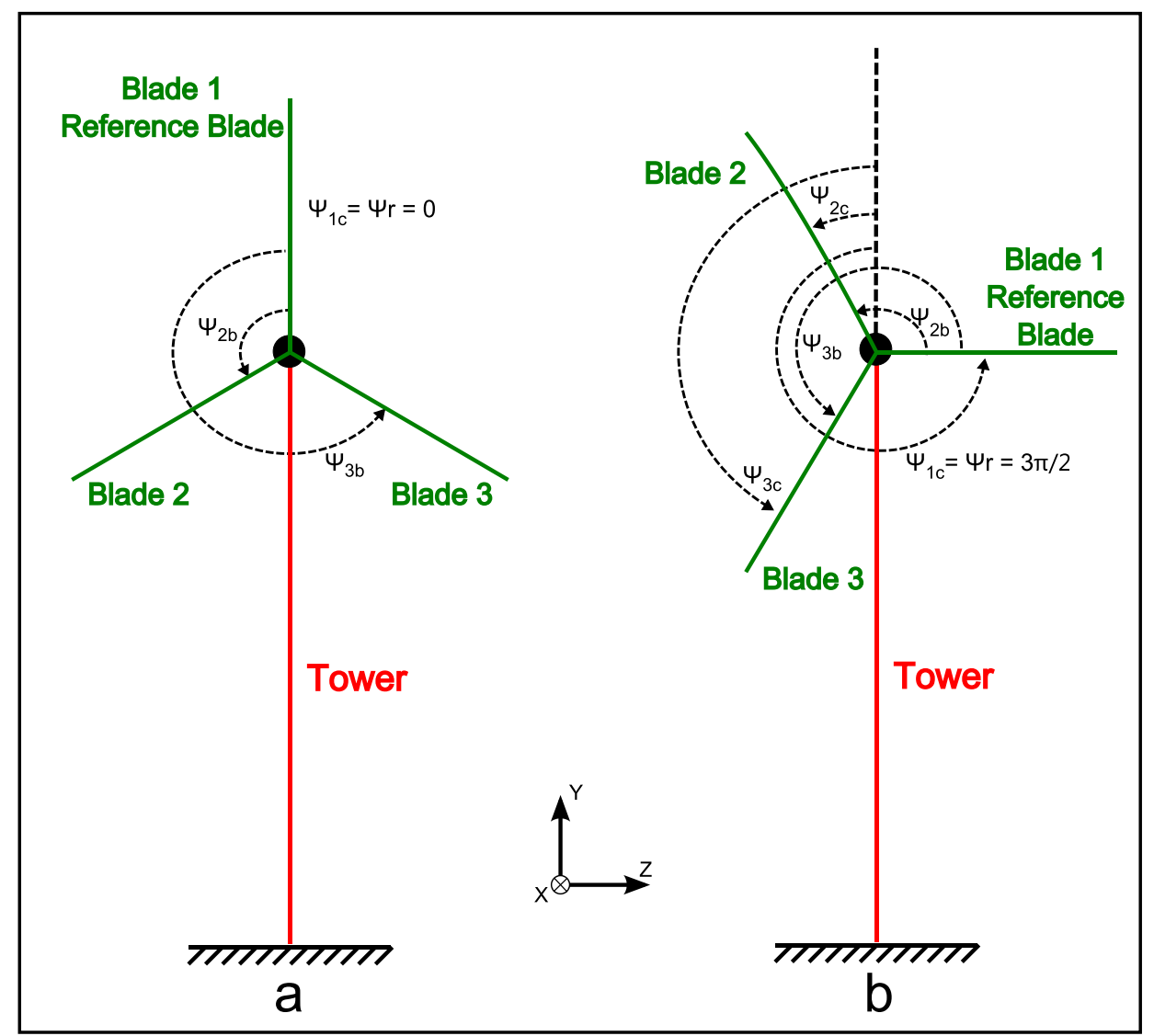

Figure 4.9. Example Blade and rotor azimuth angles used for the Generalized Rotor Model 
The blade azimuth conventions for a two blade rotor would be very similar to the azimuth coordinate system shown in Figure 4.9. The two bladed rotor azimuth conventions would also assign blade 1 as the reference blade. A two bladed rotor obviously lacks a third blade and therefore there would be no $\Psi_{3 b}$ value and the $\Psi_{2 b}$ value would be equal to $\pi$.

\subsubsection{Generalized Rotor Model Coordinate System}

The Simplified Rotor Model used two similar but separate blade models for the upper and lower blades, shown in Figure 4.6 and Figure 4.7, respectively. The Generalized Rotor Model uses only one blade model which is the same for each blade with the exception of the blade azimuth angle, $\Psi_{\mathrm{b}}$. Thus this generalized Baseline Blade Model is used as the foundation for each blade model in the Generalized Rotor Model. In the case of a three bladed rotor the Baseline Blade Model, shown in Figure 4.10, is applied to each blade by setting the $\Psi_{\mathrm{b}}$ value to $\Psi_{1 \mathrm{~b}}, \Psi_{2 \mathrm{~b}}$, and $\Psi_{3 \mathrm{~b}}$ for blades 1,2 , and 3 respectively.

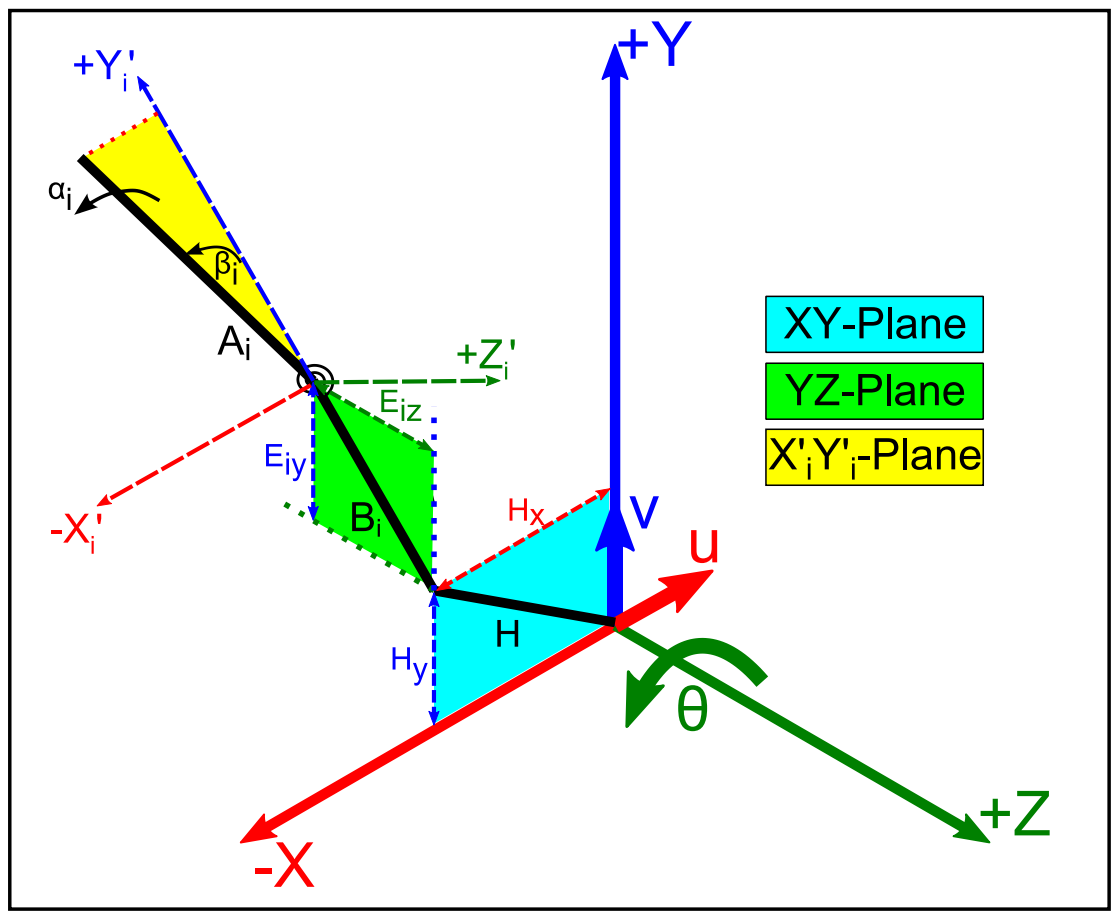

Figure 4.10. Baseline Blade Model for the i'th blade in the Generalize Rotor Model. Generalized coordinates and key distances are shown. 
Figure 4.10 displays the Baseline Blade Model used for the Generalized Rotor Model in 3D space. Note that the hub $\mathrm{H}$ is in the $\mathrm{X}-\mathrm{Y}$ plane and the blade root $\left(\right.$ section $\mathrm{B}_{\mathrm{i}}$ ) is in the $\mathrm{Y}-\mathrm{Z}$ plane. The secondary blade coordinate system $\mathrm{X}_{\mathrm{i}}{ }^{\prime}-\mathrm{Y}_{\mathrm{i}}{ }^{\prime}-\mathrm{Z}_{\mathrm{i}}{ }^{\prime}$ has its origin at the blade's hinge and rotates with the blade such that $Y_{i}{ }^{\prime}$ remains collinear with the blade base $B_{i}$. An additional constraint on the $X_{i}{ }^{\prime}-Y_{i}{ }^{\prime}-Z_{i}{ }^{\prime}$ coordinate system is that $X_{i}{ }^{\prime}$ remain parallel with $X$. The blade tip (section $A_{i}$ ) flaps with angle $\alpha_{i}$ in the $\mathrm{X}_{\mathrm{i}}{ }^{\prime}-\mathrm{Y}_{\mathrm{i}}{ }^{\prime}$ plane. The $\mathrm{X}_{\mathrm{i}}{ }^{\prime}-\mathrm{Y}_{\mathrm{i}}{ }^{\prime}-\mathrm{Z}_{\mathrm{i}}{ }^{\prime}$ coordinate system will be used to describe the motion and energy of the blade tip.

The generalized coordinates for the i'th blade in the Generalized Rotor Model are displayed in Figure 4.10 and are:

- u horizontal deflection of tower's tip

- v vertical deflection of tower's tip

- $\theta \quad$ rotation of tower's tip

- $\Psi_{\text {ic }}$ the blade's azimuth position

- $\alpha_{i} \quad$ absolute rotation of section $\mathrm{A}_{\mathrm{i}}$, in the $\mathrm{X}_{\mathrm{i}}{ }^{\prime}-\mathrm{Y}_{\mathrm{i}}{ }^{\prime}$ Plane, about the blade's hinge ...........wrt the $Y_{i}^{\prime}-Z_{i}^{\prime}$ Plane

- $\beta_{i} \quad$ relative rotation of section $\mathrm{A}_{\mathrm{i}}$, in the $\mathrm{X}_{\mathrm{i}}$ '- $\mathrm{Y}_{\mathrm{i}}$ ' Plane, about the blade's hinge wrt Section $\mathrm{B}_{\mathrm{i}}$

Key distances are also defined in Figure 4.10 and are:

- $\mathrm{H}_{\mathrm{x}}$ the distance from the tower tip to blade root due to the hub in the X-Axis

- $\mathrm{H}_{\mathrm{y}} \quad$ the distance from the tower tip to blade root due to the hub in the Y-Axis

- $\mathrm{E}_{\mathrm{iy}}$ the distance from the blade's root to the blade's hinge in the Y-Axis

- $\mathrm{E}_{\mathrm{iz}}$ the distance from the blade's root to the blade's hinge in the Z-Axis 
For simplicity dimensions $\mathrm{R}$ and $\mathrm{E}$ are not displayed because they are consistent with the dimensioning of the Simplified Blade Model as displayed in Figure 4.6.

The two coordinate systems displayed in Figure 4.10 have unit vectors associated with them. These unit vectors are displayed in Figure 4.11.

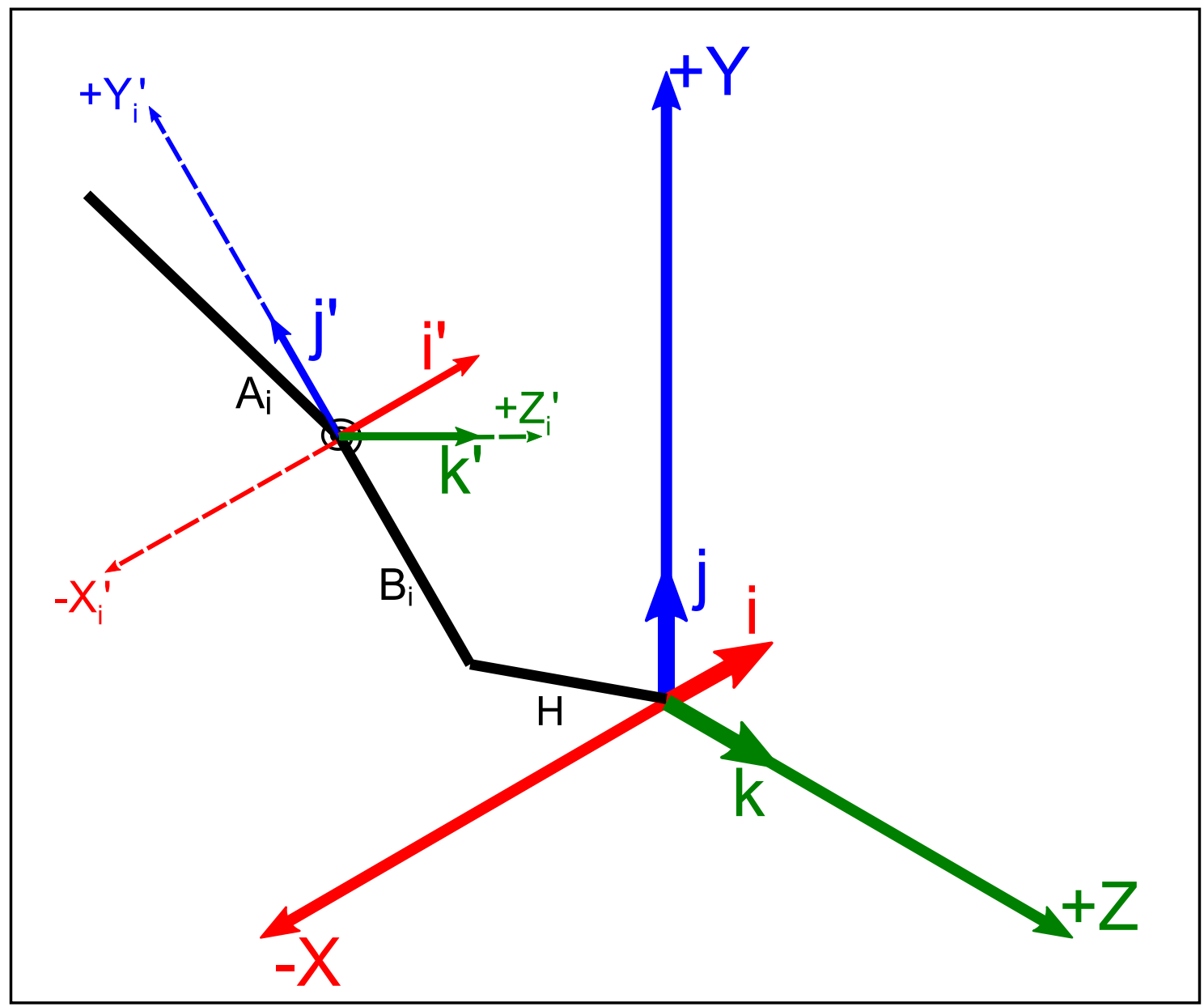

Figure 4.11. Unit vectors associated with the Baseline Blade Model.

The EOM derivation for the Generalized Rotor Blade Model starts by developing the blade kinetic and potential energies in terms of mass centered coordinate systems. Coordinate transformations must then be used to rewrite the energy expressions in terms of the generalized coordinates, this process was previously illustrated for the Simplified Rotor Model. One of the 
key differences for the Generalized Rotor Model is the added complexity associated with the 3D coordinate transformations. Figure 4.12 displays the mass centered coordinate systems for the blade sections $\mathrm{A}_{\mathrm{i}}$ and $\mathrm{B}_{\mathrm{i}}$.

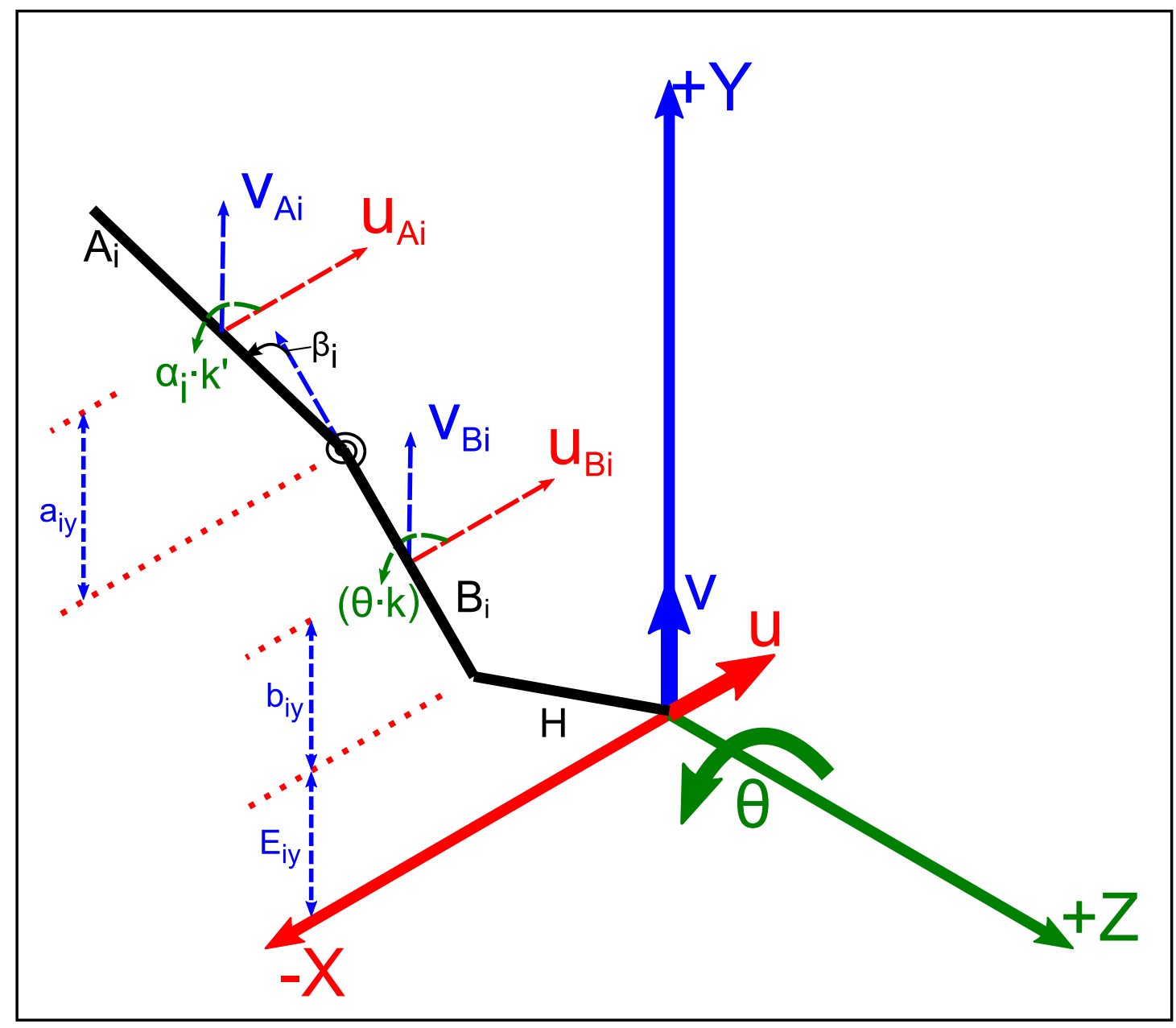

Figure 4.12. Mass centered coordinate systems for the Baseline Blade Model.

Critical distances such as $\mathrm{a}_{\mathrm{iy}}, \mathrm{b}_{\mathrm{iy}}$, and $\mathrm{E}_{\mathrm{iy}}$ are now functions of the blades azimuth position. Additionally, the motion of the each blade's tip substantially complicates the kinetic energy expressions.

\subsubsection{Generalized Rotor Blade Kinetic Energy}

The method used to determine the kinetic energy of each blade mirrors the method used for the Simplified Rotor Model. As with the Simplified Rotor Model, the kinetic energy of each blade 
section is first described in terms of the mass centered coordinates. Coordinate transformations are then used to translate the kinetic energy expressions into functions of the generalized coordinates.

The kinetic energy of the hub for the Generalized Rotor model is exactly the same as the value determined for the Simplified Rotor Model, see Eq. 42.

The kinetic energy of section A and B of the Generalized Rotor Model both differ from the kinetic energy of section B and A in the Simplified Rotor Model in two key areas:

1. The coordinate transformation used for blade motion in the $\mathrm{X}$ direction must account for the blade's azimuth position. See Section 4.4.3.1.

2. Misalignment between the blade's principal axis and the global coordinate system is induced by rotor azimuth angle $\left(\Psi_{\mathrm{r}}\right)$. Derivation of additional blade mass properties is necessary to account for this misalignment and to determine the blade's kinetic energy. See Section 0.

Both section A and B must account for items 1 and 2 above. Item 1 above will be addressed in the context of section B and item 2 will be addressed in the context of section A.

\subsubsection{Blade Motion in the X-Direction and Azimuth Position}

The coordinate transformation used for the motion of section B in the X-direction must change to account for the blade's azimuth position. The coordinate transformation used for the Simplified Rotor Model's section B motion in the X-direction was Eq. 44. The analogous transformation for the Baseline Blade Model used in the Generalized Rotor Model is:

$$
u_{B i}=u-\left(\frac{E \cdot \cos \left(\Psi_{\mathrm{ic}}\right)}{2}+H_{y}\right) \cdot \theta
$$




\subsubsection{Kinetic Energy and Additional Blade Mass Properties}

The kinetic energy expression for the blade tip, section A, is more complex than its counterpart for the Simplified Rotor Model. Previously, Eq. 47 was used to express the kinetic energy of the Simplified Rotor Model as a function of its mass-centered coordinate system. The analogous to Eq. 47 for the Baseline Blade Model used in the Generalized Rotor Model is:

$$
T_{A i}=\frac{M_{A} \cdot\left(\dot{u}_{A i}^{2}+\dot{v}_{A i}^{2}\right)+H_{A i} \cdot \omega_{A i}}{2}
$$

Where $H_{A i}$ is the angular momentum of section $\mathrm{A}_{\mathrm{i}}$ and $\omega_{A i}$ is the rotational velocity of section $\mathrm{A}_{\mathrm{i}}$. This method for determining the kinetic energy of rigid bodies undergoing 3D motion is laid out in Meriam's et al. classic work on Dynamics (27). The additional kinetic energy complexity is driven by the varying azimuth angles and the resulting misalignment between the principal axis for section A and the global axis X-Y-Z.

Again energy associated with blade rotation in azimuth is neglected. Thus for our purpose, the pertinent rotational velocity of section $\mathrm{A}_{\mathrm{i}}$ is a combination of the tower tip rotation and the rotation about the blade hinge. Consistent with Figure 4.12, the rotational velocity of section $A_{i}$ may be expressed as:

$$
\omega_{A i}=\dot{\alpha}_{\imath} \cdot k^{\prime}
$$

The rotational velocity $\dot{\alpha_{1}}$ results from the flapping motion of the blade about the hinge as well as tower tip rotation. $\dot{\alpha}_{1}$ is in the $k^{\prime}$ direction which is based on the $\mathrm{X}_{\mathrm{i}}{ }^{\prime}-\mathrm{Y}_{\mathrm{i}}{ }^{\prime}-\mathrm{Z}_{\mathrm{i}}{ }^{\prime}$ coordinate system as displayed in Figure 4.11. The following coordinate transformation is used to express $\omega_{A i}$ in terms of the global X-Y-Z coordinate system.

$$
k^{\prime}=k \cdot \cos \left(\Psi_{i c}\right)+j \cdot \sin \left(\Psi_{\mathrm{ic}}\right)
$$


Substituting Eq. 97 into Eq. 96 transforms the section $A_{i}$ rotational velocity into a function of the global coordinate system.

$$
\omega_{A i}=\dot{\alpha}_{l}\left[k \cdot \cos \left(\Psi_{i c}\right)+j \cdot \sin \left(\Psi_{\mathrm{ic}}\right)\right]
$$

Eq. 98

The section $\mathrm{A}_{\mathrm{i}}$ rotational velocity is best described by breaking it into its vector components.

$$
\begin{aligned}
& \omega_{A i y}=\dot{\alpha}_{\imath} \cdot \sin \left(\Psi_{\mathrm{ic}}\right) \cdot \mathrm{j} \\
& \omega_{A i z}=\dot{\alpha} \cdot \cos \left(\Psi_{\mathrm{ic}}\right) \cdot \mathrm{k}
\end{aligned}
$$

The momentum of section $\mathrm{A}_{\mathrm{i}}, H_{A i}$, which first appeared in Eq. 95 may be fully described by the following equation.

$$
\begin{aligned}
H_{A i} & =\left(\mathrm{I}_{\text {Aixx }} \omega_{\text {Aix }}-\mathrm{I}_{\text {Aixy }} \omega_{\text {Aiy }}-\mathrm{I}_{\text {Aixz }} \omega_{\text {Aiz }}\right) i \\
& +\left(-\mathrm{I}_{\text {Aiyx }} \omega_{\text {Aix }}+\mathrm{I}_{\text {Aiyy }} \omega_{\text {Aiy }}-\mathrm{I}_{\text {Aiyz }} \omega_{\text {Aiz }}\right) j \\
& +\left(-\mathrm{I}_{\text {Aizx }} \omega_{\text {Aix }}-\mathrm{I}_{\text {Aizy }} \omega_{\text {Aiy }}+\mathrm{I}_{\text {Aizz }} \omega_{\text {Aiz }}\right) k
\end{aligned}
$$

Eq. 101

In Eq. 95 the angular momentum appears in the following term: $\frac{1}{2} H_{A i} \cdot \omega_{A i}$. Performing the dot product and arranging terms result in the following equation.

$$
\begin{aligned}
\frac{1}{2} H_{A i} \cdot \omega_{A i}= & \frac{1}{2}\left(\mathrm{I}_{\text {Aixx }} \omega_{\text {Aix }}{ }^{2}+\mathrm{I}_{\text {Aiyy }} \omega_{\text {Aiy }}{ }^{2}+\mathrm{I}_{\text {Aizz }} \omega_{\text {Aiz }}{ }^{2}\right) \\
& -\left(\mathrm{I}_{\text {Aixy }} \omega_{\text {Aix }} \omega_{\text {Aiy }}+\mathrm{I}_{\text {Aixz }} \omega_{\text {Aix }} \omega_{\text {Aiz }}+\mathrm{I}_{\text {Aiyz }} \omega_{\text {Aiy }} \omega_{\text {Aiz }}\right)
\end{aligned}
$$

Note from Eq. 99 and Eq. 100 section A has no rotational velocity in the X-direction $\left(\omega_{\text {Aix }}=0\right.$. i). Thus Eq. 102 may be simplified by eliminating all terms containing $\omega_{\text {Aix }}$, as follows. 


$$
\frac{1}{2} H_{A i} \cdot \omega_{A i}=\frac{1}{2}\left(\mathrm{I}_{\text {Aiyy }} \omega_{\text {Aiy }}{ }^{2}+\mathrm{I}_{\text {Aizz }} \omega_{\text {Aiz }}{ }^{2}\right)-\left(\mathrm{I}_{\text {Aiyz }} \omega_{\text {Aiy }} \omega_{\text {Aiz }}\right)
$$

From Eq. 103 we see that the mass moment of inertia must be found for section $A_{i}$ about the yy and $\mathrm{zz}$ axis. Additionally, the product of inertia for section $\mathrm{A}_{\mathrm{i}}$ must be found about $\mathrm{yz}$. The noted mass properties are most easily determined by integrating about the $\mathrm{Y}^{\prime}$ axis, which follows section A. Figure 4.13 shows section A and axis Y' projected onto the global Y-Z plane.

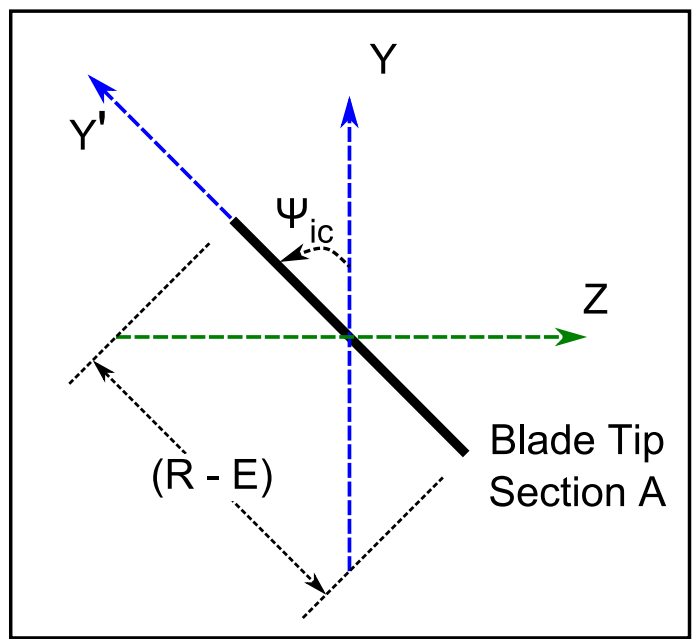

Figure 4.13. Section A from the Generalize Rotor Model - Baseline Blade Model.

The section A moment of inertia about the YY axis is found using the following formula.

$$
\mathrm{I}_{\text {Aiyy }}=\oint \mathrm{Z}^{2} \mathrm{dm}
$$

The differential mass, dm, may be related to section A length and mass by the following expression.

$$
d m=\frac{M_{A}}{(R-E)} \cdot d Y^{\prime}
$$


Substituting Eq. 105 into Eq. 104 and relating Z to Y' using simple trigonometry gives:

$$
\mathrm{I}_{\text {Aiyy }}=\frac{M_{A}}{(R-E)} \int_{\frac{-(\mathrm{R}-\mathrm{E})}{2}}^{\frac{\mathrm{R}-\mathrm{E}}{2}}\left(-\mathrm{Y}^{\prime} \cdot \sin \left(\Psi_{\mathrm{ic}}\right)\right)^{2} \mathrm{~d} \mathrm{Y}^{\prime}
$$

Simplifying Eq. 106 gives:

$$
\mathrm{I}_{\text {Aiyy }}=\frac{1}{12} M_{A}(R-E)^{2} \cdot\left(\sin \left(\Psi_{\text {ic }}\right)\right)^{2}
$$

Using a similar method for $\mathrm{I}_{\mathrm{Aizz}}$ and $\mathrm{I}_{\text {Aiyz }}$ along with the appropriate definitions of the respective mass properties yields:

$$
\begin{aligned}
& \mathrm{I}_{\mathrm{Aizz}}=\frac{1}{12} M_{A}(R-E)^{2} \cdot\left(\cos \left(\Psi_{\mathrm{ic}}\right)\right)^{2} \\
& \mathrm{I}_{\mathrm{Aiyz}}=-\frac{1}{24} M_{A}(R-E)^{2} \cdot \sin \left(2 \Psi_{\mathrm{ic}}\right)
\end{aligned}
$$

Combining equations Eq. 95, Eq. 103, Eq. 107, Eq. 108, and Eq. 109 allows for the solution of the kinetic energy of blade section A in the Generalized Rotor Model.

\subsubsection{Generalized Rotor Model Conclusion}

The Generalized Rotor Model shares many similarities with the Simplified Rotor Model, which was used to illustrate key concepts in this chapter. The major differences in the derivation of the Generalized Rotor Model and the Simplified Rotor Model were laid out in Sections 4.4.1 through 4.4.3. The detailed derivation of the three bladed and two bladed Generalized Rotor Models is presented in Appendices A and B. The final three bladed Generalized Rotor Model solution is 
listed below; the solution for the two bladed version is very similar and may be found in Appendix B.

The final three bladed Generalized Rotor Model takes on the following form:

$$
\boldsymbol{M}_{\boldsymbol{G E N}}\left\{\begin{array}{c}
\ddot{u} \\
\ddot{v} \\
\ddot{\vartheta} \\
\ddot{\alpha_{1}} \\
\ddot{\alpha_{2}} \\
\ddot{\alpha_{3}}
\end{array}\right\}+\boldsymbol{K}_{\boldsymbol{G E N}}\left\{\begin{array}{c}
u \\
v \\
\vartheta \\
\alpha_{1} \\
\alpha_{2} \\
\alpha_{3}
\end{array}\right\}=\boldsymbol{F}\left(t, u, v, \theta, \alpha_{1}, \alpha_{2}, \alpha_{3}\right)
$$

The mass and stiffness matrices are given the subscripts GRM to signify the Generalized Rotor Model. Each of the coefficient matrices is $6 \times 6$ corresponding to the 6 degrees of freedom used in the 3 bladed Generalized Rotor Model.

The three bladed Generalized Rotor Model mass matrix terms equal to zero are:

$$
\begin{aligned}
& M_{G R M 3(1,2)}=M_{G R M 3(2,1)}=0 \\
& M_{G R M 3(2,4)}=M_{G R M 3(4,2)}=0 \\
& M_{G R M 3(2,5)}=M_{G R M 3(5,2)}=0 \\
& M_{G R M 3(2,6)}=M_{G R M 3(6,2)}=0 \\
& M_{G R M 3(4,5)}=M_{G R M 3(5,4)}=0 \\
& M_{G R M 3(4,6)}=M_{G R M 3(6,4)}=0 \\
& M_{G R M 3(5,6)}=M_{G R M 3(6,5)}=0
\end{aligned}
$$


The nonzero three bladed Generalized Rotor Model mass matrix terms are:

\begin{tabular}{|c|c|}
\hline$M_{G R M 3(1,1)}=M_{G R M 3(2,2)}=M_{H}+3 M_{A}+3 M_{B}$ & Eq. 112 \\
\hline $\begin{array}{c}M_{G R M 3(1,3)}=M_{G R M 3(3,1)}=-3 \cdot H_{y} \cdot\left(M_{A}+M_{B}\right)-\left(M_{A} \cdot E_{y}+\frac{M_{B} \cdot E_{y}}{2}\right) \cdot\left(\cos \Psi_{1 \mathrm{c}}+\right. \\
\left.\cos \Psi_{2 \mathrm{c}}+\cos \Psi_{3 \mathrm{c}}\right)\end{array}$ & Eq. 113 \\
\hline$M_{G R M 3(1,4)}=M_{G R M 3(4,1)}=-M_{A} \cdot a$ & Eq. 114 \\
\hline$M_{G R M 3(1,5)}=M_{G R M 3(5,1)}=-M_{A} \cdot a$ & Eq. 115 \\
\hline$M_{G R M 3(1,6)}=M_{G R M 3(6,1)}=-M_{A} \cdot a$ & Eq. 116 \\
\hline$M_{G R M 3(2,3)}=M_{G R M 3(3,2)}=-3 \cdot H_{x} \cdot\left(M_{A}+M_{B}\right)$ & Eq. 117 \\
\hline $\begin{array}{l}M_{G R M 3(3,3)}=I_{H}+\left(I_{B}\right) \cdot\left[\left(\cos \Psi_{1 \mathrm{c}}\right)^{2}+\left(\cos \Psi_{2 \mathrm{c}}\right)^{2}+\left(\cos \Psi_{3 \mathrm{c}}\right)^{2}\right] \\
+M_{B} \cdot\left\{3 \cdot H_{x}^{2}+\left[H_{y}+\frac{1}{2} \cdot E \cdot \cos \Psi_{1 \mathrm{c}}\right]^{2}+\left[H_{y}+\frac{1}{2} \cdot E \cdot \cos \Psi_{2 \mathrm{c}}\right]^{2}\right. \\
\left.+\left[H_{y}+\frac{1}{2} \cdot E \cdot \cos \Psi_{3 \mathrm{c}}\right]^{2}\right\} \\
+M_{A} \cdot\left\{3 \cdot H_{x}^{2}+\left[H_{y}+E \cdot \cos \Psi_{1 \mathrm{c}}\right]^{2}+\left[H_{y}+E \cdot \cos \Psi_{2 \mathrm{c}}\right]^{2}\right. \\
\left.+\left[H_{y}+E \cdot \cos \Psi_{3 \mathrm{c}}\right]^{2}\right\}\end{array}$ & Eq. 118 \\
\hline$M_{G R M 3(3,4)}=M_{G R M 3(4,3)}=M_{A} \cdot a \cdot\left(H_{y}+E \cdot \cos \Psi_{1 \mathrm{c}}\right)$ & Eq. 119 \\
\hline$M_{G R M 3(3,5)}=M_{G R M 3(5,3)}=M_{A} \cdot a \cdot\left(H_{y}+E \cdot \cos \Psi_{2 c}\right)$ & Eq. 120 \\
\hline$M_{G R M 3(3,6)}=M_{G R M 3(6,3)}=M_{A} \cdot a \cdot\left(H_{y}+E \cdot \cos \Psi_{3 c}\right)$ & Eq. 121 \\
\hline $\begin{array}{l}M_{G R M 3(4,4)} \\
\qquad \begin{array}{l}M_{A} \cdot a^{2}+I_{A} \\
\quad \cdot\left[\left(\cos \Psi_{1 \mathrm{c}}\right)^{4}+\left(\sin \Psi_{1 \mathrm{c}}\right)^{4}+\left(\sin 2 \Psi_{1 \mathrm{c}}\right) \cdot\left(\cos \Psi_{1 \mathrm{c}}\right) \cdot\left(\sin \Psi_{1 \mathrm{c}}\right)\right]\end{array}\end{array}$ & Eq. 122 \\
\hline $\begin{array}{l}M_{G R M 3(5,5)} \\
\qquad \begin{array}{l}M_{A} \cdot a^{2}+I_{A} \\
\quad \cdot\left[\left(\cos \Psi_{2 c}\right)^{4}+\left(\sin \Psi_{2 c}\right)^{4}+\left(\sin 2 \Psi_{2 c}\right) \cdot\left(\cos \Psi_{2 c}\right) \cdot\left(\sin \Psi_{2 c}\right)\right]\end{array}\end{array}$ & Eq. 123 \\
\hline 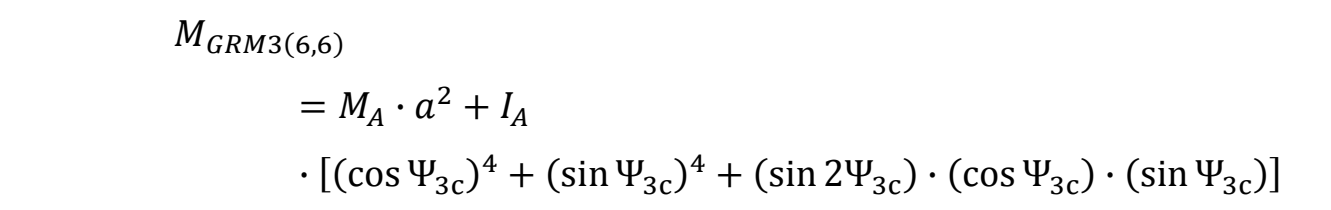 & Eq. 124 \\
\hline
\end{tabular}


All of the three bladed Generalized Rotor Model Stiffness Matrix terms are zero except the following terms, where $K_{e q}$ is defined in Eq. 75:

\begin{tabular}{|c|c|}
\hline$K_{G R M 3(3,3)}=K_{e q} \cdot\left[\left(\cos \Psi_{1 \mathrm{c}}\right)^{2}+\left(\cos \Psi_{2 \mathrm{c}}\right)^{2}+\left(\cos \Psi_{3 \mathrm{c}}\right)^{2}\right]$ & Eq. 125 \\
\hline$K_{G R M 3(4,3)}=K_{G R M 3(3,4)}=-K_{e q}\left(\cos \Psi_{1 \mathrm{c}}\right)$ & Eq. 126 \\
\hline$K_{G R M 3(5,3)}=K_{G R M 3(3,5)}=-K_{e q}\left(\cos \Psi_{2 \mathrm{c}}\right)$ & Eq. 127 \\
\hline$K_{G R M 3(6,3)}=K_{G R M 3(3,6)}=-K_{e q}\left(\cos \Psi_{3 \mathrm{c}}\right)$ & Eq. 128 \\
\hline$K_{G R M 3(4,4)}=K_{G R M 3(5,5)}=K_{G R M 3(6,6)}=K_{e q}$ & Eq. 129 \\
\hline
\end{tabular}




\section{Load Cases}

\subsection{Introduction}

Wind turbines experience complex environmental loading due to varying wind conditions. Variations include changes in wind speed, direction, gusts, turbulence, changes in wind profile as a function of height, and changes in the wind profile across the swept surface of the rotor blades. For initial design of a wind turbine tower it is possible to simplify the structural analysis process by considering only a single conservative load case that is intended to bound all conditions but this approach lacks the fidelity necessary for system optimization. To design a robust and optimized system it is necessary to consider multiple load cases, both static and dynamic, to accurately simulate the system's response to complex conditions.

Multiple Aeroelastic load cases are implemented in this work and feature a combination of design load cases specified by IEC 61400-2 (3) as well as several custom load cases. Both static and dynamic load cases are considered. Several conservative assumptions are made regarding possible wind turbine fault conditions which allow the consolidation of IEC aeroelastic load cases; justification for omitted IEC load cases is included in this chapter. The custom load cases include two simple static load cases, useful for vetting initial tower concepts, as well as a swept sine dynamic load case which is useful for detailed study of coupled tower / rotor response to dynamic loads.

To determine the loads acting on a wind turbine it is necessary to not only have a definition of the wind profile but it is also necessary to also establish the aerodynamic correlations between wind velocity and loads. Therefore this chapter will begin by establishing the aerodynamic correlations required to determine loads induced by the wind. The load cases themselves will then be detailed. 


\subsection{Aerodynamic Load Parameters}

Aeroelastic load cases are specified in terms of wind profiles. To perform an aeroelastic simulation it is necessary to determine the loads, induced by the wind profile, acting on the system. To do this an aerodynamic correlation between wind profiles and system loads must be established. The following sections detail the aerodynamic parameters implemented in this work.

\subsubsection{Tower Drag Loads}

As the wind flows past the tower an aerodynamic drag load is produced. The drag load is calculated independently for each tower element based on the geometry of the tower element and the wind velocity acting on the tower element. The drag load is characterized by Eq. 130 (3), where:

- $\mathrm{C}_{\mathrm{d}}$ is the tower aerodynamic drag coefficient [unitless]

- This value is defined by the analyst in the user input file. The recommended value, and value used in the Case Study, is 1.3. A value of 1.3 was selected to be consistent with the work done by Tom Gwon (6) and was per agreement with Dr. Lemieux. A drag coefficient of 1.3 is conservative when compared with the value of 0.7 recommended by IEC (3) for cylindrical sections with a characteristic length of $>4$ inches. The value is also conservative when compared to the $C_{d}$ range of 0.35 to 1.0 , expressed as a function of Reynolds Number, recommended by Fox et al. (28).

- $\rho$ is the density of air $\left[1 \mathrm{lbf} * \mathrm{sec}^{2} / \mathrm{in}^{4}\right.$, "slinches"/in $]$

○ This work uses $0.0766 \mathrm{lbm} / \mathrm{ft}^{3}$, based on the U.S. Standard Atmosphere at sea level and $59^{\circ} \mathrm{F}(7)$. Equivalent to $1.1478 \times 10^{-7} \mathrm{lbf}^{*} \mathrm{sec}^{2} / \mathrm{in}^{4}$ in consistent ips units.

- $\quad \mathrm{V}$ is the wind velocity [inches / second]

- Wind velocity is dependent on load case. 
- $\mathrm{A}_{\text {proj }}$ is the projected area of the tower element [inches ${ }^{2}$ ]

○ Projected area is dependent on tower element diameter and length.

$$
F_{\text {TowerDrag }}=C_{d} \cdot \frac{1}{2} \cdot \rho \cdot V^{2} A_{\text {proj }}
$$

\subsubsection{Operational Rotor Thrust Loads}

As the wind flows past an operational wind turbine a thrust load is developed that pushes the rotor downwind (+X-Axis). The rotor thrust load is characterized by Eq. 131 (11), where $\rho$ and V are as defined in Section 5.2.1, $\mathrm{C}_{\mathrm{d}}$ and $\mathrm{A}_{\mathrm{Rotor}}$ are defined as follows:

- $\mathrm{C}_{\mathrm{T}}$ is the rotor thrust coefficient [unitless]

$\circ$ This value is defined by the analyst in the user input file. The recommended value, and value used in the Case Study, is 0.5. A value of 0.5 was selected in agreement with

Dr. Lemieux and was based on the IEC recommendation (3). Note that the $\mathrm{C}_{\mathrm{T}}$ value can vary widely depending on rotor blade geometry and operational conditions. For an ideal wind turbine operating at the Betz Limit (maximum theoretical power production) the $\mathrm{C}_{\mathrm{T}}$ value is $8 / 9$ (11), as can be shown through momentum theory. For turbines operating in a turbulent wake state, which can occur at high tip speed ratios (as is the case when a constant speed turbine operates at low wind speeds), the Glauert empirical relation gives a maximum $\mathrm{Ct}$ value of 2.0 (29). As is discussed in Section 5.4.1 the $2100 \mathrm{lbf}$ thrust load cases is a very conservative load case based on a $60 \mathrm{mph}$ wind and a $\mathrm{C}_{\mathrm{T}}$ of 2.0. To avoid being overly conservative a $\mathrm{C}_{\mathrm{T}}$ of 0.5 was chosen for all other load cases to be consistent with IEC recommendations and to produce "real world" results.

- $\mathrm{A}_{\mathrm{SweptRotor}}$ is the swept area of the rotor blades [inches ${ }^{2}$ ] 
- Rotor swept area is dependent on blade length

$$
F_{\text {RotorThrust }}=C_{T} \cdot \frac{1}{2} \cdot \rho \cdot V^{2} A_{\text {SweptRotor }}
$$

\subsubsection{Parked Rotor Drag Loads}

As is discussed in Section 5.4.2 the 50 Year Extreme Wind Model load case is applied to a parked wind turbine. The resulting rotor drag loads are similar to the rotor thrust loads discussed in Section 5.2.2, since both act on the rotor blades and result in a downwind force, but a different force coefficient is used and the projected area of the blades are used instead of their swept area. The parked rotor drag load is characterized by Eq. 132 (3), where $\rho$ and V are as defined in Section 5.2.1, $\mathrm{C}_{\mathrm{f}}$ and $\mathrm{A}_{\text {ProjRotor }}$ are defined as follows:

- $\mathrm{C}_{\mathrm{f}}$ is the rotor thrust coefficient [unitless]

- This value is defined by the analyst in the user input file. The recommended value, and value used in the Case Study, is 1.5. A value of 1.5 was selected to be consistent with IEC recommendations (3).

- $\mathrm{A}_{\text {ProjRotor }}$ is the projected rotor area $\left[\right.$ inches $\left.^{2}\right]$

- This value is the total rotor projected area and includes the combined projected area for all blades, the hub, nacelle, etc...

$$
F_{\text {ParkedRotorDrag }}=C_{f} \cdot \frac{1}{2} \cdot \rho \cdot V^{2} A_{\text {ProjRotor }}
$$

\subsubsection{Rotor Blade Hinge Bending Moments}

As discussed in Chapter 4 the blades are modeled using the Hinge Offset Equivalent Blade Method. As such, a flapwise bending moment acting on the hinged portion of the blade, must be calculated for each blade. The premises of this correlation is that the centroid of the thrust force generated by each blade is located at a distance of two-thirds (2/3) of the way down the blade 
away from the blade root as shown by Manwell et al. (11). This work includes a conservative assumption that the thrust force is entirely generated by the hinged portion of each blade. The blade bending moment is characterized by Eq. 133 (11), where:

- $\mathrm{F}_{\text {RotorThrust }}$ is the total rotor thrust load [lbf]

$\circ \quad \mathrm{F}_{\text {RotorThrust }}$ is as defined in Eq. 131

- Alternatively, for a parked rotor, $F_{\text {ParkedRotorDrag }}$ as defined by Eq. 132 may be substituted for $\mathrm{F}_{\text {RotorThrust }}$

- $\quad \mathrm{B}$ is the number of blades [unitless]

- The number of blades in this work is limited to 2 or 3

- $R \cdot(1-e)$ is the hinged portion of the blade [inches]

- Where $\mathrm{R}$ is the blade radius [inches] and $\mathrm{e}$ is the hinge blade ratio as defined in Chapter 4.

$$
M_{\text {BladeHinge }}=\frac{F_{\text {RotorThrust }}}{B} \cdot \frac{2}{3} \cdot R \cdot(1-e)
$$

\subsection{IEC Small Wind Turbine Classes}

As will be discussed in Section 5.4 and Section 5.5 this work has several load cases that are consistent with Aeroelastic Design Load Cases (DLC) defined in IEC 61400-2 (3). IEC load cases are specified as functions of Small Wind Turbine (SWT) Class velocity parameters as shown in Table 5.1. SWT Class I is the most severe while SWT Class IV is the least severe (i.e., lower SWT Class \# indicates higher wind velocity). The Case Study utilized SWT Class III loads.

Note that SWT Classes should not be confused with Wind Power Classes, which are used to characterize wind power density as a function of wind speed. Unlike SWT Classes, Wind Power 
Classes range from Class 1 to Class 7 with an increase in Wind Power Class number corresponding to an increase in wind speed.

Table 5.1. IEC SWT Class Velocity Parameters

\begin{tabular}{|c|c|c|c|c|}
\hline \multirow{2}{*}{ Velocity Parameter } & \multicolumn{4}{|c|}{ SWT Class } \\
\cline { 2 - 5 } & $\mathrm{I}$ & $\mathrm{II}$ & $\mathrm{III}$ & $\mathrm{IV}$ \\
\hline $\mathrm{V}_{\text {ref }}[\mathrm{mph}]$ & 111.8 & 95.1 & 83.9 & 67.1 \\
\hline $\mathrm{V}_{\text {ave }}[\mathrm{mph}]$ & 22.4 & 19.0 & 16.8 & 13.4 \\
\hline $\mathrm{V}_{\text {ref }}[$ inches $/$ second] & 1969 & 1673 & 1476 & 1181 \\
\hline $\mathrm{V}_{\text {ave }}[$ inches $/$ second] & 394 & 335 & 295 & 236 \\
\hline
\end{tabular}

\subsection{Static Load Cases}

Static load cases are a great place to start when developing the preliminary design for a wind turbine tower. While they ignore dynamic effects they can accurately model quasi-steady state wind conditions (wind profiles with negligible time dependent variations). This work considers a total of four static load cases, two of which are IEC Aeroelastic Design Load Cases while the other two are custom load cases.

\subsubsection{Static Load Case A - Tip Load}

The Static Load Case A - Tip Load features a steady state rotor thrust load applied to the tower tip. The magnitude of the tip load is defined by the analyst in the user input file. The Case Study utilizes a $2100 \mathrm{lbf}$ tip load. As mentioned in Section 5.2.2 the $2100 \mathrm{lbf}$ tip load was derived using a $60 \mathrm{mph}(1056 \mathrm{in} / \mathrm{s})$ wind velocity along with a very conservative coefficient of rotor thrust $\left(\mathrm{C}_{\mathrm{T}}\right)$ of 2.0. Along with a blade radius (R) of 72 inches the tip load can be found using Eq. 131 and as detailed in Eq. 134.

$$
\begin{gathered}
F_{\text {RotorThrust }}=C_{T} \cdot \frac{1}{2} \cdot \rho \cdot V^{2} A_{\text {SweptRotor }} \\
F_{\text {RotorThrust }}=2.0 \cdot \frac{1}{2} \cdot\left(1.1478 \cdot 10^{-7} \frac{\mathrm{lbf} \cdot \mathrm{s}^{2}}{\mathrm{in}}\right) \cdot\left(1056 \frac{\mathrm{in}}{\mathrm{s}}\right)^{2} \cdot(72 \mathrm{in})^{2} \pi \\
F_{\text {RotorThrust }}=2084.5 \mathrm{lbf} \approx 2100 \mathrm{lbf}
\end{gathered}
$$




\subsubsection{Static Load Case B - 50 Year Extreme Wind Model (IEC DLC 5.1)}

The Static Load Case B - 50 Year Extreme Wind Model (EWM50) features a steady load profile that is consistent with Design Load Case (DLC) 5.1 in IEC 61400-2 (3) and is intended to represent the strongest peak wind expected in a 50 year period. This load cases is evaluated with the turbine in a parked configuration. In the cases of a passively controlled furling wind turbine, such as the Case Study configuration, it is assumed that the drag load defined by Eq. 132 bound the load developed on the furled rotor. The load case features a variation in wind profile as a function of distance from the ground with zero velocity at the ground and the peak velocity at the tower tip (where the rotor hub is mounted). The EWM50 wind profile is shown in Figure 5.1 and is characterized by Eq. 135, where:

- $\mathrm{y}$ is the distance from the ground [inches]

- $\mathrm{y}_{\text {hub }}$ is the distance the tower tip is from the ground [inches]

$$
V_{E W M 50}(y)=1.4 V_{\text {ref }}\left(\frac{y}{y_{\text {hub }}}\right)^{0.11}
$$

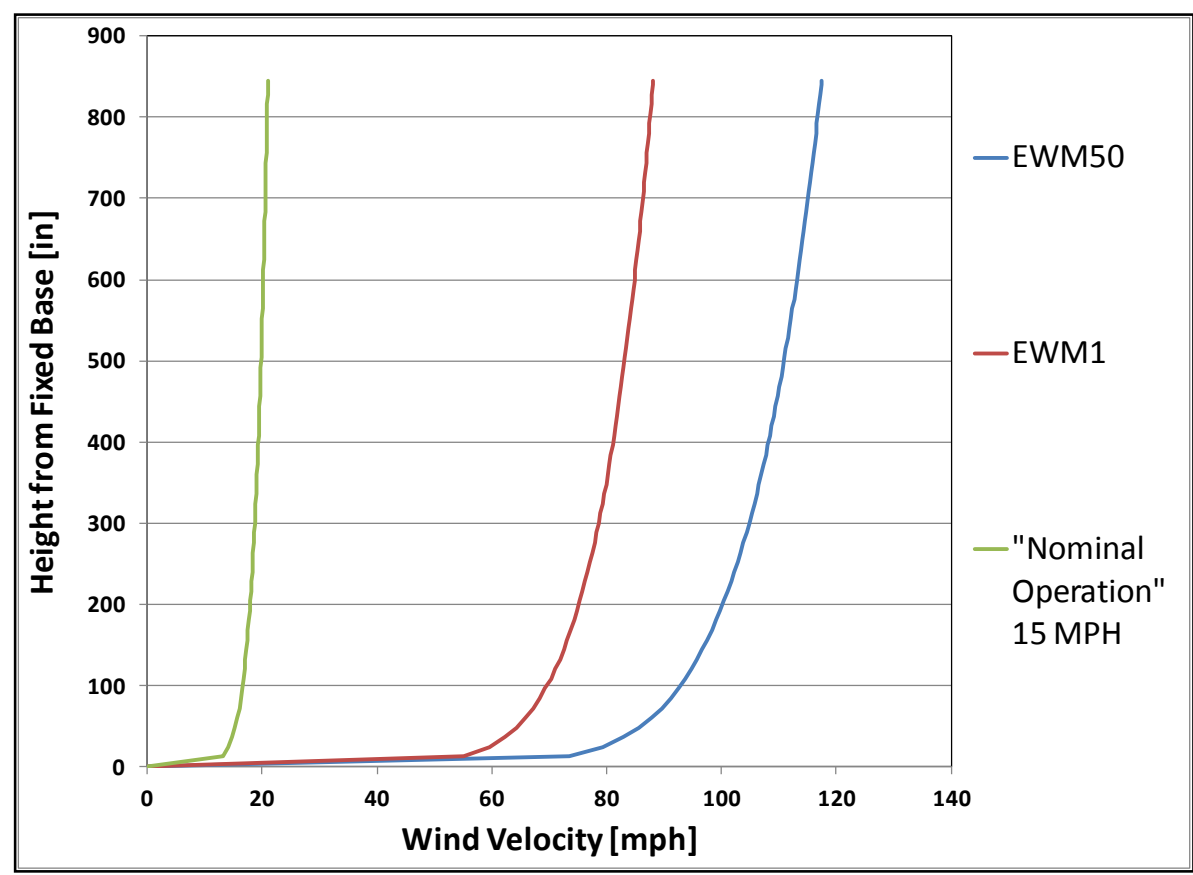

Figure 5.1. EWM50, EWM1, and "Nominal Operation" 15MPH Wind Profiles 


\subsubsection{Static Load Case C - 1 Year Extreme Wind Model (IEC DLC 6.1)}

The Static Load Case C - 1 Year Extreme Wind Model (EWM1) features a steady load profile that is consistent with Design Load Case (DLC) 6.1 in IEC 61400-2 (3) and is intended to represent the strongest peak wind expected in a 1 year period. IEC specifies that this condition applies to a parked wind turbine with a fault condition but does not specify all possible fault conditions. To be conservative this work assumes that the fault condition prevents breaking, or furling, of the wind turbine thus resulting in an operational state. Therefore Eq. 131 is used to determine the rotor thrust load. The EWM1 wind profile is simply 75\% of the EWM50 profile. The EWM1 profile is shown in Figure 5.1, and is characterized by Eq. 135, where $\mathrm{y}$ and $\mathrm{y}_{\text {hub }}$ are as defined in Section 5.4.2.

$$
V_{E W M 1}(y)=0.75 \cdot V_{E W M 50}(y)=1.05 V_{\text {ref }}\left(\frac{y}{y_{h u b}}\right)^{0.11}
$$

\subsubsection{Static Load Case D - Nominal Operation}

The Static Load Case D - Nominal Operation features a steady load profile that is intended to represent the wind profile during nominal operation. As the name suggests, this load case applies during operation so Eq. 131 is used to determine rotor thrust load. The Nominal Operation load case wind profile is the same as the EMW50 and EWM1 wind profiles but is scaled down such that the peak wind velocity is set to a user defined magnitude. For the purpose of the Case Study the Nominal Operation peak wind velocity is $15 \mathrm{mph}$ and is shown in Figure 5.1. The Nominal

Operation profile is characterized by Eq. 137, where y and $\mathrm{y}_{\text {hub }}$ are as defined in Section 5.4.2 and $\mathrm{V}_{\mathrm{UI}}[\mathrm{in} / \mathrm{sec}]$ is the User Inputted wind velocity.

$$
V_{\text {Nominaloperation }}(y)=V_{U I}\left(\frac{y}{y_{h u b}}\right)^{0.11}
$$




\subsection{Dynamic Load Cases}

Unlike static load cases, dynamic load cases feature wind profiles that change as a function of time. Dynamic load cases allow the analyst to study system transient response to realistic environmental conditions. Furthermore dynamic load cases can be used to ensure there is no detrimental resonance of the coupled tower / rotor system to dynamic environmental loads. This work considers a total of three dynamic load cases, all of which are IEC Aeroelastic Design Load Cases.

\subsubsection{Dynamic Load Case E - Extreme Operating Gust (IEC DLC 1.3)}

The Dynamic Load Case E-Extreme Operating Gust (EOG) features a load profile that is consistent with Design Load Case (DLC) 1.3 in IEC 61400-2 (3) and is intended to simulate the largest gust occurring in a 50 year period. The EOG wind profile is shown in Figure 5.2 for the SWT Class III Case Study, and is characterized by Eq. 138. Note that the formulation of Eq. 138 simplifies the IEC formulation by applying the conservative assumption that the rotor diameter is much less than the tower height (which is typical for small wind turbines), if this assumption is not true then Eq. 138 will result in a slightly stronger wind profile than the IEC formulation. Also note that in the formulation of Eq. 138 the units of velocity are in inches per second. Figure 5.2 displays the wind profile at the tower tip; the wind velocity decreases at lower tower heights in a similar manner as is shown in Figure 5.1.

$$
\begin{aligned}
& \boldsymbol{V}(\boldsymbol{t}, \boldsymbol{y})= \\
& \begin{cases}3 \boldsymbol{V}_{\text {ave }}\left(\frac{y}{y_{\text {hub }}}\right)^{0.2}-2.368\left(0.9 \cdot 2.237\left(\frac{i n}{s}\right)+0.36 V_{\text {ave }}\right) \cdot\left[\sin \left(\frac{3 \pi t}{14}\right) \cdot\left(1-\cos \left(\frac{2 \pi t}{14}\right)\right)\right] & \text { for } 0 \leq t \leq 14 \text { sec } \text { Eq. } 138 \\
3 V_{\text {ave }}\left(\frac{y}{y_{\text {hub }}}\right)^{0.2} & \text { for } t<0 \text { sec and } t>14 \text { sec }\end{cases}
\end{aligned}
$$




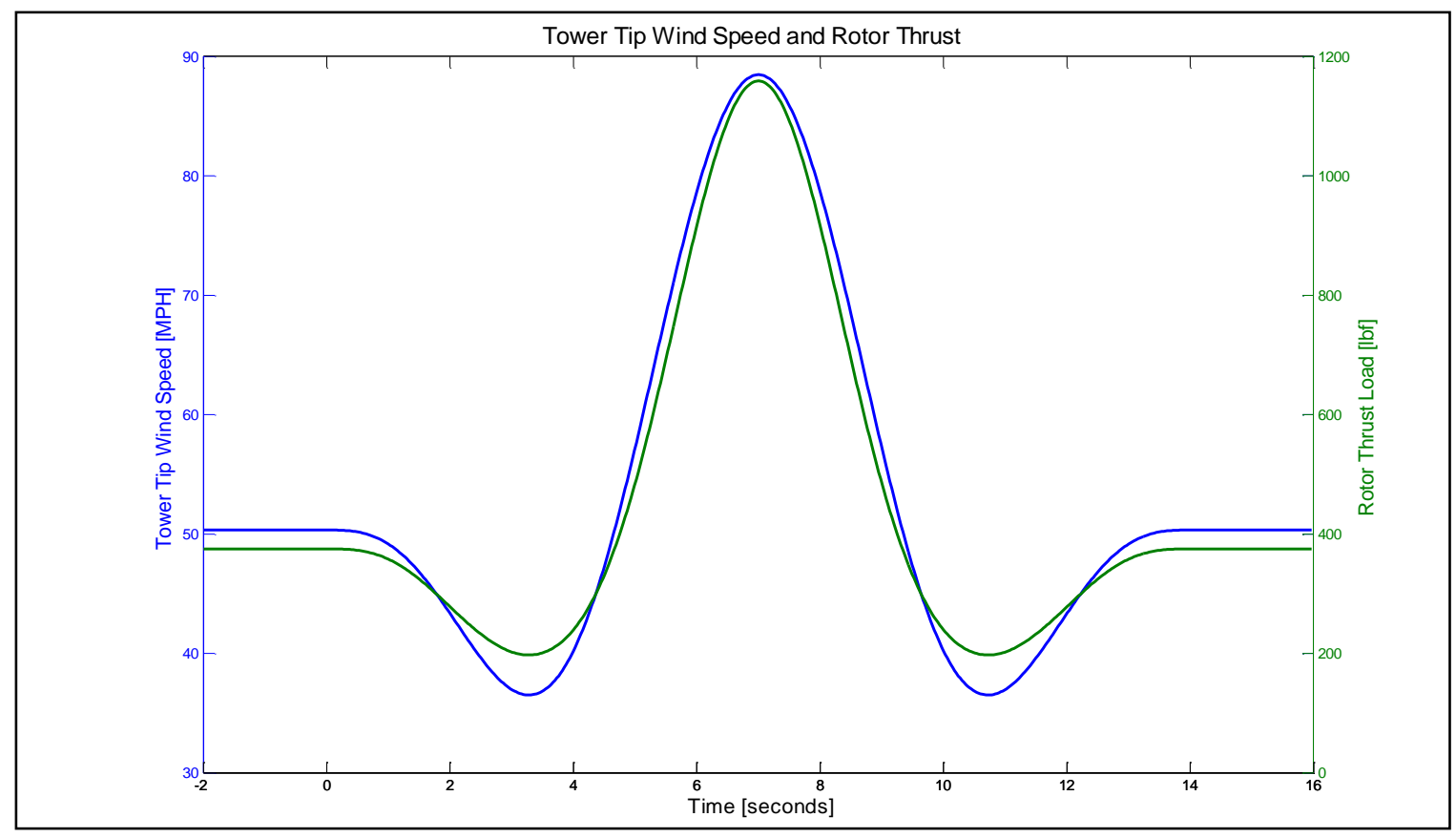

Figure 5.2. EOG Wind Profile at Tower Tip

\subsubsection{Dynamic Load Case F - Extreme Coherent Gust (IEC DLC 1.5)}

The Dynamic Load Case F-Extreme Coherent Gust (ECG) features a load profile that is consistent with Design Load Case (DLC) 1.5 in IEC 61400-2 (3). The ECG wind profile is shown in Figure 5.3 for the SWT Class III Case Study, and is characterized by Eq. 139. Note that Figure 5.3 displays the wind profile at the tower tip; the wind velocity decreases at lower tower heights in a similar manner as is shown in Figure 5.1.

$$
V(t, y)=\left\{\begin{array}{lr}
1.4 V_{\text {ave }}\left(\frac{y}{y_{\text {hub }}}\right)^{0.2} & \text { for } t \leq 10 \mathrm{sec} \\
1.4 V_{\text {ave }}\left(\frac{y}{y_{\text {hub }}}\right)^{0.2}+0.5 \cdot 590.6\left(\frac{\text { in }}{\mathrm{s}}\right)\left(1-\cos \left(\frac{\pi \mathrm{t}}{10 \mathrm{~s}}\right)\right) & \text { for } t<t<10 \mathrm{~s}
\end{array}\right.
$$




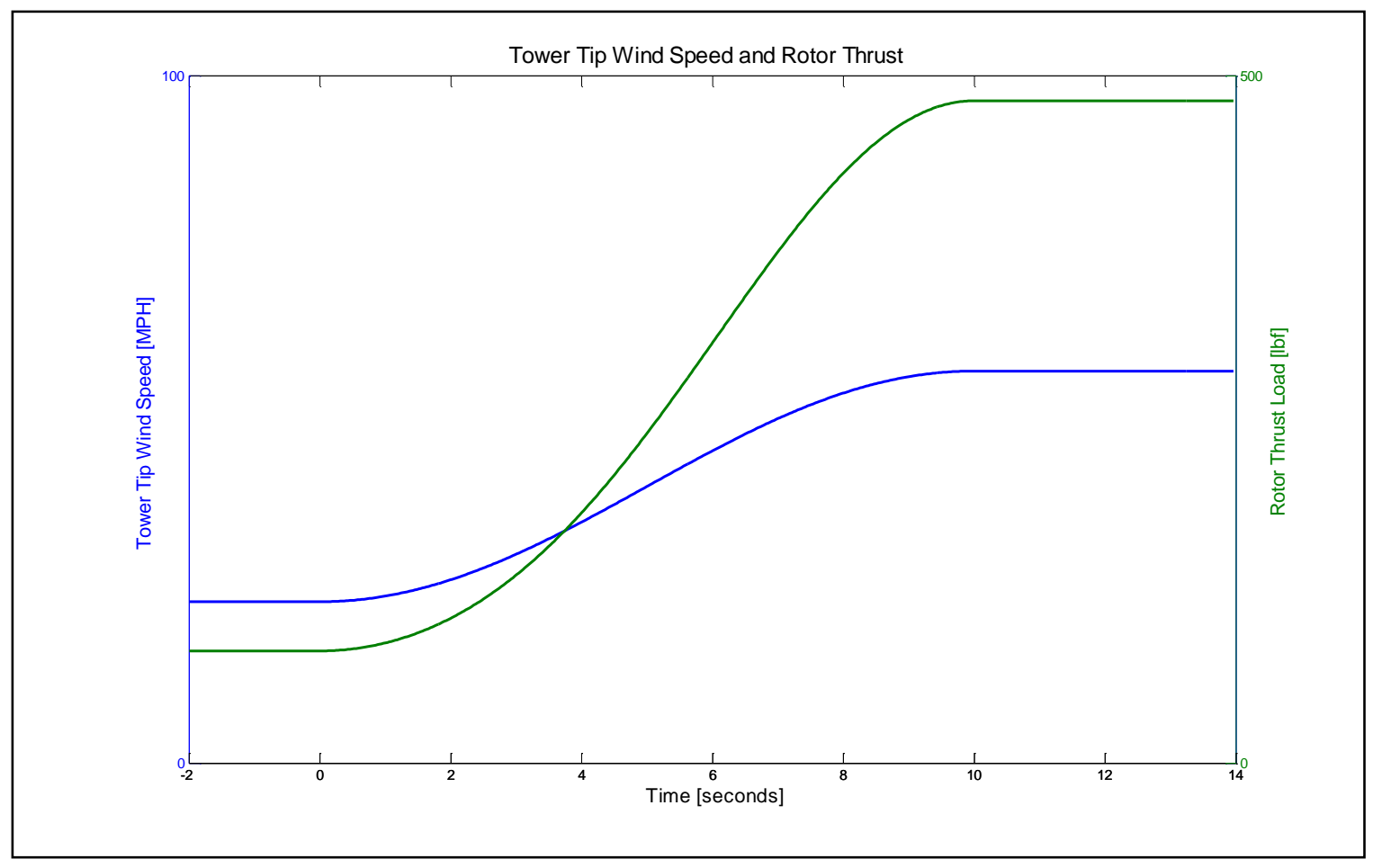

Figure 5.3. EOG Wind Profile at Tower Tip

\subsubsection{Dynamic Load Case G - Normal Turbulence Model (IEC DLC 2.2)}

The Dynamic Load Case G-Normal Turbulence Model (NTM) features a load profile that is consistent with Design Load Case (DLC) 2.2 in IEC 61400-2 (3). The ECG wind profile is shown in Figure 5.4 for the SWT Class III Case Study, and is characterized by Eq. 140. Note that Figure 5.4 displays the wind profile at the tower tip; the wind velocity decreases at lower tower heights in a similar manner as is shown in Figure 5.1.

The NTM formulation implemented in this work is a simplification of the IEC deterministic turbulence model (3).

- NTM Simplification 1: Since the simulations in this work are performed in a 2D plane, with wind velocity oriented in the in the X-Axis, a conservative approach is taken by 
using the root-sum-square (RSS) of the IEC NTM Longitudinal (X-Axis) and Lateral (ZAxis) wind profiles and then applying the resultant RSS in the X-Axis.

- $\quad$ NTM Simplification 2: The IEC NTM formulation features turbulence amplitudes that vary across the swept area of the rotor blades with peak turbulence occurring at the outer perimeter of the swept area. This work makes a conservative simplification by applying the peak turbulence levels homogeneously across the entire rotor blade swept area.

$$
V_{N T M_{-} \text {Total }}(t, y)=\sqrt{\left(V_{\text {Longitudinal }}(t, y)\right)^{2}+\left(V_{\text {Lateral }}(t, y)\right)^{2}}
$$

Where Eq. 140 utilizes the Longitudinal (X-Axis) and the Lateral (Z-Axis) wind profiles as follows:

$$
\begin{aligned}
& V_{\text {Longitudinal }}(t, y)= \\
& V_{\text {Cutout }}\left(\frac{y}{y_{\text {hub }}}\right)^{0.2}+A_{1} \sin \left(2 \pi f_{1} t\right)+A_{2}\left(\frac{R}{2}\right) \cdot \sin \left(2 \pi\left(f_{2} t+\frac{1}{4} \sin \left(2 \pi f_{3} t\right)\right)\right)+A_{2}\left(\frac{R}{2}\right) . \\
& \sin \left(2 \pi\left(f_{2} t+\frac{1}{4} \cos \left(2 \pi f_{3} t\right)\right)\right) \\
& V_{\text {Lateral }}(t, y)=A_{3} \sin \left(2 \pi\left(f_{4} t+\frac{1}{4} \sin \left(2 \pi f_{5} t\right)\right)\right)
\end{aligned}
$$

Equations Eq. 141 and Eq. 142 utilize the following amplitude parameters, where $\mathrm{V}_{\text {Cutout }}$ is the cutout wind speed $(30 \mathrm{mph}=528 \mathrm{in} / \mathrm{sec}$ for the Case Study):

$$
\begin{gathered}
A_{1}=2 \cdot\left(0.9 \cdot 2.237\left(\frac{\text { in }}{\boldsymbol{s}}\right)+0.12 \cdot V_{\text {Cutout }}\right) \\
A_{2}=\frac{A_{1}}{2 R} \\
A_{3}=0.8 \cdot A_{1}
\end{gathered}
$$


Equations Eq. 141 and Eq. 142 utilize the following frequency parameters:

$$
\begin{gathered}
f_{1}=\frac{0.0194 \cdot V_{\text {Cutout }}}{\Lambda} \\
f_{2}=4 \cdot f_{1} \\
f_{3}=\frac{f_{1}}{10} \\
f_{4}=0.6 \cdot f_{1} \\
f_{5}=\frac{f_{4}}{10}
\end{gathered}
$$

Eq. 150

Equation Eq. 146 utilizes the following turbulence scale parameter, where $\mathrm{y}_{\text {hub }}$ is the height from the ground to the tower tip (where the rotor hub is mounted):

$$
\Lambda=\left\{\begin{array}{c}
0.7 y_{\text {hub }} \text { for } y_{\text {hub }}<1181.1 \text { inches } \\
826.77 \text { inches for } y_{h u b} \geq 1181.1 \text { inches }
\end{array}\right.
$$

Note that in Eq. 143 and Eq. 146 the units of velocity are in inches per second. 


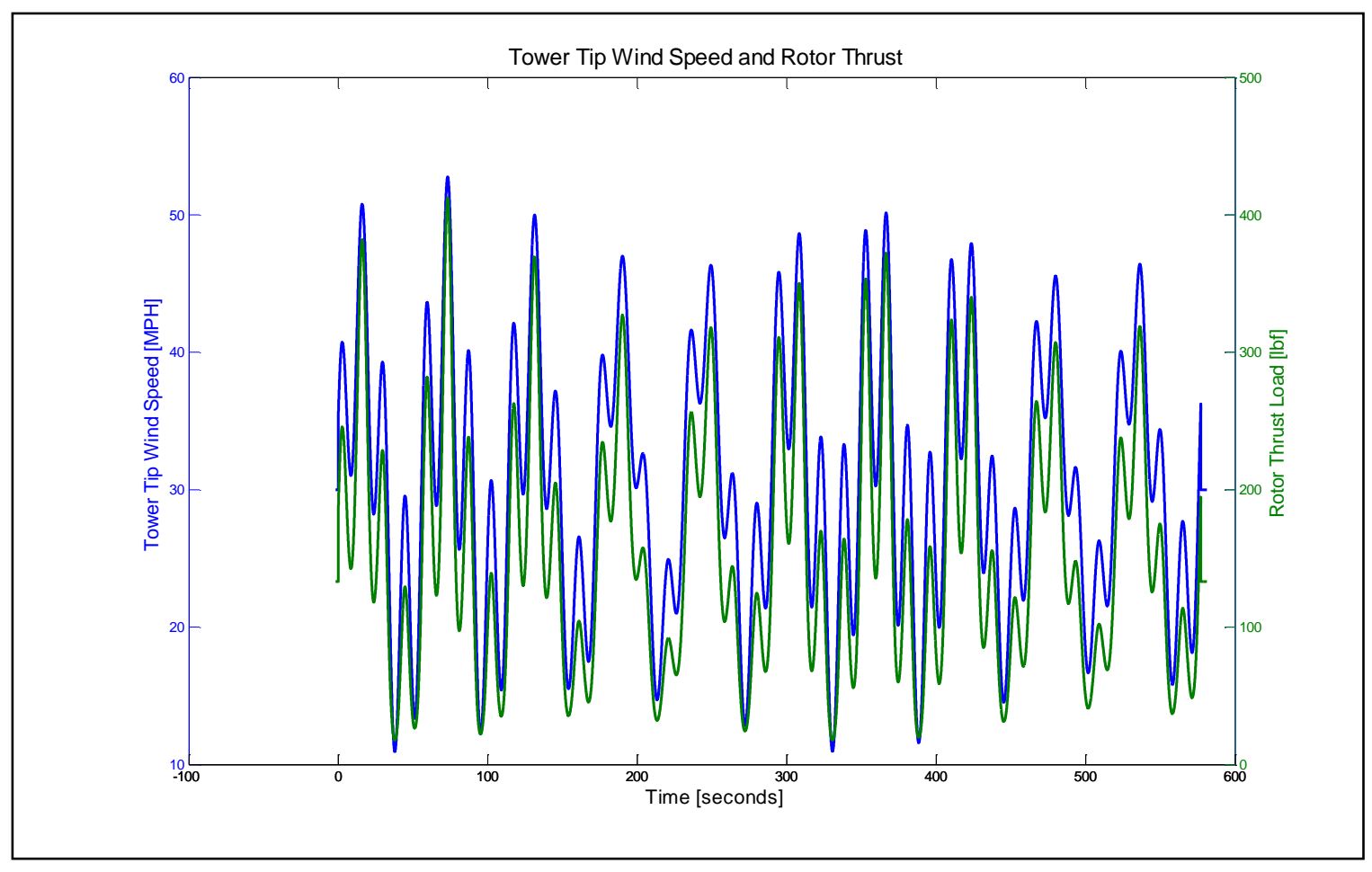

Figure 5.4. NTM Wind Profile at Tower Tip

\subsection{Specialty Load Case}

Two specialty load cases are included in this work to provide additional insight into tower / rotor coupled system dynamic response. The specialty load cases include the Swept Sine and Vortex Shedding load cases.

\subsubsection{Specialty Load Case H - Rotor Thrust Swept Sine}

The Specialty Load Case H-Rotor Thrust Swept Sine features a sinusoidal rotor thrust load with a logarithmic frequency sweep rate defined by the user. The Swept Sine load case is very useful for studying the dynamic response of the system in the frequency domain.

When reviewing results from a normal modes eigenvalue / eigenvector solution the analyst can identify system modes and mode shapes but can $\underline{N O T}$ evaluate the relative participation of those modes in system response to realistic load profiles. The Swept Sine simulation allows the analyst to evaluate coupled tower / rotor system response, and modal participation, to a rotor thrust load 
with varying frequency. This empowers the analyst with an increase level of insight into system behavior that can be useful when optimizing the system.

The Swept Sine profile used in the Case Study is shown in Figure 5.5 (note that this profile has been truncated, the actual profile used in the case study runs up to $20 \mathrm{~Hz}$ and lasts 917 seconds, over 15 minutes). The analyst should repeat the Swept Sine simulation with incrementally decreased sweep rate until convergence is achieved (a sweep rate that is too high will cause system transients that may distort the transfer function result). The Swept Sine profile is characterized by Eq. 152 and the profile frequency content is characterized by Eq. 153 (30).

$$
\begin{array}{cc}
F_{\text {ThrustSweptSine }}(t)=F_{\text {ThrustAmp }} \sin \left\{2 \pi\left[\frac{f_{s S 1} \cdot\left(-1+2^{R_{S S} \cdot t}\right)}{R_{S S} \cdot \ln (2)}\right]\right\} & \text { Eq. } 152 \\
f_{\text {spectral }}(t)=f_{\text {sS } 1} \cdot\left(2^{R_{S S} \cdot t}\right) & \text { Eq. } 153
\end{array}
$$

Eq. 152 and Eq. 153 should be evaluated over a time (t) interval of $t=0$ through $t=t_{f}$ where $t_{f}$ is defined in Eq. 154:

$$
t_{f}=\frac{\ln \left(\frac{f_{s s 2}}{f_{s s 1}}\right)}{\ln (2) \cdot R_{s s}}
$$

In Eq. 152 through Eq. 154 the following constants are defined by the analyst in the user input file and are as follows:

- $\mathrm{R}_{\mathrm{ss}}$ is the logarithmic sweep rate [octaves / second]

$\circ \quad$ The Case Study uses 0.5 octaves $/$ minute $=0.00833$ octaves $/$ second

- $\mathrm{f}_{\mathrm{ss} 1}$ is the starting frequency $[\mathrm{Hz}]$

○ The Case Study uses $0.1 \mathrm{~Hz}$ 
- $\quad \mathrm{F}_{\mathrm{ss} 2}$ is the final frequency $[\mathrm{Hz}]$

- The Case Study uses $20 \mathrm{~Hz}$

- $\mathrm{F}_{\text {ThrustAmp }}$ is the thrust load amplitude [lbf]

- The Case Study uses 100 lbf

- Not that this value is somewhat trivial as the results of the simulation are presented in terms of a transfer function (response / forcing function).

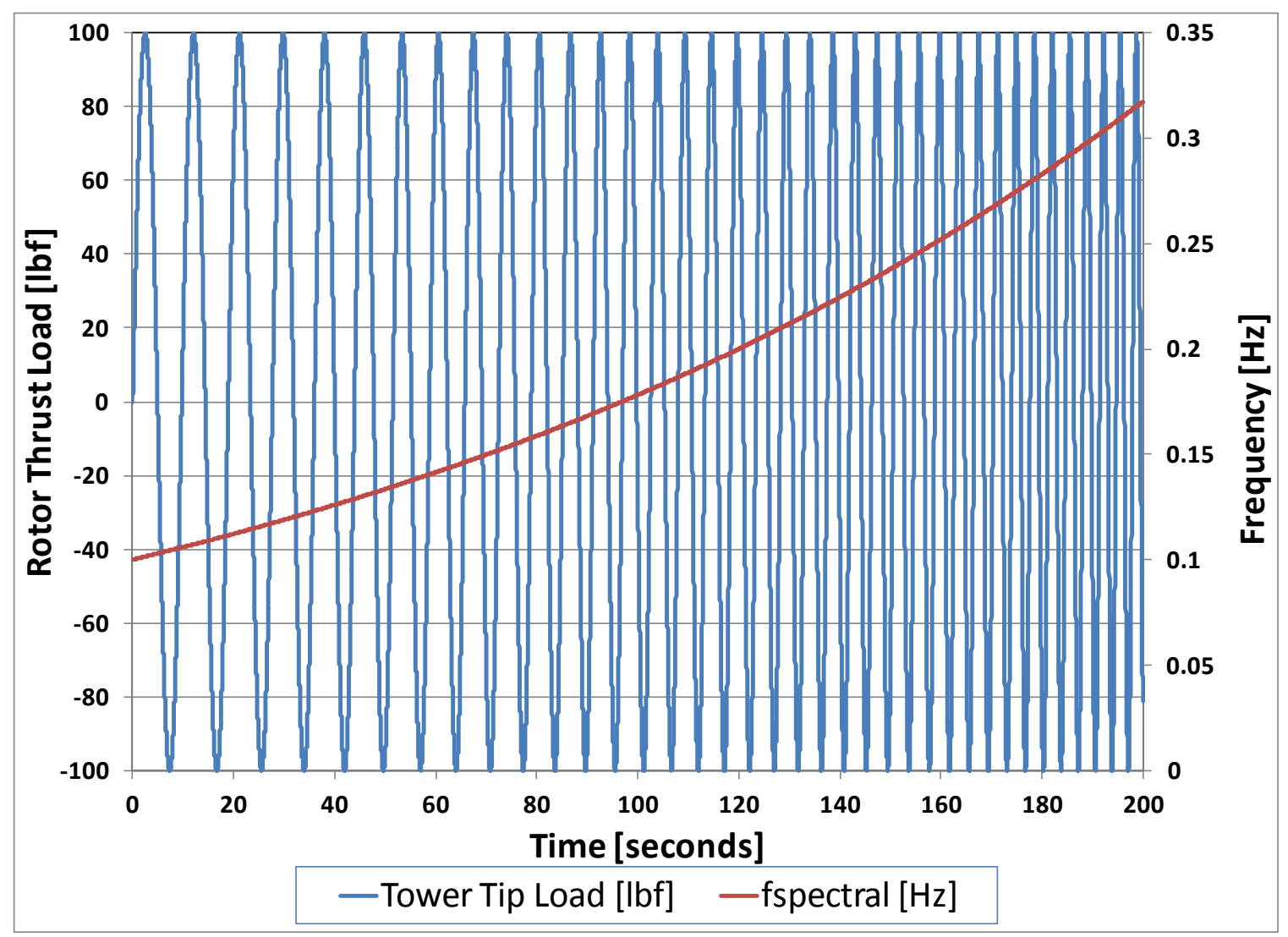

Figure 5.5. Swept Sine Rotor Thrust Load and Frequency

Note that Figure 5.5 displays the instantaneous sinusoidal Tower Tip Load magnitude as well as the instantaneous Tower Tip Load frequency (fspectral). Also note that the magnitude corresponds to the primary independent axis (left side Y-Axis) while the frequency corresponds to the secondary independent axis (right side Y-Axis). 


\subsubsection{Specialty Load Case I - Tower Vortex Shedding}

The Specialty Load Case I - Tower Vortex Shedding is not a standalone load case and is not implemented in the Matlab script accompanying this work; rather it is an empirically based approximation of tower vortex shedding frequency and loads that, along with the Swept Sine transfer function, can be used to approximate tower response due to vortex shedding.

When wind flows past a cylindrical tower it can produce cyclic excitation, orthogonal to the wind direction, due to vortex shedding. Vortex shedding frequency and loads should be evaluated to ensure it does not cause detrimental resonance of the system.

Experimental data shows that vortex shedding frequencies can be estimated using the dimensionless Strouhal number correlation shown in Eq. 155 (28), where:

- $\mathrm{f}_{\text {VortexShedding }}$ is the frequency of vortex shedding $[\mathrm{Hz}]$

- $\quad$ St is the Strouhal number and is approximately 0.21 for Reynolds numbers $>1000$.

- $\mathrm{D}_{\text {Tower }}$ is the diameter of the tower [inches]

- $\quad \mathrm{V}$ is the wind velocity [inches / second]

$\circ \quad \mathrm{V}$ should be varied between $5 \mathrm{mph}$ (speeds below $5 \mathrm{mph}$ are negligible) and the highest expected wind speed (based on EWM50, 117.5 mph for SWT Class 3)

$$
f_{\text {VortexShedding }}=\frac{S_{t} \cdot V}{D_{\text {Tower }}}
$$


The vortex shedding force $\left(\mathrm{F}_{\text {VortexShedding }}\right)[\mathrm{lbf}]$, which acts on the tower orthogonal to the wind direction, may be approximated by Eq. 156, where $\rho$ and $\mathrm{V}$ are as defined in Section 5.2.1, $\mathrm{C}_{\mathrm{L}}$ and $\mathrm{A}_{\text {ProjTower }}$ are defined as follows:

- $\mathrm{C}_{\mathrm{L}}$ is the Vortex Shedding Coefficient of Lift [unitless]

$\circ \mathrm{C}_{\mathrm{L}}$ can range from 0.2 to 0.5 (31) depending on Reynolds number (the Case

Study uses a $C_{L}$ value of 0.5 to be conservative).

- $\mathrm{A}_{\text {ProjTower }}$ is the projected area of the Tower [inches ${ }^{2}$ ]

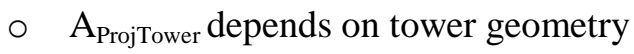

$$
F_{\text {VortexShedding }}=C_{L} \cdot \frac{1}{2} \cdot \rho \cdot V^{2} A_{\text {ProjTower }}
$$

As demonstrated in the Case Study $f_{\text {VortexShedding }}$ and $F_{\text {VortexShedding }}$ should be evaluated for all the possible wind velocities (and in the case of a tapered tower should be evaluated for all tower diameters). The resulting distributed tower force can then be conservatively applied to the tower tip and the maximum tower response can be estimated by using the Swept Sine transfer function. See Chapter 8 for results.

\subsection{Omitted IEC DLC Justification}

As mentioned in Section 2.1 several of the IEC Aeroelastic Design Load Cases have been omitted from this work. The omissions are justified because this work applies conservative assumptions (primarily related to wind turbine fault conditions) to the load cases that are included, thus the included load cases bound the excluded load cases. 


\section{IEC DLC 1.1 Omission}

a. DLC Description:

i. NTM with $\mathrm{V}=\mathrm{V}_{\text {Cutout }}$ or $3 \mathrm{~V}_{\text {ave }}$

ii. Operational State: Power Production

b. Omission Justification:

i. Equivalent to DLC 2.2 when $\mathrm{V}_{\text {Cutout }}$ is $<3 \mathrm{~V}_{\text {ave }}$ (which is valid for the Case Study) and with the assumption that the fault condition of DLC 2.2 results in full power production (which could be caused by a locked furling mechanism and a faulty break).

2. IEC DLC 1.2 Omission

a. DLC Description:

i. Extreme Coherent Gust with Direction Change (ECD) with $\mathrm{V}=1.4 \mathrm{~V}_{\text {ave }}$

ii. Operational State: Power Production

b. Omission Justification:

i. Equivalent to ECG (DLC 1.5) except ECD features a change in direction.

ii. The direction change is accounted for by running ECD in multiple orientation, relative to the tower, as is discussed in Chapter 3 (for example the Case Study is simulated in the "Strong Axis" and "Weak Axis"). Furthermore the half-wave period of the direction change is 10 seconds, equivalent to $0.05 \mathrm{~Hz}$, which is well below the natural frequency of any practical small wind turbine tower design, thus the change in direction can be modeled as quasi-steady state (i.e., multiple ECG simulations can substitute for an ECD simulation). 
3. IEC DLC 1.4 Omission

a. DLC Description:

i. Extreme Direction Change (EDC) with $\mathrm{V}=3 \mathrm{~V}_{\text {ave }}$

ii. Operational State: Power Production

b. Omission Justification:

i. EDC does not feature a change in wind magnitude, only a change in wind direction.

ii. Multiple included load cases feature wind magnitudes that bound the EDC wind magnitude.

iii. Similar to the justification listed for DLC 2.2, the half-wave period of the direction change is 6 seconds, equivalent to $0.083 \mathrm{~Hz}$, which is well below the natural frequency of any practical small wind turbine tower design, thus the change in direction can be modeled as quasi-steady state.

4. IEC DLC 2.1 Omission

a. DLC Description:

i. Normal Wind Profile (NWP) with $\mathrm{V}=2.5 \mathrm{~V}_{\text {ave }}$

ii. Operational State: Power Production plus occurrence of fault

b. Omission Justification:

i. NWP is enveloped by the EWM1 (DLC 6.1) when the fault condition of DLC 6.1 is assumed to result in full power production (an assumption implemented in this work). 
5. IEC DLC 2.3 Omission

a. DLC Description:

i. Extreme Operating Gust (EOG) with $\mathrm{V}=2.5 \mathrm{~V}_{\text {ave }}$

ii. Operational State: Power Production plus occurrence of fault

b. Omission Justification:

i. Bounded by EOG DLC 1.3 since this work assumes full power production all the way up to $\mathrm{V}=3.0 \mathrm{~V}_{\text {ave }}$ for DLC 1.3 regardless of $\mathrm{V}_{\text {Cutout }}$ (essentially the DLC 1.3 implementation in this work includes fault conditions).

6. IEC DLC 3.1 Omission

a. DLC Description:

i. Normal Turbulence Model (NTM) with $\mathrm{V}=\mathrm{V}_{\text {Cutout }}$

ii. Operational State: Normal Shutdown

b. Omission Justification:

i. Equivalent to NTM DLC 2.2; assumes that turbine shutdown does not result in tower vulnerabilities.

7. IEC DLC 3.2 Omission

a. DLC Description:

i. Extreme Operating Gust (EOG) with $\mathrm{V}=\mathrm{V}_{\text {Cutout }}$

ii. Operational State: Normal Shutdown

b. Omission Justification:

i. Bounded by EOG DLC 1.3; assumes that turbine shutdown does not result in tower vulnerabilities and that $\mathrm{V}_{\text {Cutout }}<3 \mathrm{~V}_{\text {ave }}$ (valid for the Case Study with a $\mathrm{V}_{\text {Cutout }}$ of $30 \mathrm{mph}$ ) 
8. IEC DLC 4.1 Omission

a. DLC Description:

i. Normal Turbulence Model (NTM) with $V=V_{\text {Emergency }}$ where $V_{\text {Emergency }}$ is defined by the operator and represents the maximum wind conditions for which a safe emergency / manual shutdown can be performed

ii. Operational State: Emergence / Manual Shutdown

b. Omission Justification:

i. Equivalent to NTM DLC 2.2; assuming that $\mathrm{V}_{\text {Emergency }}=\mathrm{V}_{\text {Cutout }}$ and that emergence / manual shutdown does not result in tower vulnerabilities.

9. IEC DLC 5.2 Omission

a. DLC Description:

i. Normal Turbulence Model (NTM) with $\mathrm{V}=0.7 * \mathrm{~V}_{\text {ref }}$

ii. Operational State: Parked and fault condition

b. Omission Justification:

i. Bounded by NTM DLC 2.2; assuming that tower response to DLC 5.2 (when in a parked, furled, or idling condition) is less than DLC 2.2 when in an operating condition.

10. IEC DLC 7.1 Omission

a. DLC Description:

i. Transportation, assembly, maintenance, and repair loads

b. Omission Justification:

i. Transportation, assembly, maintenance, and repair loads are beyond the scope of this work and are discussed in the work done by Tom Gwon (6). 


\section{Solution Methodology}

\subsection{Introduction}

In Chapters 3 and 4 the process for developing and coupling the tower and rotor models was established. Subsequently, in Chapter 5 the load cases were detailed. With the model developed and the load cases established, the focus is now turned to the solution methodologies that lay at the core of the simulation. An independent solution method is utilized for static, modal, and dynamic simulations. Each of these types of solution sequences will be discussed in the following sections.

\subsection{Static Solution Sequence}

Static simulations are the simplest type of simulation and subsequently the solution method is very straight forward. The goal of a static simulation is to determine system displacements due to a set of static external forces acting on the system. In Eq. $157 \boldsymbol{K}_{\boldsymbol{S}}$ is the System Stiffness Matrix, $\left\{u_{S}\right\}$ is the System Displacement Vector, and $\left\{F_{S}\right\}$ is the System Force Vector (external forces acting on the system). By "system" we are referring to the coupled tower / rotor system. Eq. 157 is the matrix formulation of Hooke's law (19).

$$
\boldsymbol{K}_{\boldsymbol{S}}\left\{u_{S}\right\}=\left\{F_{S}\right\}
$$

Note that in Eq. 157 the $\left\{u_{S}\right\}$ vector represents a set of system displacements in generalized coordinates. For example, the definition of $u$ for the Simplified Rotor Model coupled with the Simplified Tower Model (as shown in Figure 4.8 of Chapter 4) would be as shown in Eq. 158. 


$$
\{u\}=\left\{\begin{array}{l}
u_{2} \\
v_{2} \\
\theta_{2} \\
u_{3} \\
v_{3} \\
\vartheta_{3} \\
\alpha_{1} \\
\alpha_{2}
\end{array}\right\}
$$

Eq. 158

Eq. 157 represents a system of linear equations that must be solved for $\left\{u_{S}\right\}$, given $\boldsymbol{K}_{\boldsymbol{S}}$ and $\left\{F_{S}\right\}$. The traditional way to complete the solution is to compute the inverse of $\boldsymbol{K}_{\boldsymbol{S}}$ as show in Eq. 159 . Note that the static solution is independent of system mass and damping properties and is only a function of system stiffness and external forces.

$$
\left\{u_{S}\right\}=K_{S}{ }^{-1}\left\{F_{S}\right\}
$$

In practice it is typically not necessary to compute the full inverse of $\boldsymbol{K}_{\boldsymbol{S}}$ to solve for $\left\{u_{S}\right\}$. The Matlab tool accompanying this work utilizes a built-in Matlab Gaussian Elimination technique to solve the system of equations. This technique is widely used and is more efficient than computing the full inverse of $\boldsymbol{K}_{\boldsymbol{S}}(5)$.

When a guy-wire supported tower configuration is evaluated, Eq. 157 is solved twice, in a two step process as follows:

- Step 1: Solve for system displacements due to guy-wire pretension load

- Step 2: Solve for system displacements due to environmental wind load

The displacement results for Step 1 and Step 2 are then added together (based on load superposition) to determine the net displacements due to the combined influence of guy-wire pretension and environmental load. Note that this process is a linear solution sequence and cannot simulate the nonlinear effects of a slack guy-wire. Thus the guy-wire pretention must be high 
enough so that the environmental load does not overcome the guy-wire pre-tension. The analyst should review the results (reference the "Guy-Wire_Tension" tab of the MS Excel output file for each load case) to confirm that all guy-wires remain in a state of tension after the environmental load is applied, and if not the pretention should be increased and the simulation repeated.

\subsection{Modal Solution Sequence}

Identifying system mode shapes and their corresponding modal frequencies allows the analyst to develop an understanding of the system's dynamic characteristics. Furthermore, the mode shapes and modal frequencies must be identified prior to completing a dynamic simulation (since the dynamic solver utilizes this information). Mode shapes are orthogonal and linearly independent from each other (no mode shape can be expresses as a linear function of other mode shapes). In mathematical terms the mode shapes are referred to as Eigenvectors and the modal frequencies are the square root of the corresponding Eigenvalues. As such, to determine the mode shapes and modal frequencies the Eigenvector / Eigenvalue problem, shown in Eq. 160, must be solved (1).

$$
\left[\boldsymbol{K}_{\boldsymbol{S}}-\omega_{i}^{2} \boldsymbol{M}_{\boldsymbol{s}}\right]\left\{\phi_{i}\right\}=\mathbf{0}
$$

In Eq. 160:

- $\boldsymbol{K}_{\boldsymbol{s}}$ is System Stiffness Matrix

- $\boldsymbol{M}_{\boldsymbol{s}}$ is System Mass Matrix

- $\left\{\phi_{i}\right\}$ is the $i$ 'th Eigenvector (same as the mode shape)

$\circ\left\{\phi_{i}\right\}$ is normalized in this work to a maximum displacement of 1

- $\quad\left\{\omega_{i}\right\}^{2}$ is the $i$ 'th Eigenvalue

- Taking the square root gives $\omega_{i}$ which is the modal frequency of the $i$ 'th mode shape 
Note that $\omega_{1}$, which is the lowest modal frequency, is referred to as the fundamental frequency (also sometimes referred to as the natural frequency)

The modal solution in this work is implemented using the built in Matlab "eig" function. Note that the modal frequency is independent of system damping and external forces and is only a function of system stiffness and mass.

\subsection{Dynamic Solution Sequence}

The dynamic solution sequence is used to simulate transient events (i.e., events in which system response varies with time). There are multiple methods for executing a dynamic solution sequence; the method implemented in this work utilizes the Mode-Superposition Method (1). The goal of the dynamic solution sequence is to solve the System Equation of Motion (EOM) shown in Eq. 161.

$$
\boldsymbol{M}_{\boldsymbol{s}}\{\ddot{u}\}+\boldsymbol{C}_{\boldsymbol{s}}\{\dot{u}\}+\boldsymbol{K}_{\boldsymbol{s}}\{u\}=\left\{F_{s}(t)\right\}
$$

In Eq. 161:

- $\quad \boldsymbol{K}_{\boldsymbol{s}}$ and $\boldsymbol{M}_{\boldsymbol{s}}$ are as defined in Section 6.3

- $\boldsymbol{C}_{\boldsymbol{s}}$ is the System Damping Matrix

- $\left\{F_{S}(t)\right\}$ is the System External Forcing function [which is a function of time (t)]

- $\{u\}$ is the system displacement vector which expresses the displacement at each of the system's degrees of freedom in generalized coordinates as a function of time

- Accents over $u$ indicate time derivatives as follows:

- Velocity: $\{\dot{u}\}=\frac{\partial u}{\partial t}$

- Acceleration: $\{\ddot{u}\}=\frac{\partial^{2} u}{\partial t^{2}}$ 
Note that the EOM (Eq. 161) is a coupled system of second order differential equations. The Mode-Superposition Method uses the normalized mode shapes to transform the EOM from generalized coordinates to modal coordinates and in doing so decouples the EOM into a set of uncoupled second order differential equations that can be solved independently of each other. This method requires a special type of damping called Modal Damping. The Mode-Superposition Method consists of the following steps, which are each summarized in the subsequent sections:

1. Determine the normalized mode shapes and modal frequencies (see Section 6.3)

2. Use the normalized mode shapes to transform $\boldsymbol{K}_{\boldsymbol{s}}$ and $\boldsymbol{M}_{\boldsymbol{s}}$ from generalized coordinates into modal coordinates

3. Develop the System Modal Damping Matrix $\boldsymbol{C}_{\boldsymbol{s}}$

4. Independently solve the set of uncoupled second order differential equations to determine system displacements in modal coordinates

a. This work uses the $4^{\text {th }}$ Order Runge-Kutta Numerical Integration Method

5. Transform the system displacements from modal coordinates back to generalized coordinates

\subsubsection{Transformation to Modal Coordinates}

The transformation of $\boldsymbol{K}_{\boldsymbol{s}}$ and $\boldsymbol{M}_{\boldsymbol{s}}$ from generalized coordinates into modal coordinates requires the construction the Modal Matrix, $\mathbf{\Phi}$. The Modal Matrix is simply a collection of the normalized mode shape vectors such that the $i$ 'th column of the modal matrix corresponds with the $i$ 'th mode shape vector, as shown in Eq. 162.

$$
\boldsymbol{\Phi}=\left[\begin{array}{llll}
\left\{\phi_{1}\right\} & \left\{\phi_{2}\right\} & \ldots & \left\{\phi_{n}\right\}
\end{array}\right],
$$


The key to the Mode-Superposition Method is that a set of displacements $\{u\}$, in generalized coordinates, can be expressed as a function of the normalized modes and displacements $\{\eta\}$, in modal coordinates, as shown in Eq. 163.

$$
\{u\}=\boldsymbol{\Phi}\{\eta\}=\sum_{i=1}^{n}\left\{\phi_{i}\right\}\left\{\eta_{i}\right\}
$$

Substituting Eq. 163 into Eq. 161 results in the transformation of the EOM from generalized coordinates to modal coordinates as shown in Eq. 164.

$$
\widetilde{\boldsymbol{M}}_{\boldsymbol{s}}\{\ddot{\eta}\}+\widetilde{\boldsymbol{C}}_{\boldsymbol{s}}\{\dot{\eta}\}+\widetilde{\boldsymbol{K}}_{\boldsymbol{s}}\{\eta\}=\left\{\widetilde{F}_{s}(t)\right\}
$$

Where $\widetilde{\boldsymbol{M}}_{\boldsymbol{s}}, \widetilde{\boldsymbol{C}}_{\boldsymbol{s}}$, and $\widetilde{\boldsymbol{K}}_{\boldsymbol{s}}$ are the System Modal Mass, Damping, and Stiffness matrices and $\{\tilde{F}(t)\}$ is the System Modal Forcing function and are defined in Eq. 165.

$$
\begin{gathered}
\widetilde{\boldsymbol{M}}_{\boldsymbol{s}}=\boldsymbol{\Phi}^{\mathbf{T}} \boldsymbol{M}_{\boldsymbol{s}} \boldsymbol{\Phi} \\
\widetilde{\boldsymbol{C}}_{\boldsymbol{s}}=\boldsymbol{\Phi}^{\mathbf{T}} \boldsymbol{C}_{\boldsymbol{s}} \boldsymbol{\Phi} \\
\widetilde{\boldsymbol{K}}_{\boldsymbol{s}}=\boldsymbol{\Phi}^{\mathbf{T}} \boldsymbol{K}_{\boldsymbol{s}} \boldsymbol{\Phi} \\
\left\{\widetilde{F}_{S}(t)\right\}=\boldsymbol{\Phi}^{\mathbf{T}}\left\{F_{S}(t)\right\}
\end{gathered}
$$

\subsubsection{Modal Damping}

Modal Damping is a special type of viscous damping in which the damping matrix $\widetilde{\boldsymbol{C}}_{\boldsymbol{s}}$ is a diagonal matrix (non-zero terms only exist on the diagonal) which allows the EOM to be uncoupled when expressed in modal coordinates. Modal Damping is the most common type of damping used in structural dynamics analysis (1). Modal Damping assigns a user specified damping factor $\left(\zeta_{i}\right)$ to each mode for all modes considered in the analysis (modes 1 through $\mathrm{n}$ ). Equation Eq. 166 details how Modal Damping is determined in generalized coordinates. Eq. 165 may then be used to determine Modal Damping in modal coordinates. 


$$
\boldsymbol{C}_{\boldsymbol{s}}=\sum_{i=1}^{n} \frac{2 \zeta_{i} \omega_{i}}{\widetilde{M}_{s_{i}}}\left\{\boldsymbol{M}_{\boldsymbol{s}}\left\{\phi_{2}\right\}\right\}\left\{\boldsymbol{M}_{\boldsymbol{s}}\left\{\phi_{2}\right\}\right\}^{T}
$$

The modal damping factors typically fall in the range of $0.01 \leq \zeta_{i} \leq 0.1$ for most structural applications (1). A thorough review of damping parameters recommended in literature was completed by Tom Gwon (6). The case study in this work uses a damping factor of $\zeta=0.05$ for all modes to be consistent with the work completed by Tom Gwon (6).

\subsubsection{Runge-Kutta Numerical Integration}

After the EOM has been transformed into an uncoupled set of second order differential equations in modal coordinates, the equations associated with each modal coordinate are independently solved using the $4^{\text {th }}$ Order Runge-Kutta Numerical Integration Method. This method starts with rearranging the EOM in modal coordinates, Eq. 164, as shown in Eq. 167 where the $(i)$ subscript indicates that Eq. 167 represents the EOM for the $i$ 'th mode. Then Eq. 167, which is a second order differential equation, is expresses as a set of two first order differential equations as shown in Eq. 168.

$$
\begin{gathered}
\left\{\ddot{\eta}_{i}\right\}=\frac{\left\{\widetilde{F}_{s_{i}}(t)\right\}-\widetilde{\boldsymbol{C}}_{\boldsymbol{s}_{\boldsymbol{i}}}\left\{\dot{\eta}_{i}\right\}-\widetilde{\boldsymbol{K}}_{\boldsymbol{s}_{\boldsymbol{i}}}\left\{\eta_{i}\right\}}{\widetilde{\boldsymbol{M}}_{\boldsymbol{s}_{\boldsymbol{i}}}} \\
\dot{\eta}_{i}=V_{i} \\
\left\{\dot{V}_{i}\right\}=f\left(\widetilde{F}_{s_{i}}(t), V_{i}, \eta_{i}\right)=\frac{\left\{\widetilde{F}_{s_{i}}(t)\right\}-\widetilde{\boldsymbol{C}}_{\boldsymbol{s}_{\boldsymbol{i}}}\left\{V_{i}\right\}-\widetilde{\boldsymbol{K}}_{\boldsymbol{s}_{\boldsymbol{i}}}\left\{\eta_{i}\right\}}{\widetilde{\boldsymbol{M}}_{\boldsymbol{s}_{\boldsymbol{i}}}}
\end{gathered}
$$

The $4^{\text {th }}$ Order Runge-Kutta Numerical Integration Method can now be used to determine the modal displacement of the $i^{\prime}$ th mode $\left(\eta_{i}\right)$ as shown in Eq. 169 where the $(j)$ subscript indicates the $j$ 'th time step and $d t$ is the duration of a time step. As shown in Eq. 169 displacements and 
velocities for the next time step are solved for as a function of current displacements and velocities as well as the time step size.

$$
\begin{gathered}
\eta_{i_{j+1}}=\eta_{i_{j}}+\frac{1}{6}\left(m_{1}+2 m_{2}+2 m_{3}+m_{4}\right) \\
V_{i_{j+1}}=V_{i_{j}}+\frac{1}{6}\left(k_{1}+2 k_{2}+2 k_{3}+k_{4}\right) \\
\text { Where: } \\
m_{1}=(d t)\left(V_{i_{j}}\right) \\
m_{2}=(d t)\left(V_{i_{j}}+\frac{1}{2} k_{1}\right) \\
m_{3}=(d t)\left(V_{i_{j}}+\frac{1}{2} k_{2}\right) \\
m_{4}=(d t)\left(V_{i_{j}}+k_{3}\right) \\
k_{1}=(d t) f\left(\tilde{F}_{s_{i}}\left(t_{j}\right), V_{i_{j}}, \eta_{i_{j}}\right) \\
k_{2}=(d t) f\left(\tilde{F}_{s_{i}}\left(t_{j}+\frac{1}{2} d t\right), V_{i_{j}}+\frac{1}{2} k_{1}, \eta_{i_{j}}+\frac{1}{2} m_{1}\right) \\
k_{3}=(d t) f\left(\tilde{F}_{s_{i}}\left(t_{j}+\frac{1}{2} d t\right), V_{i_{j}}+\frac{1}{2} k_{2}, \eta_{i_{j}}+\frac{1}{2} m_{2}\right) \\
k_{4}=(d t) f\left(\tilde{F}_{s_{i}}\left(t_{j}+d t\right), V_{i_{j}}+k_{3}, \eta_{i_{j}}+m_{3}\right)
\end{gathered}
$$

The initial conditions $\eta_{i_{1}}$ and $V_{i_{1}}$ (at the first time step, $j=1$ ) are solved for by performing a static solution sequence using the initial force profile $\tilde{F}_{S_{i}}\left(t_{1}\right)$ and the method described in Section 6.2 .

The process is repeated for all modes from the first mode $(i=1)$ to the highest mode of interest $(i=n)$. The solution sequence is truncated such that modes beyond the $n$ 'th mode are not included. The analyst defines the value for $(n)$ and should do so by starting with a low value (such as $n=3$ ) and proceeding to higher values until result (both displacements and stresses) have 
converged. Note that convergence is typically achieved more rapidly for displacement results than for stress results, because stress is a function which is at least one order lower than displacements (32). The Case Study utilized 30 modes $(n=30)$ in order to achieve result convergence (which represents a significant truncation since the total number of modes in the Case Study is 115).

After the modal displacements $\{\eta\}$ are determined they are transformed back to the generalized coordinates $\left\{u_{S}\right\}$ using Eq. 163 .

\subsection{Results Post Processing}

\subsubsection{Tower Elemental Forces}

After displacement results in generalized coordinates $\left\{u_{S}\right\}$ are computed the nodal displacements for each element can be identified, transformed into elemental coordinates, and Eq. 170 can be used to determine elemental forces where:

- $\left\{F_{i}\right\}$ is the elemental forces of the $i$ 'th element

- $\quad \boldsymbol{K}_{\boldsymbol{i}}$ is the elemental stiffness matrix of the $i^{\prime}$ th element

- $\left\{u_{i}\right\}$ is the nodal displacements of the $i$ 'th element

$$
\left\{F_{i}\right\}=\boldsymbol{K}_{i}\left\{u_{i}\right\}
$$

\subsubsection{Tower Elemental Stresses}

After the elemental forces are determined at each tower node the corresponding nodal stresses can be computed. Consider the 2D Plane Beam Tower Element shown in Figure 3.4 of Chapter 3 and let:

- $\quad F_{u_{1}}$ be the force acting on Node 1 aligned with the $u_{1}$ DOF (i.e., shear force in the XAxis) 
- $\quad F_{v_{1}}$ be the force acting on Node 1 aligned with the $v_{1}$ DOF (i.e., axial force in the YAxis)

- $\quad M_{\theta_{1}}$ be the force acting on Node 1 aligned with the $\vartheta_{1}$ DOF (i.e., moment about the ZAxis)

The tower nodal stresses at Node 1 can then be calculated for axial stress $\left(\sigma_{A 1}\right)$, bending stress $\left(\sigma_{B 1}\right)$, and shear stress $\tau_{1}$ as shown in Eq. 171 through Eq. 173.

$$
\begin{gathered}
\sigma_{A 1}=\frac{F_{v_{1}}}{A} \\
\sigma_{B 1}=\frac{M_{\theta_{1}} \cdot\left(\frac{D_{t}}{2}\right)}{I} \cdot \cos (\Theta) \\
\tau_{1}=\frac{2 F_{u_{1}}}{A} \cdot \sin (\Theta)
\end{gathered}
$$

In Eq. 172 and Eq. 173 the $(\Theta)$ value represents the angle from the away from the $+X$-Axis (downwind) in the XZ-Plane as shown in Figure 4.13. Note that bending stress $\left(\sigma_{B 1}\right)$ is maximized at $\left(\Theta=0^{\circ}\right.$ and $\left.180^{\circ}\right)$ while shear stress $\tau_{1}$ is maximized at $\left(\Theta=90^{\circ}\right.$ and $\left.270^{\circ}\right)$. 


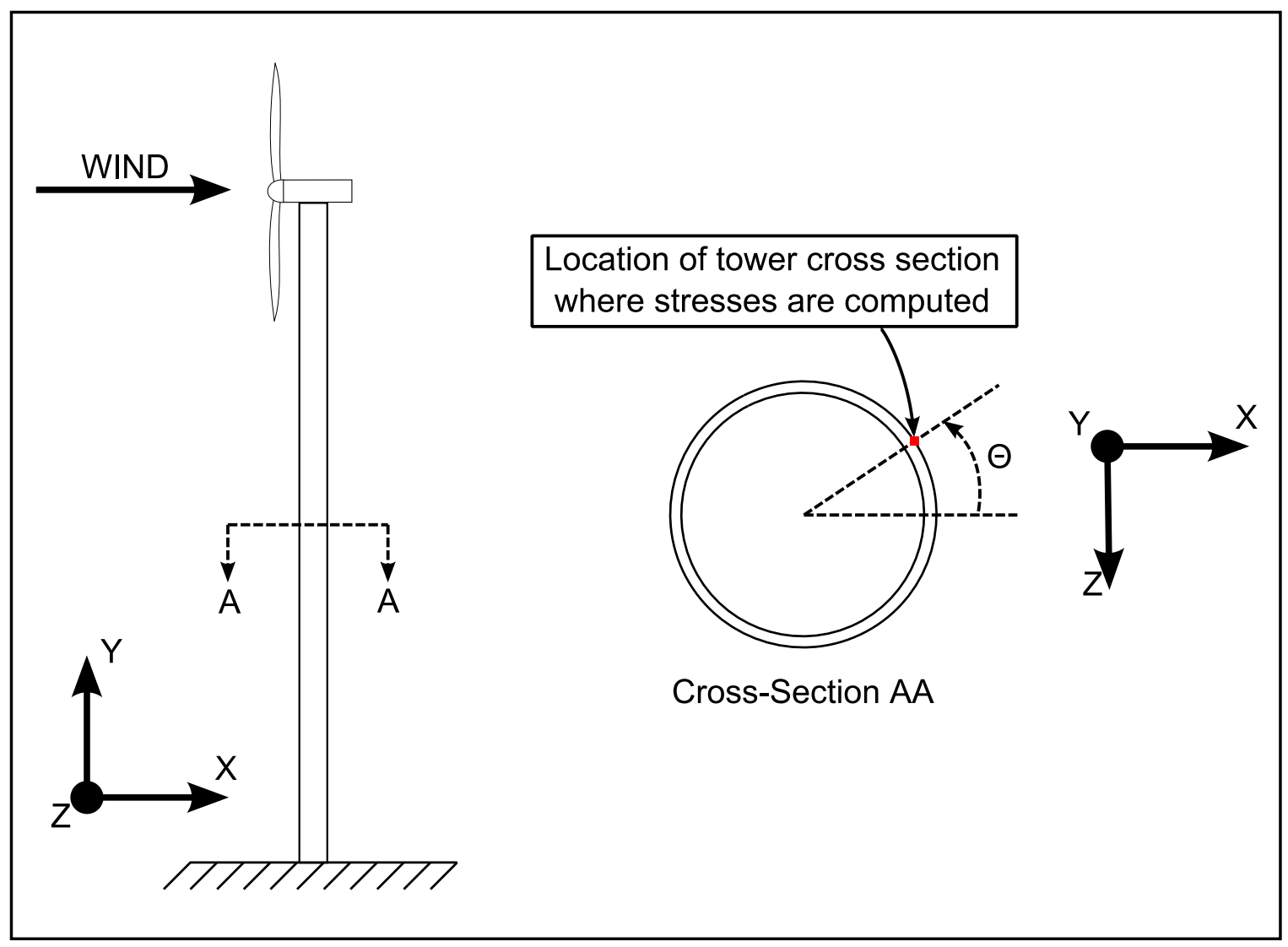

Figure 6.1. Tower Cross Section Stress Theta Location

Often times material strength properties are only available based on tensile coupon testing. Thus it is frequently desirable to transform the bending, axial, and shear stress state into an equivalent tensile stress (von Mises stress) using strain energy theory (19). The von Mises stress at Node 1 $\left(\sigma_{e 1}\right)$ is shown in Eq. 174.

$$
\sigma_{e 1}=\sqrt{\frac{2\left(\sigma_{A 1}+\sigma_{B 1}\right)^{2}+6\left(\tau_{1}\right)^{2}}{2}}
$$

The Matlab tool accompanying this work reports maximum tower von Mises stress, along with the $(\Theta)$ value for which von Mises stress is maximized, at each tower station height. 


\section{Verification}

\subsection{Introduction}

The focus of this chapter is on verifying the accuracy of the modeling and simulation methods laid out in this work. Steps taken to validate the tower model, the rotor model, and the solution methods are summarized in this chapter. Validation techniques include comparisons to theoretical closed-form solutions, comparisons to peer works, and comparisons to test results.

\subsection{Tower - Comparisons to Theoretical Models}

A simple cantilever tower with uniform cross-section and no tip mass or rotor was simulated using the methods established in this work and the results were compared to theoretical models. Comparisons were made for static loading and modal analysis. The cantilever beam is shown in Figure 7.1 and has the following features:

- Solid Circular Cross-Section with 1.0 inch diameter

- Length (L): 20 inches

- Young's Modulus (E): 10.4 MPSI

- Density $(\rho): 0.098 \mathrm{lbs} / \mathrm{in}^{3}\left(0.0002536 \mathrm{slinch} / \mathrm{in}^{3}\right)$

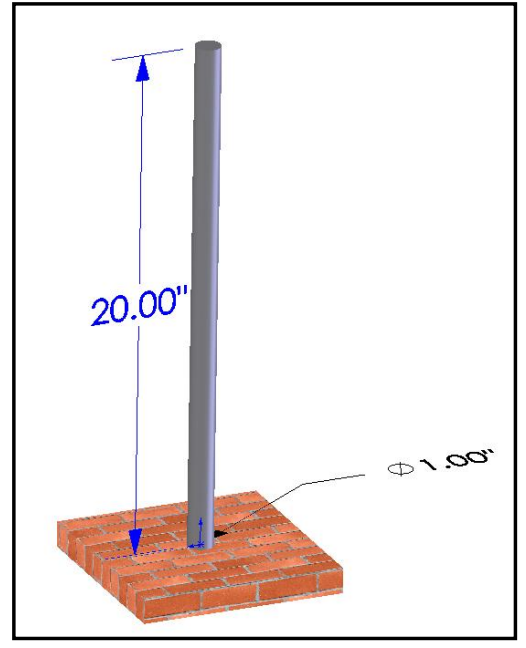

Figure 7.1. Cantilever Beam used for Verification Study 
The EOM for the cantilever beam, based on Bernoulli-Euler Beam Theory, is shown in Eq. 175. The static tip deflection induced by a transverse tip load is well established (19) and is shown in Eq. 176. A theoretical solution for the mode shapes and corresponding modal frequencies are detailed in Eq. 177 and Eq. 178, respectively (1). Eq. 177 and Eq. 178 use the constants listed in Eq. 179 and Eq. 180.

$$
\begin{gathered}
\frac{\partial^{2}}{\partial y^{2}}\left(E I \frac{\partial^{2} u}{\partial y^{2}}\right)+\rho A \frac{\partial^{2} u}{\partial t^{2}}=0 \\
u_{\max }=\frac{F L^{3}}{3 E I} \\
u_{r}(y)=\cosh \left(\lambda_{r} y\right)-\cos \left(\lambda_{r} y\right)-k_{r}\left(\sinh \left(\lambda_{r} y\right)-\sin \left(\lambda_{r} y\right)\right) \\
\omega_{r}=\frac{\left(\lambda_{r} L\right)^{2}}{L^{2}} \sqrt{\frac{E I}{\rho A}} \\
\lambda_{1} L=1.8751 \\
\lambda_{2} L=4.6941 \\
\lambda_{3} L=7.8548 \\
\lambda_{3} L=10.996 \\
\cosh \left(\lambda_{r} L\right)+\cos \left(\lambda_{r} L\right) \\
k_{r}=\frac{\sinh \left(\lambda_{r} L\right)+\sin \left(\lambda_{r} L\right)}{}
\end{gathered}
$$

Comparison of the FEM and theoretical tip deflection results under the applied $100 \mathrm{lbf}$ load is listed in Table 7.1, results match exactly. The static deformation shape FEM and theoretical results are displayed in Figure 7.2 (20 elements were used for the FEM model). Figure 7.2 also displays a comparison of FEM vs theoretical modal results including transverse mode shapes and axial mode shape (again, 20 elements were used for the FEM model). Table 7.2 displays a modal convergence study featuring a comparison of modal frequencies (FEM vs theoretical) for the first four transverse mode shapes. As shown in Table 7.2 modal frequency convergence was achieved, 
for the first four transverse mode shapes, by 20 elements. At a mesh density of 20 elements the modal frequency error is negligible $(0.002 \%)$ and the FEM vs theoretical mode shapes, as well as static deformation, are indistinguishable.

Table 7.1. Cantilever Beam Static Results Validation

\begin{tabular}{|c|c|c|}
\hline \multicolumn{3}{|c|}{ Tip Deflection Under Applied Load F [in] } \\
\hline Finite Element Method & Theoretical & $\%$ Error \\
\hline 0.522 & 0.522 & 0 \\
\hline
\end{tabular}
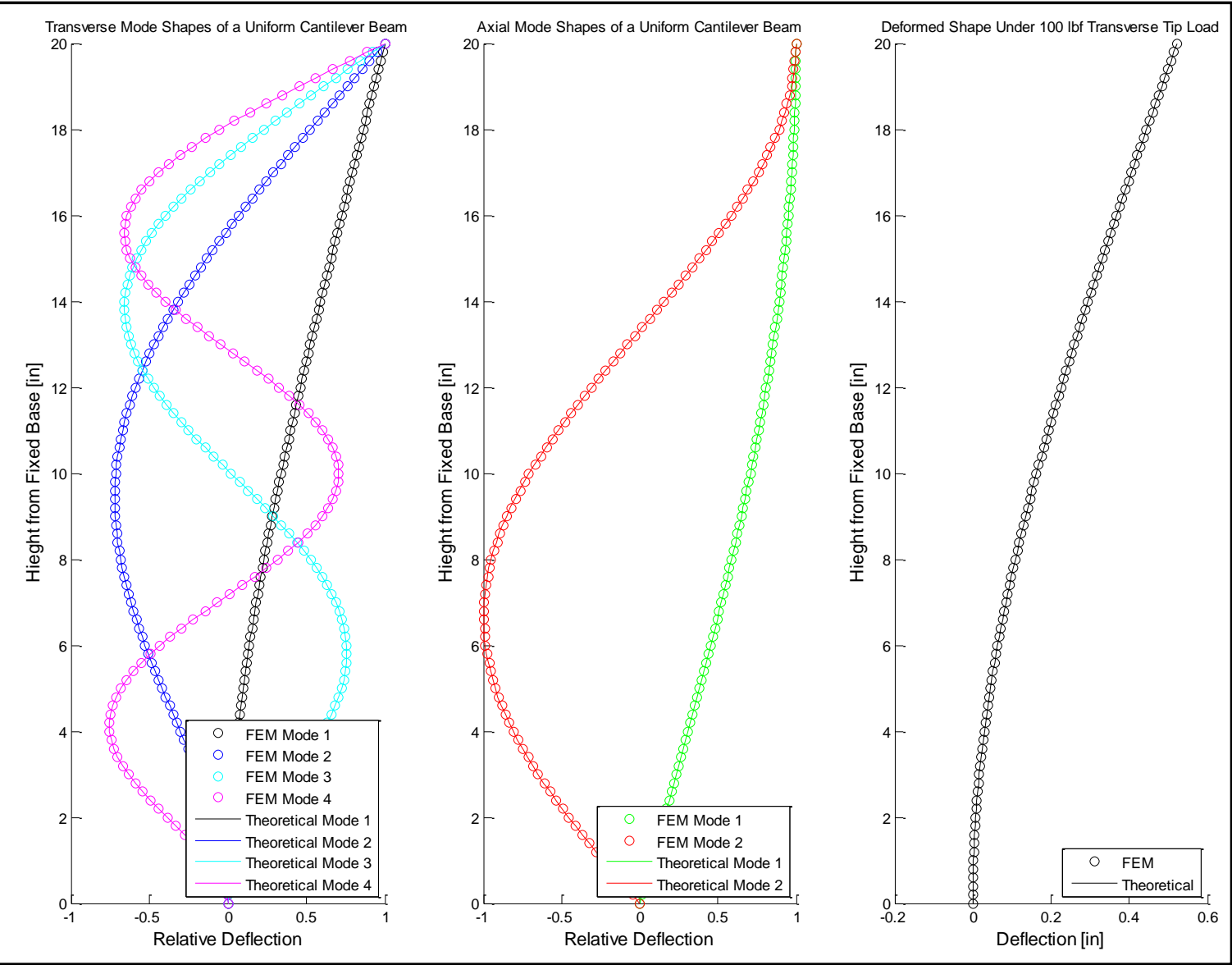

Figure 7.2. Cantilever Beam Modal and Static Results Comparison 
Table 7.2. Cantilever Beam Modal Convergence Study

\begin{tabular}{|c|c|c|c|c|c|c|c|c|c|c|c|}
\hline & & \multicolumn{8}{|c|}{ Number of elements } \\
\cline { 3 - 13 } Mode & Theoretical & \multicolumn{2}{|c|}{1} & \multicolumn{2}{|c|}{2} & \multicolumn{2}{|c|}{4} & \multicolumn{2}{c|}{10} & \multicolumn{2}{c|}{20} \\
\cline { 3 - 12 } & Freq $[\mathrm{Hz}]$ & Freq $[\mathrm{Hz}]$ & $\%$ Err & Freq $[\mathrm{Hz}]$ & $\%$ Err & Freq $[\mathrm{Hz}]$ & $\%$ Err & Freq $[\mathrm{Hz}]$ & $\%$ Err & Freq $[\mathrm{Hz}]$ & $\%$ Err \\
\hline 1 & 70.83 & 71.17 & 0.48 & 70.86 & 0.05 & 70.83 & 0.00 & 70.83 & 0.00 & 70.83 & 0.000 \\
\hline 2 & 443.87 & 701.16 & 57.97 & 447.64 & 0.85 & 444.38 & 0.11 & 443.87 & 0.00 & 443.87 & 0.000 \\
\hline 3 & 1242.86 & 2791.21 & 124.58 & 1513.99 & 21.82 & 1252.44 & 0.77 & 1243.13 & 0.02 & 1242.87 & 0.001 \\
\hline 4 & 2435.69 & NA & NA & 2596.81 & 6.62 & 2470.79 & 1.44 & 2437.75 & 0.08 & 2435.64 & -0.002 \\
\hline
\end{tabular}

\subsection{Tower - Physical Tower Testing and Model Correlation}

In Spring 2007 George Katsanis, Andrew Hamill, and William Krehbiel collaborated on a laboratory experiment for ME 517, Advanced Vibrations, under Dr. Meagher. While the experiment predated this work it was inspired by the, then budding, Cal Poly Wind Power Research Center. The experiment configuration consisted of a pinned monopole tower supported by guy-wires and featured a tip mass, as shown in Figure 7.3 and Figure 7.4.

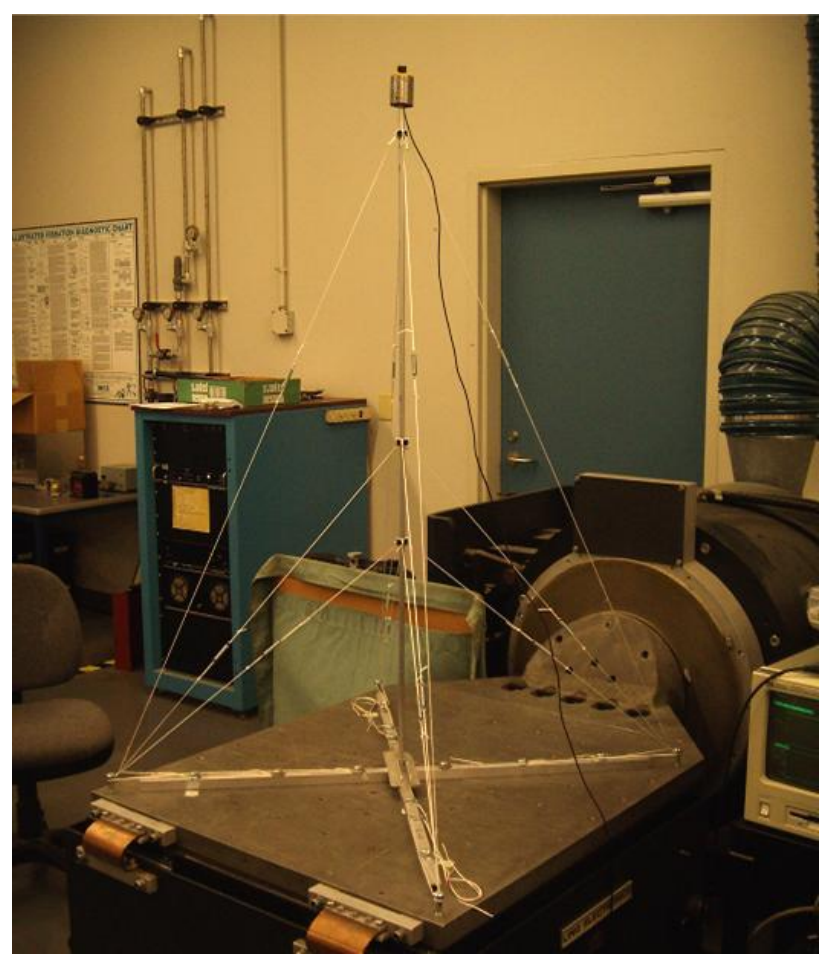

Figure 7.3. Guy-Wire Supported Tower Experiment Configuration 


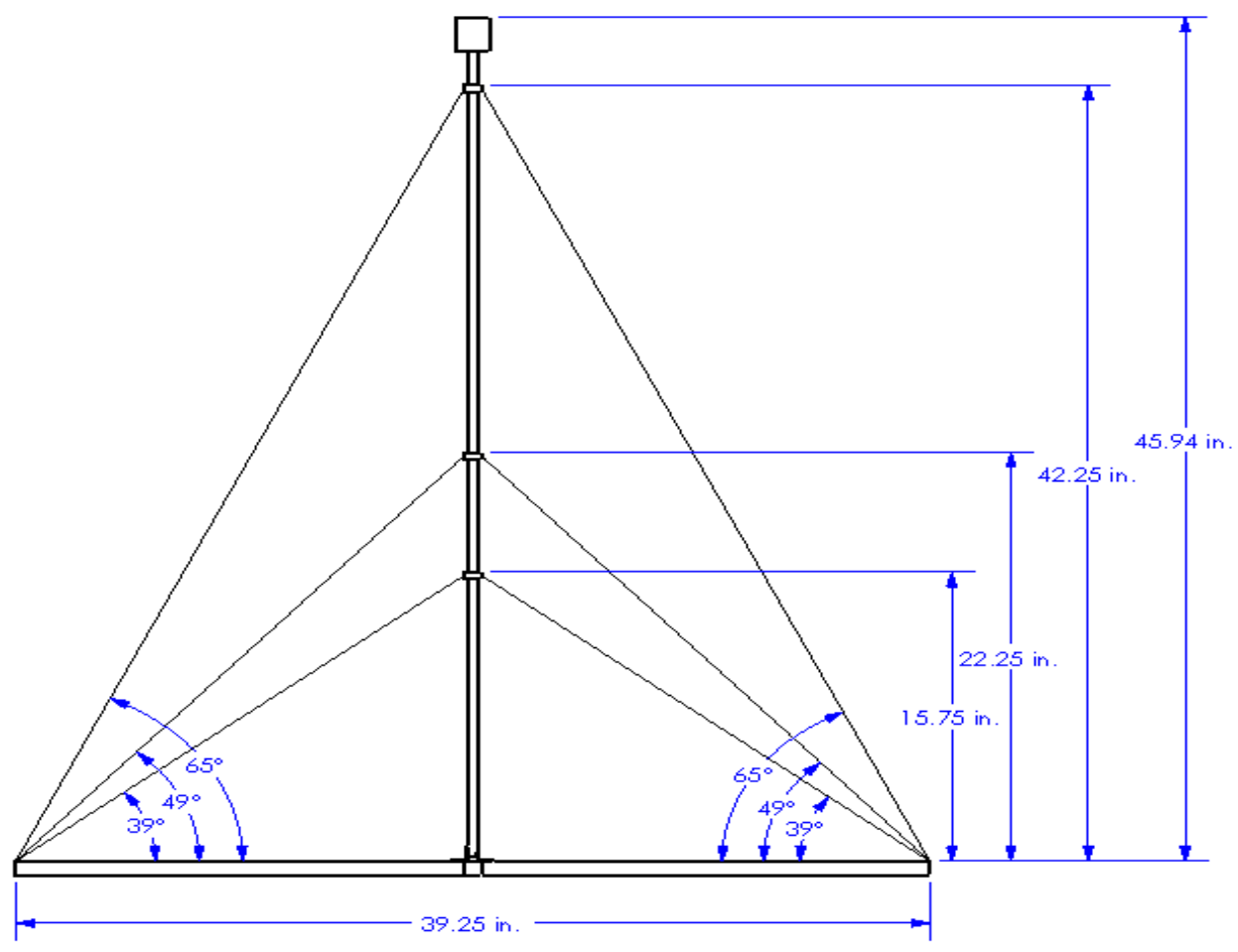

Figure 7.4. Guy-Wire Supported Tower Experiment Dimensions

The tower featured the following parameters:

- Solid Circular Cross-Section with 3/8 inch diameter

- $\quad$ Length (L): 44.25 inches

- Young's Modulus (E): 10.4 MPSI

- Density $(\rho): 0.098 \mathrm{lbs} / \mathrm{in}^{3}\left(0.0002536 \mathrm{slinch} / \mathrm{in}^{3}\right)$

- Tower Top Mass: 0.90 lbs (347.76 slinches)

Hammer tap tests, as shown in Figure 7.5, were performed to determine the tower's first three mode shapes and corresponding modal frequencies. The Star Modal Analysis program was used to process the data and produce the mode shape vector plots shown in Figure 7.6. 


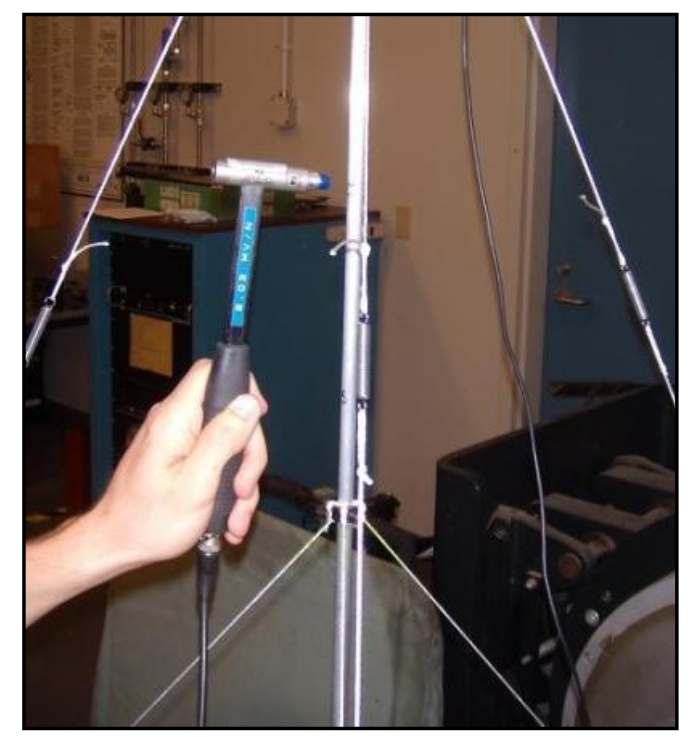

Figure 7.5. Guy-Wire Supported Tower Experiment Modal Tap Test

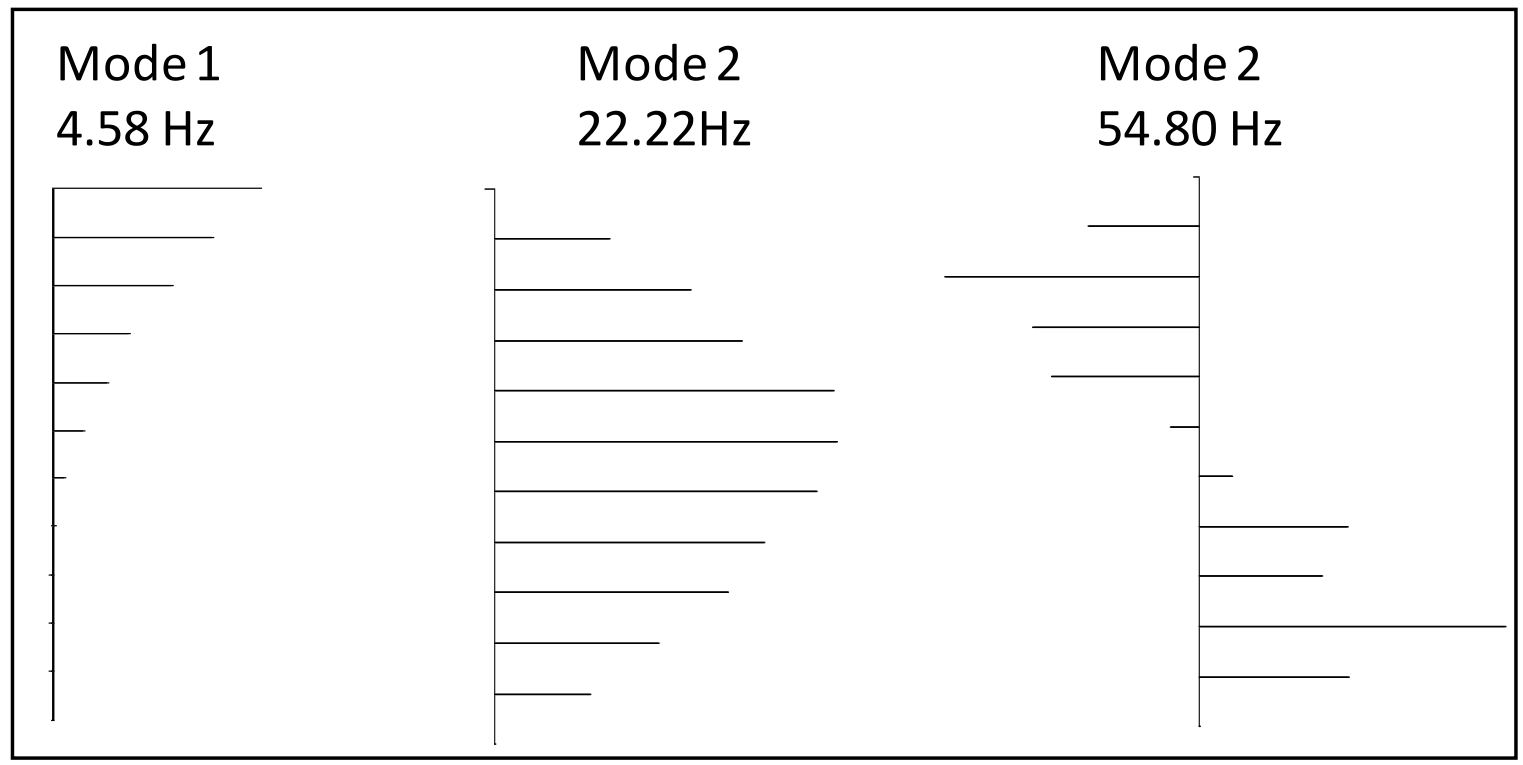

Figure 7.6. Guy-Wire Supported Tower Experiment Modal Results

The Matlab tool accompanying this work has been used to perform a modal analysis of the experimental configuration. The stiffness of the guy-wires was correlated in the model such that the $1^{\text {st }}$ modal frequency matched the experimental result. The resulting mode shapes and modal frequencies are shown in Figure 7.7. Note that the first three mode shapes in Figure 7.7 agree well with the corresponding mode experimental shapes shown in Figure 7.6. Note that the first modal 
frequency matches exactly (as expected since the Matlab model was tuned using this parameter). The second and third modal frequencies also agree well with the experimental results, with $9.3 \%$ error and $1.8 \%$ error respectively. Exact match of modal frequencies should not be expected as the experimental configuration likely featured some non-linear behavior due to the tower base support and insufficiently pretensioned guy-wires.

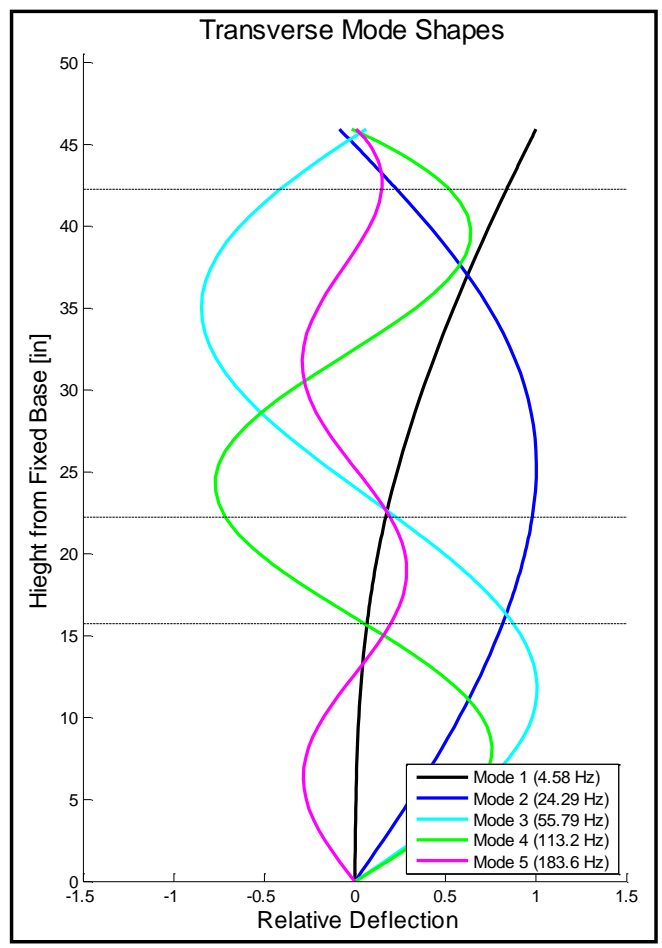

Figure 7.7. Matlab Modal Simulation of Experimental Configuration

\subsection{Tower - Case Study Comparison to Peer Work}

In Tom Gwon's work (6) Abaqus, a commercial FEA code, was used to study the Cal Poly Wind Turbine Tower response to static loading and as well as the tower's modal characteristics. The tower configuration evaluated in Gwon's work is the same configuration (without the rotor) used in Case Study of this work. In Gwon's work the wind turbine was modeled as a point mass while this work includes a flexible model for the blades along with a point mass for the other turbine 
components (nacelle, hub, generator, etc...). The results from this work correspond well with the results from Gwon's work as detailed in the following sections.

\subsubsection{Case Study Comparison to Peer Work - Static Loading}

Gwon evaluated the tower's response to the same 2,100 lbf thrust load used in Load Cases A in this work. Results are compared in Table 7.3.

Table 7.3. Comparison of Katsanis and Gwon Static 2100 lbf Static Thrust Load Results

\begin{tabular}{|c|c|c|c|c|c|c|}
\hline & \multicolumn{4}{|c|}{ Strong Axis } & \multicolumn{2}{c|}{ Weak Axis } \\
\cline { 2 - 7 } Study & $\begin{array}{c}\text { Max Tower } \\
\text { Deflection } \\
{[\text { inches] }}\end{array}$ & $\begin{array}{c}\text { Max Tower } \\
\text { Stress } \\
{[\mathrm{ksi}]}\end{array}$ & $\begin{array}{c}\text { Strut } \\
\text { Compression } \\
{[\mathrm{kips}]}\end{array}$ & $\begin{array}{c}\text { Gin-Pole } \\
\text { Tension } \\
{[\mathrm{kips}]}\end{array}$ & $\begin{array}{c}\text { Max Tower } \\
\text { Deflection } \\
{[\text { inches] }}\end{array}$ & $\begin{array}{c}\text { Max Tower } \\
\text { Stress } \\
{[\mathrm{ksi}]}\end{array}$ \\
\hline Katsanis & 41.8 & 35.1 & 12.6 & 16.7 & 69.5 & 35.1 \\
\hline Gwon & 40.0 & 36.3 & 10.0 & 6.7 & 68.8 & 37.6 \\
\hline \% Difference & 4.3 & -3.1 & 26.2 & 149.3 & 1.0 & -6.5 \\
\hline
\end{tabular}

Note that in Table 7.3 all results are in good agreement with the exception of the strut compression, and especially, the gin-pole tension induced by loading in the Strong-Axis. In Gwon's work some detailed features of the tower structure, such as gin-pole interface plates, were included which have been omitted from this work. In this work the gin-pole and strut connections to the tower, as well as to their pile-foundation, are modeled as direct pinned connections. This work does not include the influence of the interface plate that connects the gin-pole and strut to the pile-foundation or the influence of other gussets and brackets. The Cal Poly Wind Turbine Tower is an indeterminate structure (removal of the gin-pole would result in a determinate structure) and as such internal system loads can build up in the gin-pole. The modeling techniques used in this work (namely the omission of interface plates and brackets) results in an artificially stiff load path from the lower portion of the tower through the gin-pole and to the gin-pole's pilefoundation. Since load follows the stiffest path, the simplifications made in this work result in an over prediction of gin-pole loads. In reality the gin-pole interface plate and brackets add compliance to the gin-pole load path and serve to lessen the load transmitted through this path. The strength of this work does not lay in its utility to perform detailed stress analysis of 
secondary structure, but rather to evaluate macro-scale dynamic behavior of the coupled tower / rotor system, and to study how rotor parameters influence tower response.

\subsubsection{Case Study Comparison to Peer Work - Modal Evaluation}

System modal frequencies are compared in Table 7.4. The corresponding mode shapes, produced by this work, are shown in Figure 7.8. The mode shapes produced by Gwon's work are captured in Figures 7.1 through 7.10 of (6). The modal frequencies and mode shapes of modes 1, 2, 3, 5, 7, 8 , and 10 correspond well between the two works.

Modes 4, 6, and 9 appear in Gwon's work but not in this work because these modes are bending modes of the strut and gin-pole. As discussed in Chapter 3 the gin-pole and strut in this work are each modeled with a single bar element (2-force member capable of axial load only). Therefore the gin-pole and strut modes are not generated in this work. The exclusion of these modes is of little consequence when one considers the loading and likely failure modes of the gin-pole and strut. Since the gin-pole and strut are installed with pinned clevis style interfaces little to no bending moment will be transmitted to / through the gin-pole and strut. The critical failure mode for the gin-pole and strut is buckling due to compressive axial load; which is considered in both this work as well as Gwon's work.

Note that modes 11,13 , and 14, detailed in Table 7.4, are coupled tower / rotor modes with significant system response occurring in both the tower as well as rotor blade flapwise motion. Mode 12 is a "pure" rotor blade flapwise mode with negligible tower excitation.

The Case Study, detailed in Chapter 8, includes additional modal simulation results from this work including higher frequency tower axial modes. 
Table 7.4. Comparison of Katsanis and Gwon Modal Frequency Results

\begin{tabular}{|c|c|c|c|c|}
\hline \multirow{2}{*}{ Mode } & \multicolumn{2}{|c|}{ Frequency } & & \\
\cline { 2 - 3 } & Katsanis & Gwon & \% Differnce & Discription \\
\hline 1 & 0.59 & 0.58 & 1.0 & Weak Axis 1st Mode \\
\hline 2 & 0.81 & 0.83 & -2.9 & Strong Axis 1st Mode \\
\hline 3 & 2.97 & 2.81 & 5.9 & Weak Axis 2nd Mode \\
\hline 4 & N/A & 4.67 & N/A & Strut 1st Bending Mode \\
\hline 5 & 4.70 & 4.96 & -5.2 & Strong Axis 2nd Mode \\
\hline 6 & N/A & 6.38 & N/A & Strut 2nd Bending Mode \\
\hline 7 & 8.05 & 8.13 & -0.9 & Weak Axis 3rd Mode \\
\hline 8 & 12.99 & 13.90 & -6.5 & Strong Axis 3rd Mode \\
\hline 9 & N/A & 14.08 & N/A & Gin-pole 1st Bending Mode \\
\hline 10 & 15.08 & 15.61 & -3.4 & Weak-Axis 4th Mode \\
\hline 11 & 16.20 & N/A & N/A & Strong-Axis 4th Mode Coupled with Blade Flapwise Mode \\
\hline 12 & 16.25 & N/A & N/A & Pure Blade Flapwise Mode (minimal tower coupling) \\
\hline 13 & 16.38 & N/A & N/A & Weak-Axis 5th Mode Coupled with Blade Flapwise Mode \\
\hline 14 & 16.78 & N/A & N/A & Strong-Axis 5th Mode Coupled with Blade Flapwise Mode \\
\hline
\end{tabular}

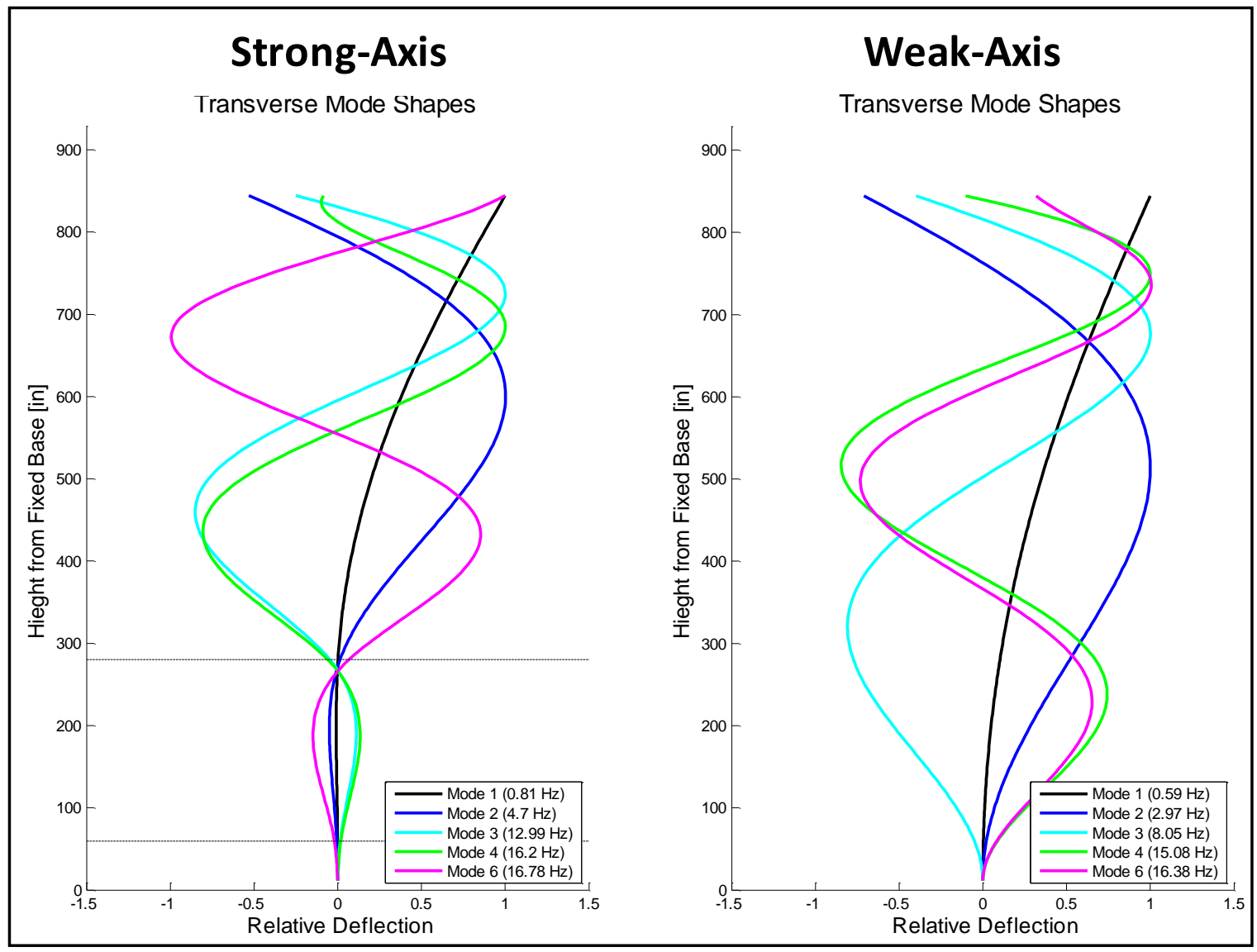

Figure 7.8. Matlab Modal Simulation of Cal Poly Wind Turbine Tower 


\subsection{Rotor - Model Checks}

Several simple rotor model checkouts were performed to validate the Case Study 3 blade rotor modal. These checkouts are summarized below:

- Static:

- All blades have a $\beta$ angle (relative flapwise rotation) of zero for a simple tower tip thrust load, such as the 2100 lbf load case where no loads are applied directly to the blades. The $\alpha$ angle (absolute flapwise rotation) is only a function of the $\theta$ angle (tower tip rotation) and $\Psi$ angle (blade's azimuth position). In other words, there is no elastic deformation of the blade and blade $\alpha$ angle is dependent only on kinematics.

- Applied 1000 in-lbf moment to blade 1 (with blade 1 oriented vertical upwards, $\Psi=0)$

- Tower deflection very similar to applying the 1000in-lbf moment directly to the tower tip in the $\theta$ direction.

- $\quad$ Blade $\beta$ angle (relative flapwise rotation) for blade 1 was $\beta_{1}=$ $1000 / \mathrm{K}_{1}(4,4)$

- Blade $\beta$ angles (relative flapwise rotation) for blades 2 and 3 were $\beta_{2}=\beta_{3}$ $=0$

- Tower bending loads were 1000 in-lbf at each node between the tower tip and the strut interface, zero shear, and zero axial load. Axial and shear loads developed at and below the strut interface - as expected.

○ Applied a +1000 in-lbf moment to each blade's hinge

- Blade $\beta$ angle (relative flapwise rotation) for all blades were

$$
\beta_{1}=\beta_{2}=\beta_{3}=1000 / \mathrm{K}_{1}(4,4)=1000 / \mathrm{K}_{2}(4,4)=1000 / \mathrm{K}_{3}(4,4)
$$


- No tower deformation / loads - as expected since the blade moment loads all sum to zero.

- Modal:

- Rotor blade flapwise modal frequencies match user input for rotating and nonrotating blade natural frequencies.

\subsection{Dynamic - Validation of Runge-Kutta Numerical Integration}

To validate the $4^{\text {th }}$ Order Runge-Kutta Numerical Integration method, used in the dynamic solver, a study has been conducted using the Single Degree of Freedom (SDOF) system shown in Figure 7.9. The EOM for this SDOF system is shown in Eq. 181. For this example a very simple force profile $\left(F_{\text {sdof }}(t)\right)$ is considered in which a force of constant amplitude is applied at time $=0$ (i.e., step function). For this type of simple force profile an exact solution can be found (1) and is shown in Eq. 182.

$$
M_{\text {sdof }} \ddot{u}+C_{\text {sdof }} \dot{u}+K_{\text {sdof }} u=F_{\text {sdof }}(t)
$$

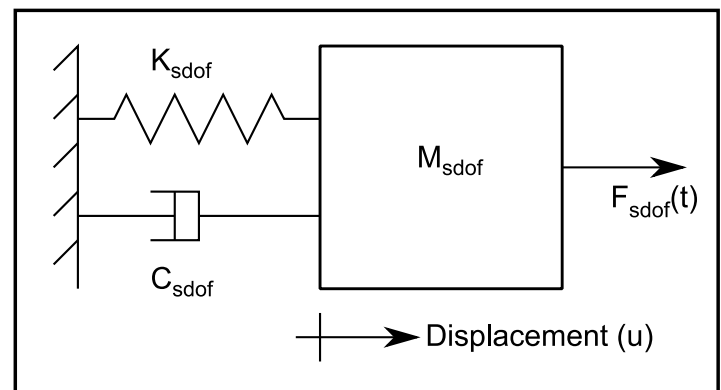

Figure 7.9. Example SDOF System

$$
u(t)=\frac{F_{\text {sdof }}}{K_{\text {sdof }}}\left[1-e^{-\zeta \omega_{n} t}\left(\cos \left(\omega_{d} t\right)+\frac{\zeta \omega_{n}}{\omega_{d}} \sin \left(\omega_{d} t\right)\right)\right]
$$

Eq. 182 
Eq. 182 uses the damping ratio $(\zeta)$, the un-damped natural frequency $\left(\omega_{n}\right)$, and the damped natural frequency $\left(\omega_{d}\right)$, as shown in Eq. 183 through Eq. 185.

$$
\begin{gathered}
\zeta=\frac{C_{\text {sdof }}}{2 \sqrt{K_{\text {sdof }} M_{\text {sdof }}}} \\
\omega_{n}=\sqrt{\frac{K_{\text {sdof }}}{M_{\text {sdof }}}} \\
\omega_{d}=\omega_{n} \sqrt{1-\zeta^{2}}
\end{gathered}
$$

For this validation study the following properties were chosen for the SDOF mass, damping, and stiffness as shown in Eq. 186.

$$
\begin{gathered}
M_{\text {sdof }}=38.64 \mathrm{lbs}=0.1 \frac{\mathrm{lbf} \cdot \mathrm{s}^{2}}{\mathrm{in}} \\
C_{\text {sdof }}=1 \frac{\mathrm{lbf} \cdot \mathrm{s}}{\mathrm{in}} \\
K_{\text {sdof }}=1000 \frac{\mathrm{lbf}}{\mathrm{in}}
\end{gathered}
$$

The parameters above are significant as they result in a damping ratio of $\zeta=0.05$, which is the same damping ratio used in the case study. The EOM of Eq. 181 was solved using the exact theoretical solution method of Eq. 182 and the $4^{\text {th }}$ Order Runge-Kutta Numerical Integration method described in Chapter 6. A convergence study was conducted by progressively decreasing the duration of the time step size used in the Runge-Kutta method. The time step sizes were chosen as a function of the un-damped SDOF period (which is 0.0629 seconds). For example the study began by using 5 time steps per period (thus the time step size is 0.0629 seconds $/ 5=$ 0.0126 seconds). The results of the convergence study are shown in Figure 7.10. The Rung-Kutta model was found to rapidly diverge when using less than 3 time steps per period. At as few as 5 
time steps per period the Runge-Kutta method does a reasonable job. At 10 time steps per period the Runge-Kutta method matches very closely with the exact solution (peak displacement percent error $<0.02 \%$ ). Using more time steps per period (e.g., 20) results in even better convergence, but such accuracy is rarely worth the computational penalty. For these reasons the User Input template recommends using a time step size which will result in 10 time steps per period for the highest modal frequency included in the simulation (as discussed in Chapter 6 the user can omit high frequency modes from the simulation using modal truncation).

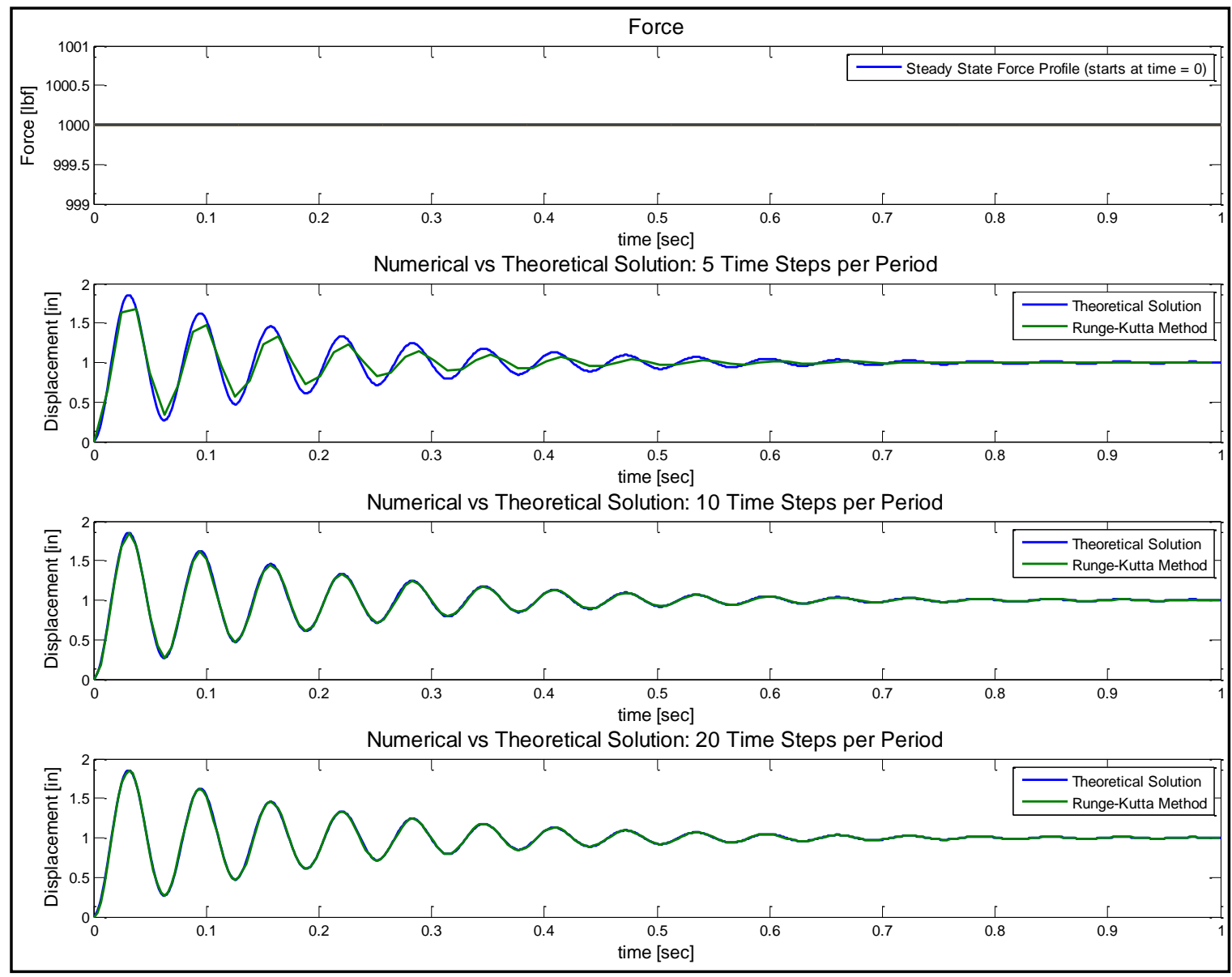

Figure 7.10. SDOF Theoretical vs Runge-Kutta Convergence Study 


\section{Case Study}

\subsection{Introduction}

The coupled tower / turbine simulation methods, established in the preceding chapters, are applied to the tower / turbine configuration used by the Cal Poly Wind Power Research Center, herein referred to as the Cal Poly System. The Cal Poly System is evaluated for all load cases discussed in Chapter 5. Several side studies were conducted utilizing the Cal Poly System including a modal truncation convergence study, a vortex shedding study, and a hypothetical guywire optimization study.

Data, plots, figures, and in-depth discussion of the Case Study are all included in Appendix C. This chapter opens with a brief overview of the Cal Poly System followed by a brief summary of the Case Study. Appendix $\mathrm{C}$ is reference throughout this chapter and the reader is directed there for further details.

\subsection{Overview of the Cal Poly System}

The Cal Poly System parameters have been detailed in depth by Gwon (6) and Edwards (9), who focused on tower and blade design, respectively. Select parameters, pertinent to this work, have been summarized in the subsequent tower and turbine sections.

The Cal Poly System is displayed in Figure 8.1 along with the rotor and tower coordinate systems. The coordinate systems are discussed further in Section 3.4.3 as well as Chart 4 of Appendix C. 


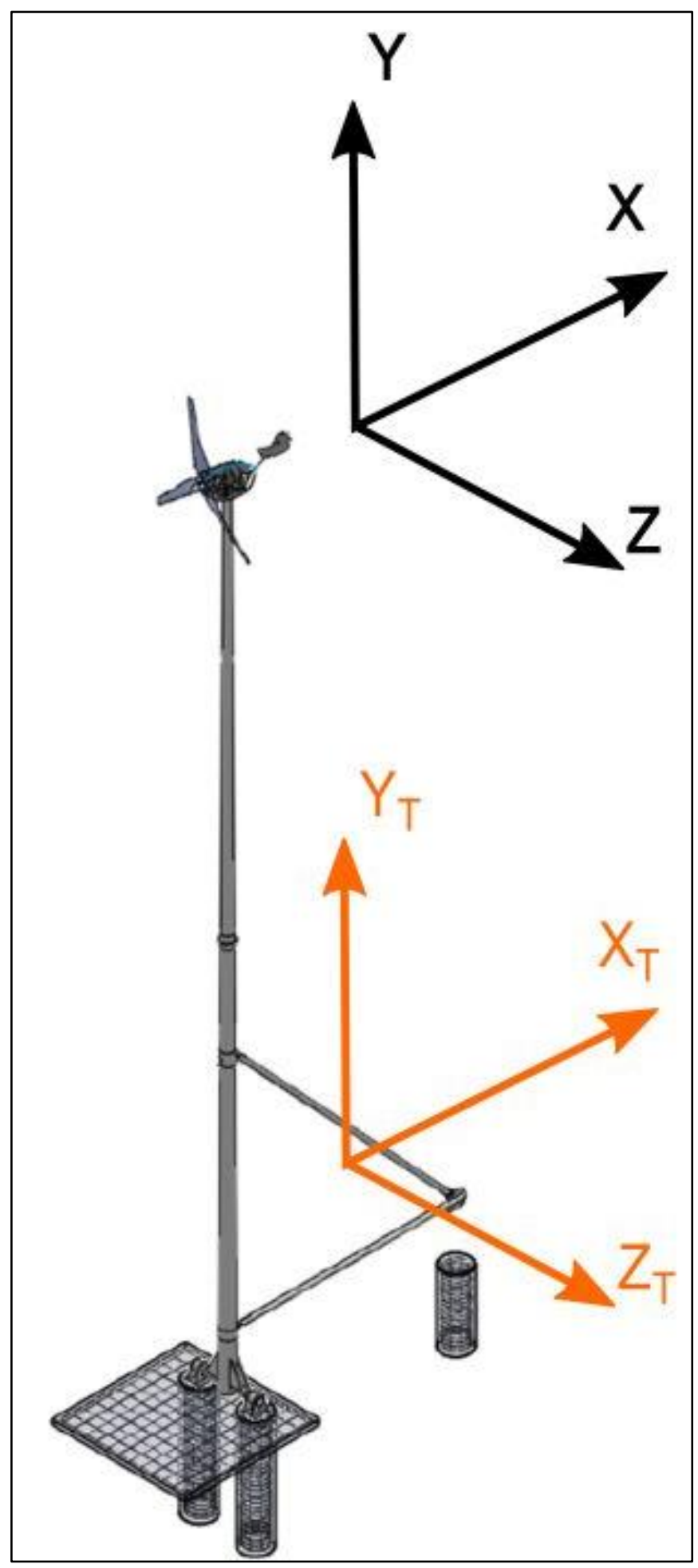

Figure 8.1. The Cal Poly System-Coordinates 


\subsubsection{Cal Poly System - Tower}

The Cal Poly System features a steel tubular tower with a gin-pole and strut support

configuration. The tower is mounted to a set of bearings at its base which, along with the gin-pole and strut support allow the tower to be erected and lowered through the use of a truck mounted cable winch. When compared to alternative tower configurations, which often require a large crane which can reach to the top of the tower, a gin-pole and strut supported tower simplifies initial installation as well as future turbine access for servicing. An additional advantage of the gin-pole and strut configuration is the lack of guy-wires which reduces potential hazards for students who will work around the system.

Special modeling considerations specific to gin-pole and strut supported towers were explored in Section 3.4.3 and apply to this case study. Loading was considered in the Strong-Axis (wind oriented in the $\mathrm{X}_{\mathrm{T}}$-Axis) as well as the Weak-Axis (wind oriented in the $\mathrm{Z}_{\mathrm{T}}$-Axis). For StrongAxis loading the base of the tower was modeled using a pinned boundary condition (only translations constrained) and overturning moments caused by the wind load are reached out through the gin-pole and strut supports. For Weak-Axis loading the base of the tower was modeled as fixed (translations and rotations constrained) and the gin-pole and struts were assumed to provide no support (overturning moment reacted out through the tower's base). Soil compliance and damping effects have been ignored in this work (i.e., the ground is modeled as perfectly rigid).

Select tower specifications are shown in Table 8.1 and dimensions are shown in Figure 8.2.

Table 8.1. Cal Poly Tower Specifications

\begin{tabular}{|c|c|}
\hline \multicolumn{2}{|c|}{ Cal Poly System - Tower Specifications } \\
\hline Tower Type & Tapered Tube with Gin-Pole Support \\
\hline Tower Height & $70 \mathrm{ft}$ \\
\hline Tower Material & ASTM-A570 Grade-50 Steel \\
\hline
\end{tabular}




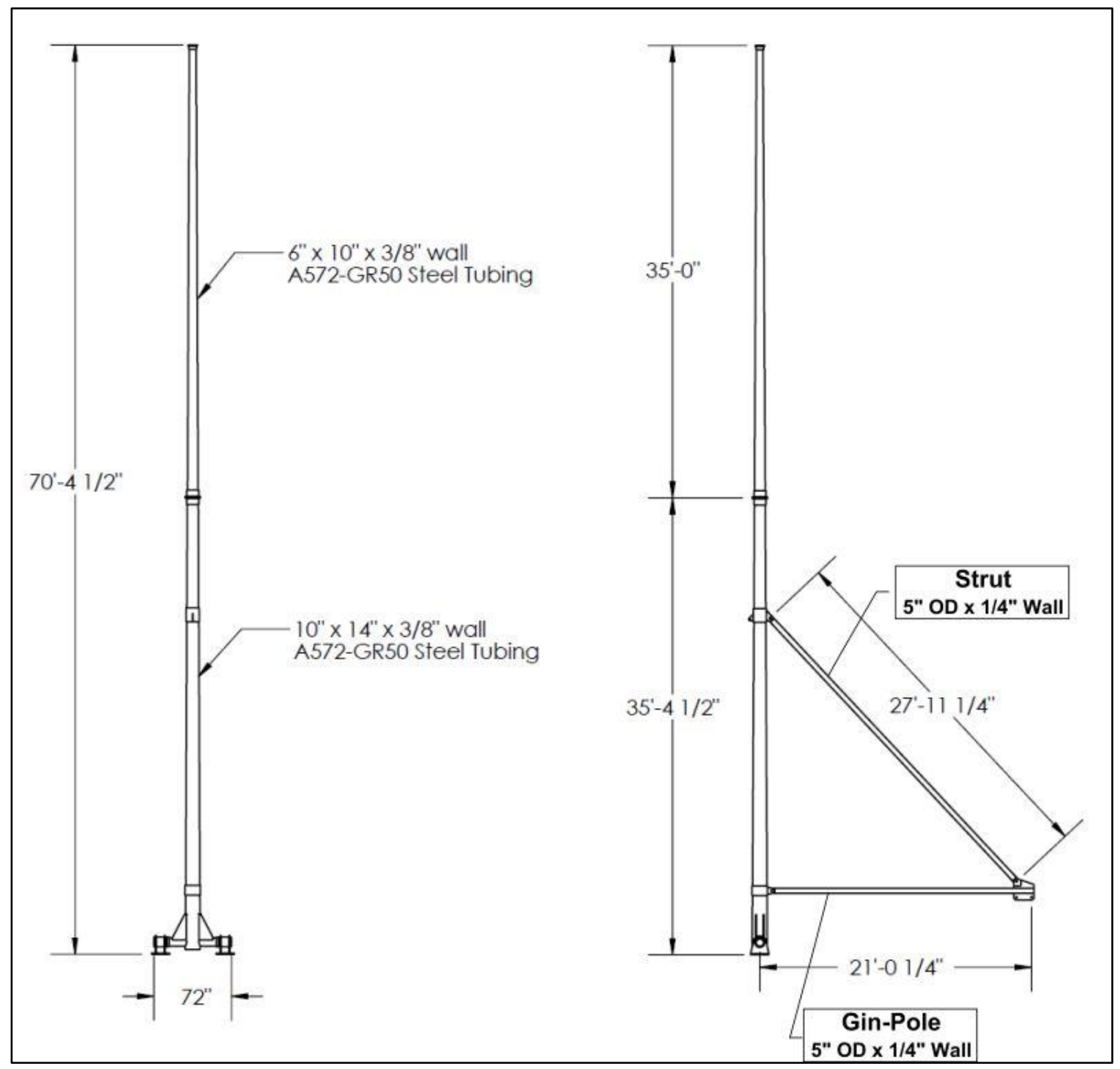

Figure 8.2. The Cal Poly System - Select Dimensions 


\subsubsection{Cal Poly System - Turbine}

Select specification of the Cal Poly Turbine are included in Table 8.2.

\section{Table 8.2. Cal Poly Turbine Specifications}

\begin{tabular}{|c|c|}
\hline \multicolumn{2}{|c|}{$\begin{array}{c}\text { Cal Poly System - Turbine Specifications } \\
\text { (partially reproduced from (6)) }\end{array}$} \\
\hline Wind Turbine Category & Small Wind Turbine (per IEC61400-2 [4]) \\
\hline Wind Turbine Type & Horizontal-Axis Wind-Turbine (HAWT) \\
\hline Wind Turbine Configuration & Stall-regulated, Passive-yawing \\
\hline Wind Turbine Weight & $460 \mathrm{lbf}$ \\
\hline Number of Blades & 3 \\
\hline Rotor Diameter & $12 \mathrm{ft}$ \\
\hline Rated Power & $3 \mathrm{~kW}$ \\
\hline Rated Rotor Speed & $230 \mathrm{rpm}$ \\
\hline Rated Wind Speed & $22.4 \mathrm{mph}$ \\
\hline Cut-off Wind Turbine Speed & $300 \mathrm{rpm}$ (Wind Speed of 30 mph) \\
\hline Stopping Mechanism & Disk Brake \\
\hline
\end{tabular}

The Cal Poly Turbine was modeled using the methods detailed in Chapter 4 . The turbine blades were modeled using Hinge Offset Equivalent Blade Theory and the other turbine components (hub, drive train, generator, bedplate, and nacelle) were modeled using a single point mass at the tower tip. The offset from the tower tip to the rotor center / blade root interface is assumed to be 16 inches in the $\mathrm{X}$-Axis and 12 inches in the Y-Axis.

The Hinge Offset Equivalent Blade Theory requires knowledge of blade rotating and non-rotating natural frequency. This information was not previously available so these parameters were estimated using the Myklestad Method (11) along with information available in Edward's work (9). The evaluation process is documented in detail in Appendix C-8. As discussed in Appendix C-8 the evaluation resulted in the following natural frequencies:

- Non-Rotating Natural Frequency: $15.69 \mathrm{~Hz}$

- Rotating Natural Frequency: $19.38 \mathrm{~Hz}$ (at operational rotor speed of 230 RPM) 
Upon development of the Hinge Offset Equivalent Blade Model, using the above parameters, it was found that a hinge ratio of $\mathrm{e}=0.84$ resulted (i.e., the hinge was much closer to the blade tip than the root). A high hinge ratio (e) results in reduced blade mass dynamic participation. To be conservative it was desired to use a lower hinge ratio, which results in increased blade mass dynamic participation. Therefore the rotating natural frequency was adjusted downward to 16.25 Hz. This adjustment resulted in a blade hinge ratio of $\mathrm{e}=0.12$ (which seems much more reasonable when considering the static blade deformation plot appearing in Appendix C-8). This illustrates an aspect of the Hinge Offset Equivalent Blade Model that the analyst must be aware of. Adjustments to blade analytical parameters should be made such that the hinge is placed in a reasonable configuration (i.e., closer to the root than to the blade tip).

As discussed in Sections 8.4.1 and 8.4.2 the Swept Sine load case was used to study the influence of blade natural frequency parameters by varying these parameters and evaluating changes in system response. As the blade flapwise natural frequency approaches the tower's fundamental frequency the system response (both tower tip displacement and blade flapwise displacement) increases dramatically. Fortunately, the blade's non-rotation natural frequency $(15.69 \mathrm{~Hz})$ was estimated to be over 4 octaves higher than the tower's Strong-Axis fundamental frequency $(0.81$ $\mathrm{Hz}$ ) putting well out of the range of concern and minimizing dynamic interaction between the tower and the blades.

\subsection{Material Strength and Partial Factors of Safety}

As noted in Table 8.1 the tower is constructed from ASTM-A570 Grade-50 High Strength Steel. The material strength as well as Partial Factors of Safety used in the case study are summarized in Table 8.3. 
Table 8.3. Cal Poly Tower Material Strength and Factors of Safety

\begin{tabular}{|l|c|l||}
\hline \multicolumn{3}{|c|}{ Tower Material Properties and Partial Factors of Safety } \\
\hline Material Properties & Value & Source \\
\hline Ultimate tensile strength & $65.3 \mathrm{ksi}$ & ASTM A572/A572M-12, Table 4 (33) \\
\hline Yield tensile strength & $50 \mathrm{ksi}$ & ASTM A572/A572M-12, Table 4 (33) \\
\hline Strut Critical Buckling Load & $27.05 \mathrm{kips}$ & (Shigley, Eq 5-47) (19) \\
\hline Partial Safety Factors & Value & Source \\
\hline Material Ultimate & 1.1 & IEC 61400-2, Table 6 (3) \\
\hline Material Yield & 1 & IEC does not specify, assume 1 \\
\hline Material Fatigue & 1.25 & IEC 61400-2, Table 6 (3) \\
\hline Material Stability (Buckling) & 1.1 & IEC does not specify, assume same as ultimate \\
\hline Load Ultimate & 1.35 & IEC 61400-2, Table 7 (3) \\
\hline Load Yield & 1 & IEC does not specify, assume 1 \\
\hline Load Fatigue & 1 & IEC 61400-2, Table 7 (3) \\
\hline Load Stability (Buckling) & 1.35 & IEC does not specify, assume same as ultimate \\
\hline
\end{tabular}

The material strengths and partial safety factors, appearing in Table 8.3, are used, along with the predicted stresses, to calculate Margin of Safety using Eq. 187. The metric for acceptability is that the Margin of Safety must be $\geq 0$. Note that Eq. 187 is based on IEC (3) guidelines.

$$
\text { Margin of Safety }=\frac{\sigma_{a}}{\sigma_{p} \cdot \gamma_{m} \cdot \gamma_{l}}-1
$$

Where:

- $\sigma_{a}$ is the allowable material stress (yield or ultimate) as defined in Table 8.3

- $\sigma_{p}$ is the predicted material stress (yield or ultimate)

- $\quad \gamma_{m}$ is the material partial factor of safety as defined in Table 8.3

- $\quad \gamma_{l}$ is the load partial factor of safety as defined in Table 8.3

\subsection{Case Study Evaluations}

The results of the Case Study are all included in Appendix C. Note that Chart 3 of Appendix C includes a Results Summary Table which reports Max Tower Deflection, Max Tower von Mises 
Stress, Strut Axial Load, and Margin of Safety for all load cases. The Fatigue Cumulative Damage Index is also reported for all Normal Turbulence Model simulations. The overall maximum tower deflection was 69.45 inches and the overall minimum Margin of Safety was 0.25, both occurring for Load Case A (2100 lbf Tip Load) in the Weak Axis.

As discussed in the preceding chapters, the loads and stresses produced from this work can be used to study system characteristics, identify tower / rotor interaction trends, and perform system structural optimization. The stresses reported by this work do not capture secondary structure or component level (i.e., gusset, bearing, bracket, etc...) behavior and do not consider stress concentration factors. As such the results from this work should always be used in conjunction with detailed stress analysis of all structural components, as performed by Gwon (6). The subsequent sections in this chapter discuss the Case Study evaluations in further detail and highlight notable findings captured in Appendix C. Many of the results were previously discussed in Chapter 7 where they were compared, for verification purposes, to Gwon's work (6).

\subsubsection{Strong Axis Load Cases}

All static and dynamic load cases discussed in Chapter 5 were evaluated for the Cal Poly System in the Strong Axis. Results for all Strong Axis simulations, including modal simulations, are included in Appendix C-1.

None of the IEC dynamic load cases feature frequency content sufficiently high to cause system resonance. Even the Normal Turbulence Model, which contains the highest frequency content, only features frequency content up to $0.1 \mathrm{~Hz}$ which is 3 octaves lower than the fundamental Strong-Axis mode of $0.81 \mathrm{~Hz}$. Furthermore, since the rotor blade non-rotating and rotating natural frequency parameters were both set to the $16 \mathrm{~Hz}$ range, they effectively act as rigid bodies for all IEC load cases. Thus the results for the IEC load case would be essentially the same even if the blades had been omitted from the model and their mass included in the tower tip point mass. As such the most interesting load case is, by far, the Swept Sine load case. 
The results from the Strong Axis Swept Sine load case are included in Appendix C-1 Charts 59 70. For the purpose of the Case Study the Swept Sine simulation was run from $0.1 \mathrm{~Hz}$ to $20 \mathrm{~Hz}$ with a logarithmic sweep rate of 0.5 octaves / minute. As discussed on Chart 61 of Appendix C-1 the simulation was repeated several times to study the influence of rotor natural frequency and load application method on system response. In Charts 63 - 65 of Appendix C-1 the thrust load is applied directly to the tower tip and no moment is applied to the blade hinge. In Chart 63 of Appendix C-1 the rotor blades are modeled as perfectly rigid and the tower's $1^{\text {st }}, 2^{\text {nd }}$, and $3^{\text {rd }}$ modes are clearly visible. In Chart 64 of Appendix C-1 the thrust load is again applied directly to the tower tip but this time the rotor blades feature a flapwise mode at $16.25 \mathrm{~Hz}$; which shows up in the tower tip displacement transfer function just beyond the tower's $3^{\text {rd }}$ mode. In chart 65 of Appendix C-1 the thrust load is once again applied directly to the tower tip but this time the blade flapwise mode has been tuned to $0.81 \mathrm{~Hz}$ to match the tower's fundamental mode; interestingly the tower tip displacement is actually reduced for this case as the blades act as vibration absorbers - a behavior discussed by Craig (1). In Charts 66 - 68 of Appendix C-1 the rotor thrust load is combined with a proportional moment acting directly on the blade hinges as discussed in Section 5.2.4. In Chart 66 of Appendix C-1 the addition of blade hinge moment has virtually no effect on the rigid blade case (which makes sense considering that the blades are not allowed to flex about their hinge when rigid). In Chart 67 of Appendix C-1 the addition of blade hinge moment is found to have a pronounced effect when the blades are modeled with a flapwise mode at $16.25 \mathrm{~Hz}$; the tower response transfer function is a magnitude of order higher than the corresponding case without blade hinge moment. Finally in Chart 68 of Appendix C-1 the addition of blade hinge moment is found to again have a pronounced effect when the blade flapwise mode is tuned to match the tower's fundamental mode; once again increasing tower tip response by approximately a magnitude of order over the corresponding case without blade hinge moment. 
When considering System response to the Swept Sine Load Case it is not only interesting to evaluate the tower tip displacement transfer function but the blade flapwise rotational displacement is also of interest. Charts 69 and 70 of Appendix C-1 include overlays of blade flapwise rotation relative to the tower tip ( $\beta$ angle). Both Charts 69 and 70 consider the case where the thrust load is combined with a proportional blade hinge moment. In Chart 69 the tower tip response transfer function is overlaid with the blade flapwise $\beta$ angle for the case in which the blade flapwise mode is $16.25 \mathrm{~Hz}$; a pronounced peak in blade $\beta$ angle clearly aligns with a peak in tower tip response at $16.25 \mathrm{~Hz}$ - this is an excellent illustration of coupled tower / rotor blade dynamic behavior. Chart 70 features a similar overlay but this time for the case in which the blade flapwise response has been tuned to $0.81 \mathrm{~Hz}$ to match the tower's fundamental mode; again tower tip resonance and blade flapwise resonance are coincident.

\subsubsection{Weak Axis Load Cases}

As with the Strong Axis all load cases were also evaluated for the Weak Axis and captured in Appendix C-2. With loading oriented in the Weak Axis the maximum peak tower tip displacement increases by roughly a factor of 1.66 over loading in the Strong Axis. Despite the larger tower displacements the corresponding tower stresses do not significantly increase when loading is oriented in the Weak Axis. The tapered geometry of the tower tube (which features a 14 inch diameter at the base and a 6 inch diameter at the tip) helps mitigate tower stress sensitivity to load direction.

The tower fundamental mode in the Weak Axis is $0.59 \mathrm{~Hz}$ which is significantly lower than the $0.81 \mathrm{~Hz}$ fundamental mode in the Strong Axis. Nevertheless, and as was the case for the Strong Axis, the IEC load cases fail to cause resonance of the system in the Weak Axis since the highest excitation frequency content is $0.1 \mathrm{~Hz}$ (over two octaves below the tower fundamental mode). As such the most interesting dynamic load case is the Swept Sine Load Case. The same six combinations of Swept Sine load application method (with and without blade hinge moment) and blade tuning (rigid, $16.25 \mathrm{~Hz}$ mode, and tuned to the tower's fundamental frequency) were 
evaluated for the Weak Axis as was done for the Strong Axis. Chart 124 of Appendix C-2 features a tower tip transfer function overlay of all six evaluations. The findings from the Swept Sine Weak Axis simulation were analogous to the Strong Axis findings, see Section 8.4.1.

\subsubsection{Modal Truncation Study}

In addition to the Strong Axis and Weak Axis Load Case studies several side studies were also performed. The first side study was a modal truncation study. As discussed in Section 6.4 the Mode-Superposition Method allows the analyst to truncate the solution sequence such that only n-number of modes are considered. For most structural systems, response due to dynamic load profiles is dominated by excitation of the first few system modes and as such higher frequency modes can often be omitted from the analysis (1).

A convergence study, discussed further in Charts 126 - 128 of Appendix C-3 was conducted to determine the minimum number of system modes that should be included in the simulation. The convergence study utilized the quasi-steady state initial portion of the Extreme Operating Gust load case and compared results from a static solution sequence to a truncated mode-superposition solution sequence; tower displacements and stresses were compared.

Only the first 7 modes are required to achieve excellent displacement convergence. When 7 modes are included the RMS Percent Error (defined in Chart 129) for displacements is only $0.04 \%$ when comparing the truncated mode-superposition solution sequence to the static solution sequence. Interestingly when 7 modes are included the RMS Percent Error for von Mises stress is $1.92 \%$; certainly not poor but not nearly as good as displacements. It was found that 25 modes are required to achieve $0.04 \%$ RMS Error for von Mises Stress, indicating that system stress converges much more slowly than displacement. This behavior is well documented (32) and is evident when one considers that stresses are proportional to local displacement gradients (i.e., the derivative of displacement). Small local changes in displacement gradient may not appear consequential when one evaluates overall macro-scale displacement but these small changes can have a big impact on local stresses. All dynamic load case simulations appearing in Appendix C-1 
and C-2 utilized 30 modes, which as shown in Appendix C-3, provides excellent convergence for both displacement and stress.

\subsubsection{Cyclic Excitation due to Vortex Shedding}

Appendix C-4 includes an evaluation of cyclic excitation due to vortex shedding. As discussed on

Chart 135 of Appendix C-4, when wind flows past a cylindrical tower vortex shedding can

produce excitation orthogonal to the wind direction. Charts 135 and 136 capture the method, and

results, for determining the vortex shedding frequency based on the Strouhal number (28). Chart

137 discusses the method for conservatively estimating the force generated by vortex shedding

(31). Chart 137 also discusses the method for using the swept sine transfer function to estimate

maximum tower response to vortex shedding. Finally Chart 138 summarizes the vortex shedding

evaluation results. Maximum tower displacement, due to vortex shedding, is estimated at 0.28

inches.

\subsubsection{Guy-Wire Optimization}

Appendix C-5 includes an example guy-wire optimization side study which considers hypothetical stiffening of the Cal Poly System's Weak Axis through the use of two sets of guywires. The guy-wires are oriented in the $\mathrm{Y}_{\mathrm{T}} \mathrm{Z}_{\mathrm{T}}$-Plane as shown in Chart 140. As discussed in Chart 141 the height of the guy-wire interfaces to the tower were optimized for several parameters including maximizing the tower's fundamental frequency, maximizing separation between the tower's $1^{\text {st }}$ and $2^{\text {nd }}$ modes, and minimizing tower stress due to environmental loading (optimization was performed separately for each parameter). Results for each optimization are shown in Charts 142 through 144. When compared with the baseline configuration the fundamental frequency increases dramatically from $0.59 \mathrm{~Hz}$ to $3.45 \mathrm{~Hz}$ when using the guy-wire configuration discussed on Chart 142 (effectively stiffening the Weak-Axis by a factor of 34).

\subsubsection{Strut Critical Buckling Load}

Appendix C-6 captures the calculation to determine the critical axial load for strut buckling. The Euler column formula for pinned-pinned boundary conditions was used (19). The critical 
buckling load was found to be 27 kips. This load was used along with a formula analogous to Eq. 187 to determine the strut buckling margins of safety that appear on Chart 3 of Appendix C.

\subsubsection{Fatigue}

Appendix C-7 summarizes the fatigue evaluation. Chart 148 summarizes the estimated Fatigue Strength Curve (i.e., "S-N" Curve) for ASTM A572 Grade 50 Steel based on empirical relations (19). Per IEC (3) the only load case included in this work that is subject to fatigue evaluation is the Normal Turbulence Model. The maximum stress (10.05 ksi) produced by the Normal Turbulence Model is less that the predicted material endurance limit. Therefore the tower's primary structure could theoretically withstand an unlimited duration of exposure to SWTC 3 Normal Turbulence Model conditions (the reader is deferred to Gwon (6) for evaluation of secondary structure). As such the Cumulative Damage Index (CDI) calculation is trivial and results in a CDI of 0 for exposure to any duration of Normal Turbulence Model conditions. If stresses above the material endurance limit had been predicted then a cycle counting method such as the Rainflow Method (11) would need to be utilized to estimate load cycle count and to calculate the CDI.

\subsubsection{Myklestad Method}

Appendix C-8 summarizes the use of the Myklestad Method to determine the rotor blade rotating and non-rotating flapwise natural frequencies. Notable features of this evaluation were previously discussed in Section 8.2.2. 


\section{Conclusion and Future Work}

\subsection{Conclusion}

A highly integrated tool has been developed to perform structural analysis and optimization studies of small wind turbine towers. The tool has been implemented in Matlab and a digital copy of the tool has been submitted along with this report. The tool can be used throughout the design phase of tower development to explore coupled tower / rotor dynamic interaction, determine tower loads and stresses due to IEC Aeroelastic Design Load Cases, and to optimize system parameters such as guy-wire placement.

A detailed summary of the theoretical foundation for the tower and rotor modeling approach, implemented by the tool, have been detailed in this report. Careful consideration was given to identify a conservative set of IEC Aeroelastic Design Load Cases and custom load cases that the designer can use to simulate realistic wind conditions. A rigorous approach was taken to verify the tool with comparison to theoretical closed form solutions, comparisons to physical laboratory test results, and comparisons to peer results. As a capstone to this project a case study was conducted by using the tool to simulate the Cal Poly Wind Power Research Center wind turbine and tower system. The case study included determination of tower deflections and margins of safety for the primary tower structure, a detailed evaluation of coupled tower / rotor dynamic interaction, and a optimization study for hypothetical guy-wire placement.

\subsection{Future Work}

The full scale physical testing projects should be considered as future work and results could be used for model correlation.

1) Correlation of Aerodynamic Parameters: As noted in Section 1.4.1 (item 3.b) the tool requires the user to input several aerodynamic parameters (tower drag coefficient, operation rotor 
thrust coefficient, and the parked rotor drag coefficient). These parameters are linearly proportional to the tower responses (deflections, loads, and stresses) produced by the tool. A project could be conducted to measure wind speed (using digital anemometers) and correlate it to tower strain (using strain gages). At a minimum the wind speed should be measured at the ground, mid-tower height, and tower tip. The digital anemometers should be offset from the tower and rotor so that their measurements are not influenced by the tower / rotor's wake. Likewise strain gages should be placed at multiple tower heights (10 or more station heights) and be oriented such that they measure bending strain. The strain data could then be used to determine the tower's deformation state and then calculate the loads required to produce that deformation state. These loads could then be compared to the measured wind speeds and the aerodynamic parameters above could be determined.

2) Correlation of System Dynamic Parameters:

a) Tower Rigid Body Mode Evaluation: Deflections of the Cal Poly wind turbine tower (in both the strong-axis and weak-axis) at low wind speeds have been observed to be larger in magnitude than predicted by the model. Furthermore a lateral swaying motion of the tower has been observed in the weak-axis even when the wind is oriented in the strongaxis. It is possible that these motions are caused by rigid body motion of the tower (i.e., tower motion that is not associated with tower strain). Such rigid body motion could be caused by clearance fittings in the tower joints. For example if the housing for the bearings located at the tower's bases were slightly oversized then a gap would exist that could allow the tower to pivot about the X-Axis (orthogonal to the bearing axis). Another cause for the observed behavior could be compliance in the ground soil and foundation. Note that these causes are simply speculation at this time. To determine if rigid body tower motion is occurring a triaxial accelerometer could be attached to the tower's tip and strain gages, as described in item (1) above, could be attached along the tower. If the magnitude of tower transverse (X-Axis and Z-Axis) accelerations are larger than could be 
explained by the observed tower strains then rigid body motion (pivoting about the tower's base) is the culprit. If such motions are believed to be caused by ground soil and foundation compliance then the simulation tool could be augmented to include this behavior. If the motion is believed to be caused by joint clearances then it would be difficult to simulate such behavior as it would be a non-linear phenomenon.

b) Tower Flexible Body Mode Evaluation: Full scale determination of the tower's flexible body modes (modes associated with tower strain) would be very useful for model correlation. Modal testing is typically performed using one of two methods: 1) a modal tap test in which a hammer is used to provide an impulse inputs to the structure at various locations or 2) an electrodynamics "stinger" type modal shaker is used to excite mode shapes. The modal hammer test is often preferred due to its simplicity. However, when performing a modal test on a large structure (such as the Cal Poly wind turbine tower) the excitation provided by a hammer hit would be so low that accelerometer signal-to-noise ratio would be poor. As such the modal shaker method would be preferred but this would result in a complicated / expensive set up, (example "stinger" type modal shakers can be found at www.modalshop.com). A possible setup would be to suspend the modal shakers from a long cable (so that their pendulum mode is much lower than the tower's natural frequency), then add some mass to the shaker (so that there is more inertia to "push" against), attach the stinger to the tower, and then perform a swept sine test and record response accelerations along the tower's height. The shaker would need to be strategically position at anti-node locations along the tower and potentially repositioned for each tower mode of interest. This test setup could also be used to determine system damping by shutting the shaker off once system resonance is achieved and observing response decay rate.

c) Rotor Blade Flexible Body Mode Evaluation: The rotor blade natural frequency is an input parameter to the simulation tool. For the purpose of the case study the blade natural 
frequency parameters were estimated using the techniques detailed in Appendix C-8.

Physical testing should be considered to determine the blades' actual natural frequency.

The best way to do this would be to remove one of the blades from the rotor and perform a modal tap test, as described in item (2)b) above, in a laboratory setting. 


\section{Bibliography}

1. Craig RR, Kurdila AJ. Fundamentals of Structural Dynamics. 2nd. Hoboken : John Wiley \& Sons, 2006.

2. Eggleston DM, Stoddard FS. Wind Turbine Engineering Design. New York : Van Nostrand Reinhold Company Inc., 1987.

3. IEC. Wind turbine generator systems - Part 2: Design requirements for small wind turbines. s.1. : IEC, 2006. IEC 61400-2.

4. Cook, Robert D. Finite Element Modeling for Stress Analysis. New York, NY : John Wiley \& Sons, Inc., 1995.

5. Sauer, Timothy. Numerical Analysis. Boston, MA : Pearson Education, Inc, 2006.

6. Gwon, Tae-gyun (Tom). Structural Analyses of Wind Turbine Tower for $3 \mathrm{~kW}$ HorizontalAxis Wind Turbine. Mechanical Engineering. San Luis Obispo : California Polytechnic State University, 2011. Masters Thesis.

7. U.S. Standard Atmosphere. Washington, D.C. : U.S. Government Printing Office, 1976.

8. Nosti, Christopher. PERFORMANCE ANALYSIS AND LIFE PREDICTION FOR SMALL WIND TURBINE BLADES: A WOOD LAMINATE CASE STUDY. Mechanical Engineering. San Luis Obispo : California Polytechnic State University, 2009. Masters Thesis.

9. Edwards, Bryan Kyle. Composite Manufacturing of Small Wind Turbine Blades. Mechanical Engineering. San Luis Obispo : California Polytechnic State University, 2009. Masters Thesis.

10. Seidman, David. The Complete Sailor. 2. Camden, Maine : International Marine / Ragged Mountain Press, 2011. 
11. Manwell JF, McGowan JG, Rogers AL. Wind Energy Explained - Theory, Design and Application. West Sussex : John Wiley \& Sons Ltd., 2002.

12. The Boeing Company. Boeing History MOD-2/MOD-5B Wind Turbines. [Online] http://www.boeing.com/boeing/history/boeing/windturbine.page.

13. Kenward, Alyson. Forecast Dims for Further Growth in Wind Power. Climate Central. [Online] March 12, 2013. http://www.climatecentral.org/news/forecast-dims-for-future-growthin-wind-power-15721.

14. Energy Information Administration (EIA). Electric Power Monthly with Data for February 2013. Washington, DC : U.S. Department of Energy, 2013.

15. Levitan, Dave. Wind Power Tax Credit Survives Fiscal Cliff Deal. [Online] Forbes, January 2, 2013. http://www.forbes.com/sites/davelevitan/2013/01/02/wind-power-tax-credit-survivesfiscal-cliff-deal/.

16. U.S. Department of Energy. 20\% Wind Energy by 2030; Increasing Wind Energy's Contribution to U.S. Electricity Supply. Washington. D.C. : U.S. Department of Energy, 2008. DOE/GO-102008-2567.

17. E. Lantz, M. Hand, R. Wiser. The Past and Future Cost of Wind Energy. Golden, Co : National Renewable Energy Laboratory (NREL), 2012. NREL/CP.

18. Software, MSC. MSC Nastran Quick Reference Guide. Santa Ana, Ca : MSC Software Corporation, 2011.

19. Shigley, Joseph E., Mischke, Charles R. and Budynas, Richard G. Mechanical Engineering Design. New York, NY : McGraw-Hill, 2004. 
20. Structural design optimization of wind turbine towers. Negm HM, Maalawi KY. 74, 2000, Computers and Structures, pp. 649-666.

21. Rao, J. S. Turbomachine Blade Vibration. New Delhi : New Age Publishers, 1991.

22. Structural Design Optimization of Wind Turbine Towers. Negm HM, Maalawi KY. Cairo, Egypt : Computers \& Structures, 2000, Vol. 74.

23. Optimisation of a Steel Tower for a Wind Turbine Structure. Uys PE, Farkas J, Jarmai K, Tonder FV. Pretoria, South Africa : Elsevier, 2007, Engineering Structures, Vol. 29.

24. A, Ahlstrom. Aeroelastic Simulation of Wind Trubine Dynamics. Department of Mechanics, Royal Institute of Technology. Stockholm, Sweden : s.n., 2005. Doctoral Thesis.

25. Multi-Flexible-Body Dynamic Analysis of Horizontal Axis Wind Turbines. Lee D, Hodges DH, Patil MJ. Atlanta, Georgia : John Wiley \& Sons, 2002, Wind Energy, Vol. 5.

26. A New Multibody Modelling Methodology for wind Turbine Structures Using a Cardanic Joint Beam Element. Zhao X, MiaBer P, Wu J. Bochum, Germany : Elsevier, 2007, Renewable Energy, Vol. 32.

27. Meriam JL, Kraige LG. Engineering Mechanics Dynamics. 4th Edition. s.l. : John Wiley \& Sons, Inc, 2002.

28. Fox, Robert W., McDonald, Alan T. and Pritchard, Philip J. Introduction to Fluid Mechanics. 6. Hoboken, NJ : John Wiley \& Sons, 2004.

29. Moriarty, Patrick J. AeroDyn Theory Manual. Golden, Colorado : National Renewable Energy Laboratory (NREL), 2005. NREL/EL-5.

30. Irvine, Tom. Sine Sweep Frequency Parameters. [Online] 1998. http://www.vibrationdata.com/. 
31. Canadian Highway Bridge Design Code. s.l. : Canadian Standards Association, 2005.

\section{CAN/CSA-S6-0.}

32. Lepi, Steven. Practical Guide to Finite Elements: A Solid Mechanics Approach. New York, NY : CRC Press, 1998.

33. ASTM International. West Conshohocken, PA : ASTM International, 2012. A572/A572M-

12. 


\title{
Appendix A
}

\section{Derivation of 3 Blade Generalized Rotor Model}

\author{
MathCAD Printout
}

Formatted for Electronic Viewing 


\section{GENERALIZED ROTOR MODEL - 3 BLADED ROTOR}

FORMATTED FOR ELECTRONIC VIEWING - ZOOM IN TO SEE EQUATIONS

\section{Blade One Equation of Motion Derivation}

Relate Motion of the center of gravity of member A to the system DOF velocities Position

$\operatorname{Xag}\left(v, \theta, \alpha_{1}\right):=-\mathrm{a} \cdot \alpha_{1}+v-\left(\right.$ Ey $\left.\cos \left(\Psi_{1 \mathrm{~b}}+\Psi_{\mathrm{r}}\right)+\mathrm{Hy}\right) \cdot \theta$

$\operatorname{Yag}(\nu, \theta):=\nu-\mathrm{Hx} \cdot \theta$

Velocity

$\operatorname{Xag}_{\mathrm{t}}\left(v_{\mathrm{t}}, \theta_{\mathrm{t}}, \alpha_{1 \mathrm{t}}\right):=-\mathrm{a} \cdot \alpha_{1 \mathrm{t}}+v_{\mathrm{t}}-\left(\mathrm{Ey} \cdot \cos \left(\Psi_{1 \mathrm{~b}}+\Psi_{\mathrm{r}}\right)+\mathrm{Hy}\right) \cdot \theta_{\mathrm{t}}$

$\operatorname{Yag}_{\mathrm{t}}\left(\nu_{\mathrm{t}}, \theta_{\mathrm{t}}\right):=\nu_{\mathrm{t}}-\mathrm{Hx} \cdot \theta_{\mathrm{t}}$

Relate Motion of the center of gravity of member B to the system DOF velocities

Position

$\operatorname{Xbg}(v, \theta):=v-\left(\frac{\mathrm{Ey} \cdot \cos \left(\Psi_{1 \mathrm{~b}}+\Psi_{\mathrm{r}}\right)}{2}+\mathrm{Hy}\right) \cdot \theta$

$\operatorname{Ybg}(\nu, \theta):=\nu-\mathrm{Hx} \cdot \theta$

Velocity

$\operatorname{Xbg}_{\mathrm{t}}\left(v_{\mathrm{t}}, \theta_{\mathrm{t}}\right):=v_{\mathrm{t}}-\left(\frac{\mathrm{Ey} \cdot \cos \left(\Psi_{1 \mathrm{~b}}+\Psi_{\mathrm{r}}\right)}{2}+\mathrm{Hy}\right) \cdot \theta_{\mathrm{t}}$

$\operatorname{Ybg}_{\mathrm{t}}\left(\nu_{\mathrm{t}}, \theta_{\mathrm{t}}\right):=\nu_{\mathrm{t}}-\mathrm{Hx} \cdot \theta_{\mathrm{t}}$

Relate the rotation of $\alpha_{1}$ about local unit vector $K_{\text {hat }}$ to global unit vectors $j_{\text {hat }}$ and $k_{\text {hat }}$ (This relation is used to define the rotational velocity of member $A$.

$\mathrm{K}_{\text {hat }}\left(\mathrm{j}_{\text {hat }}, \mathrm{k}_{\text {hat }}\right):=\mathrm{j}_{\text {hat }} \cdot \sin \left(\Psi_{1 \mathrm{~b}}+\Psi_{\mathrm{r}}\right)+\mathrm{k}_{\text {hat }} \cdot \cos \left(\Psi_{1 \mathrm{~b}}+\Psi_{\mathrm{r}}\right)$

$\mathrm{K}_{\text {yhat }}:=\frac{\mathrm{d}}{\mathrm{dj}_{\text {hat }}} \mathrm{K}_{\text {hat }}\left(\mathrm{j}_{\text {hat }}, \mathrm{k}_{\text {hat }}\right) \quad \mathrm{K}_{\text {yhat }} \rightarrow \sin \left(\Psi_{\mathrm{r}}+\Psi_{1 \mathrm{~b}}\right)$

$\mathrm{K}_{\mathrm{zhat}}:=\frac{\mathrm{d}}{\mathrm{dk}_{\text {hat }}} \mathrm{K}_{\text {hat }}\left(\mathrm{j}_{\text {hat }}, \mathrm{k}_{\text {hat }}\right) \quad \mathrm{K}_{\mathrm{zhat}} \rightarrow \cos \left(\Psi_{\mathrm{r}}+\Psi_{1 \mathrm{~b}}\right)$

Rotational Velocity of member A

$$
\begin{array}{ll}
\omega_{\text {ay }}\left(\alpha_{1 \mathrm{t}}\right):=\alpha_{1 \mathrm{t}} \cdot \mathrm{K}_{\text {yhat }} & \omega_{\text {ay }}\left(\alpha_{1 \mathrm{t}}\right) \rightarrow \alpha_{1 \mathrm{t}} \cdot \sin \left(\Psi_{\mathrm{r}}+\Psi_{1 \mathrm{~b}}\right) \\
\omega_{\text {az }}\left(\alpha_{1 \mathrm{t}}\right):=\alpha_{1 \mathrm{t}} \cdot \mathrm{K}_{\text {zhat }} & \omega_{\text {az }}\left(\alpha_{1 \mathrm{t}}\right) \rightarrow \alpha_{1 \mathrm{t}} \cdot \cos \left(\Psi_{\mathrm{r}}+\Psi_{1 \mathrm{~b}}\right)
\end{array}
$$

Note: time derivatives of variables are annotated with subscripts. For example $u_{t}$ is the first time derivative of $u$ while $u_{t t}$ is the second time derivative of $u$.

Rotational Velocity of member B

$$
\omega_{\text {bz }}\left(\theta_{\mathrm{t}}\right):=\theta_{\mathrm{t}} \quad \omega_{\mathrm{bz}}\left(\theta_{\mathrm{t}}\right) \rightarrow \theta_{\mathrm{t}}
$$

\section{Moments and Products of Inertia of Rotor Member A}

$$
\begin{aligned}
& \mathrm{I}_{\text {ayy }}:=\mathrm{I}_{\mathrm{a}} \cdot\left(\sin \left(\Psi_{1 \mathrm{~b}}+\Psi_{\mathrm{r}}\right)\right)^{2} \\
& \mathrm{I}_{\mathrm{azz}}:=\mathrm{I}_{\mathrm{a}} \cdot\left(\cos \left(\Psi_{1 \mathrm{~b}}+\Psi_{\mathrm{r}}\right)\right)^{2} \\
& \mathrm{I}_{\text {ayz }}:=\frac{-\mathrm{I}_{\mathrm{a}}}{2} \cdot \sin \left[2 \cdot\left(\Psi_{1 \mathrm{~b}}+\Psi_{\mathrm{r}}\right)\right]
\end{aligned}
$$

\section{Moments and Products of Inertia of Rotor Member B}

$$
\begin{aligned}
& \mathrm{I}_{\text {byy }}:=\mathrm{I}_{\mathrm{b}} \cdot\left(\sin \left(\Psi_{1 \mathrm{~b}}+\Psi_{\mathrm{r}}\right)\right)^{2} \\
& \mathrm{I}_{\mathrm{bzz}}:=\mathrm{I}_{\mathrm{b}} \cdot\left(\cos \left(\Psi_{1 \mathrm{~b}}+\Psi_{\mathrm{r}}\right)\right)^{2} \\
& \mathrm{I}_{\mathrm{byz}}:=\frac{-\mathrm{I}_{\mathrm{b}}}{2} \cdot \sin \left[2 \cdot\left(\Psi_{1 \mathrm{~b}}+\Psi_{\mathrm{r}}\right)\right]
\end{aligned}
$$




\section{Kinetic Energy $(T)$}

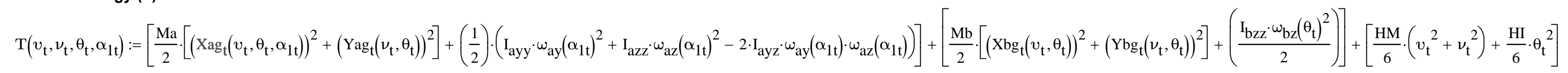

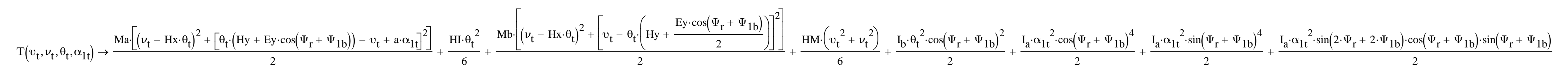

\section{Take the derivative of Kinetic Energy wrt System DOF velocities}

$\frac{\mathrm{d}}{\mathrm{d} v_{\mathrm{t}}} \mathrm{T}\left(v_{\mathrm{t}}, \nu_{\mathrm{t}}, \theta_{\mathrm{t}}, \alpha_{1 \mathrm{t}}\right) \mid \begin{aligned} & \text { collect } \\ & v_{\mathrm{t}}, \nu_{\mathrm{t}}, \theta_{\mathrm{t}}, \alpha_{1 \mathrm{t}}\end{aligned}\left(\frac{\mathrm{HM}}{3}+\mathrm{Ma}+\mathrm{Mb}\right) \cdot v_{\mathrm{t}}+\left[-\mathrm{Mb} \cdot\left(\mathrm{Hy}+\frac{\mathrm{Ey} \cdot \cos \left(\Psi_{\mathrm{r}}+\Psi_{1 \mathrm{~b}}\right)}{2}\right)-\frac{\mathrm{Ma} \cdot\left(2 \cdot \mathrm{Hy}+2 \cdot \mathrm{Ey} \cdot \cos \left(\Psi_{\mathrm{r}}+\Psi_{1 \mathrm{~b}}\right)\right)}{2}\right] \cdot \theta_{\mathrm{t}}+(-\mathrm{Ma} \cdot \mathrm{a}) \cdot \alpha_{1 \mathrm{t}}$

$\frac{\mathrm{d}}{\mathrm{d} \nu_{t}} \mathrm{~T}\left(v_{\mathrm{t}}, \nu_{\mathrm{t}}, \theta_{\mathrm{t}}, \alpha_{1 \mathrm{t}}\right) \mid \begin{aligned} & \text { collect } \\ & v_{\mathrm{t}}, \nu_{\mathrm{t}}, \theta_{\mathrm{t}}, \mathrm{\alpha}_{1 \mathrm{t}}\end{aligned} \rightarrow\left(\frac{\mathrm{HM}}{3}+\mathrm{Ma}+\mathrm{Mb}\right) \cdot v_{\mathrm{t}}+(-\mathrm{Hx} \cdot \mathrm{Ma}-\mathrm{Hx} \cdot \mathrm{Mb}) \cdot \theta_{\mathrm{t}}$

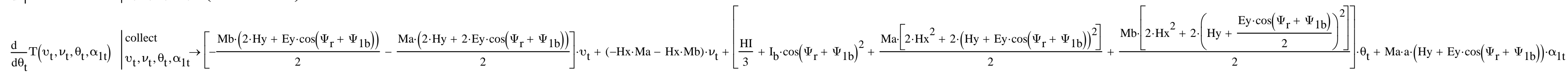

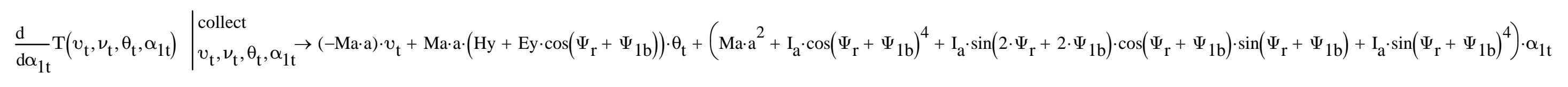

\section{Take the time derivative of the above terms by substituting in special accelerations}

${ }^{\mathrm{T}} v_{\mathrm{tt}}\left(v_{\mathrm{tt}}, v_{\mathrm{tt}}, \theta_{\mathrm{tt}}, \alpha_{1 \mathrm{tt}}\right):=\frac{\mathrm{d}}{\mathrm{d} v_{\mathrm{tt}}} \mathrm{T}\left(v_{\mathrm{tt}}, v_{\mathrm{tt}}, \theta_{\mathrm{tt}}, \alpha_{1 \mathrm{tt}}\right)$

$\mathrm{T}_{\nu \mathrm{t}}\left(v_{\mathrm{tt}}, v_{\mathrm{tt}}, \theta_{\mathrm{tt}}, \alpha_{1 \mathrm{tt}}\right):=\frac{\mathrm{d}}{\mathrm{d} \nu_{\mathrm{tt}}} \mathrm{T}\left(v_{\mathrm{tt}}, v_{\mathrm{tt}}, \theta_{\mathrm{tt}}, \alpha_{1 \mathrm{tt}}\right)$

$\mathrm{T}_{\theta t}\left(v_{\mathrm{tt}}, v_{\mathrm{tt}}, \theta_{\mathrm{tt}}, \alpha_{1 \mathrm{tt}}\right):=\frac{\mathrm{d}}{\mathrm{d} \theta_{\mathrm{tt}}} \mathrm{T}\left(v_{\mathrm{tt}}, v_{\mathrm{tt}}, \theta_{\mathrm{tt}}, \alpha_{1 \mathrm{tt}}\right)$

$\mathrm{T}_{1 \alpha \mathrm{t}}\left(v_{\mathrm{tt}}, v_{\mathrm{tt}}, \theta_{\mathrm{tt}}, \alpha_{1 \mathrm{tt}}\right):=\frac{\mathrm{d}}{\mathrm{d} \alpha_{1 \mathrm{tt}}} \mathrm{T}\left(v_{\mathrm{tt}}, v_{\mathrm{tt}}, \theta_{\mathrm{tt}}, \alpha_{1 \mathrm{tt}}\right)$

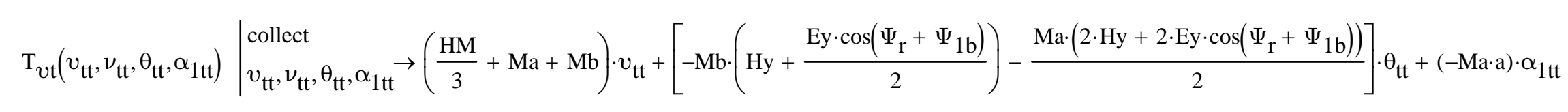

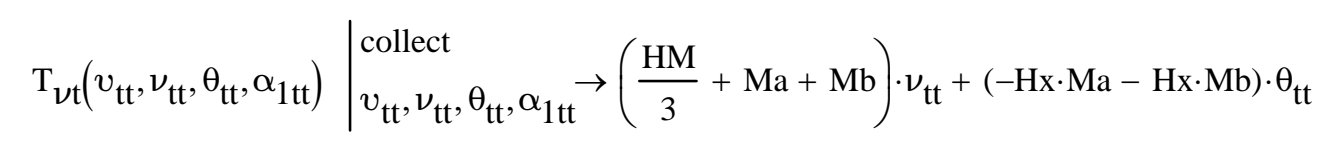

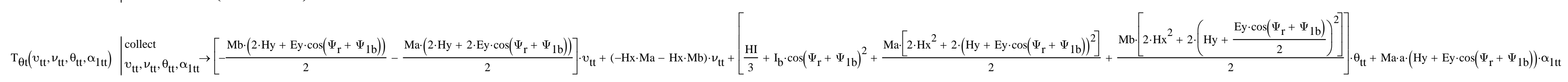

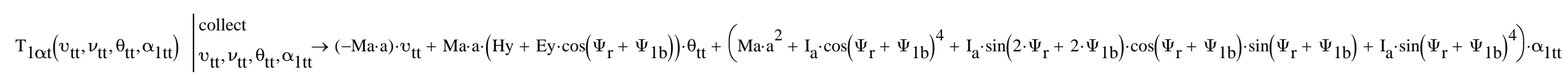




\section{Potential Energy}

$\mathrm{V}\left(v, \nu, \theta, \alpha_{1}\right):=\frac{1}{2} \cdot \mathrm{K} \cdot\left(\alpha_{1}\right)^{2}+\frac{1}{2} \cdot \mathrm{K} \cdot\left(\theta \cdot \cos \left(\Psi_{1 \mathrm{~b}}+\Psi_{\mathrm{r}}\right)\right)^{2}-\mathrm{K} \cdot \alpha_{1} \cdot \theta \cdot \cos \left(\Psi_{1 \mathrm{~b}}+\Psi_{\mathrm{r}}\right)+\mathrm{Ma} \cdot \mathrm{g} \cdot \mathrm{Yag}(\nu, \theta)+\mathrm{Mb} \cdot \mathrm{g} \cdot \mathrm{Ybg}(\nu, \theta)+\frac{1}{3} \mathrm{HM} \cdot \mathrm{g} \cdot \nu+\frac{1}{4} \cdot \Omega^{2} \cdot \mathrm{Ma} \cdot \mathrm{R} \cdot \frac{\mathrm{Ey}}{\mathrm{R}} \cdot\left(1-\frac{\mathrm{Ey}}{\mathrm{R}}\right) \cdot\left(\alpha_{1}-\theta \cdot \cos \left(\Psi_{1 \mathrm{~b}}+\Psi_{\mathrm{r}}\right)\right)^{2}+\frac{4}{2} \cdot \Omega^{2} \cdot \mathrm{I}_{\mathrm{a}} \cdot\left(\alpha_{1}-\theta \cdot \cos \left(\Psi_{1 \mathrm{~b}}+\Psi_{\mathrm{r}}\right)\right)^{2}$

$\mathrm{V}\left(v, \nu, \theta, \alpha_{1}\right) \rightarrow \frac{\mathrm{K} \cdot \alpha_{1}^{2}}{2}+\frac{\mathrm{HM} \cdot \mathrm{g} \cdot \nu}{3}+\mathrm{Ma} \cdot \mathrm{g} \cdot(\nu-\mathrm{Hx} \cdot \theta)+\mathrm{Mb} \cdot \mathrm{g} \cdot(\nu-\mathrm{Hx} \cdot \theta)+2 \cdot \mathrm{I}_{\mathrm{a}} \cdot \Omega^{2} \cdot\left(\alpha_{1}-\theta \cdot \cos \left(\Psi_{\mathrm{r}}+\Psi_{1 b}\right)\right)^{2}+\frac{\mathrm{K} \cdot \theta^{2} \cdot \cos \left(\Psi_{\mathrm{r}}+\Psi_{1 \mathrm{~b}}\right)^{2}}{2}-\mathrm{K} \cdot \theta \cdot \alpha_{1} \cdot \cos \left(\Psi_{\mathrm{r}}+\Psi_{1 \mathrm{~b}}\right)-\frac{\mathrm{Ey} \cdot \mathrm{Ma} \cdot \mathrm{R} \cdot \Omega^{2} \cdot\left(\frac{\mathrm{Ey}}{\mathrm{R}}-1\right) \cdot\left(\alpha_{1}-\theta \cdot \cos \left(\Psi_{\mathrm{r}}+\Psi_{1 \mathrm{~b}}\right)\right)^{2}}{4}$

\section{Take the derivative of Potential Energy wrt System DOF cord.}

$\mathrm{V}_{v}\left(v, \nu, \theta, \alpha_{1}\right):=\frac{\mathrm{d}}{\mathrm{d} v} \mathrm{~V}\left(v, \nu, \theta, \alpha_{1}\right)$

$\mathrm{V}_{\nu}\left(v, \nu, \theta, \alpha_{1}\right):=\frac{\mathrm{d}}{\mathrm{d} \nu} \mathrm{V}\left(\nu, \nu, \theta, \alpha_{1}\right)$

$\mathrm{V}_{\theta}\left(v, \nu, \theta, \alpha_{1}\right):=\frac{\mathrm{d}}{\mathrm{d} \theta} \mathrm{V}\left(v, \nu, \theta, \alpha_{1}\right)$

$\mathrm{V}_{1 \alpha}\left(v, \nu, \theta, \alpha_{1}\right):=\frac{\mathrm{d}}{\mathrm{d} \alpha_{1}} \mathrm{~V}\left(v, \nu, \theta, \alpha_{1}\right)$

$\mathrm{v}_{v}\left(v, \nu, \theta, \alpha_{1}\right) \mid \begin{aligned} & \text { collect } \\ & v, \nu, \theta, \alpha_{1} \rightarrow 0\end{aligned}$

$\mathrm{V}_{\nu}\left(v, \nu, \theta, \alpha_{1}\right) \mid \begin{aligned} & \text { collect } \\ & v, \nu, \theta, \alpha_{1}\end{aligned} \rightarrow \frac{\mathrm{HM} \cdot \mathrm{g}}{3}+\mathrm{Ma} \cdot \mathrm{g}+\mathrm{Mb} \cdot \mathrm{g}$

$\mathrm{V}_{\theta}\left(v, \nu, \theta, \alpha_{1}\right) \mid \begin{aligned} & \operatorname{collect} \\ & v, \nu, \theta, \alpha_{1}\end{aligned} \rightarrow\left[\mathrm{K} \cdot \cos \left(\Psi_{\mathrm{r}}+\Psi_{1 \mathrm{~b}}\right)^{2}+4 \cdot \mathrm{I}_{\mathrm{a}} \cdot \Omega^{2} \cdot \cos \left(\Psi_{\mathrm{r}}+\Psi_{1 \mathrm{~b}}\right)^{2}-\frac{\text { Ey } \cdot \mathrm{Ma} \cdot \mathrm{R} \cdot \Omega^{2} \cdot \cos \left(\Psi_{\mathrm{r}}+\Psi_{1 \mathrm{~b}}\right)^{2} \cdot\left(\frac{\mathrm{Ey}}{\mathrm{R}}-1\right)}{2}\right] \cdot \theta+\left[\frac{\mathrm{Ey} \cdot \mathrm{Ma} \cdot \mathrm{R} \cdot \Omega^{2} \cdot \cos \left(\Psi_{\mathrm{r}}+\Psi_{1 \mathrm{~b}}\right) \cdot\left(\frac{\mathrm{Ey}}{\mathrm{R}}-1\right)}{2}-4 \cdot \mathrm{I}_{\mathrm{a}} \cdot \Omega^{2} \cdot \cos \left(\Psi_{\mathrm{r}}+\Psi_{1 \mathrm{~b}}\right)-\mathrm{K} \cdot \cos \left(\Psi_{\mathrm{r}}+\Psi_{1 \mathrm{~b}}\right)\right] \cdot \alpha_{1}-(\mathrm{Hx} \cdot \mathrm{Ma} \cdot \mathrm{g}+\mathrm{Hx} \cdot \mathrm{Mb} \cdot \mathrm{g})$

$\mathrm{V}_{1 \alpha}\left(v, \nu, \theta, \alpha_{1}\right) \mid \begin{aligned} & \operatorname{collect} \\ & v, \nu, \theta, \alpha_{1}\end{aligned} \rightarrow\left[\frac{\text { Ey·Ma } \cdot \mathrm{R} \cdot \Omega^{2} \cdot \cos \left(\Psi_{\mathrm{r}}+\Psi_{1 \mathrm{~b}}\right) \cdot\left(\frac{\mathrm{Ey}}{\mathrm{R}}-1\right)}{2}-4 \cdot \mathrm{I}_{\mathrm{a}} \cdot \Omega^{2} \cdot \cos \left(\Psi_{\mathrm{r}}+\Psi_{1 \mathrm{~b}}\right)-\mathrm{K} \cdot \cos \left(\Psi_{\mathrm{r}}+\Psi_{1 \mathrm{~b}}\right)\right] \cdot \theta+\left[\mathrm{K}+4 \cdot \mathrm{I}_{\mathrm{a}} \cdot \Omega^{2}-\frac{\text { Ey } \cdot \mathrm{Ma} \cdot \mathrm{R} \cdot \Omega^{2} \cdot\left(\frac{\text { Ey }}{\mathrm{R}}-1\right)}{2}\right] \cdot \alpha_{1}$ 


\section{Define Blade One Mass and Stiffness Matrices}

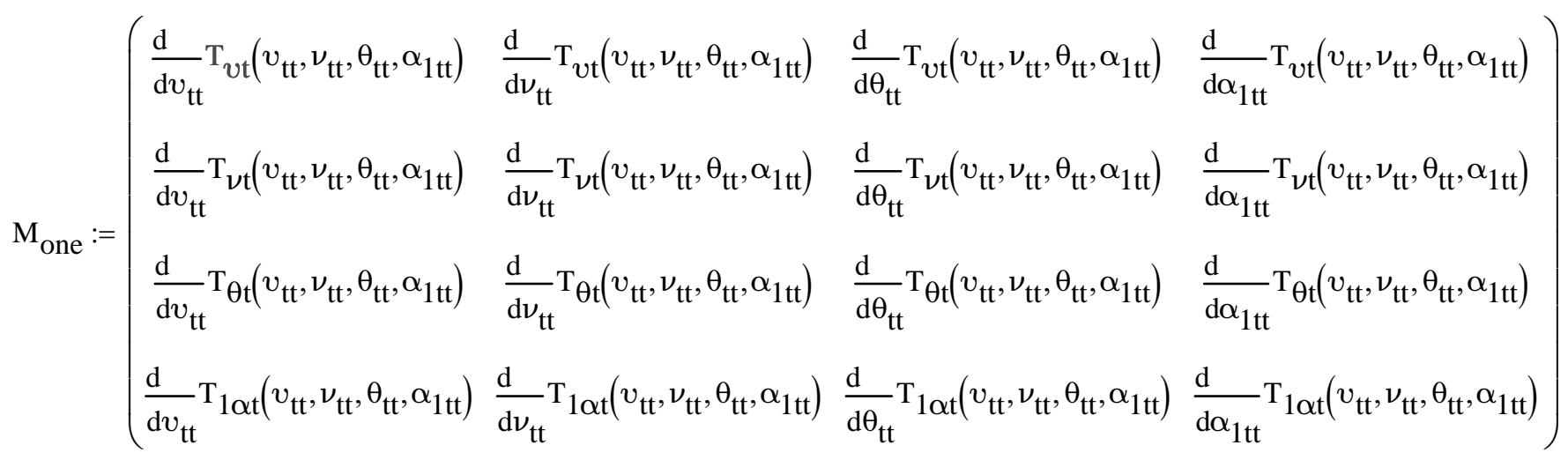

$$
\begin{aligned}
& \mathrm{K}_{\text {one }}:=\left(\begin{array}{llll}
\frac{\mathrm{d}}{\mathrm{d} v} \mathrm{~V}_{v}\left(v, \nu, \theta, \alpha_{1}\right) & \frac{\mathrm{d}}{\mathrm{d} \nu} \mathrm{V}_{v}\left(v, \nu, \theta, \alpha_{1}\right) & \frac{\mathrm{d}}{\mathrm{d} \theta} \mathrm{V}_{v}\left(v, \nu, \theta, \alpha_{1}\right) & \frac{\mathrm{d}}{\mathrm{d} \alpha_{1}} \mathrm{~V}_{v}\left(v, \nu, \theta, \alpha_{1}\right) \\
\frac{\mathrm{d}}{\mathrm{d} v} \mathrm{~V}_{\nu}\left(v, \nu, \theta, \alpha_{1}\right) & \frac{\mathrm{d}}{\mathrm{d} \nu} \mathrm{V}_{\nu}\left(v, \nu, \theta, \alpha_{1}\right) & \frac{\mathrm{d}}{\mathrm{d} \theta} \mathrm{V}_{\nu}\left(v, \nu, \theta, \alpha_{1}\right) & \frac{\mathrm{d}}{\mathrm{d} \alpha_{1}} \mathrm{~V}_{\nu}\left(v, \nu, \theta, \alpha_{1}\right) \\
\frac{\mathrm{d}}{\mathrm{d} v} \mathrm{~V}_{\theta}\left(v, \nu, \theta, \alpha_{1}\right) & \frac{\mathrm{d}}{\mathrm{d} \nu} \mathrm{V}_{\theta}\left(v, \nu, \theta, \alpha_{1}\right) & \frac{\mathrm{d}}{\mathrm{d} \theta} \mathrm{V}_{\theta}\left(v, \nu, \theta, \alpha_{1}\right) & \frac{\mathrm{d}}{\mathrm{d} \alpha_{1}} \mathrm{~V}_{\theta}\left(v, \nu, \theta, \alpha_{1}\right) \\
\frac{\mathrm{d}}{\mathrm{d} v} \mathrm{~V}_{1 \alpha}\left(v, \nu, \theta, \alpha_{1}\right) & \frac{\mathrm{d}}{\mathrm{d} \nu} \mathrm{V}_{1 \alpha}\left(v, \nu, \theta, \alpha_{1}\right) & \frac{\mathrm{d}}{\mathrm{d} \theta} \mathrm{V}_{1 \alpha}\left(v, \nu, \theta, \alpha_{1}\right) & \frac{\mathrm{d}}{\mathrm{d} \alpha_{1}} \mathrm{~V}_{1 \alpha}\left(v, \nu, \theta, \alpha_{1}\right)
\end{array}\right)
\end{aligned}
$$

\section{Blade One Mass Matrix}

For clarity each term of Mone is explicitly stated

$$
\begin{aligned}
& \mathrm{M}_{\text {one }_{0,0}} \rightarrow \frac{\mathrm{HM}}{3}+\mathrm{Ma}+\mathrm{Mb} \\
& \mathrm{M}_{\text {one }_{0,2}} \rightarrow-\frac{\mathrm{Mb} \cdot\left(2 \cdot \mathrm{Hy}+\mathrm{Ey} \cdot \cos \left(\Psi_{\mathrm{r}}+\Psi_{1 \mathrm{~b}}\right)\right)}{2}-\frac{\mathrm{Ma} \cdot\left(2 \cdot \mathrm{Hy}+2 \cdot \mathrm{Ey} \cdot \cos \left(\Psi_{\mathrm{r}}+\Psi_{1 \mathrm{~b}}\right)\right)}{2} \\
& \mathrm{M}_{\text {one }_{0,3}} \rightarrow-\mathrm{Ma} \cdot \mathrm{a} \\
& \mathrm{M}_{\text {one }_{1,1}} \rightarrow \frac{\mathrm{HM}}{3}+\mathrm{Ma}+\mathrm{Mb} \\
& \mathrm{M}_{\text {one }_{1,2}} \rightarrow-\mathrm{Hx} \cdot \mathrm{Ma}-\mathrm{Hx} \cdot \mathrm{Mb} \\
& \mathrm{M}_{\mathrm{One}_{2,2}} \rightarrow \frac{\mathrm{HI}}{3}+\mathrm{I}_{\mathrm{b}} \cdot \cos \left(\Psi_{\mathrm{r}}+\Psi_{1 \mathrm{~b}}\right)^{2}+\frac{\mathrm{Ma} \cdot\left[2 \cdot \mathrm{Hx}^{2}+2 \cdot\left(\mathrm{Hy}+\mathrm{Ey} \cdot \cos \left(\Psi_{\mathrm{r}}+\Psi_{1 \mathrm{~b}}\right)\right)^{2}\right]}{2}+\frac{\mathrm{Mb} \cdot\left[2 \cdot \mathrm{Hx}^{2}+2 \cdot\left(\mathrm{Hy}+\frac{\left.\left.\mathrm{Ey} \cdot \cos \left(\Psi_{\mathrm{r}}+\Psi_{1 \mathrm{~b}}\right)\right)^{2}\right]}{2}\right.\right.}{2} \\
& \mathrm{M}_{\text {one }_{2,3}} \rightarrow \text { Ma } \cdot \mathrm{a} \cdot\left(\mathrm{Hy}+\mathrm{Ey} \cdot \cos \left(\Psi_{\mathrm{r}}+\Psi_{1 \mathrm{~b}}\right)\right) \\
& \mathrm{M}_{\text {one }_{3,3}} \rightarrow \text { Ma } \cdot \mathrm{a}^{2}+\mathrm{I}_{\mathrm{a}} \cdot \cos \left(\Psi_{\mathrm{r}}+\Psi_{1 \mathrm{~b}}\right)^{4}+\mathrm{I}_{\mathrm{a}} \cdot \sin \left(2 \cdot \Psi_{\mathrm{r}}+2 \cdot \Psi_{1 \mathrm{~b}}\right) \cdot \cos \left(\Psi_{\mathrm{r}}+\Psi_{1 \mathrm{~b}}\right) \cdot \sin \left(\Psi_{\mathrm{r}}+\Psi_{1 \mathrm{~b}}\right)+\mathrm{I}_{\mathrm{a}} \cdot \sin \left(\Psi_{\mathrm{r}}+\Psi_{1 \mathrm{~b}}\right)^{4}
\end{aligned}
$$

\section{Blade One Stiffness Matrix}

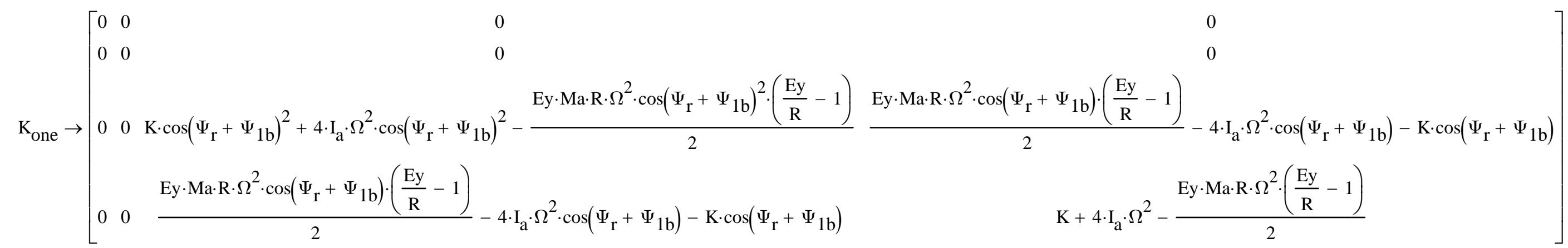




\section{Blade Two Equation of Motion Derivation}

\section{Relate Motion of the center of gravity of member A to the system DOF velocities} Position

$\operatorname{Xag}\left(v, \theta, \alpha_{2}\right):=-\mathrm{a} \cdot \alpha_{2}+v-\left(\right.$ Ey $\cdot \cos \left(\Psi_{2 b}+\Psi_{\mathrm{r}}\right)+$ Hy $) \cdot \theta$

$\operatorname{Yag}(\nu, \theta):=\nu-\mathrm{Hx} \cdot \theta$

Velocity

$\operatorname{Xag}_{\mathrm{t}}\left(v_{\mathrm{t}}, \theta_{\mathrm{t}}, \alpha_{2 \mathrm{t}}\right):=-\mathrm{a} \cdot \alpha_{2 \mathrm{t}}+v_{\mathrm{t}}-\left(\mathrm{Ey} \cdot \cos \left(\Psi_{2 \mathrm{~b}}+\Psi_{\mathrm{r}}\right)+\mathrm{Hy}\right) \cdot \theta_{\mathrm{t}}$

$\operatorname{Yag}_{\mathrm{t}}\left(\nu_{\mathrm{t}}, \theta_{\mathrm{t}}\right):=\nu_{\mathrm{t}}-\mathrm{Hx} \cdot \theta_{\mathrm{t}}$

Relate Motion of the center of gravity of member B to the system DOF velocities

Position

$\operatorname{Xbg}(v, \theta):=v-\left(\frac{E y \cdot \cos \left(\Psi_{2 b}+\Psi_{\mathrm{r}}\right)}{2}+\mathrm{Hy}\right) \cdot \theta$

$\operatorname{Ybg}(\nu, \theta):=\nu-\operatorname{Hx} \cdot \theta$

Velocity

$\operatorname{Xbg}_{\mathrm{t}}\left(v_{\mathrm{t}}, \theta_{\mathrm{t}}\right):=v_{\mathrm{t}}-\left(\frac{\mathrm{Ey} \cdot \cos \left(\Psi_{2 \mathrm{~b}}+\Psi_{\mathrm{r}}\right)}{2}+\mathrm{Hy}\right) \cdot \theta_{\mathrm{t}}$

$\operatorname{Ybg}_{t}\left(\nu_{t}, \theta_{t}\right):=\nu_{t}-H x \cdot \theta_{t}$

Relate the rotation of $\alpha_{1}$ about local unit vector $K_{\text {hat }}$ to global unit vectors $j_{\text {hat }}$ and $k_{\text {hat }}$ (This relation is used to define the rotational velocity of member $A$.

$\mathrm{K}_{\text {hat }}\left(\mathrm{j}_{\text {hat }}, \mathrm{k}_{\text {hat }}\right):=\mathrm{j}_{\text {hat }} \cdot \sin \left(\Psi_{2 \mathrm{~b}}+\Psi_{\mathrm{r}}\right)+\mathrm{k}_{\text {hat }} \cdot \cos \left(\Psi_{2 \mathrm{~b}}+\Psi_{\mathrm{r}}\right)$

$\mathrm{K}_{\text {yhat }}:=\frac{\mathrm{d}}{\mathrm{dj}_{\text {hat }}} \mathrm{K}_{\text {hat }}\left(\mathrm{j}_{\text {hat }}, \mathrm{k}_{\text {hat }}\right) \quad \mathrm{K}_{\text {yhat }} \rightarrow \sin \left(\Psi_{\mathrm{r}}+\Psi_{2 \mathrm{~b}}\right)$

$\mathrm{K}_{\text {zhat }}:=\frac{\mathrm{d}}{\mathrm{dk}_{\text {hat }}} \mathrm{K}_{\text {hat }}\left(\mathrm{j}_{\text {hat }}, \mathrm{k}_{\text {hat }}\right) \quad \mathrm{K}_{\mathrm{zhat}} \rightarrow \cos \left(\Psi_{\mathrm{r}}+\Psi_{2 \mathrm{~b}}\right)$

Rotational Velocity of member A

$$
\begin{array}{ll}
\omega_{\text {ay }}\left(\alpha_{2 t}\right):=\alpha_{2 t} \cdot K_{\text {yhat }} & \omega_{\text {ay }}\left(\alpha_{2 t}\right) \rightarrow \alpha_{2 t} \cdot \sin \left(\Psi_{\mathrm{r}}+\Psi_{2 b}\right) \\
\omega_{\text {az }}\left(\alpha_{2 t}\right):=\alpha_{2 t} \cdot K_{\text {zhat }} & \omega_{\text {az }}\left(\alpha_{2 t}\right) \rightarrow \alpha_{2 t} \cdot \cos \left(\Psi_{r}+\Psi_{2 b}\right)
\end{array}
$$

\section{Rotational Velocity of member B}

$$
\begin{aligned}
& \stackrel{\omega_{\text {mbar }}}{ }\left(\theta_{t}\right):=\theta_{t} \\
& \omega_{\mathrm{bz}}\left(\theta_{\mathrm{t}}\right) \rightarrow \theta_{\mathrm{t}}
\end{aligned}
$$

\section{Moments and Products of Inertia of Rotor Member A}

$$
\begin{aligned}
& \mathrm{I}_{\mathrm{ayy}}:=\mathrm{I}_{\mathrm{a}} \cdot\left(\sin \left(\Psi_{2 \mathrm{~b}}+\Psi_{\mathrm{r}}\right)\right)^{2} \\
& \mathrm{I}_{\mathrm{azz}}:=\mathrm{I}_{\mathrm{a}} \cdot\left(\cos \left(\Psi_{2 \mathrm{~b}}+\Psi_{\mathrm{r}}\right)\right)^{2} \\
& \mathrm{I}_{\mathrm{ayz}}:=\frac{-\mathrm{I}_{\mathrm{a}}}{2} \cdot \sin \left[2 \cdot\left(\Psi_{2 \mathrm{~b}}+\Psi_{\mathrm{r}}\right)\right]
\end{aligned}
$$

\section{Moments and Products of Inertia of Rotor Member B}

$$
\begin{aligned}
& \mathrm{I}_{\text {byy }}:=\mathrm{I}_{\mathrm{b}} \cdot\left(\sin \left(\Psi_{2 \mathrm{~b}}+\Psi_{\mathrm{r}}\right)\right)^{2} \\
& \mathrm{I}_{\mathrm{bzz}}:=\mathrm{I}_{\mathrm{b}} \cdot\left(\cos \left(\Psi_{2 \mathrm{~b}}+\Psi_{\mathrm{r}}\right)\right)^{2} \\
& \mathrm{I}_{\mathrm{byz}}:=\frac{-\mathrm{I}_{\mathrm{b}}}{2} \cdot \sin \left[2 \cdot\left(\Psi_{2 \mathrm{~b}}+\Psi_{\mathrm{r}}\right)\right]
\end{aligned}
$$




\section{Potential Energy}

$\mathrm{V}\left(v, \nu, \theta, \alpha_{2}\right):=\frac{1}{2} \cdot \mathrm{K} \cdot\left(\alpha_{2}\right)^{2}+\frac{1}{2} \cdot \mathrm{K} \cdot\left(\theta \cdot \cos \left(\Psi_{2 \mathrm{~b}}+\Psi_{\mathrm{r}}\right)\right)^{2}-\mathrm{K} \cdot \alpha_{2} \cdot \theta \cdot \cos \left(\Psi_{2 \mathrm{~b}}+\Psi_{\mathrm{r}}\right)+\mathrm{Ma} \cdot \mathrm{g} \cdot \mathrm{Yag}(\nu, \theta)+\mathrm{Mb} \cdot \mathrm{g} \cdot \mathrm{Ybg}(\nu, \theta)+\frac{1}{3} \mathrm{HM} \cdot \mathrm{g} \cdot \nu+\frac{1}{4} \cdot \Omega^{2} \cdot \mathrm{Ma} \cdot \mathrm{R} \cdot \frac{\mathrm{Ey}}{\mathrm{R}} \cdot\left(1-\frac{\mathrm{Ey}}{\mathrm{R}}\right) \cdot\left(\alpha_{2}-\theta \cdot \cos \left(\Psi_{2 \mathrm{~b}}+\Psi_{\mathrm{r}}\right)\right)^{2}+\frac{4}{2} \cdot \Omega^{2} \cdot \mathrm{I}_{\mathrm{a}} \cdot\left(\alpha_{2}-\theta \cdot \cos \left(\Psi_{2 \mathrm{~b}}+\Psi_{\mathrm{r}}\right)\right)^{2}$

$\mathrm{V}\left(v, \nu, \theta, \alpha_{2}\right) \rightarrow \frac{\mathrm{K} \cdot \mathrm{\alpha}_{2}{ }^{2}}{2}+\frac{\mathrm{HM} \cdot \mathrm{g} \cdot \nu}{3}+\mathrm{Ma} \cdot \mathrm{g} \cdot(\nu-\mathrm{Hx} \cdot \theta)+\mathrm{Mb} \cdot \mathrm{g} \cdot(\nu-\mathrm{Hx} \cdot \theta)+2 \cdot \mathrm{I}_{\mathrm{a}} \cdot \Omega^{2} \cdot\left(\alpha_{2}-\theta \cdot \cos \left(\Psi_{\mathrm{r}}+\Psi_{2 b}\right)\right)^{2}+\frac{\mathrm{K} \cdot \theta^{2} \cdot \cos \left(\Psi_{\mathrm{r}}+\Psi_{2 \mathrm{~b}}\right)^{2}}{2}-\mathrm{K} \cdot \theta \cdot \alpha_{2} \cdot \cos \left(\Psi_{\mathrm{r}}+\Psi_{2 \mathrm{~b}}\right)-\frac{\mathrm{Ey} \cdot \mathrm{Ma} \cdot \mathrm{R} \cdot \Omega^{2} \cdot\left(\frac{\mathrm{Ey}}{\mathrm{R}}-1\right) \cdot\left(\alpha_{2}-\theta \cdot \cos \left(\Psi_{\mathrm{r}}+\Psi_{2 \mathrm{~b}}\right)\right)^{2}}{4}$

\section{Take the derivative of Potential Energy wrt System DOF cord.}

$\mathrm{V}_{v}\left(v, \nu, \theta, \alpha_{2}\right):=\frac{\mathrm{d}}{\mathrm{d} v} \mathrm{~V}\left(v, \nu, \theta, \alpha_{2}\right)$

$\mathrm{V}_{\nu}\left(v, \nu, \theta, \alpha_{2}\right):=\frac{\mathrm{d}}{\mathrm{d} \nu} \mathrm{V}\left(\nu, \nu, \theta, \alpha_{2}\right)$

$\mathrm{V}_{\theta}\left(v, \nu, \theta, \alpha_{2}\right):=\frac{\mathrm{d}}{\mathrm{d} \theta} \mathrm{V}\left(v, \nu, \theta, \alpha_{2}\right)$

$\mathrm{V}_{2 \alpha}\left(v, \nu, \theta, \alpha_{1}\right):=\frac{\mathrm{d}}{\mathrm{do}_{2}} \mathrm{~V}\left(v, \nu, \theta, \alpha_{2}\right)$

$\mathrm{V}_{v}\left(v, \nu, \theta, \alpha_{2}\right) \mid \begin{aligned} & \text { collect } \\ & v, \nu, \theta, \alpha_{2} \rightarrow 0\end{aligned}$

$\mathrm{V}_{\nu}\left(v, \nu, \theta, \alpha_{2}\right) \mid \begin{aligned} & \text { collect } \\ & v, \nu, \theta, \alpha_{2}\end{aligned} \rightarrow \frac{\mathrm{HM} \cdot \mathrm{g}}{3}+\mathrm{Ma} \cdot \mathrm{g}+\mathrm{Mb} \cdot \mathrm{g}$

$\mathrm{V}_{\theta}\left(v, \nu, \theta, \alpha_{2}\right) \mid \begin{aligned} & \operatorname{collect} \\ & v, \nu, \theta, \alpha_{2}\end{aligned} \rightarrow\left[K \cdot \cos \left(\Psi_{\mathrm{r}}+\Psi_{2 \mathrm{~b}}\right)^{2}+4 \cdot \mathrm{I}_{\mathrm{a}} \cdot \Omega^{2} \cdot \cos \left(\Psi_{\mathrm{r}}+\Psi_{2 \mathrm{~b}}\right)^{2}-\frac{\text { Ey } \cdot \mathrm{Ma} \cdot \mathrm{R} \cdot \Omega^{2} \cdot \cos \left(\Psi_{\mathrm{r}}+\Psi_{2 \mathrm{~b}}\right)^{2} \cdot\left(\frac{\mathrm{Ey}}{\mathrm{R}}-1\right)}{2}\right] \cdot \theta+\left[\frac{\mathrm{Ey} \cdot \mathrm{Ma} \cdot \mathrm{R} \cdot \Omega^{2} \cdot \cos \left(\Psi_{\mathrm{r}}+\Psi_{2 \mathrm{~b}}\right) \cdot\left(\frac{\mathrm{Ey}}{\mathrm{R}}-1\right)}{2}-4 \cdot \mathrm{I}_{\mathrm{a}} \cdot \Omega^{2} \cdot \cos \left(\Psi_{\mathrm{r}}+\Psi_{2 \mathrm{~b}}\right)-\mathrm{K} \cdot \cos \left(\Psi_{\mathrm{r}}+\Psi_{2 \mathrm{~b}}\right) \cdot \cdot \alpha_{2}-(\mathrm{Hx} \cdot \mathrm{Ma} \cdot \mathrm{g}+\mathrm{Hx} \cdot \mathrm{Mb} \cdot \mathrm{g})\right.$

$\mathrm{V}_{2 \alpha}\left(v, \nu, \theta, \alpha_{2}\right) \mid \begin{aligned} & \operatorname{collect} \\ & v, \nu, \theta, \alpha_{2}\end{aligned} \rightarrow\left[\frac{\text { Ey } \cdot \text { Ma } \cdot \mathrm{R} \cdot \Omega^{2} \cdot \cos \left(\Psi_{\mathrm{r}}+\Psi_{2 \mathrm{~b}}\right) \cdot\left(\frac{\mathrm{Ey}}{\mathrm{R}}-1\right)}{2}-4 \cdot \mathrm{I}_{\mathrm{a}} \cdot \Omega^{2} \cdot \cos \left(\Psi_{\mathrm{r}}+\Psi_{2 \mathrm{~b}}\right)-\mathrm{K} \cdot \cos \left(\Psi_{\mathrm{r}}+\Psi_{2 \mathrm{~b}}\right)\right] \cdot \theta+\left[\mathrm{K}+4 \cdot \mathrm{I}_{\mathrm{a}} \cdot \Omega^{2}-\frac{\text { Ey } \cdot \mathrm{Ma} \cdot \mathrm{R} \cdot \Omega^{2} \cdot\left(\frac{\text { Ey }}{\mathrm{R}}-1\right)}{2}\right] \cdot \alpha_{2}$ 


\section{Define Blade Two Mass and Stiffness Matrices}

$$
\begin{aligned}
& \left(\frac{\mathrm{d}}{\mathrm{d} v_{\mathrm{tt}}} \mathrm{T}_{v \mathrm{t}}\left(v_{\mathrm{tt}}, v_{\mathrm{tt}}, \theta_{\mathrm{tt}}, \alpha_{2 \mathrm{tt}}\right) \quad \frac{\mathrm{d}}{\mathrm{d} v_{\mathrm{tt}}} \mathrm{T}_{v \mathrm{t}}\left(v_{\mathrm{tt}}, v_{\mathrm{tt}}, \theta_{\mathrm{tt}}, \alpha_{2 \mathrm{tt}}\right) \quad \frac{\mathrm{d}}{\mathrm{d} \theta_{\mathrm{tt}}} \mathrm{T}_{v \mathrm{t}}\left(v_{\mathrm{tt}}, v_{\mathrm{tt}}, \theta_{\mathrm{tt}}, \alpha_{2 \mathrm{tt}}\right) \quad \frac{\mathrm{d}}{\mathrm{d} \alpha_{2 \mathrm{tt}}} \mathrm{T}_{v \mathrm{t}}\left(v_{\mathrm{tt}}, v_{\mathrm{tt}}, \theta_{\mathrm{tt}}, \alpha_{2 \mathrm{tt}}\right)\right.
\end{aligned}
$$

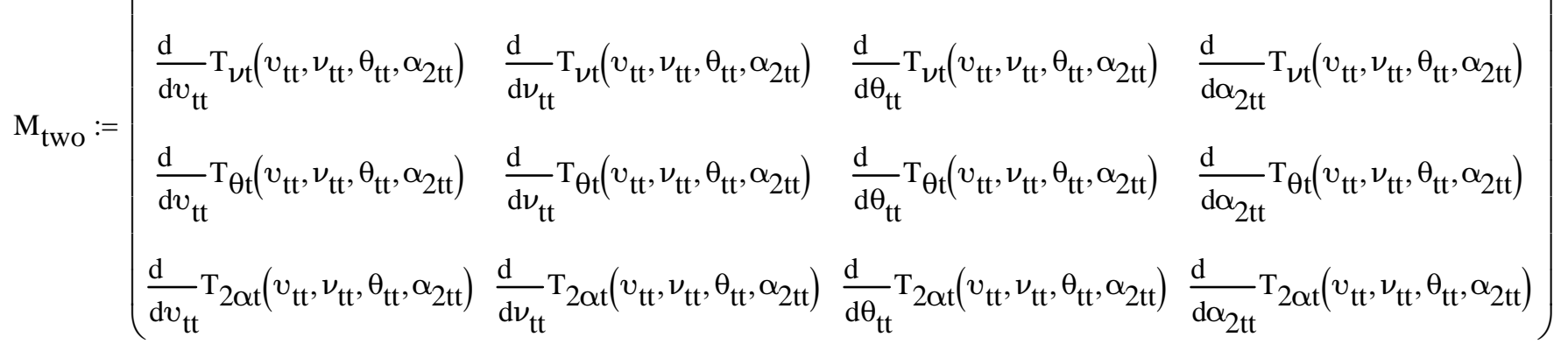

$$
\begin{aligned}
& \mathrm{K}_{\mathrm{two}}:=\left(\begin{array}{llll}
\frac{\mathrm{d}}{\mathrm{d} v} \mathrm{~V}_{v}\left(v, \nu, \theta, \alpha_{2}\right) & \frac{\mathrm{d}}{\mathrm{d} \nu} \mathrm{V}_{v}\left(v, \nu, \theta, \alpha_{2}\right) & \frac{\mathrm{d}}{\mathrm{d} \theta} \mathrm{V}_{v}\left(v, \nu, \theta, \alpha_{2}\right) & \frac{\mathrm{d}}{\mathrm{d} \alpha_{2}} \mathrm{~V}_{v}\left(v, \nu, \theta, \alpha_{2}\right) \\
\frac{\mathrm{d}}{\mathrm{d} v} \mathrm{~V}_{\nu}\left(v, \nu, \theta, \alpha_{2}\right) & \frac{\mathrm{d}}{\mathrm{d} \nu} \mathrm{V}_{\nu}\left(v, \nu, \theta, \alpha_{2}\right) & \frac{\mathrm{d}}{\mathrm{d} \theta} \mathrm{V}_{\nu}\left(v, \nu, \theta, \alpha_{2}\right) & \frac{\mathrm{d}}{\mathrm{d} \alpha_{2}} \mathrm{~V}_{\nu}\left(v, \nu, \theta, \alpha_{2}\right) \\
\frac{\mathrm{d}}{\mathrm{d} v} \mathrm{~V}_{\theta}\left(v, \nu, \theta, \alpha_{2}\right) & \frac{\mathrm{d}}{\mathrm{d} \nu} \mathrm{V}_{\theta}\left(v, \nu, \theta, \alpha_{2}\right) & \frac{\mathrm{d}}{\mathrm{d} \theta} \mathrm{V}_{\theta}\left(v, \nu, \theta, \alpha_{2}\right) & \frac{\mathrm{d}}{\mathrm{d} \alpha_{2}} \mathrm{~V}_{\theta}\left(v, \nu, \theta, \alpha_{2}\right) \\
\frac{\mathrm{d}}{\mathrm{d} v} \mathrm{~V}_{2 \alpha}\left(v, \nu, \theta, \alpha_{2}\right) & \frac{\mathrm{d}}{\mathrm{d} \nu} \mathrm{V}_{2 \alpha}\left(v, \nu, \theta, \alpha_{2}\right) & \frac{\mathrm{d}}{\mathrm{d} \theta} \mathrm{V}_{2 \alpha}\left(v, \nu, \theta, \alpha_{2}\right) & \frac{\mathrm{d}}{\mathrm{d} \alpha_{2}} \mathrm{~V}_{2 \alpha}\left(v, \nu, \theta, \alpha_{2}\right)
\end{array}\right)
\end{aligned}
$$

\section{Blade Two Mass Matrix}

For clarity each term of Mone is explicitly stated

$\mathrm{M}_{\mathrm{two}_{0,0}} \rightarrow \frac{\mathrm{HM}}{3}+\mathrm{Ma}+\mathrm{Mb}$

$\mathrm{M}_{\mathrm{two}_{0,2}} \rightarrow-\frac{\mathrm{Mb} \cdot\left(2 \cdot \mathrm{Hy}+\mathrm{Ey} \cdot \cos \left(\Psi_{\mathrm{r}}+\Psi_{2 \mathrm{~b}}\right)\right)}{2}-\frac{\mathrm{Ma} \cdot\left(2 \cdot \mathrm{Hy}+2 \cdot \mathrm{Ey} \cdot \cos \left(\Psi_{\mathrm{r}}+\Psi_{2 \mathrm{~b}}\right)\right)}{2}$

$\mathrm{M}_{\mathrm{two}_{0,3}} \rightarrow-\mathrm{Ma} \cdot \mathrm{a}$

$\mathrm{M}_{\mathrm{two}_{1,1}} \rightarrow \frac{\mathrm{HM}}{3}+\mathrm{Ma}+\mathrm{Mb}$

$\mathrm{M}_{\mathrm{two}_{1,2}} \rightarrow-\mathrm{Hx} \cdot \mathrm{Ma}-\mathrm{Hx} \cdot \mathrm{Mb}$

$\mathrm{M}_{\mathrm{two}_{2,2}} \rightarrow \frac{\mathrm{HI}}{3}+\mathrm{I}_{\mathrm{b}} \cdot \cos \left(\Psi_{\mathrm{r}}+\Psi_{2 \mathrm{~b}}\right)^{2}+\frac{\mathrm{Ma} \cdot\left[2 \cdot \mathrm{Hx}^{2}+2 \cdot\left(\mathrm{Hy}+\mathrm{Ey} \cdot \cos \left(\Psi_{\mathrm{r}}+\Psi_{2 \mathrm{~b}}\right)\right)^{2}\right]}{2}+\frac{\mathrm{Mb} \cdot\left[2 \cdot \mathrm{Hx}^{2}+2 \cdot\left(\mathrm{Hy}+\frac{\mathrm{Ey} \cdot \cos \left(\Psi_{\mathrm{r}}+\Psi_{2 \mathrm{~b}}\right)}{2}\right)^{2}\right]}{2}$

$\mathrm{M}_{\mathrm{two}_{2,3}} \rightarrow$ Ma·a $\left(\mathrm{Hy}+\mathrm{Ey} \cdot \cos \left(\Psi_{\mathrm{r}}+\Psi_{2 \mathrm{~b}}\right)\right)$

$\mathrm{M}_{\mathrm{two}_{3,3}} \rightarrow \mathrm{Ma} \cdot \mathrm{a}^{2}+\mathrm{I}_{\mathrm{a}} \cdot \cos \left(\Psi_{\mathrm{r}}+\Psi_{2 \mathrm{~b}}\right)^{4}+\mathrm{I}_{\mathrm{a}} \cdot \sin \left(2 \cdot \Psi_{\mathrm{r}}+2 \cdot \Psi_{2 \mathrm{~b}}\right) \cdot \cos \left(\Psi_{\mathrm{r}}+\Psi_{2 \mathrm{~b}}\right) \cdot \sin \left(\Psi_{\mathrm{r}}+\Psi_{2 \mathrm{~b}}\right)+\mathrm{I}_{\mathrm{a}} \cdot \sin \left(\Psi_{\mathrm{r}}+\Psi_{2 \mathrm{~b}}\right)^{4}$

\section{Blade Two Stiffness Matrix}

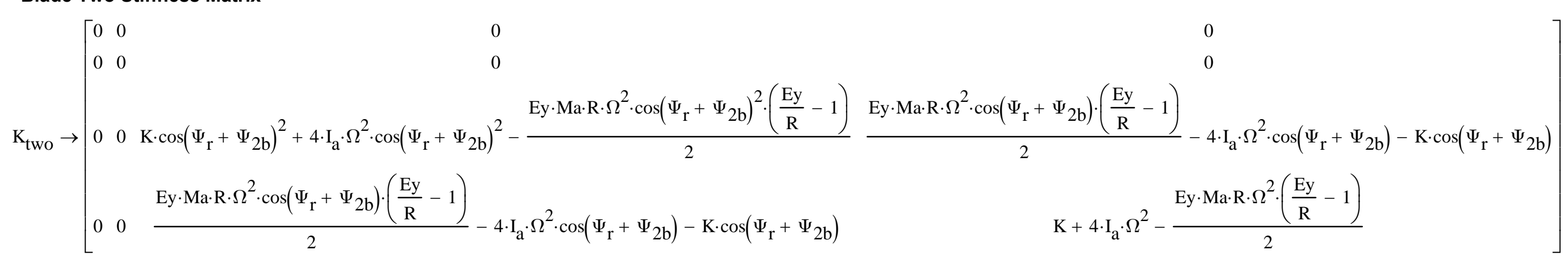




\section{Blade Three Equation of Motion Derivation}

\section{Relate Motion of the center of gravity of member A to the system DOF velocities} Position

$\operatorname{Xag}\left(v, \theta, \alpha_{3}\right):=-\mathrm{a} \cdot \alpha_{3}+v-\left(\right.$ Ey $\left.\cdot \cos \left(\Psi_{3 \mathrm{~b}}+\Psi_{\mathrm{r}}\right)+\mathrm{Hy}\right) \cdot \theta$

$\operatorname{Yag}(\nu, \theta):=\nu-\mathrm{Hx} \cdot \theta$

Velocity

$\operatorname{Xag}_{\mathrm{t}}\left(v_{\mathrm{t}}, \theta_{\mathrm{t}}, \alpha_{3 \mathrm{t}}\right):=-\mathrm{a} \cdot \alpha_{3 \mathrm{t}}+v_{\mathrm{t}}-\left(\mathrm{Ey} \cdot \cos \left(\Psi_{3 \mathrm{~b}}+\Psi_{\mathrm{r}}\right)+\mathrm{Hy}\right) \cdot \theta_{\mathrm{t}}$

$\operatorname{Yag}_{\mathrm{t}}\left(\nu_{\mathrm{t}}, \theta_{\mathrm{t}}\right):=\nu_{\mathrm{t}}-\mathrm{Hx} \cdot \theta_{\mathrm{t}}$

Relate Motion of the center of gravity of member B to the system DOF velocities Position

$\operatorname{Xbg}(v, \theta):=v-\left(\frac{E y \cdot \cos \left(\Psi_{3 \mathrm{~b}}+\Psi_{\mathrm{r}}\right)}{2}+\mathrm{Hy}\right) \cdot \theta$

$\operatorname{Ybg}(\nu, \theta):=\nu-\mathrm{Hx} \cdot \theta$

Velocity

$\operatorname{Xbg}_{\mathrm{t}}\left(v_{\mathrm{t}}, \theta_{\mathrm{t}}\right):=v_{\mathrm{t}}-\left(\frac{\mathrm{Ey} \cdot \cos \left(\Psi_{3 \mathrm{~b}}+\Psi_{\mathrm{r}}\right)}{2}+\mathrm{Hy}\right) \cdot \theta_{\mathrm{t}}$

$\operatorname{Ybg}_{\mathrm{t}}\left(\nu_{\mathrm{t}}, \theta_{\mathrm{t}}\right):=\nu_{\mathrm{t}}-\mathrm{Hx} \cdot \theta_{\mathrm{t}}$

Relate the rotation of $\alpha_{3}$ about local unit vector $K_{\text {hat }}$ to global unit vectors $j_{\text {hat }}$ and $k_{\text {hat }}$ (This relation is used to define the rotational velocity of member $A$.

$\mathrm{K}_{\text {hat }}\left(\mathrm{j}_{\text {hat }}, \mathrm{k}_{\text {hat }}\right):=\mathrm{j}_{\text {hat }} \cdot \sin \left(\Psi_{3 \mathrm{~b}}+\Psi_{\mathrm{r}}\right)+\mathrm{k}_{\text {hat }} \cdot \cos \left(\Psi_{3 \mathrm{~b}}+\Psi_{\mathrm{r}}\right)$

$\mathrm{K}_{\text {yhat }}:=\frac{\mathrm{d}}{\mathrm{dj}_{\text {hat }}} \mathrm{K}_{\text {hat }}\left(\mathrm{j}_{\text {hat }}, \mathrm{k}_{\text {hat }}\right) \quad \mathrm{K}_{\mathrm{yhat}} \rightarrow \sin \left(\Psi_{\mathrm{r}}+\Psi_{3 \mathrm{~b}}\right)$

$\mathrm{K}_{\text {zhat }}:=\frac{\mathrm{d}}{\mathrm{dk}_{\text {hat }}} \mathrm{K}_{\text {hat }}\left(\mathrm{j}_{\text {hat }}, \mathrm{k}_{\text {hat }}\right) \quad \mathrm{K}_{\mathrm{zhat}} \rightarrow \cos \left(\Psi_{\mathrm{r}}+\Psi_{3 \mathrm{~b}}\right)$

Rotational Velocity of member A
$\omega_{\text {ay }}\left(\alpha_{3 t}\right):=\alpha_{3 t} \cdot K_{\text {yhat }}$
$\omega_{\text {ay }}\left(\alpha_{2 \mathrm{t}}\right) \rightarrow \alpha_{2 \mathrm{t}} \cdot \sin \left(\Psi_{\mathrm{r}}+\Psi_{3 \mathrm{~b}}\right)$
$\omega_{\mathrm{az}}\left(\alpha_{3 \mathrm{t}}\right):=\alpha_{3 \mathrm{t}} \cdot \mathrm{K}_{\mathrm{zhat}}$
$\omega_{\mathrm{az}}\left(\alpha_{2 \mathrm{t}}\right) \rightarrow \alpha_{2 \mathrm{t}} \cdot \cos \left(\Psi_{\mathrm{r}}+\Psi_{3 \mathrm{~b}}\right)$

\section{Rotational Velocity of member B}

$$
\begin{aligned}
& \underset{\omega}{\omega_{\text {Mbrat }}}\left(\theta_{\mathrm{t}}\right):=\theta_{\mathrm{t}} \\
& \omega_{\mathrm{bz}}\left(\theta_{\mathrm{t}}\right) \rightarrow \theta_{\mathrm{t}}
\end{aligned}
$$

\section{Moments and Products of Inertia of Rotor Member A}

$$
\begin{aligned}
& \mathrm{I}_{\mathrm{ayy}}:=\mathrm{I}_{\mathrm{a}} \cdot\left(\sin \left(\Psi_{3 \mathrm{~b}}+\Psi_{\mathrm{r}}\right)\right)^{2} \\
& \mathrm{I}_{\mathrm{azz}}:=\mathrm{I}_{\mathrm{a}} \cdot\left(\cos \left(\Psi_{3 \mathrm{~b}}+\Psi_{\mathrm{r}}\right)\right)^{2} \\
& \mathrm{I}_{\mathrm{ayz}}:=\frac{-\mathrm{I}_{\mathrm{a}}}{2} \cdot \sin \left[2 \cdot\left(\Psi_{3 \mathrm{~b}}+\Psi_{\mathrm{r}}\right)\right]
\end{aligned}
$$

\section{Moments and Products of Inertia of Rotor Member B}

$$
\begin{aligned}
& \mathrm{I}_{\mathrm{byy}}:=\mathrm{I}_{\mathrm{b}} \cdot\left(\sin \left(\Psi_{3 \mathrm{~b}}+\Psi_{\mathrm{r}}\right)\right)^{2} \\
& \mathrm{I}_{\mathrm{bzz}}:=\mathrm{I}_{\mathrm{b}} \cdot\left(\cos \left(\Psi_{3 \mathrm{~b}}+\Psi_{\mathrm{r}}\right)\right)^{2} \\
& \mathrm{I}_{\mathrm{byz}}:=\frac{-\mathrm{I}_{\mathrm{b}}}{2} \cdot \sin \left[2 \cdot\left(\Psi_{3 \mathrm{~b}}+\Psi_{\mathrm{r}}\right)\right]
\end{aligned}
$$




\section{Potential Energy}

$\mathrm{V}\left(v, \nu, \theta, \alpha_{3}\right):=\frac{1}{2} \cdot \mathrm{K} \cdot\left(\alpha_{3}\right)^{2}+\frac{1}{2} \cdot \mathrm{K} \cdot\left(\theta \cdot \cos \left(\Psi_{3 \mathrm{~b}}+\Psi_{\mathrm{r}}\right)\right)^{2}-\mathrm{K} \cdot \alpha_{3} \cdot \theta \cdot \cos \left(\Psi_{3 \mathrm{~b}}+\Psi_{\mathrm{r}}\right)+\mathrm{Ma} \cdot \mathrm{g} \cdot \mathrm{Yag}(\nu, \theta)+\mathrm{Mb} \cdot \mathrm{g} \cdot \mathrm{Ybg}(\nu, \theta)+\frac{1}{3} \mathrm{HM} \cdot \mathrm{g} \cdot \nu+\frac{1}{4} \cdot \Omega^{2} \cdot \mathrm{Ma} \cdot \mathrm{R} \cdot \frac{\mathrm{Ey}}{\mathrm{R}} \cdot\left(1-\frac{\mathrm{Ey}}{\mathrm{R}}\right) \cdot\left(\alpha_{3}-\theta \cdot \cos \left(\Psi_{3 \mathrm{~b}}+\Psi_{\mathrm{r}}\right)\right)^{2}+\frac{4}{2} \cdot \Omega^{2} \cdot \mathrm{I}_{\mathrm{a}} \cdot\left(\alpha_{3}-\theta \cdot \cos \left(\Psi_{3 \mathrm{~b}}+\Psi_{\mathrm{r}}\right)\right)^{2}$

$\mathrm{V}\left(v, \nu, \theta, \alpha_{3}\right) \rightarrow \frac{\mathrm{K} \cdot \alpha_{3}{ }^{2}}{2}+\frac{\mathrm{HM} \cdot \mathrm{g} \cdot \nu}{3}+\mathrm{Ma} \cdot \mathrm{g} \cdot(\nu-\mathrm{Hx} \cdot \theta)+\mathrm{Mb} \cdot \mathrm{g} \cdot(\nu-\mathrm{Hx} \cdot \theta)+2 \cdot \mathrm{I}_{\mathrm{a}} \cdot \Omega^{2} \cdot\left(\alpha_{3}-\theta \cdot \cos \left(\Psi_{\mathrm{r}}+\Psi_{3 \mathrm{~b}}\right)\right)^{2}+\frac{\mathrm{K} \cdot \theta^{2} \cdot \cos \left(\Psi_{\mathrm{r}}+\Psi_{3 \mathrm{~b}}\right)^{2}}{2}-\mathrm{K} \cdot \theta \cdot \alpha_{3} \cdot \cos \left(\Psi_{\mathrm{r}}+\Psi_{3 \mathrm{~b}}\right)-\frac{\mathrm{Ey} \cdot \mathrm{Ma} \cdot \mathrm{R} \cdot \Omega^{2} \cdot\left(\frac{\mathrm{Ey}}{\mathrm{R}}-1\right) \cdot\left(\alpha_{3}-\theta \cdot \cos \left(\Psi_{\mathrm{r}}+\Psi_{3 \mathrm{~b}}\right)\right)^{2}}{4}$

\section{Take the derivative of Potential Energy wrt System DOF cord.}

$\mathrm{V}_{v}\left(v, \nu, \theta, \alpha_{3}\right):=\frac{\mathrm{d}}{\mathrm{d} v} \mathrm{~V}\left(v, \nu, \theta, \alpha_{3}\right)$

$\mathrm{V}_{\nu}\left(\nu, \nu, \theta, \alpha_{3}\right):=\frac{\mathrm{d}}{\mathrm{d} \nu} \mathrm{v}\left(\nu, \nu, \theta, \alpha_{3}\right)$

$\mathrm{V}_{\theta}\left(v, \nu, \theta, \alpha_{3}\right):=\frac{\mathrm{d}}{\mathrm{d} \theta} \mathrm{V}\left(v, \nu, \theta, \alpha_{3}\right)$

$\mathrm{V}_{3 \alpha}\left(v, \nu, \theta, \alpha_{3}\right):=\frac{\mathrm{d}}{\mathrm{d} \alpha_{3}} \mathrm{~V}\left(v, \nu, \theta, \alpha_{3}\right)$

$\mathrm{V}_{v}\left(v, \nu, \theta, \alpha_{3}\right) \mid \begin{aligned} & \text { collect } \\ & v, \nu, \theta, \alpha_{3} \rightarrow 0\end{aligned}$

$\mathrm{V}_{\nu}\left(v, \nu, \theta, \alpha_{3}\right) \mid \begin{aligned} & \text { collect } \\ & v, \nu, \theta, \alpha_{3}\end{aligned} \rightarrow \frac{\mathrm{HM} \cdot \mathrm{g}}{3}+\mathrm{Ma} \cdot \mathrm{g}+\mathrm{Mb} \cdot \mathrm{g}$

$\mathrm{V}_{\theta}\left(v, \nu, \theta, \alpha_{3}\right) \mid \begin{aligned} & \operatorname{collect} \\ & v, \nu, \theta, \alpha_{3}\end{aligned} \rightarrow\left[K \cdot \cos \left(\Psi_{\mathrm{r}}+\Psi_{3 \mathrm{~b}}\right)^{2}+4 \cdot \mathrm{I}_{\mathrm{a}} \cdot \Omega^{2} \cdot \cos \left(\Psi_{\mathrm{r}}+\Psi_{3 \mathrm{~b}}\right)^{2}-\frac{\text { Ey } \cdot \mathrm{Ma} \cdot \mathrm{R} \cdot \Omega^{2} \cdot \cos \left(\Psi_{\mathrm{r}}+\Psi_{3 \mathrm{~b}}\right)^{2} \cdot\left(\frac{\mathrm{Ey}}{\mathrm{R}}-1\right)}{2}\right] \cdot \theta+\left[\frac{\mathrm{Ey} \cdot \mathrm{Ma} \cdot \mathrm{R} \cdot \Omega^{2} \cdot \cos \left(\Psi_{\mathrm{r}}+\Psi_{3 \mathrm{~b}}\right) \cdot\left(\frac{\mathrm{Ey}}{\mathrm{R}}-1\right)}{2}-4 \cdot \mathrm{I}_{\mathrm{a}} \cdot \Omega^{2} \cdot \cos \left(\Psi_{\mathrm{r}}+\Psi_{3 \mathrm{~b}}\right)-\mathrm{K} \cdot \cos \left(\Psi_{\mathrm{r}}+\Psi_{3 \mathrm{~b}}\right) \cdot \cdot \alpha_{3}-(\mathrm{Hx} \cdot \mathrm{Ma} \cdot \mathrm{g}+\mathrm{Hx} \cdot \mathrm{Mb} \cdot \mathrm{g})\right.$

$\mathrm{V}_{3 \alpha}\left(v, \nu, \theta, \alpha_{3}\right) \mid \begin{aligned} & \operatorname{collect} \\ & v, \nu, \theta, \alpha_{3}\end{aligned} \rightarrow\left[\frac{\text { Ey } \cdot \mathrm{Ma} \cdot \mathrm{R} \cdot \Omega^{2} \cdot \cos \left(\Psi_{\mathrm{r}}+\Psi_{3 \mathrm{~b}}\right) \cdot\left(\frac{\mathrm{Ey}}{\mathrm{R}}-1\right)}{2}-4 \cdot \mathrm{I}_{\mathrm{a}} \cdot \Omega^{2} \cdot \cos \left(\Psi_{\mathrm{r}}+\Psi_{3 \mathrm{~b}}\right)-\mathrm{K} \cdot \cos \left(\Psi_{\mathrm{r}}+\Psi_{3 \mathrm{~b}}\right)\right] \cdot \theta+\left[\mathrm{K}+4 \cdot \mathrm{I}_{\mathrm{a}} \cdot \Omega^{2}-\frac{\text { Ey } \cdot \mathrm{Ma} \cdot \mathrm{R} \cdot \Omega^{2} \cdot\left(\frac{\text { Ey }}{\mathrm{R}}-1\right)}{2}\right] \cdot \alpha_{3}$ 


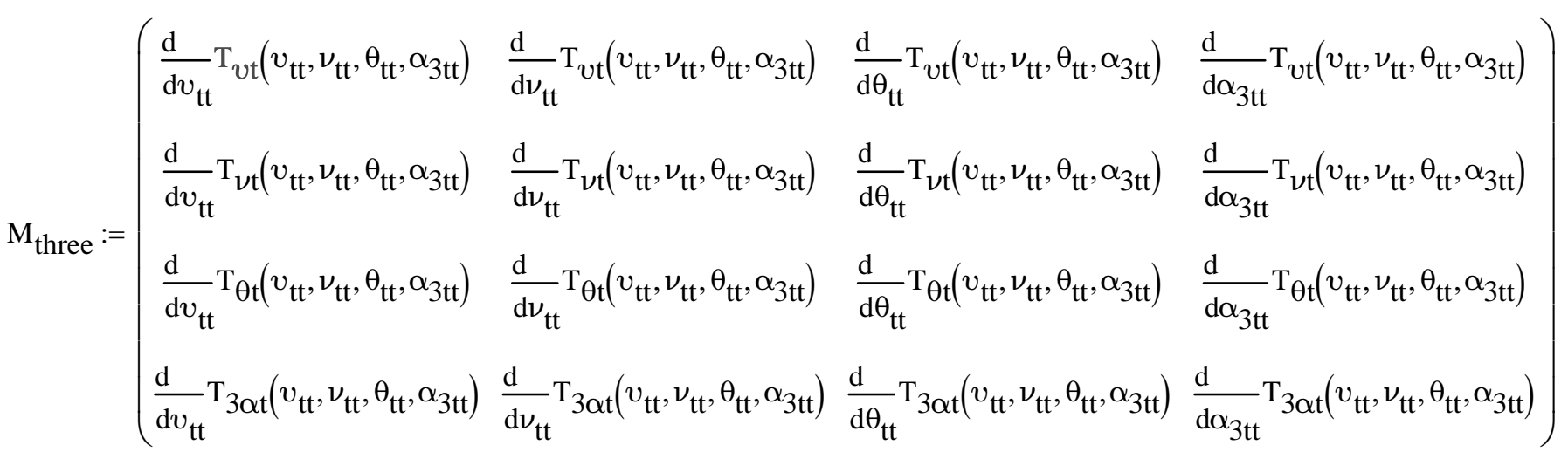

$$
\begin{aligned}
& \mathrm{K}_{\text {three }}:=\left(\begin{array}{llll}
\frac{\mathrm{d}}{\mathrm{d} v} \mathrm{~V}_{v}\left(v, \nu, \theta, \alpha_{3}\right) & \frac{\mathrm{d}}{\mathrm{d} \nu} \mathrm{V}_{v}\left(v, \nu, \theta, \alpha_{3}\right) & \frac{\mathrm{d}}{\mathrm{d} \theta} \mathrm{V}_{v}\left(v, \nu, \theta, \alpha_{3}\right) & \frac{\mathrm{d}}{\mathrm{d} \alpha_{3}} \mathrm{~V}_{v}\left(v, \nu, \theta, \alpha_{3}\right) \\
\frac{\mathrm{d}}{\mathrm{d} v} \mathrm{~V}_{\nu}\left(v, \nu, \theta, \alpha_{3}\right) & \frac{\mathrm{d}}{\mathrm{d} \nu} \mathrm{V}_{\nu}\left(v, \nu, \theta, \alpha_{3}\right) & \frac{\mathrm{d}}{\mathrm{d} \theta} \mathrm{V}_{\nu}\left(v, \nu, \theta, \alpha_{3}\right) & \frac{\mathrm{d}}{\mathrm{d} \alpha_{3}} \mathrm{~V}_{\nu}\left(v, \nu, \theta, \alpha_{3}\right) \\
\frac{\mathrm{d}}{\mathrm{d} v} \mathrm{~V}_{\theta}\left(v, \nu, \theta, \alpha_{3}\right) & \frac{\mathrm{d}}{\mathrm{d} \nu} \mathrm{V}_{\theta}\left(v, \nu, \theta, \alpha_{3}\right) & \frac{\mathrm{d}}{\mathrm{d} \theta} \mathrm{V}_{\theta}\left(v, \nu, \theta, \alpha_{3}\right) & \frac{\mathrm{d}}{\mathrm{d} \alpha_{3}} \mathrm{~V}_{\theta}\left(v, \nu, \theta, \alpha_{3}\right) \\
\frac{\mathrm{d}}{\mathrm{d} v} \mathrm{~V}_{3 \alpha}\left(v, \nu, \theta, \alpha_{3}\right) & \frac{\mathrm{d}}{\mathrm{d} \nu} \mathrm{V}_{3 \alpha}\left(v, \nu, \theta, \alpha_{3}\right) & \frac{\mathrm{d}}{\mathrm{d} \theta} \mathrm{V}_{3 \alpha}\left(v, \nu, \theta, \alpha_{3}\right) & \frac{\mathrm{d}}{\mathrm{d} \alpha_{3}} \mathrm{~V}_{3 \alpha}\left(v, \nu, \theta, \alpha_{3}\right)
\end{array}\right)
\end{aligned}
$$

\section{Blade Three Mass Matrix}

For clarity each term of Mone is explicitly stated

$$
\begin{aligned}
& \mathrm{M}_{\text {three }_{0,0}} \rightarrow \frac{\mathrm{HM}}{3}+\mathrm{Ma}+\mathrm{Mb} \\
& \mathrm{M}_{\text {three }_{0,2}} \rightarrow-\frac{\mathrm{Mb} \cdot\left(2 \cdot \mathrm{Hy}+\mathrm{Ey} \cdot \cos \left(\Psi_{\mathrm{r}}+\Psi_{3 \mathrm{~b}}\right)\right)}{2}-\frac{\mathrm{Ma} \cdot\left(2 \cdot \mathrm{Hy}+2 \cdot \mathrm{Ey} \cdot \cos \left(\Psi_{\mathrm{r}}+\Psi_{3 \mathrm{~b}}\right)\right)}{2} \\
& \text { M }_{\text {three }_{0,3}} \rightarrow-\text { Ma·a } \\
& \text { M }_{\text {three }_{1,1}} \rightarrow \frac{\mathrm{HM}}{3}+\mathrm{Ma}+\mathrm{Mb} \\
& \mathrm{M}_{\text {three }_{1,2}} \rightarrow-\mathrm{Hx} \cdot \mathrm{Ma}-\mathrm{Hx} \cdot \mathrm{Mb} \\
& \mathrm{M}_{\text {three }_{2,2}} \rightarrow \frac{\mathrm{HI}}{3}+\mathrm{I}_{\mathrm{b}} \cdot \cos \left(\Psi_{\mathrm{r}}+\Psi_{3 \mathrm{~b}}\right)^{2}+\frac{\mathrm{Ma} \cdot\left[2 \cdot \mathrm{Hx}^{2}+2 \cdot\left(\mathrm{Hy}+\mathrm{Ey} \cdot \cos \left(\Psi_{\mathrm{r}}+\Psi_{3 \mathrm{~b}}\right)\right)^{2}\right]}{2}+\frac{\mathrm{Mb} \cdot\left[2 \cdot \mathrm{Hx}{ }^{2}+2 \cdot\left(\mathrm{Hy}+\frac{\mathrm{Ey} \cdot \cos \left(\Psi_{\mathrm{r}}+\Psi_{3 \mathrm{~b}}\right)}{2}\right)^{2}\right]}{2} \\
& \mathrm{M}_{\text {three }_{2,3}} \rightarrow \mathrm{Ma} \cdot \mathrm{a} \cdot\left(\mathrm{Hy}+\mathrm{Ey} \cdot \cos \left(\Psi_{\mathrm{r}}+\Psi_{3 \mathrm{~b}}\right)\right) \\
& \mathrm{M}_{\text {three }_{3,3}} \rightarrow \text { Ma } \mathrm{a}^{2}+\mathrm{I}_{\mathrm{a}} \cdot \cos \left(\Psi_{\mathrm{r}}+\Psi_{3 \mathrm{~b}}\right)^{4}+\mathrm{I}_{\mathrm{a}} \cdot \sin \left(2 \cdot \Psi_{\mathrm{r}}+2 \cdot \Psi_{3 \mathrm{~b}}\right) \cdot \cos \left(\Psi_{\mathrm{r}}+\Psi_{3 \mathrm{~b}}\right) \cdot \sin \left(\Psi_{\mathrm{r}}+\Psi_{3 \mathrm{~b}}\right)+\mathrm{I}_{\mathrm{a}} \cdot \sin \left(\Psi_{\mathrm{r}}+\Psi_{3 \mathrm{~b}}\right)^{4}
\end{aligned}
$$

\section{Blade Three Stiffness Matrix}

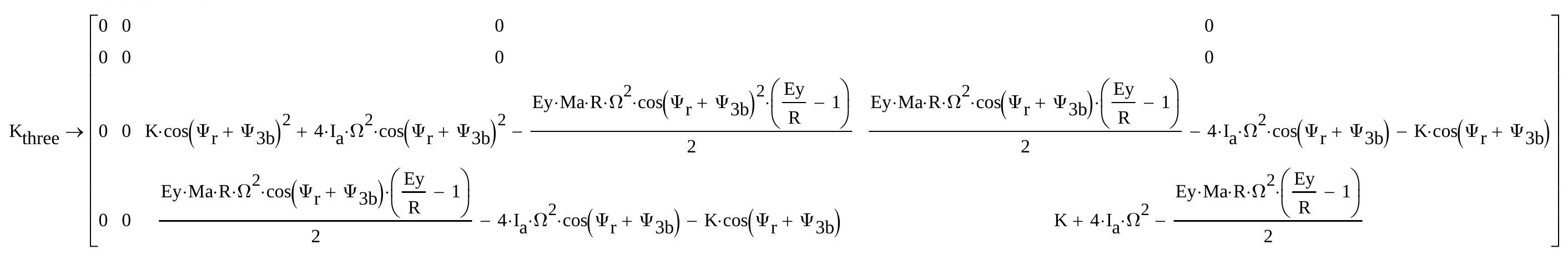




\section{Total Rotor System Equations of Motion Mass and Stiffness Matrices}

The following mass and stiffness matrices were developed my summing the one and lower stiffness matrices over the appropriate degrees of freedom. The system matrices feature the following degrees of freedom and in the stated order: $u, v, \theta, \alpha_{1}, \alpha_{2}, \alpha_{3}$

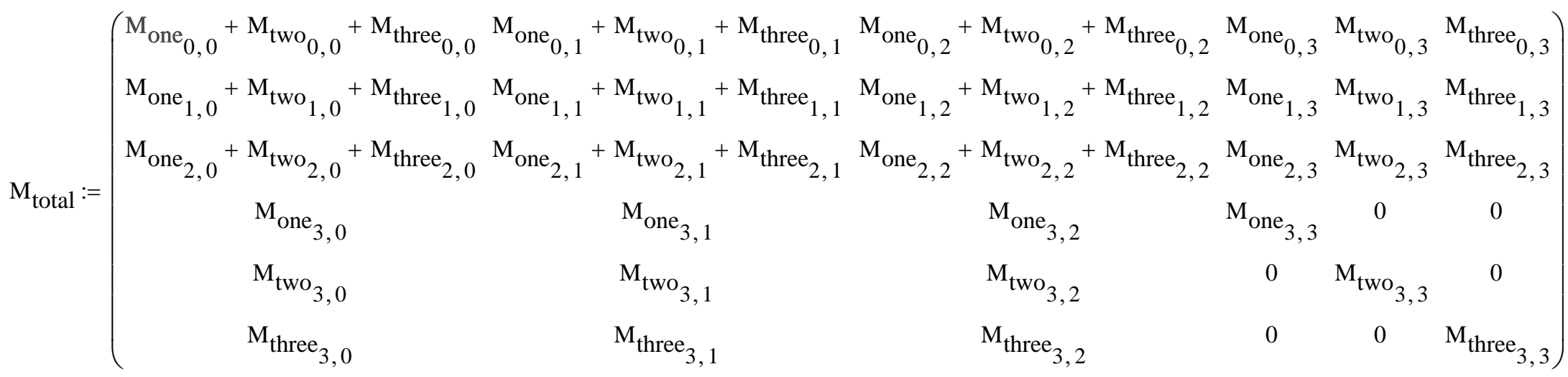

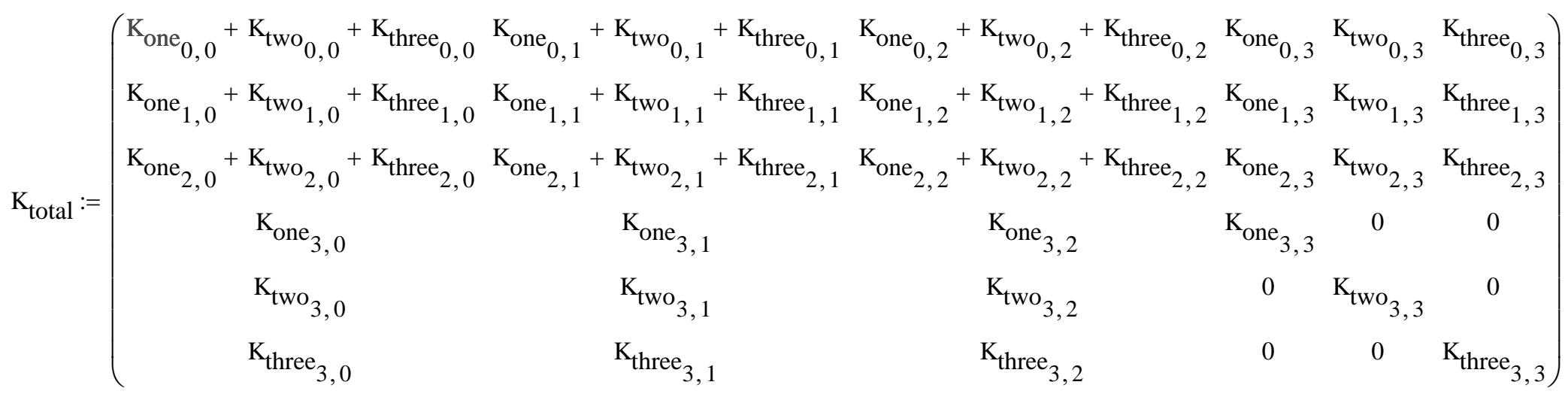


$\mathrm{M}_{\text {total }_{0,0}} \rightarrow \mathrm{HM}+3 \cdot \mathrm{Ma}+3 \cdot \mathrm{Mb}$

$\mathrm{M}_{\text {total }_{0,1}} \rightarrow 0$

$\mathrm{M}_{\mathrm{total}}, 2 \rightarrow-\frac{\mathrm{Mb} \cdot\left(2 \cdot \mathrm{Hy}+\mathrm{Ey} \cdot \cos \left(\Psi_{\mathrm{r}}+\Psi_{1 \mathrm{~b}}\right)\right)}{2}-\frac{\mathrm{Ma} \cdot\left(2 \cdot \mathrm{Hy}+2 \cdot \mathrm{Ey} \cdot \cos \left(\Psi_{\mathrm{r}}+\Psi_{1 \mathrm{~b}}\right)\right)}{2}-\frac{\mathrm{Mb} \cdot\left(2 \cdot \mathrm{Hy}+\mathrm{Ey} \cdot \cos \left(\Psi_{\mathrm{r}}+\Psi_{2 \mathrm{~b}}\right)\right)}{2}-\frac{\mathrm{Ma} \cdot\left(2 \cdot \mathrm{Hy}+2 \cdot \mathrm{Ey} \cdot \cos \left(\Psi_{\mathrm{r}}+\Psi_{2 b}\right)\right)}{2}-\frac{\mathrm{Mb} \cdot\left(2 \cdot \mathrm{Hy}+\mathrm{Ey} \cdot \cos \left(\Psi_{\mathrm{r}}+\Psi_{3 b}\right)\right)}{2}-\frac{\mathrm{Ma} \cdot\left(2 \cdot \mathrm{Hy}+2 \cdot \mathrm{Ey} \cdot \cos \left(\Psi_{\mathrm{r}}+\Psi_{3 b}\right)\right)}{2}$

$\mathrm{M}_{\text {total }_{0,3} \rightarrow-\text { Ma-a }}$

$\mathrm{M}_{\text {total }_{0,4} \rightarrow-\text { Mara }}$

$\mathrm{M}_{\text {total }_{0,5} \rightarrow-\text { Mara }}$

$\mathrm{M}_{\text {total }_{1,1}} \rightarrow \mathrm{HM}+3 \cdot \mathrm{Ma}+3 \cdot \mathrm{Mb}$

$\mathrm{M}_{\mathrm{total}_{1,2}} \rightarrow-3 \cdot \mathrm{H} \times \mathrm{Ma}-3 \cdot \mathrm{H} \times \mathrm{Mb}$

$\mathrm{M}_{\text {total }_{1,3}} \rightarrow 0$

$\mathrm{M}_{\text {total }_{1,4} \rightarrow 0}$

$\mathrm{M}_{\text {total }_{1,5}} \rightarrow 0$

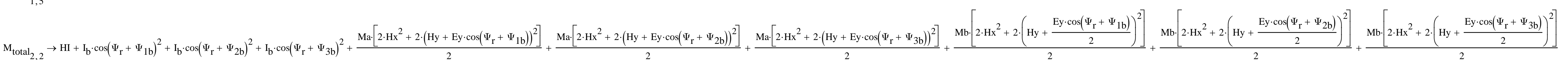
$\mathrm{M}_{\mathrm{total}_{2,3}} \rightarrow \mathrm{Ma} \cdot \mathrm{a} \cdot\left(\mathrm{Hy}+\mathrm{Ey} \cdot \cos \left(\Psi_{\mathrm{r}}+\Psi_{1 \mathrm{~b}}\right)\right)$

$\mathrm{M}_{\mathrm{total}_{2,4}} \rightarrow \mathrm{Ma} \cdot \mathrm{a} \cdot\left(\mathrm{Hy}+\mathrm{Ey} \cdot \cos \left(\Psi_{\mathrm{r}}+\Psi_{2 \mathrm{~b}}\right)\right)$

$\mathrm{M}_{\text {total }_{2,5}} \rightarrow \mathrm{Ma} \cdot \mathrm{a} \cdot\left(\mathrm{Hy}+\mathrm{Ey} \cdot \cos \left(\Psi_{\mathrm{r}}+\Psi_{3 \mathrm{~b}}\right)\right)$

$\mathrm{M}_{\text {total }} \rightarrow 3 \rightarrow \mathrm{Ma}^{2}{ }^{2}+\mathrm{I}_{\mathrm{a}} \cdot \cos \left(\Psi_{\mathrm{r}}+\Psi_{1 b}\right)^{4}+\mathrm{I}_{\mathrm{a}} \cdot \sin \left(2 \cdot \Psi_{\mathrm{r}}+2 \cdot \Psi_{1 b}\right) \cdot \cos \left(\Psi_{\mathrm{r}}+\Psi_{1 b}\right) \cdot \sin \left(\Psi_{\mathrm{r}}+\Psi_{1 b}\right)+\mathrm{I}_{\mathrm{a}} \cdot \sin \left(\Psi_{\mathrm{r}}+\Psi_{1 b}\right)^{4}$

$\mathrm{M}_{\text {total }_{3,4} \rightarrow 0} \rightarrow 0$

$\mathrm{M}_{\text {total }_{3,5} \rightarrow 0}$

$\mathrm{M}_{\text {total }_{4,4}} \rightarrow$ Ma-a ${ }^{2}+\mathrm{I}_{\mathrm{a}} \cdot \cos \left(\Psi_{\mathrm{r}}+\Psi_{2 \mathrm{~b}}\right)^{4}+\mathrm{I}_{\mathrm{a}} \cdot \sin \left(2 \cdot \Psi_{\mathrm{r}}+2 \cdot \Psi_{2 b}\right) \cdot \cos \left(\Psi_{\mathrm{r}}+\Psi_{2 \mathrm{~b}}\right) \cdot \sin \left(\Psi_{\mathrm{r}}+\Psi_{2 \mathrm{~b}}\right)+\mathrm{I}_{\mathrm{a}} \cdot \sin \left(\Psi_{\mathrm{r}}+\Psi_{2 \mathrm{~b}}\right)^{4}$

$\mathrm{M}_{\text {total }_{4,5}} \rightarrow 0$

$\mathrm{M}_{\text {total }} \rightarrow \mathrm{Ma}_{5}{ }^{2}+\mathrm{I}_{\mathrm{a}} \cdot \cos \left(\Psi_{\mathrm{r}}+\Psi_{3 \mathrm{~b}}\right)^{4}+\mathrm{I}_{\mathrm{a}} \cdot \sin \left(2 \cdot \Psi_{\mathrm{r}}+2 \cdot \Psi_{3 \mathrm{~b}}\right) \cdot \cos \left(\Psi_{\mathrm{r}}+\Psi_{3 \mathrm{~b}}\right) \cdot \sin \left(\Psi_{\mathrm{r}}+\Psi_{3 \mathrm{~b}}\right)+\mathrm{I}_{\mathrm{a}} \cdot \sin \left(\Psi_{\mathrm{r}}+\Psi_{3 \mathrm{~b}}\right)^{4}$ 


\section{Total Stiffness Matrix}

$$
\begin{aligned}
& \mathrm{K}_{\mathrm{total}_{0,0}} \rightarrow 0 \\
& \mathrm{~K}_{\mathrm{total}_{0,1}} \rightarrow 0 \\
& \mathrm{~K}_{\mathrm{total}_{0,2}} \rightarrow 0 \\
& \mathrm{~K}_{\mathrm{total}_{0,3}} \rightarrow 0
\end{aligned}
$$

$\mathrm{K}_{\text {total }_{0,4} \rightarrow 0} \rightarrow$

$\mathrm{K}_{\text {total }}, \rightarrow 0$

$\mathrm{K}_{\text {total }_{1,1} \rightarrow 0}$

$\mathrm{K}_{\mathrm{total}_{1,2}} \rightarrow 0$

$\mathrm{K}_{\text {total }_{1,3} \rightarrow 0}$

$\mathrm{K}_{\text {total. . . } \rightarrow 0}$

$\mathrm{K}_{\text {total }_{2,2}} \rightarrow \mathrm{K} \cdot \cos \left(\Psi_{\mathrm{r}}+\Psi_{1 b}\right)^{2}+\mathrm{K} \cdot \cos \left(\Psi_{\mathrm{r}}+\Psi_{2 b}\right)^{2}+\mathrm{K} \cdot \cos \left(\Psi_{\mathrm{r}}+\Psi_{3 b}\right)^{2}+4 \cdot \mathrm{I}_{\mathrm{a}} \cdot \Omega^{2} \cdot \cos \left(\Psi_{\mathrm{r}}+\Psi_{1 b}\right)^{2}+4$
$\mathrm{~K}_{\mathrm{total}_{2,3}} \rightarrow \frac{\text { Ey } \cdot \mathrm{Ma} \cdot \mathrm{R} \cdot \Omega^{2} \cdot \cos \left(\Psi_{\mathrm{r}}+\Psi_{1 b}\right) \cdot\left(\frac{\mathrm{Ey}}{\mathrm{R}}-1\right)}{2}-4 \cdot \mathrm{I}_{\mathrm{a}} \cdot \Omega^{2} \cdot \cos \left(\Psi_{\mathrm{r}}+\Psi_{1 b}\right)-\mathrm{K} \cdot \cos \left(\Psi_{\mathrm{r}}+\Psi_{1 b}\right)$

$\mathrm{K}_{\text {totat }}, \frac{\text { Ey }}{2,4} \cdot \frac{\mathrm{Ma} \cdot \mathrm{R} \cdot \Omega^{2} \cdot \cos \left(\Psi_{\mathrm{r}}+\Psi_{2 \mathrm{~b}}\right)\left(\frac{\mathrm{Ey}}{\mathrm{R}}-1\right)}{2}-4 \cdot \mathrm{I}_{\mathrm{a}} \cdot \Omega^{2} \cdot \cos \left(\Psi_{\mathrm{r}}+\Psi_{2 \mathrm{~b}}\right)-\mathrm{K} \cdot \cos \left(\Psi_{\mathrm{r}}+\Psi_{2 \mathrm{~b}}\right)$

$\mathrm{K}_{\text {total }}, 2 \rightarrow \frac{\text { Ey } \cdot \mathrm{Ma} \cdot \mathrm{R} \cdot \Omega^{2} \cdot \cos \left(\Psi_{\mathrm{r}}+\Psi_{3 \mathrm{~b}}\right) \cdot\left(\frac{\mathrm{Ey}}{\mathrm{R}}-1\right)}{2}-4 \cdot \mathrm{I}_{\mathrm{a}} \cdot \Omega^{2} \cdot \cos \left(\Psi_{\mathrm{r}}+\Psi_{3 \mathrm{~b}}\right)-\mathrm{K} \cdot \cos \left(\Psi_{\mathrm{r}}+\Psi_{3 \mathrm{~b}}\right)$

$\mathrm{K}_{\text {total }_{3,3}} \rightarrow \mathrm{K}+4 \cdot \mathrm{I} \cdot \mathrm{I} \cdot \Omega^{2}-\frac{\mathrm{Ey} \cdot \mathrm{Ma} \cdot \mathrm{R} \cdot \Omega^{2} \cdot\left(\frac{\mathrm{H}}{\mathrm{R}}-1\right)}{2}$

$\mathrm{K}_{\text {total }_{3,4} \rightarrow 0}$

$\mathrm{K}_{\text {total }}, \rightarrow 0$

$\mathrm{K}_{\text {total }_{4,4}} \rightarrow \mathrm{K}+4 \cdot \mathrm{I}_{\mathrm{a}} \cdot \Omega^{2}-\frac{\mathrm{Ey} \cdot \mathrm{Ma} \cdot \mathrm{R} \cdot \Omega^{2} \cdot\left(\frac{\mathrm{Ey}}{\mathrm{R}}-1\right)}{2}$

$\mathrm{K}_{\text {total }_{4,5}} \rightarrow 0$

$\mathrm{K}_{\mathrm{total}_{5,5}} \rightarrow \mathrm{K}+4 \cdot \mathrm{I} \cdot \mathrm{I}_{\mathrm{a}} \cdot \Omega^{2}-\frac{\text { Ey } \mathrm{Ma} \cdot \mathrm{R} \cdot \Omega^{2} \cdot\left(\frac{\mathrm{Ey}}{\mathrm{R}}-1\right)}{2}$

$\frac{E y \cdot M a \cdot R \cdot \Omega^{2} \cdot \cos \left(\Psi_{r}+\Psi_{1 b}\right)^{2} \cdot\left(\frac{E y}{R}-1\right)}{2}-\frac{E y \cdot M a \cdot R \cdot \Omega^{2} \cdot \cos \left(\Psi_{r}+\Psi_{2 b}\right)^{2} \cdot\left(\frac{E y}{R}-1\right)}{2}-\frac{E y \cdot M a \cdot R \cdot \Omega^{2} \cdot \cos \left(\Psi_{r}+\Psi_{3 b}\right)^{2} \cdot\left(\frac{E y}{R}-1\right)}{2}$ 


\title{
Appendix B
}

\section{Derivation of 2 Blade Generalized Rotor Model}

\author{
MathCAD Printout
}

Formatted for Electronic Viewing 


\section{GENERALIZED ROTOR MODEL - 2 BLADED ROTOR FORMATTED FOR ELECTRONIC VIEWING - ZOOM IN TO SEE EQUATIONS}

\section{Blade One Equation of Motion Derivation}

Relate Motion of the center of gravity of member A to the system DOF velocities Position

$\operatorname{Xag}\left(v, \theta, \alpha_{1}\right):=-\mathrm{a} \cdot \alpha_{1}+v-\left(\right.$ Ey $\left.\cdot \cos \left(\Psi_{1 \mathrm{~b}}+\Psi_{\mathrm{r}}\right)+\mathrm{Hy}\right) \cdot \theta$

$\operatorname{Yag}(\nu, \theta):=\nu-\mathrm{Hx} \cdot \theta$

\section{Velocity}

$\operatorname{Xag}_{\mathrm{t}}\left(v_{\mathrm{t}}, \theta_{\mathrm{t}}, \alpha_{1 \mathrm{t}}\right):=-\mathrm{a} \cdot \alpha_{1 \mathrm{t}}+v_{\mathrm{t}}-\left(\mathrm{Ey} \cdot \cos \left(\Psi_{1 \mathrm{~b}}+\Psi_{\mathrm{r}}\right)+\mathrm{Hy}\right) \cdot \theta_{\mathrm{t}}$

$\operatorname{Yag}_{\mathrm{t}}\left(\nu_{\mathrm{t}}, \theta_{\mathrm{t}}\right):=\nu_{\mathrm{t}}-\mathrm{Hx} \cdot \theta_{\mathrm{t}}$

Relate Motion of the center of gravity of member B to the system DOF velocities Position

$\operatorname{Xbg}(v, \theta):=v-\left(\frac{E y \cdot \cos \left(\Psi_{1 \mathrm{~b}}+\Psi_{\mathrm{r}}\right)}{2}+\mathrm{Hy}\right) \cdot \theta$

$\operatorname{Ybg}(\nu, \theta):=\nu-\mathrm{Hx} \cdot \theta$

Velocity

$\operatorname{Xbg}_{\mathrm{t}}\left(v_{\mathrm{t}}, \theta_{\mathrm{t}}\right):=v_{\mathrm{t}}-\left(\frac{\mathrm{Ey} \cdot \cos \left(\Psi_{1 \mathrm{~b}}+\Psi_{\mathrm{r}}\right)}{2}+\mathrm{Hy}\right) \cdot \theta_{\mathrm{t}}$

$\operatorname{Ybg}_{\mathrm{t}}\left(\nu_{\mathrm{t}}, \theta_{\mathrm{t}}\right):=\nu_{\mathrm{t}}-\mathrm{Hx} \cdot \theta_{\mathrm{t}}$

Relate the rotation of $\alpha_{1}$ about local unit vector $K_{\text {hat }}$ to global unit vectors $j_{\text {hat }}$ and $k_{\text {hat" }}$ (This relation is used to define the rotational velocity of member $A$.

$\mathrm{K}_{\text {hat }}\left(\mathrm{j}_{\text {hat }}, \mathrm{k}_{\text {hat }}\right):=\mathrm{j}_{\text {hat }} \cdot \sin \left(\Psi_{1 \mathrm{~b}}+\Psi_{\mathrm{r}}\right)+\mathrm{k}_{\text {hat }} \cdot \cos \left(\Psi_{1 \mathrm{~b}}+\Psi_{\mathrm{r}}\right)$

$\mathrm{K}_{\text {yhat }}:=\frac{\mathrm{d}}{\mathrm{dj}_{\text {hat }}} \mathrm{K}_{\text {hat }}\left(\mathrm{j}_{\text {hat }}, \mathrm{k}_{\text {hat }}\right) \quad \mathrm{K}_{\text {yhat }} \rightarrow \sin \left(\Psi_{\mathrm{r}}+\Psi_{1 \mathrm{~b}}\right)$

$\mathrm{K}_{\mathrm{zhat}}:=\frac{\mathrm{d}}{\mathrm{dk}_{\text {hat }}} \mathrm{K}_{\text {hat }}\left(\mathrm{j}_{\text {hat }}, \mathrm{k}_{\text {hat }}\right) \quad \mathrm{K}_{\mathrm{zhat}} \rightarrow \cos \left(\Psi_{\mathrm{r}}+\Psi_{1 \mathrm{~b}}\right)$

\section{Rotational Velocity of member A}

$$
\begin{array}{ll}
\omega_{\text {ay }}\left(\alpha_{1 \mathrm{t}}\right):=\alpha_{1 \mathrm{t}} \cdot \mathrm{K}_{\text {yhat }} & \omega_{\text {ay }}\left(\alpha_{1 \mathrm{t}}\right) \rightarrow \alpha_{1 \mathrm{t}} \cdot \sin \left(\Psi_{\mathrm{r}}+\Psi_{1 \mathrm{~b}}\right) \\
\omega_{\mathrm{az}}\left(\alpha_{1 \mathrm{t}}\right):=\alpha_{1 \mathrm{t}} \cdot \mathrm{K}_{\text {zhat }} & \omega_{\mathrm{az}}\left(\alpha_{1 \mathrm{t}}\right) \rightarrow \alpha_{1 \mathrm{t}} \cdot \cos \left(\Psi_{\mathrm{r}}+\Psi_{1 \mathrm{~b}}\right)
\end{array}
$$

\section{Rotational Velocity of member B}

$$
\omega_{\mathrm{bz}}\left(\theta_{\mathrm{t}}\right):=\theta_{\mathrm{t}} \quad \omega_{\mathrm{bz}}\left(\theta_{\mathrm{t}}\right) \rightarrow \theta_{\mathrm{t}}
$$

Moments and Products of Inertia of Rotor Member A

$$
\begin{aligned}
& \mathrm{I}_{\text {ayy }}:=\mathrm{I}_{\mathrm{a}} \cdot\left(\sin \left(\Psi_{1 \mathrm{~b}}+\Psi_{\mathrm{r}}\right)\right)^{2} \\
& \mathrm{I}_{\mathrm{azz}}:=\mathrm{I}_{\mathrm{a}} \cdot\left(\cos \left(\Psi_{1 \mathrm{~b}}+\Psi_{\mathrm{r}}\right)\right)^{2} \\
& \mathrm{I}_{\mathrm{ayz}}:=\frac{-\mathrm{I}_{\mathrm{a}}}{2} \cdot \sin \left[2 \cdot\left(\Psi_{1 \mathrm{~b}}+\Psi_{\mathrm{r}}\right)\right]
\end{aligned}
$$

\section{Moments and Products of Inertia of Rotor Member B}

$$
\begin{aligned}
& \mathrm{I}_{\text {byy }}:=\mathrm{I}_{\mathrm{b}} \cdot\left(\sin \left(\Psi_{1 \mathrm{~b}}+\Psi_{\mathrm{r}}\right)\right)^{2} \\
& \mathrm{I}_{\mathrm{bzz}}:=\mathrm{I}_{\mathrm{b}} \cdot\left(\cos \left(\Psi_{1 \mathrm{~b}}+\Psi_{\mathrm{r}}\right)\right)^{2} \\
& \mathrm{I}_{\mathrm{byz}}:=\frac{-\mathrm{I}_{\mathrm{b}}}{2} \cdot \sin \left[2 \cdot\left(\Psi_{1 \mathrm{~b}}+\Psi_{\mathrm{r}}\right)\right]
\end{aligned}
$$

Note: time derivatives of variables are annotated with subscripts. For example $u_{t}$ is the first time derivative of $u$ while $u_{t t}$ is the second time derivative of $u$. 


\section{Kinetic Energy $(T)$}

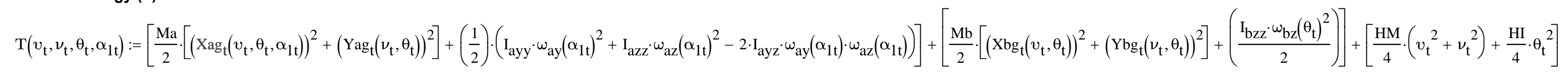

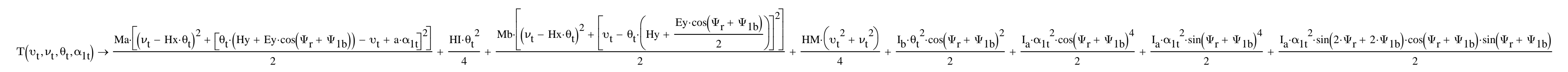

\section{Take the derivative of Kinetic Energy wrt System DOF velocities}

$\frac{\mathrm{d}}{\mathrm{d} v_{\mathrm{t}}} \mathrm{T}\left(v_{\mathrm{t}}, \nu_{\mathrm{t}}, \theta_{\mathrm{t}}, \alpha_{1 \mathrm{t}}\right) \mid \begin{aligned} & \text { collect } \\ & v_{\mathrm{t}}, \nu_{\mathrm{t}}, \theta_{\mathrm{t}}, \alpha_{1 \mathrm{t}}\end{aligned}\left(\frac{\mathrm{HM}}{2}+\mathrm{Ma}+\mathrm{Mb}\right) \cdot v_{\mathrm{t}}+\left[-\mathrm{Mb} \cdot\left(\mathrm{Hy}+\frac{\mathrm{Ey} \cdot \cos \left(\Psi_{\mathrm{r}}+\Psi_{1 \mathrm{~b}}\right)}{2}\right)-\frac{\mathrm{Ma} \cdot\left(2 \cdot \mathrm{Hy}+2 \cdot \mathrm{Ey} \cdot \cos \left(\Psi_{\mathrm{r}}+\Psi_{1 \mathrm{~b}}\right)\right)}{2}\right] \cdot \theta_{\mathrm{t}}+(-\mathrm{Ma} \cdot \mathrm{a}) \cdot \alpha_{1 \mathrm{t}}$

$\frac{\mathrm{d}}{\mathrm{d} \nu_{t}} \mathrm{~T}\left(v_{\mathrm{t}}, \nu_{\mathrm{t}}, \theta_{\mathrm{t}}, \alpha_{1 \mathrm{t}}\right) \mid \begin{aligned} & \text { collect } \\ & v_{\mathrm{t}}, \nu_{\mathrm{t}}, \theta_{\mathrm{t}}, \mathrm{\alpha}_{1 \mathrm{t}}\end{aligned} \rightarrow\left(\frac{\mathrm{HM}}{2}+\mathrm{Ma}+\mathrm{Mb}\right) \cdot v_{\mathrm{t}}+(-\mathrm{Hx} \cdot \mathrm{Ma}-\mathrm{Hx} \cdot \mathrm{Mb}) \cdot \theta_{\mathrm{t}}$

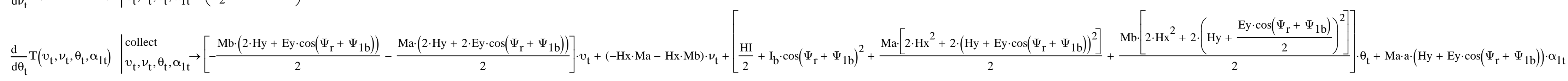

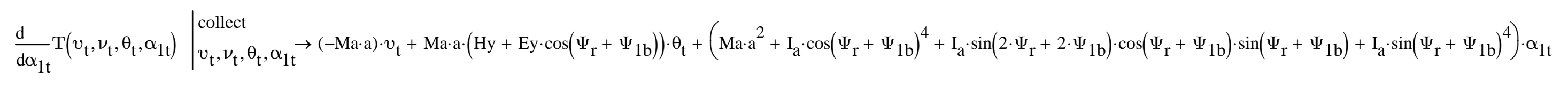

\section{Take the time derivative of the above terms by substituting in special accelerations}

${ }^{\mathrm{T}} v_{\mathrm{tt}}\left(v_{\mathrm{tt}}, v_{\mathrm{tt}}, \theta_{\mathrm{tt}}, \alpha_{1 \mathrm{tt}}\right):=\frac{\mathrm{d}}{\mathrm{d} v_{\mathrm{tt}}} \mathrm{T}\left(v_{\mathrm{tt}}, v_{\mathrm{tt}}, \theta_{\mathrm{tt}}, \alpha_{1 \mathrm{tt}}\right)$

$\mathrm{T}_{\nu \mathrm{t}}\left(v_{\mathrm{tt}}, v_{\mathrm{tt}}, \theta_{\mathrm{tt}}, \alpha_{1 \mathrm{tt}}\right):=\frac{\mathrm{d}}{\mathrm{d} \nu_{\mathrm{tt}}} \mathrm{T}\left(v_{\mathrm{tt}}, v_{\mathrm{tt}}, \theta_{\mathrm{tt}}, \alpha_{1 \mathrm{tt}}\right)$

$\mathrm{T}_{\theta t}\left(v_{\mathrm{tt}}, v_{\mathrm{tt}}, \theta_{\mathrm{tt}}, \alpha_{1 \mathrm{tt}}\right):=\frac{\mathrm{d}}{\mathrm{d} \theta_{\mathrm{tt}}} \mathrm{T}\left(v_{\mathrm{tt}}, v_{\mathrm{tt}}, \theta_{\mathrm{tt}}, \alpha_{1 \mathrm{tt}}\right)$

$\mathrm{T}_{1 \alpha \mathrm{t}}\left(v_{\mathrm{tt}}, v_{\mathrm{tt}}, \theta_{\mathrm{tt}}, \alpha_{1 \mathrm{tt}}\right):=\frac{\mathrm{d}}{\mathrm{d} \alpha_{1 \mathrm{tt}}} \mathrm{T}\left(v_{\mathrm{tt}}, v_{\mathrm{tt}}, \theta_{\mathrm{tt}}, \alpha_{1 \mathrm{tt}}\right)$

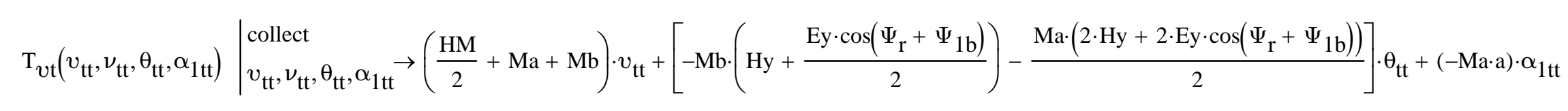

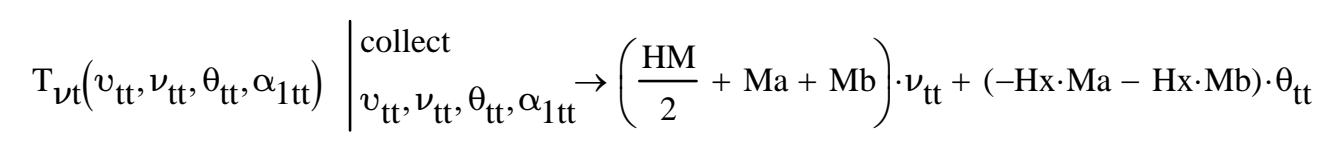

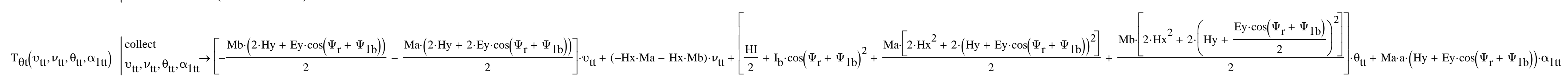

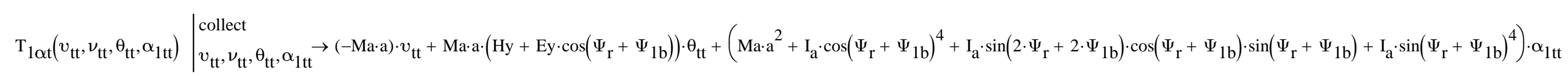




\section{Potential Energy}

$\mathrm{V}\left(v, \nu, \theta, \alpha_{1}\right):=\frac{1}{2} \cdot \mathrm{K} \cdot\left(\alpha_{1}\right)^{2}+\frac{1}{2} \cdot \mathrm{K} \cdot\left(\theta \cdot \cos \left(\Psi_{1 \mathrm{~b}}+\Psi_{\mathrm{r}}\right)\right)^{2}-\mathrm{K} \cdot \alpha_{1} \cdot \theta \cdot \cos \left(\Psi_{1 \mathrm{~b}}+\Psi_{\mathrm{r}}\right)+\mathrm{Ma} \cdot \mathrm{g} \cdot \mathrm{Yag}(\nu, \theta)+\mathrm{Mb} \cdot \mathrm{g} \cdot \mathrm{Ybg}(\nu, \theta)+\frac{1}{2} \mathrm{HM} \cdot \mathrm{g} \cdot \nu+\frac{1}{4} \cdot \Omega^{2} \cdot \mathrm{Ma} \cdot \mathrm{R} \cdot \frac{\mathrm{Ey}}{\mathrm{R}} \cdot\left(1-\frac{\mathrm{Ey}}{\mathrm{R}}\right) \cdot\left(\alpha_{1}-\theta \cdot \cos \left(\Psi_{1 \mathrm{~b}}+\Psi_{\mathrm{r}}\right)\right)^{2}+\frac{4}{2} \cdot \Omega^{2} \cdot \mathrm{I}_{\mathrm{a}} \cdot\left(\alpha_{1}-\theta \cdot \cos \left(\Psi_{1 \mathrm{~b}}+\Psi_{\mathrm{r}}\right)\right)^{2}$

$\mathrm{V}\left(v, \nu, \theta, \alpha_{1}\right) \rightarrow \frac{\mathrm{K} \cdot \alpha_{1}^{2}}{2}+\frac{\mathrm{HM} \cdot \mathrm{g} \cdot \nu}{2}+\mathrm{Ma} \cdot \mathrm{g} \cdot(\nu-\mathrm{Hx} \cdot \theta)+\mathrm{Mb} \cdot \mathrm{g} \cdot(\nu-\mathrm{Hx} \cdot \theta)+2 \cdot \mathrm{I}_{\mathrm{a}} \cdot \Omega^{2} \cdot\left(\alpha_{1}-\theta \cdot \cos \left(\Psi_{\mathrm{r}}+\Psi_{1 b}\right)\right)^{2}+\frac{\mathrm{K} \cdot \theta^{2} \cdot \cos \left(\Psi_{\mathrm{r}}+\Psi_{1 \mathrm{~b}}\right)^{2}}{2}-\mathrm{K} \cdot \theta \cdot \alpha_{1} \cdot \cos \left(\Psi_{\mathrm{r}}+\Psi_{1 \mathrm{~b}}\right)-\frac{\mathrm{Ey} \cdot \mathrm{Ma} \cdot \mathrm{R} \cdot \Omega^{2} \cdot\left(\frac{\mathrm{Ey}}{\mathrm{R}}-1\right) \cdot\left(\alpha_{1}-\theta \cdot \cos \left(\Psi_{\mathrm{r}}+\Psi_{1 \mathrm{~b}}\right)\right)^{2}}{4}$

\section{Take the derivative of Potential Energy wrt System DOF cord.}

$\mathrm{V}_{v}\left(v, \nu, \theta, \alpha_{1}\right):=\frac{\mathrm{d}}{\mathrm{d} v} \mathrm{~V}\left(v, \nu, \theta, \alpha_{1}\right)$

$\mathrm{V}_{\nu}\left(v, \nu, \theta, \alpha_{1}\right):=\frac{\mathrm{d}}{\mathrm{d} \nu} \mathrm{V}\left(\nu, \nu, \theta, \alpha_{1}\right)$

$\mathrm{V}_{\theta}\left(v, \nu, \theta, \alpha_{1}\right):=\frac{\mathrm{d}}{\mathrm{d} \theta} \mathrm{V}\left(v, \nu, \theta, \alpha_{1}\right)$

$\mathrm{V}_{1 \alpha}\left(v, \nu, \theta, \alpha_{1}\right):=\frac{\mathrm{d}}{\mathrm{d} \alpha_{1}} \mathrm{~V}\left(v, \nu, \theta, \alpha_{1}\right)$

$\mathrm{v}_{v}\left(v, \nu, \theta, \alpha_{1}\right) \mid \begin{aligned} & \text { collect } \\ & v, \nu, \theta, \alpha_{1} \rightarrow 0\end{aligned}$

$\mathrm{V}_{\nu}\left(v, \nu, \theta, \alpha_{1}\right) \mid \begin{aligned} & \text { collect } \\ & v, \nu, \theta, \alpha_{1}\end{aligned} \rightarrow \frac{\mathrm{HM} \cdot \mathrm{g}}{2}+\mathrm{Ma} \cdot \mathrm{g}+\mathrm{Mb} \cdot \mathrm{g}$

$\mathrm{V}_{\theta}\left(v, \nu, \theta, \alpha_{1}\right) \mid \begin{aligned} & \operatorname{collect} \\ & v, \nu, \theta, \alpha_{1}\end{aligned} \rightarrow\left[\mathrm{K} \cdot \cos \left(\Psi_{\mathrm{r}}+\Psi_{1 \mathrm{~b}}\right)^{2}+4 \cdot \mathrm{I}_{\mathrm{a}} \cdot \Omega^{2} \cdot \cos \left(\Psi_{\mathrm{r}}+\Psi_{1 \mathrm{~b}}\right)^{2}-\frac{\text { Ey } \cdot \mathrm{Ma} \cdot \mathrm{R} \cdot \Omega^{2} \cdot \cos \left(\Psi_{\mathrm{r}}+\Psi_{1 \mathrm{~b}}\right)^{2} \cdot\left(\frac{\mathrm{Ey}}{\mathrm{R}}-1\right)}{2}\right] \cdot \theta+\left[\frac{\mathrm{Ey} \cdot \mathrm{Ma} \cdot \mathrm{R} \cdot \Omega^{2} \cdot \cos \left(\Psi_{\mathrm{r}}+\Psi_{1 \mathrm{~b}}\right) \cdot\left(\frac{\mathrm{Ey}}{\mathrm{R}}-1\right)}{2}-4 \cdot \mathrm{I}_{\mathrm{a}} \cdot \Omega^{2} \cdot \cos \left(\Psi_{\mathrm{r}}+\Psi_{1 \mathrm{~b}}\right)-\mathrm{K} \cdot \cos \left(\Psi_{\mathrm{r}}+\Psi_{1 \mathrm{~b}}\right)\right] \cdot \alpha_{1}-(\mathrm{Hx} \cdot \mathrm{Ma} \cdot \mathrm{g}+\mathrm{Hx} \cdot \mathrm{Mb} \cdot \mathrm{g})$

$\mathrm{V}_{1 \alpha}\left(v, \nu, \theta, \alpha_{1}\right) \mid \begin{aligned} & \operatorname{collect} \\ & v, \nu, \theta, \alpha_{1}\end{aligned} \rightarrow\left[\frac{\text { Ey·Ma } \cdot \mathrm{R} \cdot \Omega^{2} \cdot \cos \left(\Psi_{\mathrm{r}}+\Psi_{1 \mathrm{~b}}\right) \cdot\left(\frac{\mathrm{Ey}}{\mathrm{R}}-1\right)}{2}-4 \cdot \mathrm{I}_{\mathrm{a}} \cdot \Omega^{2} \cdot \cos \left(\Psi_{\mathrm{r}}+\Psi_{1 \mathrm{~b}}\right)-\mathrm{K} \cdot \cos \left(\Psi_{\mathrm{r}}+\Psi_{1 \mathrm{~b}}\right)\right] \cdot \theta+\left[\mathrm{K}+4 \cdot \mathrm{I}_{\mathrm{a}} \cdot \Omega^{2}-\frac{\text { Ey } \cdot \mathrm{Ma} \cdot \mathrm{R} \cdot \Omega^{2} \cdot\left(\frac{\mathrm{Ey}}{\mathrm{R}}-1\right)}{2}\right] \cdot \alpha_{1}$ 


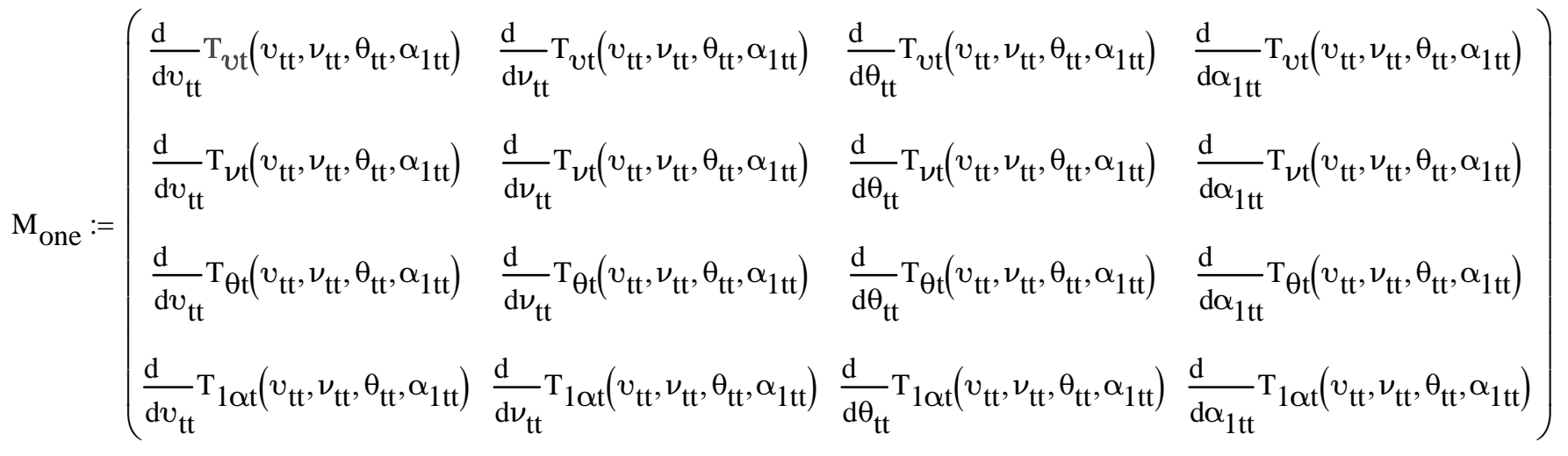

$$
\begin{aligned}
& \mathrm{K}_{\text {one }}:=\left(\begin{array}{llll}
\frac{\mathrm{d}}{\mathrm{d} v} \mathrm{~V}_{v}\left(v, \nu, \theta, \alpha_{1}\right) & \frac{\mathrm{d}}{\mathrm{d} \nu} \mathrm{V}_{v}\left(v, \nu, \theta, \alpha_{1}\right) & \frac{\mathrm{d}}{\mathrm{d} \theta} \mathrm{V}_{v}\left(v, \nu, \theta, \alpha_{1}\right) & \frac{\mathrm{d}}{\mathrm{d} \alpha_{1}} \mathrm{~V}_{v}\left(v, \nu, \theta, \alpha_{1}\right) \\
\frac{\mathrm{d}}{\mathrm{d} v} \mathrm{~V}_{\nu}\left(v, \nu, \theta, \alpha_{1}\right) & \frac{\mathrm{d}}{\mathrm{d} \nu} \mathrm{V}_{\nu}\left(v, \nu, \theta, \alpha_{1}\right) & \frac{\mathrm{d}}{\mathrm{d} \theta} \mathrm{V}_{\nu}\left(v, \nu, \theta, \alpha_{1}\right) & \frac{\mathrm{d}}{\mathrm{d} \alpha_{1}} \mathrm{~V}_{\nu}\left(v, \nu, \theta, \alpha_{1}\right) \\
\frac{\mathrm{d}}{\mathrm{d} v} \mathrm{~V}_{\theta}\left(v, \nu, \theta, \alpha_{1}\right) & \frac{\mathrm{d}}{\mathrm{d} \nu} \mathrm{V}_{\theta}\left(v, \nu, \theta, \alpha_{1}\right) & \frac{\mathrm{d}}{\mathrm{d} \theta} \mathrm{V}_{\theta}\left(v, \nu, \theta, \alpha_{1}\right) & \frac{\mathrm{d}}{\mathrm{d} \alpha_{1}} \mathrm{~V}_{\theta}\left(v, \nu, \theta, \alpha_{1}\right) \\
\frac{\mathrm{d}}{\mathrm{d} v} \mathrm{~V}_{1 \alpha}\left(v, \nu, \theta, \alpha_{1}\right) & \frac{\mathrm{d}}{\mathrm{d} \nu} \mathrm{V}_{1 \alpha}\left(v, \nu, \theta, \alpha_{1}\right) & \frac{\mathrm{d}}{\mathrm{d} \theta} \mathrm{V}_{1 \alpha}\left(v, \nu, \theta, \alpha_{1}\right) & \frac{\mathrm{d}}{\mathrm{d} \alpha_{1}} \mathrm{~V}_{1 \alpha}\left(v, \nu, \theta, \alpha_{1}\right)
\end{array}\right)
\end{aligned}
$$

\section{Blade One Mass Matrix}

For clarity each term of Mone is explicitly stated

$$
\begin{aligned}
& \mathrm{M}_{\text {one }_{0,0}} \rightarrow \frac{\mathrm{HM}}{2}+\mathrm{Ma}+\mathrm{Mb} \\
& \mathrm{M}_{\text {one }_{0,2}} \rightarrow-\frac{\mathrm{Mb} \cdot\left(2 \cdot \mathrm{Hy}+\mathrm{Ey} \cdot \cos \left(\Psi_{\mathrm{r}}+\Psi_{1 \mathrm{~b}}\right)\right)}{2}-\frac{\mathrm{Ma} \cdot\left(2 \cdot \mathrm{Hy}+2 \cdot \mathrm{Ey} \cdot \cos \left(\Psi_{\mathrm{r}}+\Psi_{1 \mathrm{~b}}\right)\right)}{2} \\
& \mathrm{M}_{\text {one }_{0,3}} \rightarrow-\mathrm{Ma} \cdot \mathrm{a} \\
& \mathrm{M}_{\text {one }_{1,1}} \rightarrow \frac{\mathrm{HM}}{2}+\mathrm{Ma}+\mathrm{Mb} \\
& \mathrm{M}_{\text {one }_{1,2}} \rightarrow-\mathrm{Hx} \cdot \mathrm{Ma}-\mathrm{Hx} \cdot \mathrm{Mb} \\
& \mathrm{M}_{\text {one }_{2,2}} \rightarrow \frac{\mathrm{HI}}{2}+\mathrm{I}_{\mathrm{b}} \cdot \cos \left(\Psi_{\mathrm{r}}+\Psi_{1 \mathrm{~b}}\right)^{2}+\frac{\mathrm{Ma} \cdot\left[2 \cdot \mathrm{Hx}{ }^{2}+2 \cdot\left(\mathrm{Hy}+\mathrm{Ey} \cdot \cos \left(\Psi_{\mathrm{r}}+\Psi_{1 \mathrm{~b}}\right)\right)^{2}\right]}{2}+\frac{\mathrm{Mb} \cdot\left[2 \cdot \mathrm{Hx}{ }^{2}+2 \cdot\left(\mathrm{Hy}+\frac{\mathrm{Ey} \cdot \cos \left(\Psi_{\mathrm{r}}+\Psi_{1 \mathrm{~b}}\right)}{2}\right)^{2}\right]}{2} \\
& \mathrm{M}_{\text {one }_{2,3}} \rightarrow \mathrm{Ma} \cdot \mathrm{a} \cdot\left(\mathrm{Hy}+\mathrm{Ey} \cdot \cos \left(\Psi_{\mathrm{r}}+\Psi_{1 \mathrm{~b}}\right)\right) \\
& \mathrm{M}_{\text {one }_{3,3}} \rightarrow \mathrm{Ma} \cdot \mathrm{a}^{2}+\mathrm{I}_{\mathrm{a}} \cdot \cos \left(\Psi_{\mathrm{r}}+\Psi_{1 \mathrm{~b}}\right)^{4}+\mathrm{I}_{\mathrm{a}} \cdot \sin \left(2 \cdot \Psi_{\mathrm{r}}+2 \cdot \Psi_{1 \mathrm{~b}}\right) \cdot \cos \left(\Psi_{\mathrm{r}}+\Psi_{1 \mathrm{~b}}\right) \cdot \sin \left(\Psi_{\mathrm{r}}+\Psi_{1 \mathrm{~b}}\right)+\mathrm{I}_{\mathrm{a}} \cdot \sin \left(\Psi_{\mathrm{r}}+\Psi_{1 \mathrm{~b}}\right)^{4}
\end{aligned}
$$

\section{Blade One Stiffness Matrix}
$\mathrm{K}_{\text {one }} \rightarrow$
$\frac{\mathrm{Ey} \cdot \mathrm{Ma} \cdot \mathrm{R} \cdot \Omega^{2} \cdot \cos \left(\Psi_{\mathrm{r}}+\Psi_{1 \mathrm{~b}}\right) \cdot\left(\frac{\mathrm{Ey}}{\mathrm{R}}-1\right)}{2}$ $\mathrm{Ey} \cdot \mathrm{Ma} \cdot \mathrm{R} \cdot \Omega^{2} \cdot \cos \left(\Psi_{\mathrm{r}}+\Psi_{1 \mathrm{~b}}\right) \cdot\left(\frac{\mathrm{Ey}}{\mathrm{R}}-1\right)$

$$
\mathrm{K}+4 \cdot \mathrm{I}_{\mathrm{a}} \cdot \Omega^{2}-\frac{\mathrm{Ey} \cdot \mathrm{Ma} \cdot \mathrm{R} \cdot \Omega^{2} \cdot\left(\frac{\mathrm{Ey}}{\mathrm{R}}-1\right)}{2}
$$




\section{Blade Two Equation of Motion Derivation}

\section{Relate Motion of the center of gravity of member A to the system DOF velocities} Position

$\operatorname{Xag}\left(v, \theta, \alpha_{2}\right):=-\mathrm{a} \cdot \alpha_{2}+v-\left(\right.$ Ey $\cdot \cos \left(\Psi_{2 b}+\Psi_{\mathrm{r}}\right)+$ Hy $) \cdot \theta$

$\operatorname{Yag}(\nu, \theta):=\nu-\mathrm{Hx} \cdot \theta$

Velocity

$\operatorname{Xag}_{\mathrm{t}}\left(v_{\mathrm{t}}, \theta_{\mathrm{t}}, \alpha_{2 \mathrm{t}}\right):=-\mathrm{a} \cdot \alpha_{2 \mathrm{t}}+v_{\mathrm{t}}-\left(\mathrm{Ey} \cdot \cos \left(\Psi_{2 \mathrm{~b}}+\Psi_{\mathrm{r}}\right)+\mathrm{Hy}\right) \cdot \theta_{\mathrm{t}}$

$\operatorname{Yag}_{\mathrm{t}}\left(\nu_{\mathrm{t}}, \theta_{\mathrm{t}}\right):=\nu_{\mathrm{t}}-\mathrm{Hx} \cdot \theta_{\mathrm{t}}$

Relate Motion of the center of gravity of member B to the system DOF velocities

Position

$\operatorname{Xbg}(v, \theta):=v-\left(\frac{E y \cdot \cos \left(\Psi_{2 b}+\Psi_{\mathrm{r}}\right)}{2}+\mathrm{Hy}\right) \cdot \theta$

$\operatorname{Ybg}(\nu, \theta):=\nu-\operatorname{Hx} \cdot \theta$

Velocity

$\operatorname{Xbg}_{\mathrm{t}}\left(v_{\mathrm{t}}, \theta_{\mathrm{t}}\right):=v_{\mathrm{t}}-\left(\frac{\mathrm{Ey} \cdot \cos \left(\Psi_{2 \mathrm{~b}}+\Psi_{\mathrm{r}}\right)}{2}+\mathrm{Hy}\right) \cdot \theta_{\mathrm{t}}$

$\operatorname{Ybg}_{t}\left(\nu_{t}, \theta_{t}\right):=\nu_{t}-H x \cdot \theta_{t}$

Relate the rotation of $\alpha_{1}$ about local unit vector $K_{\text {hat }}$ to global unit vectors $j_{\text {hat }}$ and $k_{\text {hat }}$ (This relation is used to define the rotational velocity of member $A$.

$\mathrm{K}_{\text {hat }}\left(\mathrm{j}_{\text {hat }}, \mathrm{k}_{\text {hat }}\right):=\mathrm{j}_{\text {hat }} \cdot \sin \left(\Psi_{2 \mathrm{~b}}+\Psi_{\mathrm{r}}\right)+\mathrm{k}_{\text {hat }} \cdot \cos \left(\Psi_{2 \mathrm{~b}}+\Psi_{\mathrm{r}}\right)$

$\mathrm{K}_{\text {yhat }}:=\frac{\mathrm{d}}{\mathrm{dj}_{\text {hat }}} \mathrm{K}_{\text {hat }}\left(\mathrm{j}_{\text {hat }}, \mathrm{k}_{\text {hat }}\right) \quad \mathrm{K}_{\text {yhat }} \rightarrow \sin \left(\Psi_{\mathrm{r}}+\Psi_{2 \mathrm{~b}}\right)$

$\mathrm{K}_{\text {zhat }}:=\frac{\mathrm{d}}{\mathrm{dk}_{\text {hat }}} \mathrm{K}_{\text {hat }}\left(\mathrm{j}_{\text {hat }}, \mathrm{k}_{\text {hat }}\right) \quad \mathrm{K}_{\mathrm{zhat}} \rightarrow \cos \left(\Psi_{\mathrm{r}}+\Psi_{2 \mathrm{~b}}\right)$

Rotational Velocity of member A

$$
\begin{array}{ll}
\omega_{\text {ay }}\left(\alpha_{2 t}\right):=\alpha_{2 t} \cdot K_{\text {yhat }} & \omega_{\text {ay }}\left(\alpha_{2 t}\right) \rightarrow \alpha_{2 t} \cdot \sin \left(\Psi_{\mathrm{r}}+\Psi_{2 b}\right) \\
\omega_{\text {az }}\left(\alpha_{2 t}\right):=\alpha_{2 t} \cdot K_{\text {zhat }} & \omega_{\text {az }}\left(\alpha_{2 t}\right) \rightarrow \alpha_{2 t} \cdot \cos \left(\Psi_{r}+\Psi_{2 b}\right)
\end{array}
$$

\section{Rotational Velocity of member B}

$$
\begin{aligned}
& \stackrel{\omega_{\text {mbar }}}{ }\left(\theta_{t}\right):=\theta_{t} \\
& \omega_{\mathrm{bz}}\left(\theta_{\mathrm{t}}\right) \rightarrow \theta_{\mathrm{t}}
\end{aligned}
$$

\section{Moments and Products of Inertia of Rotor Member A}

$$
\begin{aligned}
& \mathrm{I}_{\mathrm{ayy}}:=\mathrm{I}_{\mathrm{a}} \cdot\left(\sin \left(\Psi_{2 \mathrm{~b}}+\Psi_{\mathrm{r}}\right)\right)^{2} \\
& \mathrm{I}_{\mathrm{azz}}:=\mathrm{I}_{\mathrm{a}} \cdot\left(\cos \left(\Psi_{2 \mathrm{~b}}+\Psi_{\mathrm{r}}\right)\right)^{2} \\
& \mathrm{I}_{\mathrm{ayz}}:=\frac{-\mathrm{I}_{\mathrm{a}}}{2} \cdot \sin \left[2 \cdot\left(\Psi_{2 \mathrm{~b}}+\Psi_{\mathrm{r}}\right)\right]
\end{aligned}
$$

\section{Moments and Products of Inertia of Rotor Member B}

$$
\begin{aligned}
& \mathrm{I}_{\text {byy }}:=\mathrm{I}_{\mathrm{b}} \cdot\left(\sin \left(\Psi_{2 \mathrm{~b}}+\Psi_{\mathrm{r}}\right)\right)^{2} \\
& \mathrm{I}_{\mathrm{bzz}}:=\mathrm{I}_{\mathrm{b}} \cdot\left(\cos \left(\Psi_{2 \mathrm{~b}}+\Psi_{\mathrm{r}}\right)\right)^{2} \\
& \mathrm{I}_{\mathrm{byz}}:=\frac{-\mathrm{I}_{\mathrm{b}}}{2} \cdot \sin \left[2 \cdot\left(\Psi_{2 \mathrm{~b}}+\Psi_{\mathrm{r}}\right)\right]
\end{aligned}
$$




\section{Potential Energy}

$\mathrm{V}\left(v, \nu, \theta, \alpha_{2}\right):=\frac{1}{2} \cdot \mathrm{K} \cdot\left(\alpha_{2}\right)^{2}+\frac{1}{2} \cdot \mathrm{K} \cdot\left(\theta \cdot \cos \left(\Psi_{2 \mathrm{~b}}+\Psi_{\mathrm{r}}\right)\right)^{2}-\mathrm{K} \cdot \alpha_{2} \cdot \theta \cdot \cos \left(\Psi_{2 \mathrm{~b}}+\Psi_{\mathrm{r}}\right)+\mathrm{Ma} \cdot \mathrm{g} \cdot \mathrm{Yag}(\nu, \theta)+\mathrm{Mb} \cdot \mathrm{g} \cdot \mathrm{Ybg}(\nu, \theta)+\frac{1}{2} \mathrm{HM} \cdot \mathrm{g} \cdot \nu+\frac{1}{4} \cdot \Omega^{2} \cdot \mathrm{Ma} \cdot \mathrm{R} \cdot \frac{\mathrm{Ey}}{\mathrm{R}} \cdot\left(1-\frac{\mathrm{Ey}}{\mathrm{R}}\right) \cdot\left(\alpha_{2}-\theta \cdot \cos \left(\Psi_{2 \mathrm{~b}}+\Psi_{\mathrm{r}}\right)\right)^{2}+\frac{4}{2} \cdot \Omega^{2} \cdot \mathrm{I}_{\mathrm{a}} \cdot\left(\alpha_{2}-\theta \cdot \cos \left(\Psi_{2 \mathrm{~b}}+\Psi_{\mathrm{r}}\right)\right)^{2}$

$\mathrm{V}\left(v, \nu, \theta, \alpha_{2}\right) \rightarrow \frac{\mathrm{K} \cdot \mathrm{\alpha}_{2}{ }^{2}}{2}+\frac{\mathrm{HM} \cdot \mathrm{g} \cdot \nu}{2}+\mathrm{Ma} \cdot \mathrm{g} \cdot(\nu-\mathrm{Hx} \cdot \theta)+\mathrm{Mb} \cdot \mathrm{g} \cdot(\nu-\mathrm{Hx} \cdot \theta)+2 \cdot \mathrm{I}_{\mathrm{a}} \cdot \Omega^{2} \cdot\left(\alpha_{2}-\theta \cdot \cos \left(\Psi_{\mathrm{r}}+\Psi_{2 b}\right)\right)^{2}+\frac{\mathrm{K} \cdot \theta^{2} \cdot \cos \left(\Psi_{\mathrm{r}}+\Psi_{2 \mathrm{~b}}\right)^{2}}{2}-\mathrm{K} \cdot \theta \cdot \alpha_{2} \cdot \cos \left(\Psi_{\mathrm{r}}+\Psi_{2 \mathrm{~b}}\right)-\frac{\mathrm{Ey} \cdot \mathrm{Ma} \cdot \mathrm{R} \cdot \Omega^{2} \cdot\left(\frac{\mathrm{Ey}}{\mathrm{R}}-1\right) \cdot\left(\alpha_{2}-\theta \cdot \cos \left(\Psi_{\mathrm{r}}+\Psi_{2 \mathrm{~b}}\right)\right)^{2}}{4}$

\section{Take the derivative of Potential Energy wrt System DOF cord.}

$\mathrm{V}_{v}\left(v, \nu, \theta, \alpha_{2}\right):=\frac{\mathrm{d}}{\mathrm{d} v} \mathrm{~V}\left(v, \nu, \theta, \alpha_{2}\right)$

$\mathrm{V}_{\nu}\left(\nu, \nu, \theta, \alpha_{2}\right):=\frac{\mathrm{d}}{\mathrm{d} \nu} \mathrm{v}\left(\nu, \nu, \theta, \alpha_{2}\right)$

$\mathrm{V}_{\theta}\left(v, \nu, \theta, \alpha_{2}\right):=\frac{\mathrm{d}}{\mathrm{d} \theta} \mathrm{V}\left(v, \nu, \theta, \alpha_{2}\right)$

$\mathrm{V}_{2 \alpha}\left(v, \nu, \theta, \alpha_{1}\right):=\frac{\mathrm{d}}{\mathrm{d} \alpha_{2}} \mathrm{~V}\left(v, \nu, \theta, \alpha_{2}\right)$

$\mathrm{V}_{v}\left(v, \nu, \theta, \alpha_{2}\right) \mid \begin{aligned} & \text { collect } \\ & v, \nu, \theta, \alpha_{2} \rightarrow 0\end{aligned}$

$\mathrm{V}_{\nu}\left(v, \nu, \theta, \alpha_{2}\right) \mid \begin{aligned} & \text { collect } \\ & v, \nu, \theta, \alpha_{2}\end{aligned} \rightarrow \frac{\mathrm{HM} \cdot \mathrm{g}}{2}+\mathrm{Ma} \cdot \mathrm{g}+\mathrm{Mb} \cdot \mathrm{g}$

$\mathrm{V}_{\theta}\left(v, \nu, \theta, \alpha_{2}\right) \mid \begin{aligned} & \operatorname{collect} \\ & v, \nu, \theta, \alpha_{2}\end{aligned} \rightarrow\left[\mathrm{K} \cdot \cos \left(\Psi_{\mathrm{r}}+\Psi_{2 \mathrm{~b}}\right)^{2}+4 \cdot \mathrm{I}_{\mathrm{a}} \cdot \Omega^{2} \cdot \cos \left(\Psi_{\mathrm{r}}+\Psi_{2 \mathrm{~b}}\right)^{2}-\frac{\left.\text { Ey } \cdot \mathrm{Ma} \cdot \mathrm{R} \cdot \Omega^{2} \cdot \cos \left(\Psi_{\mathrm{r}}+\Psi_{2 \mathrm{~b}}\right)^{2} \cdot\left(\frac{\mathrm{Ey}}{\mathrm{R}}-1\right)\right]}{2}\right] \cdot \theta+\left[\frac{\mathrm{Ey} \cdot \mathrm{Ma} \cdot \mathrm{R} \cdot \Omega^{2} \cdot \cos \left(\Psi_{\mathrm{r}}+\Psi_{2 \mathrm{~b}}\right) \cdot\left(\frac{\mathrm{Ey}}{\mathrm{R}}-1\right)}{2}-4 \cdot \mathrm{I}_{\mathrm{a}} \cdot \Omega^{2} \cdot \cos \left(\Psi_{\mathrm{r}}+\Psi_{2 \mathrm{~b}}\right)-\mathrm{K} \cdot \cos \left(\Psi_{\mathrm{r}}+\Psi_{2 \mathrm{~b}}\right) \cdot \alpha_{2}-(\mathrm{Hx} \cdot \mathrm{Ma} \cdot \mathrm{g}+\mathrm{Hx} \cdot \mathrm{Mb} \cdot \mathrm{g})\right.$

$\mathrm{V}_{2 \alpha}\left(v, \nu, \theta, \alpha_{2}\right) \mid \begin{aligned} & \operatorname{collect} \\ & v, \nu, \theta, \alpha_{2}\end{aligned} \rightarrow\left[\frac{\text { Ey } \cdot \mathrm{Ma} \cdot \mathrm{R} \cdot \Omega^{2} \cdot \cos \left(\Psi_{\mathrm{r}}+\Psi_{2 \mathrm{~b}}\right) \cdot\left(\frac{\mathrm{Ey}}{\mathrm{R}}-1\right)}{2}-4 \cdot \mathrm{I}_{\mathrm{a}} \cdot \Omega^{2} \cdot \cos \left(\Psi_{\mathrm{r}}+\Psi_{2 \mathrm{~b}}\right)-\mathrm{K} \cdot \cos \left(\Psi_{\mathrm{r}}+\Psi_{2 \mathrm{~b}}\right)\right] \cdot \theta+\left[\mathrm{K}+4 \cdot \mathrm{I}_{\mathrm{a}} \cdot \Omega^{2}-\frac{\text { Ey } \cdot \mathrm{Ma} \cdot \mathrm{R} \cdot \Omega^{2} \cdot\left(\frac{\mathrm{Ey}}{\mathrm{R}}-1\right)}{2}\right] \cdot \alpha_{2}$ 


\section{Define Blade Two Mass and Stiffness Matrices}

$$
\begin{aligned}
& \left(\frac{\mathrm{d}}{\mathrm{d} v_{\mathrm{tt}}} \mathrm{T}_{v \mathrm{t}}\left(v_{\mathrm{tt}}, \nu_{\mathrm{tt}}, \theta_{\mathrm{tt}}, \alpha_{2 \mathrm{tt}}\right) \quad \frac{\mathrm{d}}{\mathrm{d} \nu_{\mathrm{tt}}} \mathrm{T}_{v \mathrm{t}}\left(v_{\mathrm{tt}}, \nu_{\mathrm{tt}}, \theta_{\mathrm{tt}}, \alpha_{2 \mathrm{tt}}\right) \quad \frac{\mathrm{d}}{\mathrm{d} \theta_{\mathrm{tt}}} \mathrm{T}_{v \mathrm{t}}\left(v_{\mathrm{tt}}, \nu_{\mathrm{tt}}, \theta_{\mathrm{tt}}, \alpha_{2 \mathrm{tt}}\right) \quad \frac{\mathrm{d}}{\mathrm{d} \alpha_{2 \mathrm{tt}}} \mathrm{T}_{v \mathrm{t}}\left(v_{\mathrm{tt}}, \nu_{\mathrm{tt}}, \theta_{\mathrm{tt}}, \alpha_{2 \mathrm{tt}}\right)\right. \\
& \frac{\mathrm{d}}{\mathrm{d} v_{\mathrm{tt}}} \mathrm{T}_{\nu \mathrm{t}}\left(v_{\mathrm{tt}}, \nu_{\mathrm{tt}}, \theta_{\mathrm{tt}}, \alpha_{2 \mathrm{tt}}\right) \quad \frac{\mathrm{d}}{\mathrm{d} \nu_{\mathrm{tt}}} \mathrm{T}_{\nu \mathrm{t}}\left(v_{\mathrm{tt}}, \nu_{\mathrm{tt}}, \theta_{\mathrm{tt}}, \alpha_{2 \mathrm{tt}}\right) \quad \frac{\mathrm{d}}{\mathrm{d} \theta_{\mathrm{tt}}} \mathrm{T}_{\nu \mathrm{t}}\left(v_{\mathrm{tt}}, \nu_{\mathrm{tt}}, \theta_{\mathrm{tt}}, \alpha_{2 \mathrm{tt}}\right) \quad \frac{\mathrm{d}}{\mathrm{d} \alpha_{2 \mathrm{tt}}} \mathrm{T}_{\nu \mathrm{t}}\left(v_{\mathrm{tt}}, \nu_{\mathrm{tt}}, \theta_{\mathrm{tt}}, \alpha_{2 \mathrm{tt}}\right) \\
& \frac{\mathrm{d}}{\mathrm{d} v_{\mathrm{tt}}} \mathrm{T}_{\theta \mathrm{t}}\left(v_{\mathrm{tt}}, \nu_{\mathrm{tt}}, \theta_{\mathrm{tt}}, \alpha_{2 \mathrm{tt}}\right) \quad \frac{\mathrm{d}}{\mathrm{d} \nu_{\mathrm{tt}}} \mathrm{T}_{\theta \mathrm{t}}\left(v_{\mathrm{tt}}, \nu_{\mathrm{tt}}, \theta_{\mathrm{tt}}, \alpha_{2 \mathrm{tt}}\right) \quad \frac{\mathrm{d}}{\mathrm{d} \theta_{\mathrm{tt}}} \mathrm{T}_{\theta \mathrm{t}}\left(v_{\mathrm{tt}}, v_{\mathrm{tt}}, \theta_{\mathrm{tt}}, \alpha_{2 \mathrm{tt}}\right) \quad \frac{\mathrm{d}}{\mathrm{d} \alpha_{2 \mathrm{tt}}} \mathrm{T}_{\theta \mathrm{t}}\left(v_{\mathrm{tt}}, v_{\mathrm{tt}}, \theta_{\mathrm{tt}}, \alpha_{2 \mathrm{tt}}\right) \\
& \frac{\mathrm{d}}{\mathrm{d} v_{\mathrm{tt}}} \mathrm{T}_{2 \alpha \mathrm{t}}\left(v_{\mathrm{tt}}, \nu_{\mathrm{tt}}, \theta_{\mathrm{tt}}, \alpha_{2 \mathrm{tt}}\right) \frac{\mathrm{d}}{\mathrm{d} \nu_{\mathrm{tt}}} \mathrm{T}_{2 \alpha \mathrm{t}}\left(v_{\mathrm{tt}}, \nu_{\mathrm{tt}}, \theta_{\mathrm{tt}}, \alpha_{2 \mathrm{tt}}\right) \frac{\mathrm{d}}{\mathrm{d} \theta_{\mathrm{tt}}} \mathrm{T}_{2 \alpha \mathrm{t}}\left(v_{\mathrm{tt}}, \nu_{\mathrm{tt}}, \theta_{\mathrm{tt}}, \alpha_{2 \mathrm{tt}}\right) \frac{\mathrm{d}}{\mathrm{d} \alpha_{2 t \mathrm{tt}}} \mathrm{T}_{2 \alpha \mathrm{t}}\left(v_{\mathrm{tt}}, \nu_{\mathrm{tt}}, \theta_{\mathrm{tt}}, \alpha_{2 \mathrm{tt}}\right) \\
& \mathrm{K}_{\mathrm{two}}:=\left(\begin{array}{llll}
\frac{\mathrm{d}}{\mathrm{d} v} \mathrm{~V}_{v}\left(v, \nu, \theta, \alpha_{2}\right) & \frac{\mathrm{d}}{\mathrm{d} \nu} \mathrm{V}_{v}\left(v, \nu, \theta, \alpha_{2}\right) & \frac{\mathrm{d}}{\mathrm{d} \theta} \mathrm{V}_{v}\left(v, \nu, \theta, \alpha_{2}\right) & \frac{\mathrm{d}}{\mathrm{d} \alpha_{2}} \mathrm{~V}_{v}\left(v, \nu, \theta, \alpha_{2}\right) \\
\frac{\mathrm{d}}{\mathrm{d} v} \mathrm{~V}_{\nu}\left(v, \nu, \theta, \alpha_{2}\right) & \frac{\mathrm{d}}{\mathrm{d} \nu} \mathrm{V}_{\nu}\left(v, \nu, \theta, \alpha_{2}\right) & \frac{\mathrm{d}}{\mathrm{d} \theta} \mathrm{V}_{\nu}\left(v, \nu, \theta, \alpha_{2}\right) & \frac{\mathrm{d}}{\mathrm{d} \alpha_{2}} \mathrm{~V}_{\nu}\left(v, \nu, \theta, \alpha_{2}\right) \\
\frac{\mathrm{d}}{\mathrm{d} v} \mathrm{~V}_{\theta}\left(v, \nu, \theta, \alpha_{2}\right) & \frac{\mathrm{d}}{\mathrm{d} \nu} \mathrm{V}_{\theta}\left(v, \nu, \theta, \alpha_{2}\right) & \frac{\mathrm{d}}{\mathrm{d} \theta} \mathrm{V}_{\theta}\left(v, \nu, \theta, \alpha_{2}\right) & \frac{\mathrm{d}}{\mathrm{d} \alpha_{2}} \mathrm{~V}_{\theta}\left(v, \nu, \theta, \alpha_{2}\right) \\
\frac{\mathrm{d}}{\mathrm{d} v} \mathrm{~V}_{2 \alpha}\left(v, \nu, \theta, \alpha_{2}\right) & \frac{\mathrm{d}}{\mathrm{d} \nu} \mathrm{V}_{2 \alpha}\left(v, \nu, \theta, \alpha_{2}\right) & \frac{\mathrm{d}}{\mathrm{d} \theta} \mathrm{V}_{2 \alpha}\left(v, \nu, \theta, \alpha_{2}\right) & \frac{\mathrm{d}}{\mathrm{d} \alpha_{2}} \mathrm{~V}_{2 \alpha}\left(v, \nu, \theta, \alpha_{2}\right)
\end{array}\right)
\end{aligned}
$$

\section{Blade Two Mass Matrix}

For clarity each term of Mone is explicitly stated

$$
\begin{aligned}
& \mathrm{M}_{\text {two }_{0,0}} \rightarrow \frac{\mathrm{HM}}{2}+\mathrm{Ma}+\mathrm{Mb} \\
& \mathrm{M}_{\mathrm{two}_{0,2}} \rightarrow-\frac{\mathrm{Mb} \cdot\left(2 \cdot \mathrm{Hy}+\mathrm{Ey} \cdot \cos \left(\Psi_{\mathrm{r}}+\Psi_{2 \mathrm{~b}}\right)\right)}{2}-\frac{\mathrm{Ma} \cdot\left(2 \cdot \mathrm{Hy}+2 \cdot \mathrm{Ey} \cdot \cos \left(\Psi_{\mathrm{r}}+\Psi_{2 \mathrm{~b}}\right)\right)}{2} \\
& \mathrm{M}_{\text {two }_{0,3}} \rightarrow-\text { Ma·a } \\
& \mathrm{M}_{\mathrm{two}_{1,1}} \rightarrow \frac{\mathrm{HM}}{2}+\mathrm{Ma}+\mathrm{Mb} \\
& \mathrm{M}_{\mathrm{two}_{1,2}} \rightarrow-\mathrm{Hx} \cdot \mathrm{Ma}-\mathrm{Hx} \cdot \mathrm{Mb} \\
& \mathrm{M}_{\mathrm{two}_{2,2}} \rightarrow \frac{\mathrm{HI}}{2}+\mathrm{I}_{\mathrm{b}} \cdot \cos \left(\Psi_{\mathrm{r}}+\Psi_{2 \mathrm{~b}}\right)^{2}+\frac{\mathrm{Ma} \cdot\left[2 \cdot \mathrm{Hx}^{2}+2 \cdot\left(\mathrm{Hy}+\mathrm{Ey} \cdot \cos \left(\Psi_{\mathrm{r}}+\Psi_{2 \mathrm{~b}}\right)\right)^{2}\right]}{2}+\frac{\mathrm{Mb} \cdot\left[2 \cdot \mathrm{Hx}^{2}+2 \cdot\left(\mathrm{Hy}+\frac{\left.\mathrm{Ey} \cdot \cos \left(\Psi_{\mathrm{r}}+\Psi_{2 \mathrm{~b}}\right)\right)^{2}}{2}\right]\right.}{2} \\
& \mathrm{M}_{\mathrm{two}_{2,3}} \rightarrow \mathrm{Ma} \cdot \mathrm{a} \cdot\left(\mathrm{Hy}+\mathrm{Ey} \cdot \cos \left(\Psi_{\mathrm{r}}+\Psi_{2 \mathrm{~b}}\right)\right) \\
& \mathrm{M}_{\mathrm{two}_{3,3}} \rightarrow \mathrm{Ma} \cdot \mathrm{a}^{2}+\mathrm{I}_{\mathrm{a}} \cdot \cos \left(\Psi_{\mathrm{r}}+\Psi_{2 \mathrm{~b}}\right)^{4}+\mathrm{I}_{\mathrm{a}} \cdot \sin \left(2 \cdot \Psi_{\mathrm{r}}+2 \cdot \Psi_{2 \mathrm{~b}}\right) \cdot \cos \left(\Psi_{\mathrm{r}}+\Psi_{2 \mathrm{~b}}\right) \cdot \sin \left(\Psi_{\mathrm{r}}+\Psi_{2 \mathrm{~b}}\right)+\mathrm{I}_{\mathrm{a}} \cdot \sin \left(\Psi_{\mathrm{r}}+\Psi_{2 \mathrm{~b}}\right)^{4}
\end{aligned}
$$

\section{Blade Two Stiffness Matrix}

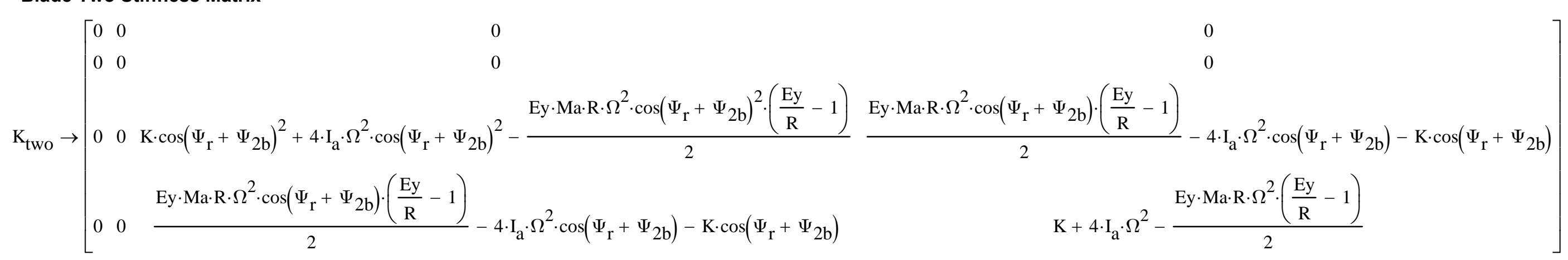




\section{Total Rotor System Equations of Motion Mass and Stiffness Matrices}

The following mass and stiffness matrices were developed my summing the one and lower stiffness matrices over the appropriate degrees of freedom. The system matrices feature the following degrees of freedom and in the stated order: $u, v, \theta, \alpha_{1}, \alpha_{2}$

$\mathrm{M}_{\text {total }}:=\left(\begin{array}{ccccc}\mathrm{M}_{\text {one }_{0,0}}+\mathrm{M}_{\mathrm{two}_{0,0}} & \mathrm{M}_{\mathrm{one}_{0,1}}+\mathrm{M}_{\mathrm{two}_{0,1}} & \mathrm{M}_{\mathrm{one}_{0,2}}+\mathrm{M}_{\mathrm{two}_{0,2}} & \mathrm{M}_{\mathrm{one}_{0,3}} & \mathrm{M}_{\mathrm{two}_{0,3}} \\ \mathrm{M}_{\mathrm{one}_{1,0}}+\mathrm{M}_{\mathrm{two}_{1,0}} & \mathrm{M}_{\mathrm{one}_{1,1}}+\mathrm{M}_{\mathrm{two}_{1,1}} & \mathrm{M}_{\mathrm{one}_{1,2}}+\mathrm{M}_{\mathrm{two}_{1,2}} & \mathrm{M}_{\mathrm{one}_{1,3}} & \mathrm{M}_{\mathrm{two}_{1,3}} \\ \mathrm{M}_{\text {one }_{2,0}}+\mathrm{M}_{\mathrm{two}_{2,0}} & \mathrm{M}_{\mathrm{one}_{2,1}}+\mathrm{M}_{\mathrm{two}_{2,1}} & \mathrm{M}_{\mathrm{one}_{2,2}}+\mathrm{M}_{\mathrm{two}_{2,2}} & \mathrm{M}_{\mathrm{one}_{2,3}} & \mathrm{M}_{\mathrm{two}_{2,3}} \\ \mathrm{M}_{\mathrm{one}_{3,0}} & \mathrm{M}_{\mathrm{one}_{3,1}} & \mathrm{M}_{\mathrm{one}_{3,2}} & \mathrm{M}_{\mathrm{one}_{3,3}} & 0 \\ \mathrm{M}_{\mathrm{two}_{3,0}} & \mathrm{M}_{\mathrm{two}_{3,1}} & \mathrm{M}_{\mathrm{two}_{3,2}} & 0 & \mathrm{M}_{\mathrm{two}_{3,3}}\end{array}\right)$

$$
\mathrm{K}_{\text {total }}:=\left(\begin{array}{ccccc}
\mathrm{K}_{\text {one }_{0,0}}+\mathrm{K}_{\mathrm{two}_{0,0}} & \mathrm{~K}_{\mathrm{one}_{0,1}}+\mathrm{K}_{\mathrm{two}_{0,1}} & \mathrm{~K}_{\mathrm{one}_{0,2}}+\mathrm{K}_{\mathrm{two}_{0,2}} & \mathrm{~K}_{\mathrm{one}_{0,3}} & \mathrm{~K}_{\mathrm{two}_{0,3}} \\
\mathrm{~K}_{\mathrm{one}_{1,0}}+\mathrm{K}_{\mathrm{two}_{1,0}} & \mathrm{~K}_{\mathrm{one}_{1,1}}+\mathrm{K}_{\mathrm{two}_{1,1}} & \mathrm{~K}_{\mathrm{one}_{1,2}}+\mathrm{K}_{\mathrm{two}_{1,2}} & \mathrm{~K}_{\mathrm{one}_{1,3}} & \mathrm{~K}_{\mathrm{two}_{1,3}} \\
\mathrm{~K}_{\mathrm{one}_{2,0}+\mathrm{K}_{\mathrm{two}_{2,0}}} \mathrm{~K}_{\mathrm{one}_{2,1}+\mathrm{K}_{\mathrm{two}_{2,1}}} & \mathrm{~K}_{\mathrm{one}_{2,2}}+\mathrm{K}_{\mathrm{two}_{2,2}} & \mathrm{~K}_{\mathrm{one}_{2,3}} & \mathrm{~K}_{\mathrm{two}_{2,3}} \\
\mathrm{~K}_{\mathrm{one}_{3,0}} & \mathrm{~K}_{\mathrm{one}_{3,1}} & \mathrm{~K}_{\mathrm{one}_{3,2}} & \mathrm{~K}_{\mathrm{one}_{3,3}} & 0 \\
\mathrm{~K}_{\mathrm{two}_{3,0}} & \mathrm{~K}_{\mathrm{two}_{3,1}} & \mathrm{~K}_{\mathrm{two}_{3,2}} & 0 & \mathrm{~K}_{\mathrm{two}_{3,3}}
\end{array}\right)
$$


$\mathrm{M}_{\text {total }_{0,0}} \rightarrow \mathrm{HM}+2 \cdot \mathrm{Ma}+2 \cdot \mathrm{Mb}$

$\mathrm{M}_{\text {total }_{0,1}} \rightarrow 0$

$\mathrm{M}_{\mathrm{total}_{0,2}} \rightarrow-\frac{\mathrm{Mb} \cdot\left(2 \cdot \mathrm{Hy}+\mathrm{Ey} \cdot \cos \left(\Psi_{\mathrm{r}}+\Psi_{1 \mathrm{~b}}\right)\right)}{2}-\frac{\mathrm{Ma} \cdot\left(2 \cdot \mathrm{Hy}+2 \cdot \operatorname{Ey} \cdot \cos \left(\Psi_{\mathrm{r}}+\Psi_{1 \mathrm{~b}}\right)\right)}{2}-\frac{\mathrm{Mb} \cdot\left(2 \cdot \mathrm{Hy}+\mathrm{Ey} \cdot \cos \left(\Psi_{\mathrm{r}}+\Psi_{2 \mathrm{~b}}\right)\right)}{2}-\frac{\mathrm{Ma} \cdot\left(2 \cdot \mathrm{Hy}+2 \cdot \mathrm{Ey} \cdot \cos \left(\Psi_{\mathrm{r}}+\Psi_{2 \mathrm{~b}}\right)\right)}{2}$

$\mathrm{M}_{\text {total }_{0,3} \rightarrow-\text { Ma a }}$

$\mathrm{M}_{\text {total }_{0,4} \rightarrow-\text { Ma.a }}$

$\mathrm{M}_{\text {total }_{1,1}} \rightarrow \mathrm{HM}+2 \cdot \mathrm{Ma}+2 \cdot \mathrm{Mb}$

$\mathrm{M}_{\mathrm{total}_{1,2}} \rightarrow-2 \cdot \mathrm{Hx} \cdot \mathrm{Ma}-2 \cdot \mathrm{Hx} \cdot \mathrm{Mb}$

$\mathrm{M}_{\text {total }_{1,3} \rightarrow 0}$

$\mathrm{M}_{\text {total }_{1,4} \rightarrow 0}$

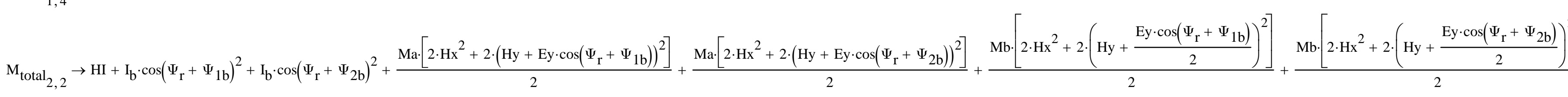

$\mathrm{M}_{\text {total }_{2,3}} \rightarrow$ Ma-a. $\left(\mathrm{Hy}+\mathrm{Ey} \cdot \cos \left(\Psi_{\mathrm{r}}+\Psi_{1 \mathrm{~b}}\right)\right)$

$\mathrm{M}_{\text {total }_{2,4}} \rightarrow$ Ma-a. $\left(\mathrm{Hy}+\mathrm{Ey} \cdot \cos \left(\Psi_{\mathrm{r}}+\Psi_{2 \mathrm{~b}}\right)\right)$

$\mathrm{M}_{\text {total }_{3,3}} \rightarrow \mathrm{Ma} \cdot \mathrm{a}^{2}+\mathrm{I}_{\mathrm{a}} \cdot \cos \left(\Psi_{\mathrm{r}}+\Psi_{1 \mathrm{~b}}\right)^{4}+\mathrm{I}_{\mathrm{a}} \cdot \sin \left(2 \cdot \Psi_{\mathrm{r}}+2 \cdot \Psi_{1 \mathrm{~b}}\right) \cdot \cos \left(\Psi_{\mathrm{r}}+\Psi_{1 \mathrm{~b}}\right) \cdot \sin \left(\Psi_{\mathrm{r}}+\Psi_{1 \mathrm{~b}}\right)+\mathrm{I}_{\mathrm{a}} \cdot \sin \left(\Psi_{\mathrm{r}}+\Psi_{1 \mathrm{~b}}\right)^{4}$

$\mathrm{M}_{\text {total }_{3,4} \rightarrow 0}$

$\mathrm{M}_{\text {total }_{4,4}} \rightarrow$ Ma-a ${ }^{2}+\mathrm{I}_{\mathrm{a}} \cdot \cos \left(\Psi_{\mathrm{r}}+\Psi_{2 \mathrm{~b}}\right)^{4}+\mathrm{I}_{\mathrm{a}} \cdot \sin \left(2 \cdot \Psi_{\mathrm{r}}+2 \cdot \Psi_{2 \mathrm{~b}}\right) \cdot \cos \left(\Psi_{\mathrm{r}}+\Psi_{2 \mathrm{~b}}\right) \cdot \sin \left(\Psi_{\mathrm{r}}+\Psi_{2 \mathrm{~b}}\right)+\mathrm{I}_{\mathrm{a}} \cdot \sin \left(\Psi_{\mathrm{r}}+\Psi_{2 \mathrm{~b}}\right)^{4}$

Total Stiffness Matrix

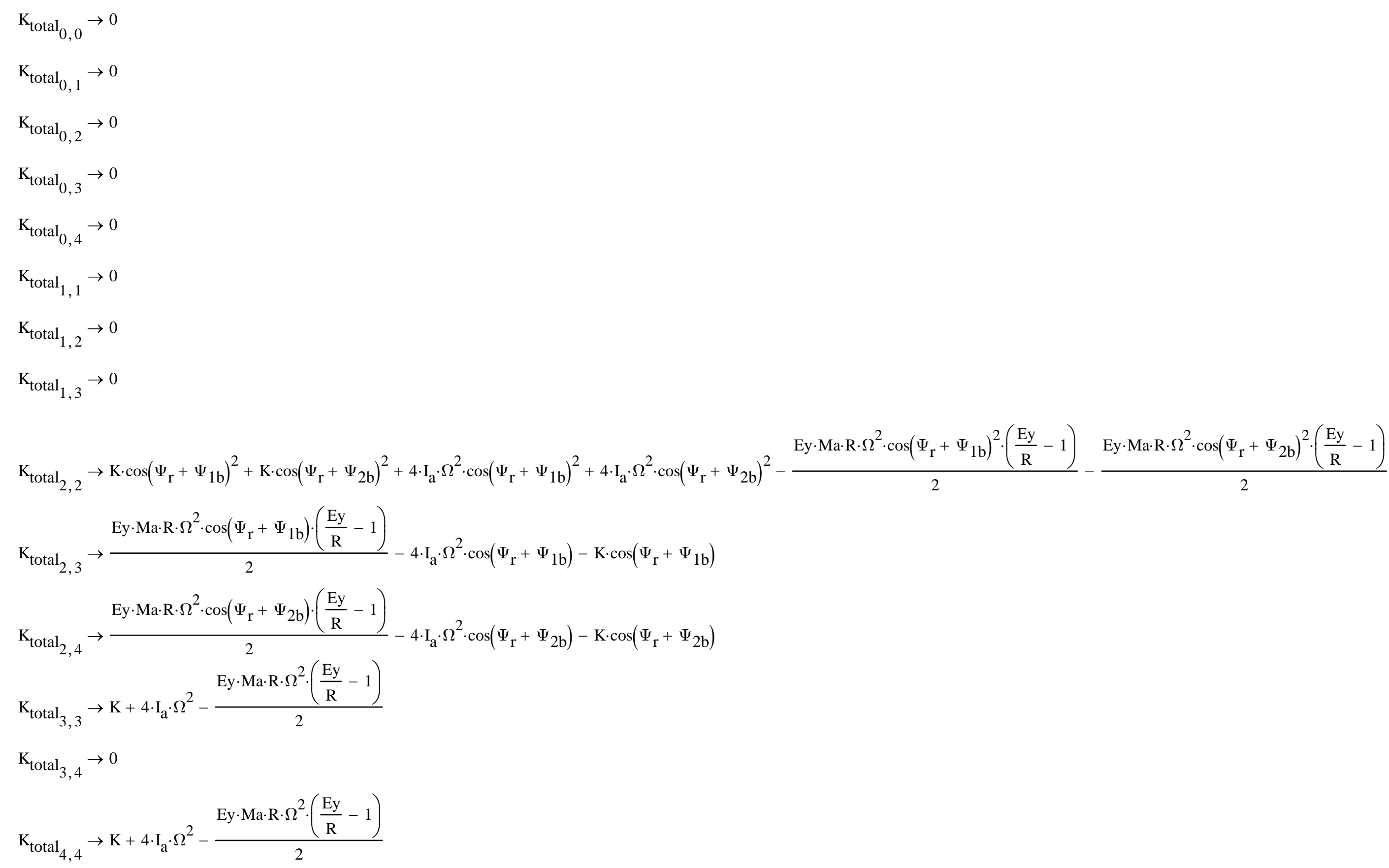




\section{Appendix C}

\section{Results Summary Charts}




\section{Appendix C - Contents}

Transient Small Wind Turbine Tower Structural Analysis with Coupled Rotor Dynamic Interaction

- Appendix C - Axis Definitions

- Appendix C1 - Strong Axis Results

- Static

- Load Case A - $2100 \mathrm{lbf}$ Tip Load

- Load Case B - 50 Year Extreme Wind Model (EWM50, Parked)

- Load Case C - 1 Year Extreme Wind Model (EWM1)

- Load Case D - 15 MPH "Nominal Operation"

- Dynamic

- Modal Evaluation

- Load Case E - Extreme Operating Gust (EOG)

- Load Case F - Extreme Coherent Gust (ECG)

- Load Case G - Normal Turbulence Model (NTM)

- Load Case H-Swept Sine

- Appendix C2 - Weak Axis Results

- Static

- Load Case A - 2100 lbf Tip Load

- $\quad$ Load Case B - 50 Year Extreme Wind Model (EWM50)

- $\quad$ Load Case C - 1 Year Extreme Wind Model (EWM1)

- Load Case D - 15 MPH "Nominal Operation"

- Dynamic

- Modal Evaluation

- Load Case E - Extreme Operating Gust (EOG)

- Load Case F - Extreme Coherent Gust (ECG)

- Load Case G - Normal Turbulence Model (NTM)

- Load Case H - Swept Sine

- Appendix C3 - Modal Truncation Convergence Study

- Appendix C4 - Cyclic Excitation Due to Vortex Shedding

- Appendix C5 - Example Guy-Wire Optimization

- Appendix C6 - Strut Critical Buckling Load

- Appendix C7 - Fatique

- Appendix C8 - Myklestad Method

- Appendix C9 - Tower Mesh Convergence Study

- Appendix C10 - Isotropic Nature of Guy-Wire Support Stiffness 
Transient Small Wind Turbine Tower Structural Analysis with Coupled Rotor Dynamic Interaction

\begin{tabular}{|c|c|c|c|c|c|c|c|}
\hline \multirow[b]{2}{*}{ Load Case } & \multirow[b]{2}{*}{$\begin{array}{c}\text { Max Tower } \\
\text { Deflection [in] }\end{array}$} & \multirow{2}{*}{$\begin{array}{c}\text { Max Tower } \\
\text { von Mises } \\
\text { Stress [Ksi] }\end{array}$} & \multirow{2}{*}{$\begin{array}{c}\text { Strut Max } \\
\text { Axial Load } \\
\text { [kips] }\end{array}$} & \multicolumn{3}{|c|}{ Margin of Safety } & \multirow[b]{2}{*}{$\begin{array}{c}\text { Fatigue } \\
\text { CDI }\end{array}$} \\
\hline & & & & Ultimate & Yield & $\begin{array}{c}\text { Strut } \\
\text { Buckling }\end{array}$ & \\
\hline Overall Max & 69.45 & 35.14 & 12.62 & 0.25 & 0.42 & 0.44 & 0.00 \\
\hline Strong Axis Results & 41.77 & 35.14 & 12.62 & 0.25 & 0.42 & 0.44 & 0.00 \\
\hline Static & 41.77 & 35.14 & 12.62 & 0.25 & 0.42 & 0.44 & - \\
\hline Load Case A - $2100 \mathrm{lbf}$ Tip Load & 41.77 & 35.14 & 12.62 & 0.25 & 0.42 & 0.44 & - \\
\hline Load Case B - 50 Year Extreme Wind Model (EWM50, Parked) & 23.33 & 23.04 & 9.59 & 0.91 & 1.17 & 0.90 & - \\
\hline Load Case C-1 Year Extreme Wind Model (EWM1) & 27.78 & 25.3 & 9.82 & 0.74 & 0.98 & 0.85 & - \\
\hline Load Case D - 15 MPH Nominal Operation & 0.8 & 0.88 & 0.29 & 48.9 & 55.8 & 62.2 & - \\
\hline Dynamic & 28.57 & 26.01 & 10.10 & 0.69 & 0.92 & 0.80 & 0.00 \\
\hline Load Case E-Extreme Operating Gust (EOG) & 28.57 & 26.01 & 10.10 & 0.69 & 0.92 & 0.80 & - \\
\hline Load Case F-Extreme Coherent Gust (ECG) & 11.7 & 10.77 & 4.17 & 3.08 & 3.64 & 3.37 & - \\
\hline Load Case G-Normal Turbulence Model (NTM) & 9.998 & 9.202 & 3.54 & 3.78 & 4.43 & 4.15 & 0.00 \\
\hline Weak Axis Results & 69.45 & 35.14 & - & 0.25 & 0.42 & - & 0.00 \\
\hline Static & 69.45 & 35.14 & - & 0.25 & 0.42 & - & - \\
\hline Load Case A - $2100 \mathrm{lbf}$ Tip Load & 69.45 & 35.14 & - & 0.25 & 0.42 & - & - \\
\hline Load Case B - 50 Year Extreme Wind Model (EWM50, Parked) & 44.53 & 27.86 & - & 0.58 & 0.79 & - & - \\
\hline Load Case C-1 Year Extreme Wind Model (EWM1) & 49.42 & 27.49 & - & 0.60 & 0.82 & - & - \\
\hline Load Case D- 15 MPH Nominal Operation & 1.43 & 0.98 & - & 43.7 & 49.8 & - & - \\
\hline Dynamic & 51.81 & 28.8 & - & 0.53 & 0.74 & - & 0.00 \\
\hline Load Case E-Extreme Operating Gust (EOG) & 51.81 & 28.8 & - & 0.53 & 0.74 & - & - \\
\hline Load Case F-Extreme Coherent Gust (ECG) & 20.93 & 11.82 & - & 2.72 & 3.23 & - & - \\
\hline Load Case G-Normal Turbulence Model (NTM) & 17.85 & 10.05 & - & 3.38 & 3.98 & - & 0.00 \\
\hline
\end{tabular}

\section{Positive Margins and Fatigue Life for all Load Cases}




\section{Coordinate Definitions}

- XYZ-Coordinate System

- Fixed to the rotor

- X-Axis: Aligned with rotor spine axis and positive downwind

- Y-Axis: Aligned with tower centerline and positive upward

- Z-Axis: $X \times Y$ (i.e., right hand rule)

- $\mathrm{X}_{\mathrm{T}} \mathrm{Y}_{\mathrm{T}} \mathrm{Z}_{\mathrm{T}}$-Coordinate System

- Fixed to the tower

- X-Axis: Parallel to the Gin-Pole

- Y-Axis: Aligned with the Y-Axis

- Z-Axis: $X_{T} \times Y_{T}$ (i.e., right hand rule)

- The angle between the $X$-Axis and $X_{T}$-Axis is $\xi$.

- "Strong-Axis" Load

- Wind is oriented in the $X_{T}$-Axis

- $\xi=0$ deg (configuration shown)

- "Weak-Axis" Load

- Wind is oriented in the $Z_{\mathrm{T}}$-Axis

- $\xi= \pm 90 \mathrm{deg}$

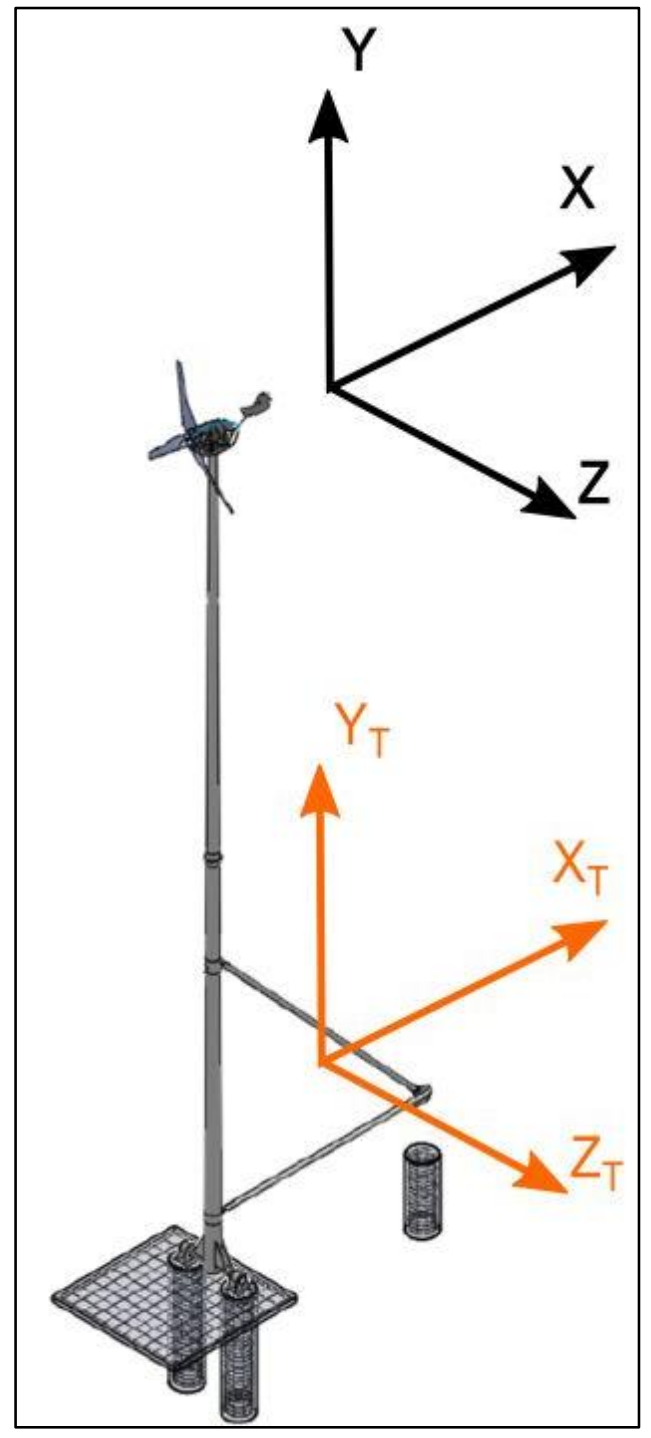




\section{Strong Axis Load Case A - Static 2100 Ibf Tip Load}




\section{Load Case A: Overview}

- $2100 \mathrm{lbf}$ load applied to tower tip

- Simulates a steady-state $60 \mathrm{mph}$ wind load

- Rotor Thrust Coefficient: $\mathrm{C}_{\mathrm{t}}=\mathbf{2 . 0}$

- Tower Drag Coefficient: $\mathrm{C}_{\mathrm{d}}=\mathbf{0 . 0}$

- Load applied in "strong axis" (the load is parallel to the plane formed by the tower, gin-pole, and strut).

- Details of the analysis parameters are summarized and defined in the attached UserInputs.xIsx file. 


\section{Load Case A: Tower Deflection}

Transverse Tower Deflection

Max Disp: 41.77 in, at hight: 844.5 in

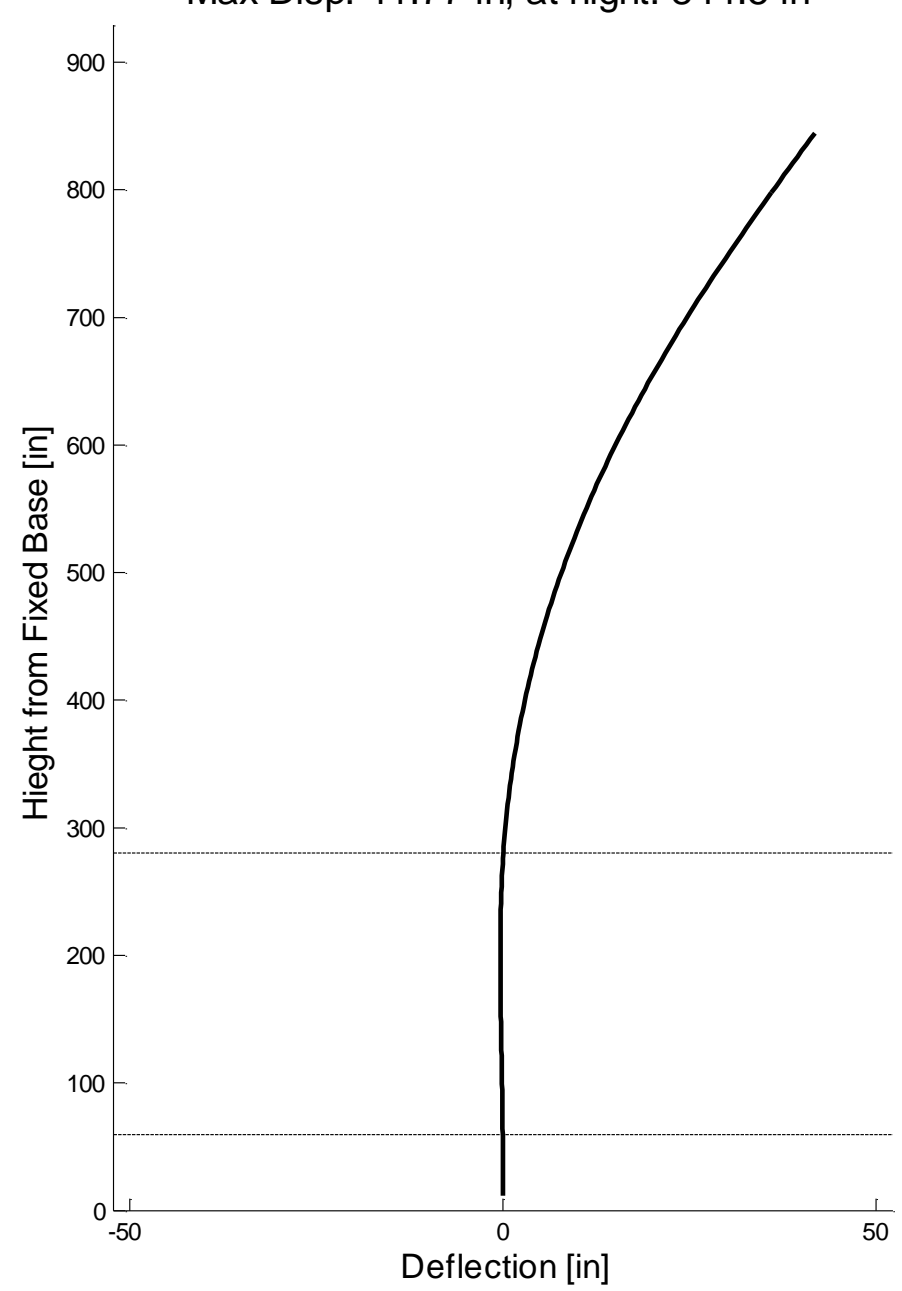

Axial Tower Deflection

Max Disp: 0.003706 in, at hight: 280.3 in

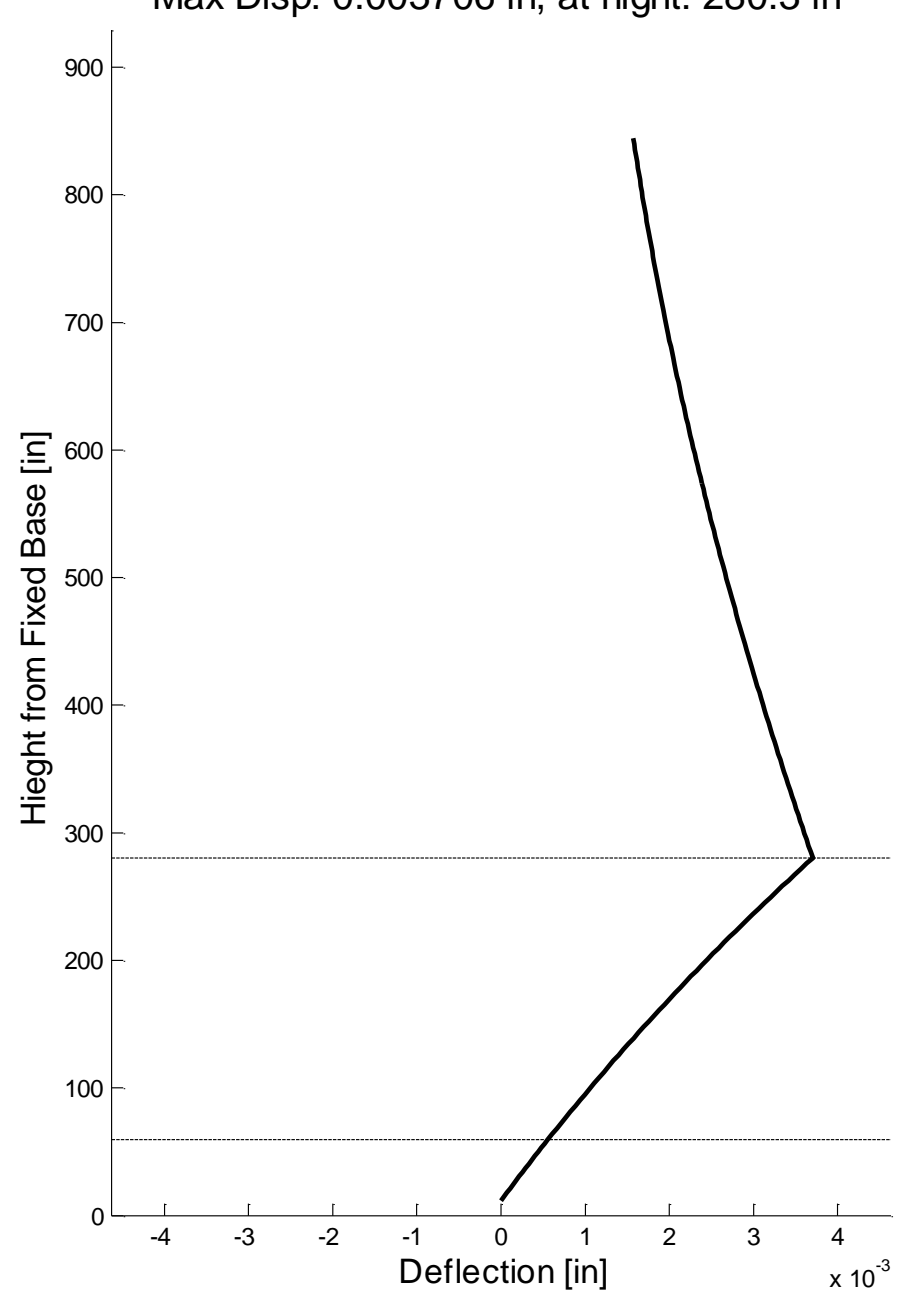




\section{Load Case A: Principle Stresses}

Transient Small Wind Turbine Tower Structural Analysis with Coupled Rotor Dynamic Interaction

Tower Shear Stress Profile

Max: $1202 \mathrm{lbf} / \mathrm{in}^{2}$, at hight: 43.5 in

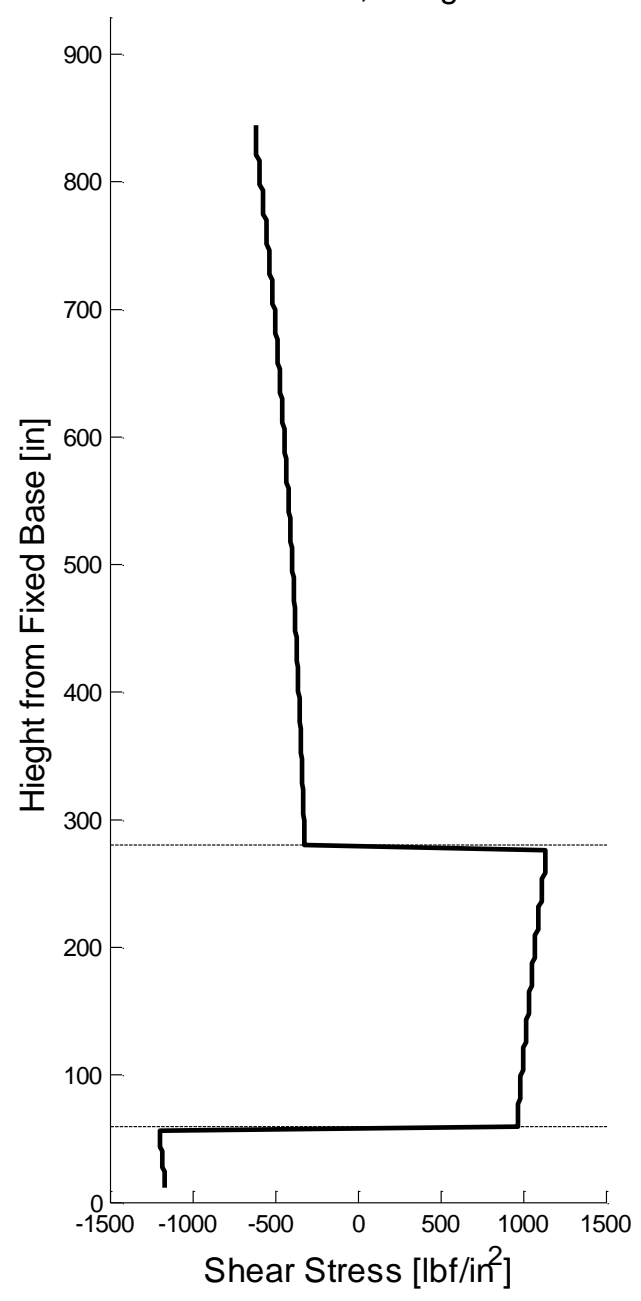

Tower Bending Stress Profile

Max: $3.499 \mathrm{e}+004 \mathrm{lbf} / \mathrm{in}^{2}$, at hight: $280.3 \mathrm{in}$

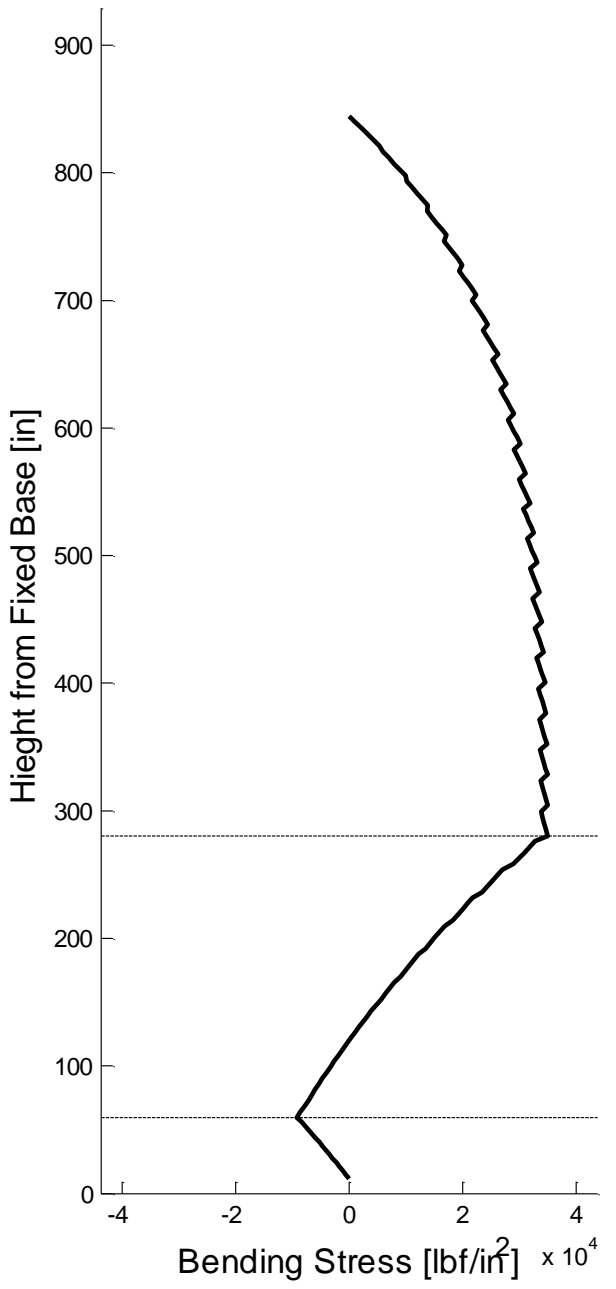

Tower Axial Stress Profile

Max: $479.6 \mathrm{lbf} / \mathrm{in}^{2}$, at hight: $258.2 \mathrm{in}$

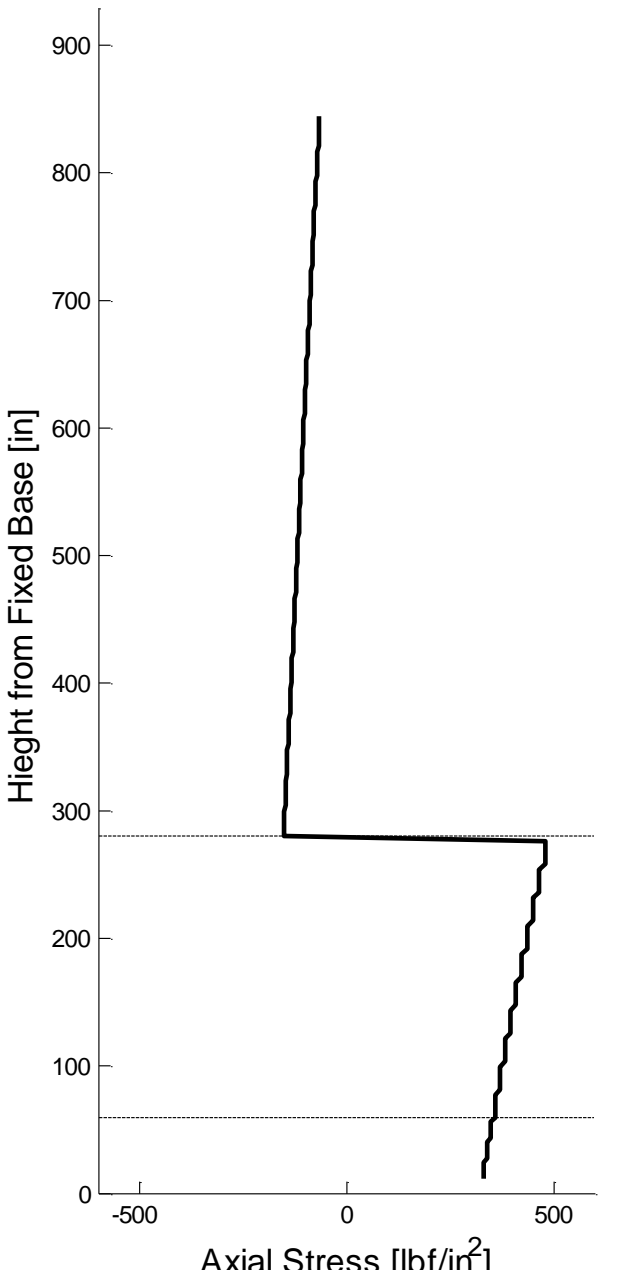




\section{Load Case A: Equivalent von Mises Stress}

Transient Small Wind Turbine Tower Structural Analysis with Coupled Rotor Dynamic Interaction

Max Tower von Mises Stress Profile

Max: $3.514 \mathrm{e}+004 \mathrm{lbf} / \mathrm{in}^{2}$, at hight: 280.3 in

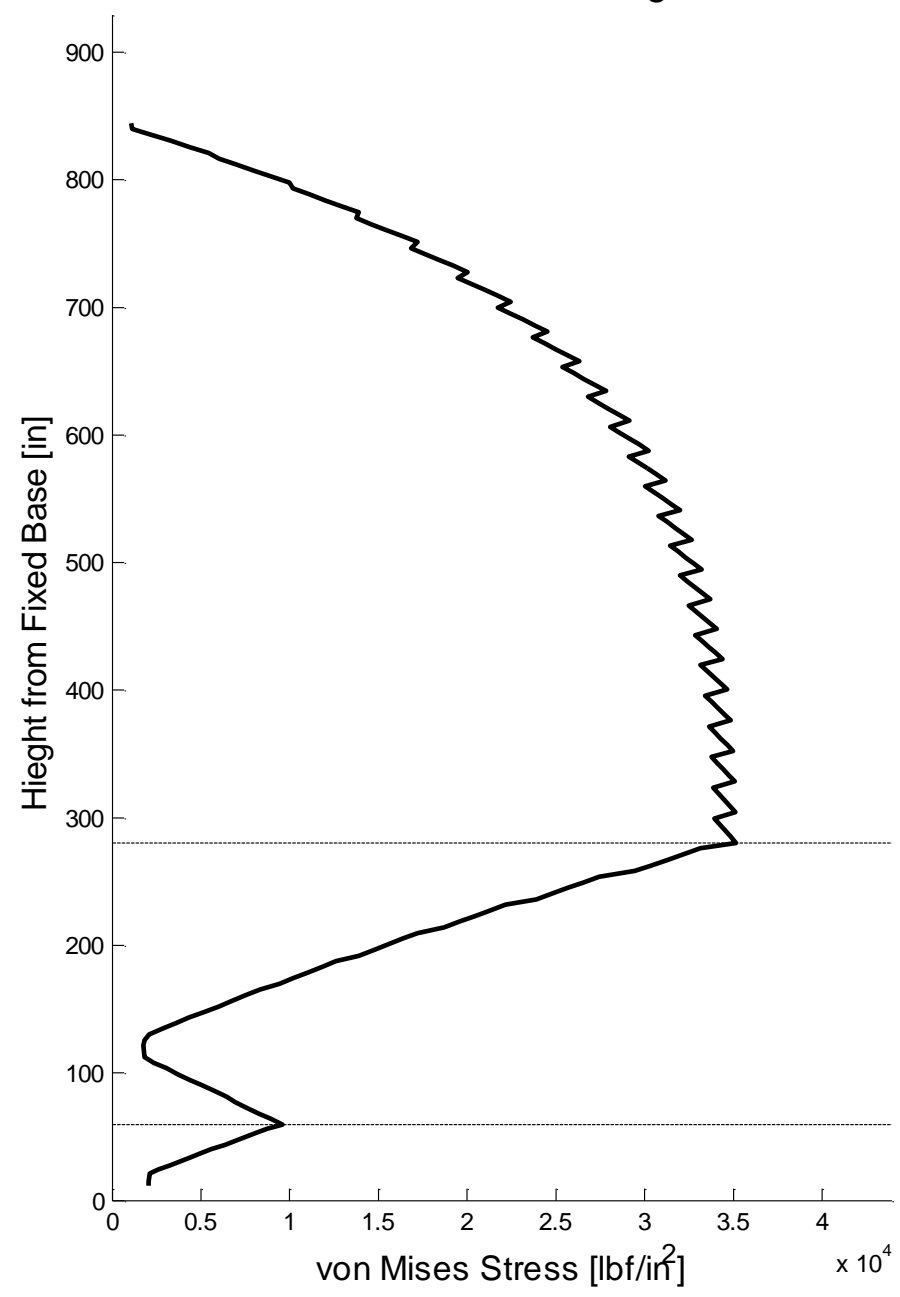

Max Tower von Mises Cross-Sectional Location (Theta) Profile

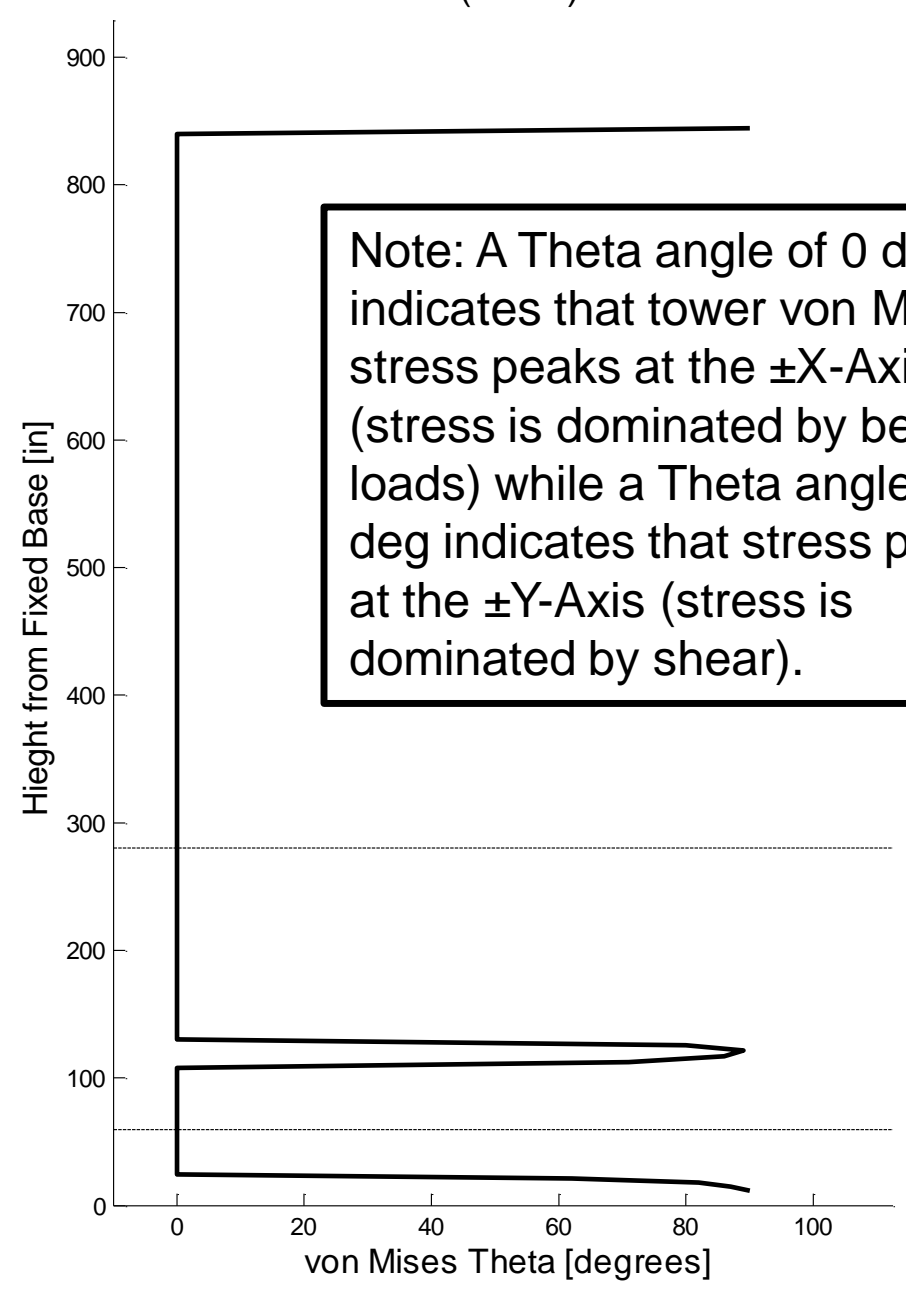




\section{Strong Axis \\ Load Case B - Static \\ 50 Year Extreme Wind \\ Model (EWM50) \\ IEC 61400-2 DLC 5.1 \\ (Parked Rotor)}

California Polytechnic State University - San Luis Obispo 


\section{Load Case B: Overview}

- Distributed load case (including tower and rotor loads)

- Small Wind Turbine Class (SWTC) 3 system

- Developed in accordance with IEC 61400-2 Design Load Case (DLC) 5.1 (Table 4)

- Rotor Force Coefficient: $C_{f}=1.5$

- Tower Drag Coefficient: $\mathrm{C}_{\mathrm{d}}=1.3$

- Assumed rotor state is parked with rotor plane orthogonal to wind direction (i.e., disk break activated but fault with furling system).

- Load applied in "strong axis" (the load is parallel to the plane formed by the tower, gin-pole, and strut).

- Details of the analysis parameters are summarized and defined in the attached UserInputs.xIsx file. 


\section{Load Case B: Tower Deflection}

Transverse Tower Deflection

Max Disp: 23.33 in, at hight: 844.5 in

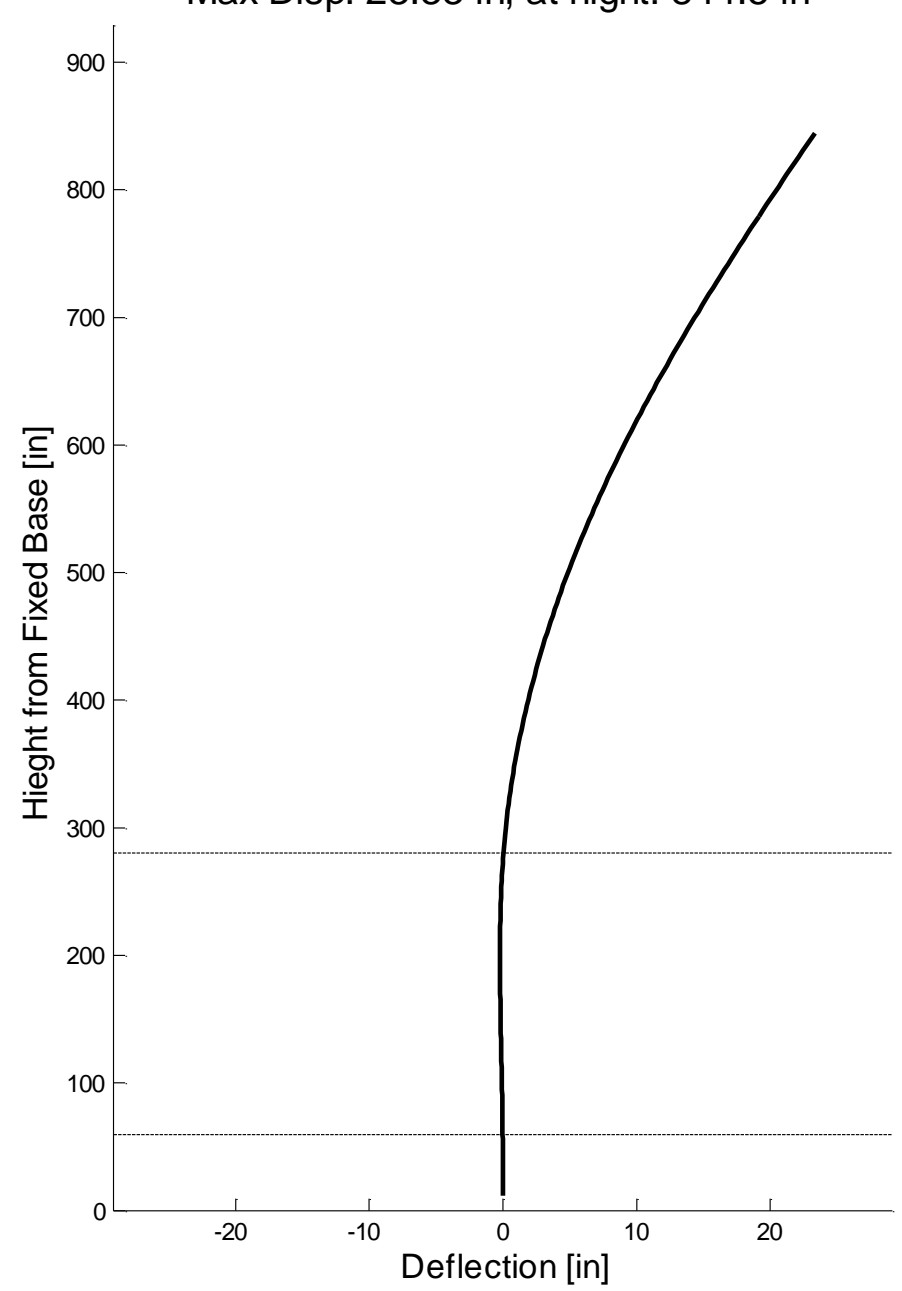

Axial Tower Deflection

Max Disp: 0.002433 in, at hight: 280.3 in

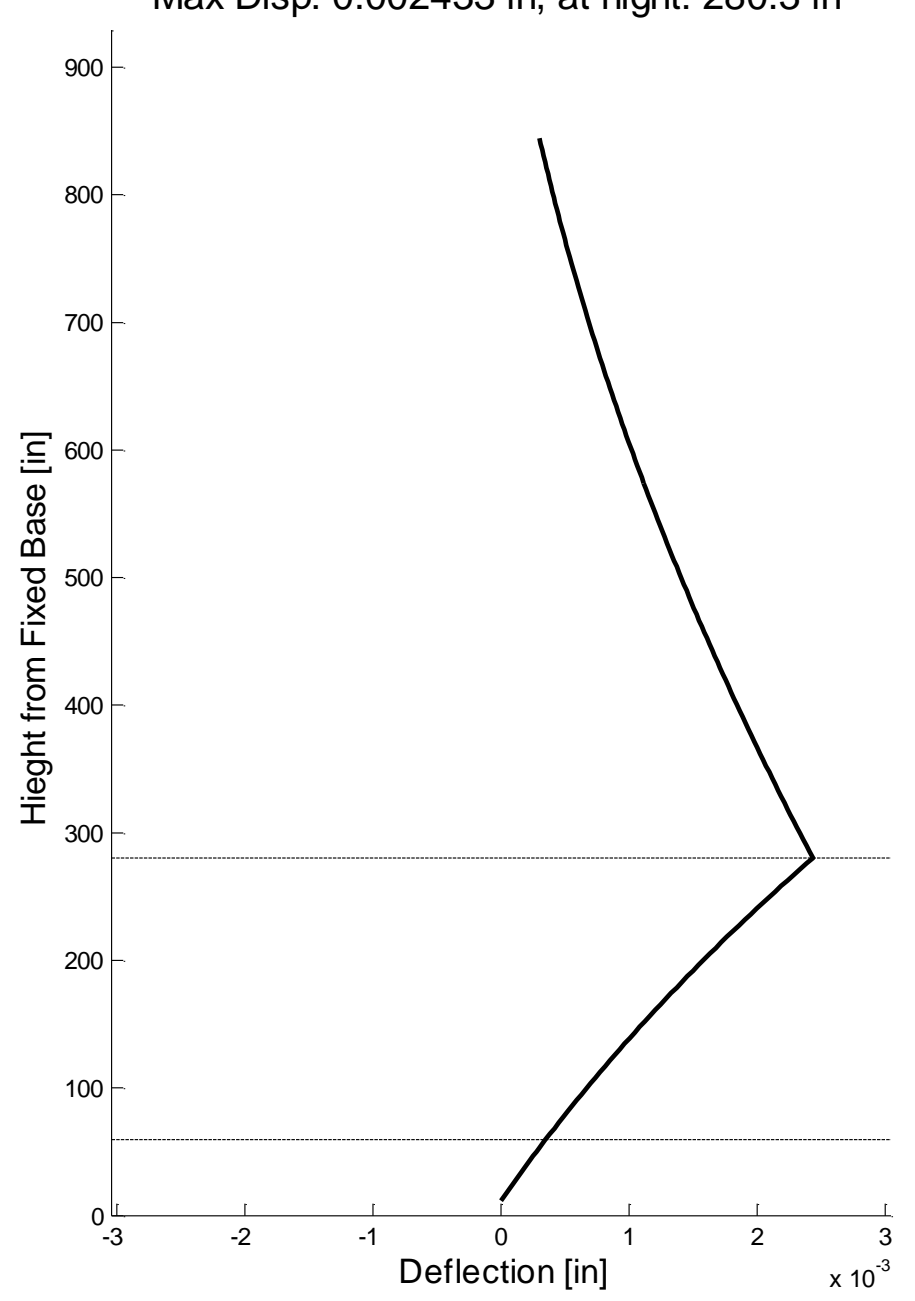




\section{Load Case B: Principle Stresses}

Transient Small Wind Turbine Tower Structural Analysis with Coupled Rotor Dynamic Interaction

Tower Shear Stress Profile

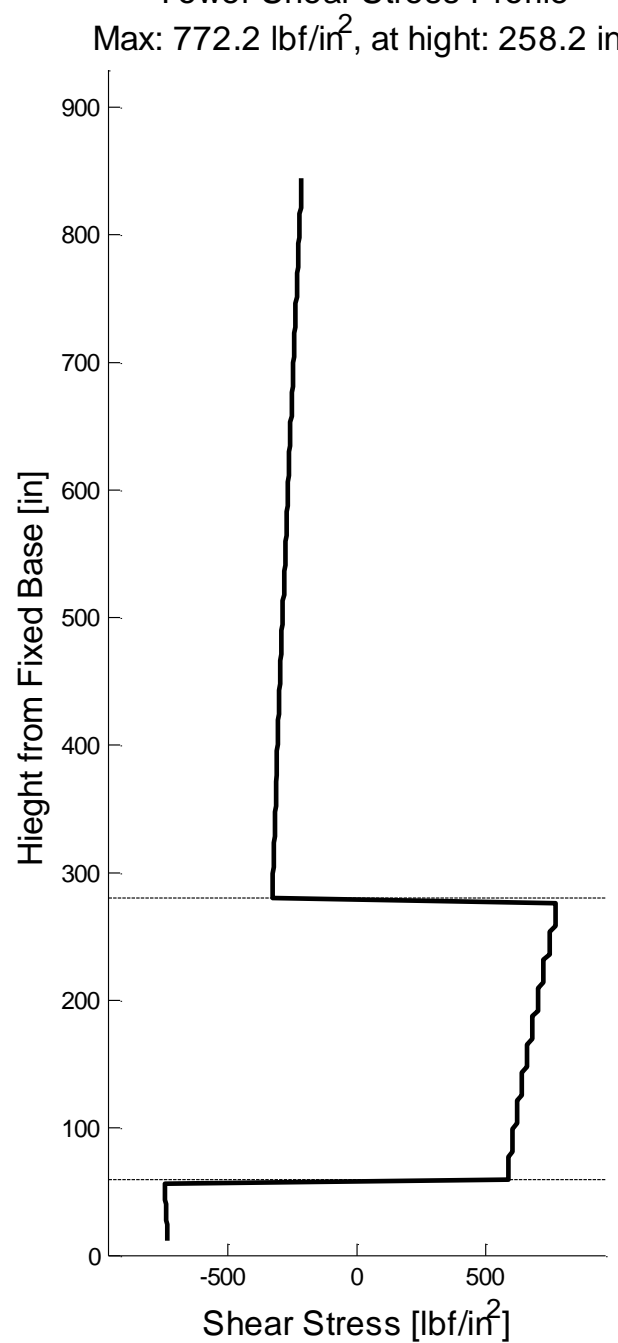

Tower Bending Stress Profile

Max: $2.289 \mathrm{e}+004 \mathrm{lbf} / \mathrm{in}^{2}$, at hight: $280.3 \mathrm{in}$

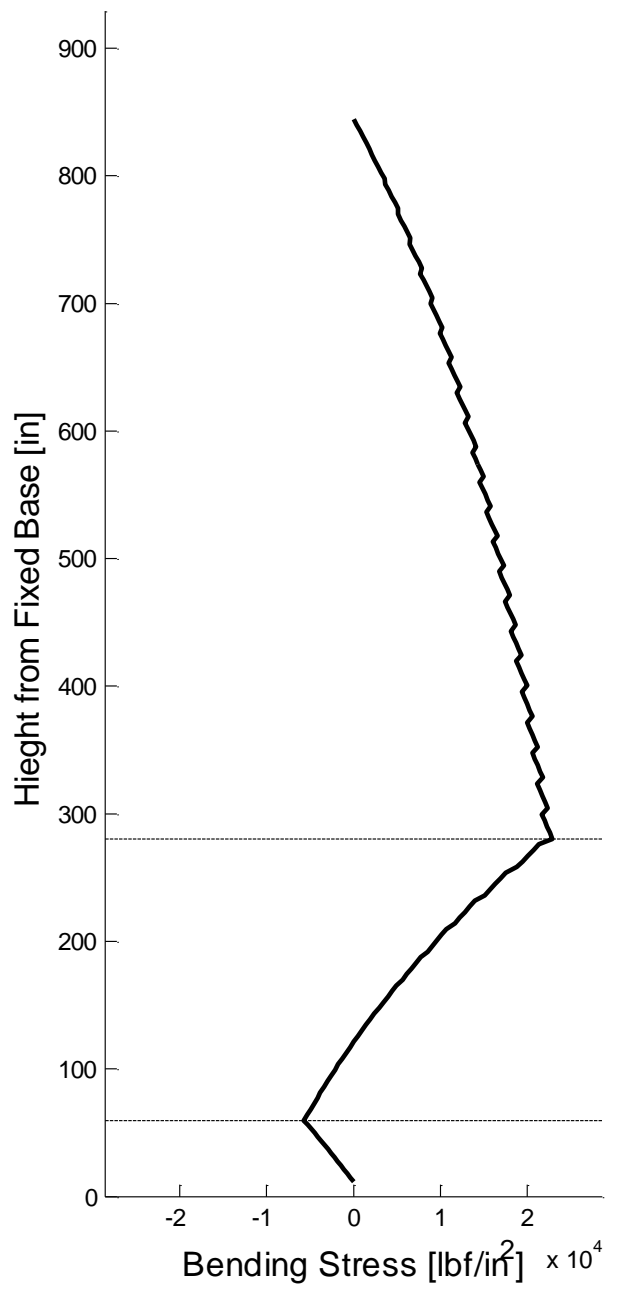

Tower Axial Stress Profile Max: $327.3 \mathrm{lbf} / \mathrm{in}^{2}$, at hight: $258.2 \mathrm{in}$

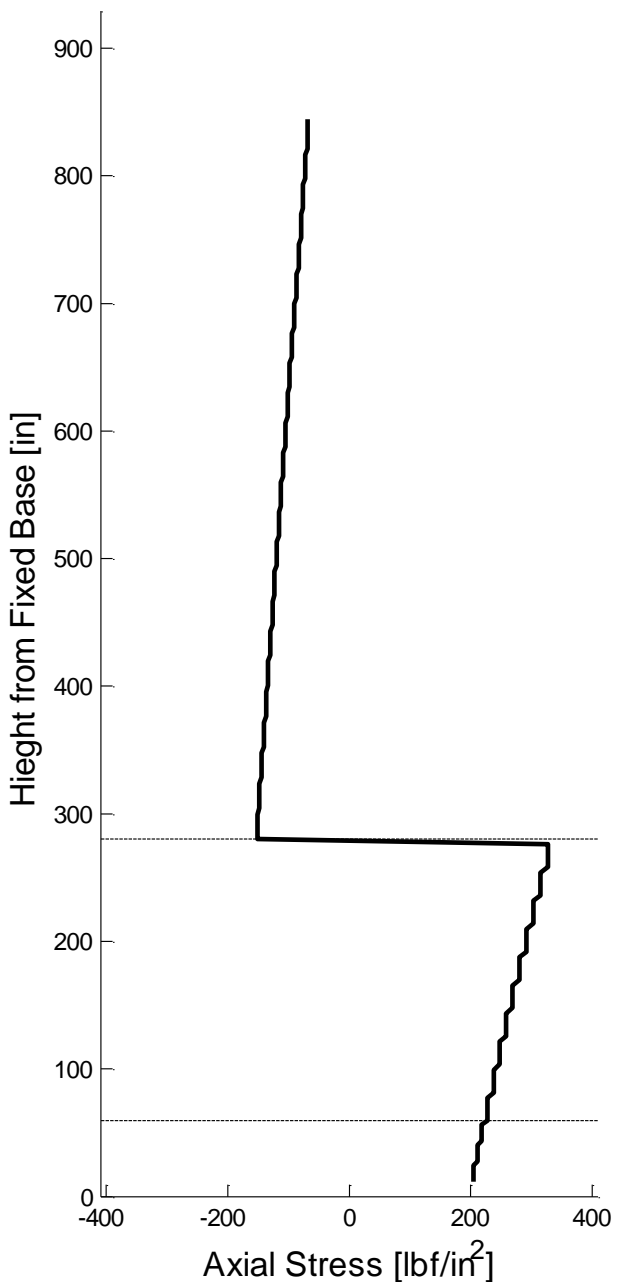




\section{Load Case B: Equivalent von Mises Stress}

Transient Small Wind Turbine Tower Structural Analysis with Coupled Rotor Dynamic Interaction

Max Tower von Mises Stress Profile

Max: $2.304 \mathrm{e}+004 \mathrm{lbf} / \mathrm{in}^{2}$, at hight: 280.3 in

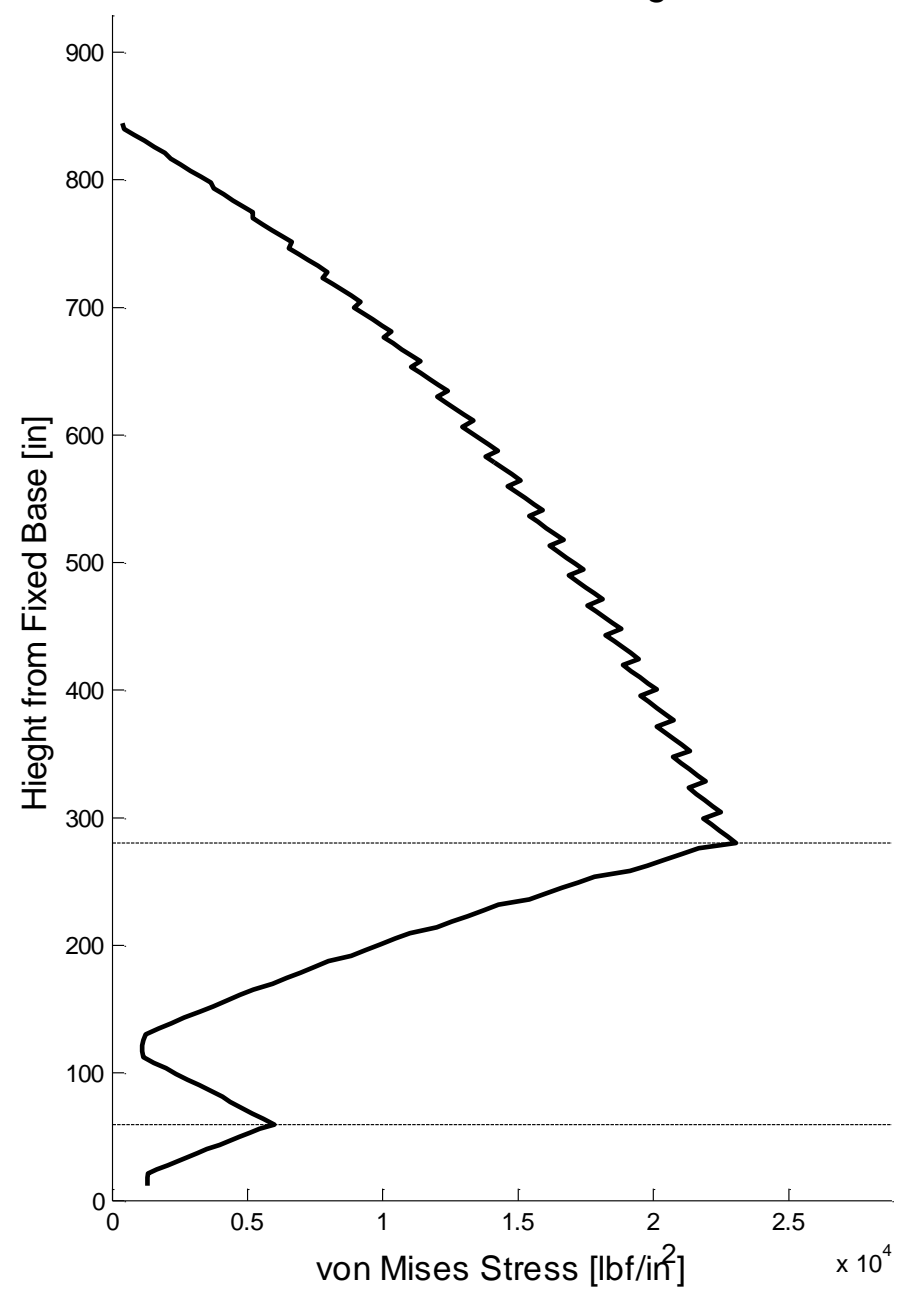

Max Tower von Mises Cross-Sectional Location (Theta) Profile

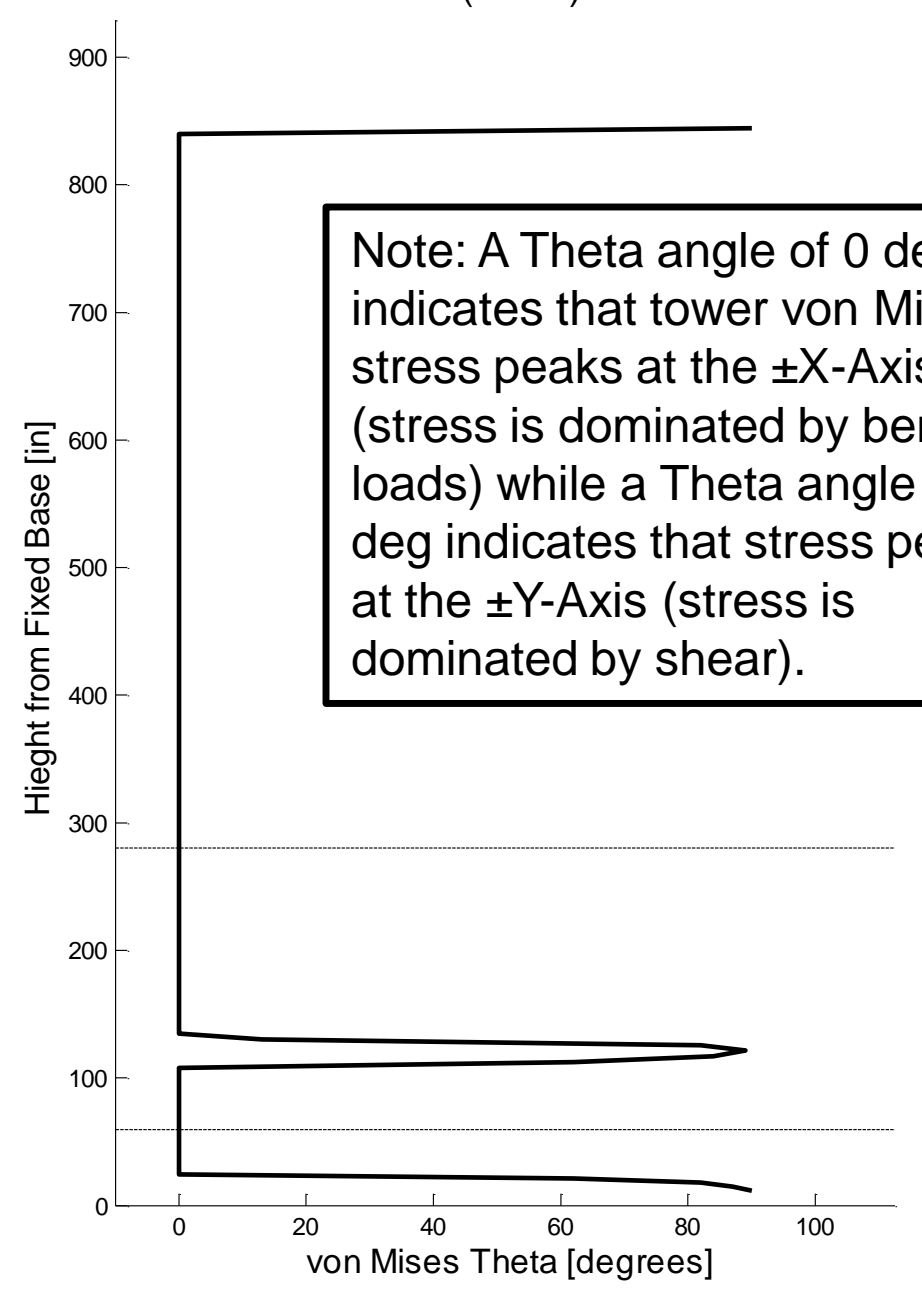




\section{Strong Axis Load Case C - Static \\ 1 Year Extreme Wind Model (EWM1) \\ IEC 61400-2 DLC 6.1}




\section{Load Case C: Overview}

Transient Small Wind Turbine Tower Structural Analysis with Coupled Rotor Dynamic Interaction

- Distributed load case (including tower and rotor loads)

- Small Wind Turbine Class (SWTC) 3 system

- Developed in accordance with IEC 61400-2 Design Load Case (DLC) 6.1 (Table 4)

- Rotor Thrust Coefficient: $C_{t}=0.5$

- Tower Drag Coefficient: $\mathrm{C}_{\mathrm{d}}=1.3$

- Assumed rotor state is power production (more conservative than IEC 61400-2 which specifies parked or idling for this DLC); effective assumption is a fault in the furling system and disk break

- Load applied in "strong axis" (the load is parallel to the plane formed by the tower, gin-pole, and strut).

- Details of the analysis parameters are summarized and defined in the attached UserInputs.xIsx file. 


\section{Load Case C: Tower Deflection}

Transverse Tower Deflection

Max Disp: 27.78 in, at hight: 844.5 in

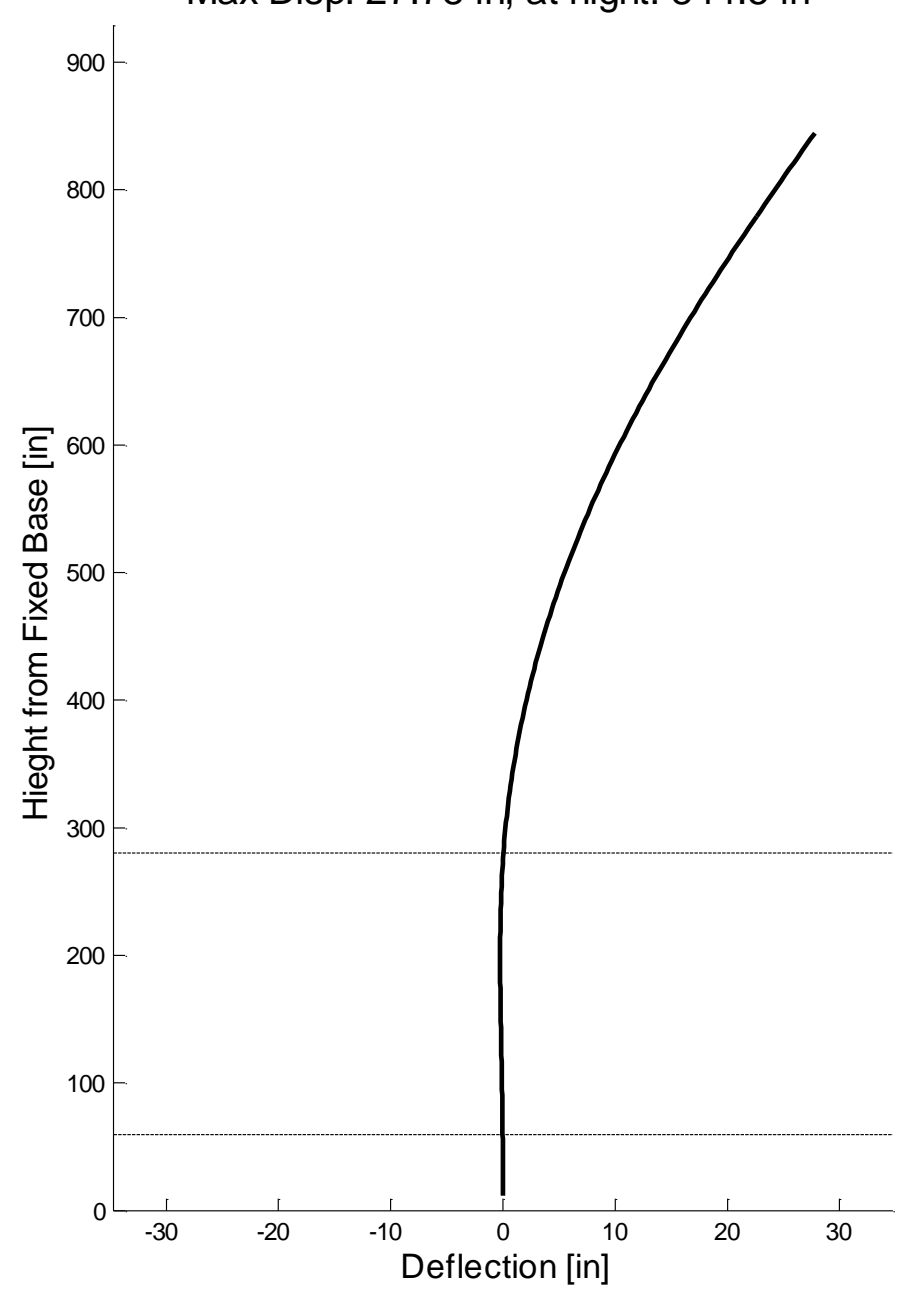

Axial Tower Deflection

Max Disp: 0.002531 in, at hight: 280.3 in

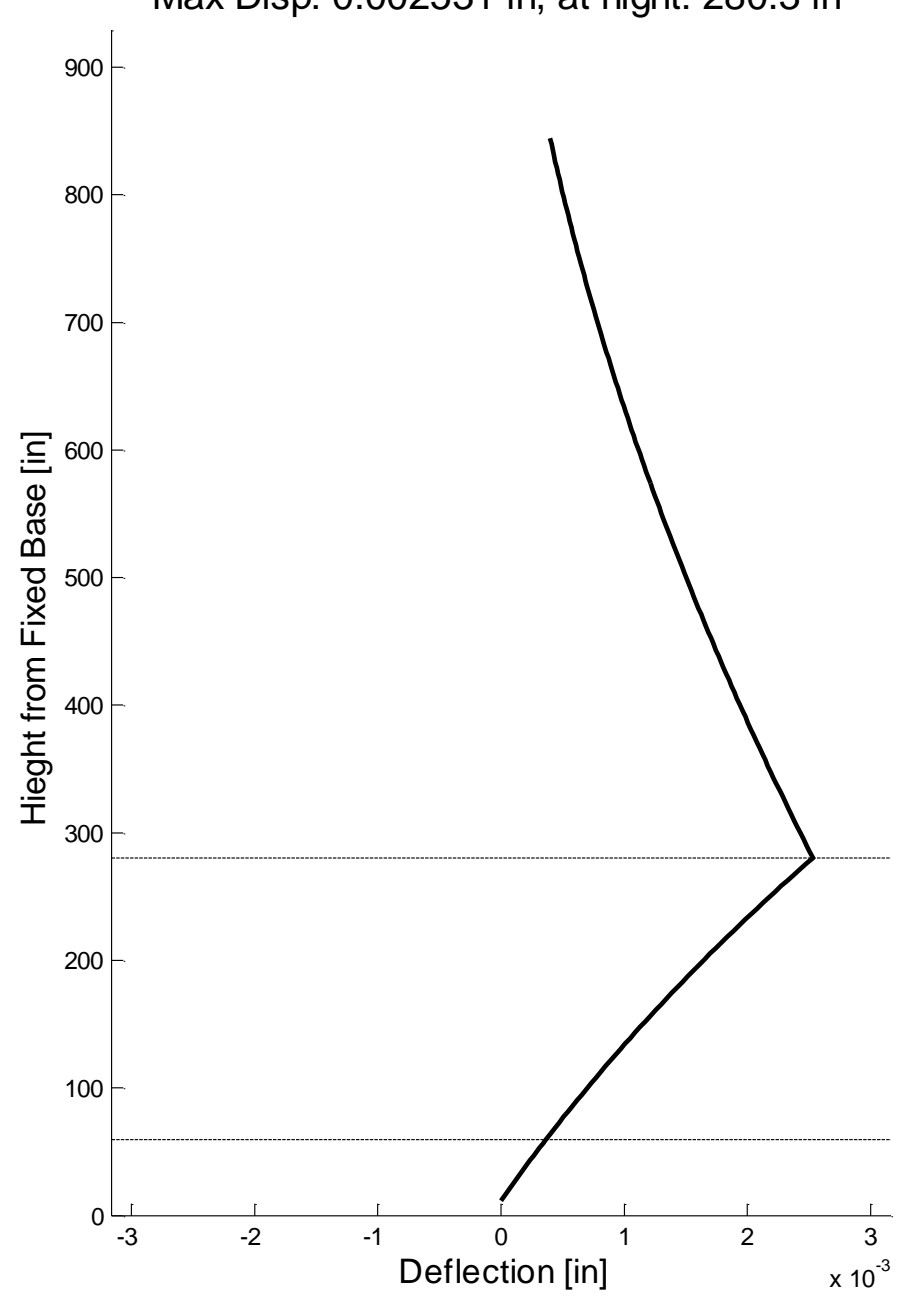




\section{Load Case C: Principle Stresses}

Transient Small Wind Turbine Tower Structural Analysis with Coupled Rotor Dynamic Interaction

Tower Shear Stress Profile

Max: $840.9 \mathrm{lbf} / \mathrm{in}^{2}$, at hight: $43.5 \mathrm{in}$

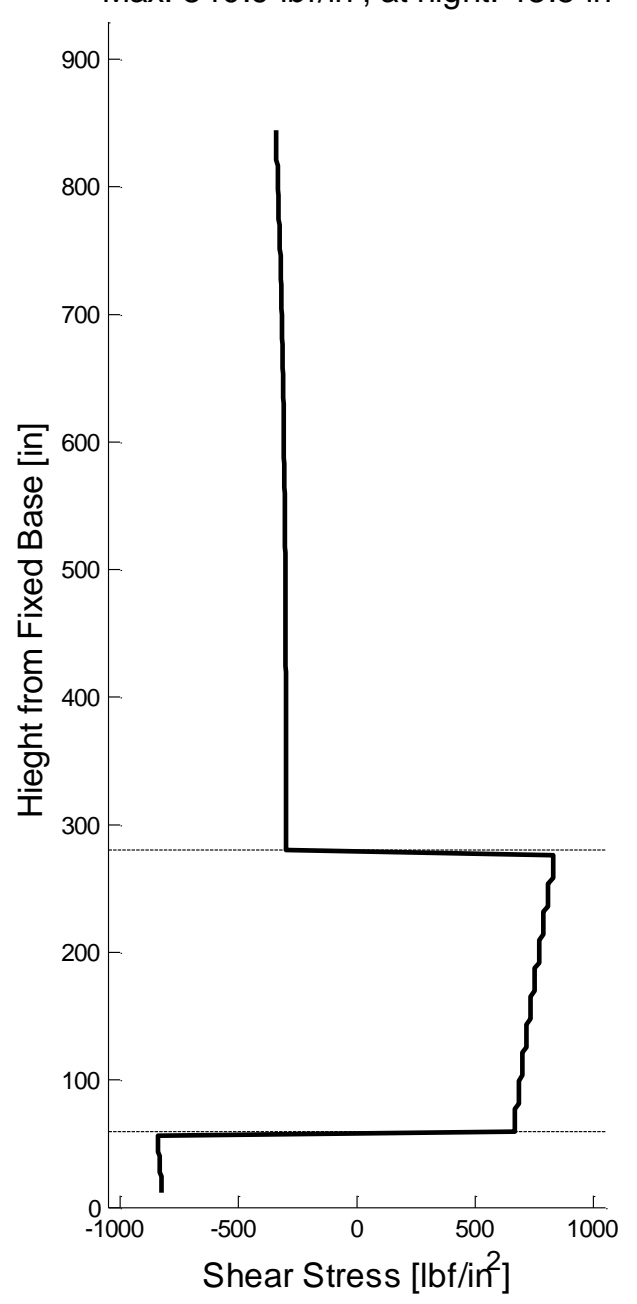

Tower Bending Stress Profile

Max: $2.515 \mathrm{e}+004 \mathrm{lbf} / \mathrm{ir}^{2}$, at hight: $280.3 \mathrm{in}$

$$
900
$$

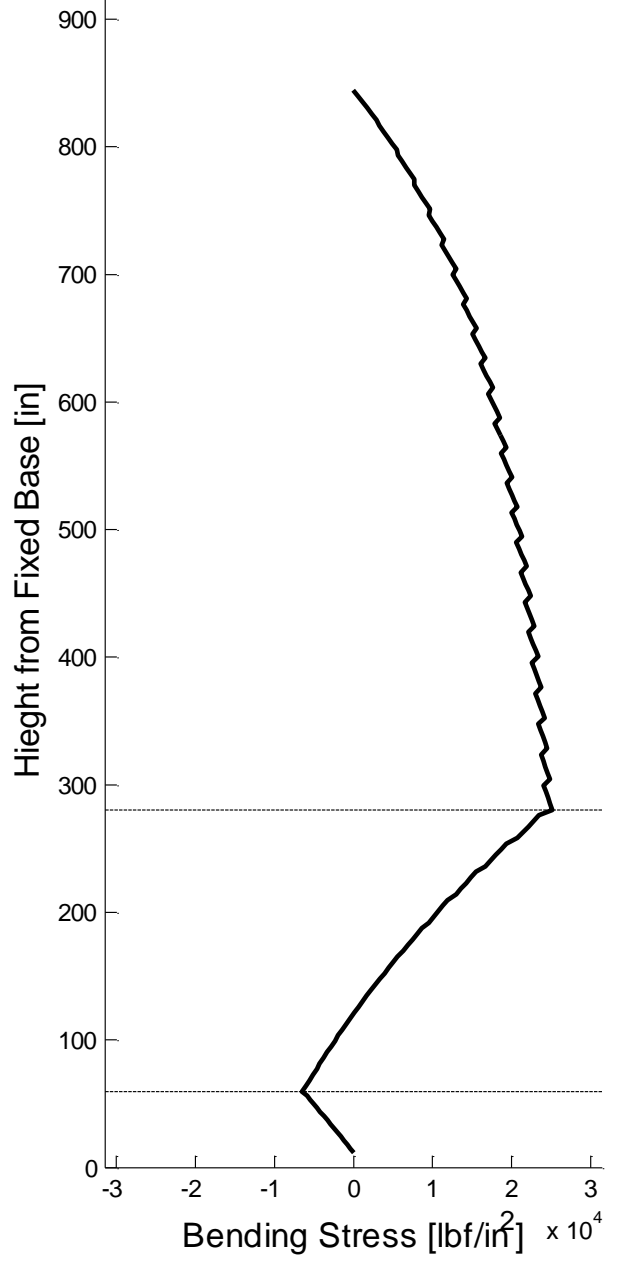

Tower Axial Stress Profile

Max: $339 \mathrm{lbf} / \mathrm{in}^{2}$, at hight: 258.2 in

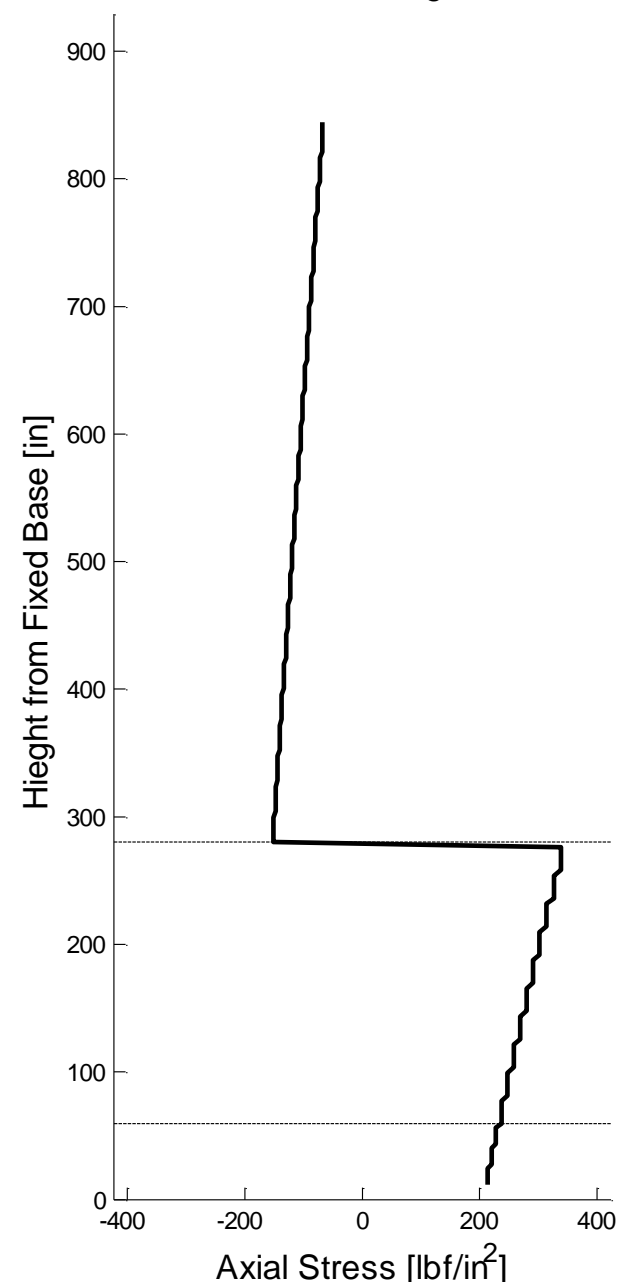




\section{Load Case C: Equivalent von Mises Stress}

Transient Small Wind Turbine Tower Structural Analysis with Coupled Rotor Dynamic Interaction

Max Tower von Mises Stress Profile

Max: $2.53 \mathrm{e}+004 \mathrm{lbf} / \mathrm{in}^{2}$, at hight: $280.3 \mathrm{in}$

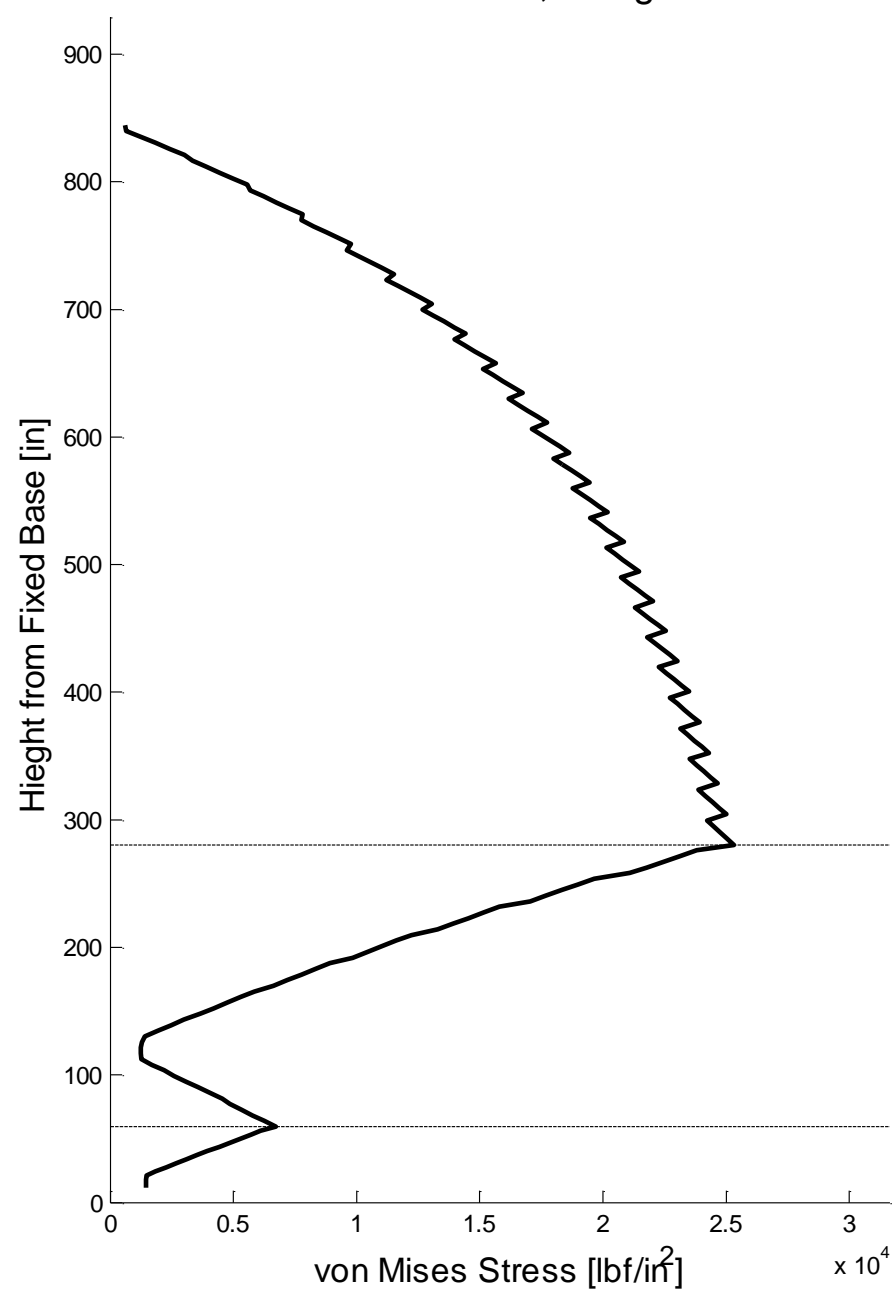

Max Tower von Mises Cross-Sectional Location (Theta) Profile

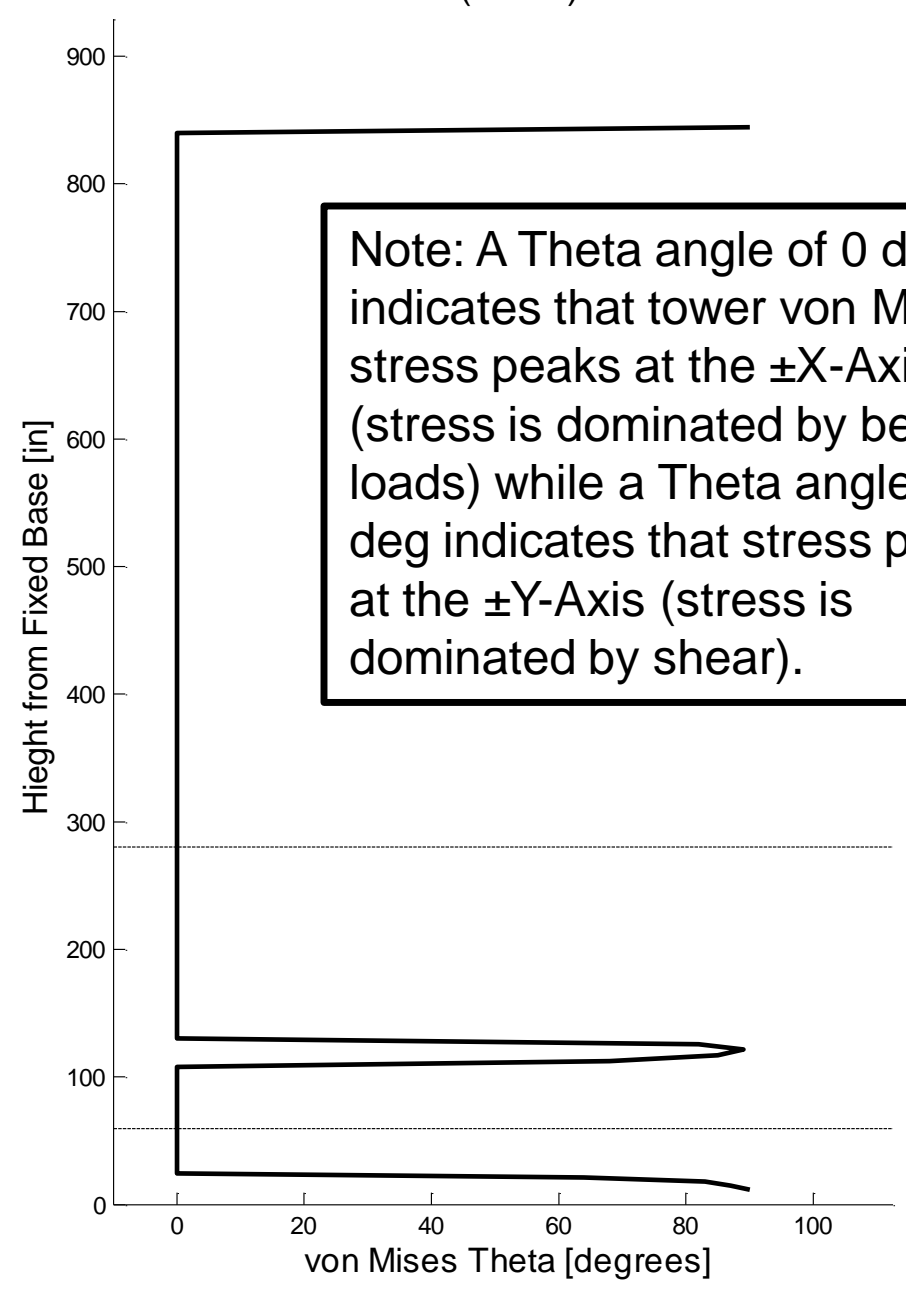




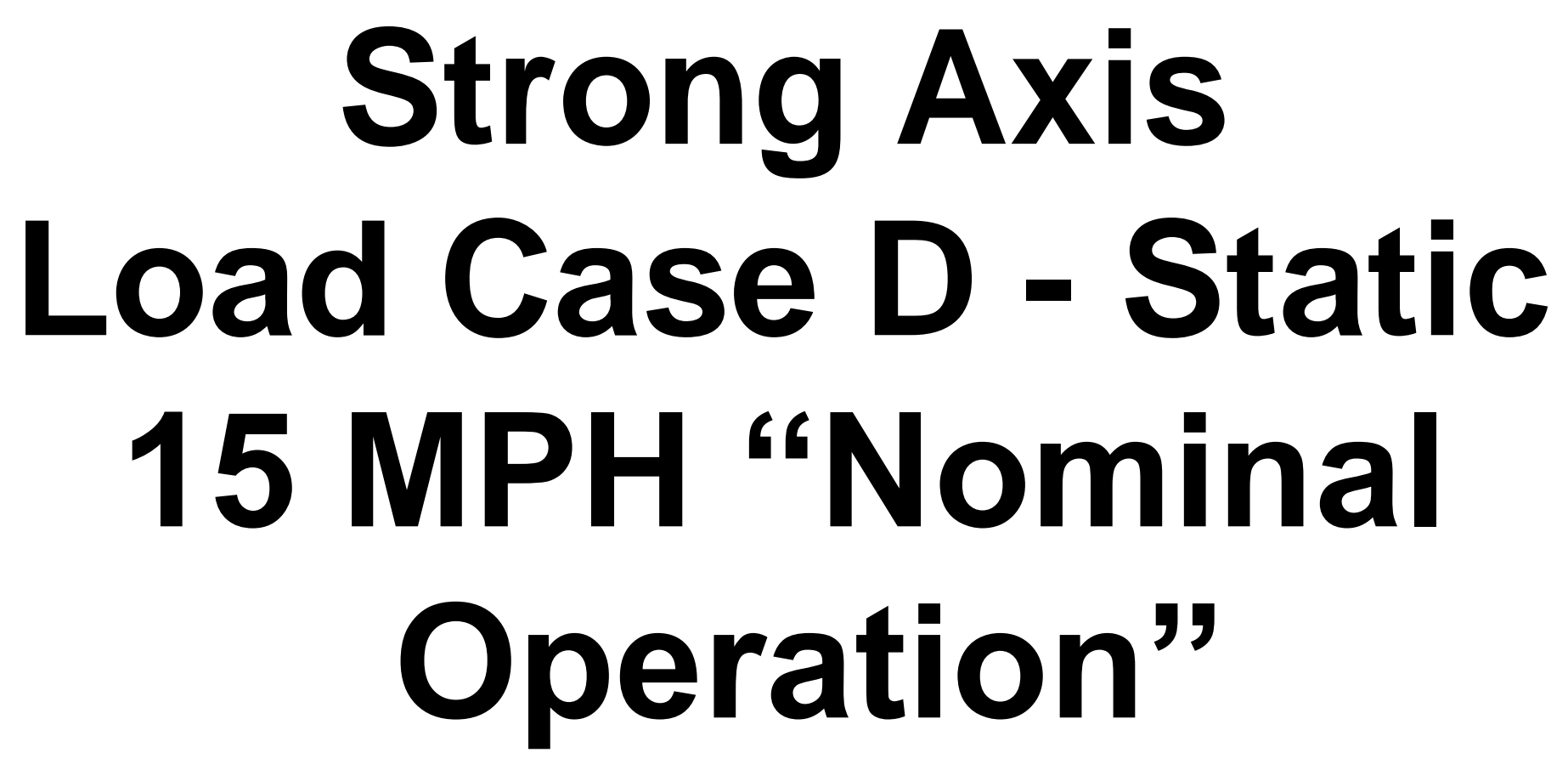




\section{Load Case D: Overview}

- Distributed load case (including tower and rotor loads)

- Small Wind Turbine Class (SWTC) 3 system

- Wind profile is same as EWM1 but scaled down such that the wind velocity at the tower tip is $15 \mathrm{MPH}$, representing a state of "nominal operation"

- Developed in accordance with IEC 61400-2 Design Load Case (DLC) 6.1 (Table 4)

- Rotor Thrust Coefficient: $C_{t}=0.5$

- Tower Drag Coefficient: $C_{d}=1.3$

- Assumed rotor state is power production.

- Load applied in "strong axis" (the load is parallel to the plane formed by the tower, gin-pole, and strut).

- Details of the analysis parameters are summarized and defined in the attached UserInputs.xIsx file. 


\section{Load Case D: Tower Deflection}

Transverse Tower Deflection

Max Disp: 0.7996 in, at hight: 844.5 in

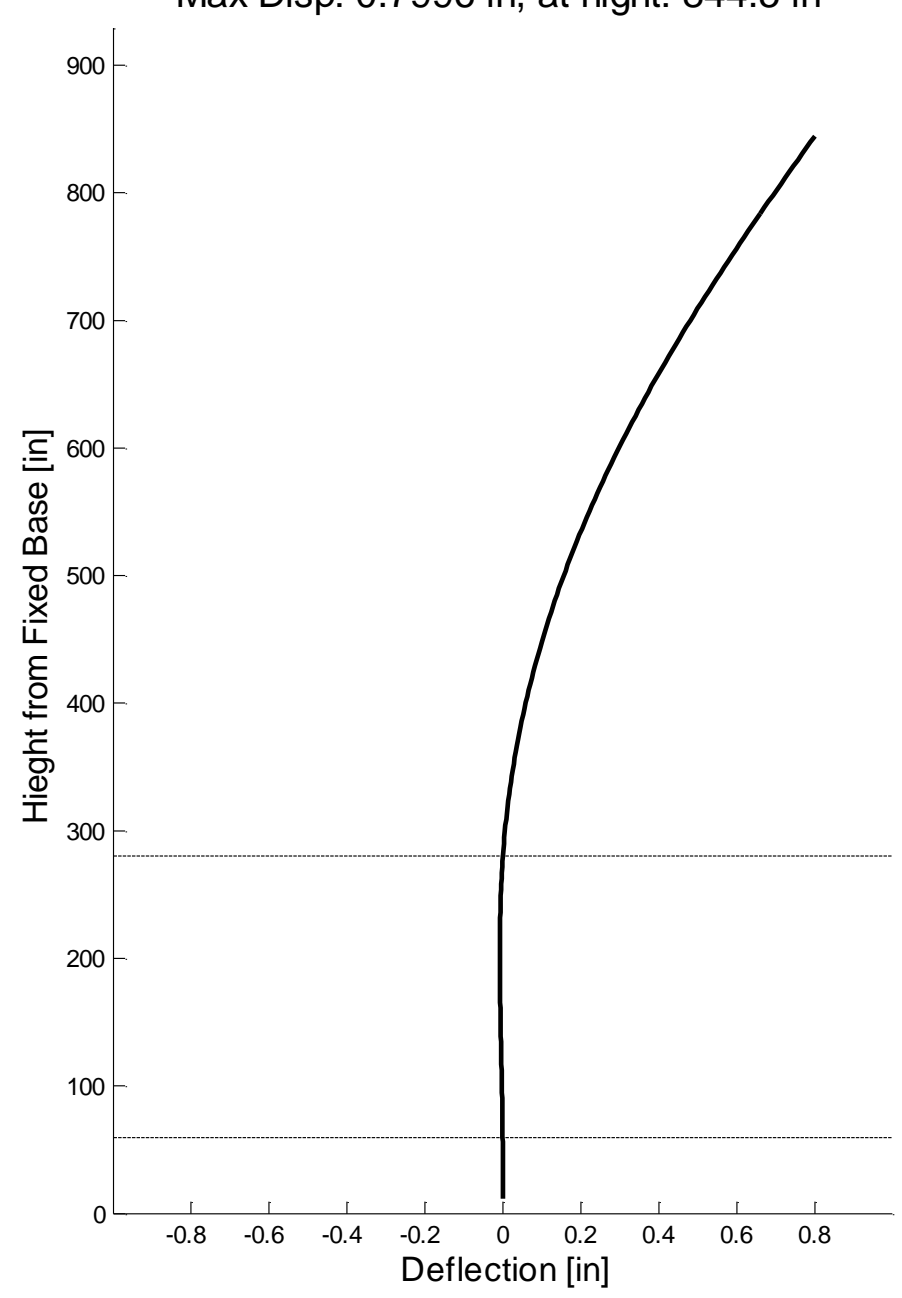

Axial Tower Deflection

Max Disp: 0.003608 in, at hight: 844.5 in

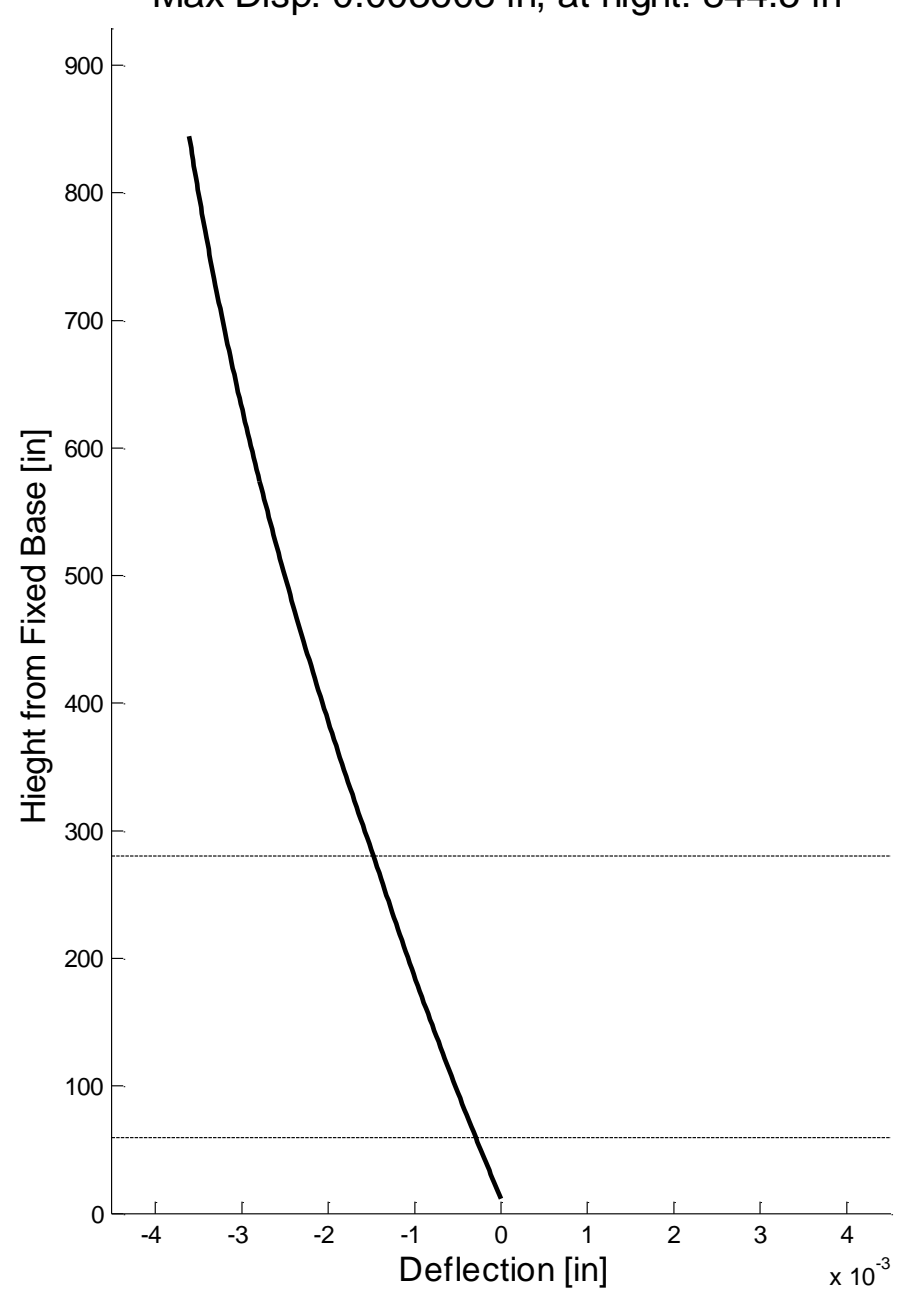




\section{Load Case D: Principle Stresses}

Transient Small Wind Turbine Tower Structural Analysis with Coupled Rotor Dynamic Interaction

Tower Shear Stress Profile

Max: $25.78 \mathrm{lbf} / \mathrm{in}^{2}$, at hight: $43.5 \mathrm{in}$

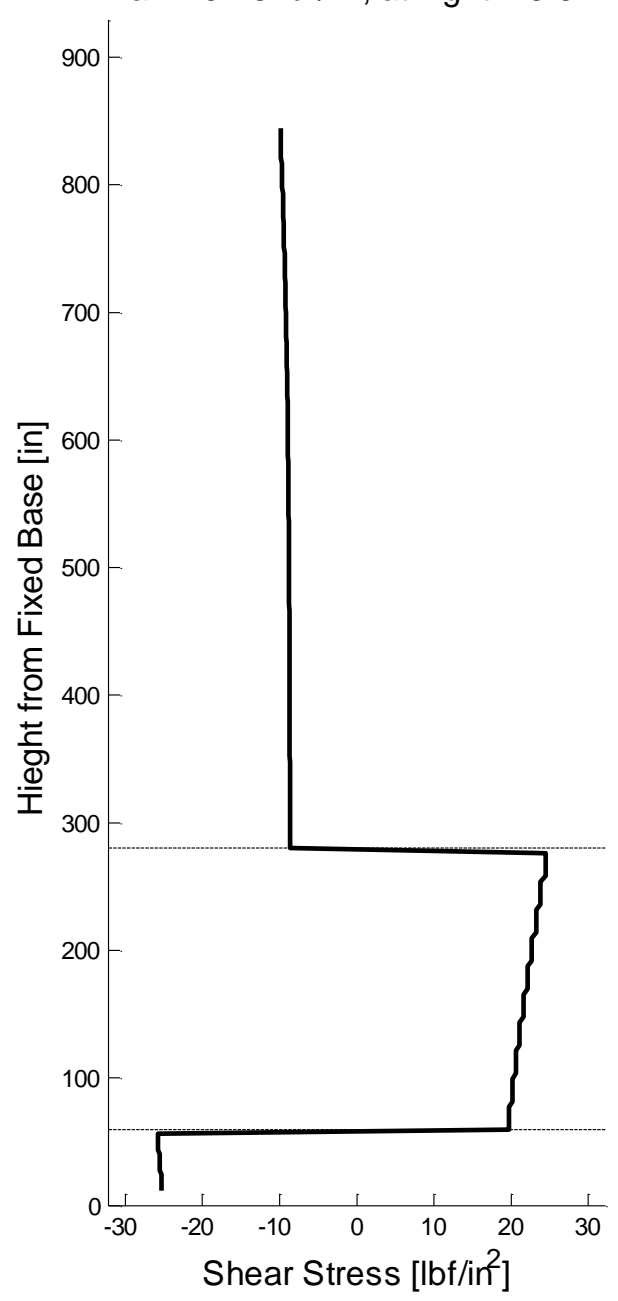

Tower Bending Stress Profile

Max: $729.5 \mathrm{lbf} / \mathrm{in}^{2}$, at hight: $280.3 \mathrm{in}$

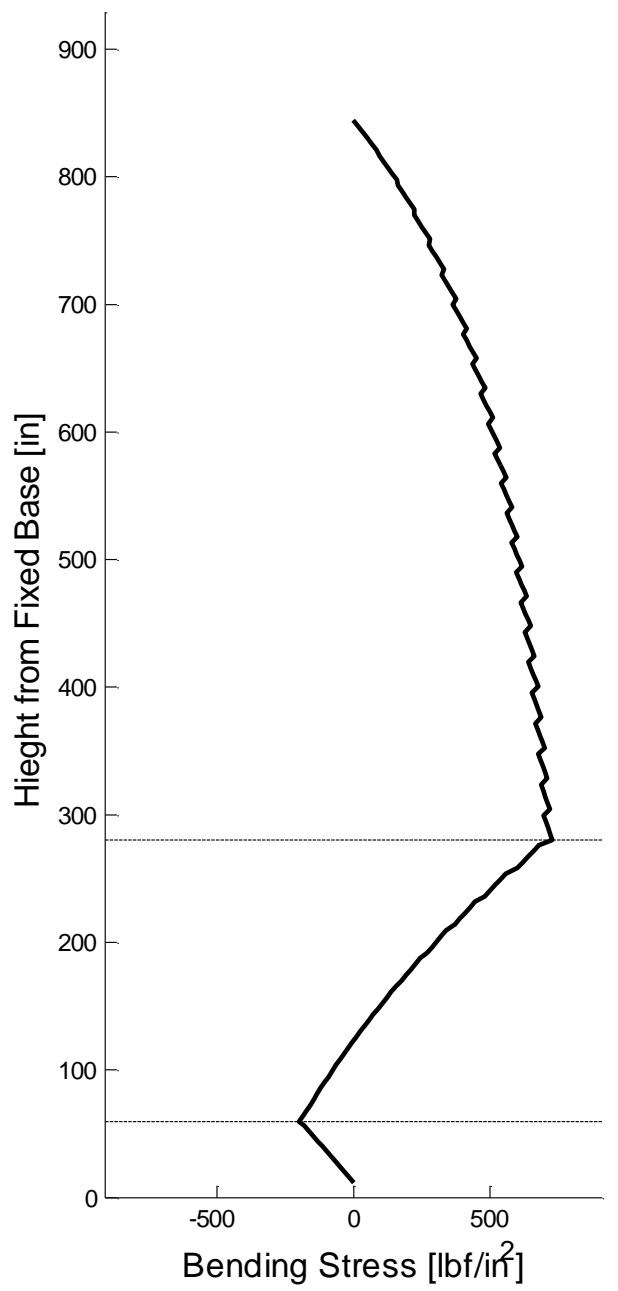

Tower Axial Stress Profile

Max: $180.7 \mathrm{lbf} / \mathrm{in}^{2}$, at hight: $11.5 \mathrm{in}$

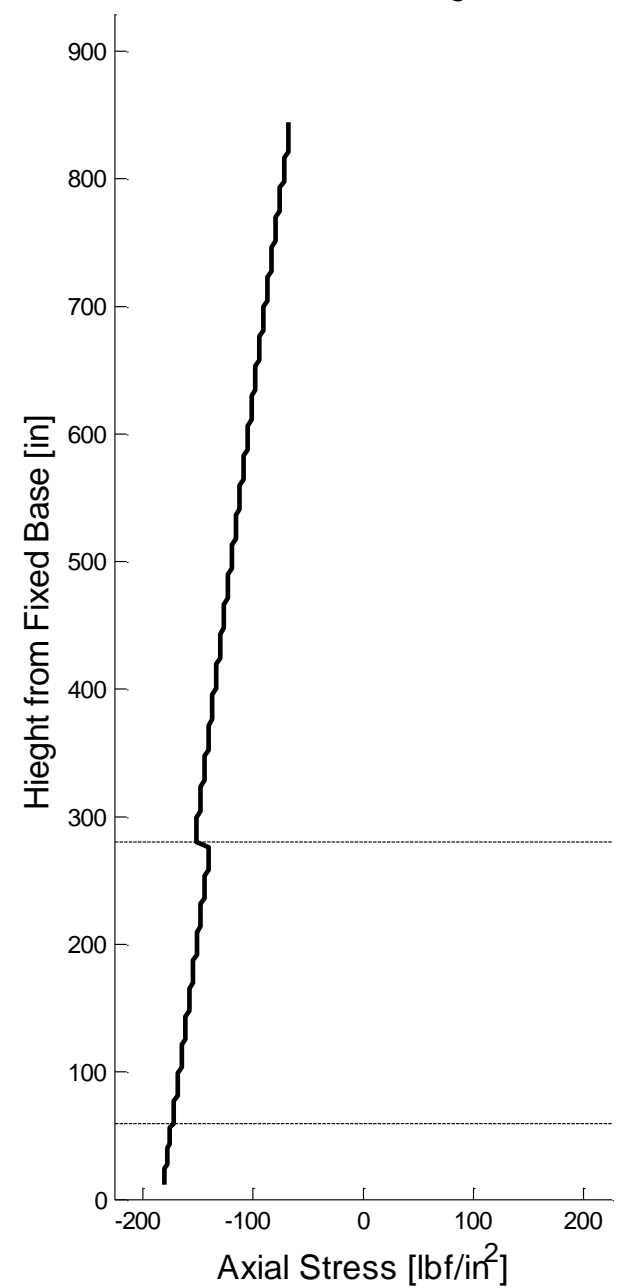




\section{Load Case D: Equivalent von Mises Stress}

Transient Small Wind Turbine Tower Structural Analysis with Coupled Rotor Dynamic Interaction

Max Tower von Mises Stress Profile

Max: $880.8 \mathrm{lbf} / \mathrm{in}^{2}$, at hight: 280.3 in

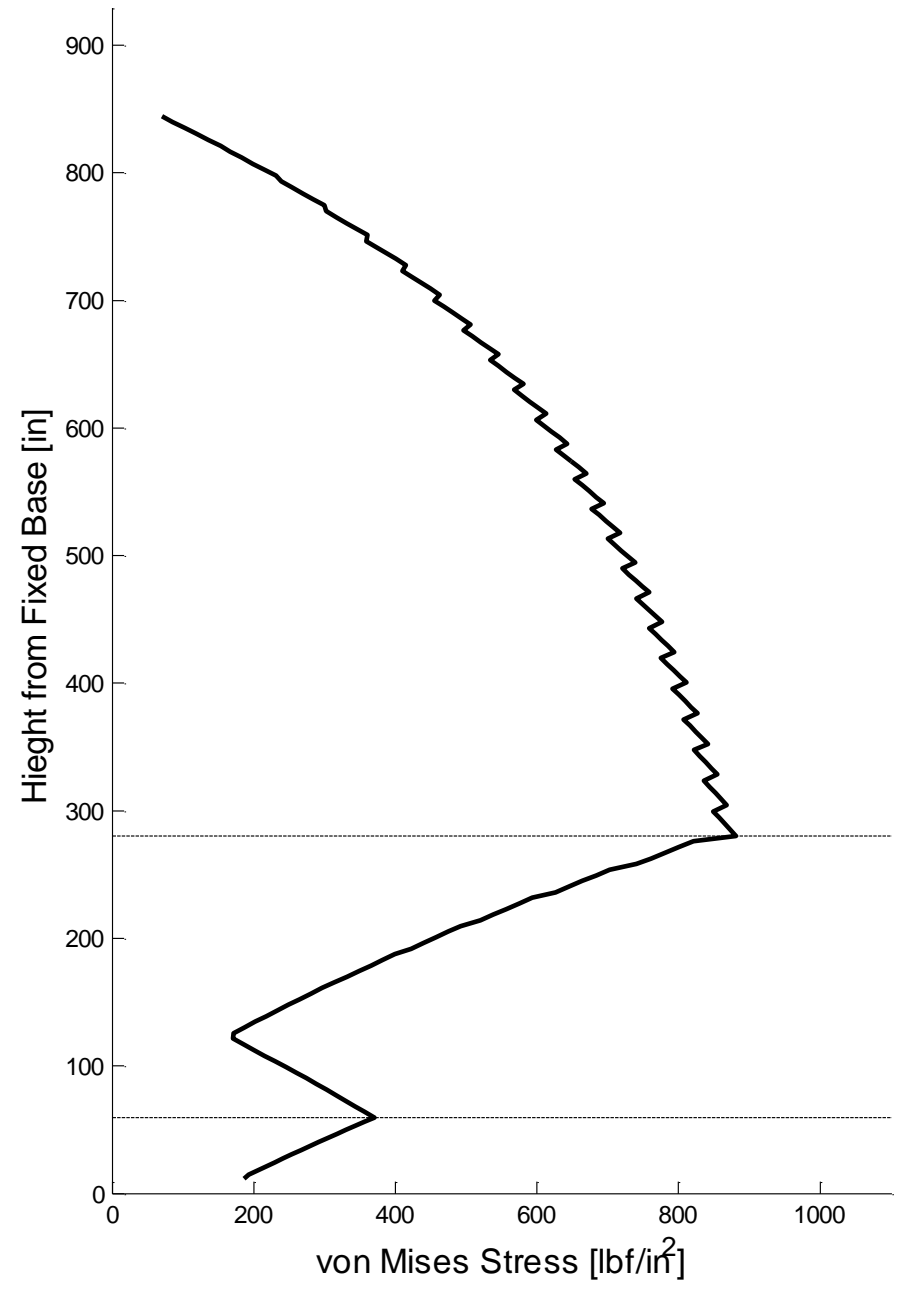

Max Tower von Mises Cross-Sectional Location (Theta) Profile

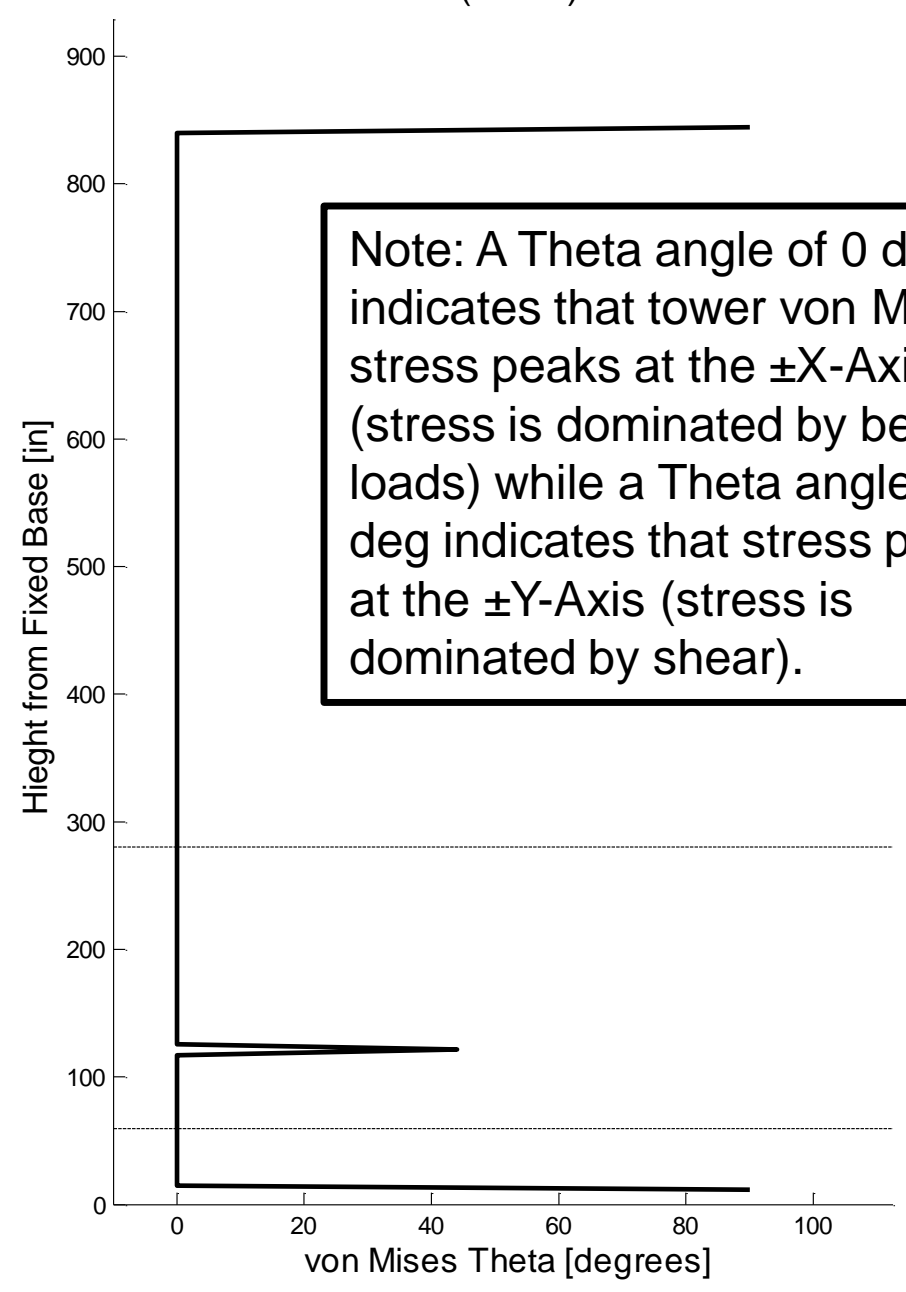




\section{Strong Axis}

Modal Evaluation 


\section{Modal Evaluation: Overview}

- Results of normal modes using an Eigenvalue / Eigenvector evaluation are displayed on the next page.

- The lowest five mode shapes are shown in the Transverse direction (X-Axis, in the Strong Axis direction) and in the Axial direction (Y-Axis).

- Note that the Transverse mode shape plot skips Mode \# 5.

- Mode \# 5 is the rotor flapwise blade mode and is at $16.25 \mathrm{~Hz}$ (this frequency was estimated using the Myklestad Method). 


\section{Modal Evaluation}
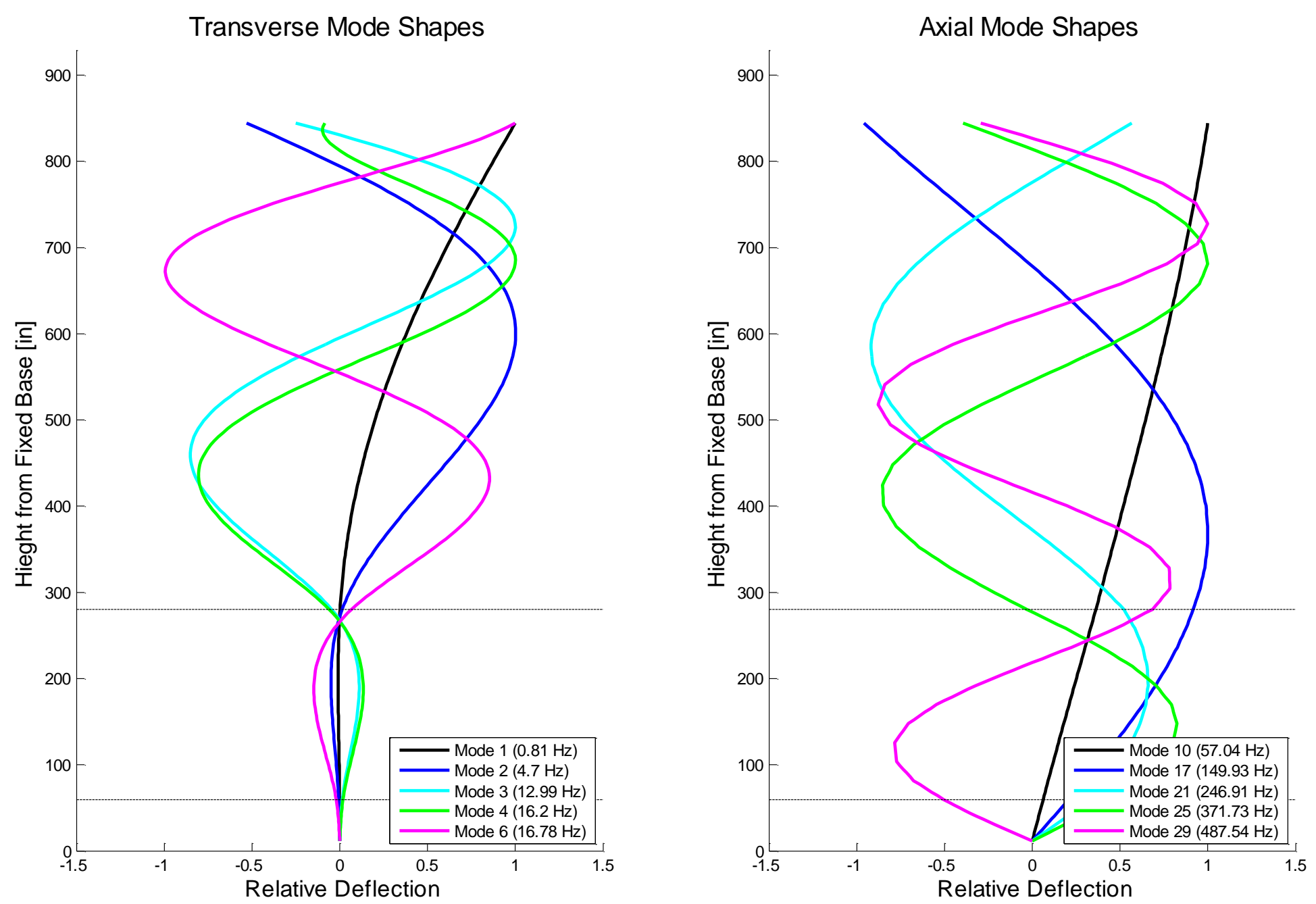

California Polytechnic State University - San Luis Obispo 


\section{Strong Axis \\ Load Case E - Dynamic Extreme Operating Gust (EOG) \\ IEC 61400-2 DLC 1.3}




\section{Load Case E: Overview}

- Distributed dynamic load case (including tower and rotor loads)

- Small Wind Turbine Class (SWTC) 3 system

- Developed in accordance with IEC 61400-2 Design Load Case (DLC) 1.3 (Table 4)

- Rotor Thrust Coefficient: $C_{t}=0.5$

- Tower Drag Coefficient: $C_{d}=1.3$

- Nhat $=30$, see Appendix A for details

- Load applied in "strong axis" (the load is parallel to the plane formed by the tower, gin-pole, and strut).

- Details of the analysis parameters are summarized and defined in the attached UserInputs.xIsx file. 


\section{Load Case E: Tower Tip Wind Speed and Rotor Thrust}

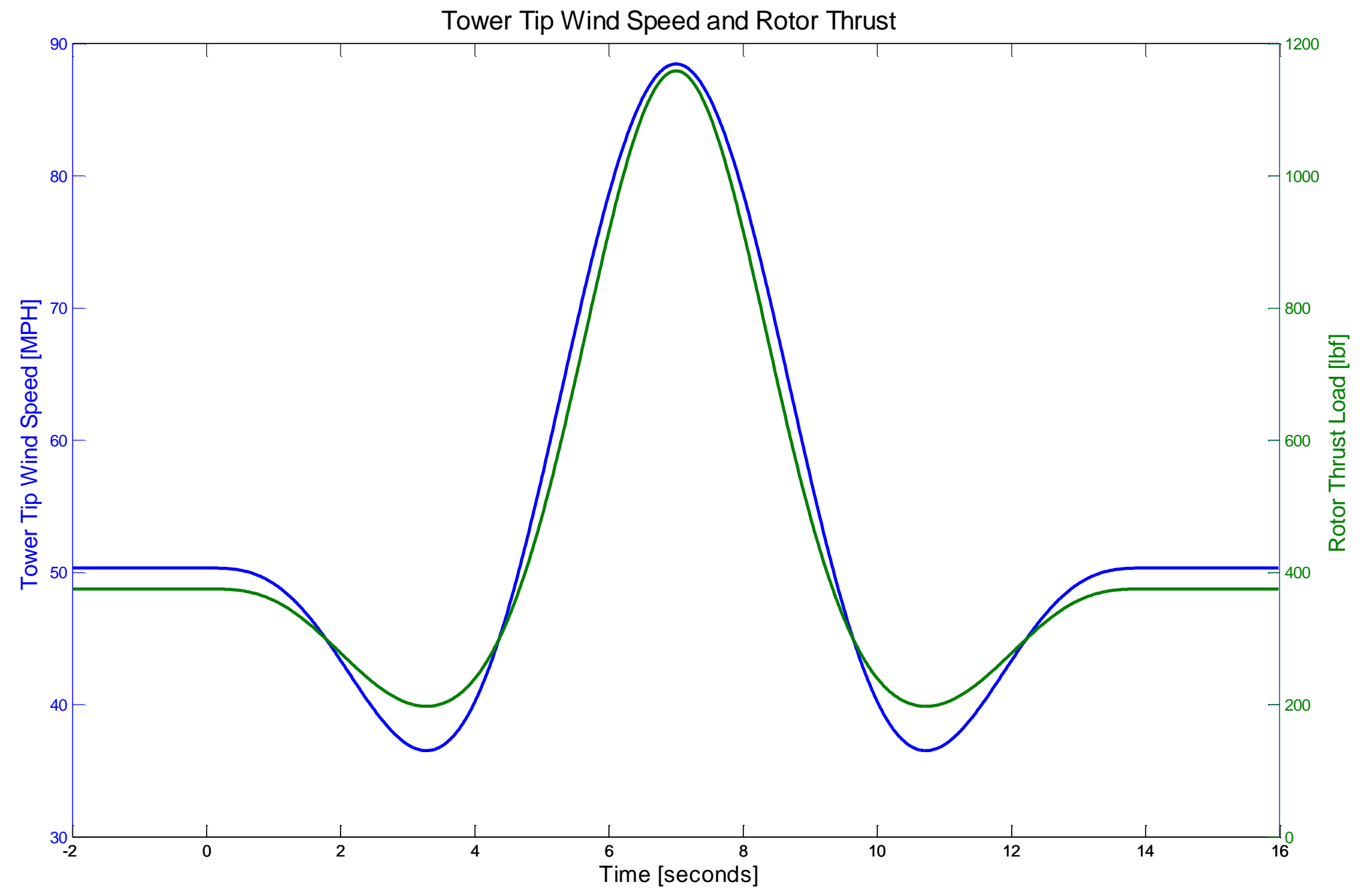

California Polytechnic State University - San Luis Obispo 


\section{Load Case E: Tower Tip Transient Deflection}

Transverse Tower Tip Deflection

Max Disp: 28.57 in

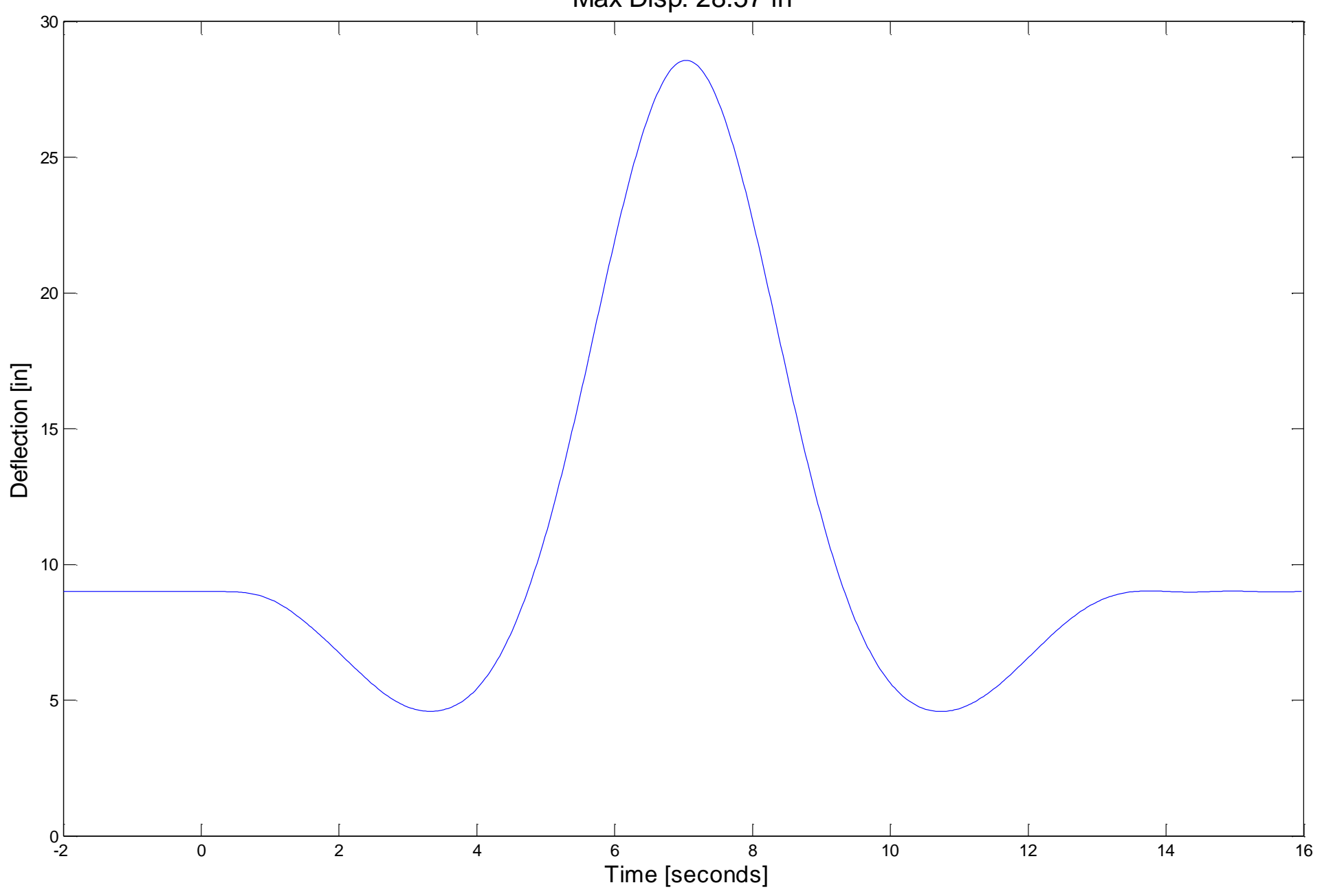

California Polytechnic State University - San Luis Obispo 


\section{Load Case E: Tower von Mises Stress Profille}
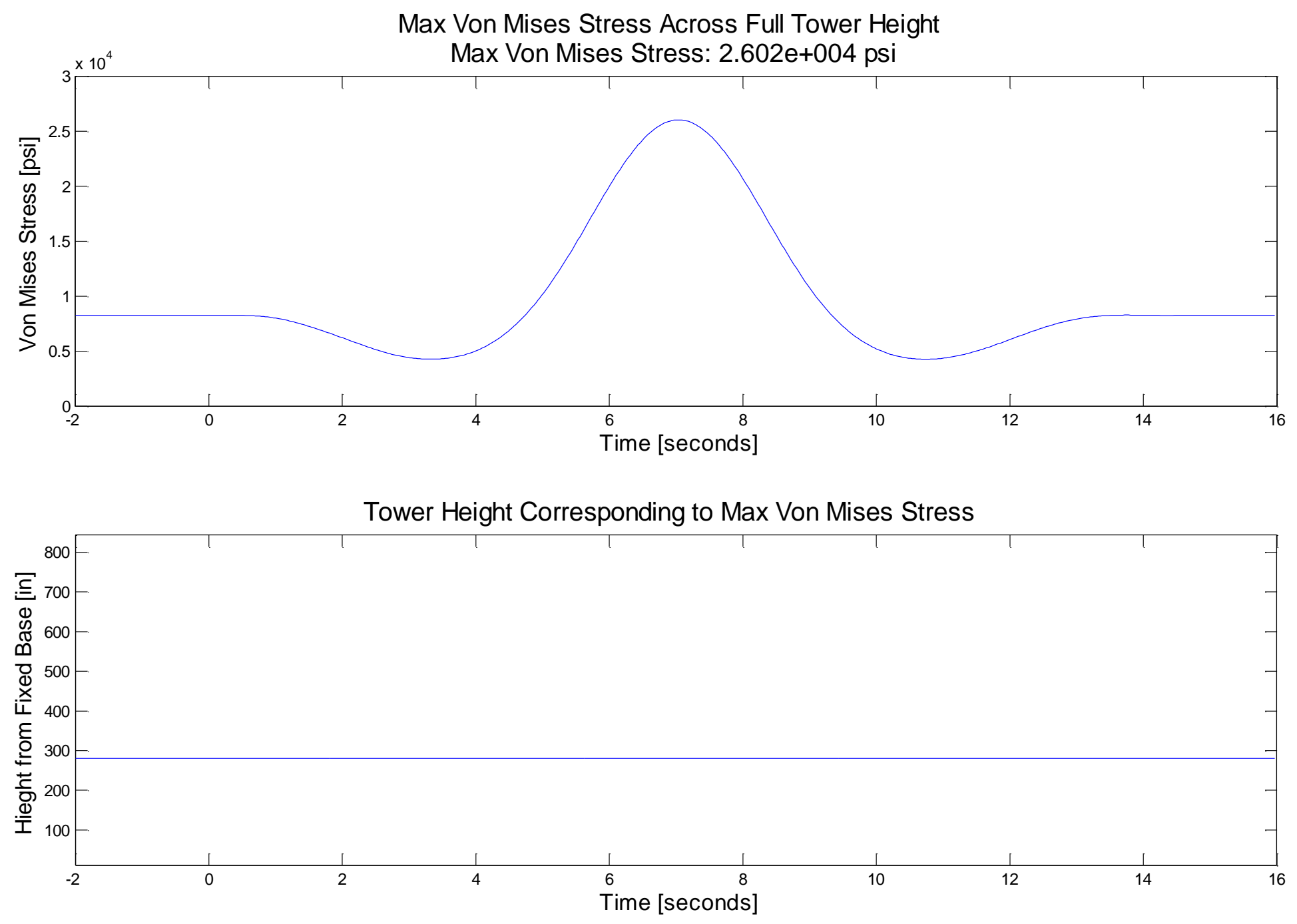


\section{Load Case E: Gin-Pole and Strut Tensille Load}

Gin-Ple and Strut Tensile Load Max Absolute Gin-Pole Load: 1.198e+004 lbf

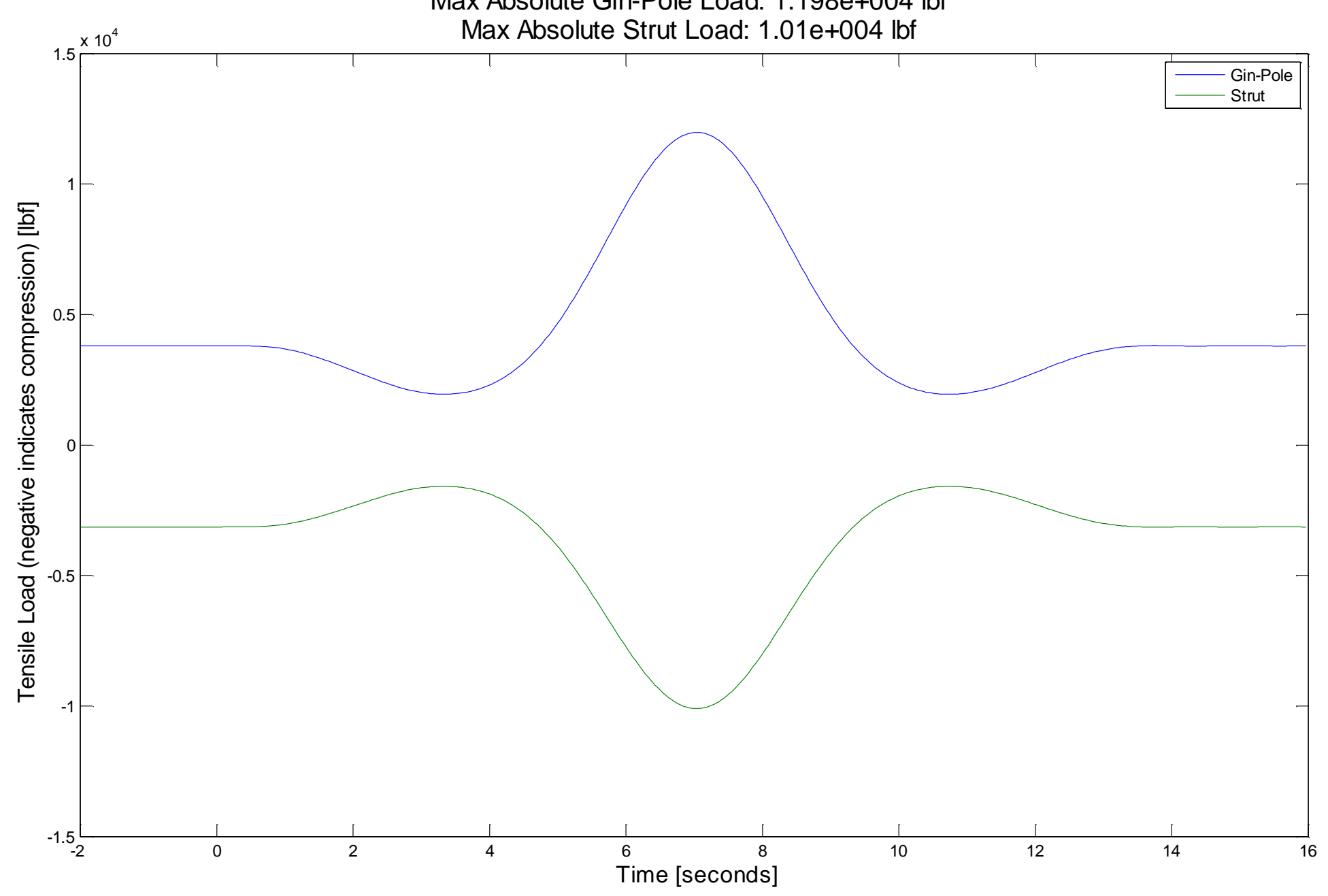




\section{Load Case E: Blade Beta Angle (relative flapwise angle)}

Blade Beta Hinge Displacement Angle Max Absolute Blade 1: 0.04555 radians Max Absolute Blade 2: 0.04556 radians Max Absolute Blade 3: 0.04556 radians

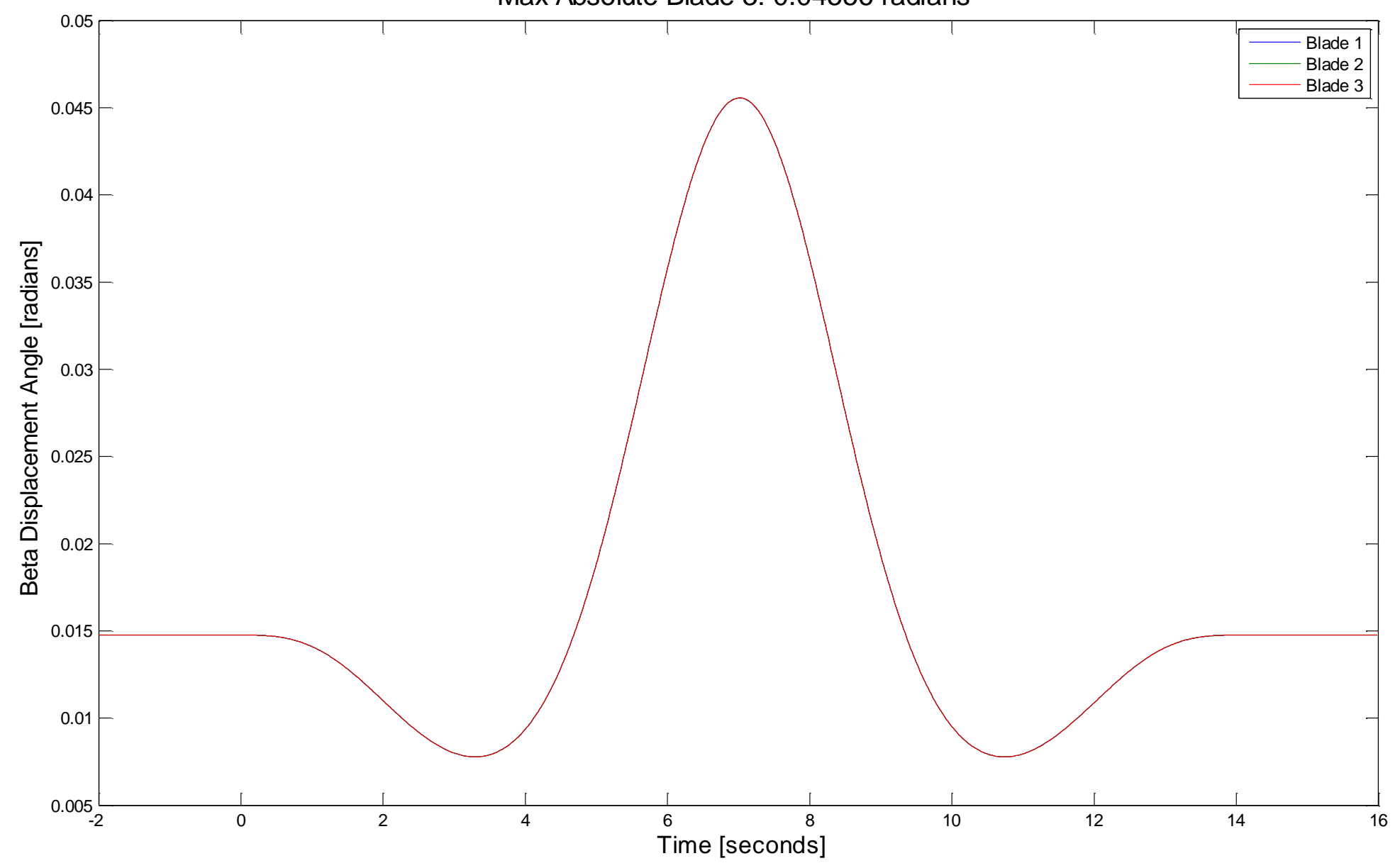




\section{Load Case E: Tower Displacement Profille at 7 Seconds}

Transient Small Wind Turbine Tower Structural Analysis with Coupled Rotor Dynamic Interaction

Transverse Tower Deflection

Max Disp: 28.57 in, at hight: 844.5 in

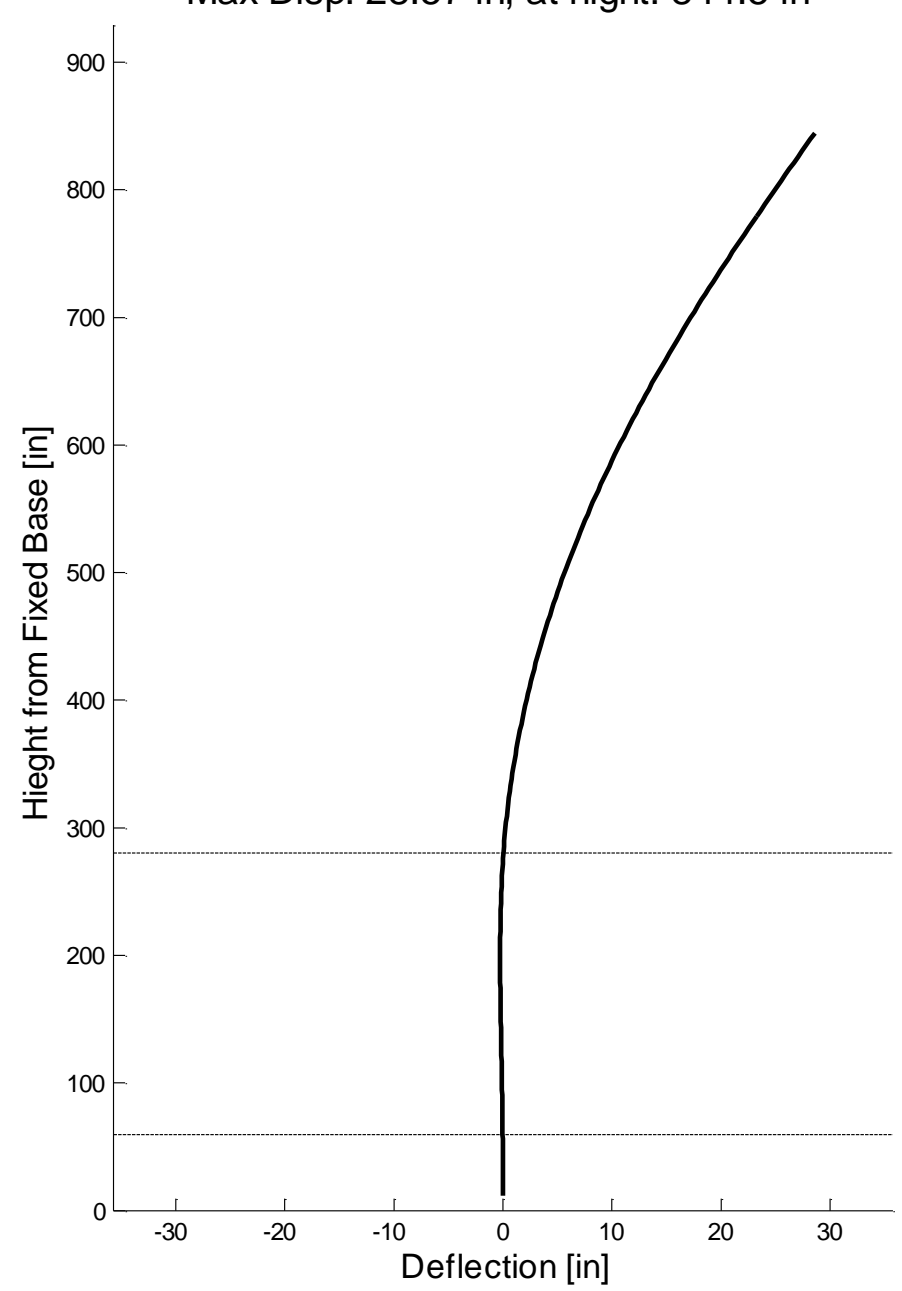

Axial Tower Deflection

Max Disp: 0.002644 in, at hight: 280.3 in

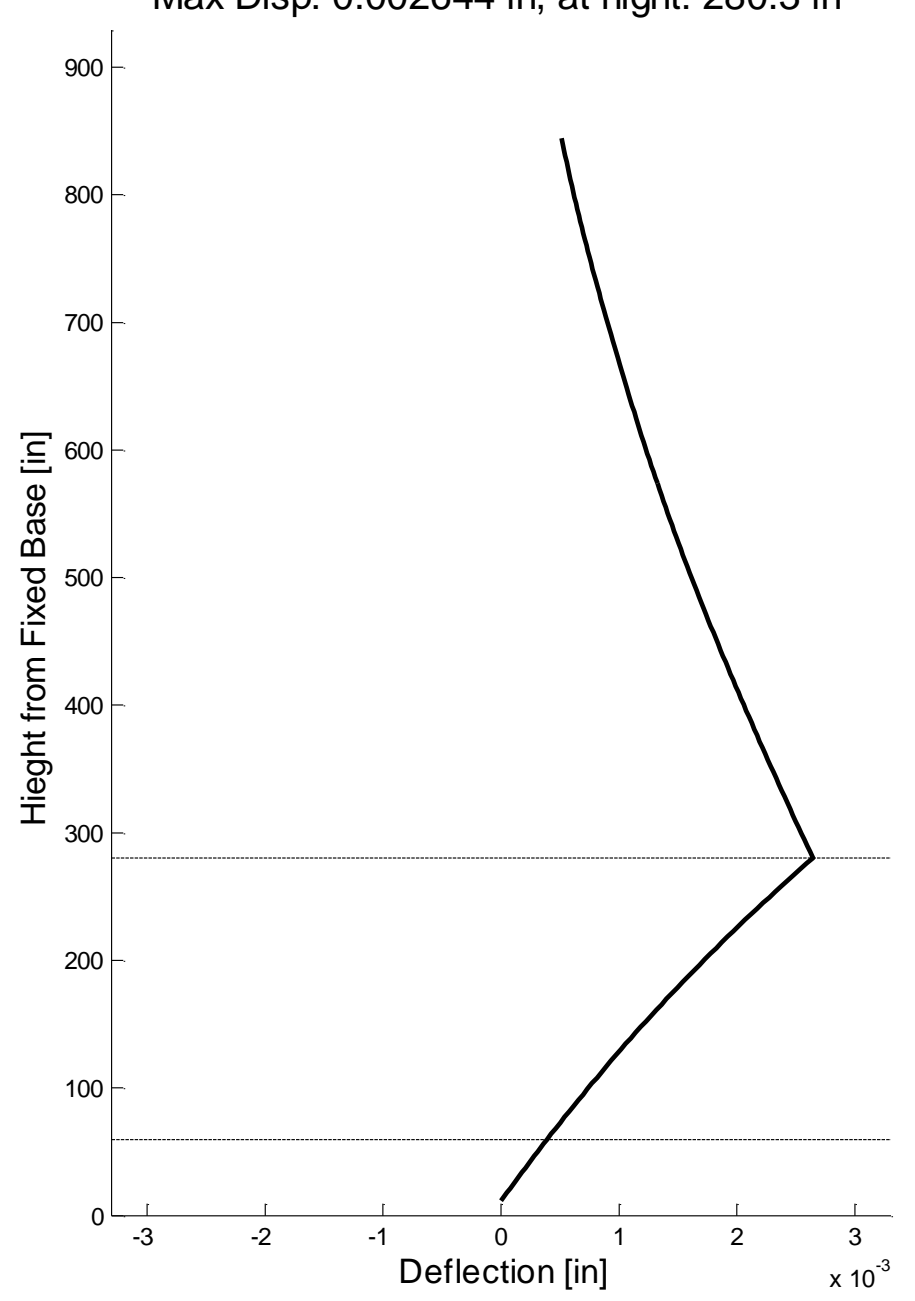




\section{Load Case E: Tower Principle Stresses at 7 Seconds}

Transient Small Wind Turbine Tower Structural Analysis with Coupled Rotor Dynamic Interaction

Tower Shear Stress Profile

Max: $867.5 \mathrm{lbf} / \mathrm{in}^{2}$, at hight: $43.5 \mathrm{in}$

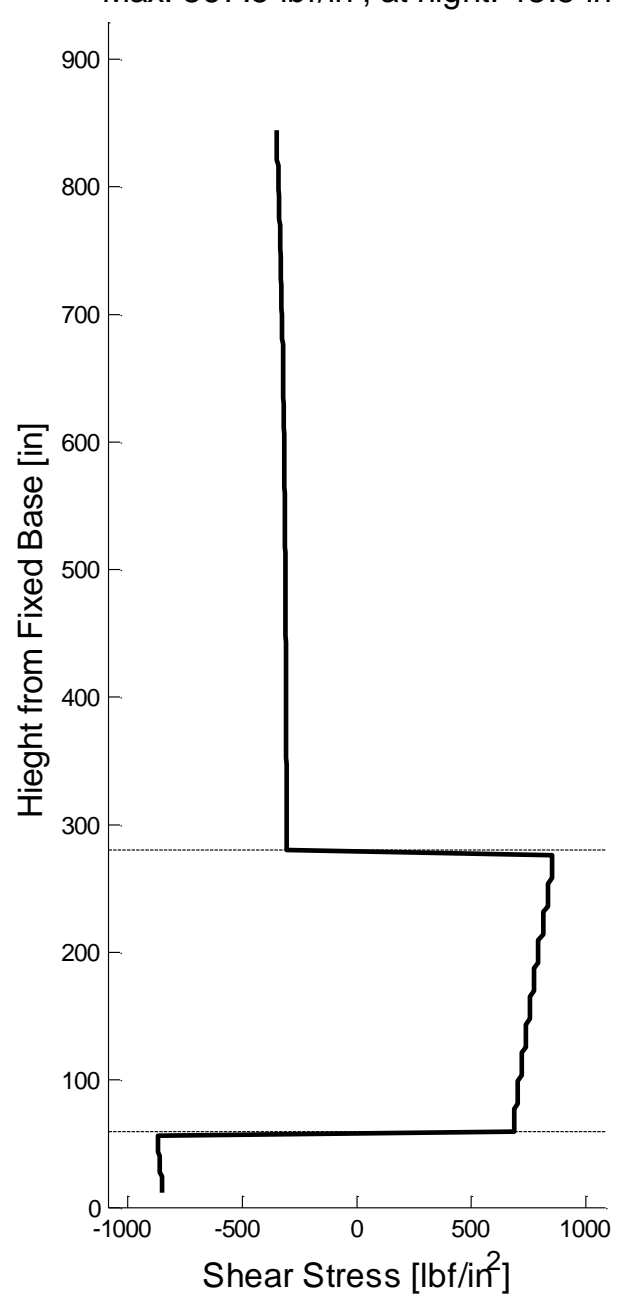

Tower Bending Stress Profile

Max: $2.586 \mathrm{e}+004 \mathrm{lbf} / \mathrm{ir}^{2}$, at hight: $280.3 \mathrm{in}$

$$
900
$$

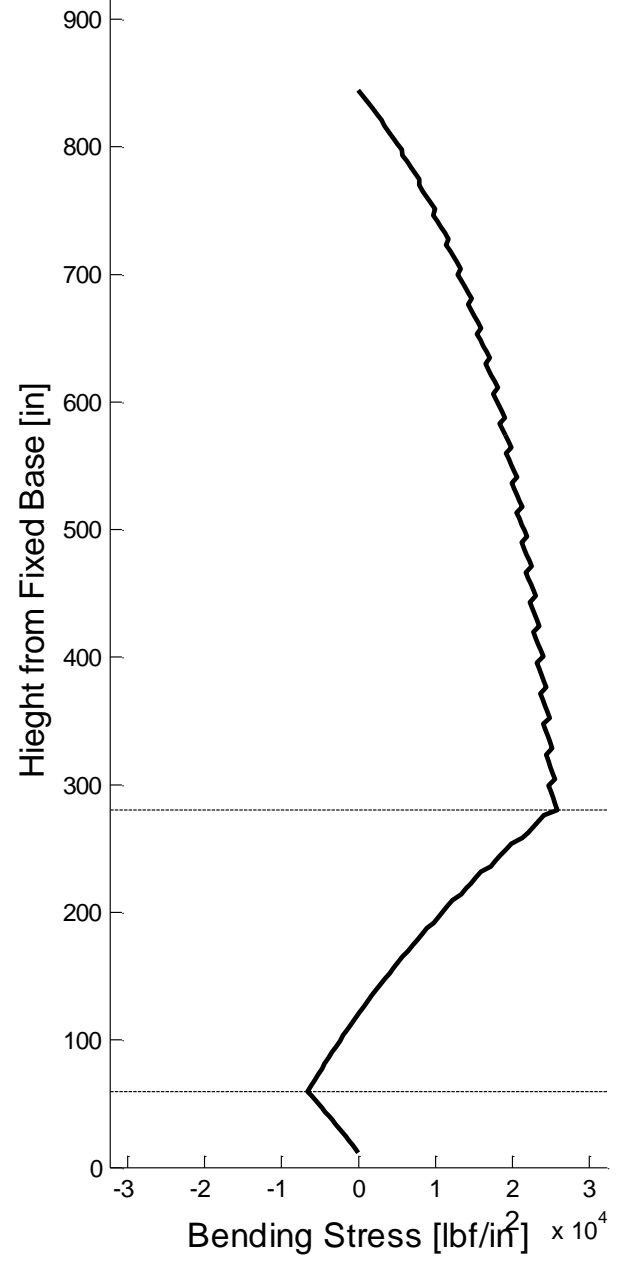

Tower Axial Stress Profile

Max: $352.6 \mathrm{lbf} / \mathrm{in}^{2}$, at hight: 258.2 in

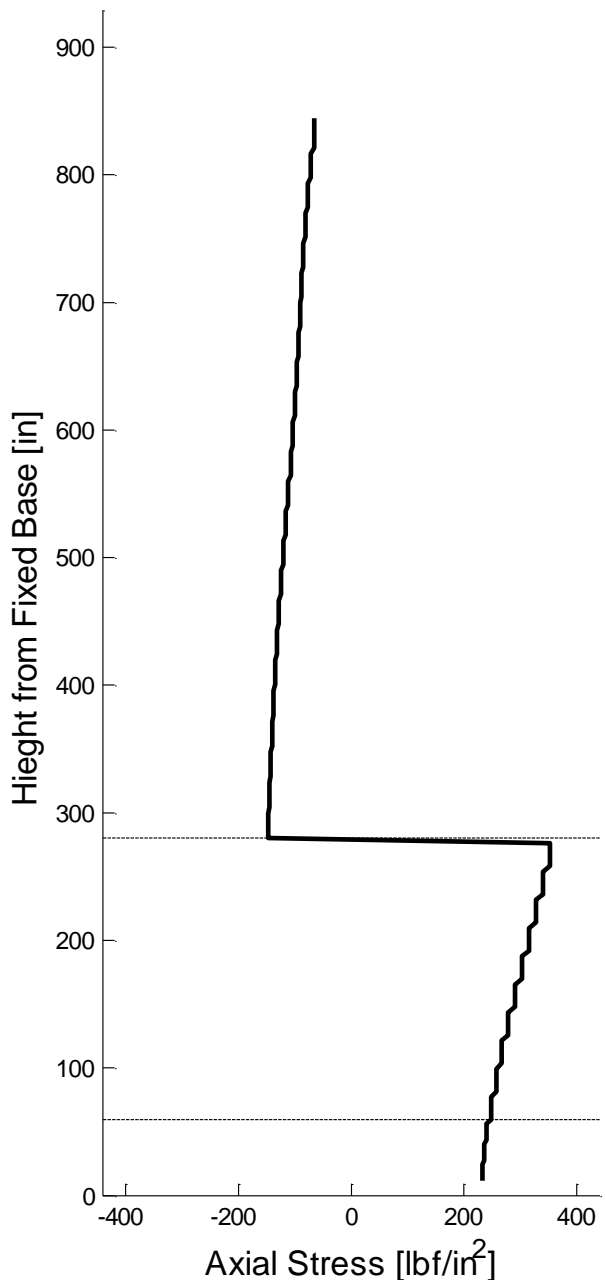




\section{Load Case E: Tower von Mises Stress at 7 Seconds}

Transient Small Wind Turbine Tower Structural Analysis with Coupled Rotor Dynamic Interaction

Max Tower von Mises Stress Profile

Max: 2.601e+004 lbf/in ${ }^{2}$, at hight: 280.3 in

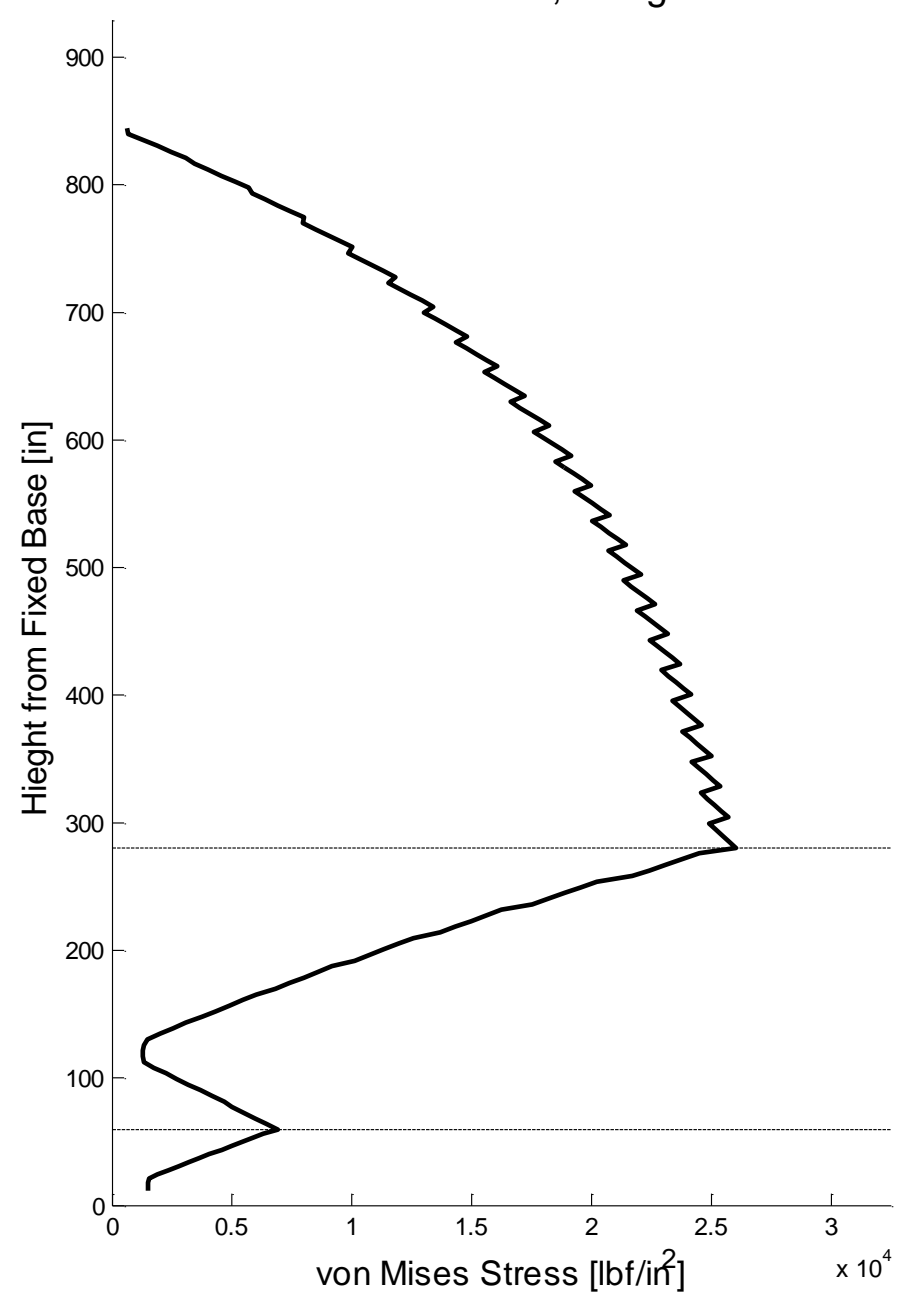

Max Tower von Mises Cross-Sectional Location (Theta) Profile

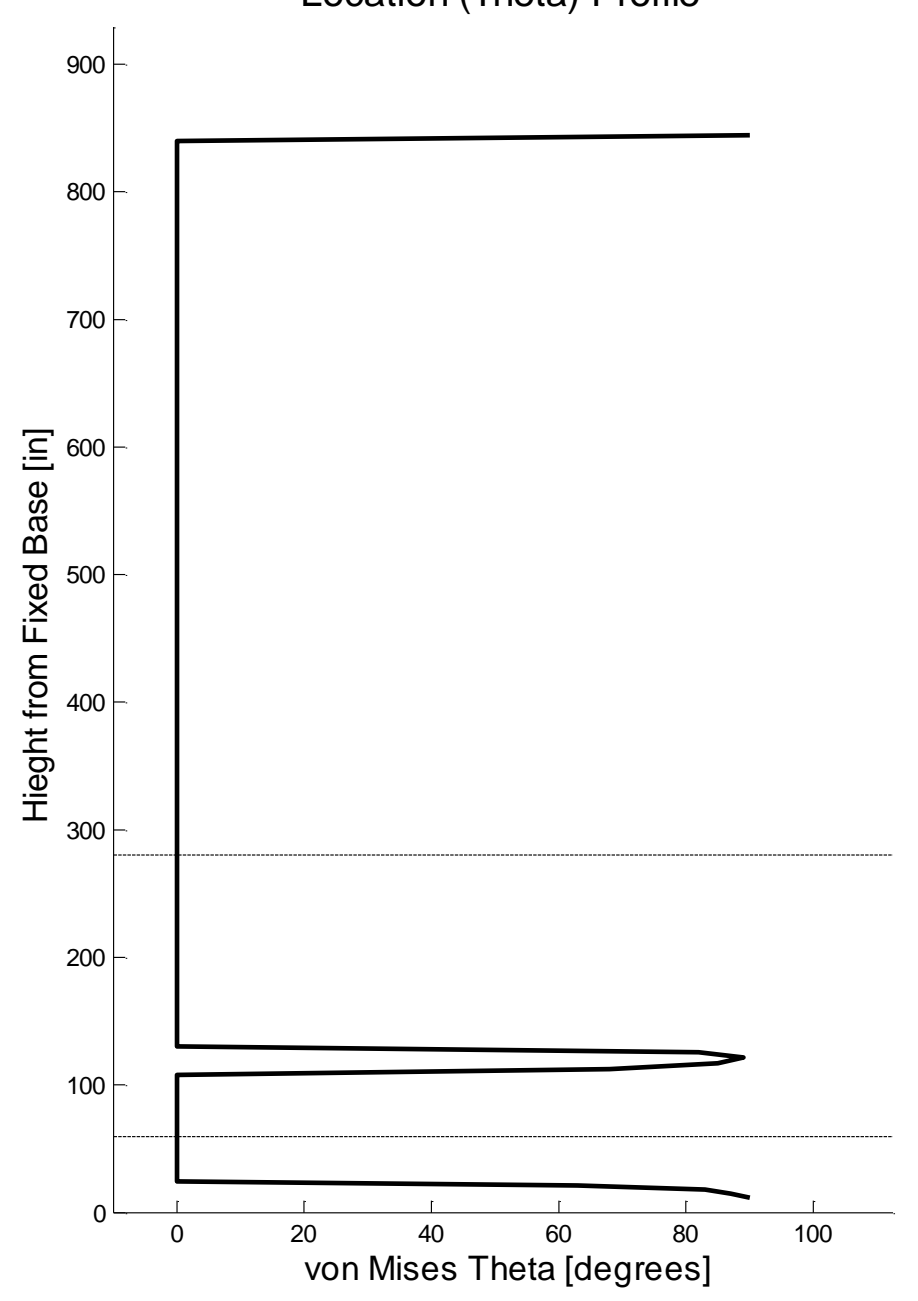




\section{Strong Axis}

Load Case F - Dynamic

Extreme Coherent Gust

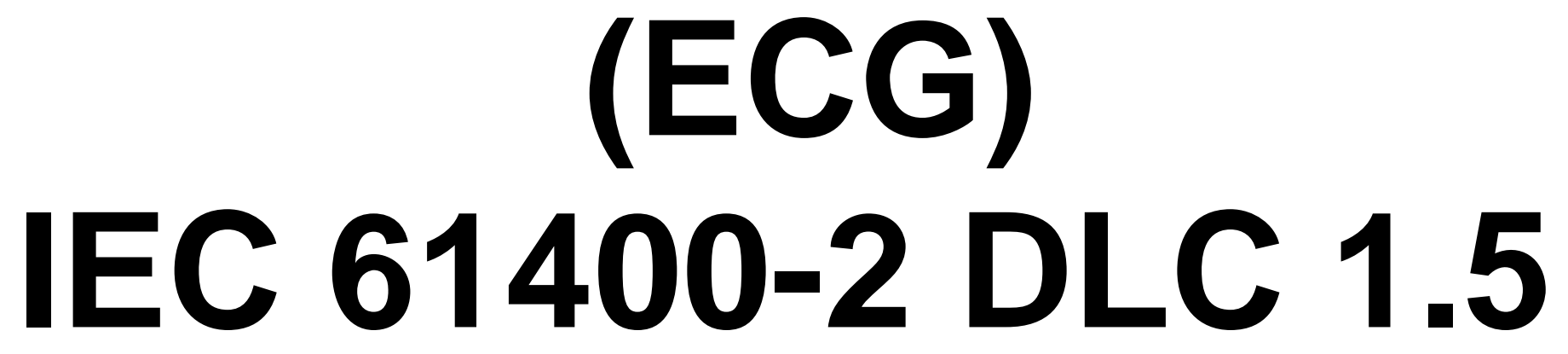




\section{Load Case F: Overview}

- Distributed dynamic load case (including tower and rotor loads)

- Small Wind Turbine Class (SWTC) 3 system

- Developed in accordance with IEC 61400-2 Design Load Case (DLC) 1.5 (Table 4)

- Rotor Thrust Coefficient: $C_{t}=0.5$

- Tower Drag Coefficient: $C_{d}=1.3$

- Nhat $=30$, see Appendix A for details

- Load applied in "strong axis" (the load is parallel to the plane formed by the tower, gin-pole, and strut).

- Details of the analysis parameters are summarized and defined in the attached UserInputs.xIsx file. 


\section{Load Case F: Tower Tip Wind Speed and Rotor Thrust}

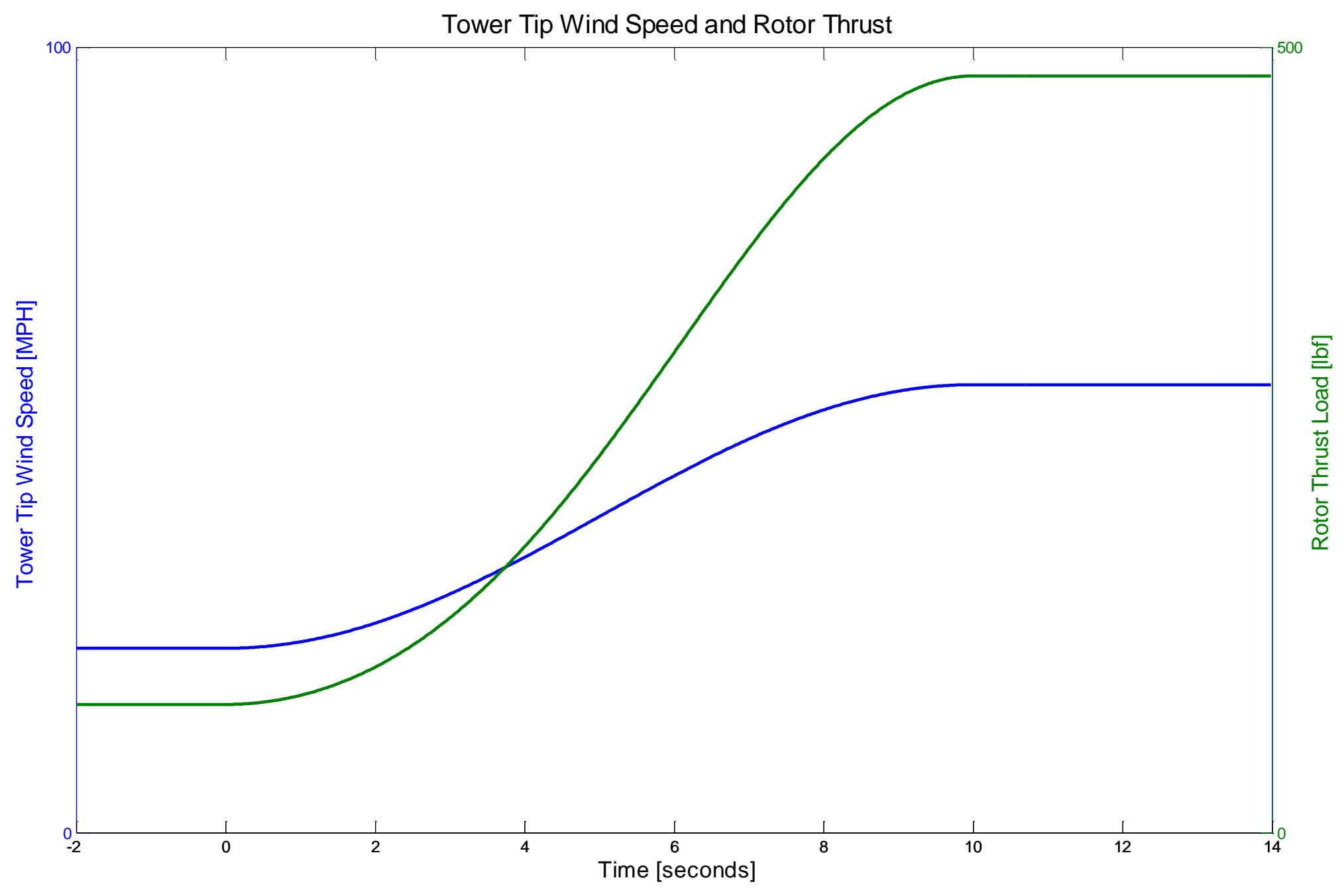

California Polytechnic State University - San Luis Obispo 


\section{Load Case F: Tower Tip Transient Deflection}

Transverse Tower Tip Deflection Max Disp: 11.7 in

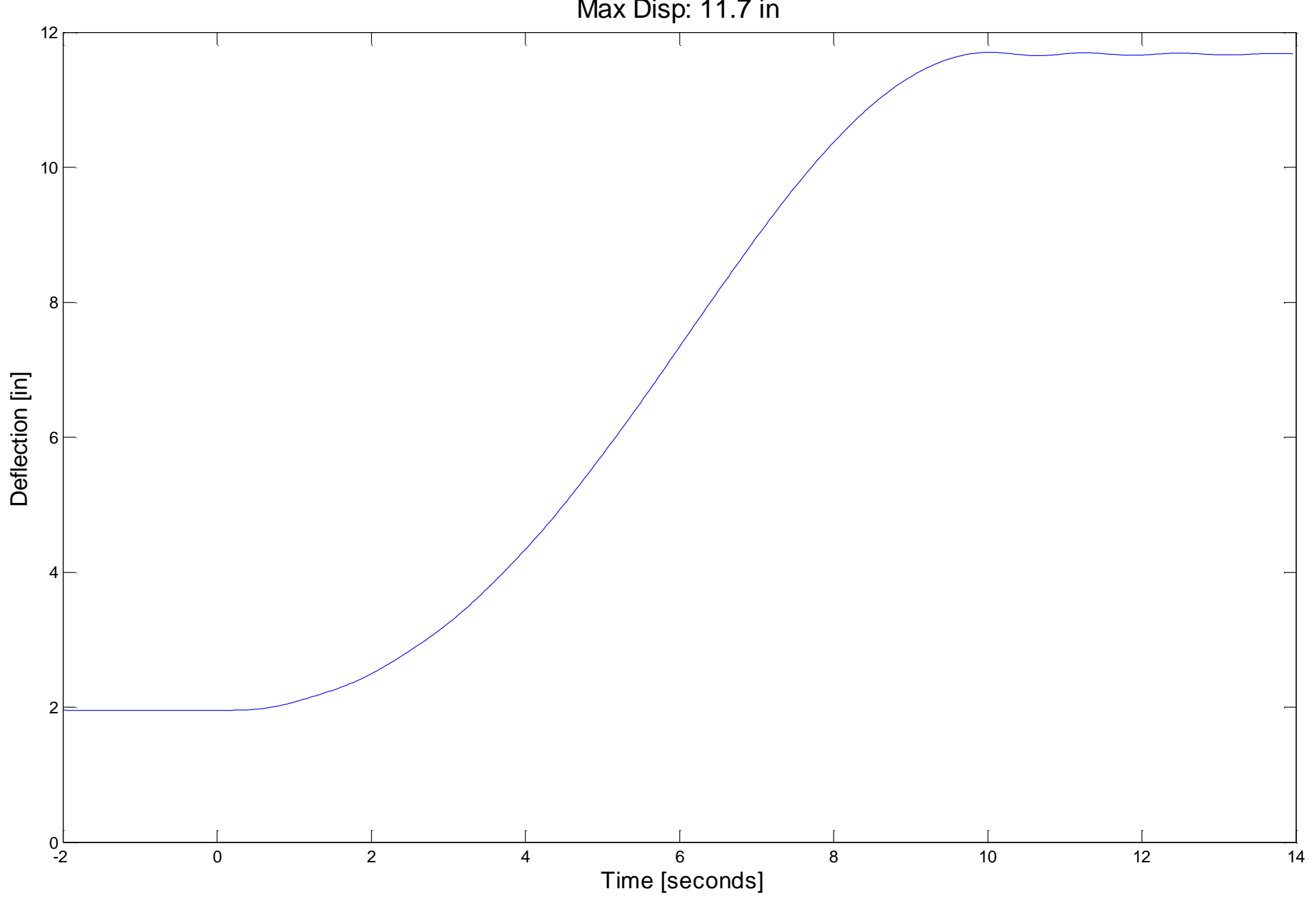

California Polytechnic State University - San Luis Obispo 


\section{Load Case F: Tower von Mises Stress Profile}

Max Von Mises Stress Across Full Tower Height Max Von Mises Stress: 1.077e+004 psi

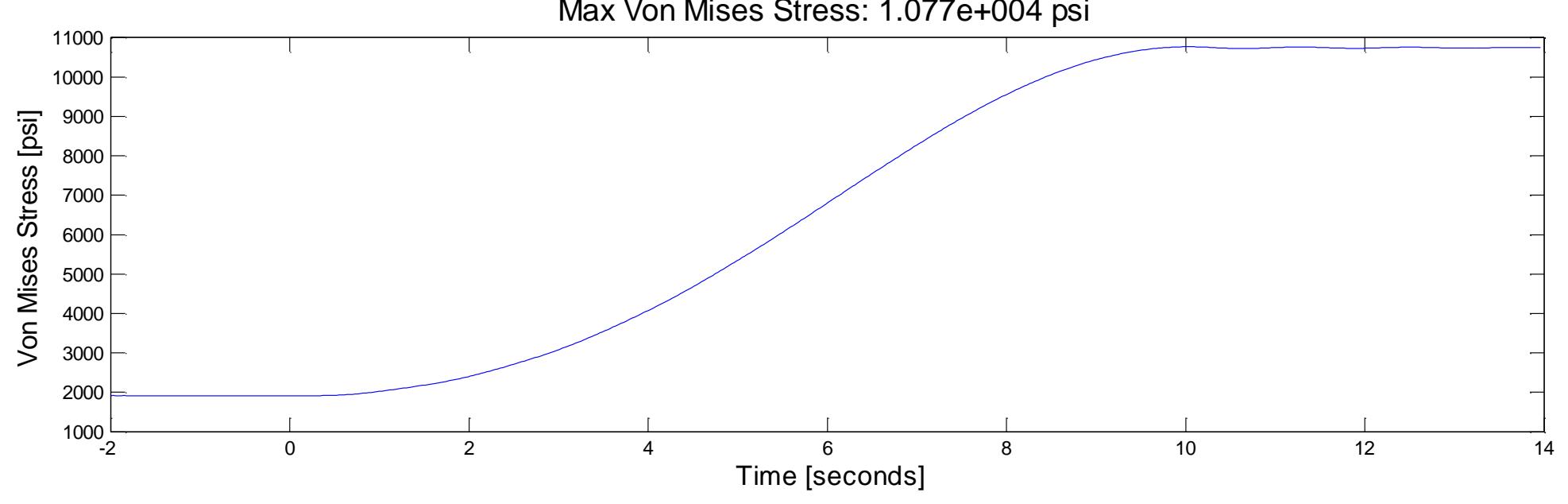

Tower Height Corresponding to Max Von Mises Stress

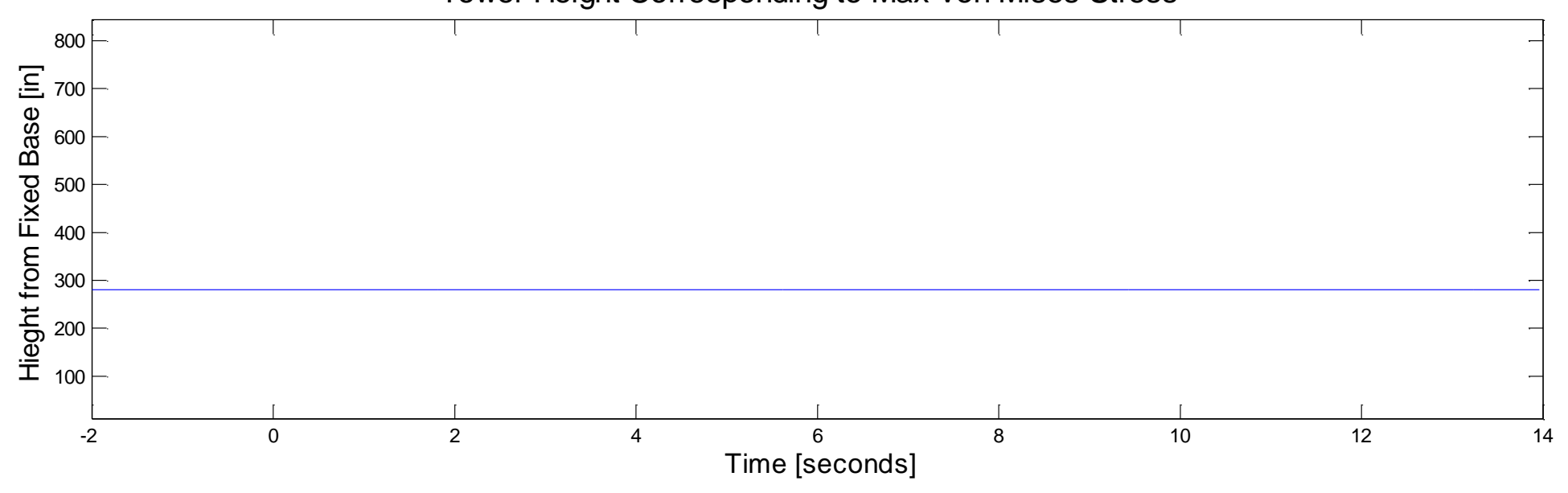




\section{Load Case F: Gin-Pole and Strut Tensile Load}

Gin-Ple and Strut Tensile Load

Max Absolute Gin-Pole Load: 4904 lbf

Max Absolute Strut Load: $4167 \mathrm{lbf}$

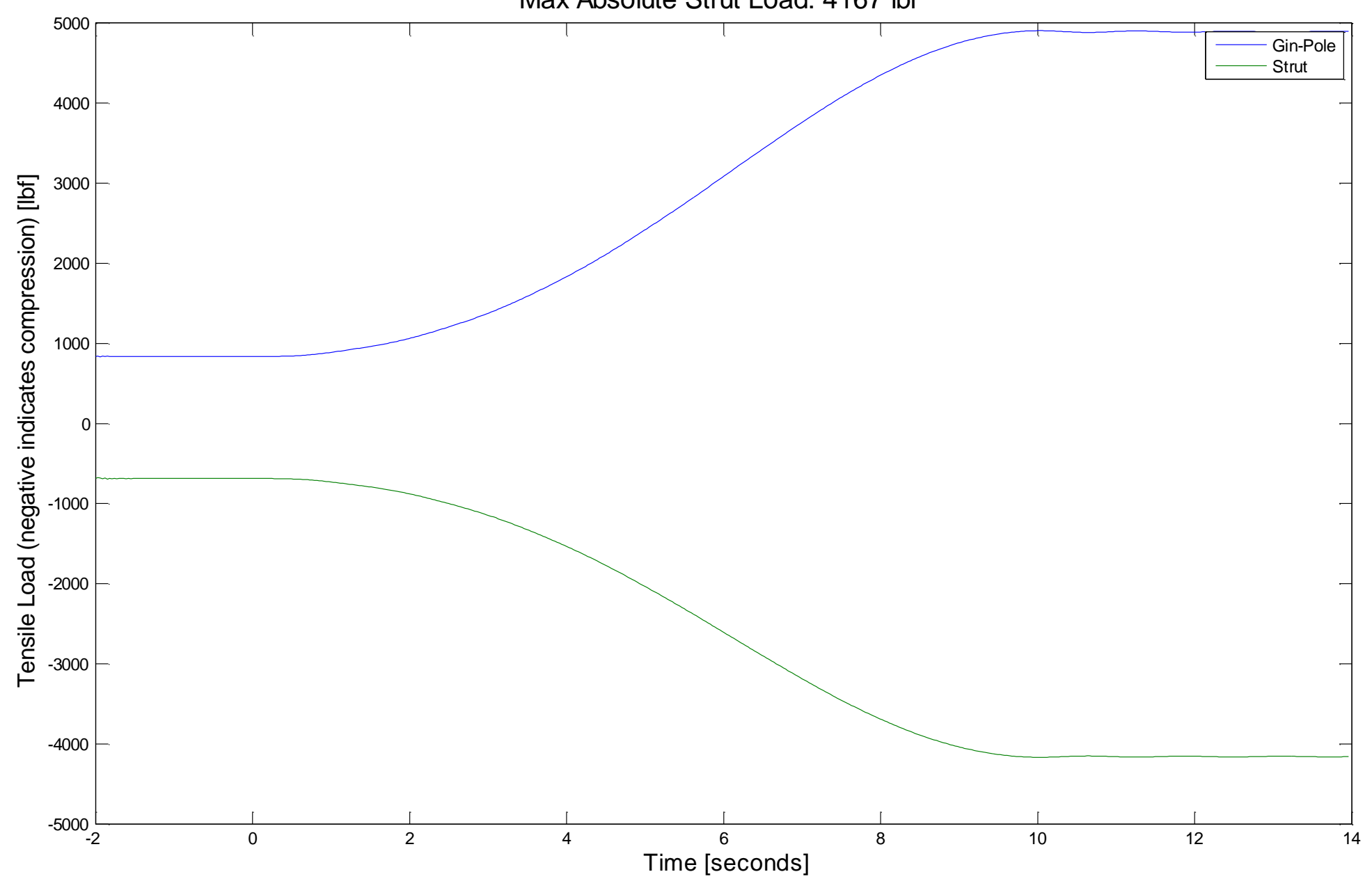

California Polytechnic State University - San Luis Obispo 


\section{Load Case F: Blade Beta Angle (relative flapwise angle)}

Blade Beta Hinge Displacement Angle Max Absolute Blade 1: 0.01895 radians Max Absolute Blade 2: 0.01895 radians

Max Absolute Blade 3: 0.01895 radians

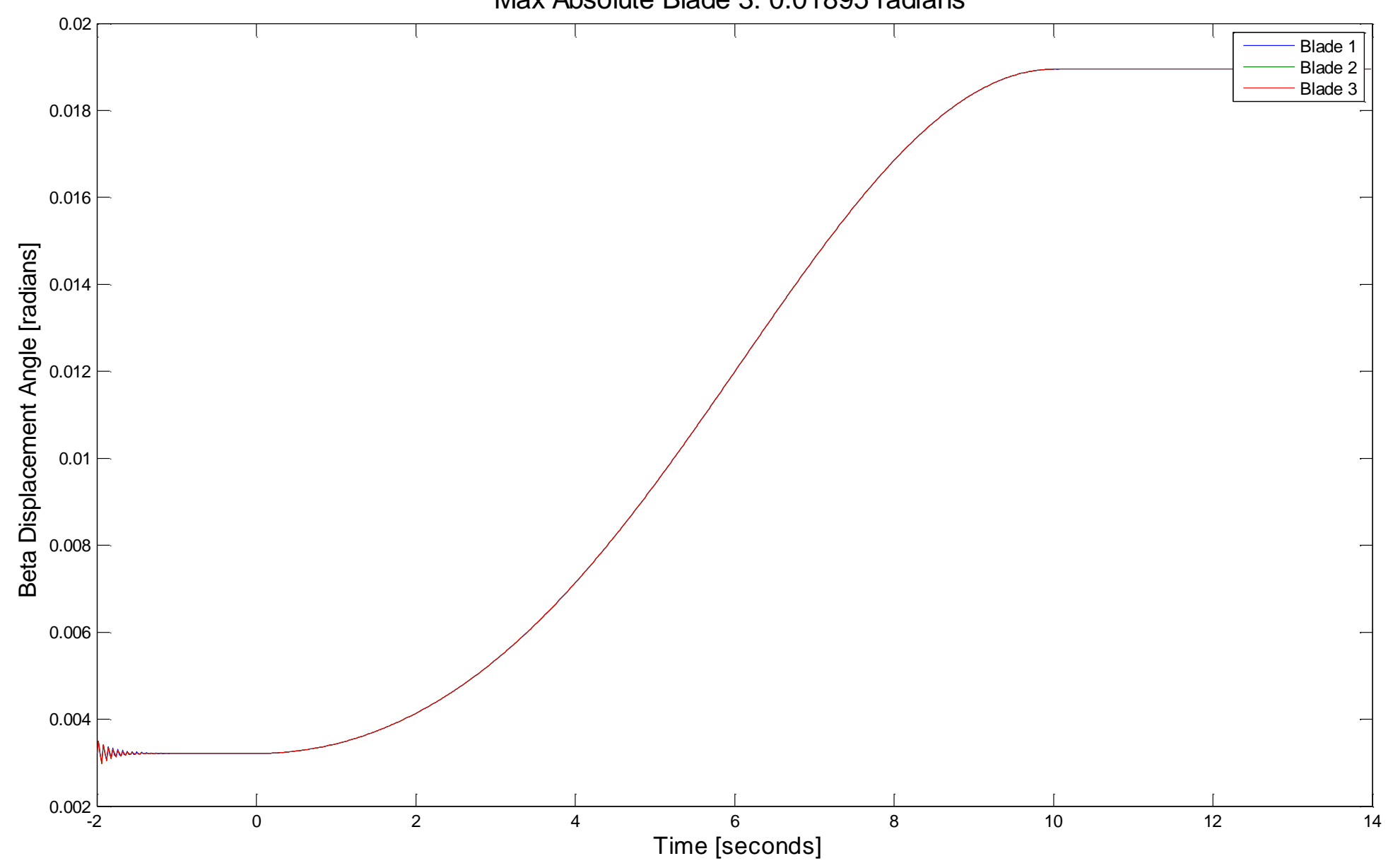




\section{Load Case F: Tower Displacement Proffle at 10 Seconds}

Transient Small Wind Turbine Tower Structural Analysis with Coupled Rotor Dynamic Interaction

Transverse Tower Deflection

Max Disp: 11.7 in, at hight: 844.5 in

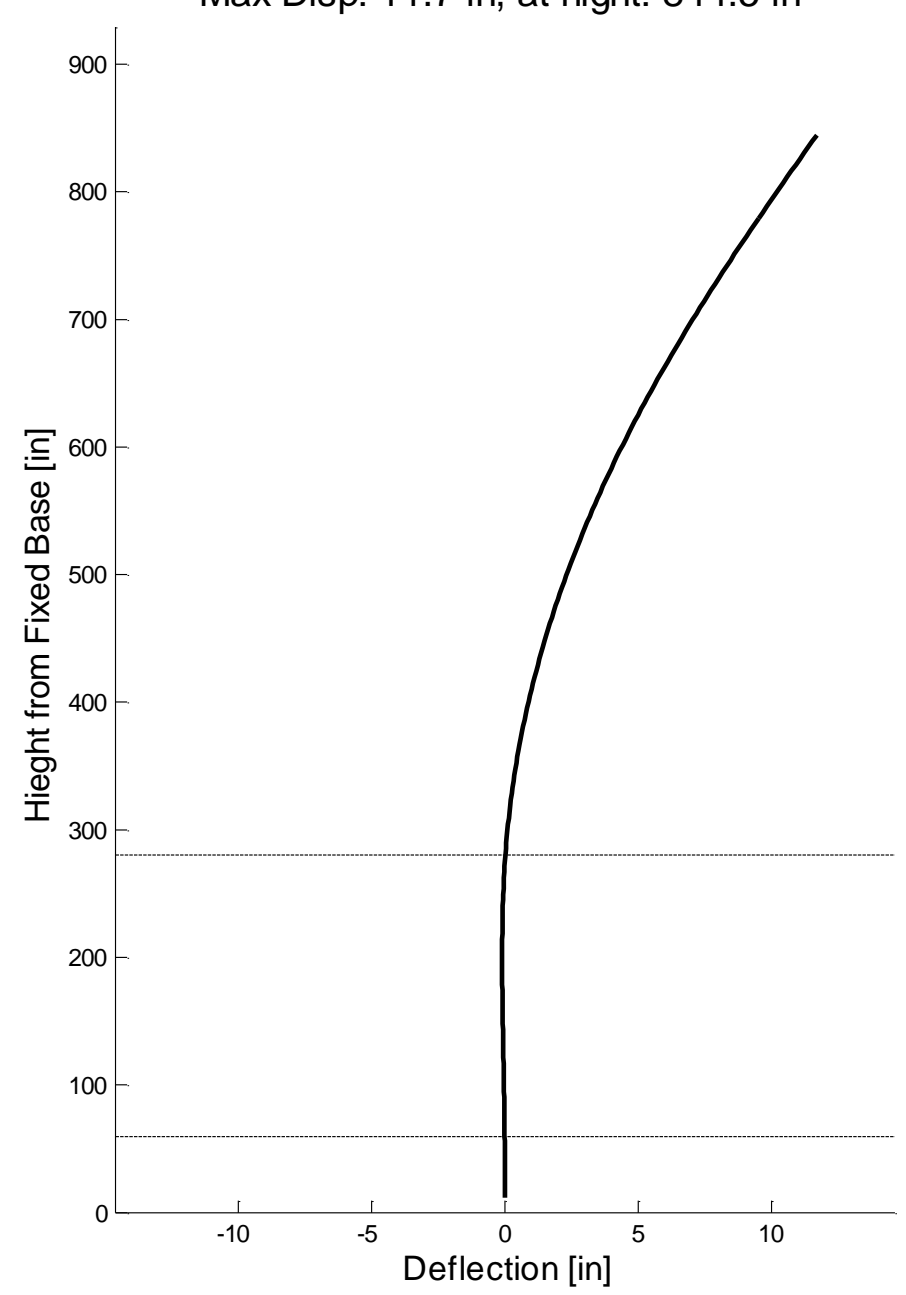

Axial Tower Deflection

Max Disp: 0.001979 in, at hight: 844.5 in

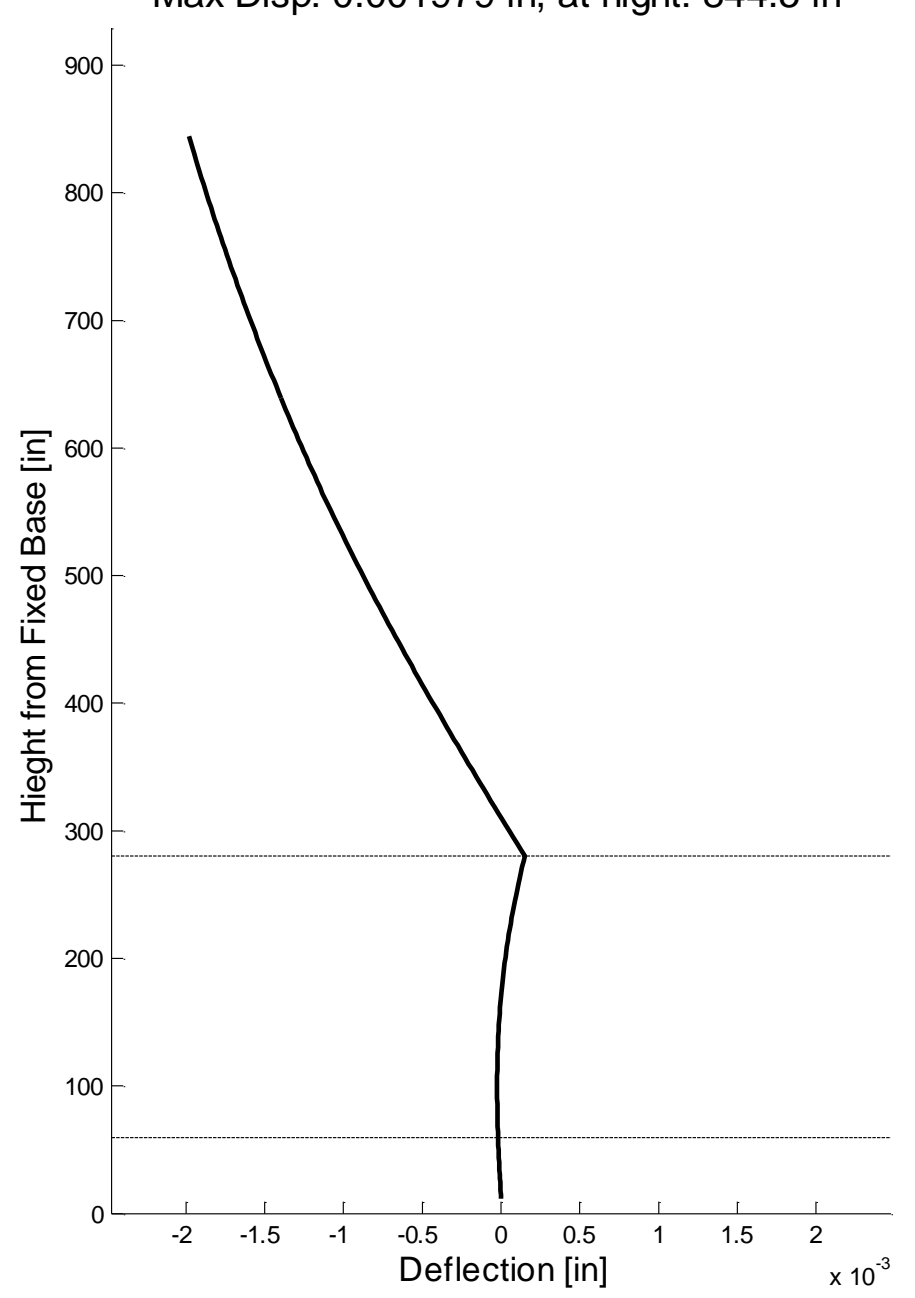




\section{Load Case F: Tower Principle Stresses at 10 Seconds}

Transient Small Wind Turbine Tower Structural Analysis with Coupled Rotor Dynamic Interaction

Tower Shear Stress Profile

Max: $356.9 \mathrm{lbf} / \mathrm{in}^{2}$, at hight: $43.5 \mathrm{in}$

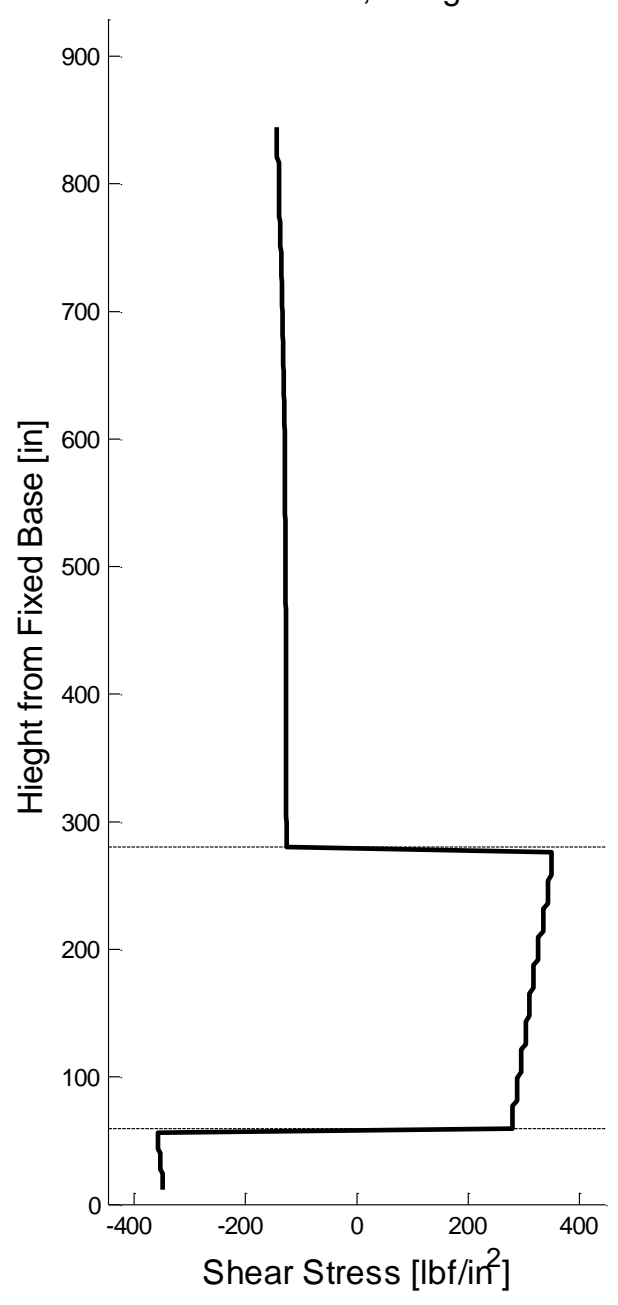

Tower Bending Stress Profile

Max: $1.062 \mathrm{e}+004 \mathrm{lbf} / \mathrm{ir}^{2}$, at hight: $280.3 \mathrm{in}$

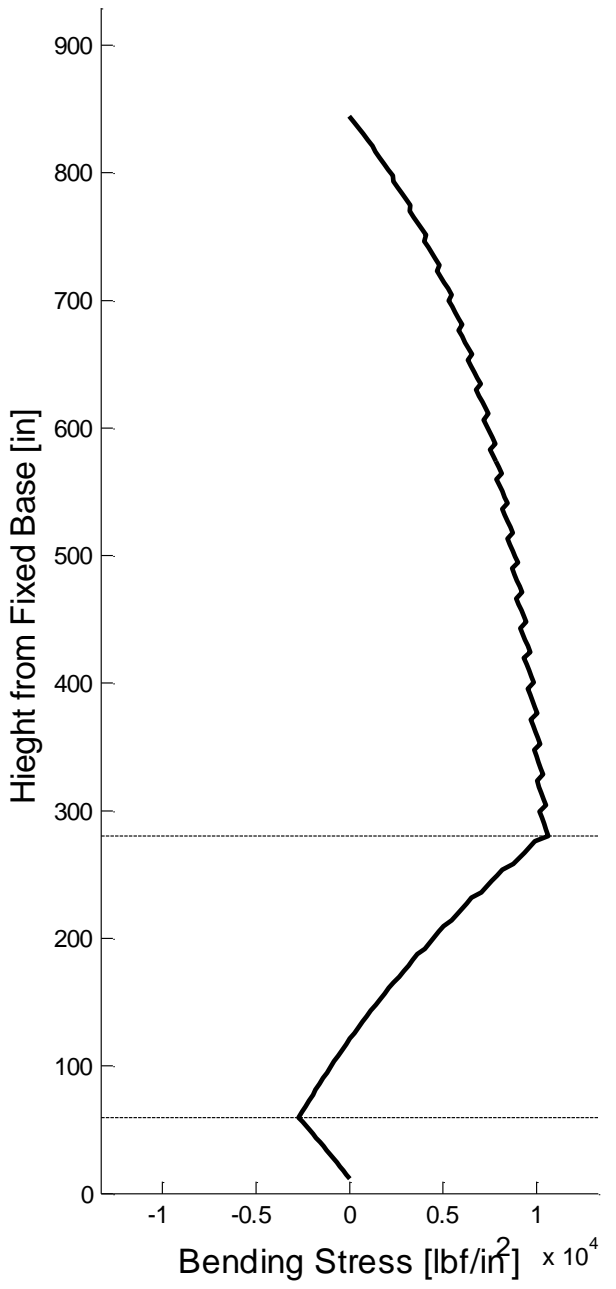

Tower Axial Stress Profile

Max: $147.3 \mathrm{lbf} / \mathrm{in}^{2}$, at hight: $280.3 \mathrm{in}$

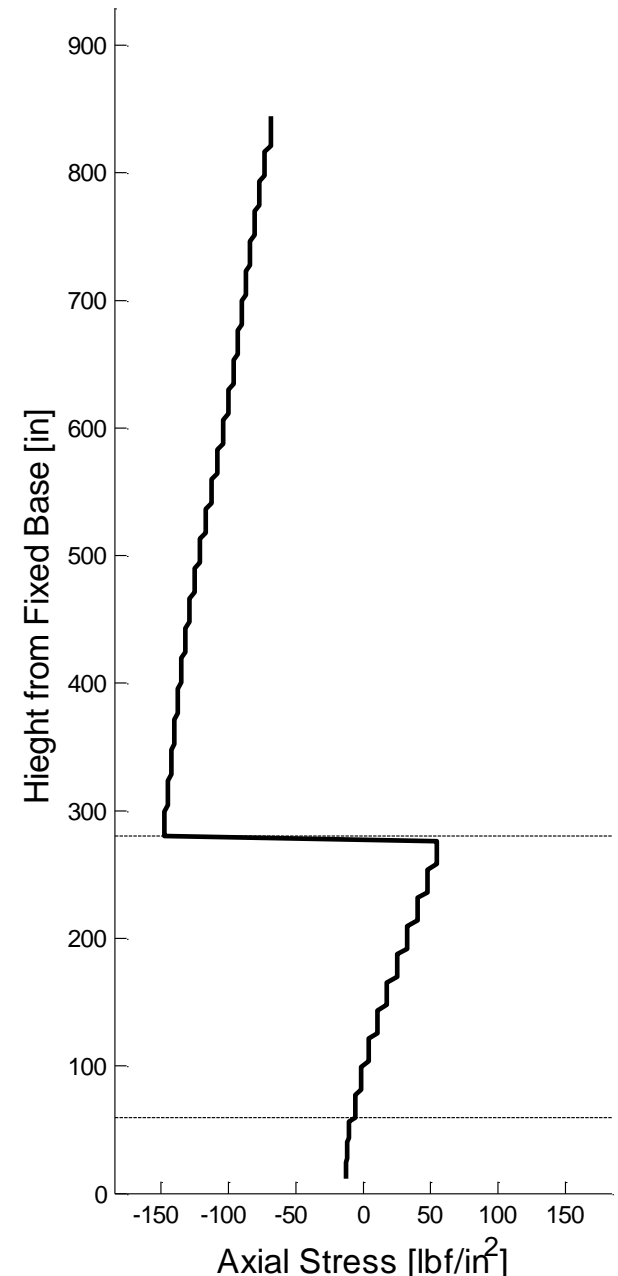




\section{Load Case F: Tower von Mises Stress at 10 Seconds}

Transient Small Wind Turbine Tower Structural Analysis with Coupled Rotor Dynamic Interaction

Max Tower von Mises Stress Profile

Max: $1.077 \mathrm{e}+004 \mathrm{lbf} / \mathrm{in}^{2}$, at hight: 280.3 in

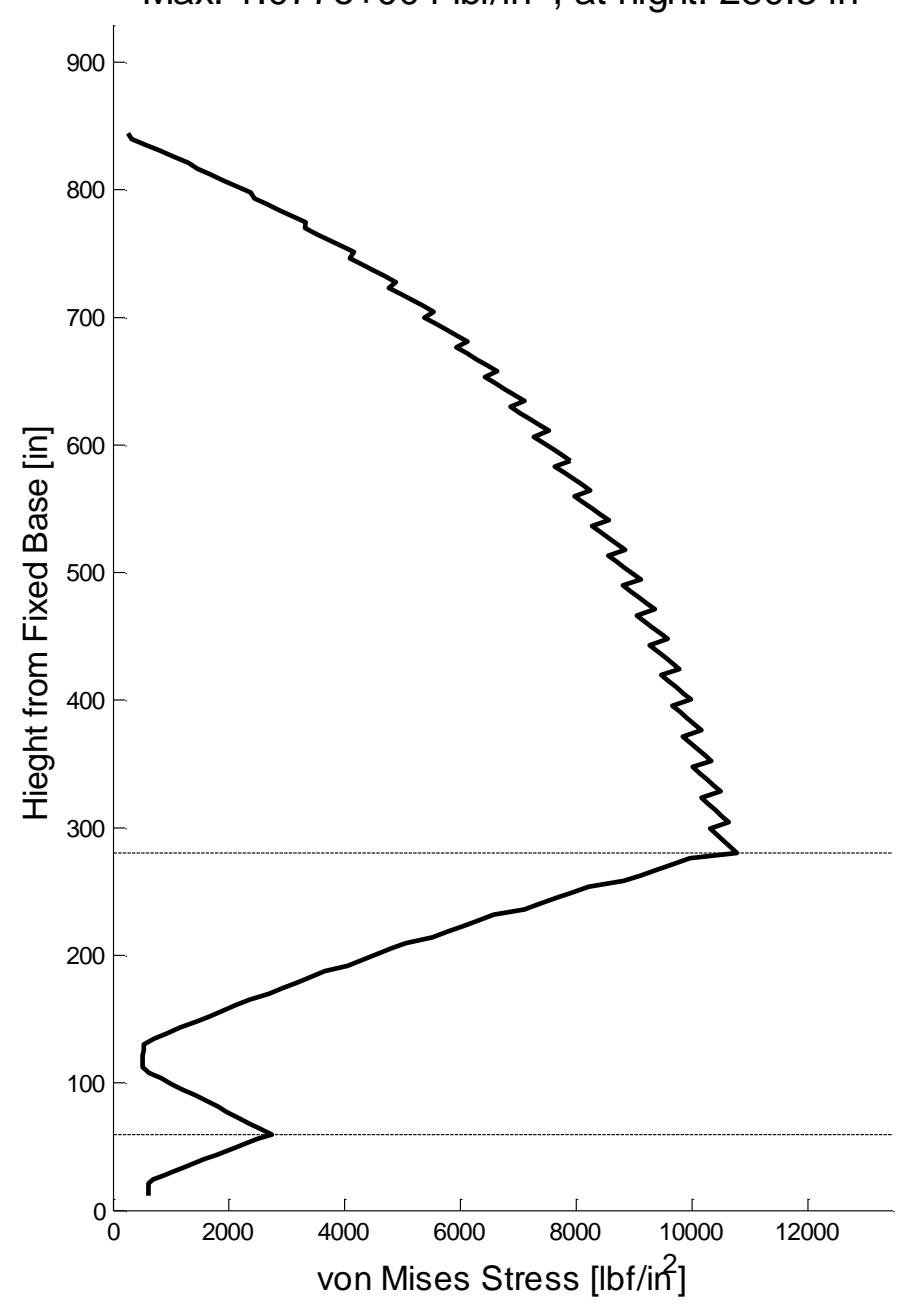

Max Tower von Mises Cross-Sectional Location (Theta) Profile

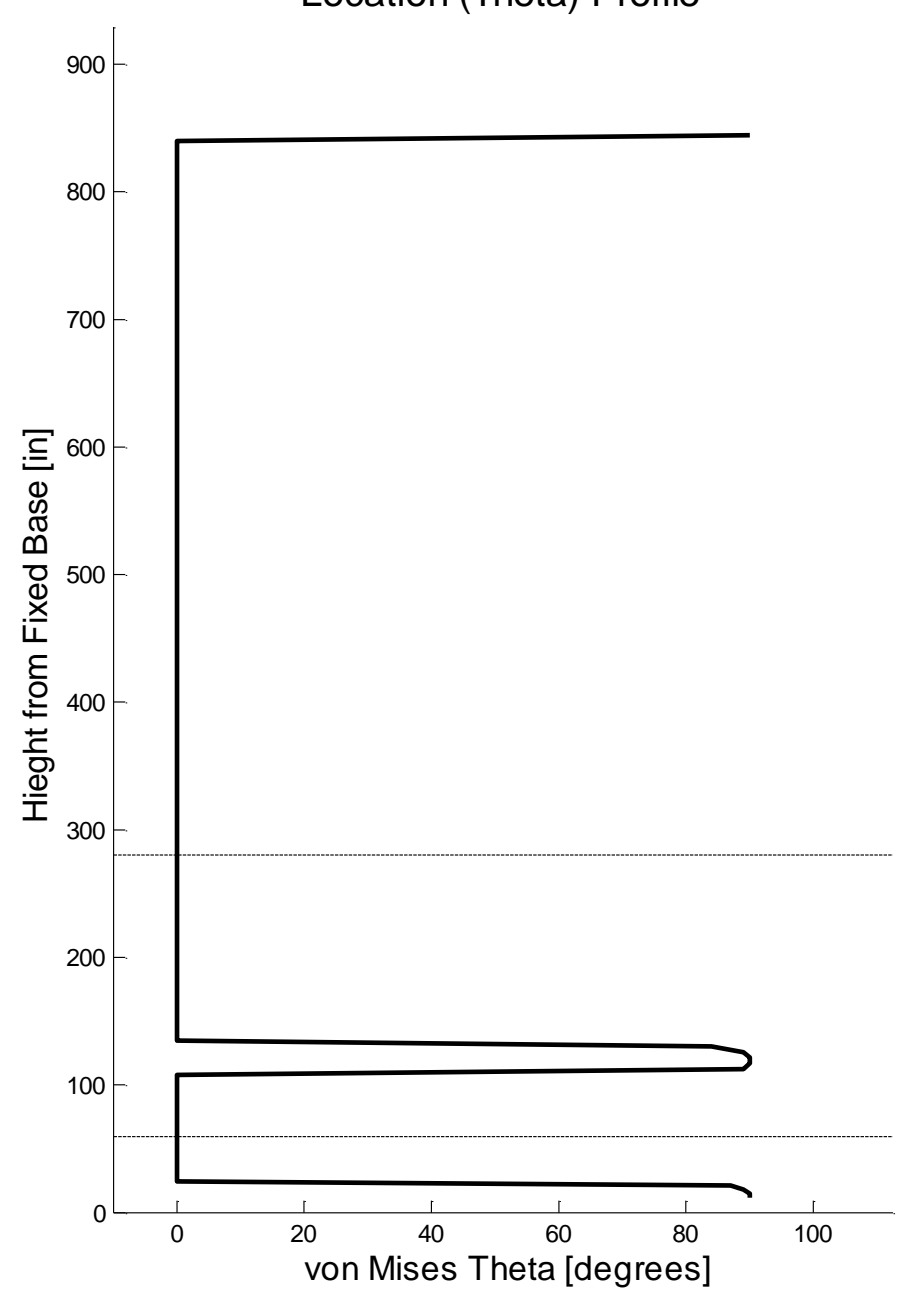




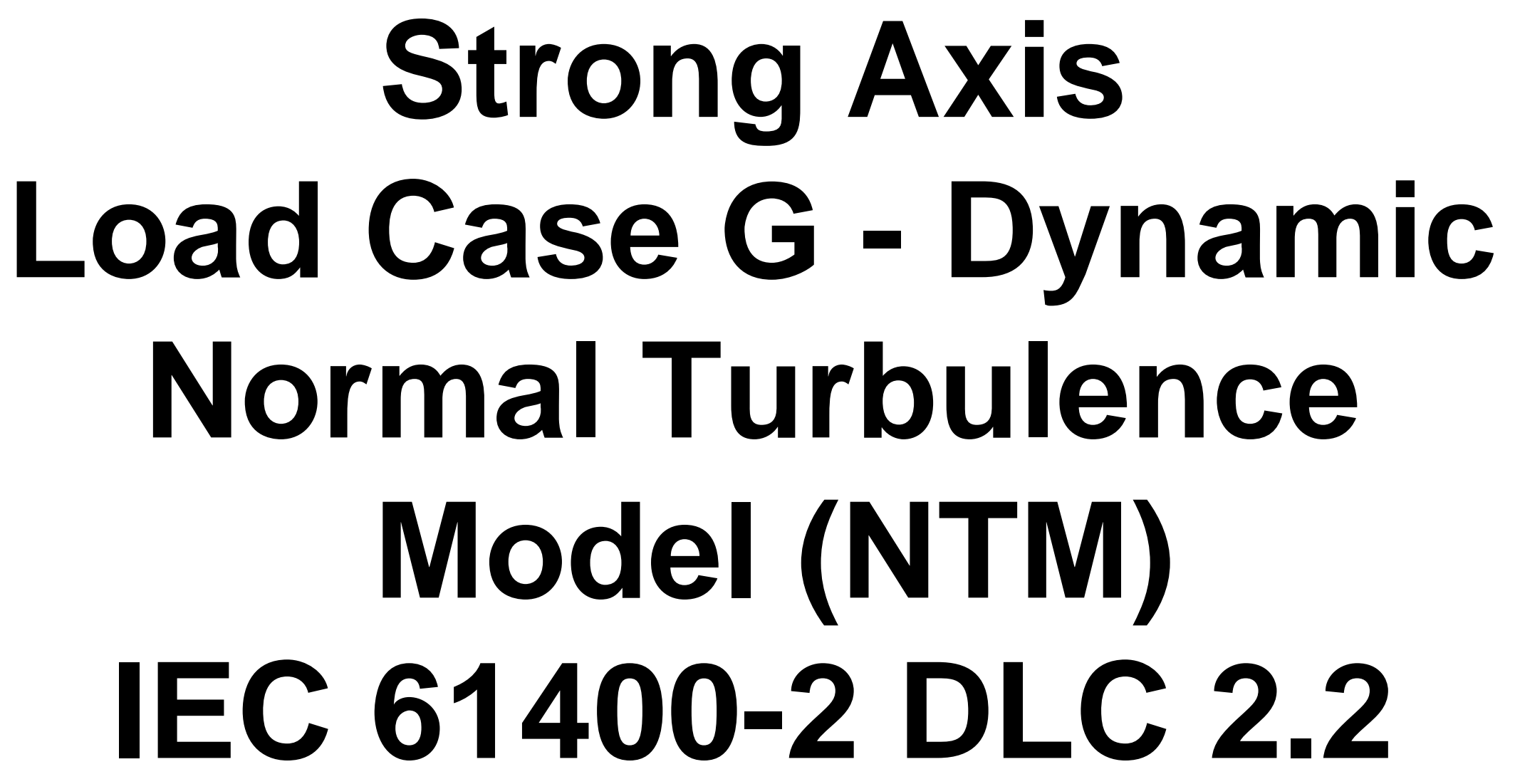




\section{Load Case G: Overview}

- Distributed dynamic load case (including tower and rotor loads)

- Small Wind Turbine Class (SWTC) 3 system

- Developed in accordance with IEC 61400-2 Design Load Case (DLC) 2.2 (Table 4)

- Rotor Thrust Coefficient: $C_{t}=0.5$

- Tower Drag Coefficient: $C_{d}=1.3$

- Nhat $=30$, see Appendix A for details

- Load applied in "strong axis" (the load is parallel to the plane formed by the tower, gin-pole, and strut).

- Details of the analysis parameters are summarized and defined in the attached UserInputs.xIsx file; note: except DSRatio was increased to 40. 


\section{Load Case G: Tower Tip Wind Speed and Rotor Thrust}

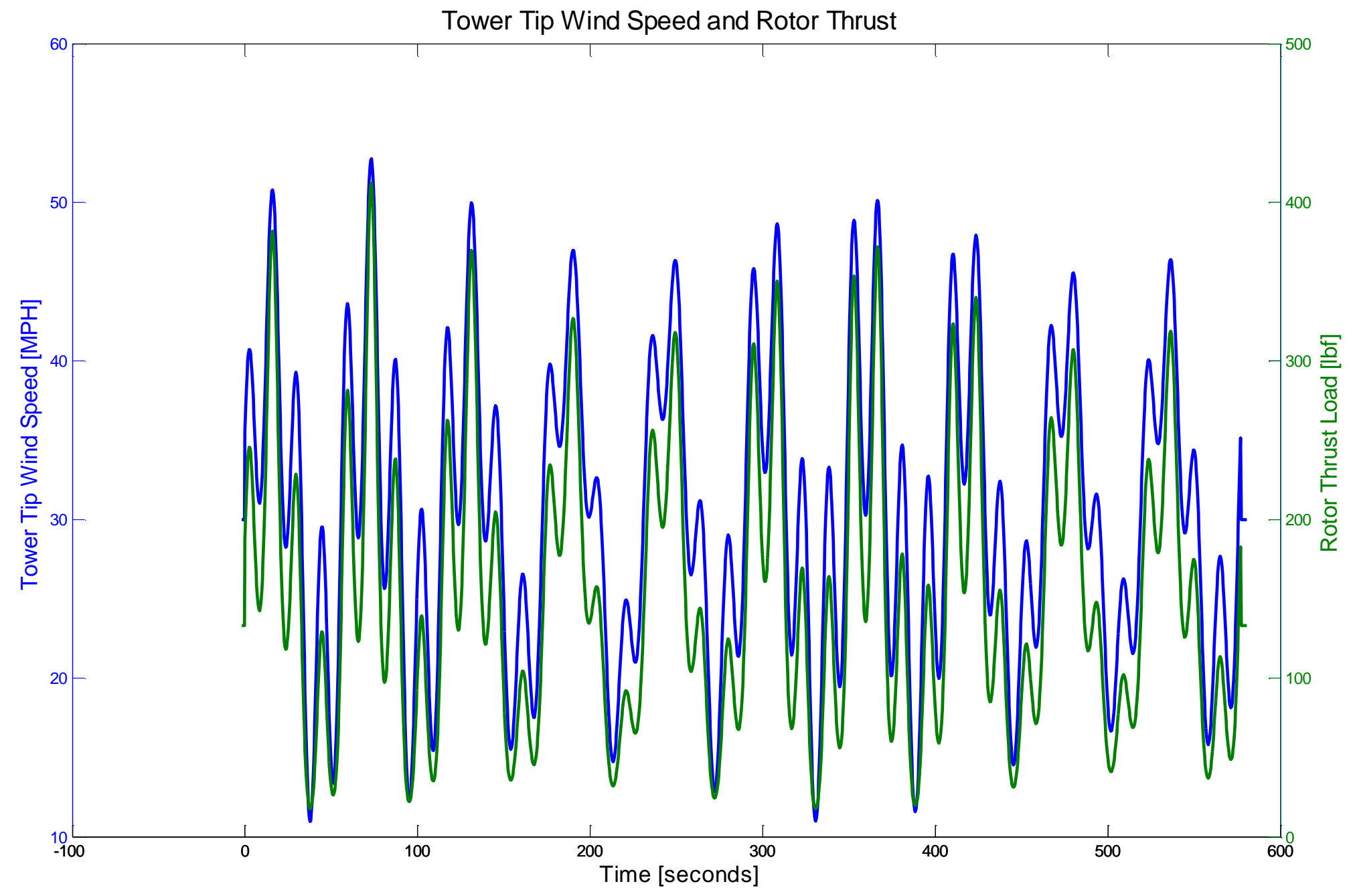




\section{Load Case G: Tower Tip Transient Deflection}

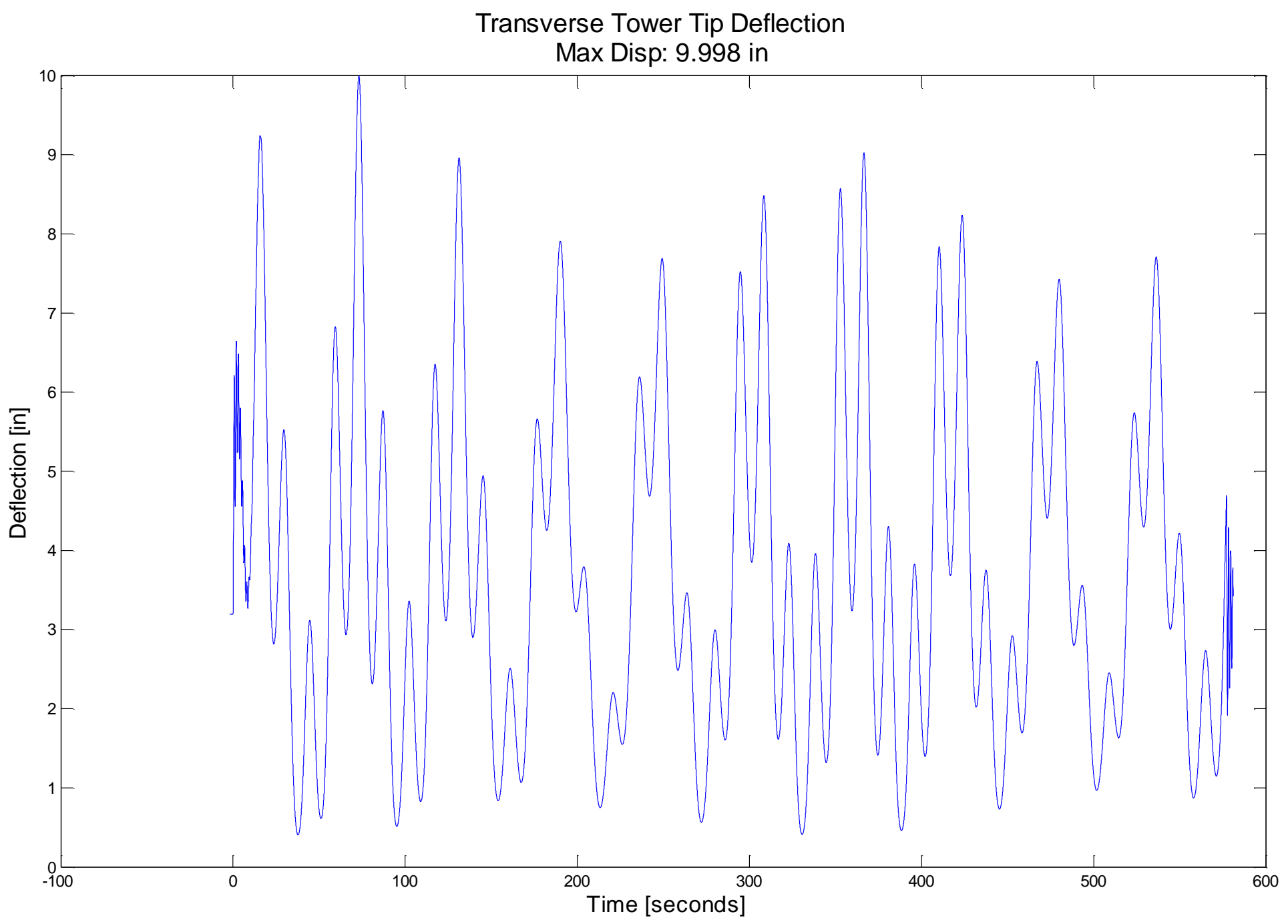

California Polytechnic State University - San Luis Obispo 


\section{Load Case G: Tower von Mises Stress Proffle}

Max Von Mises Stress Across Full Tower Height Max Von Mises Stress: 9202 psi

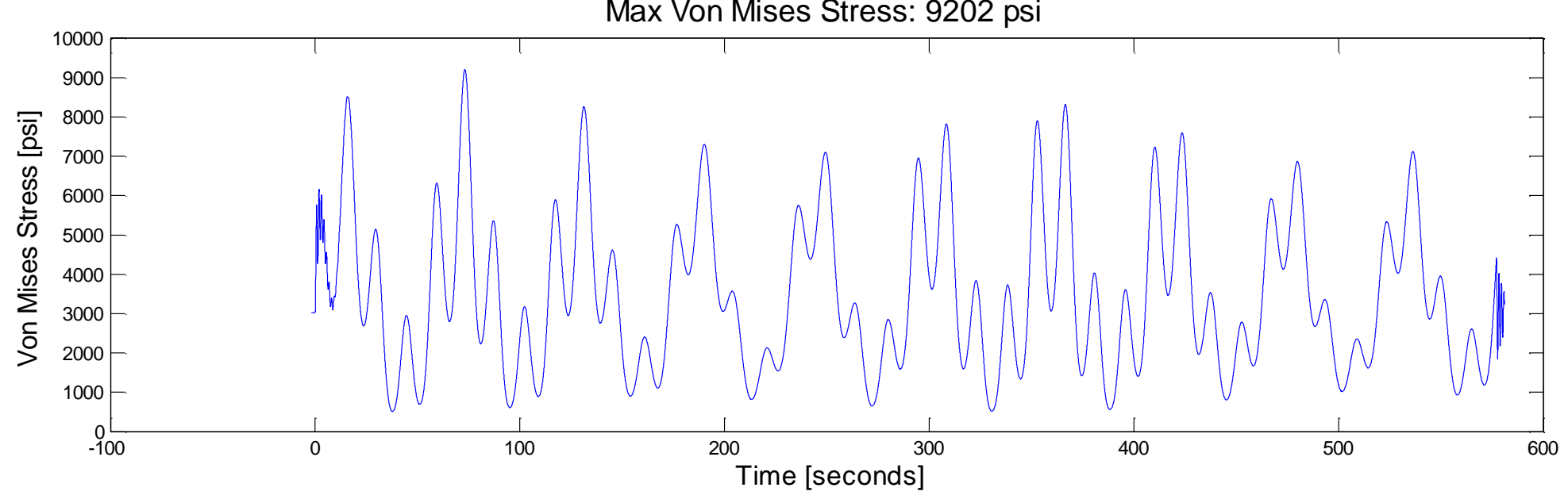

Tower Height Corresponding to Max Von Mises Stress

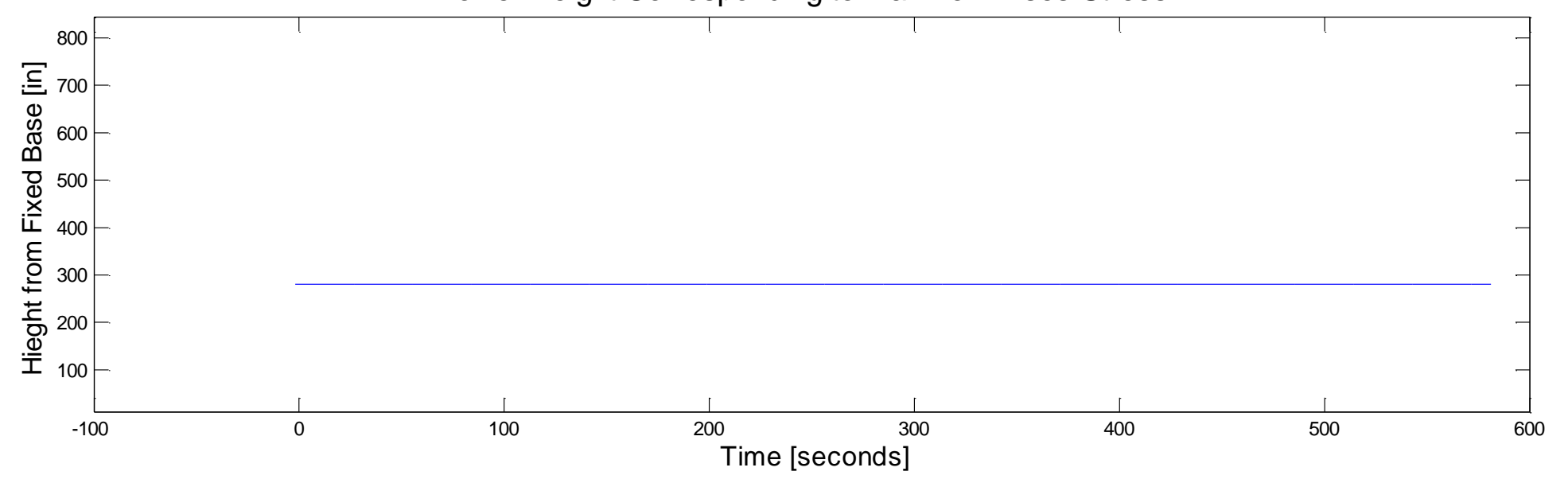




\section{Load Case G: Gin-Pole and Strut Tensile Load}

Gin-Ple and Strut Tensile Load

Max Absolute Gin-Pole Load: $4199 \mathrm{lbf}$

Max Absolute Strut Load: 3541 lbf

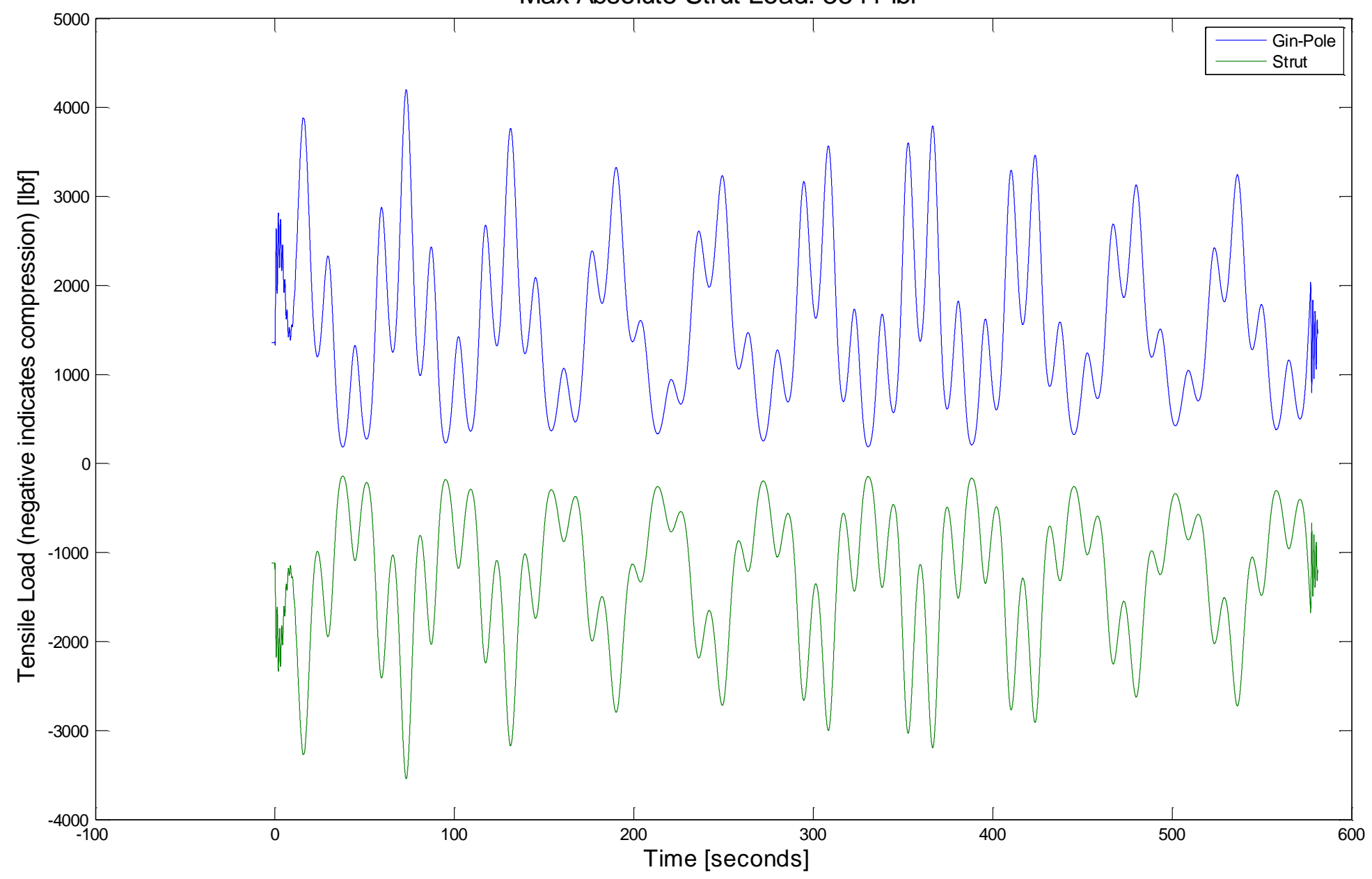

California Polytechnic State University - San Luis Obispo 


\section{Load Case G: Blade Beta Angle (relative flapwise angle)}

Blade Beta Hinge Displacement Angle Max Absolute Blade 1: 0.01621 radians Max Absolute Blade 2: 0.01621 radians Max Absolute Blade 3: 0.01621 radians

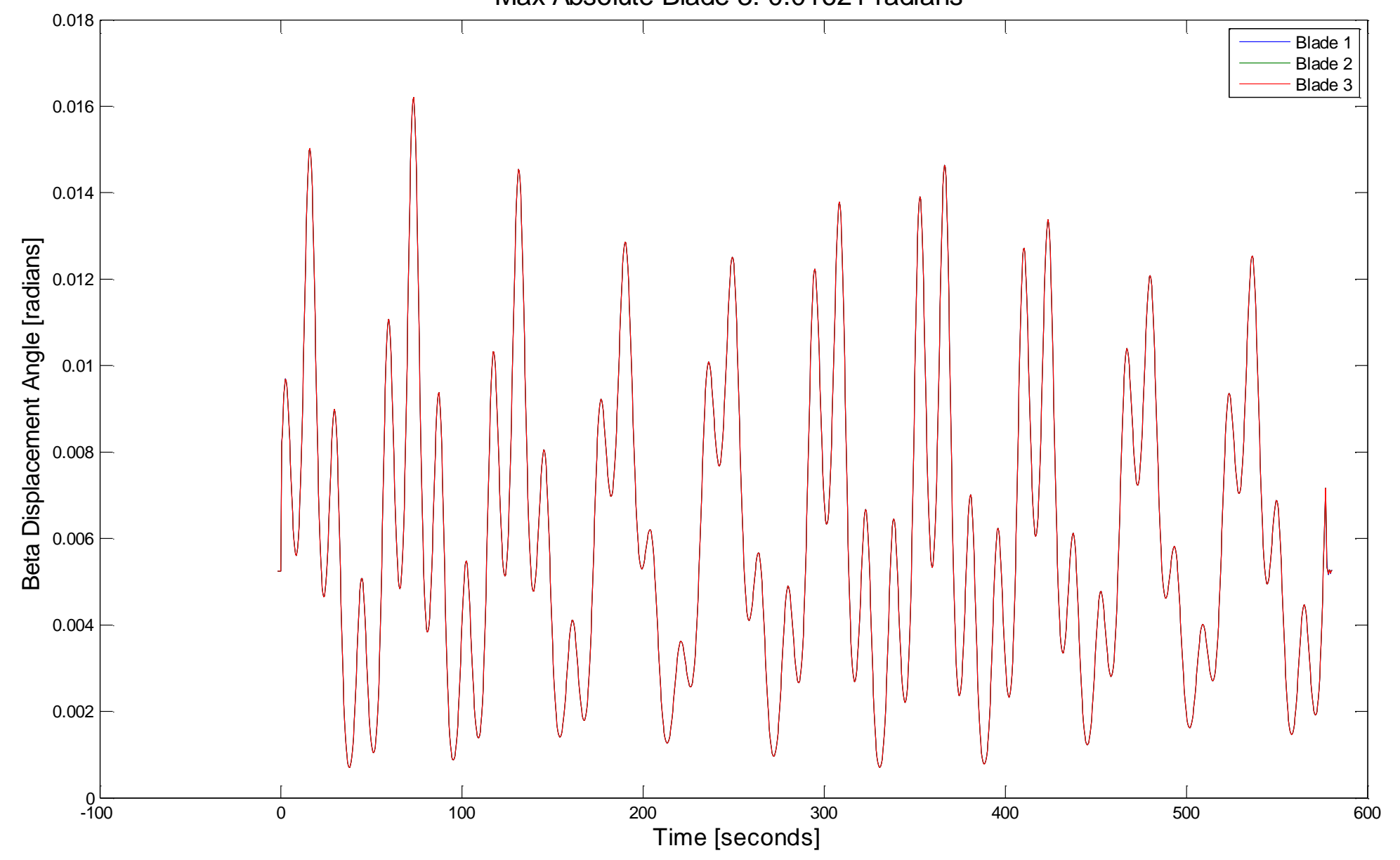

California Polytechnic State University - San Luis Obispo 


\section{Load Case G: Tower Displacement Profille at 70 Seconds}

Transient Small Wind Turbine Tower Structural Analysis with Coupled Rotor Dynamic Interaction

Transverse Tower Deflection

Max Disp: 9.998 in, at hight: 844.5 in

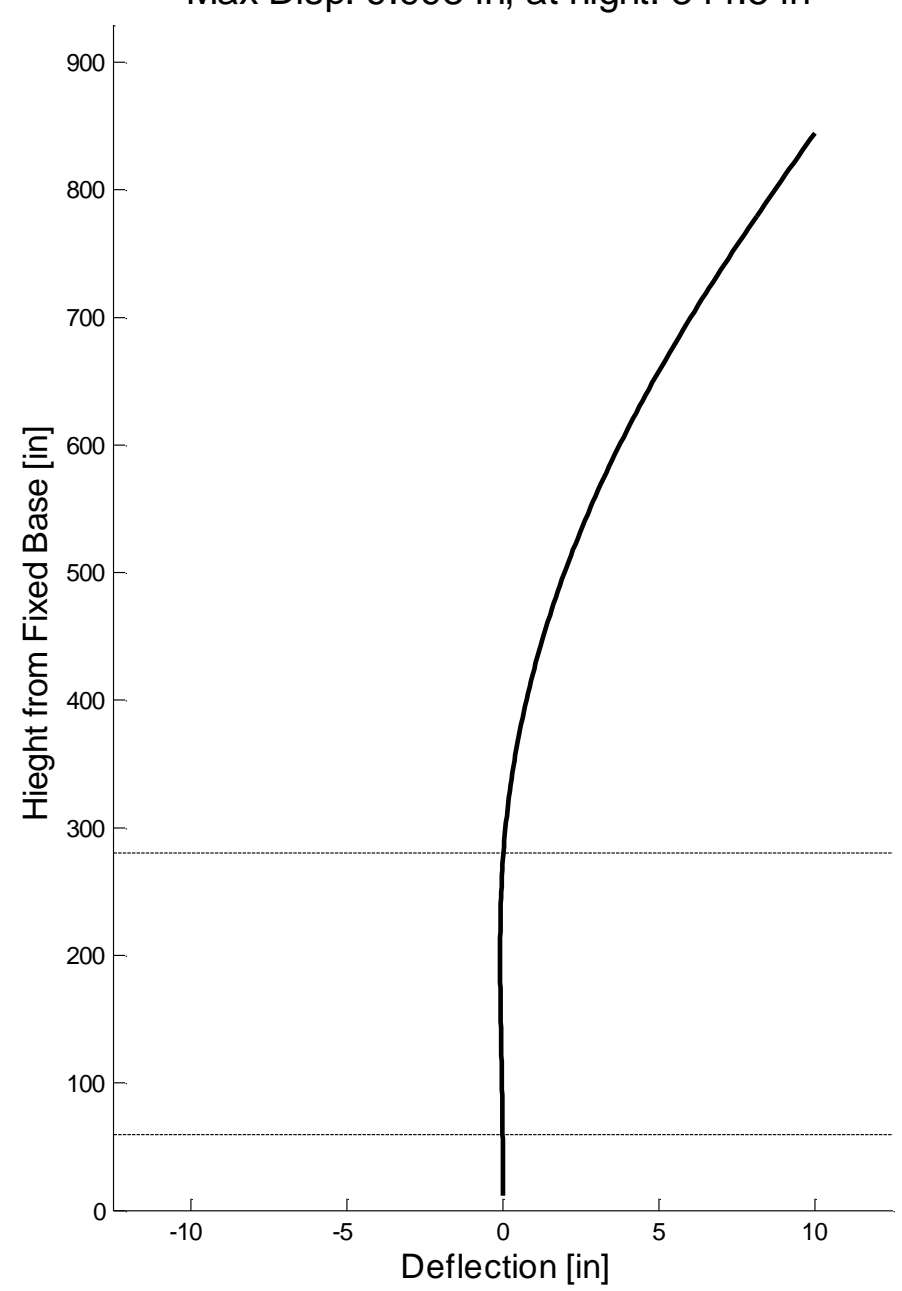

Axial Tower Deflection

Max Disp: 0.002242 in, at hight: 844.5 in

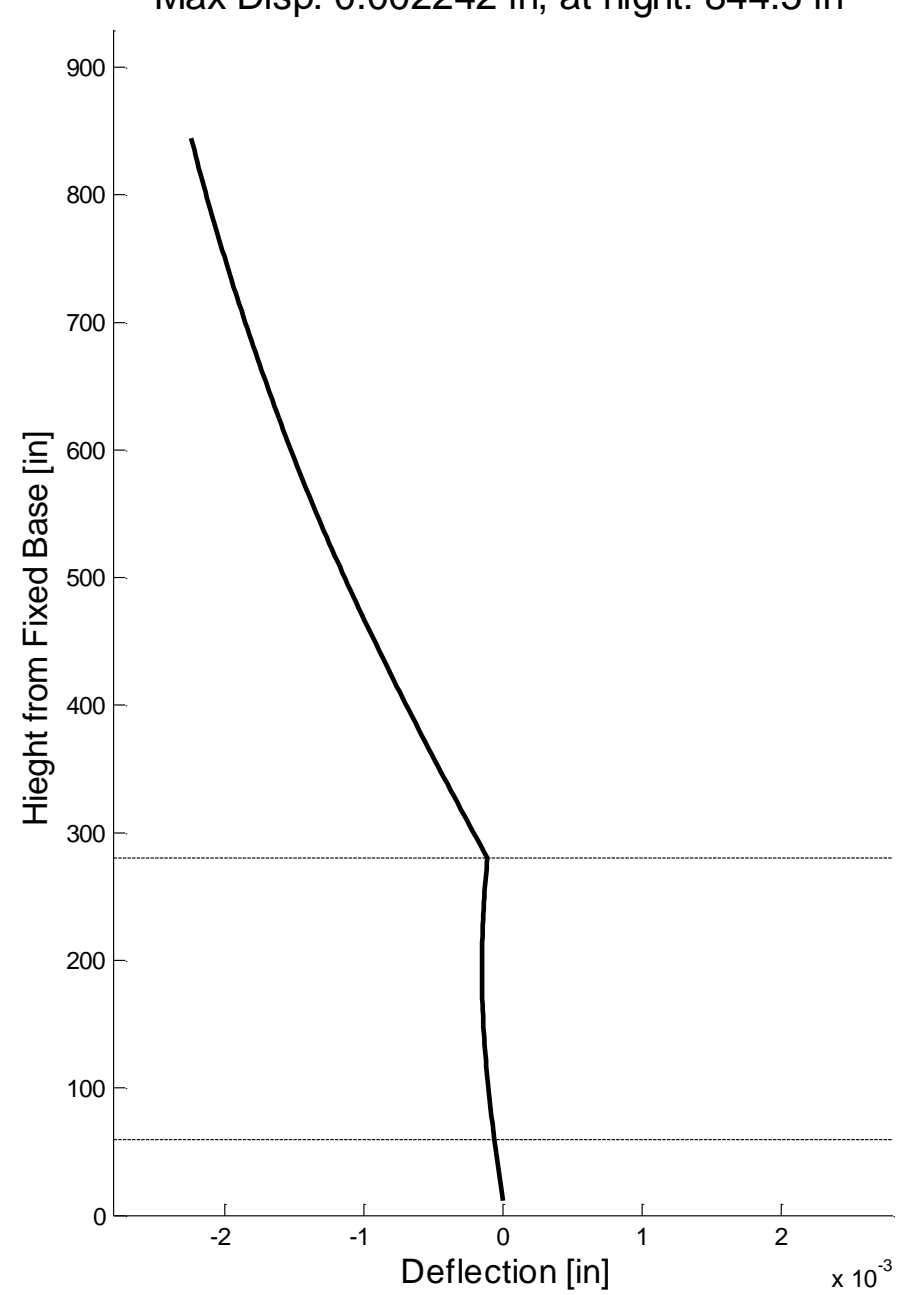




\section{Load Case G: Tower Principle Stresses at 70 Seconds}

Transient Small Wind Turbine Tower Structural Analysis with Coupled Rotor Dynamic Interaction

Tower Shear Stress Profile

Max: $305.5 \mathrm{lbf} / \mathrm{in}^{2}$, at hight: $43.5 \mathrm{in}$

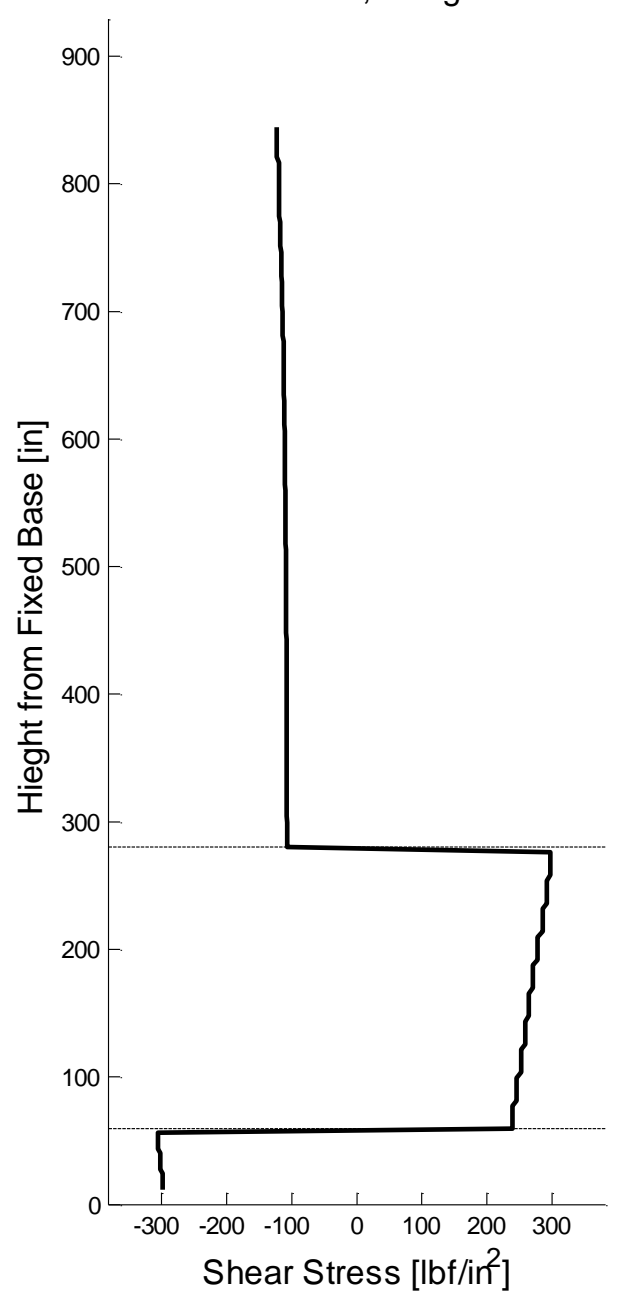

Tower Bending Stress Profile

Max: $9055 \mathrm{lbf} / \mathrm{in}^{2}$, at hight: $280.3 \mathrm{in}$

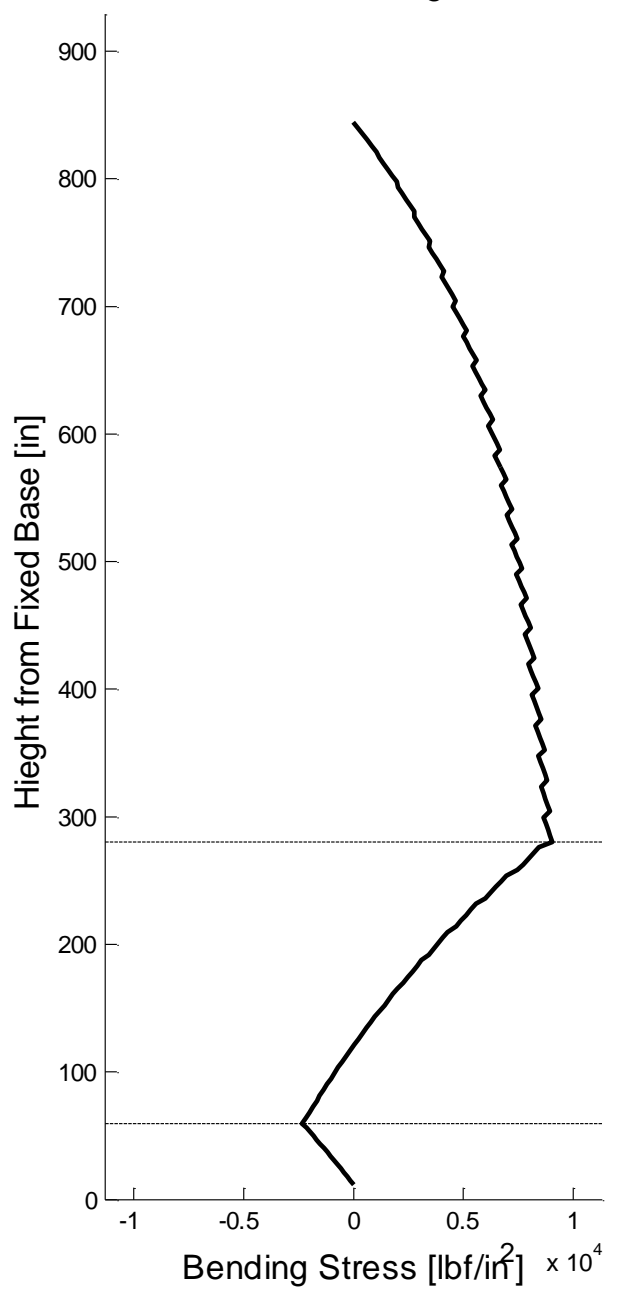

Tower Axial Stress Profile

Max: $147.3 \mathrm{lbf} / \mathrm{in}^{2}$, at hight: 280.3 in

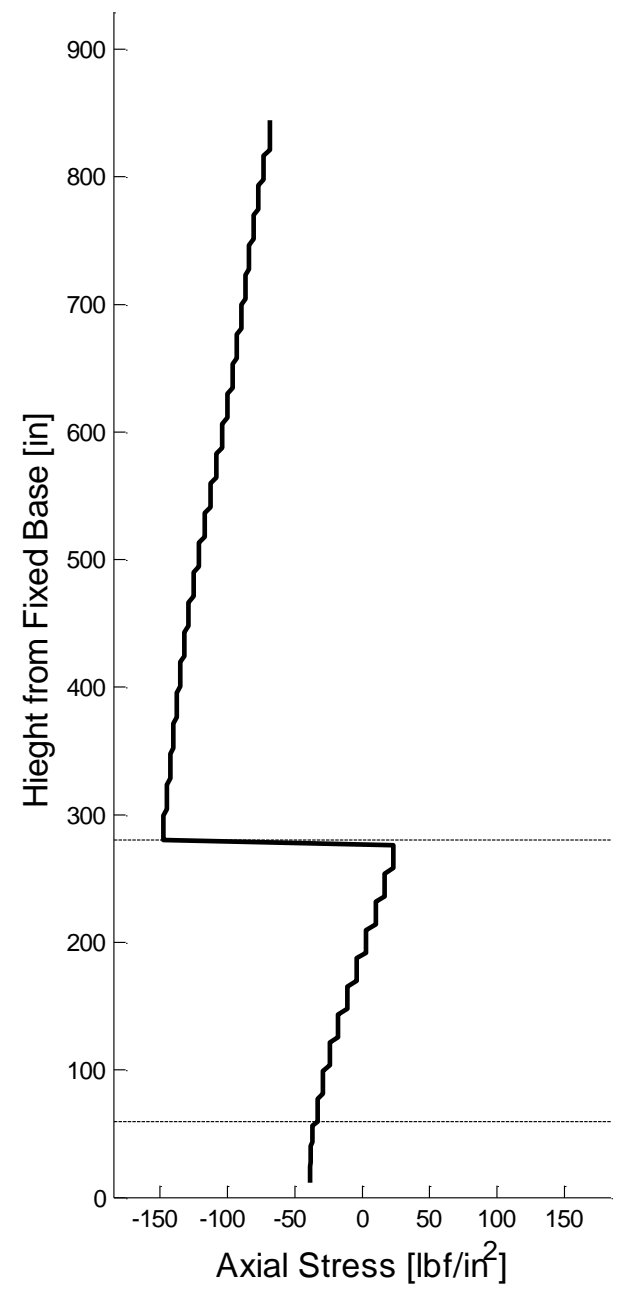




\section{Load Case G: Tower von Mises Stress at 70 Seconds}

Transient Small Wind Turbine Tower Structural Analysis with Coupled Rotor Dynamic Interaction

Max Tower von Mises Stress Profile

Max: $9202 \mathrm{lbf} / \mathrm{in}^{2}$, at hight: $280.3 \mathrm{in}$

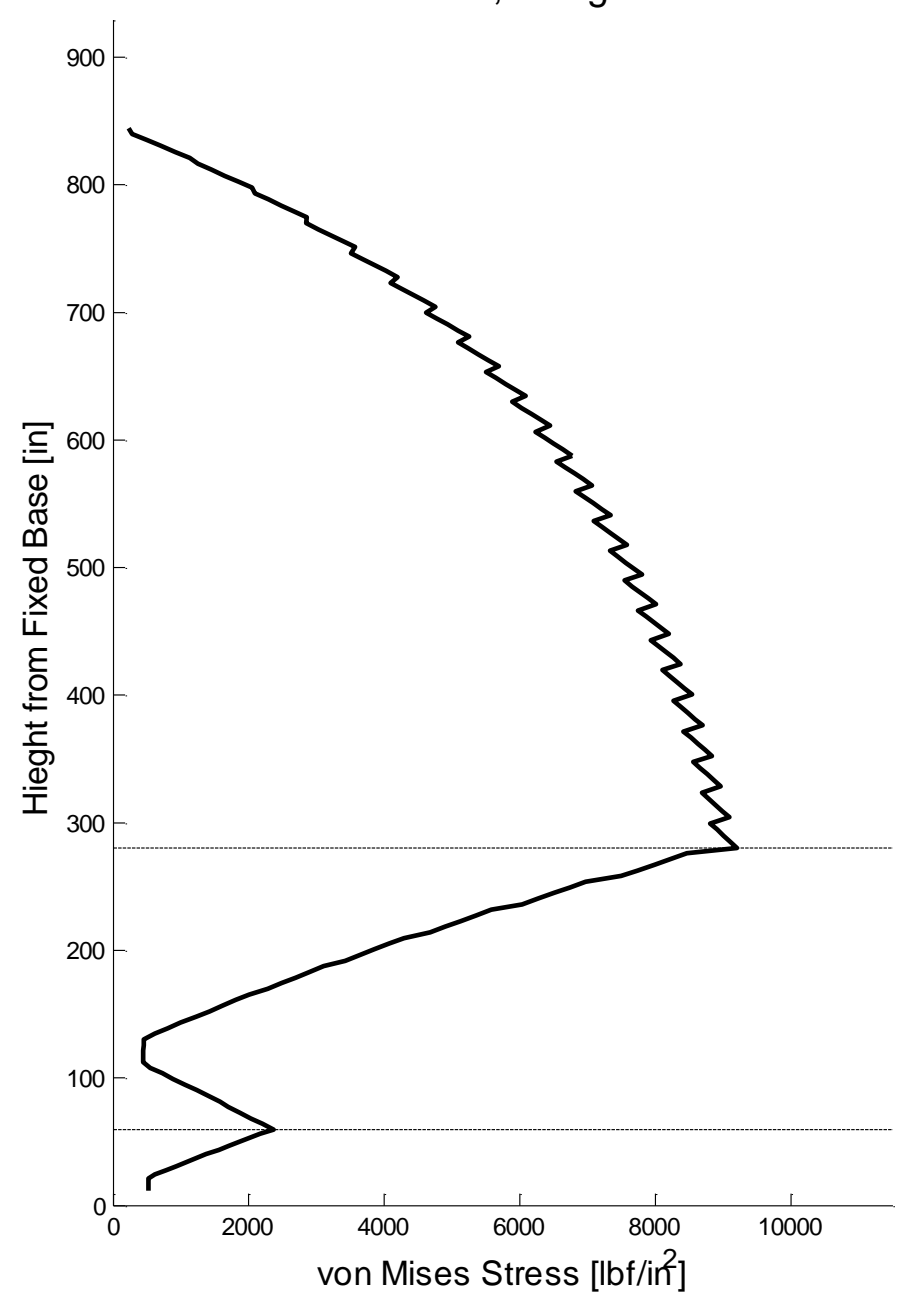

Max Tower von Mises Cross-Sectional Location (Theta) Profile

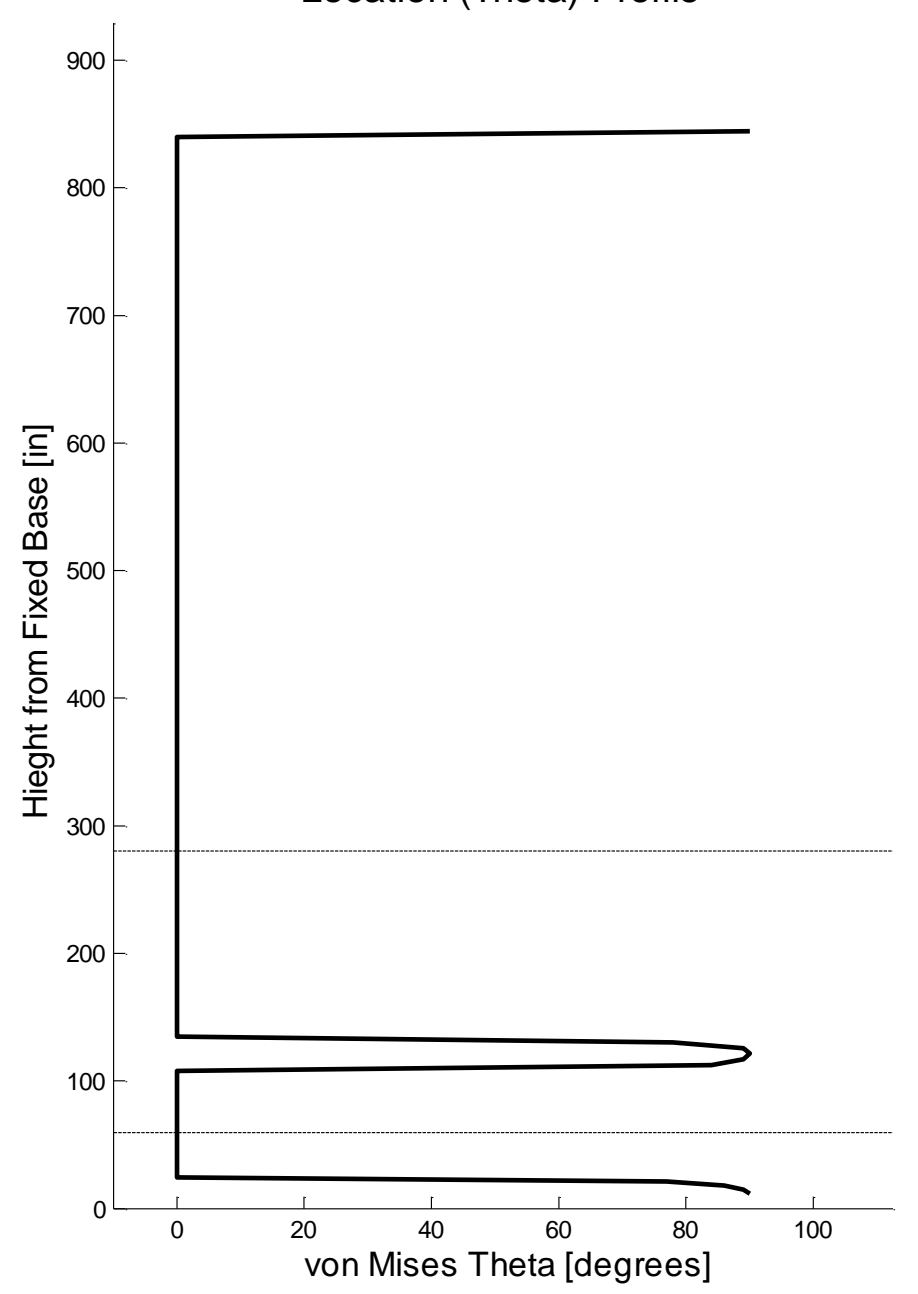




\section{Strong Axis Load Case H - Dynamic Swept Sine}




\section{Load Case H: Overview}

- Basic Parameters

- Sinusoidal tower tip load applied in the X-Axis.

- Results are displayed normalized to tower tip load amplitude

- Starting Frequency: $0.10 \mathrm{~Hz}$

- Ending Frequency: $20 \mathrm{~Hz}$

- Logarithmic Sweep Rate: 0.5 Octaves / Minute

- Nhat $=30$, see Appendix A for details

- Load applied in "strong axis" (the load is parallel to the plane formed by the tower, gin-pole, and strut).

- Details of the analysis parameters are summarized and defined in the attached UserInputs.xIsx file; note.

- Rotor Modal and Load Parameters

- See next chart 


\section{Load Case H: Various Combinations of Tower Load and}

Rotor Blade Natural Frequency

Transient Small Wind Turbine Tower Structural Analysis with Coupled Rotor Dynamic Interaction

- Various combinations of tower load and rotor blade natural frequency were studied for their effects on the tower swept sine response.

- Rotor Blade Natural Frequency:

- 1) Rotor blades modeled as rigid

- 2) Rotor blade flapwise natural frequency $=16.25 \mathrm{~Hz}$

- Realistic estimate based on the Myklestad Method

- 3) Rotor blade flapwise natural frequency $=0.81 \mathrm{~Hz}$

- Tuned to match tower $1^{\text {st }}$ mode

- Load Application:

- A) $100 \mathrm{lbf}$ thrust load without moment acting at blade hinge

- B) $\mathbf{1 0 0 ~ l b f ~ t h r u s t ~ l o a d ~ w i t h ~ m o m e n t ~ a c t i n g ~ a t ~ b l a d e ~ h i n g e ~}$

- Bladed moment based on equation 4.3.3 (Manwell et al., 2002, p163) which assumes that the resultant of the thrust force acting on each blade is centered at $2 / 3 *$ Blade Radius.

- The results of this study are shown on the following charts.

- The effect of rotor blade natural frequency (methods 1, 2, and 3) on tower transverse mode shapes is shown on the next chart.

- The effect of load application (methods A, and B) are shown on the subsequent chart.

- The legend on the following charts uses designators which reference the list above. For example case $1 \mathrm{~A}$ features a rigid rotor blade without any moment acting at the blade hinge. 


\section{Effect of Rotor Blade Flapwise Natural Freq on Tower Transverse Mode Shapes / Freq}

Transient Small Wind Turbine Tower Structural Analysis with Coupled Rotor Dynamic Interaction

1) Rotor blades modeled as rigid

Transverse Mode Shapes

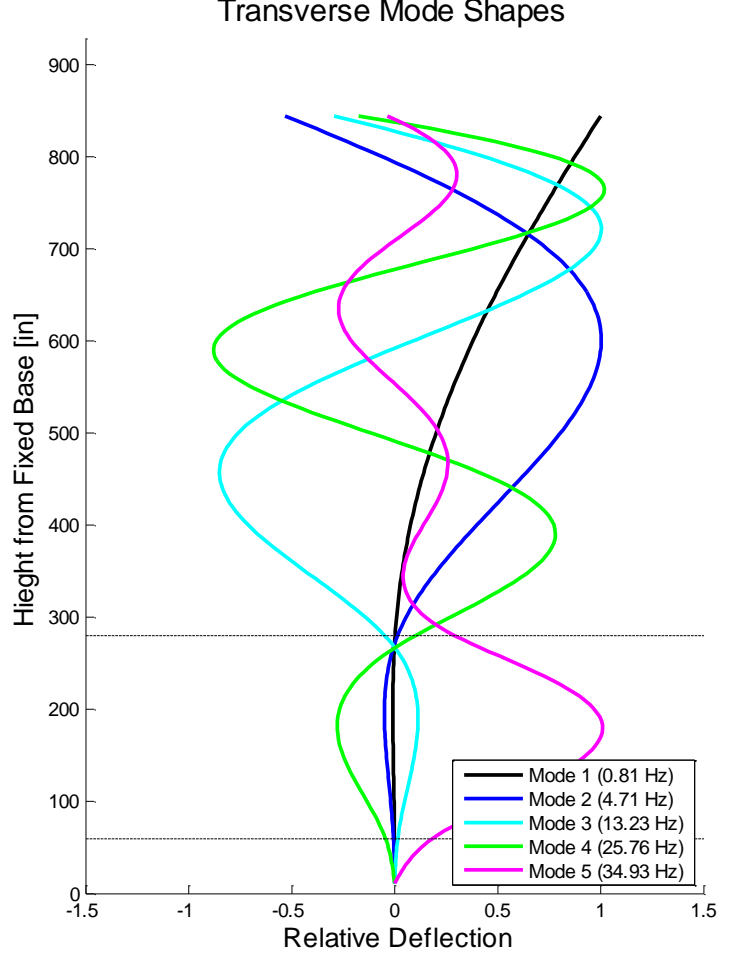

2) Rotor blade flapwise natural frequency $=16.25 \mathrm{~Hz}$

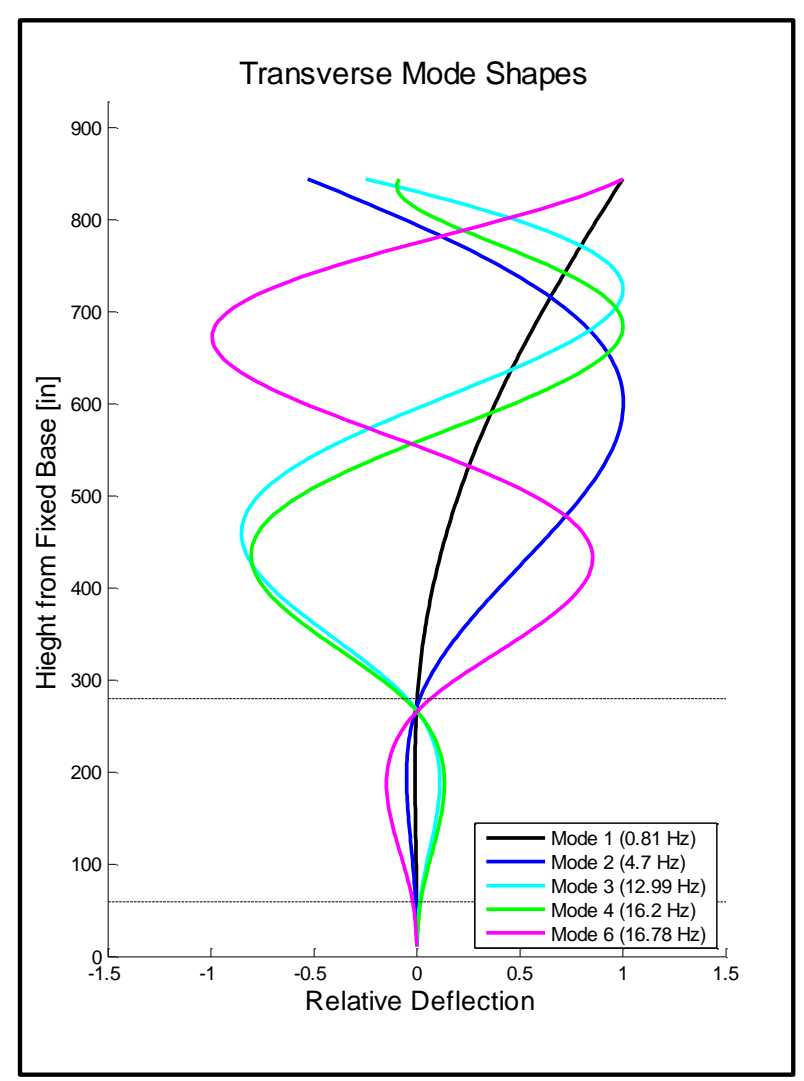

3) Rotor blade flapwise natural frequency $=0.81 \mathrm{~Hz}$

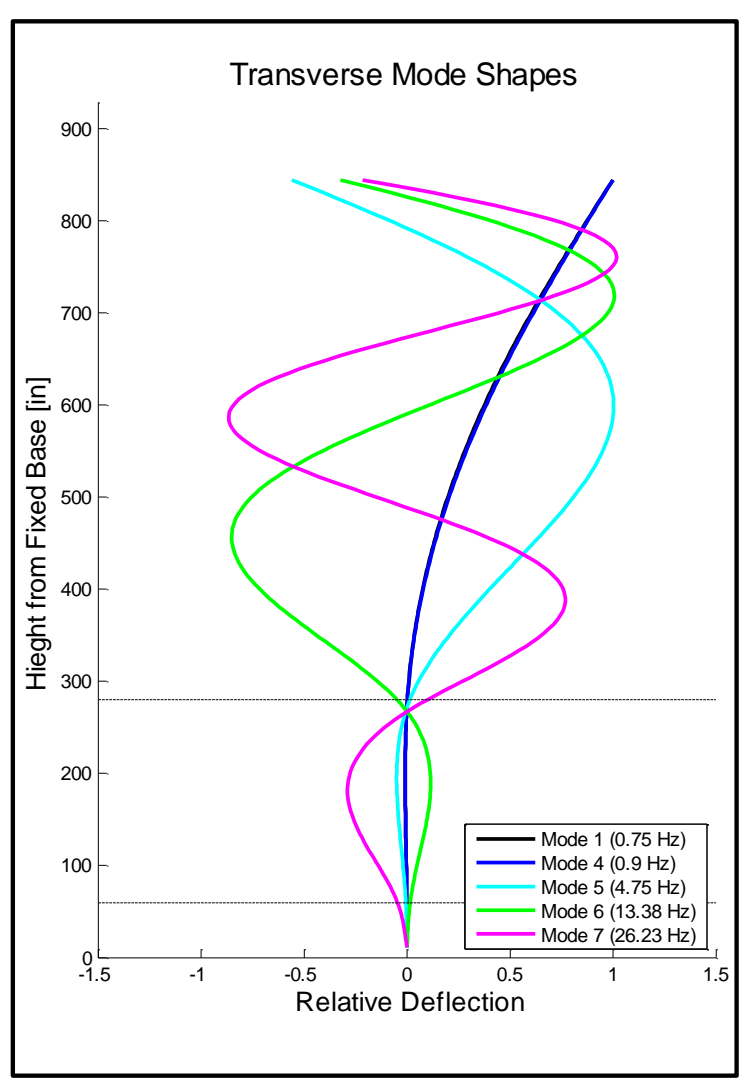




\section{Load Case H: Swept Sine - Tower Response 1A) Blades Modeled as Rigid, Thrust Load Only}

Transient Small Wind Turbine Tower Structural Analysis with Coupled Rotor Dynamic Interaction

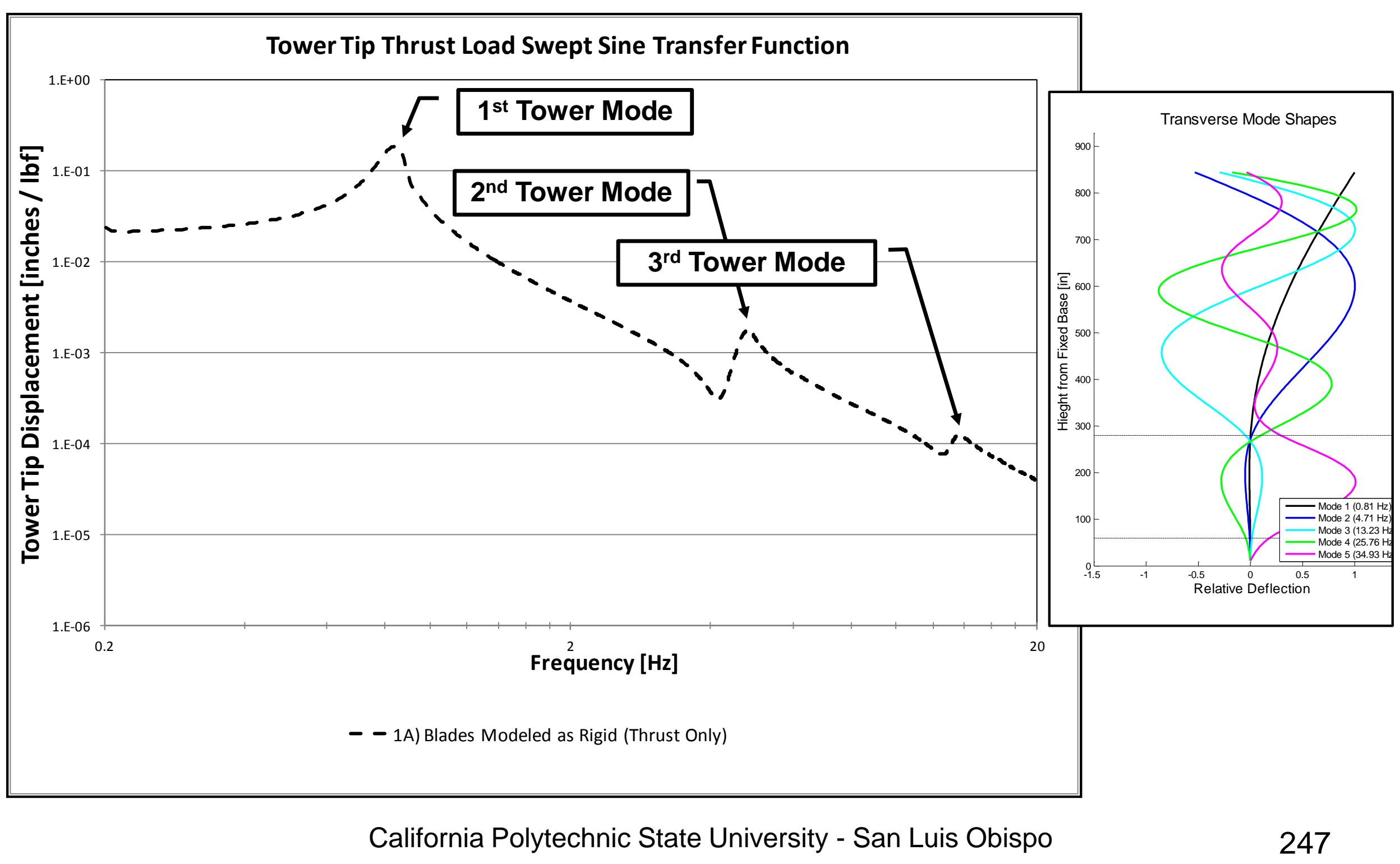




\section{Load Case H: Swept Sine - Tower Response 3A) Blade Flapwise Mode $=0.81 \mathrm{~Hz}$, Thrust Load Only}

Transient Small Wind Turbine Tower Structural Analysis with Coupled Rotor Dynamic Interaction

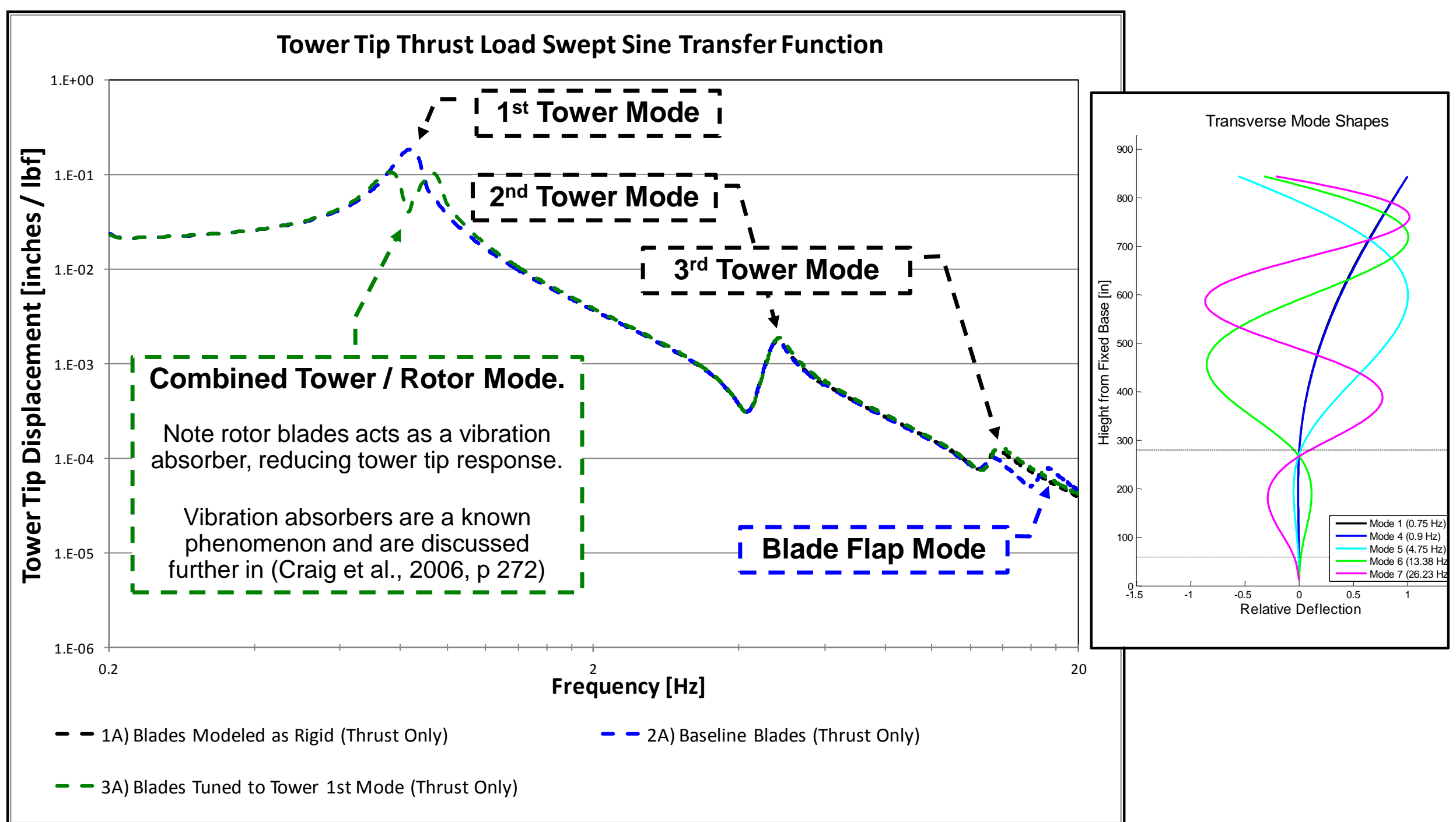




\section{Load Case H: Swept Sine - Tower Response 1A) Blades Modeled as Rigid, Thrust \& Moment Load}

Transient Small Wind Turbine Tower Structural Analysis with Coupled Rotor Dynamic Interaction

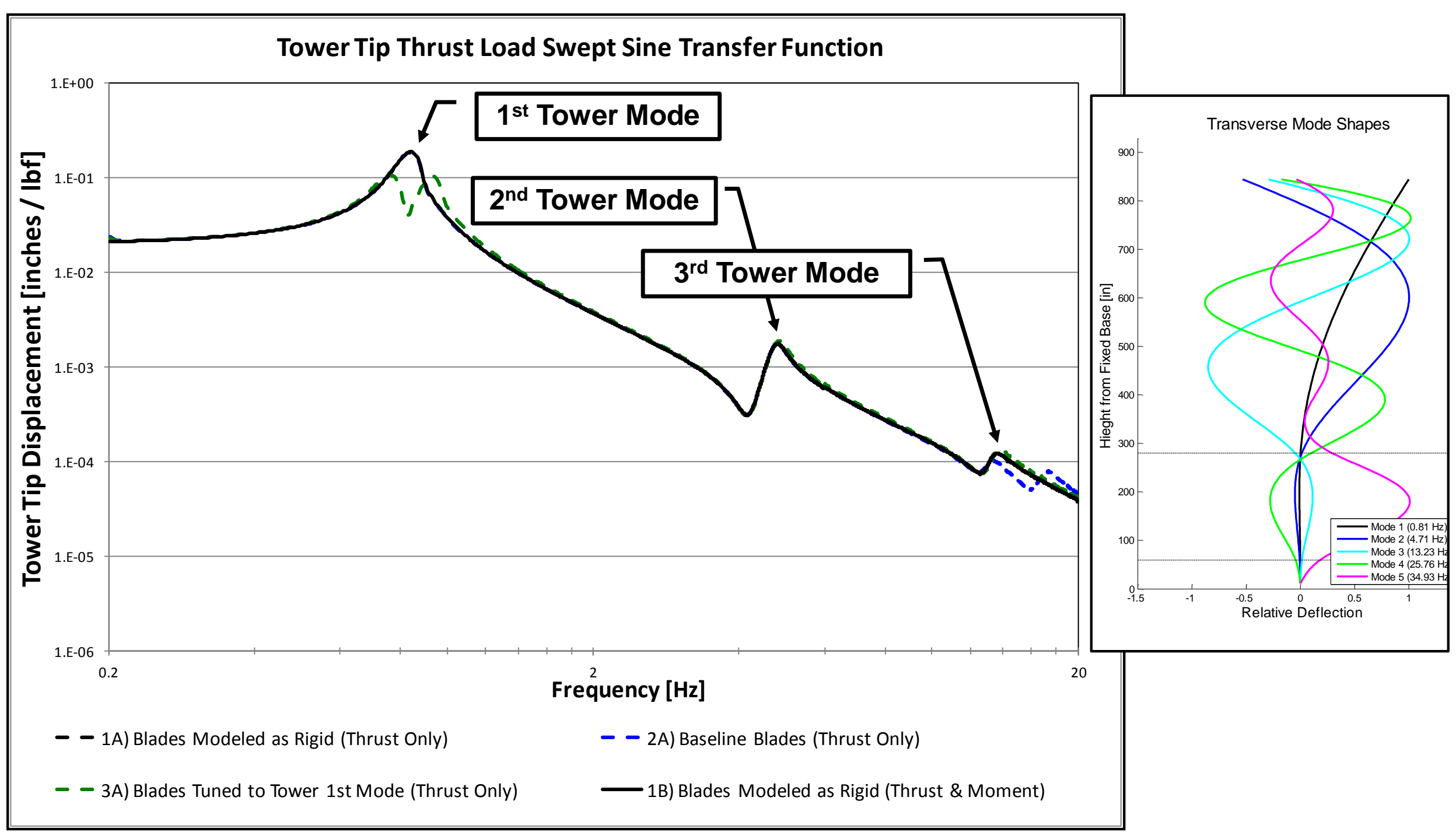




\section{Load Case H: Swept Sine - Tower Response 2B) Blade Flapwise Mode = $16.25 \mathrm{~Hz}$, Thrust \& Moment Load}

Transient Small Wind Turbine Tower Structural Analysis with Coupled Rotor Dynamic Interaction

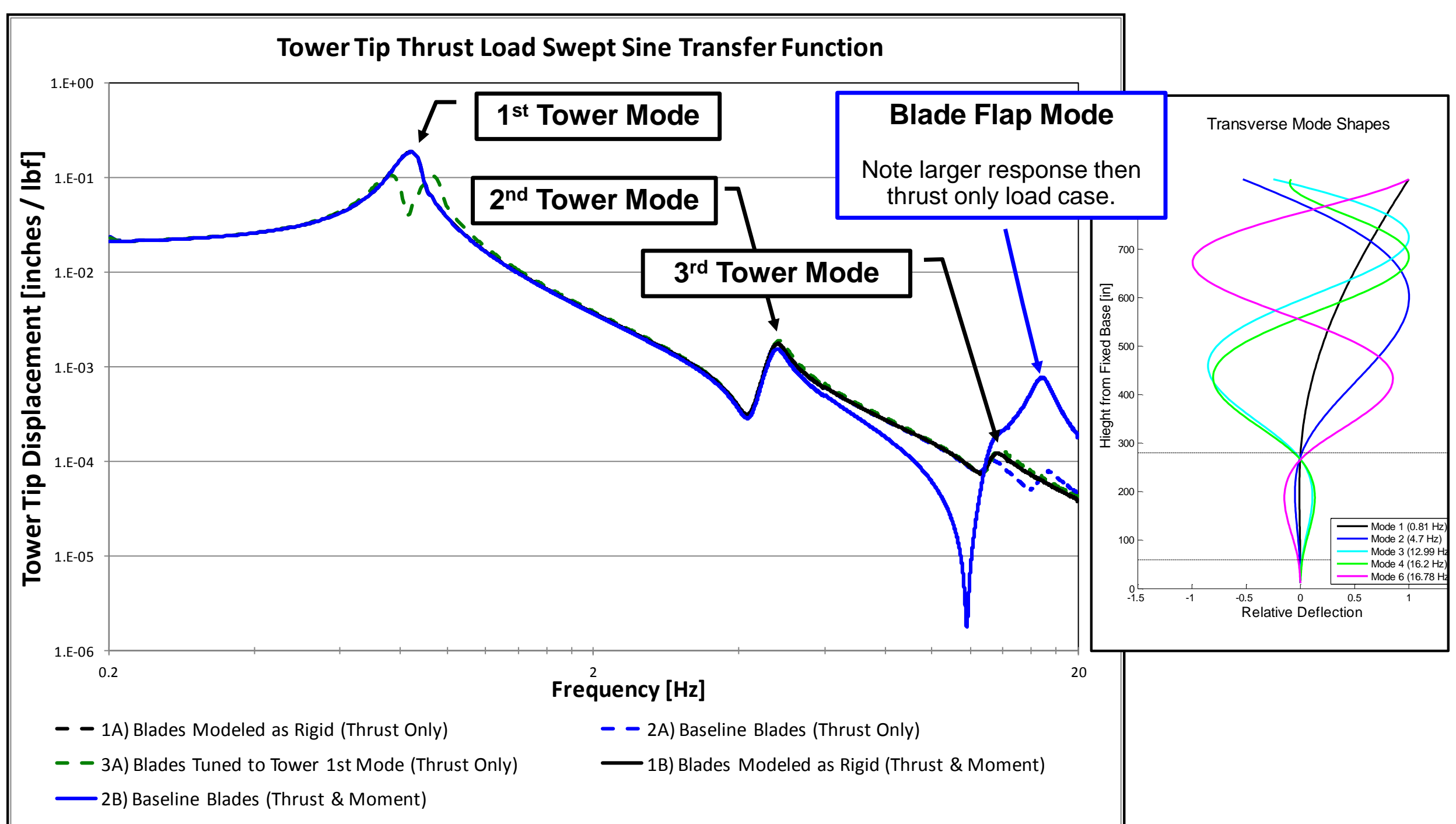




\section{Load Case H: Swept Sine - Tower Response 3B) Blade Flapwise Mode $=0.81 \mathrm{~Hz}$, Thrust \& Moment Load}

Transient Small Wind Turbine Tower Structural Analysis with Coupled Rotor Dynamic Interaction

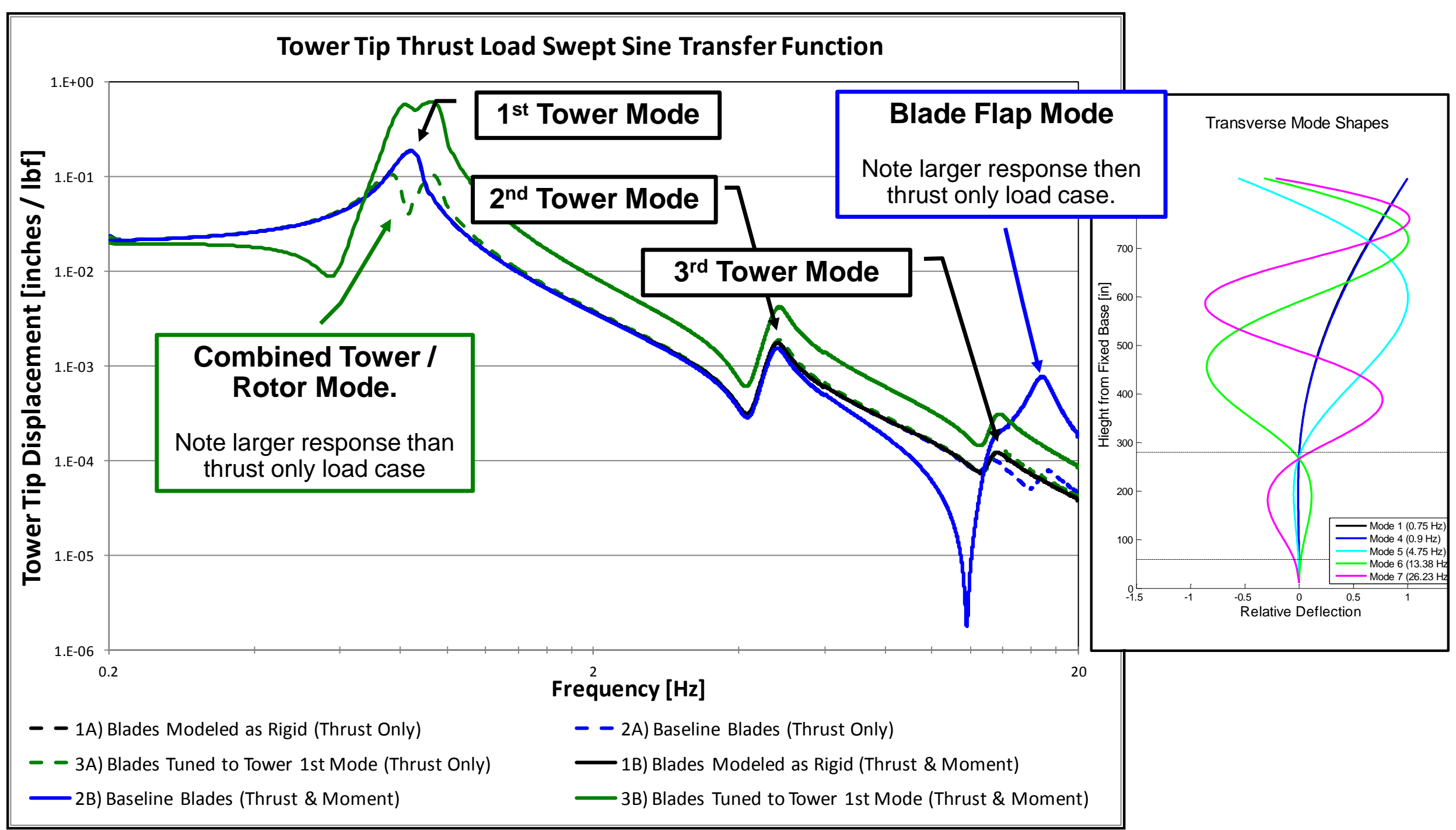




\section{Load Case H: Swept Sine - Tower vs Blade Response 2B) Blade Flapwise Mode = $16.25 \mathrm{~Hz}$, Thrust \& Moment Load}

Transient Small Wind Turbine Tower Structural Analysis with Coupled Rotor Dynamic Interaction

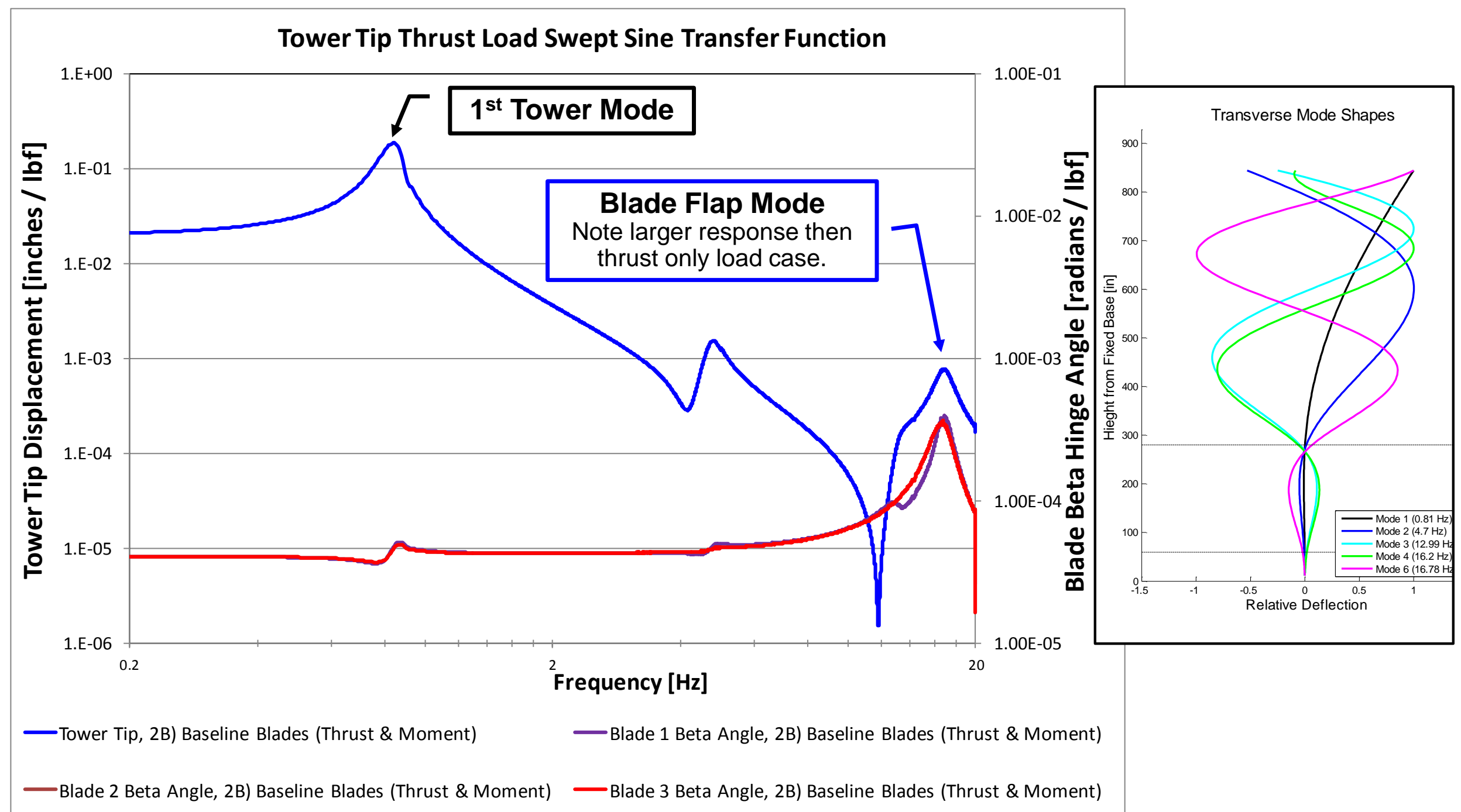




\section{Load Case H: Swept Sine - Tower vs Blade Response 3B) Blade Flapwise Mode $=0.81 \mathrm{~Hz}$, Thrust \& Moment Load}

Transient Small Wind Turbine Tower Structural Analysis with Coupled Rotor Dynamic Interaction

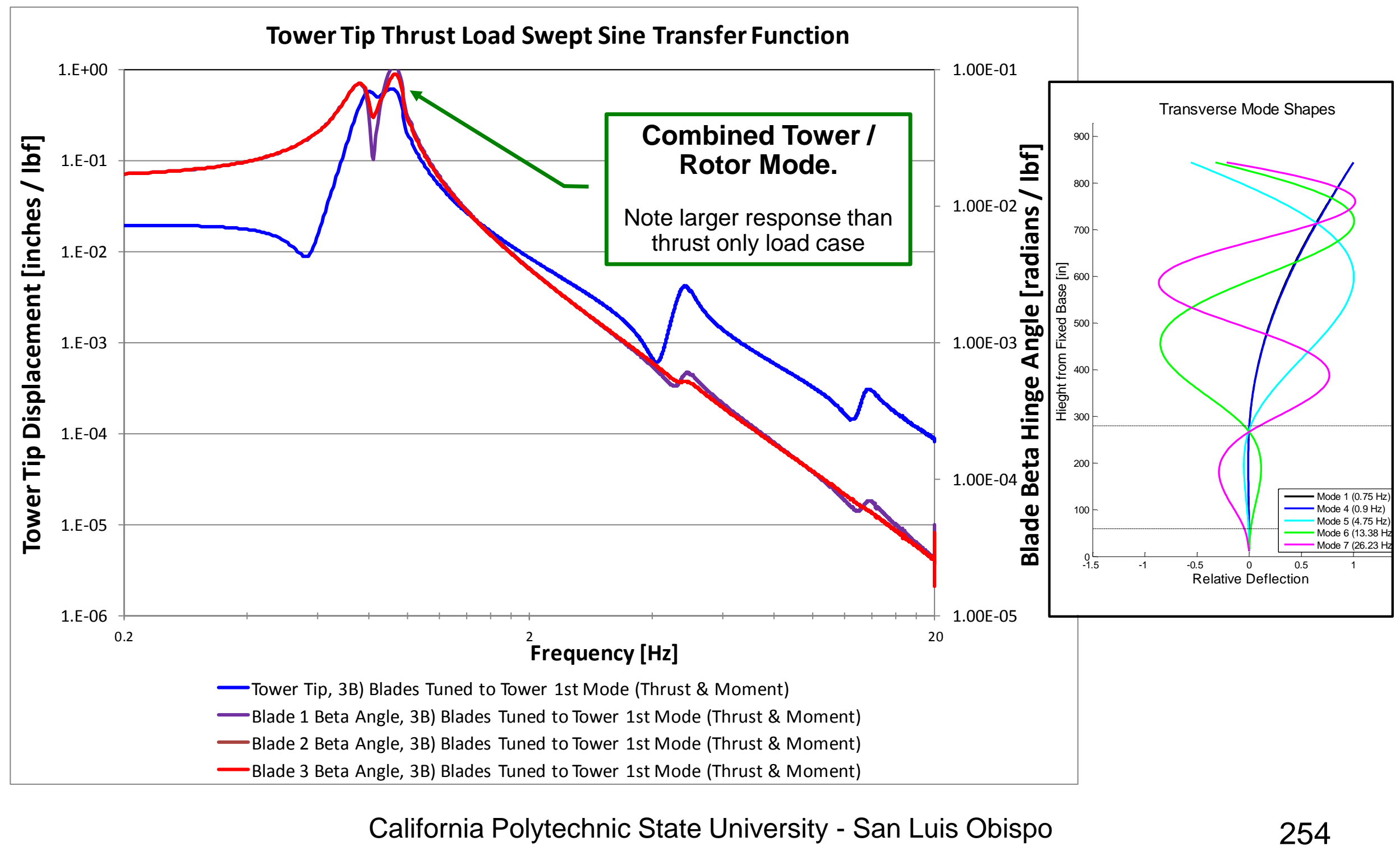




\section{Weak Axis Load Case A - Static 2100 lbf Tip Load}




\section{Load Case A: Overview}

- $2100 \mathrm{lbf}$ load applied to tower tip

- Simulates a steady-state $60 \mathrm{mph}$ wind load

- Rotor Thrust Coefficient: $C_{t}=2.0$

- Tower Drag Coefficient: $\mathrm{C}_{\mathrm{d}}=\mathbf{0 . 0}$

- Load applied in "Weak axis" (the load is parallel to the plane formed by the tower, gin-pole, and strut).

- Details of the analysis parameters are summarized and defined in the attached UserInputsWeak.xlsx file. 


\section{Load Case A: Tower Deflection}

Transverse Tower Deflection

Max Disp: 69.45 in, at hight: 844.5 in

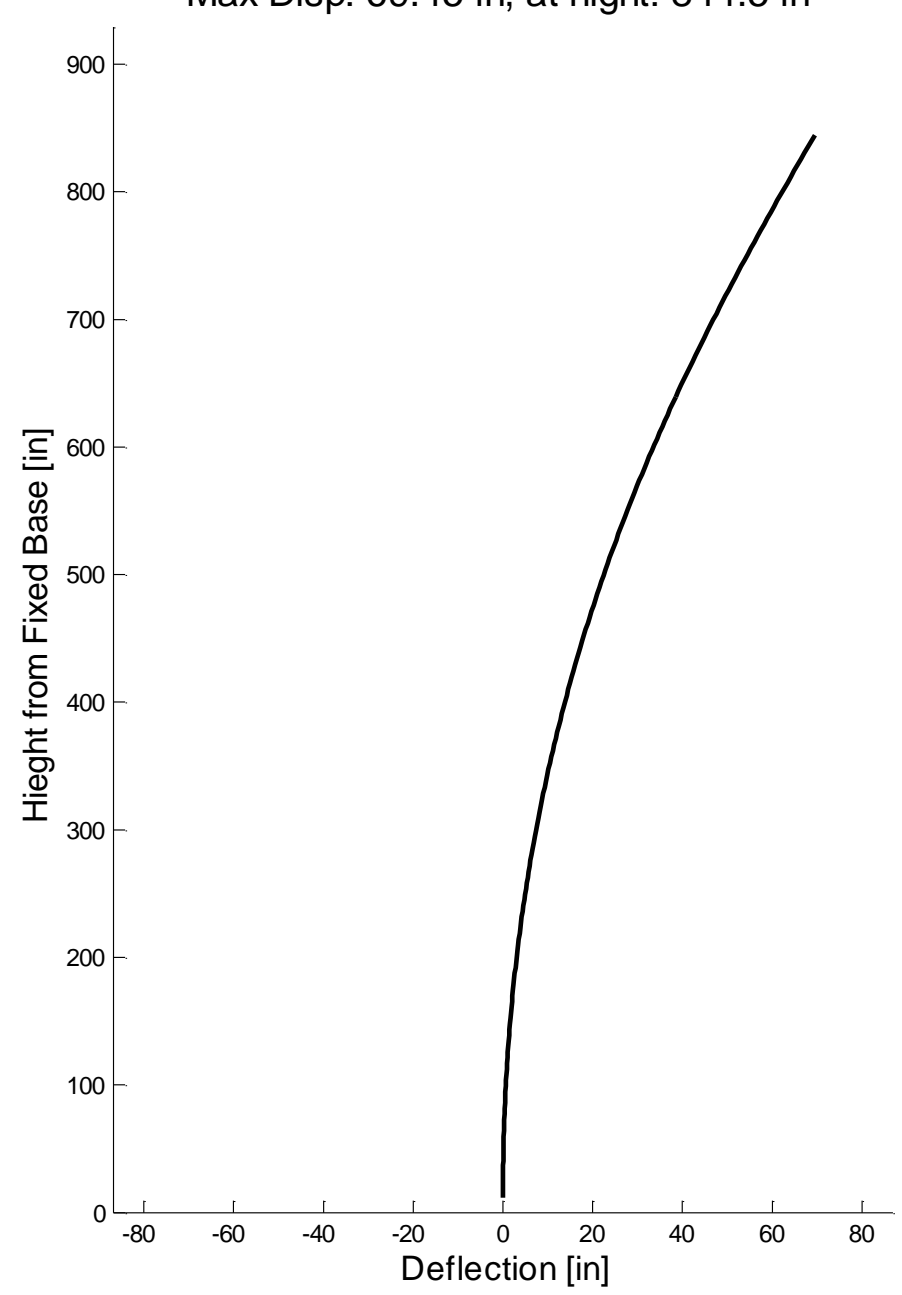

Axial Tower Deflection

Max Disp: 0.003729 in, at hight: 844.5 in

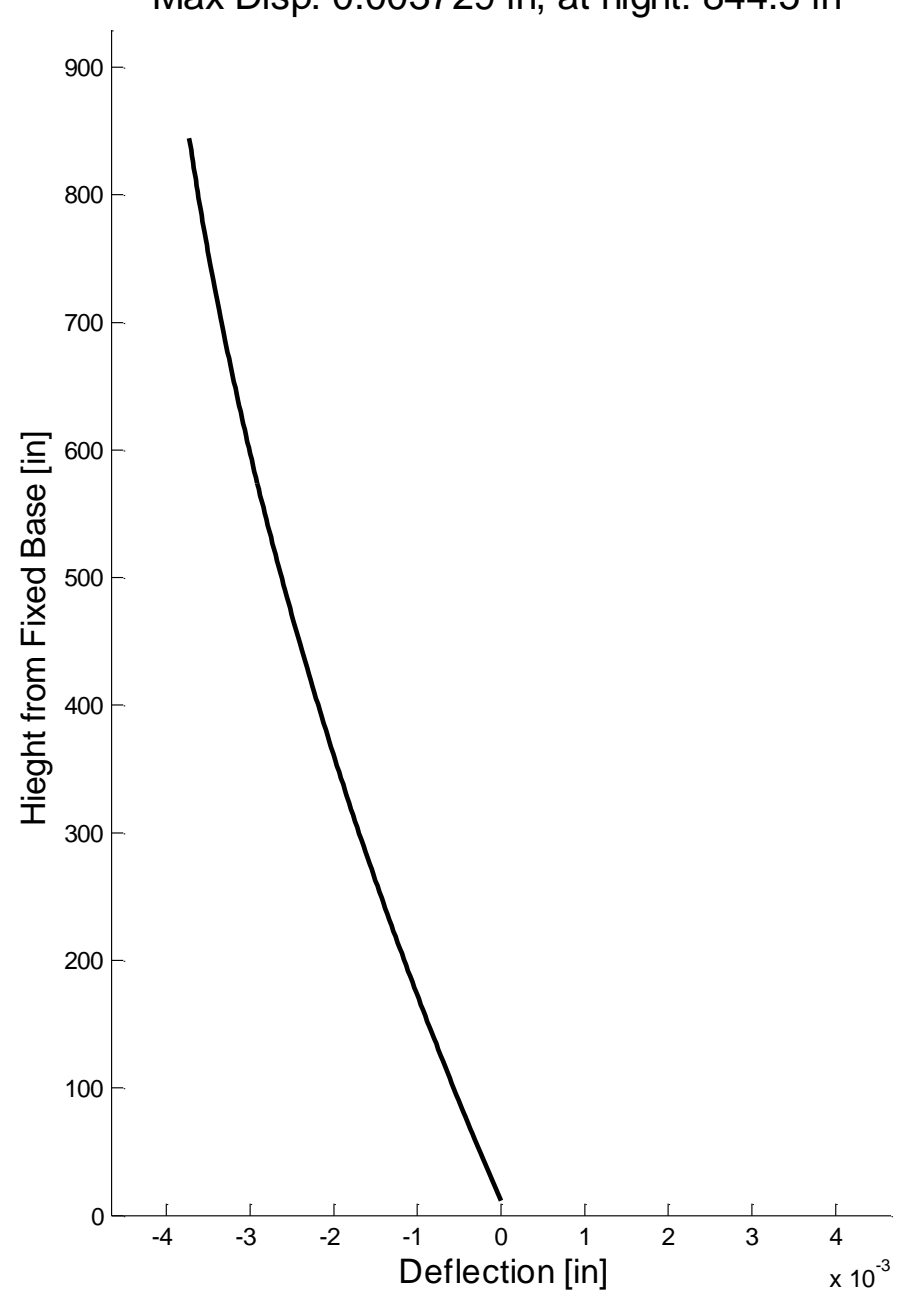




\section{Load Case A: Principle Stresses}

Transient Small Wind Turbine Tower Structural Analysis with Coupled Rotor Dynamic Interaction

Tower Shear Stress Profile

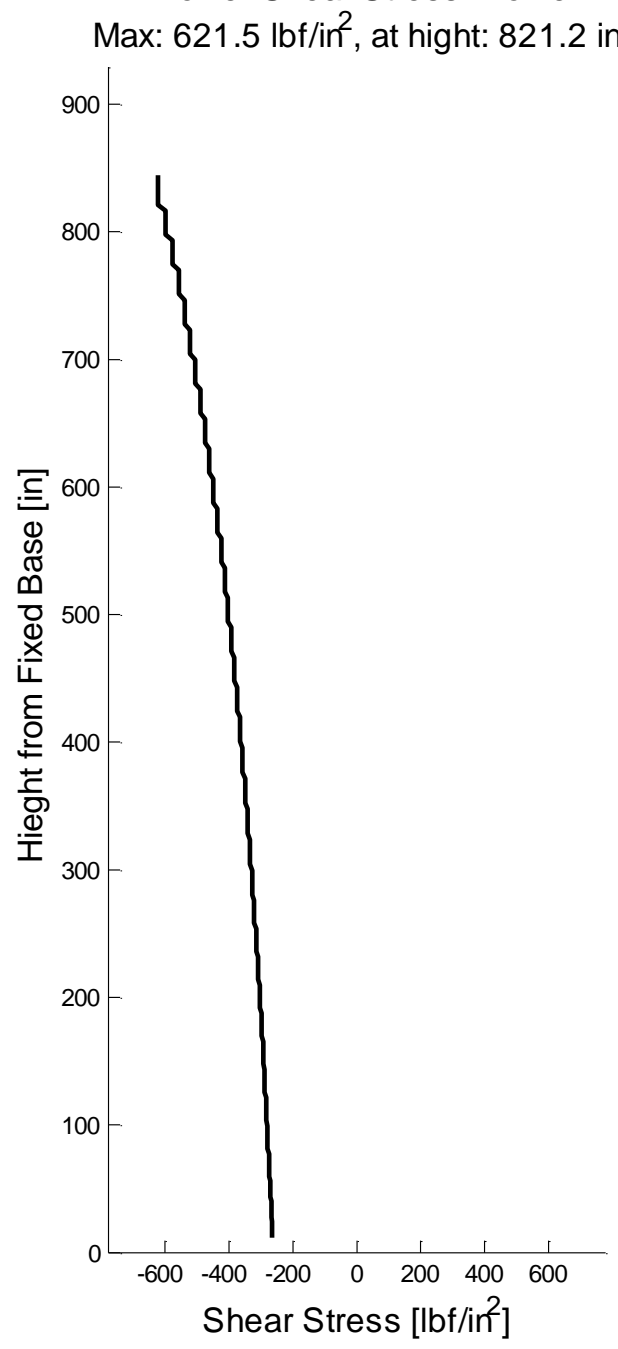

Tower Bending Stress Profile

Max: $3.499 \mathrm{e}+004 \mathrm{lbf} / \mathrm{in}^{2}$, at hight: $280.3 \mathrm{in}$

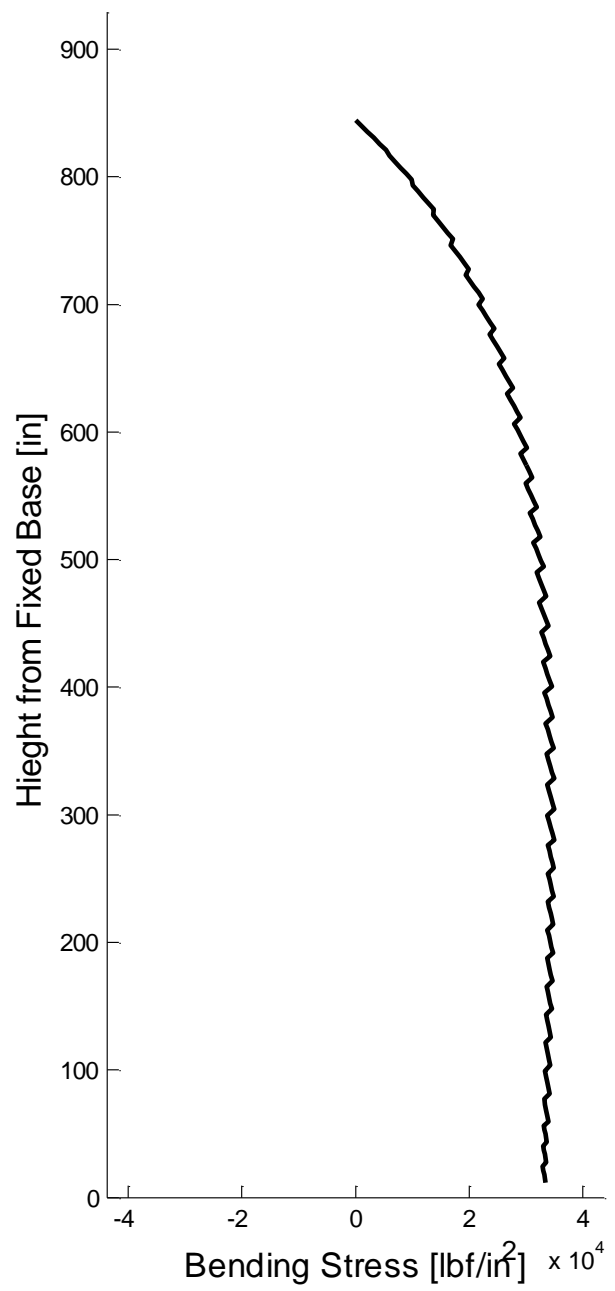

Tower Axial Stress Profile

Max: $192.6 \mathrm{lbf} / \mathrm{in}^{2}$, at hight: $11.5 \mathrm{in}$

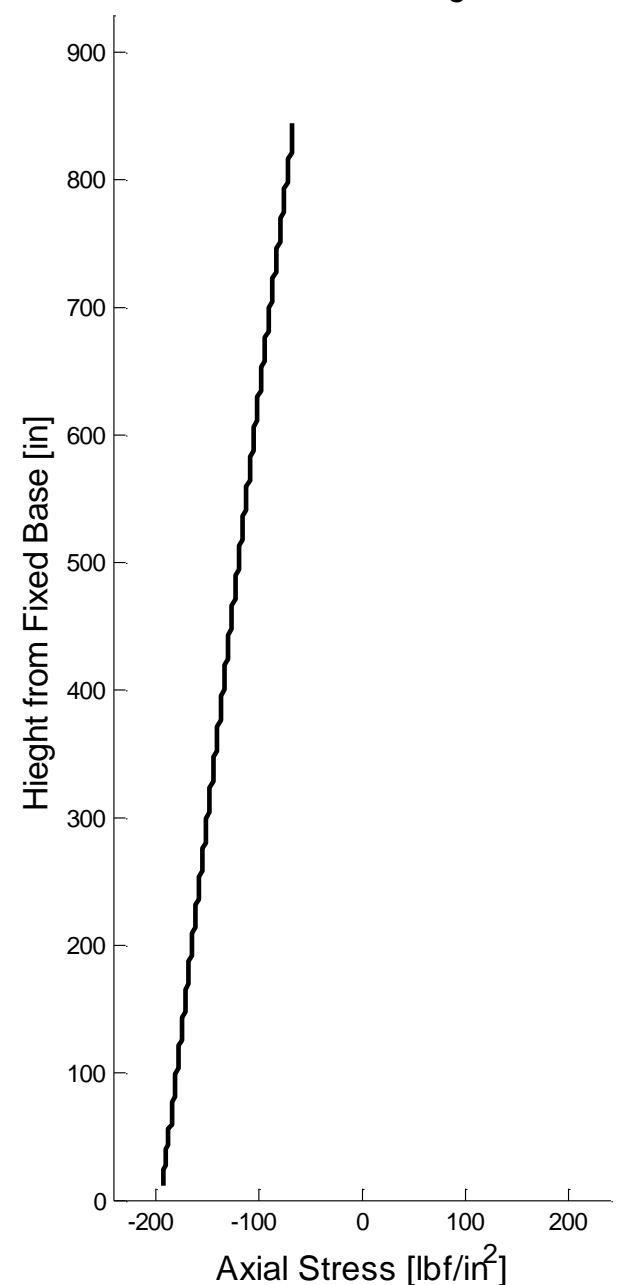




\section{Load Case A: Equivalent von Mises Stress}

Transient Small Wind Turbine Tower Structural Analysis with Coupled Rotor Dynamic Interaction

Max Tower von Mises Stress Profile

Max: $3.514 \mathrm{e}+004 \mathrm{lbf} / \mathrm{in}^{2}$, at hight: 280.3 in

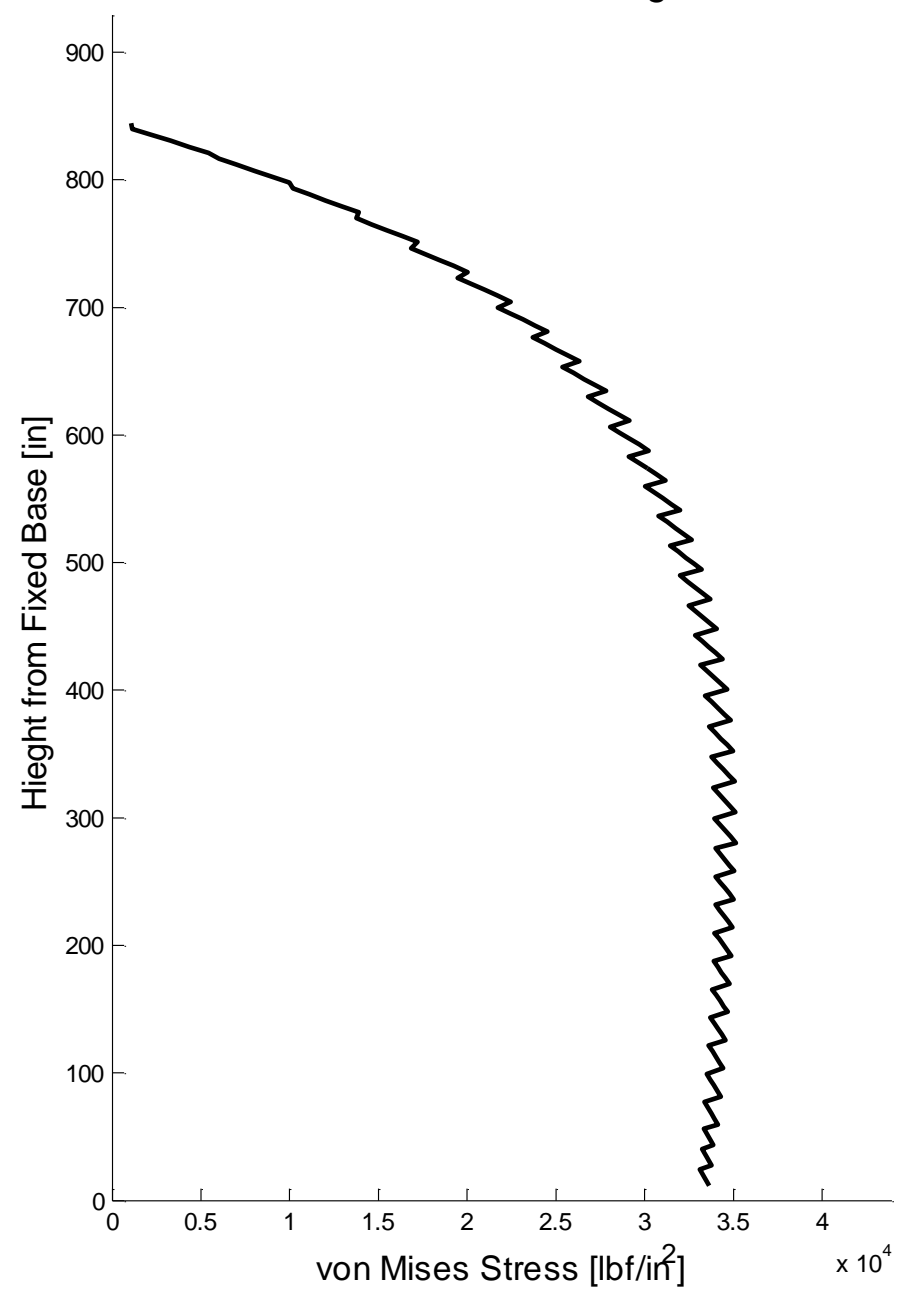

Max Tower von Mises Cross-Sectional Location (Theta) Profile

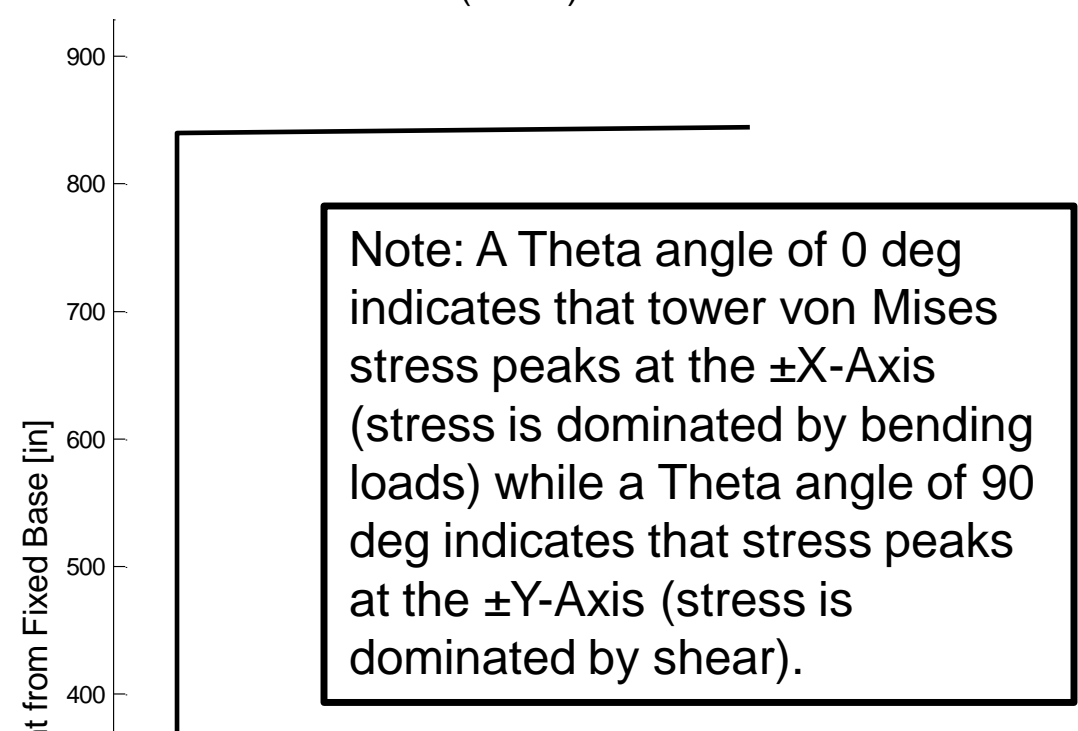




\section{Weak Axis \\ Load Case B - Static \\ 50 Year Extreme Wind \\ Model (EWM50) \\ IEC 61400-2 DLC 5.1 \\ (Parked Rotor)}

California Polytechnic State University - San Luis Obispo 


\section{Load Case B: Overview}

- Distributed load case (including tower and rotor loads)

- Small Wind Turbine Class (SWTC) 3 system

- Developed in accordance with IEC 61400-2 Design Load Case (DLC) 5.1 (Table 4)

- Rotor Force Coefficient: $C_{f}=1.5$

- Tower Drag Coefficient: $C_{d}=1.3$

- Assumed rotor state is parked with rotor plane orthogonal to wind direction (i.e., disk break activated but fault with furling system).

- Load applied in "Weak axis" (the load is orthogonal to the plane formed by the tower, gin-pole, and strut).

- Details of the analysis parameters are summarized and defined in the attached UserlnputsWeak.xIsx file. 


\section{Load Case B: Tower Deflection}

Transverse Tower Deflection

Max Disp: 44.53 in, at hight: 844.5 in

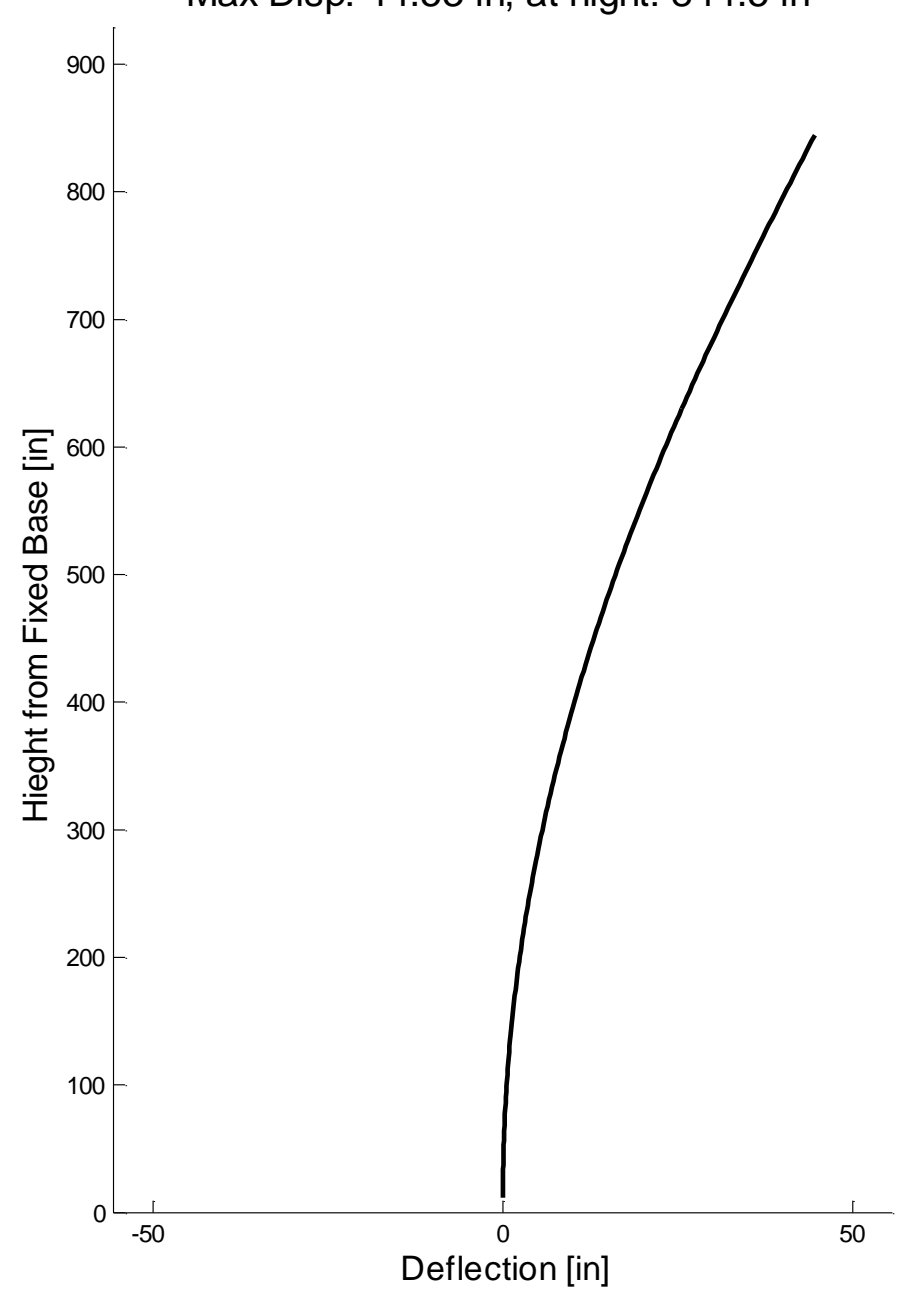

Axial Tower Deflection

Max Disp: 0.003729 in, at hight: 844.5 in

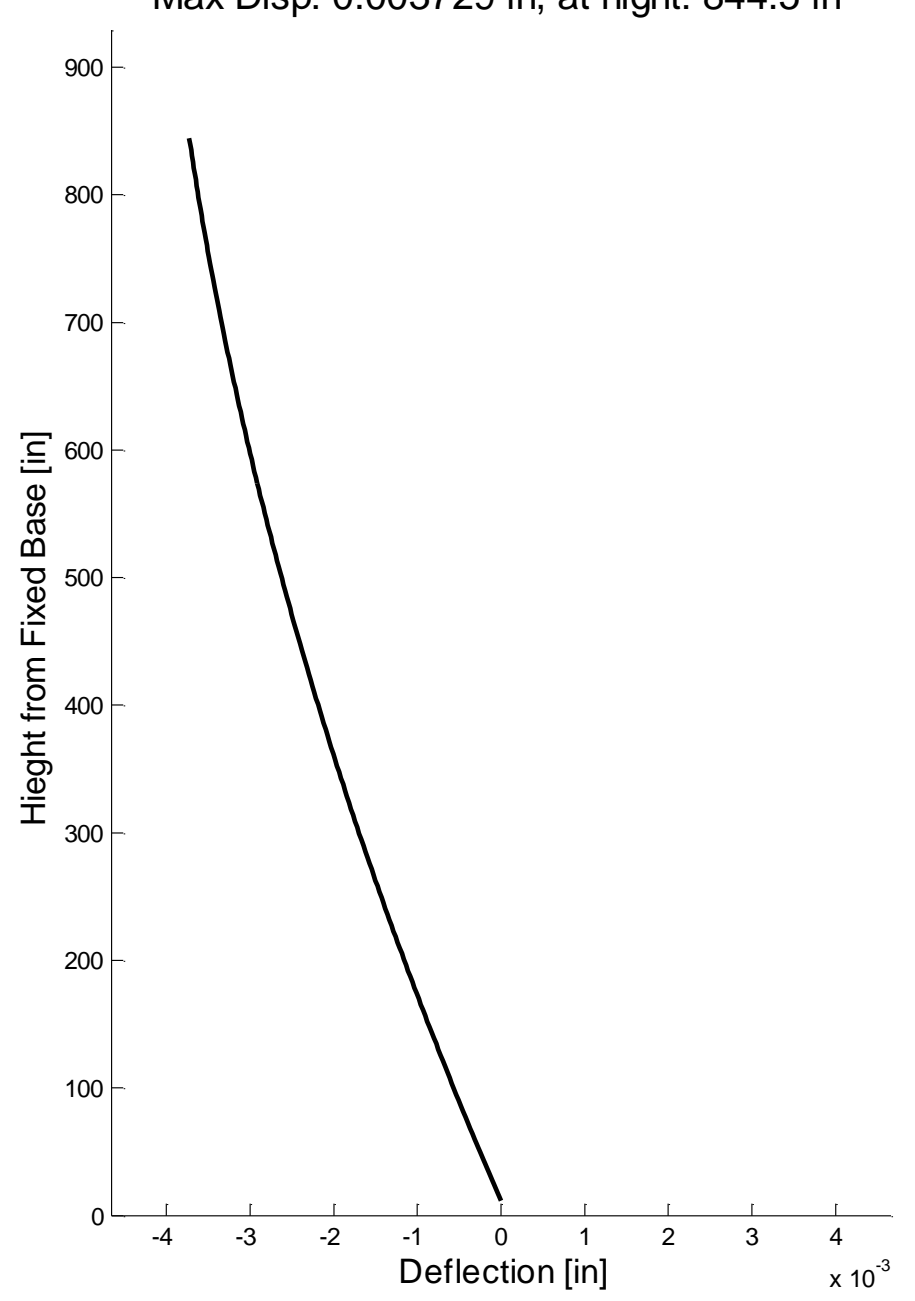




\section{Load Case B: Principle Stresses}

Transient Small Wind Turbine Tower Structural Analysis with Coupled Rotor Dynamic Interaction
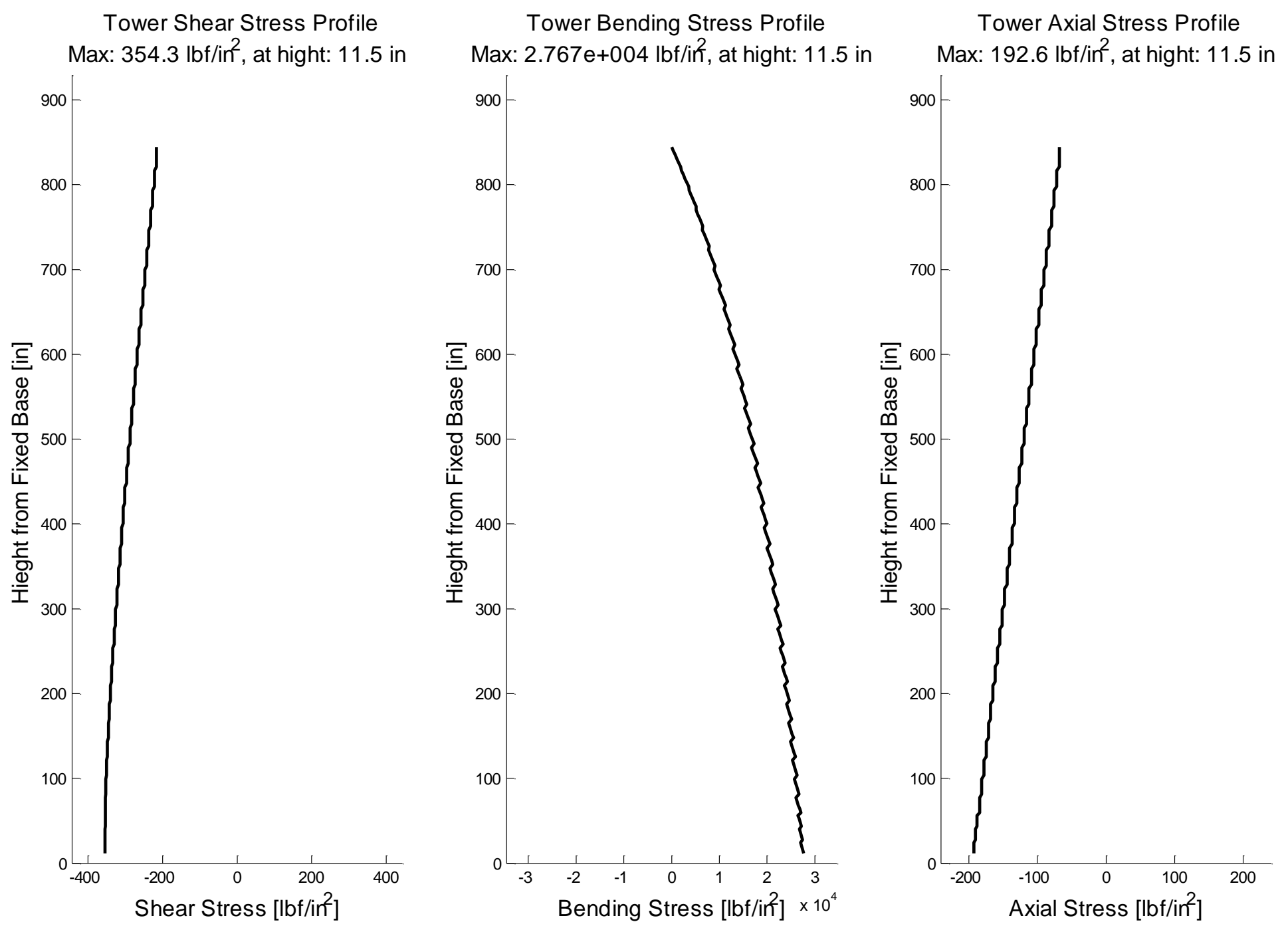


\section{Load Case B: Equivalent von Mises Stress}

Transient Small Wind Turbine Tower Structural Analysis with Coupled Rotor Dynamic Interaction

Max Tower von Mises Stress Profile

Max: $2.786 \mathrm{e}+004 \mathrm{lbf} / \mathrm{in}^{2}$, at hight: $11.5 \mathrm{in}$

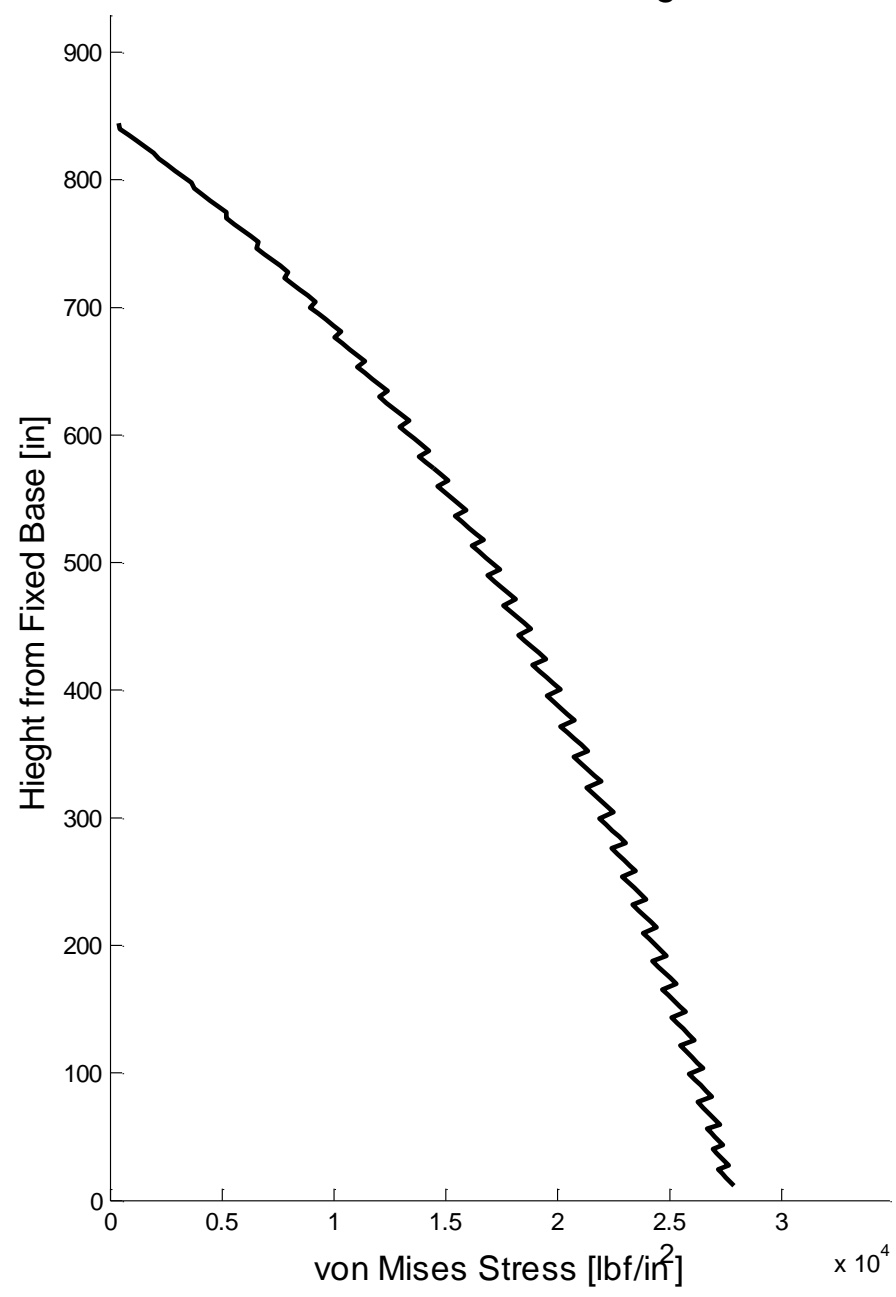

Max Tower von Mises Cross-Sectional Location (Theta) Profile

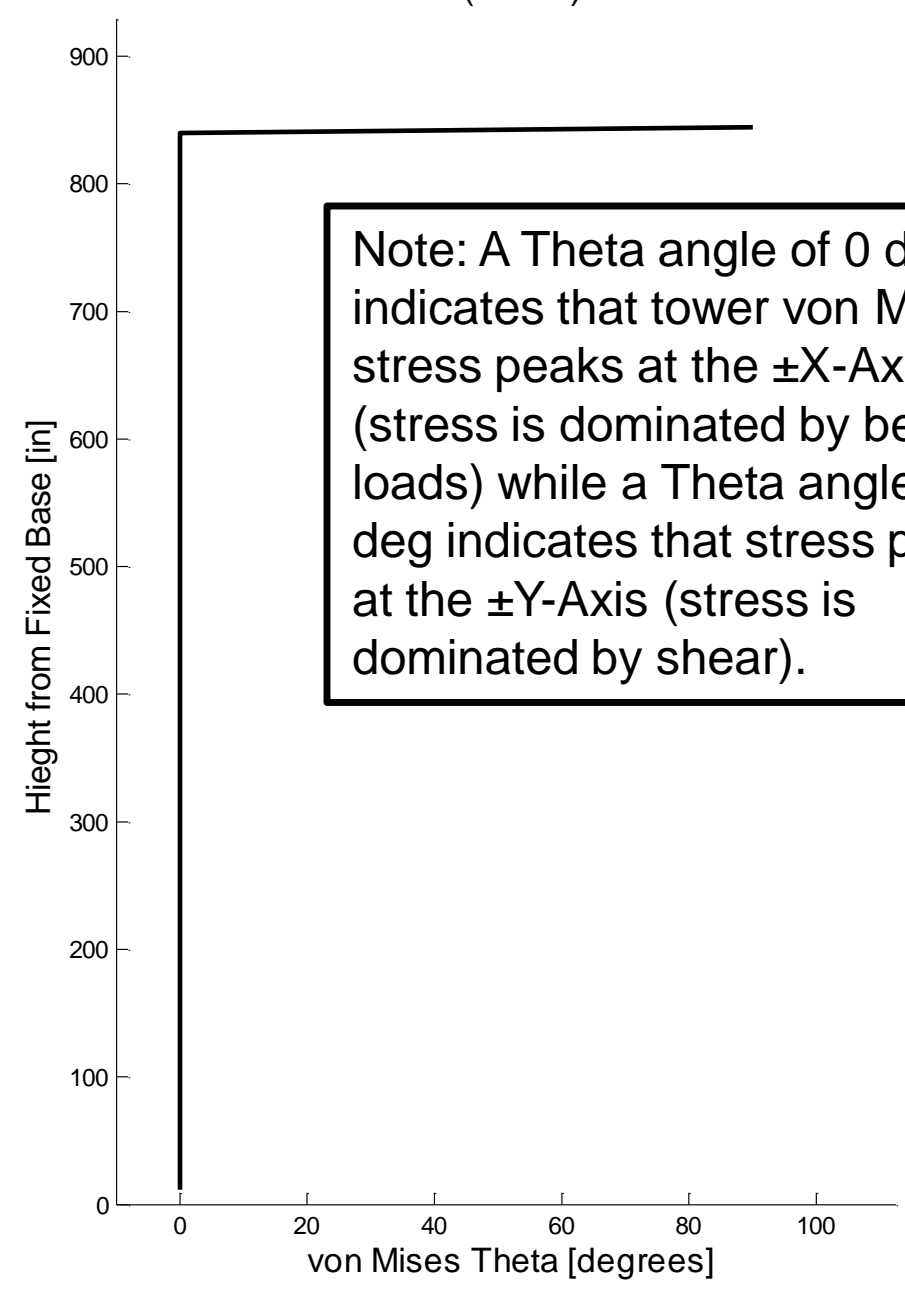




\section{Weak Axis}

Load Case C - Static

1 Year Extreme Wind

Model (EWM1)

IEC 61400-2 DLC 6.1 


\section{Load Case C: Overview}

Transient Small Wind Turbine Tower Structural Analysis with Coupled Rotor Dynamic Interaction

- Distributed load case (including tower and rotor loads)

- Small Wind Turbine Class (SWTC) 3 system

- Developed in accordance with IEC 61400-2 Design Load Case (DLC) 6.1 (Table 4)

- Rotor Thrust Coefficient: $C_{t}=0.5$

- Tower Drag Coefficient: $C_{d}=1.3$

- Assumed rotor state is power production (more conservative than IEC 61400-2 which specifies parked or idling for this DLC); effective assumption is a fault in the furling system and disk break

- Load applied in "Weak axis" (the load is orthogonal to the plane formed by the tower, gin-pole, and strut).

- Details of the analysis parameters are summarized and defined in the attached UserInputsWeak.xIsx file. 


\section{Load Case C: Tower Deflection}

Transverse Tower Deflection

Max Disp: 49.42 in, at hight: 844.5 in

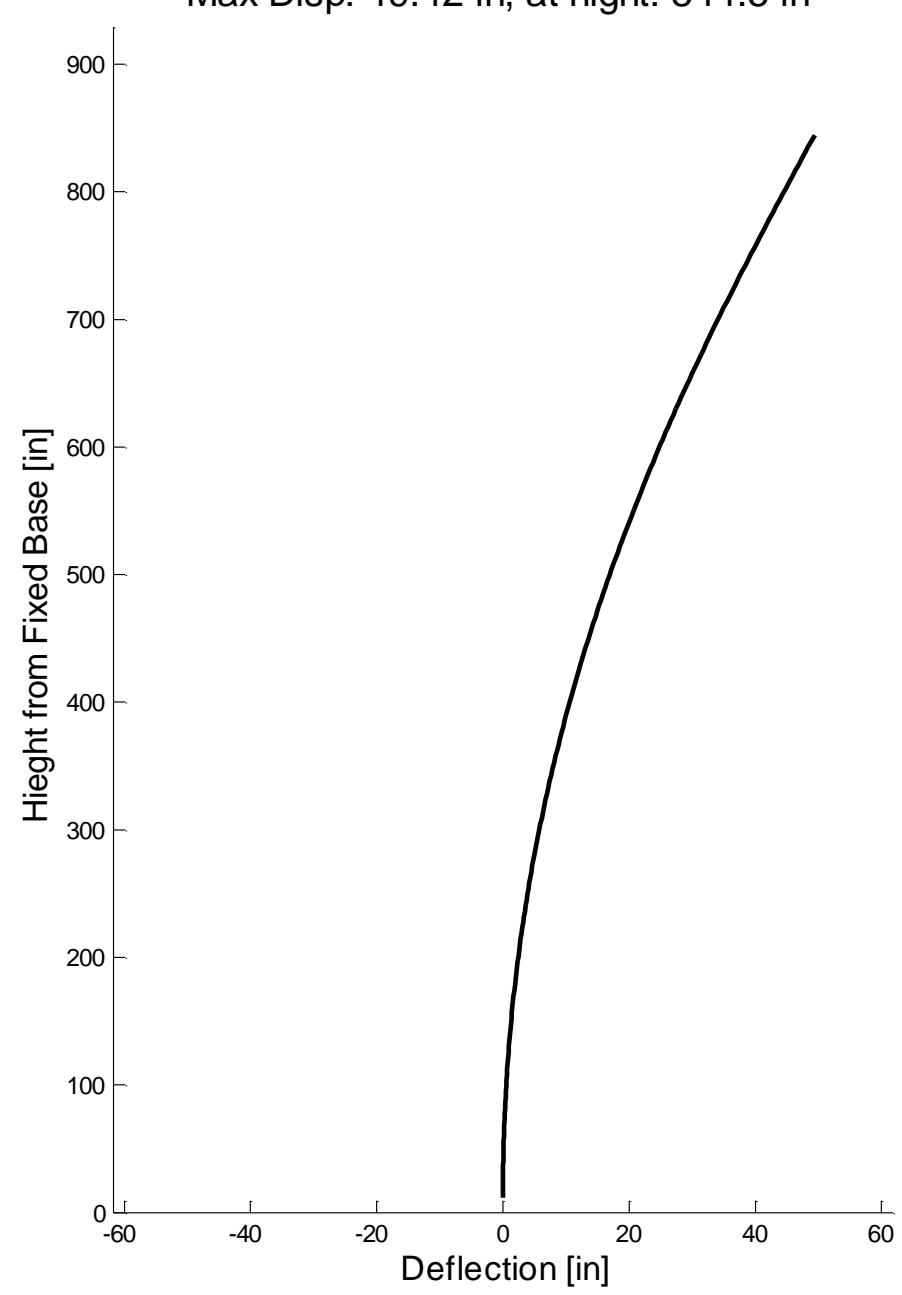

Axial Tower Deflection

Max Disp: 0.003729 in, at hight: 844.5 in

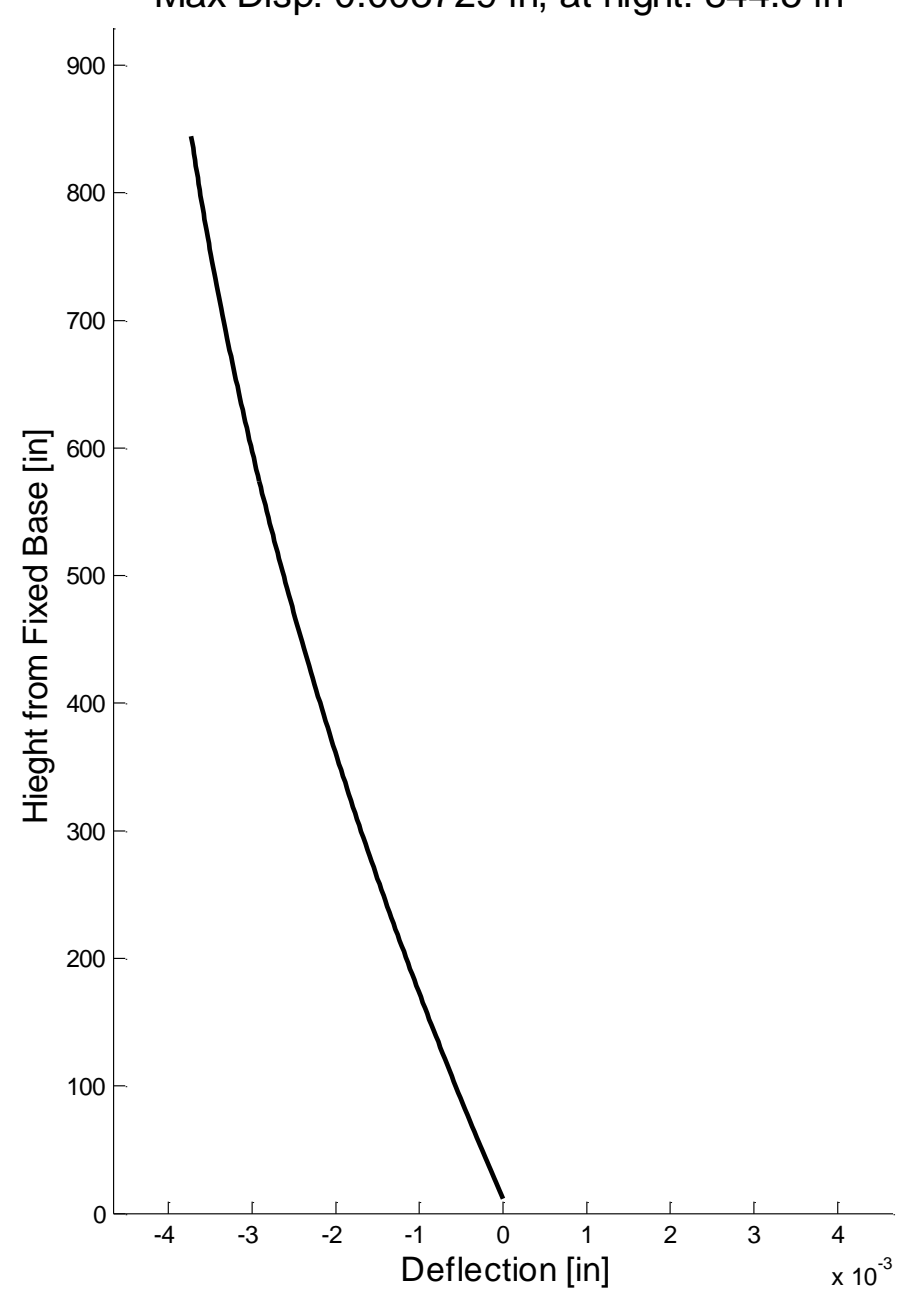




\section{Load Case C: Principle Stresses}

Transient Small Wind Turbine Tower Structural Analysis with Coupled Rotor Dynamic Interaction

Tower Shear Stress Profile

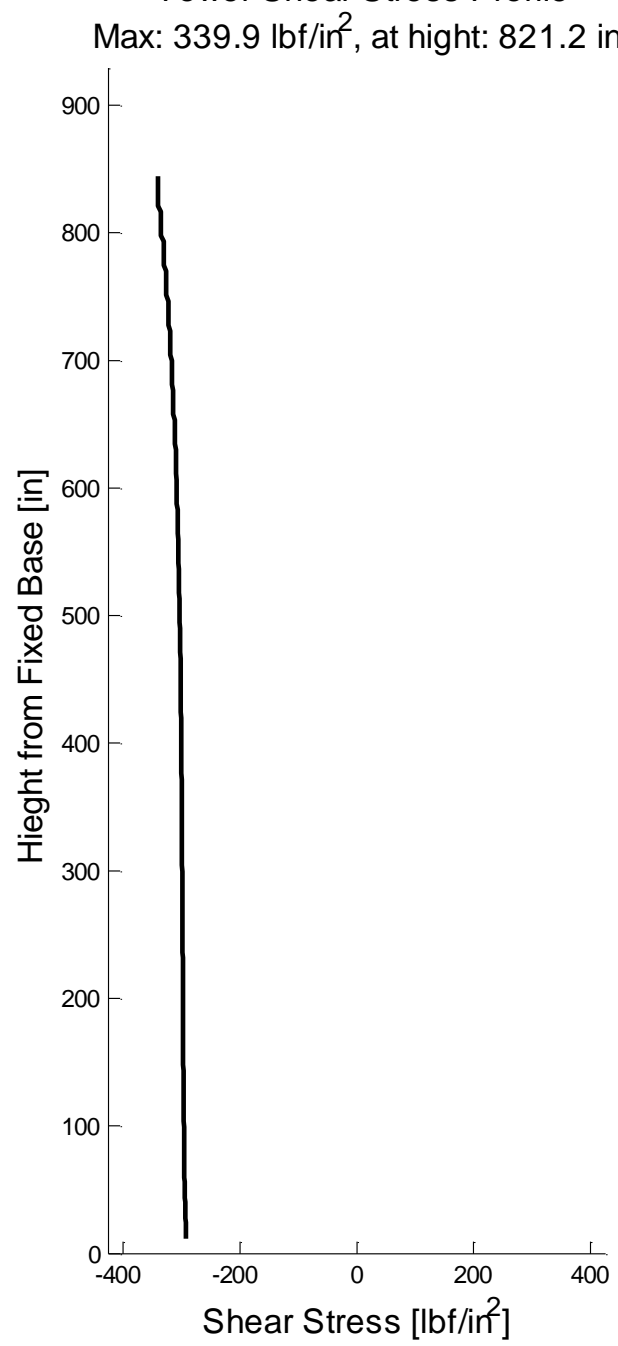

Tower Bending Stress Profile

Max: $2.73 \mathrm{e}+004 \mathrm{lbf} / \mathrm{in}^{2}$, at hight: $11.5 \mathrm{in}$

$$
900
$$

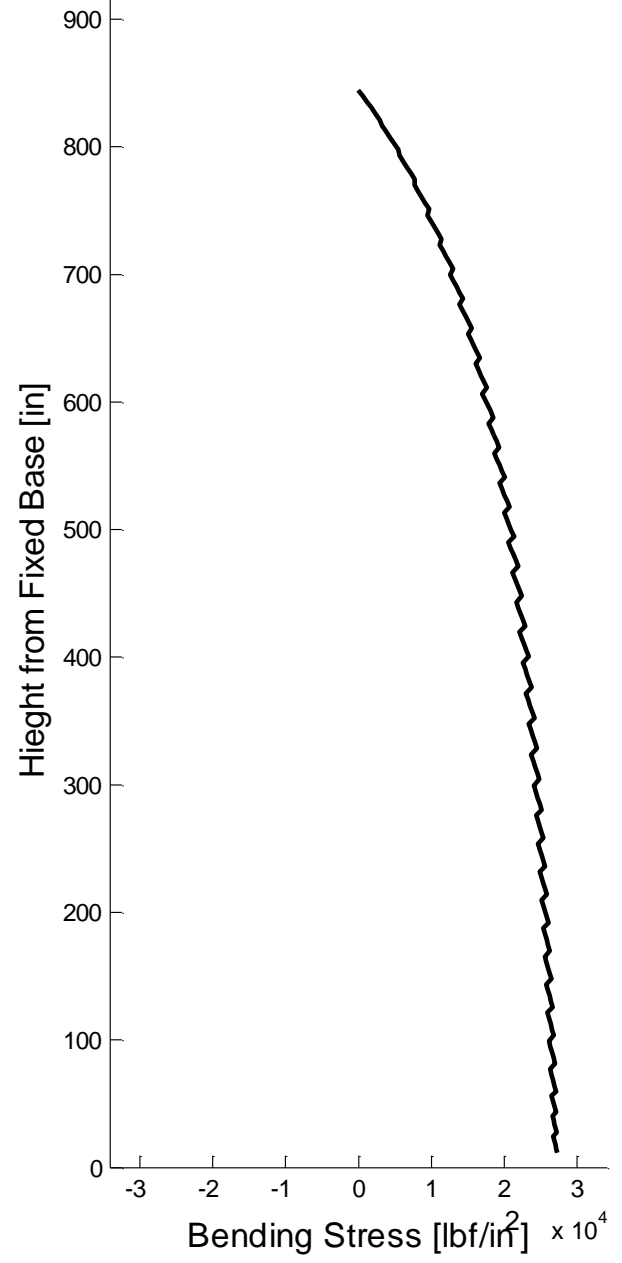

Tower Axial Stress Profile

Max: $192.6 \mathrm{lbf} / \mathrm{in}^{2}$, at hight: $11.5 \mathrm{in}$

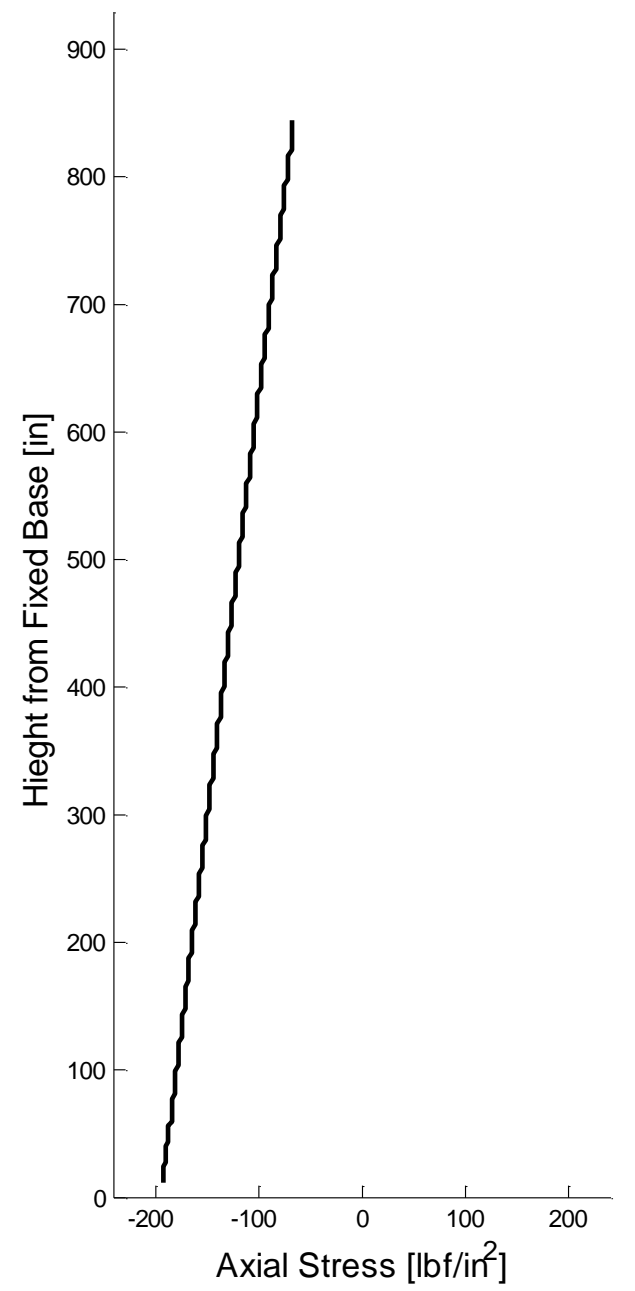




\section{Load Case C: Equivalent von Mises Stress}

Transient Small Wind Turbine Tower Structural Analysis with Coupled Rotor Dynamic Interaction

Max Tower von Mises Stress Profile

Max: $2.749 \mathrm{e}+004 \mathrm{lbf} / \mathrm{in}^{2}$, at hight: $11.5 \mathrm{in}$

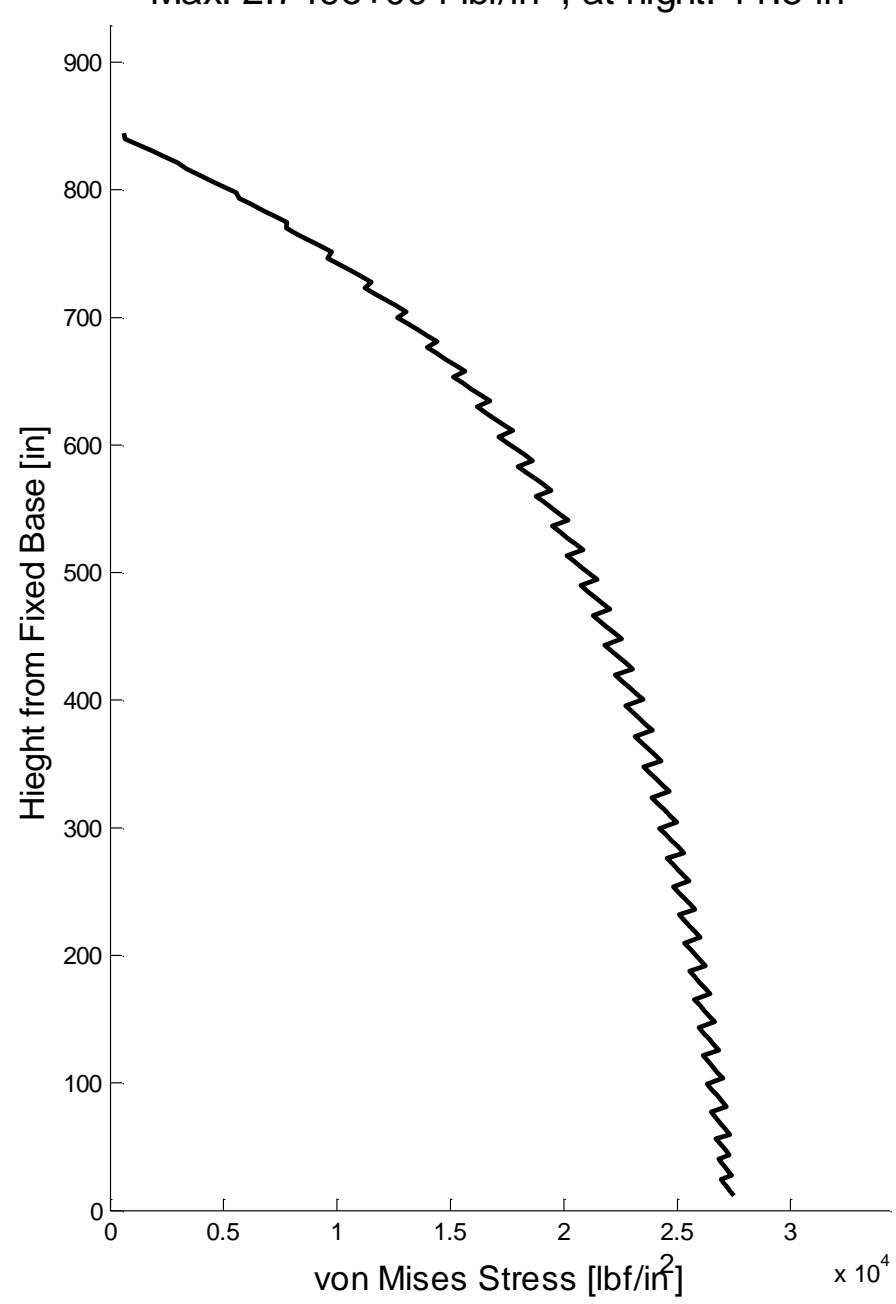

Max Tower von Mises Cross-Sectional Location (Theta) Profile

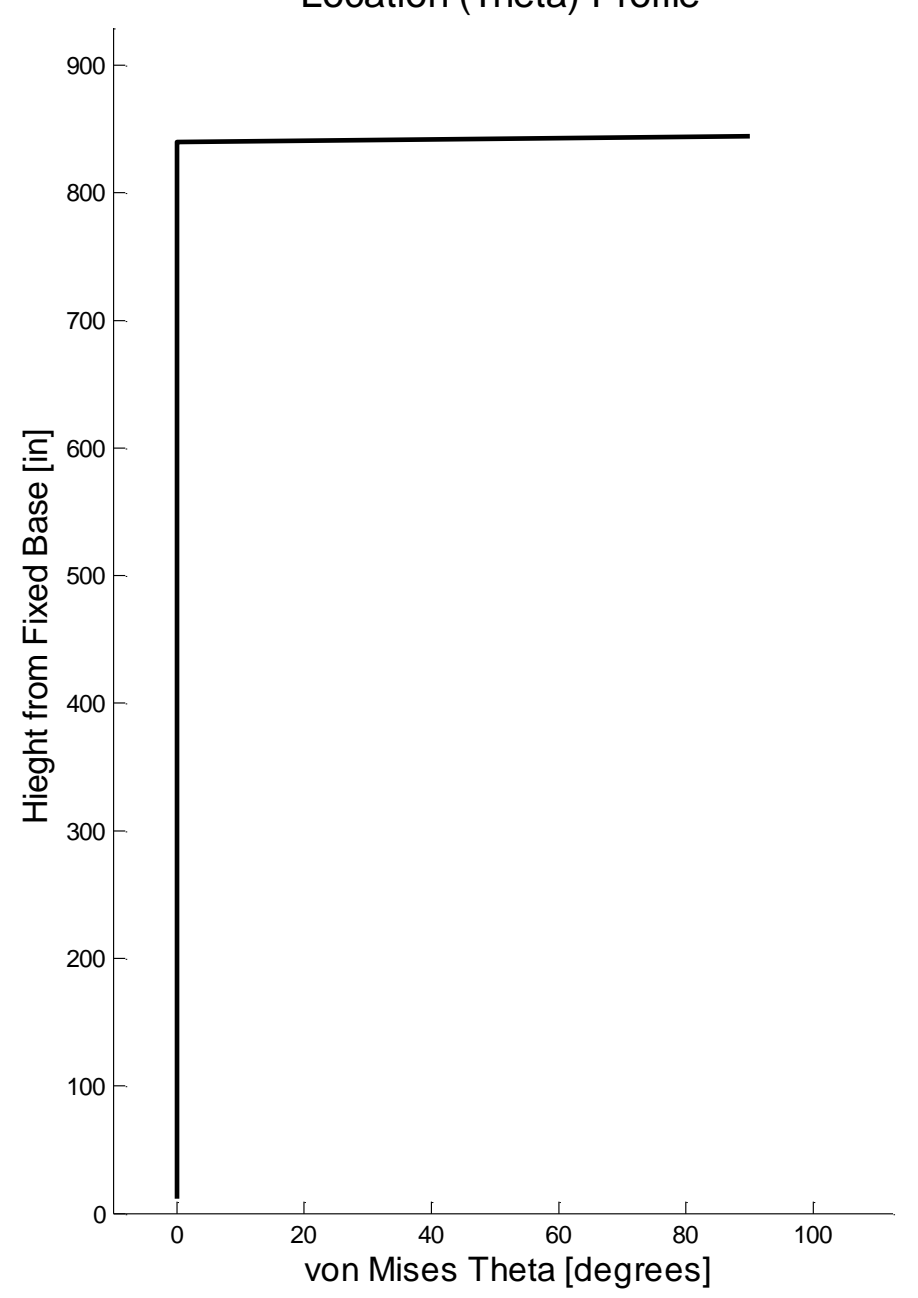




\section{Weak Axis \\ Load Case D - Static \\ 15 MPH "Nominal \\ Operation"}




\section{Load Case D: Overview}

- Distributed load case (including tower and rotor loads)

- Small Wind Turbine Class (SWTC) 3 system

- Wind profile is same as EWM1 but scaled down such that the wind velocity at the tower tip is $15 \mathrm{MPH}$, representing a state of "nominal operation"

- Developed in accordance with IEC 61400-2 Design Load Case (DLC) 6.1 (Table 4)

- Rotor Thrust Coefficient: $C_{t}=0.5$

- Tower Drag Coefficient: $C_{d}=1.3$

- Assumed rotor state is power production.

- Load applied in "Weak axis" (the load is orthogonal to the plane formed by the tower, gin-pole, and strut).

- Details of the analysis parameters are summarized and defined in the attached UserInputsWeak.xIsx file. 


\section{Load Case D: Tower Deflection}

Transverse Tower Deflection

Max Disp: 1.433 in, at hight: 844.5 in

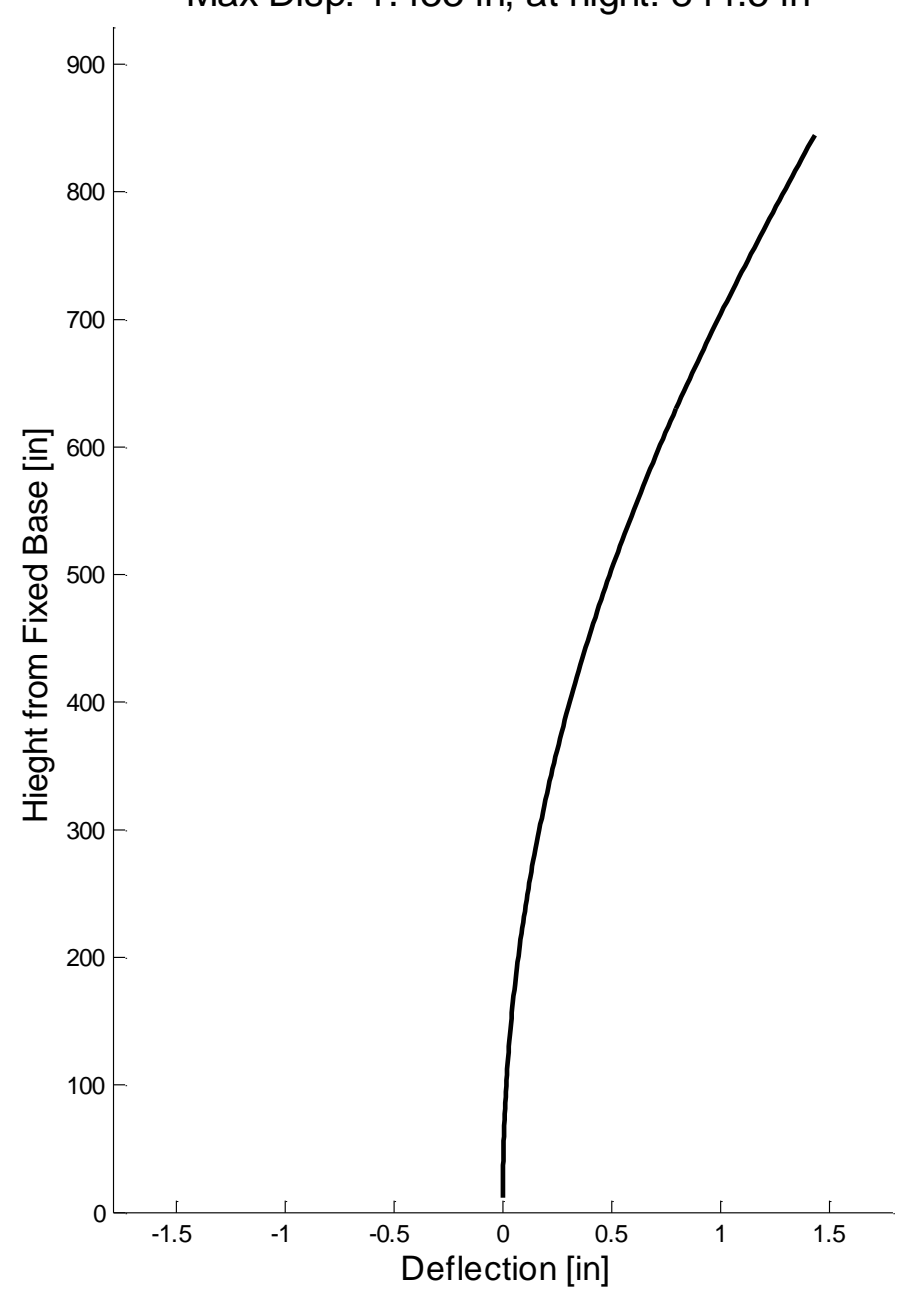

Axial Tower Deflection

Max Disp: 0.003729 in, at hight: 844.5 in

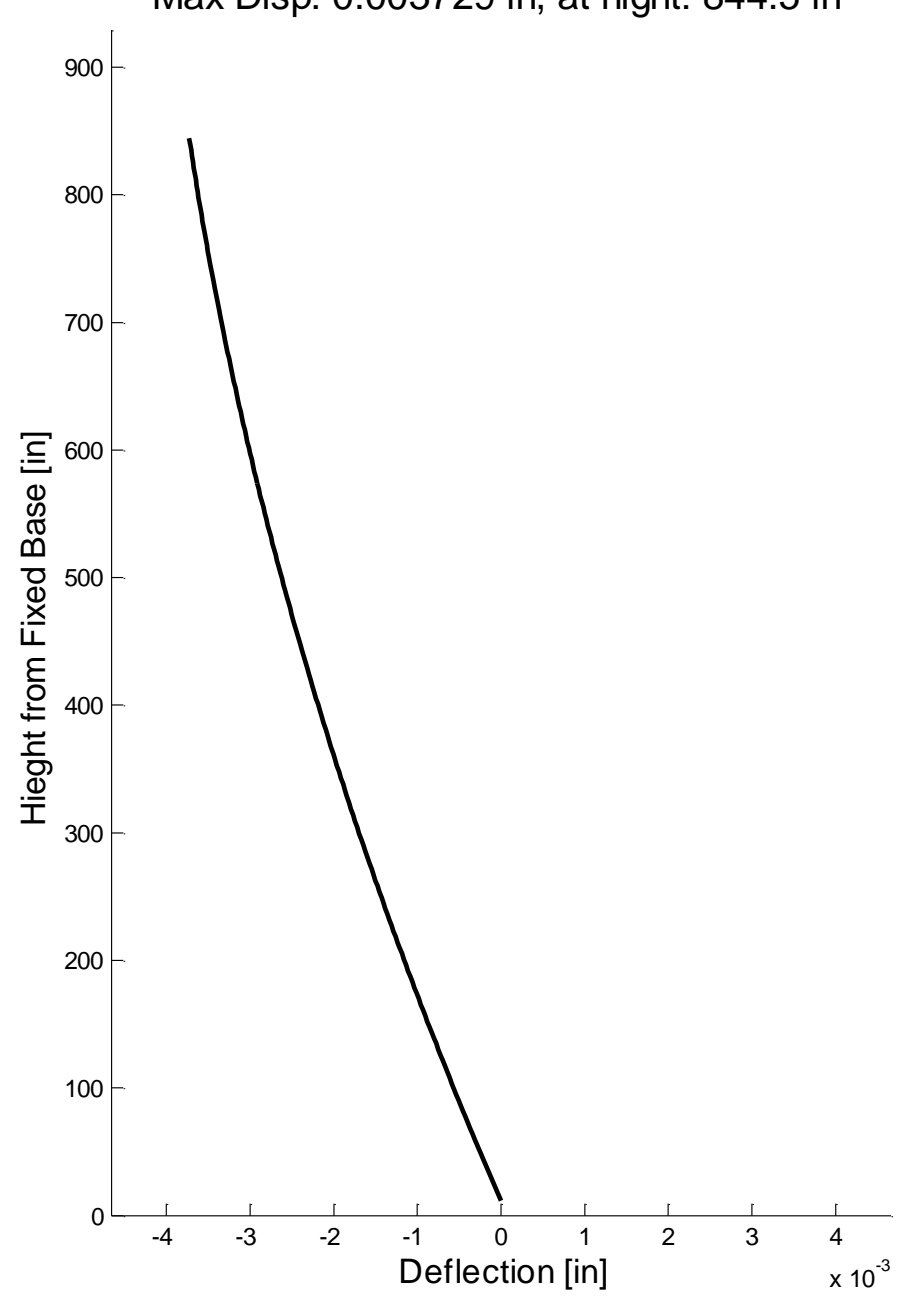




\section{Load Case D: Principle Stresses}

Transient Small Wind Turbine Tower Structural Analysis with Coupled Rotor Dynamic Interaction

Tower Shear Stress Profile

Max: $9.859 \mathrm{lbf} / \mathrm{in}^{2}$, at hight: $821.2 \mathrm{in}$

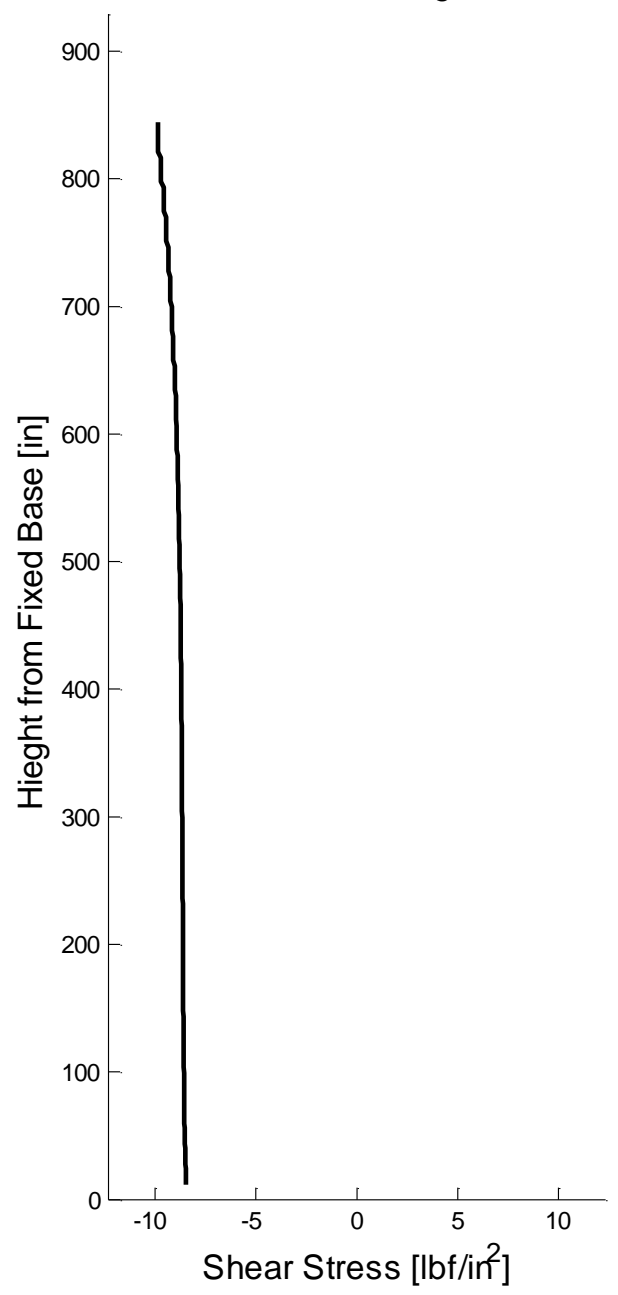

Tower Bending Stress Profile

Max: $791.8 \mathrm{lbf} / \mathrm{in}^{2}$, at hight: $11.5 \mathrm{in}$

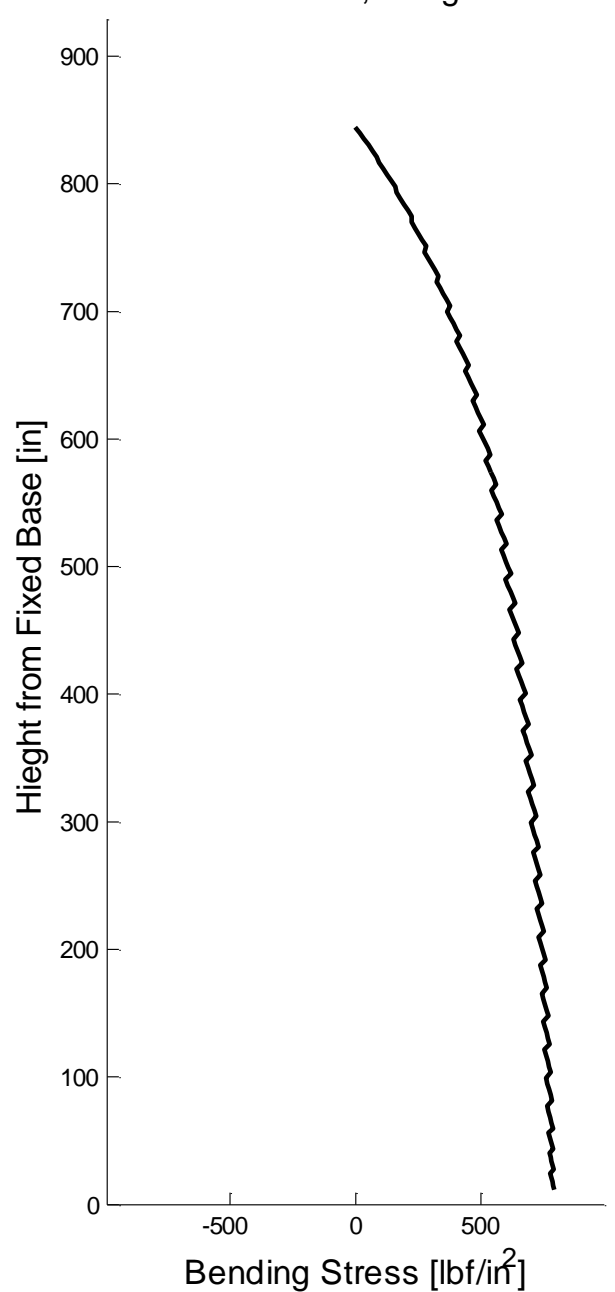

Tower Axial Stress Profile

Max: $192.6 \mathrm{lbf} / \mathrm{in}^{2}$, at hight: $11.5 \mathrm{in}$

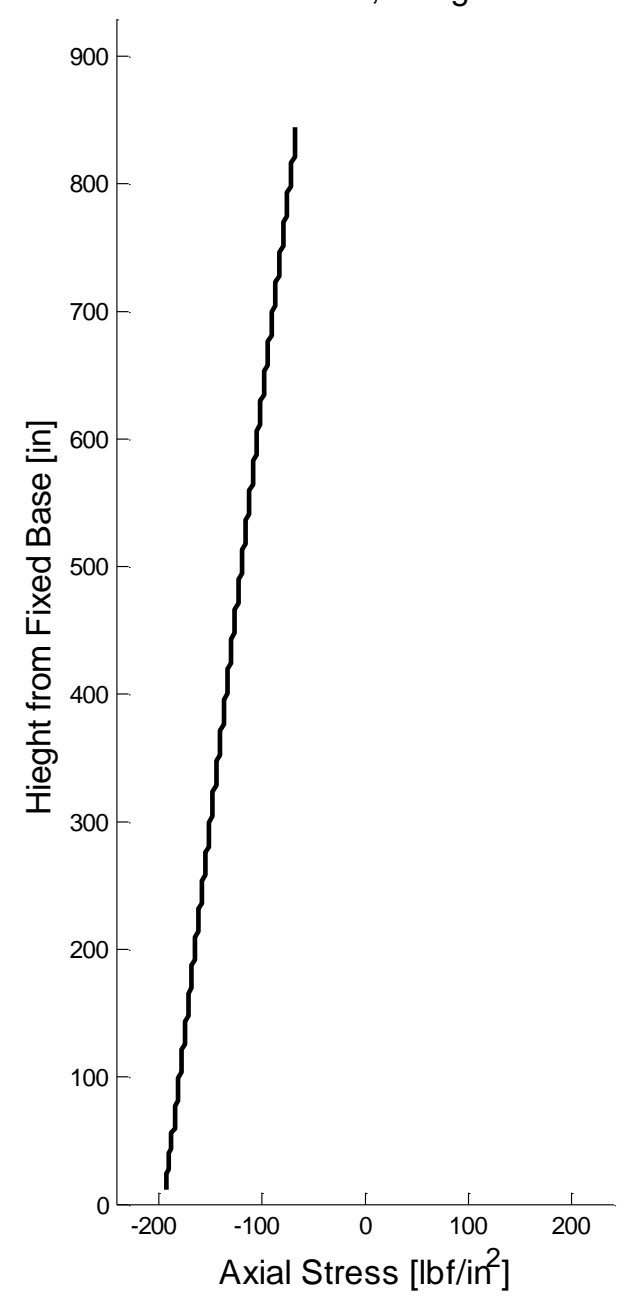




\section{Load Case D: Equivalent von Mises Stress}

Transient Small Wind Turbine Tower Structural Analysis with Coupled Rotor Dynamic Interaction

Max Tower von Mises Stress Profile

Max: $984.4 \mathrm{lbf} / \mathrm{in}^{2}$, at hight: $11.5 \mathrm{in}$

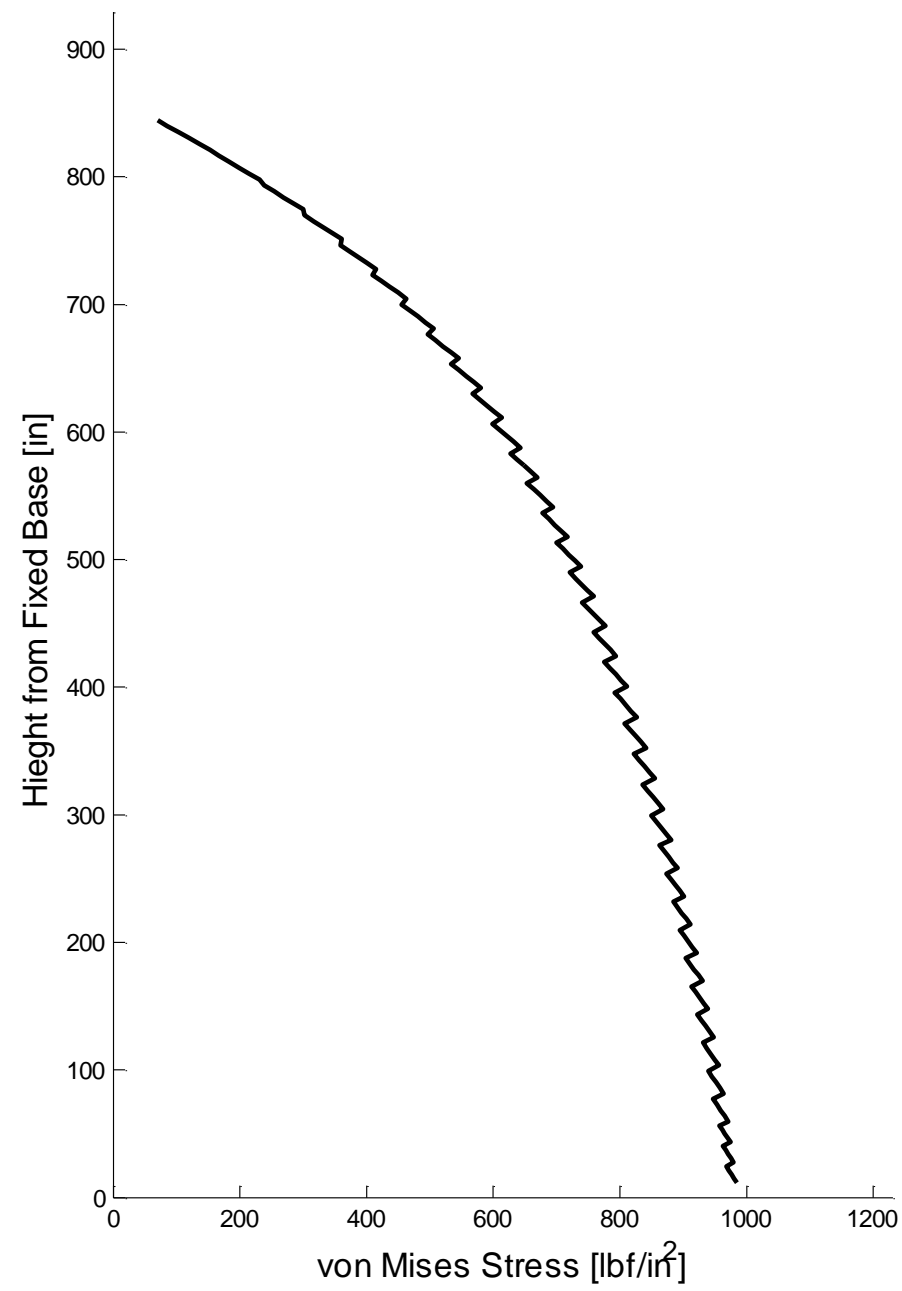

Max Tower von Mises Cross-Sectional Location (Theta) Profile

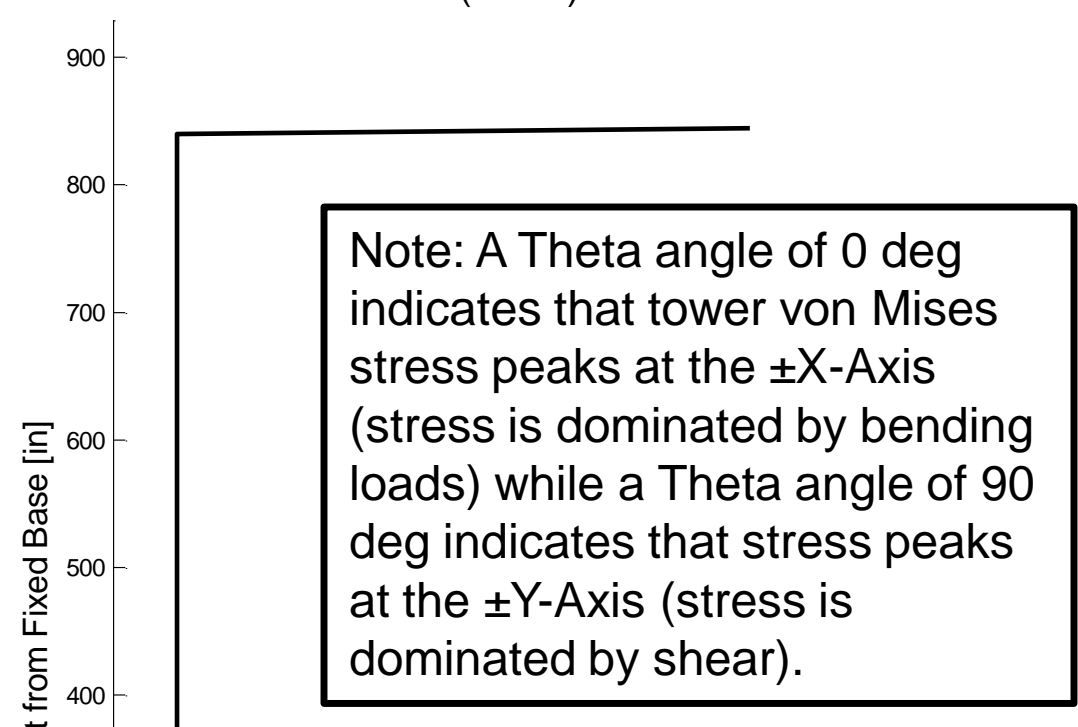




\section{Weak Axis}

Modal Evaluation 


\section{Modal Evaluation: Overview}

- Results of normal modes using an Eigenvalue / Eigenvector evaluation are displayed on the next page.

- The lowest five mode shapes are shown in the Transverse direction (X-Axis, in the Weak Axis direction) and in the Axial direction (Y-Axis).

- Note that the Transverse mode shape plot skips Mode \# 5.

- Mode \# 5 is the rotor flapwise blade mode and is at $16.25 \mathrm{~Hz}$ (this frequency was estimated using the Myklestad Method and blade). 


\section{Modal Evaluation}
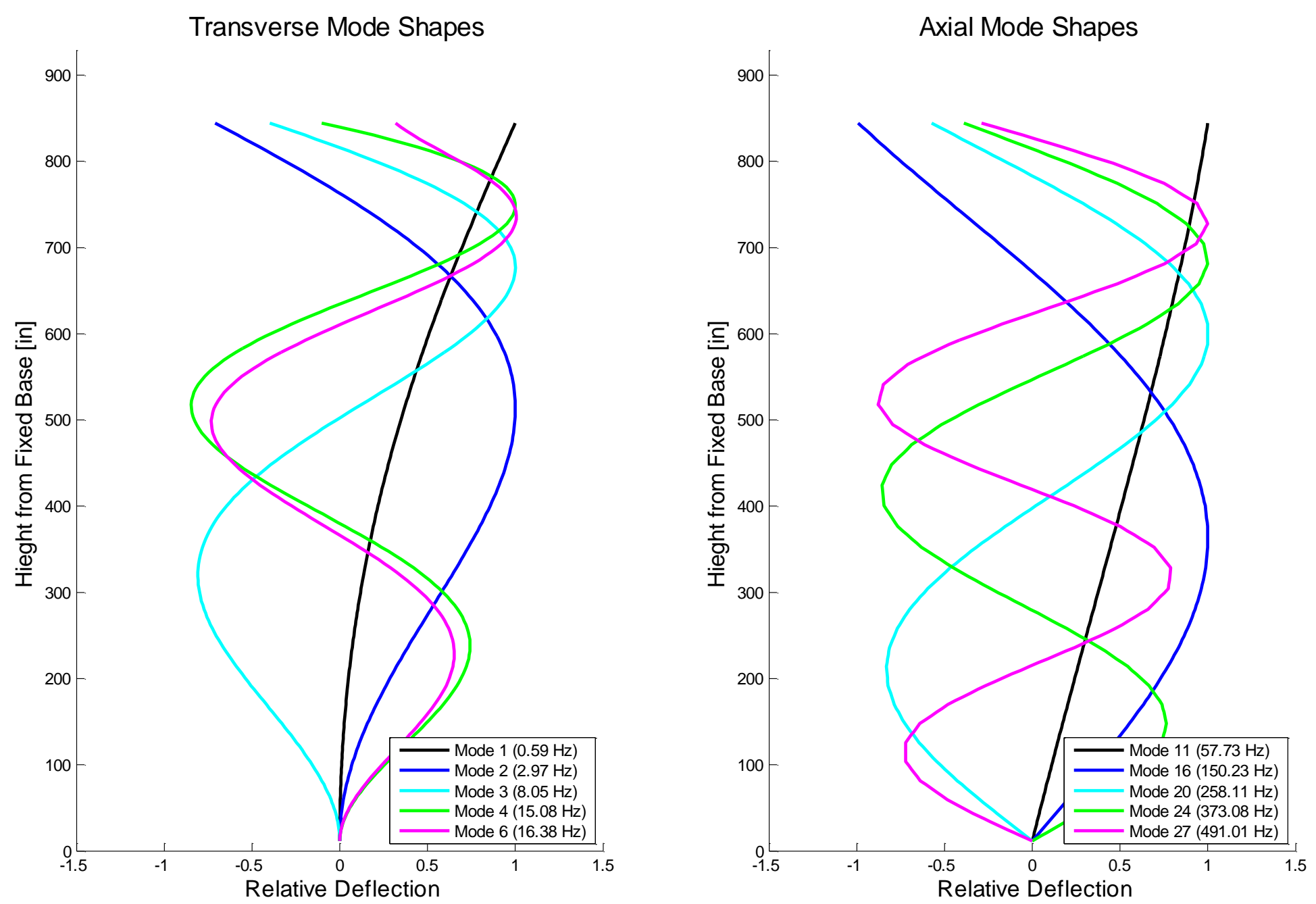

California Polytechnic State University - San Luis Obispo 


\section{Weak Axis}

Load Case E - Dynamic Extreme Operating Gust (EOG)

IEC $61400-2$ DLC

1.3 


\section{Load Case E: Overview}

- Distributed dynamic load case (including tower and rotor loads)

- Small Wind Turbine Class (SWTC) 3 system

- Developed in accordance with IEC 61400-2 Design Load Case (DLC) 1.3 (Table 4)

- Rotor Thrust Coefficient: $C_{t}=0.5$

- Tower Drag Coefficient: $C_{d}=1.3$

- Nhat $=30$, see Appendix A for details

- Load applied in "Weak axis" (the load is orthogonal to the plane formed by the tower, gin-pole, and strut).

- Details of the analysis parameters are summarized and defined in the attached UserInputsWeak.xIsx file. 


\section{Load Case E: Tower Tip Wind Speed and Rotor Thrust}

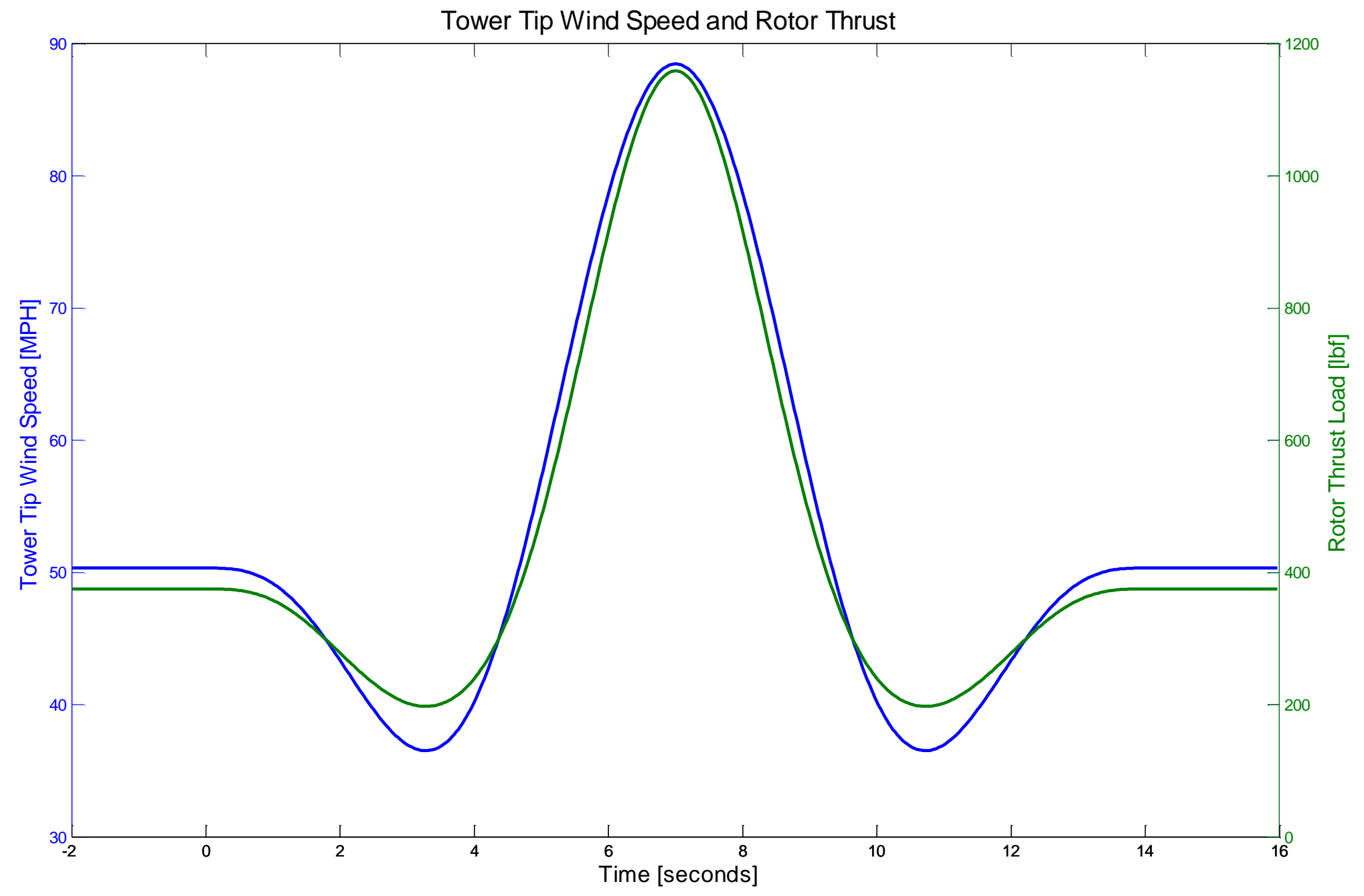

California Polytechnic State University - San Luis Obispo 


\section{Load Case E: Tower Tip Transient Deflection}

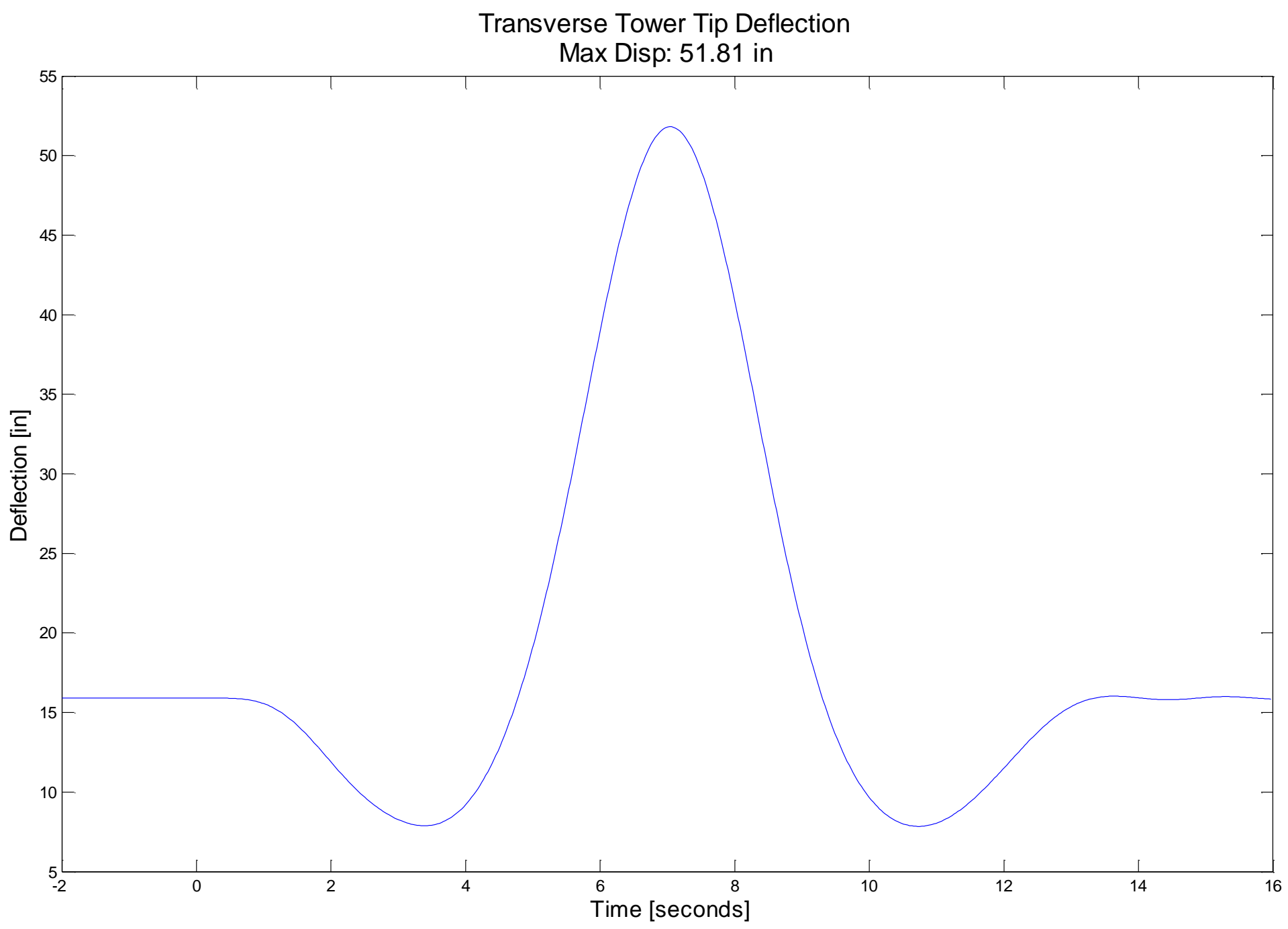

California Polytechnic State University - San Luis Obispo 


\section{Load Case E: Tower von Mises Stress Profille}
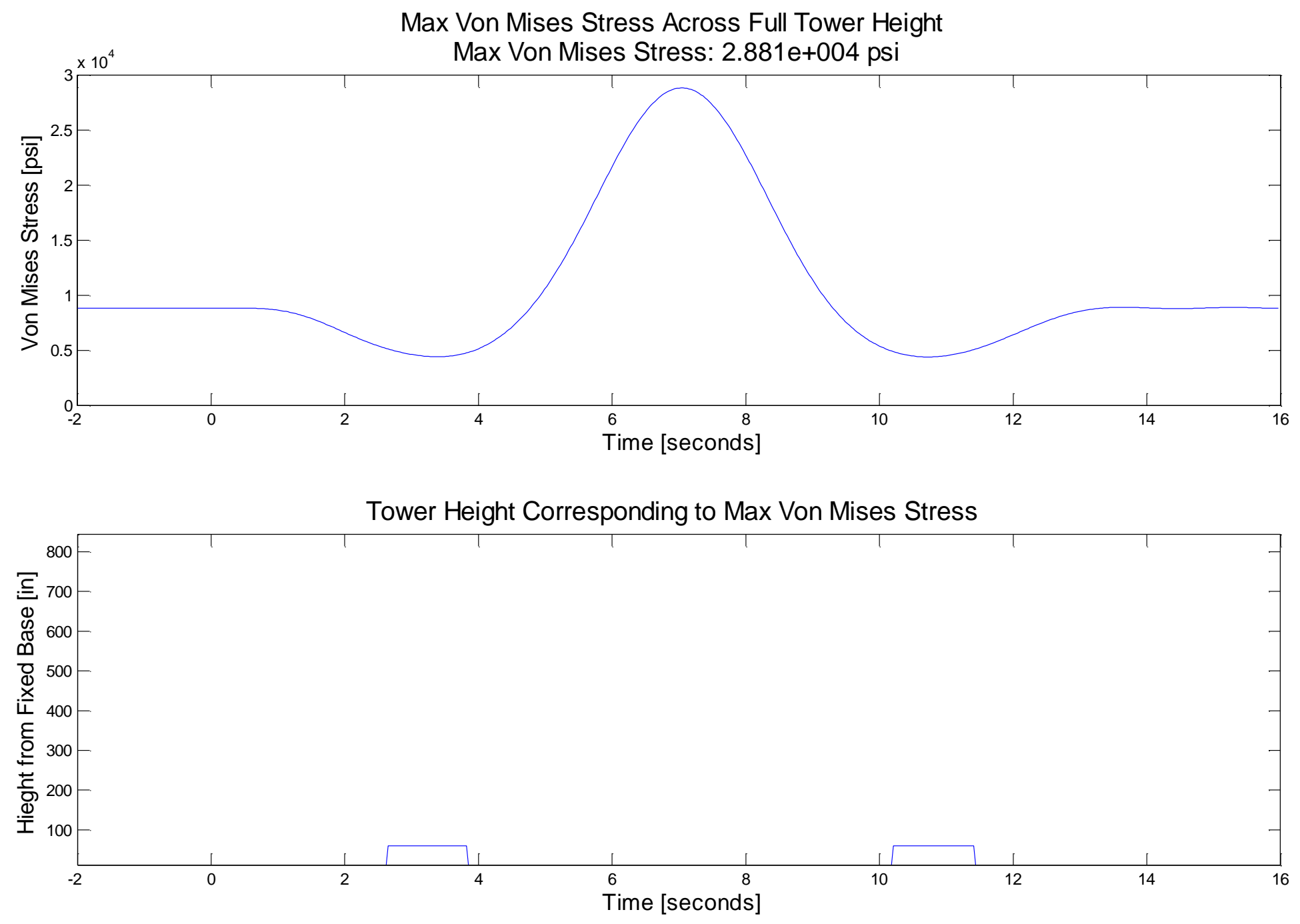


\section{Load Case E: Blade Beta Angle (relative flapwise angle)}

Blade Beta Hinge Displacement Angle Max Absolute Blade 1: 0.04552 radians Max Absolute Blade 2: 0.04553 radians Max Absolute Blade 3: 0.04553 radians

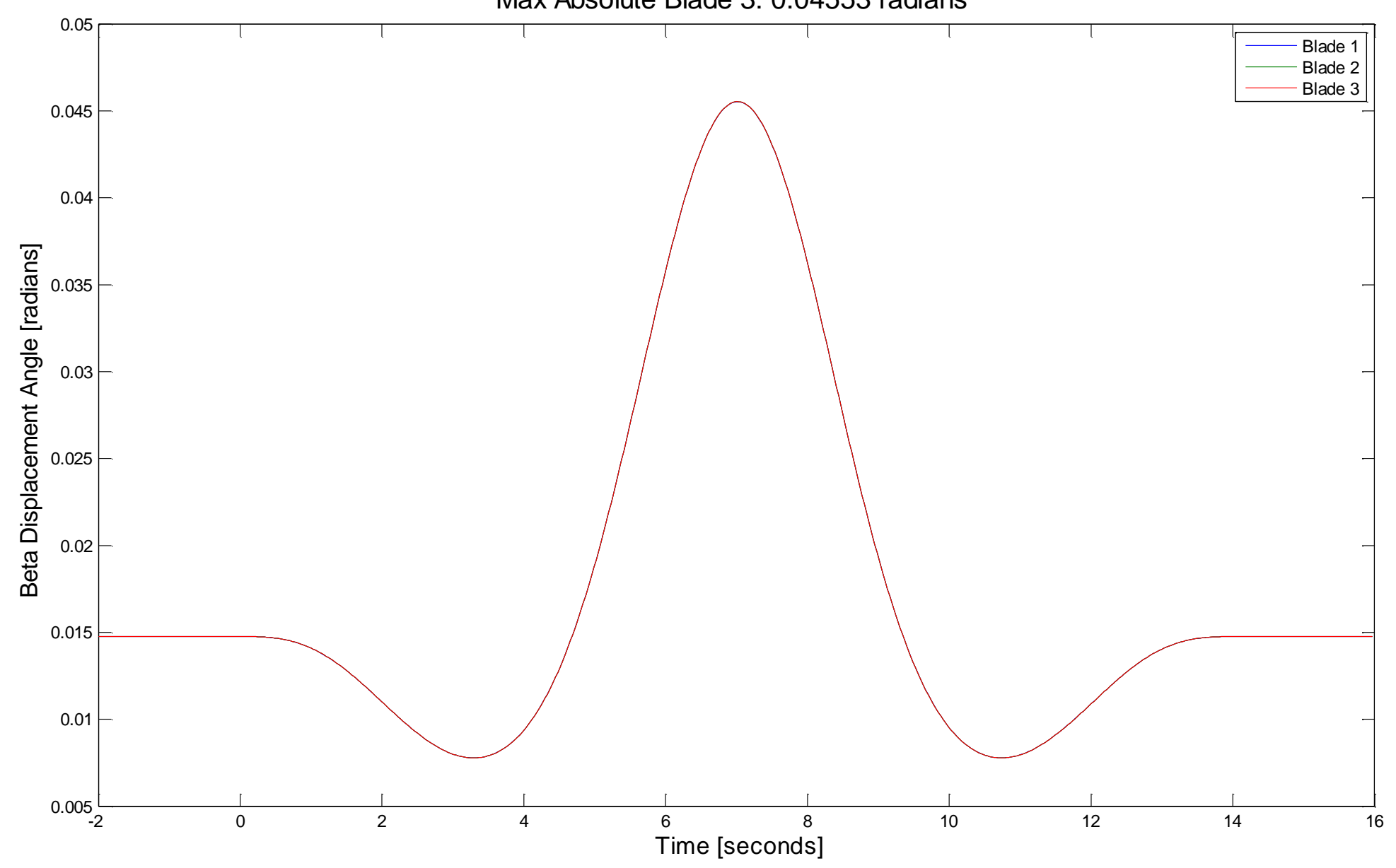




\section{Load Case E: Tower Displacement Profille at 7 Seconds}

Transient Small Wind Turbine Tower Structural Analysis with Coupled Rotor Dynamic Interaction

Transverse Tower Deflection

Max Disp: 51.81 in, at hight: 844.5 in

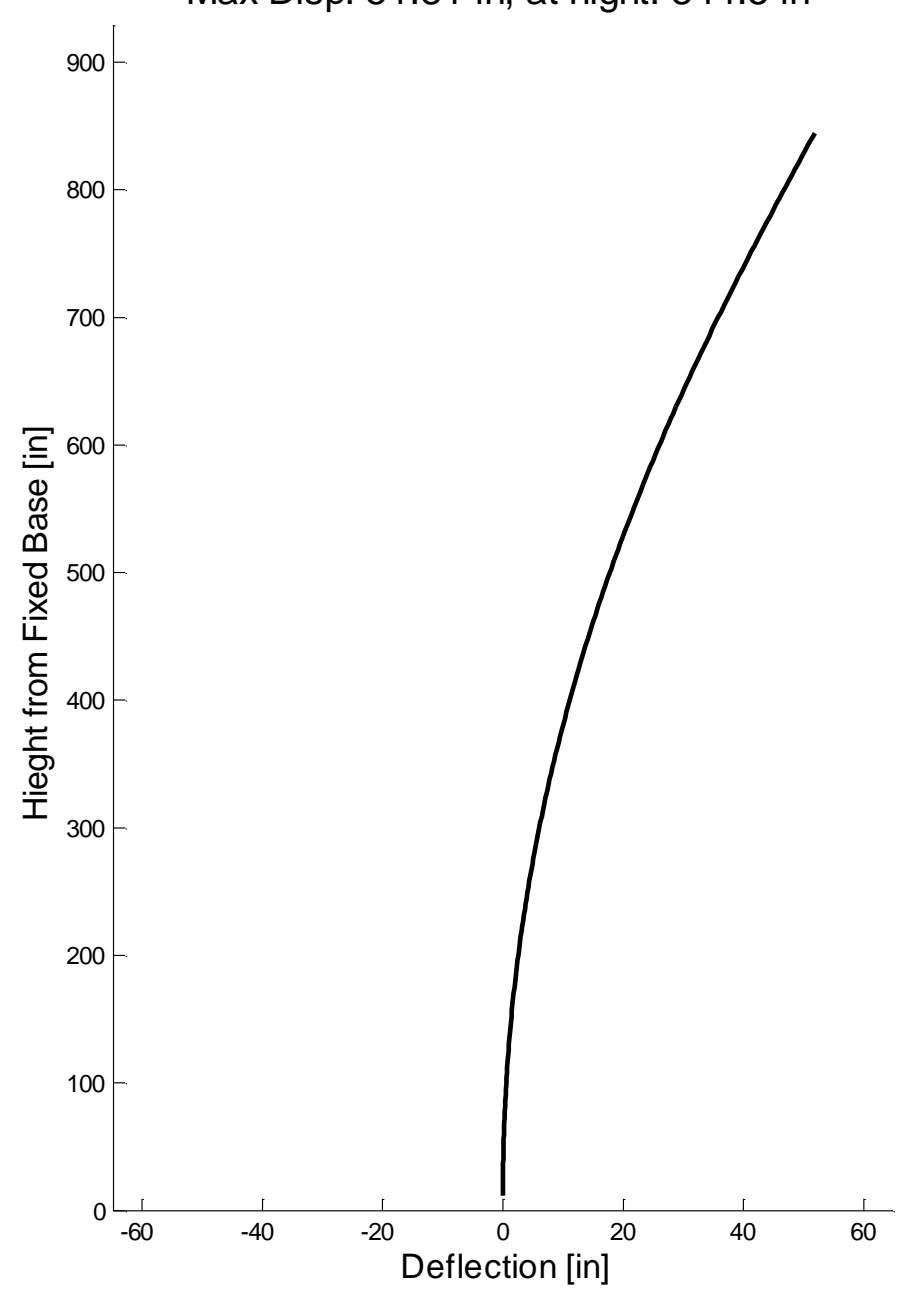

Axial Tower Deflection

Max Disp: 0.003728 in, at hight: 844.5 in

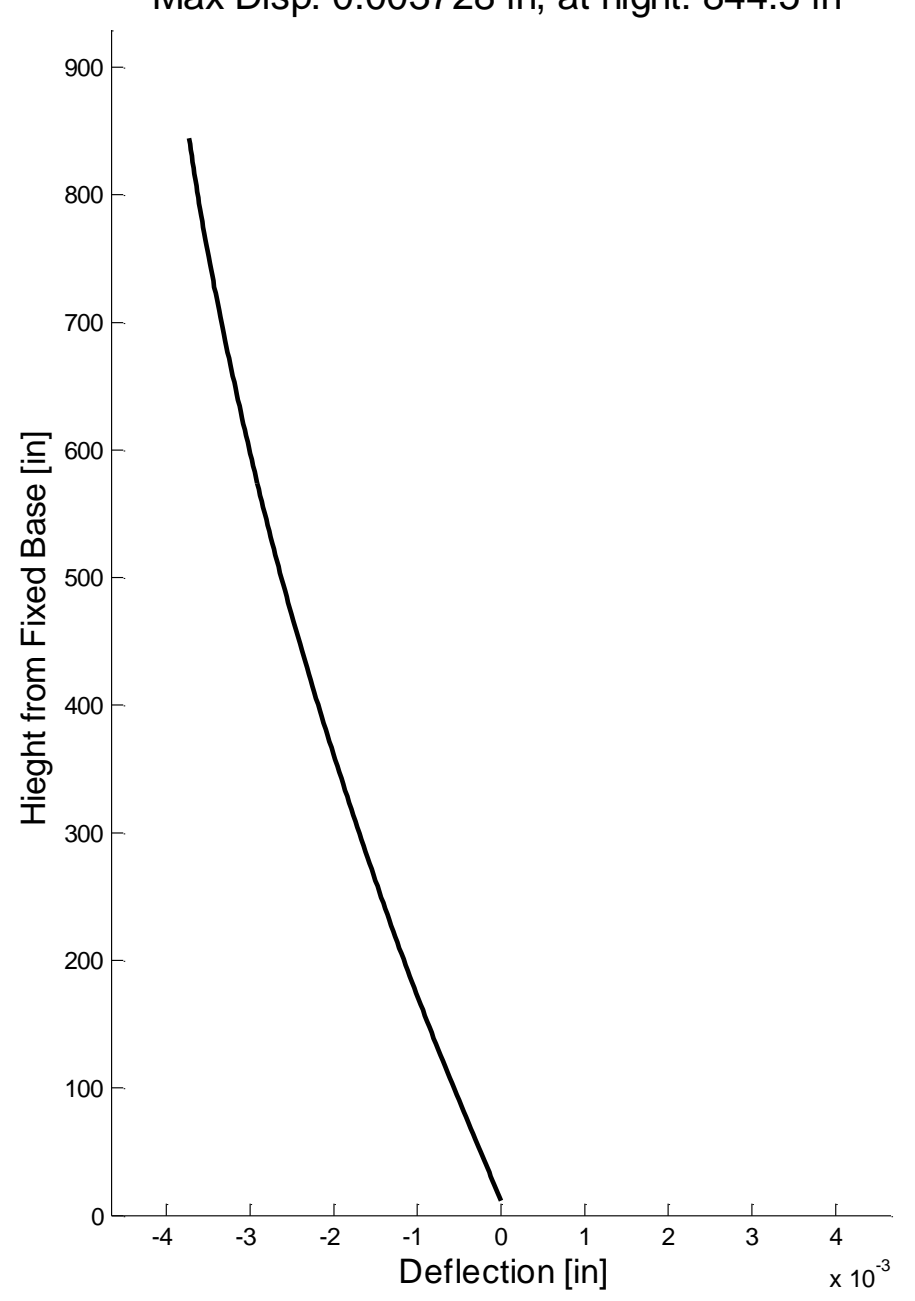




\section{Load Case E: Tower Principle Stresses at 7 Seconds}

Transient Small Wind Turbine Tower Structural Analysis with Coupled Rotor Dynamic Interaction

Tower Shear Stress Profile

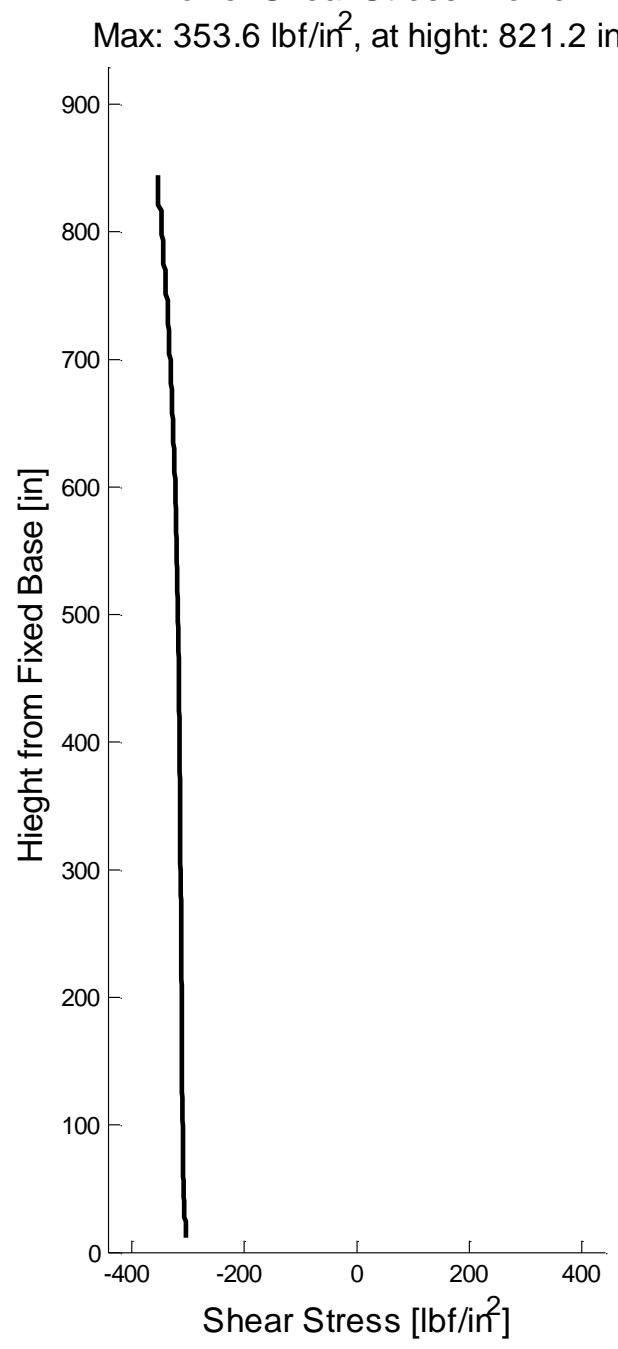

Tower Bending Stress Profile

Max: $2.862 \mathrm{e}+004 \mathrm{lbf} / \mathrm{in}^{2}$, at hight: $11.5 \mathrm{in}$

900

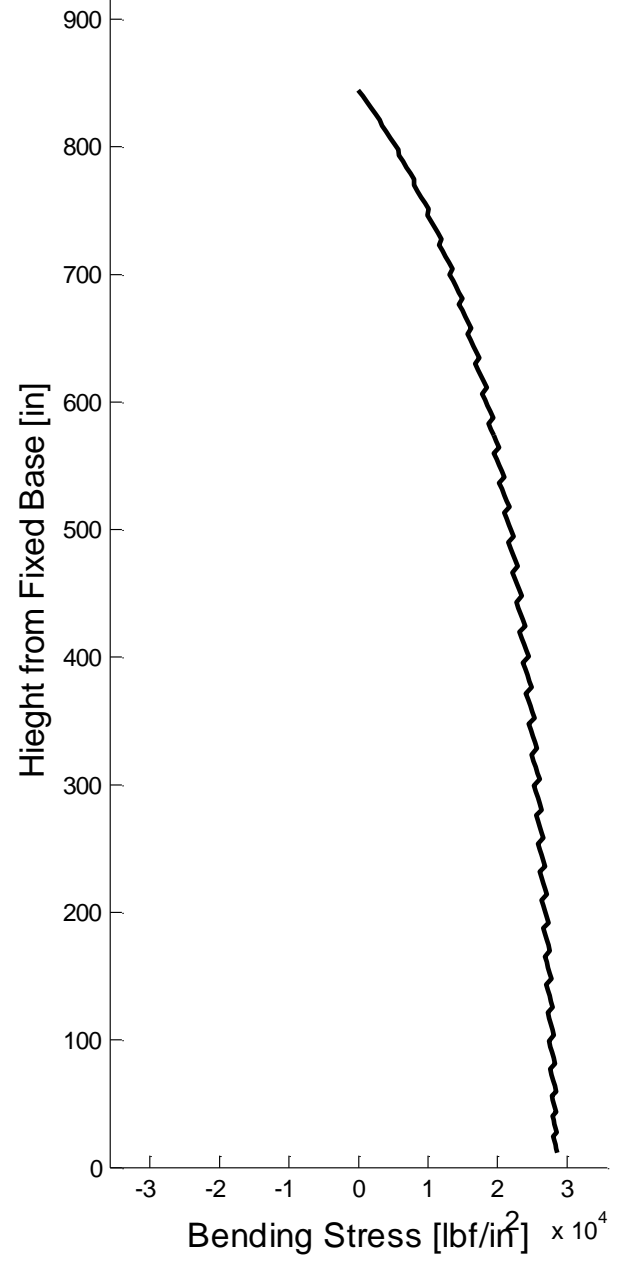

Tower Axial Stress Profile

Max: $187 \mathrm{lbf} / \mathrm{in}^{2}$, at hight: $43.5 \mathrm{in}$

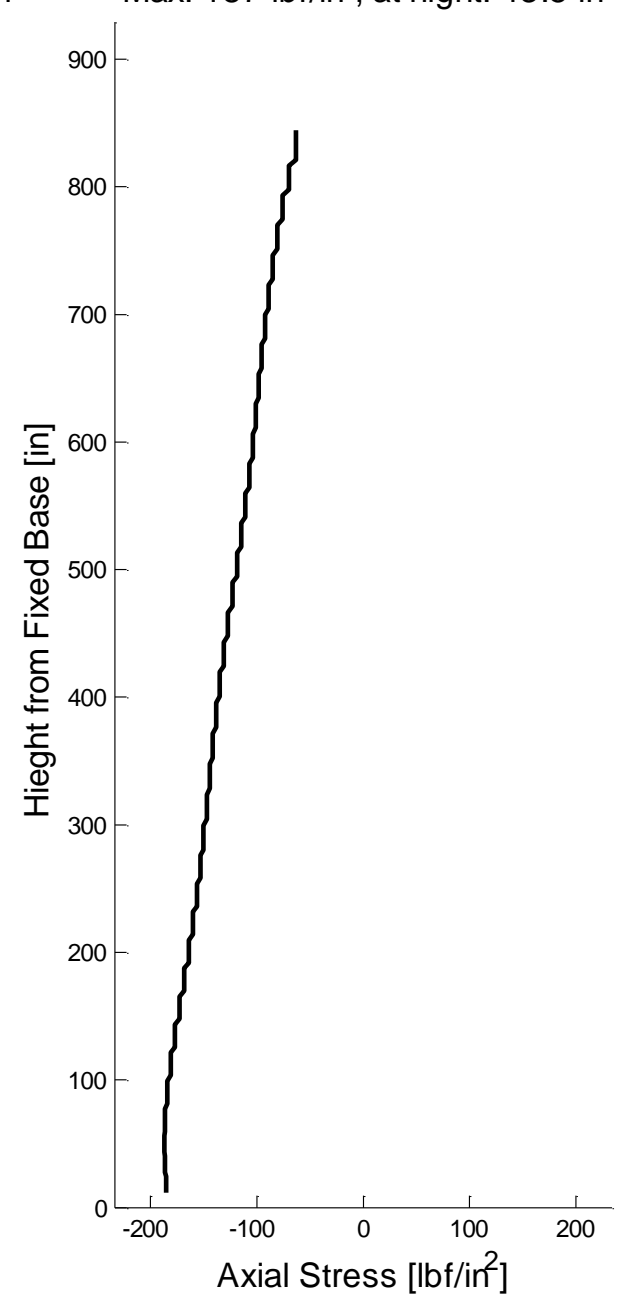




\section{Load Case E: Tower von Mises Stress at 7 Seconds}

Transient Small Wind Turbine Tower Structural Analysis with Coupled Rotor Dynamic Interaction

Max Tower von Mises Stress Profile

Max: $2.88 \mathrm{e}+004 \mathrm{lbf} / \mathrm{in}^{2}$, at hight: $11.5 \mathrm{in}$

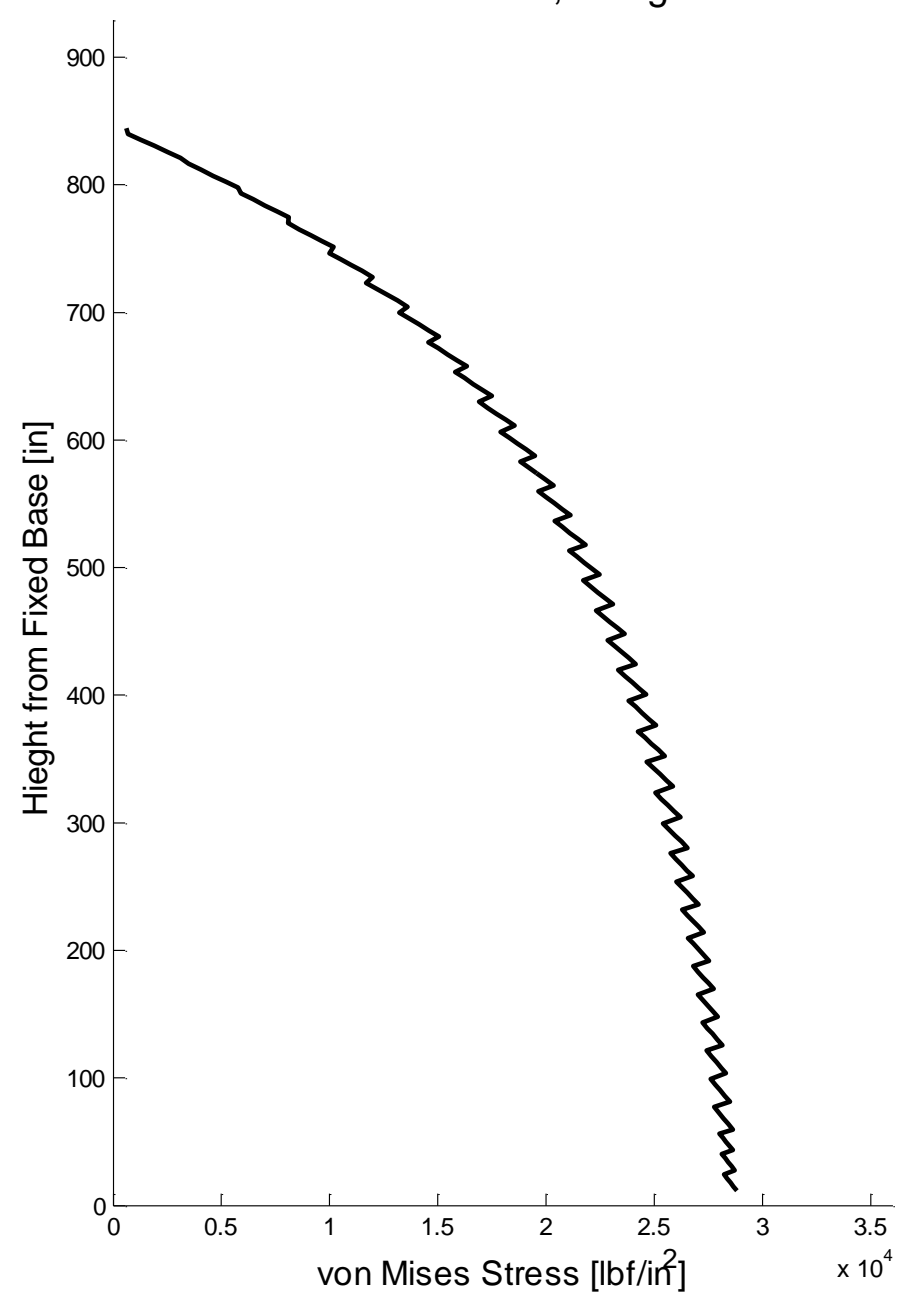

Max Tower von Mises Cross-Sectional

Location (Theta) Profile

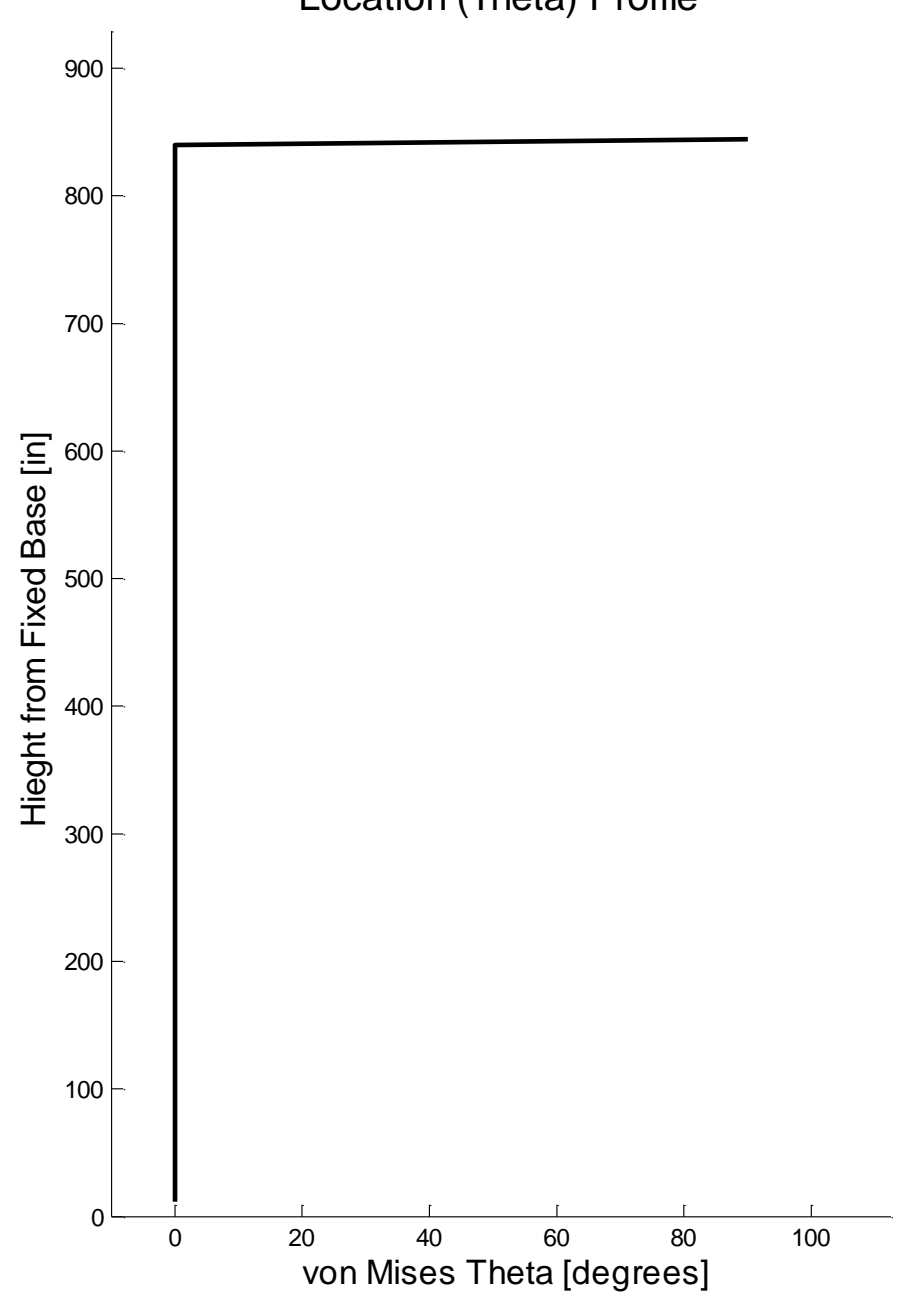




\section{Weak Axis}

Load Case F - Dynamic Extreme Coherent Gust

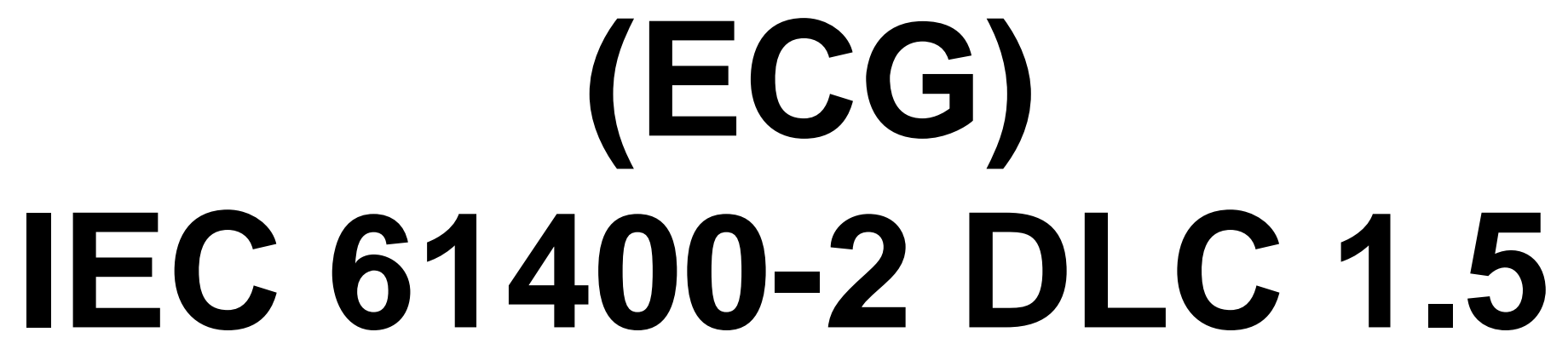




\section{Load Case F: Overview}

- Distributed dynamic load case (including tower and rotor loads)

- Small Wind Turbine Class (SWTC) 3 system

- Developed in accordance with IEC 61400-2 Design Load Case (DLC) 1.5 (Table 4)

- Rotor Thrust Coefficient: $C_{t}=0.5$

- Tower Drag Coefficient: $C_{d}=1.3$

- Nhat $=30$, see Appendix A for details

- Load applied in "Weak axis" (the load is orthogonal to the plane formed by the tower, gin-pole, and strut).

- Details of the analysis parameters are summarized and defined in the attached UserInputsWeak.xIsx file. 


\section{Load Case F: Tower Tip Wind Speed and Rotor Thrust}

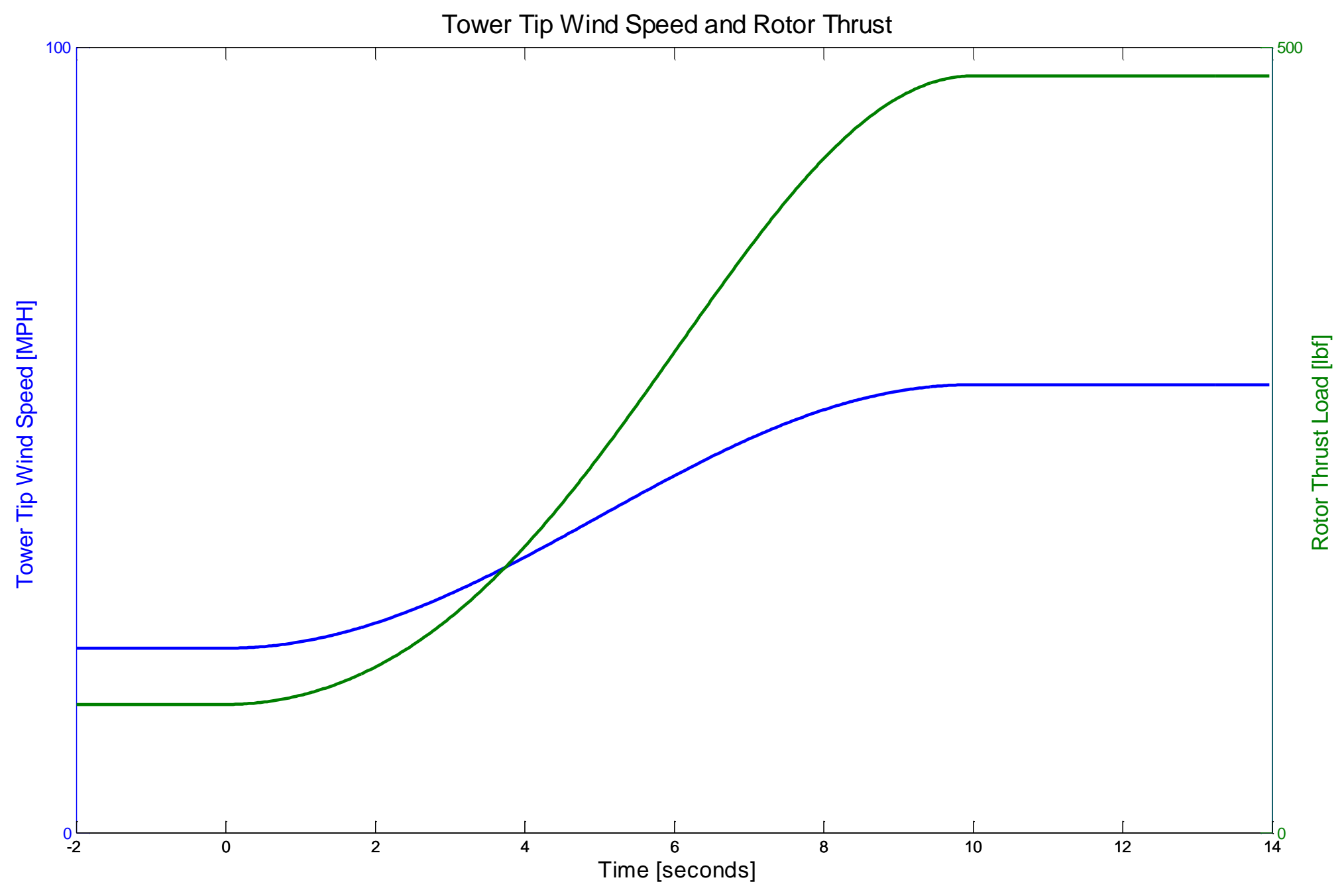

California Polytechnic State University - San Luis Obispo 


\section{Load Case F: Tower Tip Transient Deflection}

Transverse Tower Tip Deflection

Max Disp: 20.93 in

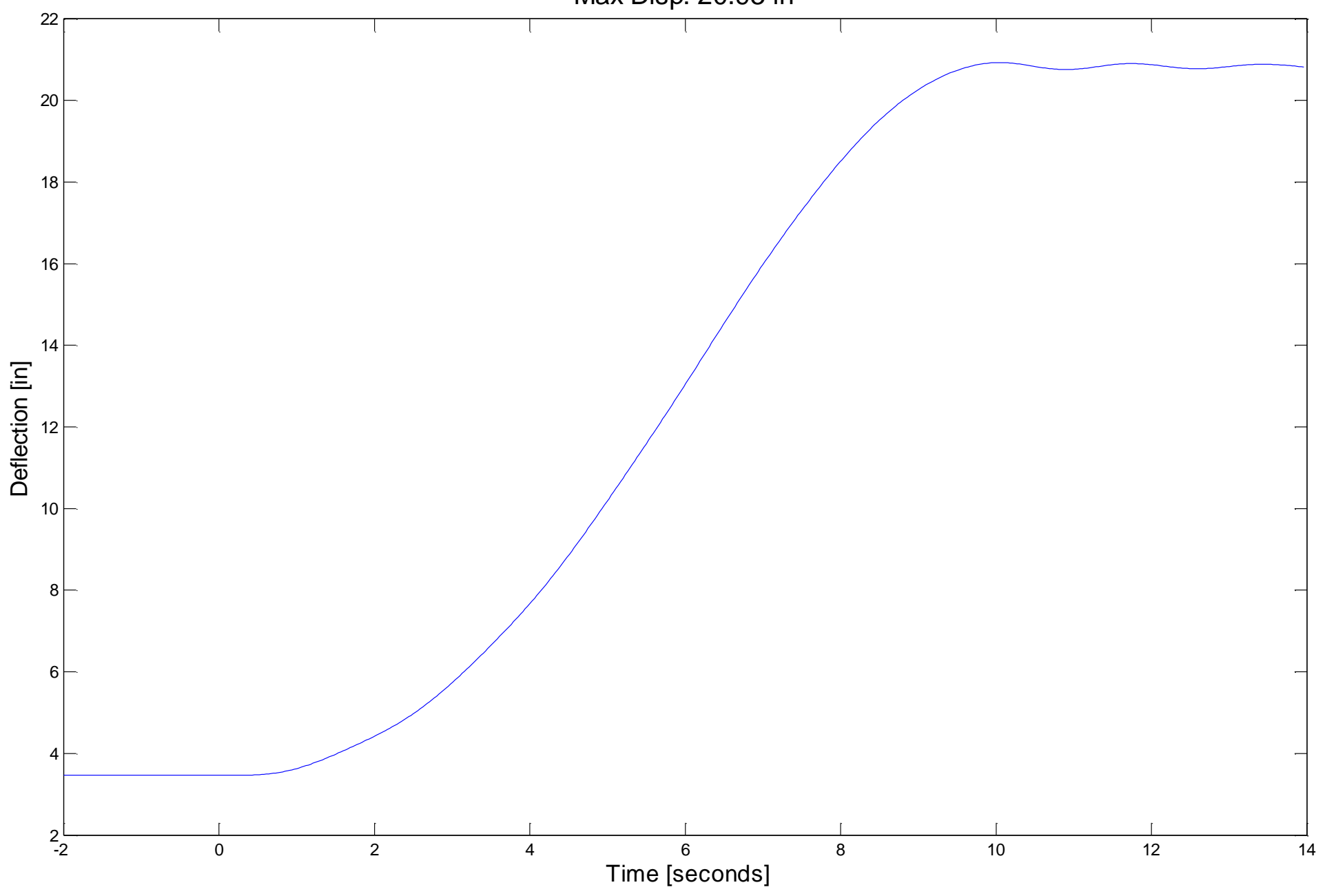

California Polytechnic State University - San Luis Obispo 


\section{Load Case F: Tower von Mises Stress Profille}

Max Von Mises Stress Across Full Tower Height Max Von Mises Stress: 1.182e+004 psi

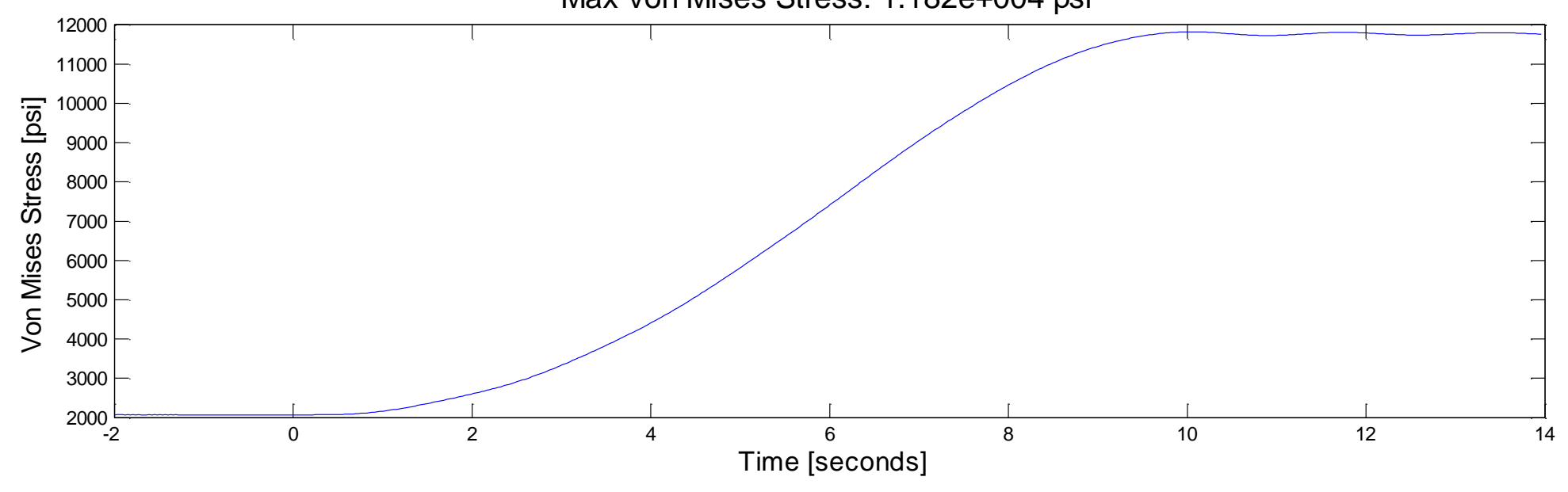

Tower Height Corresponding to Max Von Mises Stress

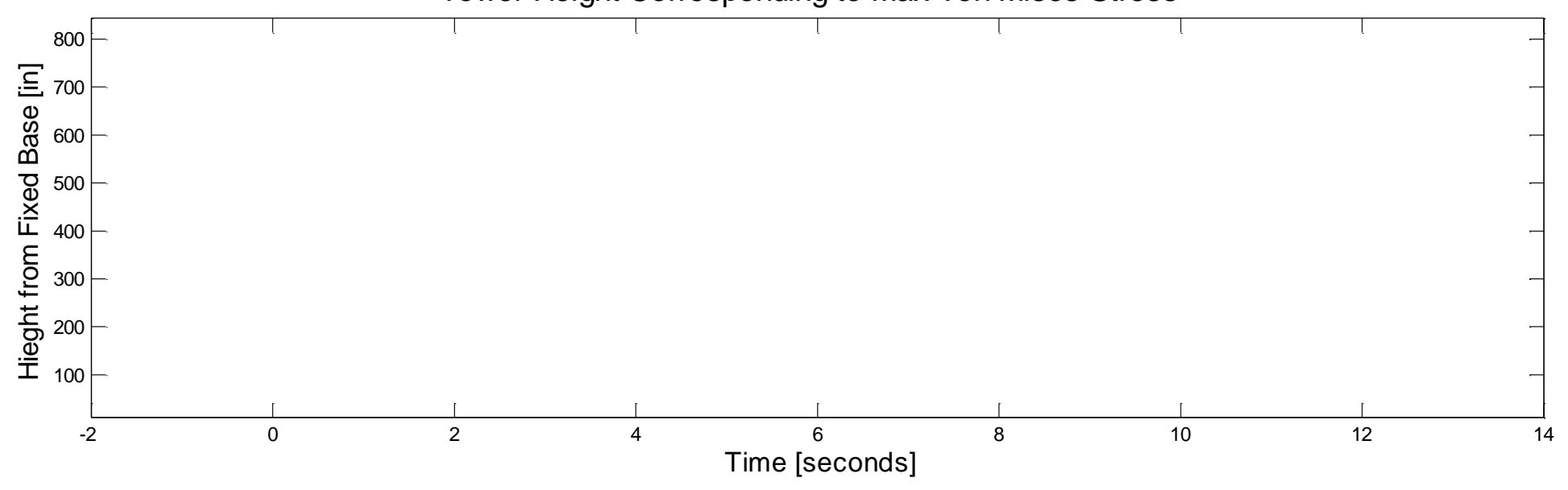




\section{Load Case F: Blade Beta Angle (relative flapwise angle)}

Blade Beta Hinge Displacement Angle Max Absolute Blade 1: 0.01895 radians Max Absolute Blade 2: 0.01895 radians

Max Absolute Blade 3: 0.01895 radians

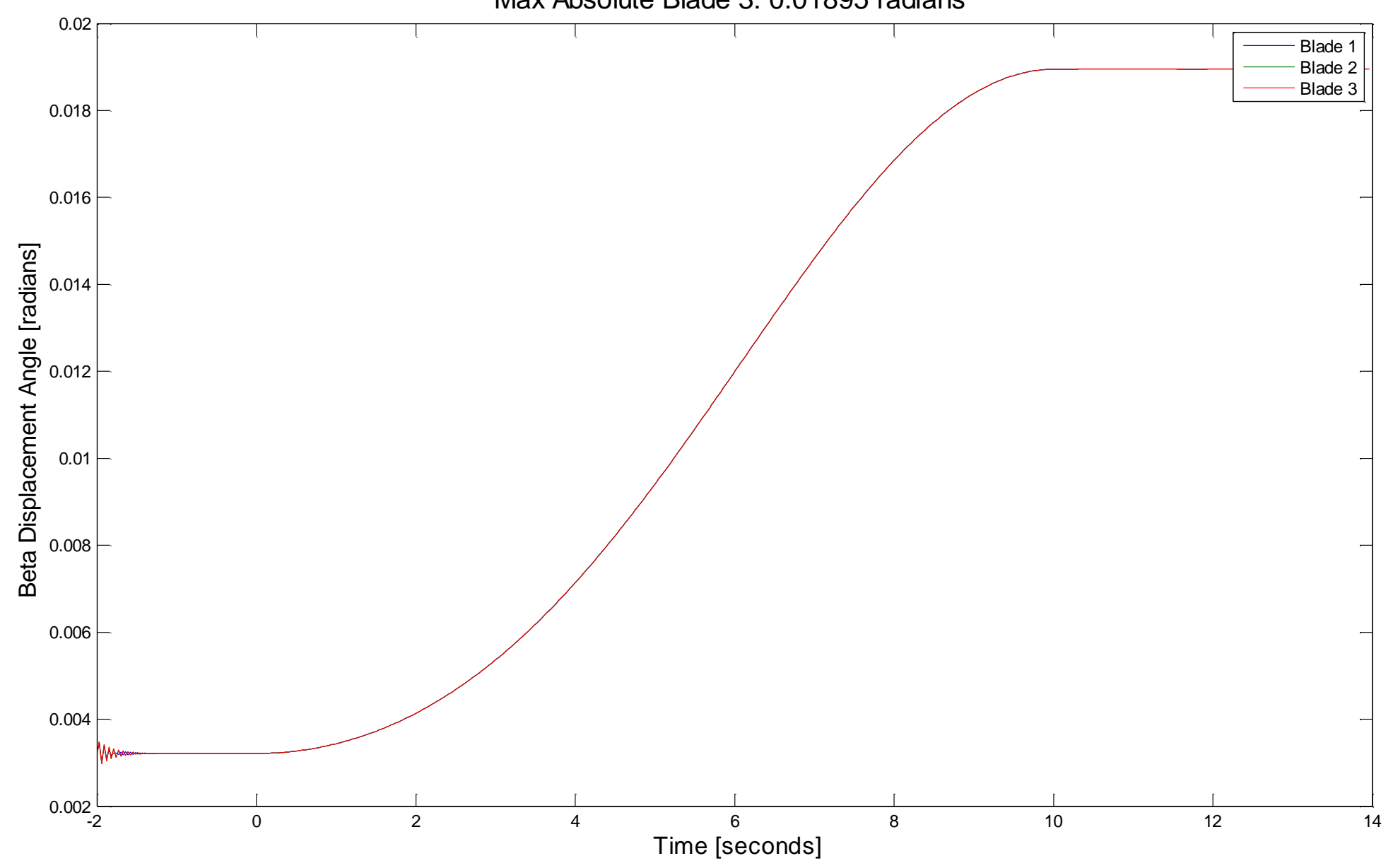




\section{Load Case F: Tower Displacement Proffle at 10 Seconds}

Transient Small Wind Turbine Tower Structural Analysis with Coupled Rotor Dynamic Interaction

Transverse Tower Deflection

Max Disp: 20.93 in, at hight: 844.5 in

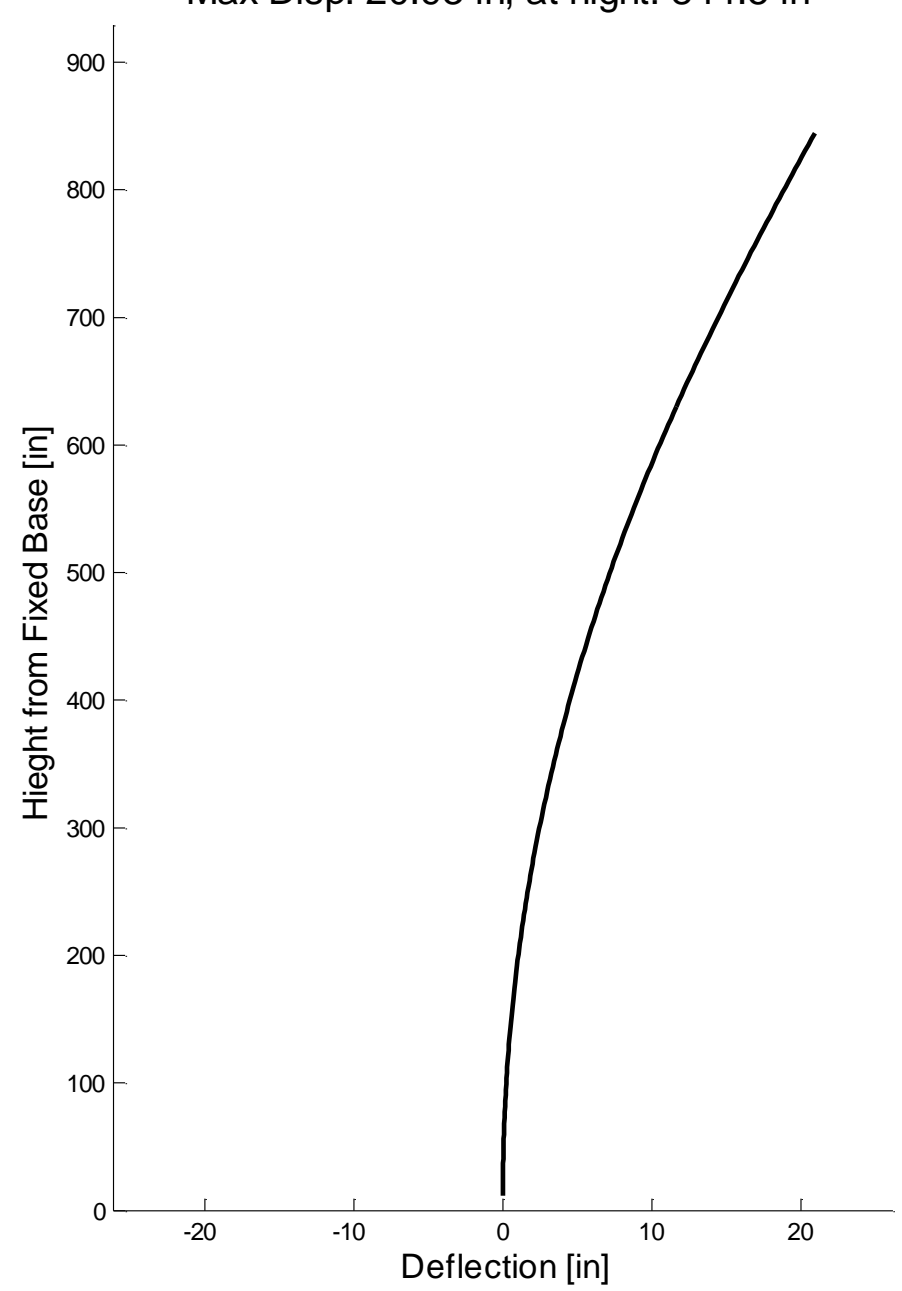

Axial Tower Deflection

Max Disp: 0.003728 in, at hight: 844.5 in

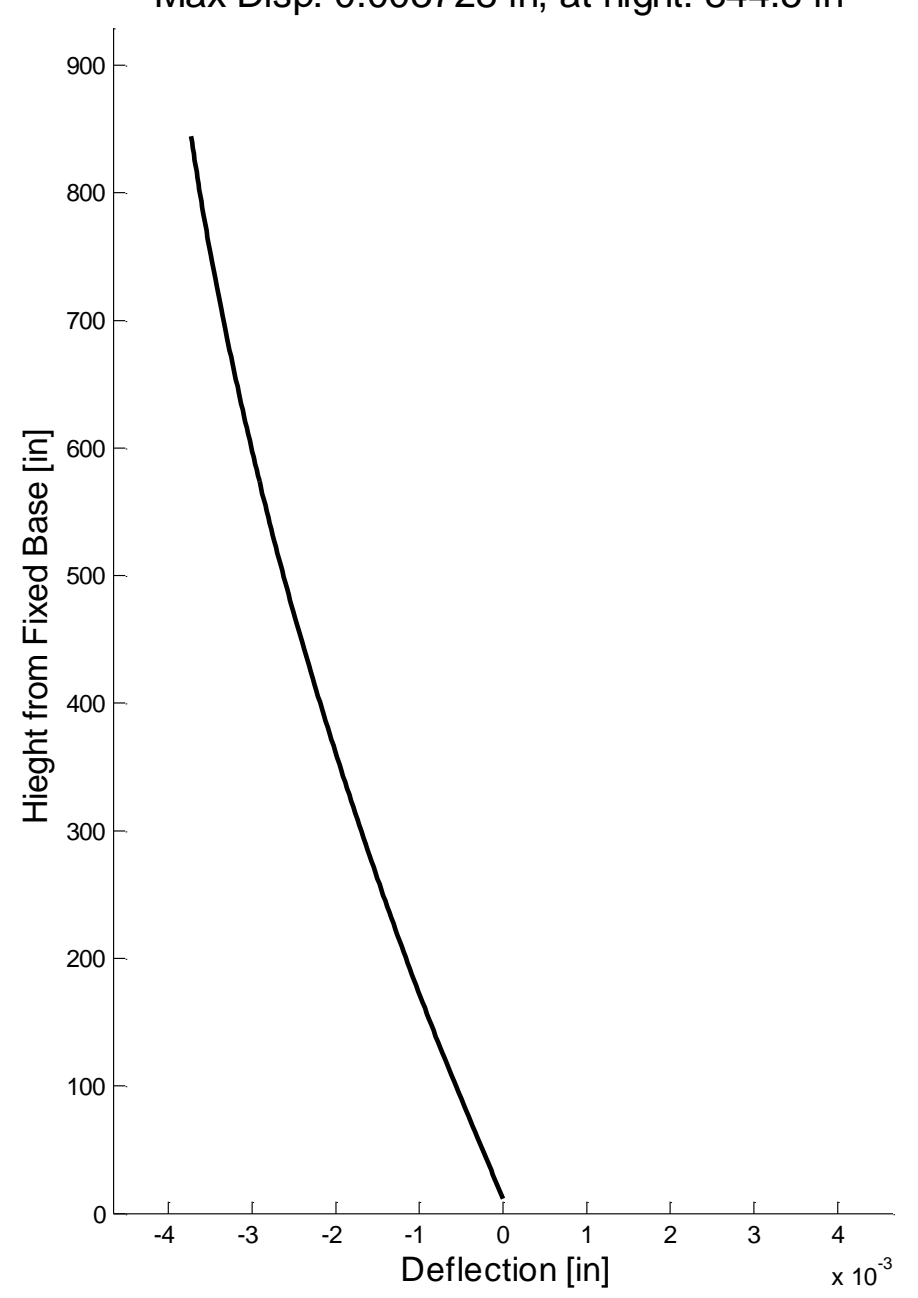




\section{Load Case F: Tower Principle Stresses at 10 Seconds}

Transient Small Wind Turbine Tower Structural Analysis with Coupled Rotor Dynamic Interaction

Tower Shear Stress Profile

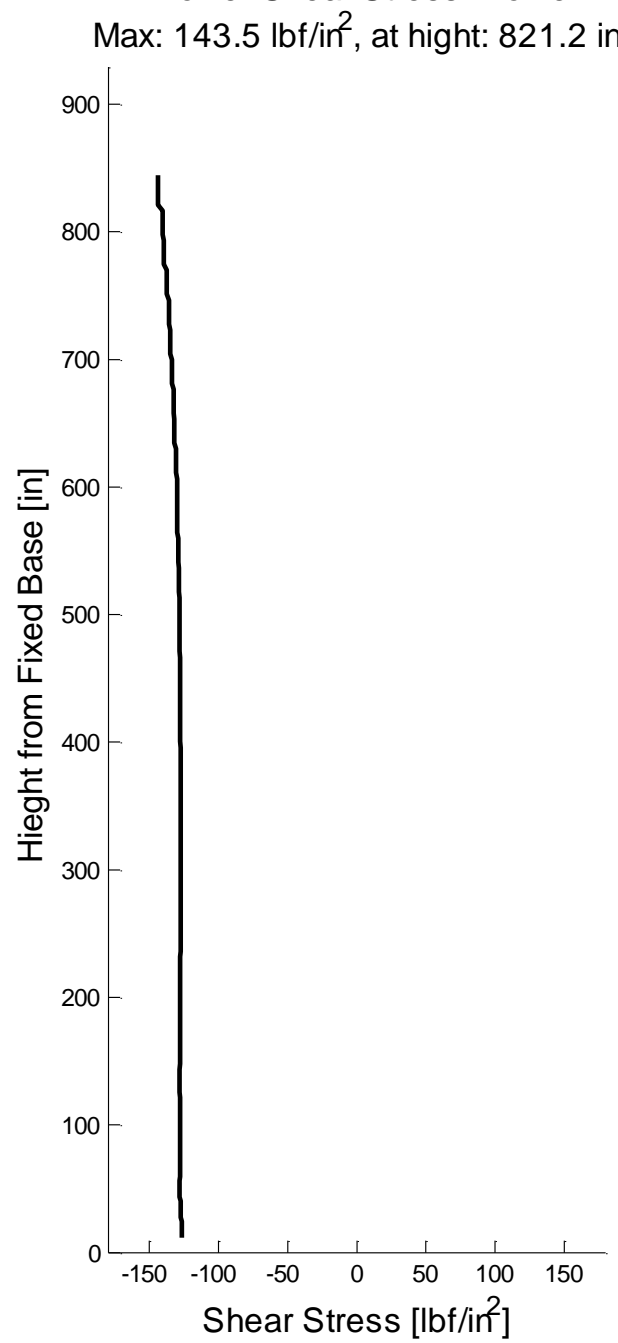

Tower Bending Stress Profile

Max: $1.164 \mathrm{e}+004 \mathrm{lbf} / \mathrm{in}^{2}$, at hight: $11.5 \mathrm{in}$

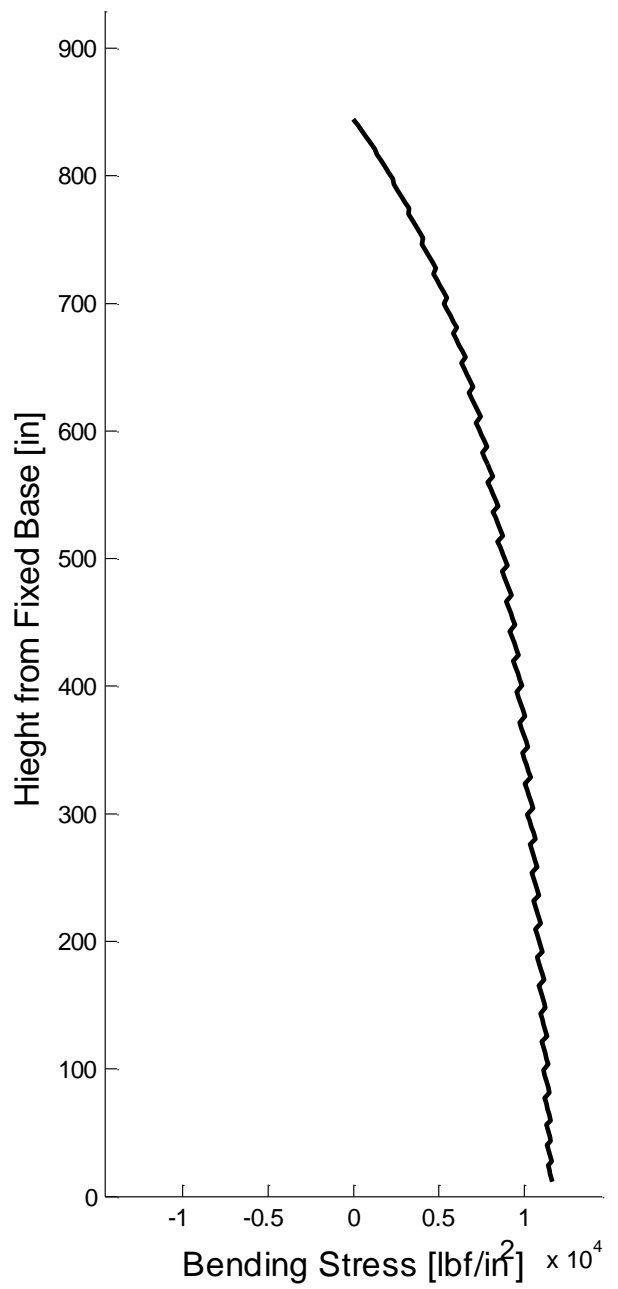

Tower Axial Stress Profile

Max: $187.1 \mathrm{lbf} / \mathrm{in}^{2}$, at hight: $43.5 \mathrm{in}$

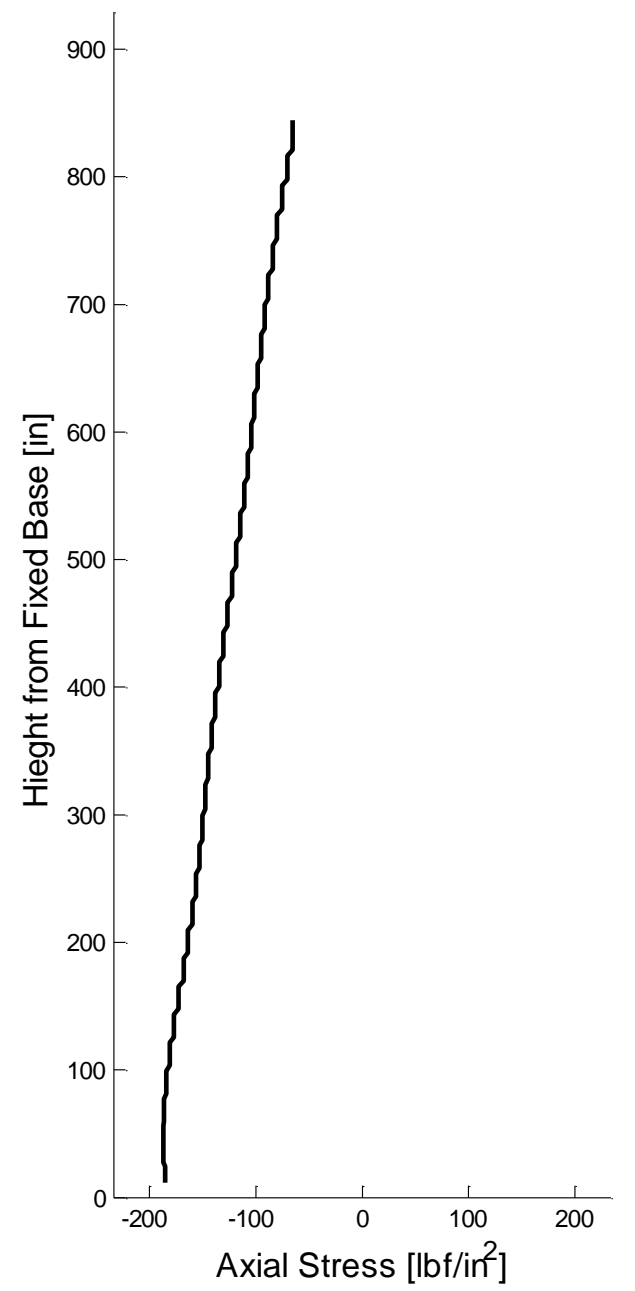




\section{Load Case F: Tower von Mises Stress at 10 Seconds}

Transient Small Wind Turbine Tower Structural Analysis with Coupled Rotor Dynamic Interaction

Max Tower von Mises Stress Profile

Max: $1.182 \mathrm{e}+004 \mathrm{lbf} / \mathrm{in}^{2}$, at hight: $11.5 \mathrm{in}$

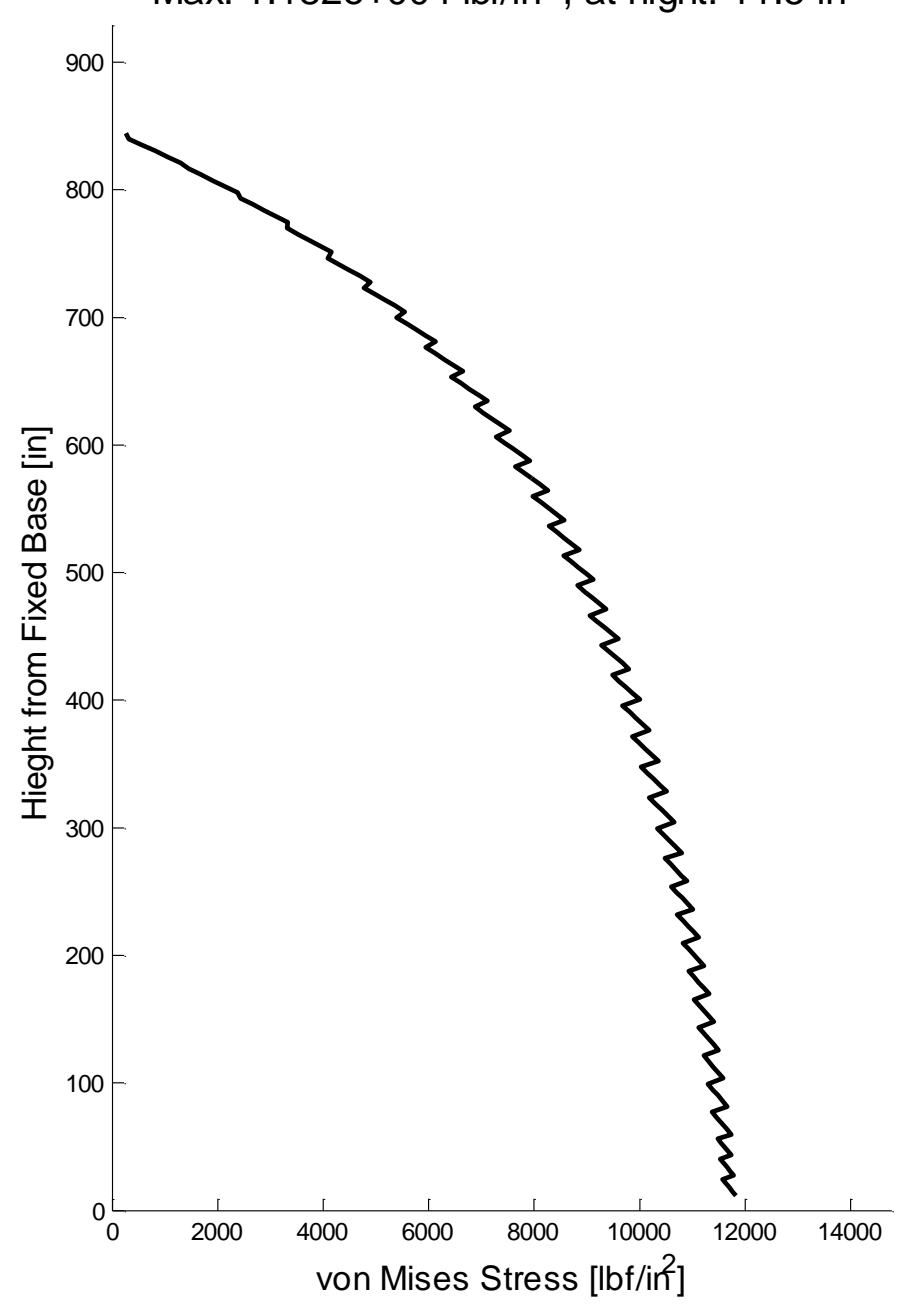

Max Tower von Mises Cross-Sectional Location (Theta) Profile

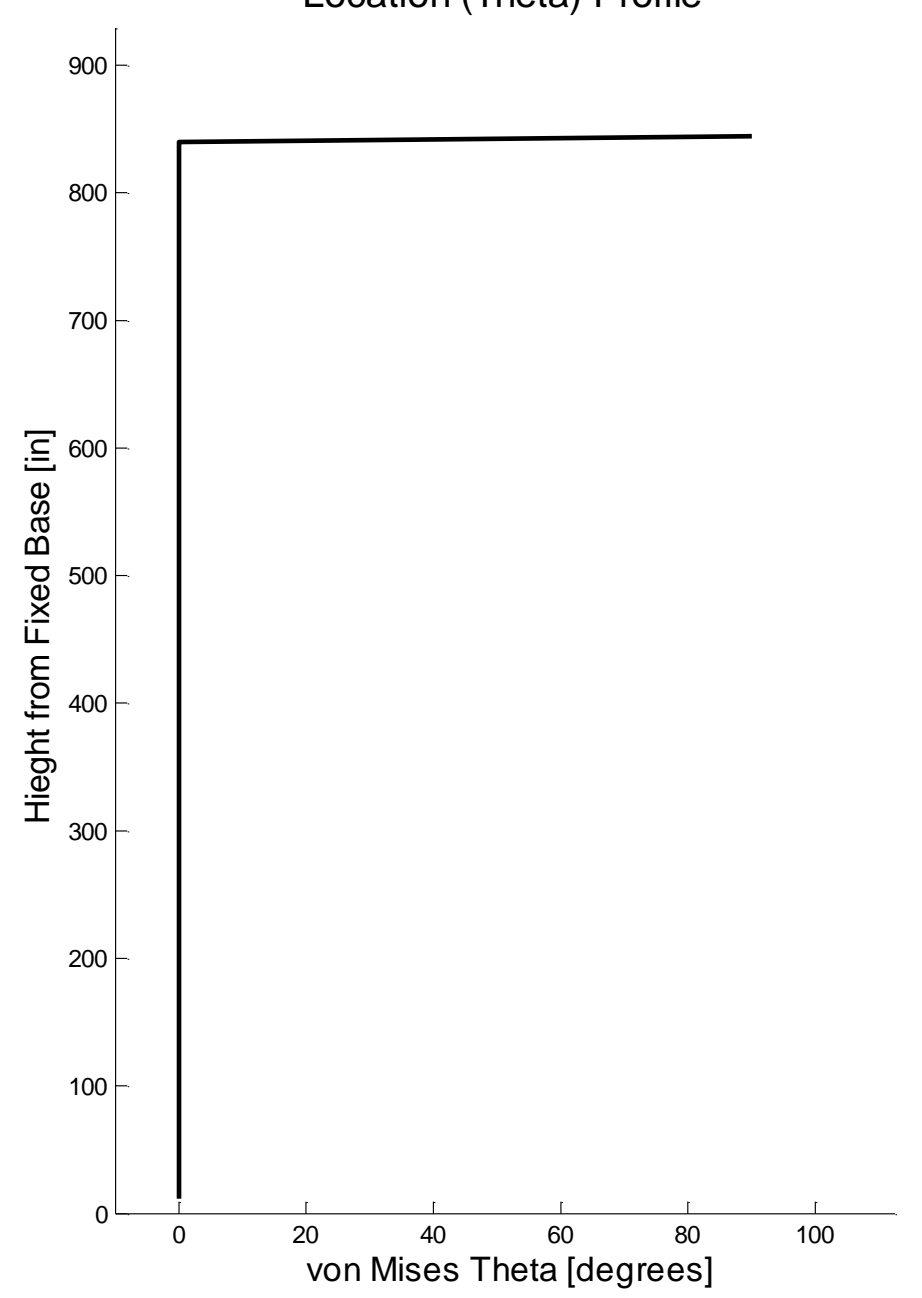




\section{Weak Axis}

Load Case G - Dynamic

Normal Turbulence Model (NTM)

IEC 61400-2 DLC 2.2 


\section{Load Case G: Overview}

- Distributed dynamic load case (including tower and rotor loads)

- Small Wind Turbine Class (SWTC) 3 system

- Developed in accordance with IEC 61400-2 Design Load Case (DLC) 2.2 (Table 4)

- Rotor Thrust Coefficient: $C_{t}=0.5$

- Tower Drag Coefficient: $C_{d}=1.3$

- Nhat $=30$, see Appendix A for details

- Load applied in "Weak axis" (the load is orthogonal to the plane formed by the tower, gin-pole, and strut).

- Details of the analysis parameters are summarized and defined in the attached UserInputsWeak.xIsx file; note: except DSRatio was increased to 40. 


\section{Load Case G: Tower Tip Wind Speed and Rotor Thrust}

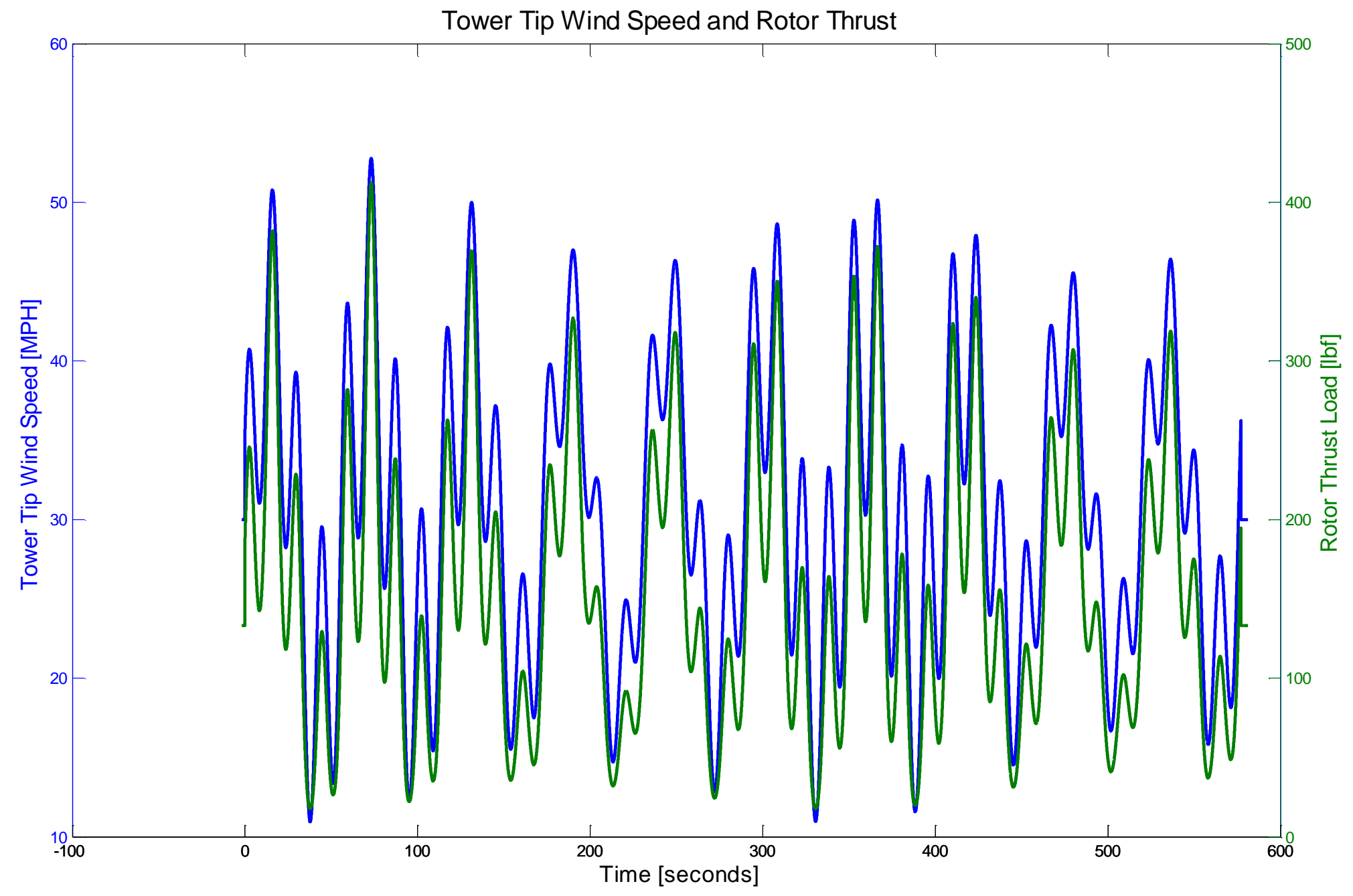




\section{Load Case G: Tower Tip Transient Deflection}

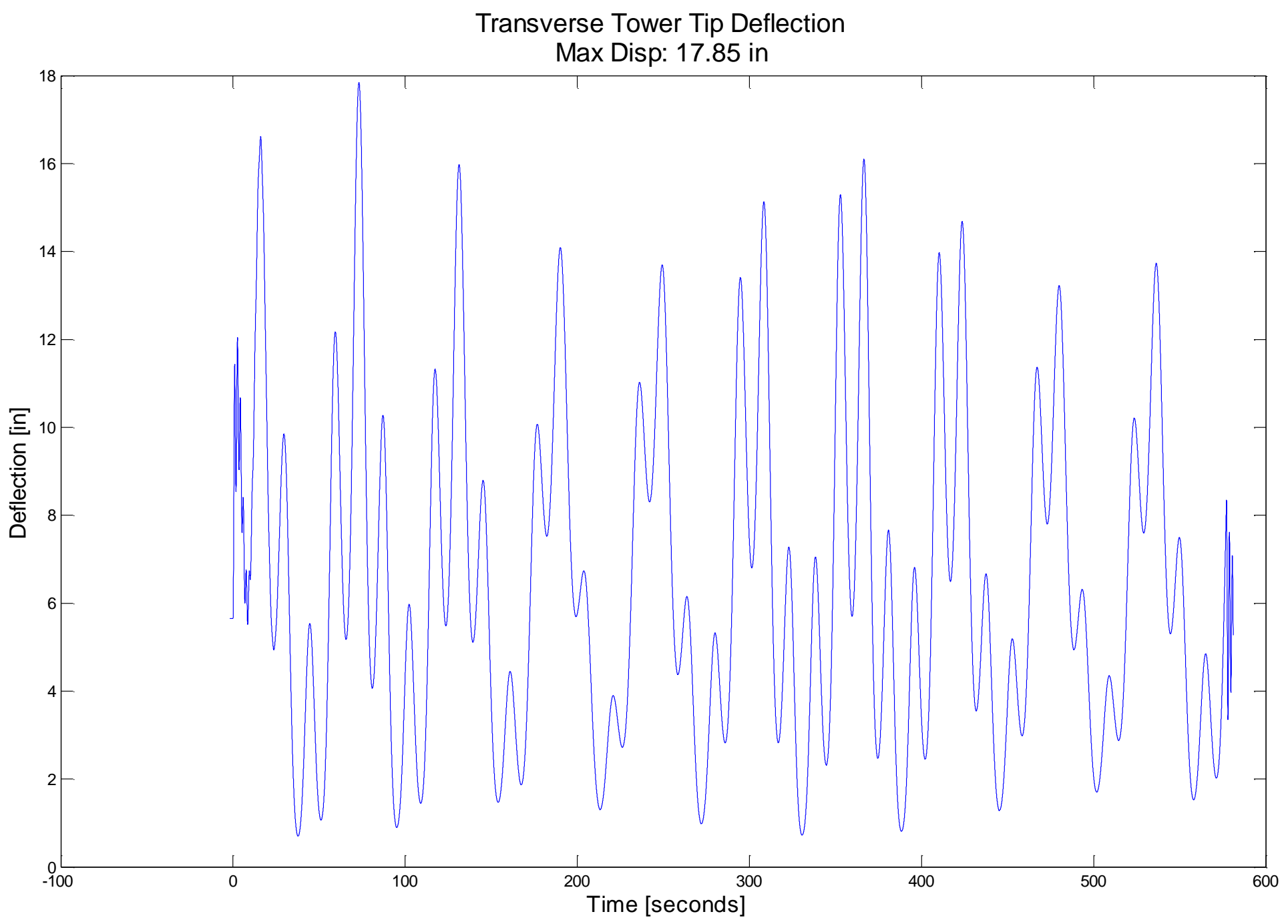

California Polytechnic State University - San Luis Obispo 


\section{Load Case G: Tower von Mises Stress Proffle}

Max Von Mises Stress Across Full Tower Height

Max Von Mises Stress: 1.005e+004 psi

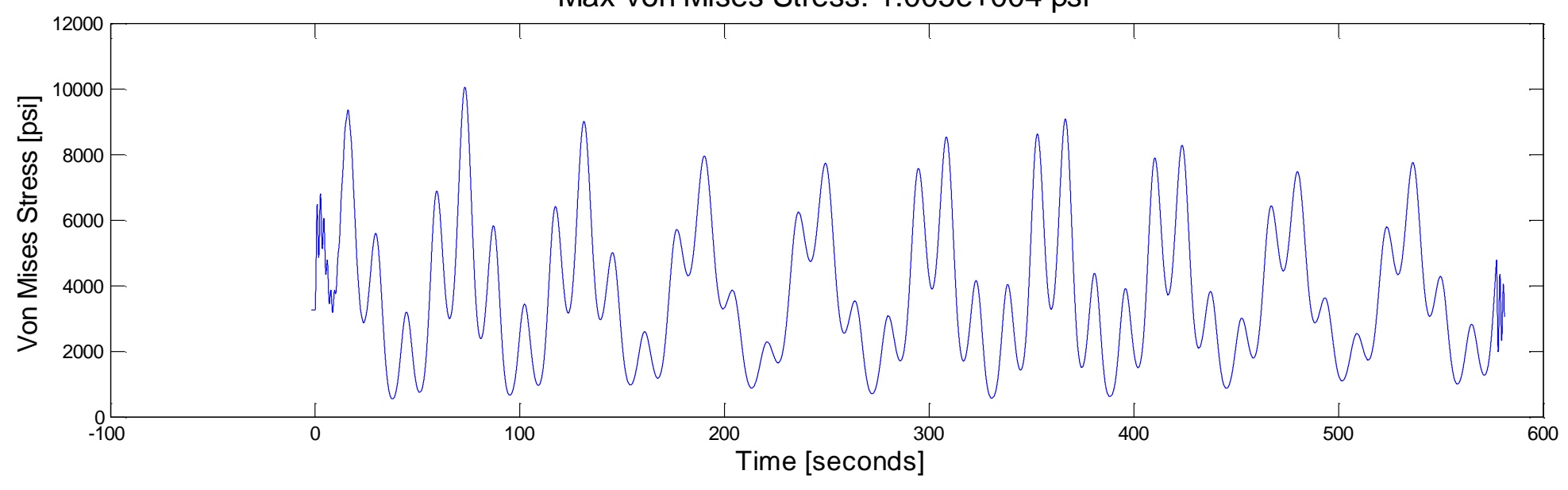

Tower Height Corresponding to Max Von Mises Stress

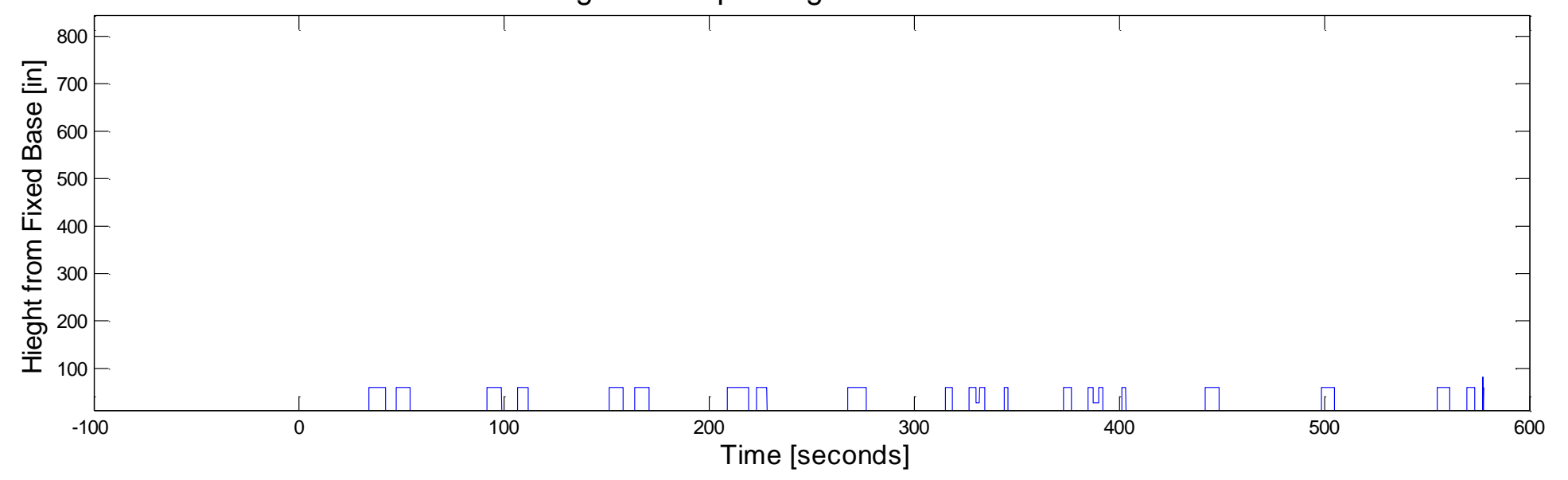

California Polytechnic State University - San Luis Obispo 


\section{Load Case G: Blade Beta Angle (relative flapwise angle)}

Blade Beta Hinge Displacement Angle Max Absolute Blade 1: 0.01621 radians Max Absolute Blade 2: 0.01621 radians Max Absolute Blade 3: 0.01621 radians

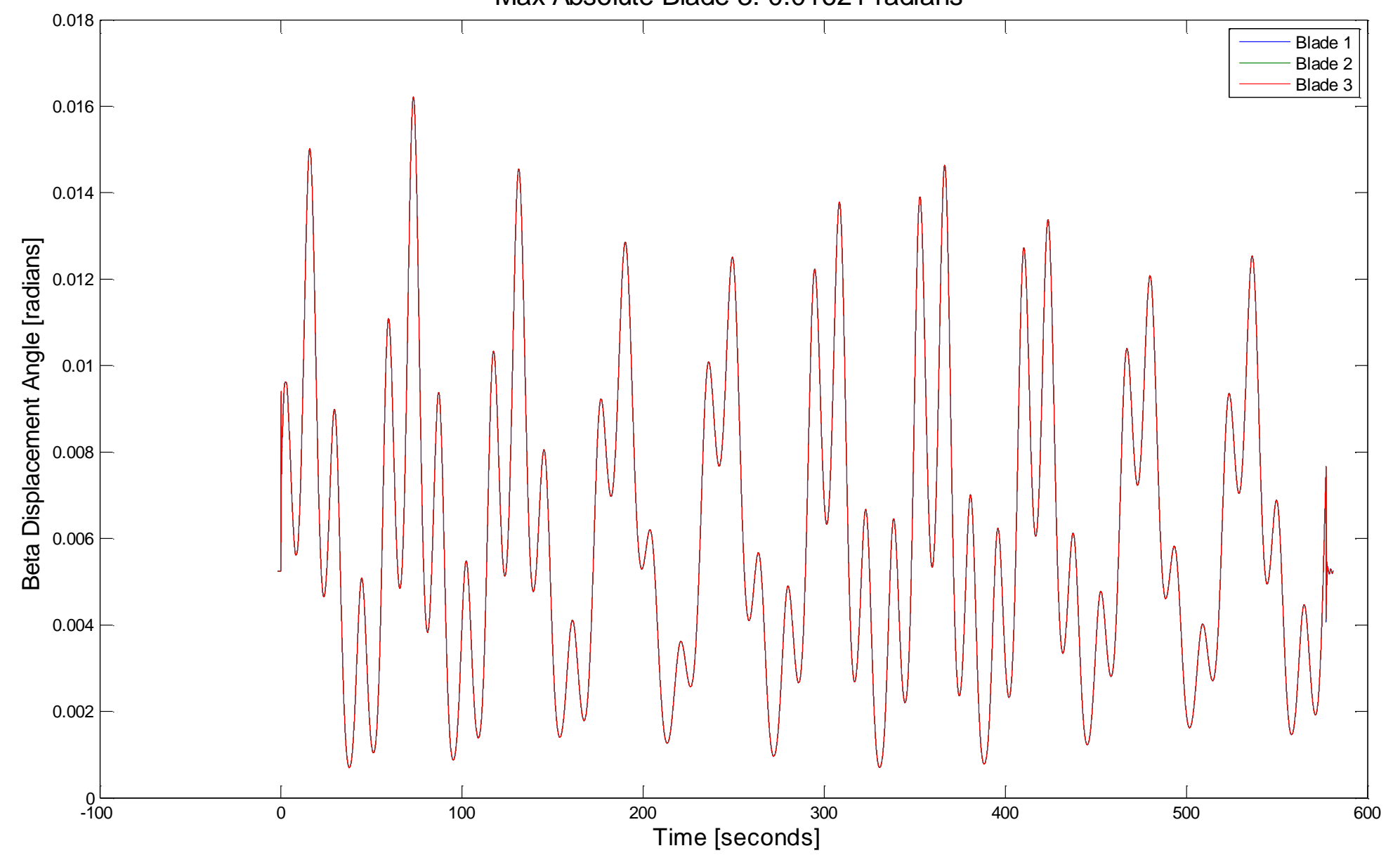

California Polytechnic State University - San Luis Obispo 


\section{Load Case G: Tower Displacement Profille at 70 Seconds}

Transverse Tower Deflection

Max Disp: 17.85 in, at hight: 844.5 in

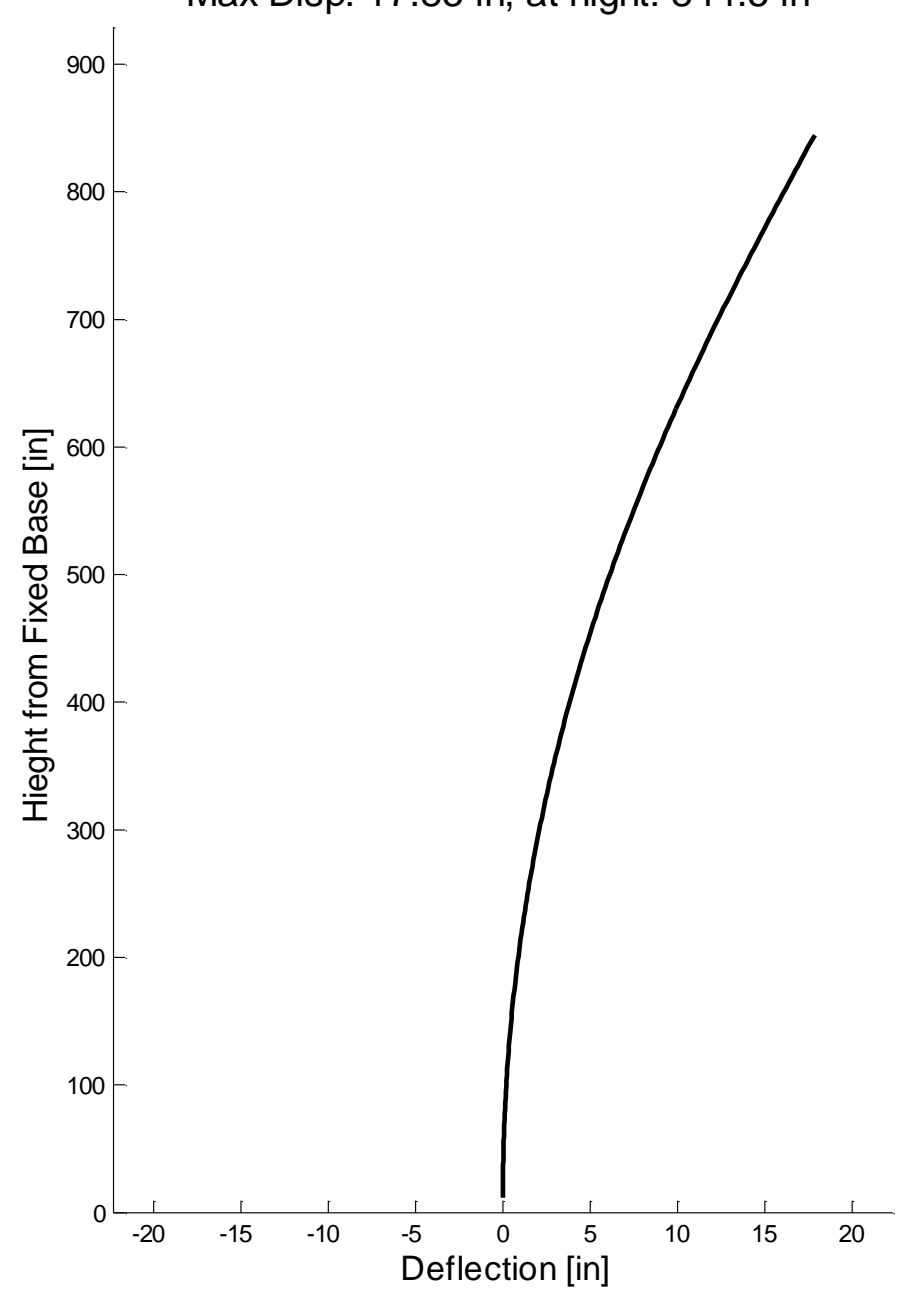

Axial Tower Deflection

Max Disp: 0.003728 in, at hight: 844.5 in

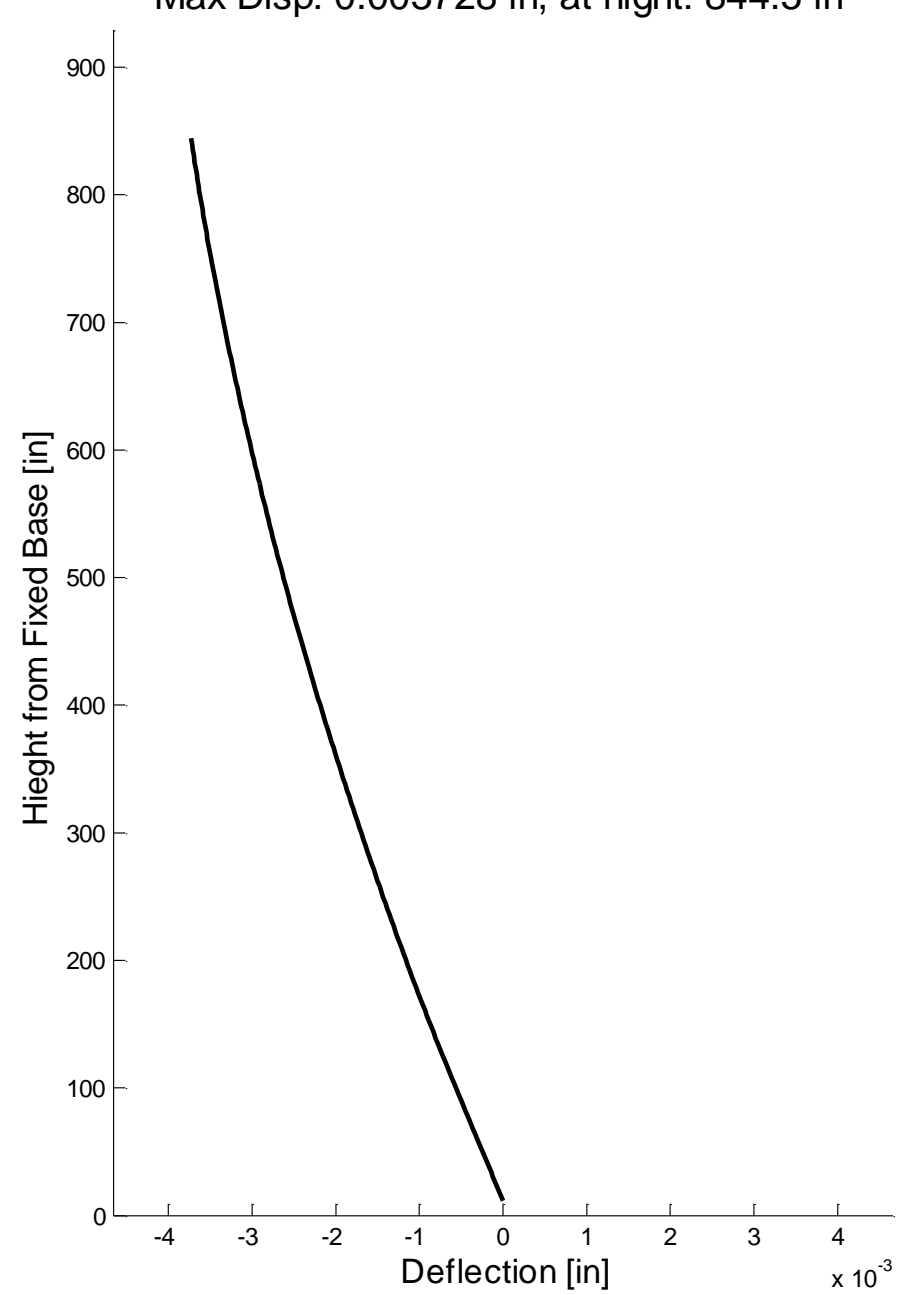




\section{Load Case G: Tower Principle Stresses at 70 Seconds}

Transient Small Wind Turbine Tower Structural Analysis with Coupled Rotor Dynamic Interaction
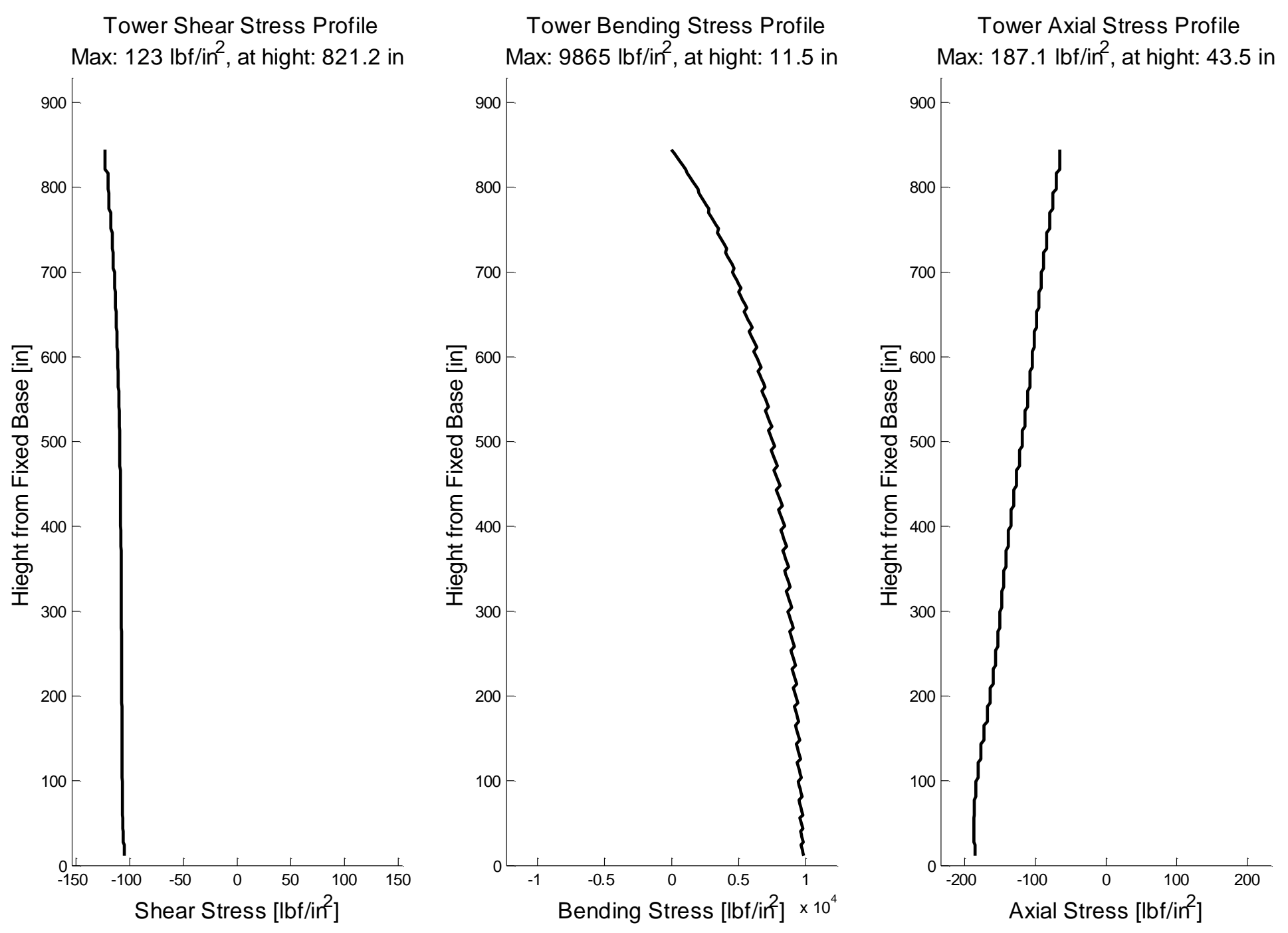


\section{Load Case G: Tower von Mises Stress at 70 Seconds}

Transient Small Wind Turbine Tower Structural Analysis with Coupled Rotor Dynamic Interaction

Max Tower von Mises Stress Profile

Max: $1.005 \mathrm{e}+004 \mathrm{lbf} / \mathrm{in}^{2}$, at hight: $11.5 \mathrm{in}$

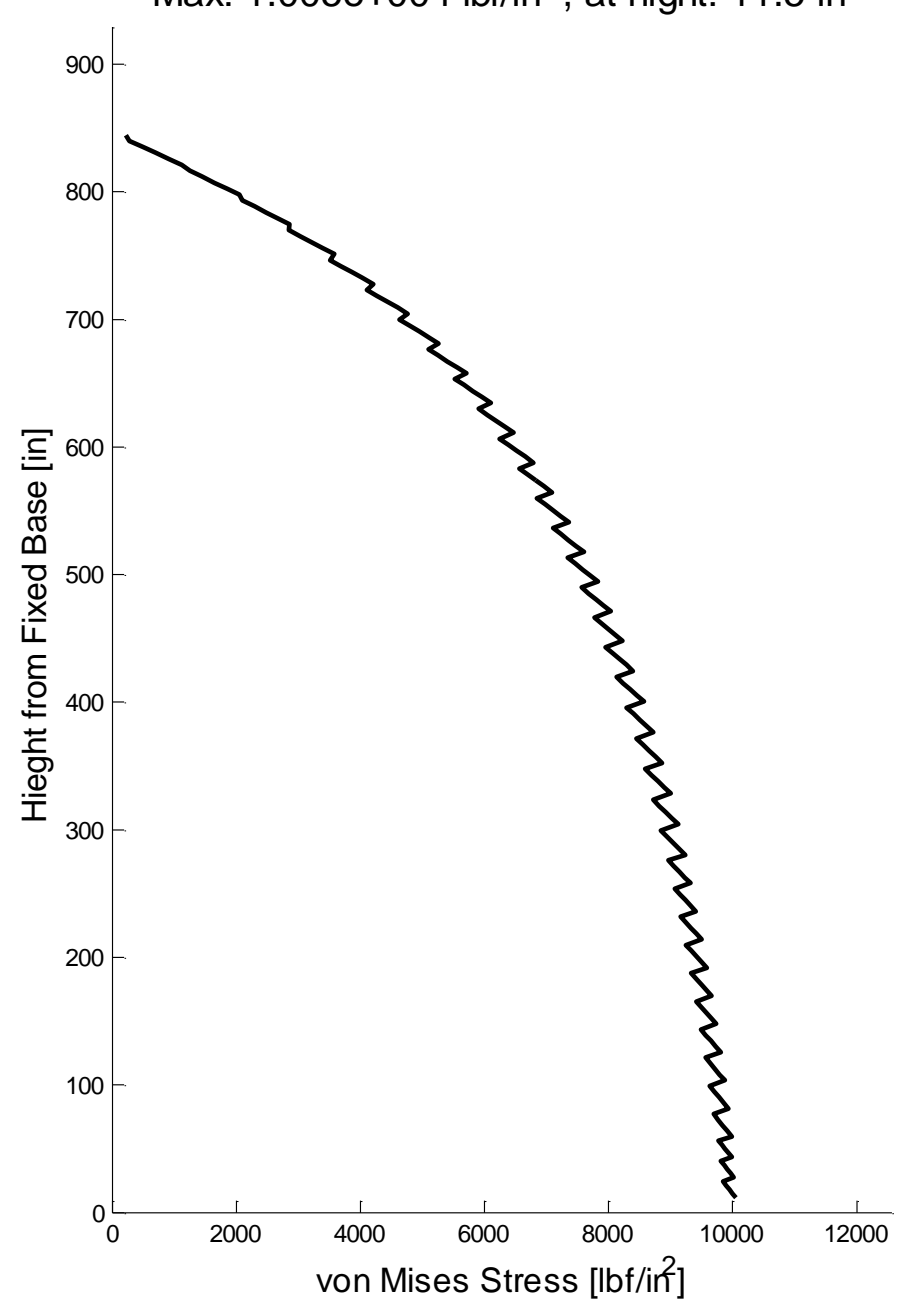

Max Tower von Mises Cross-Sectional Location (Theta) Profile

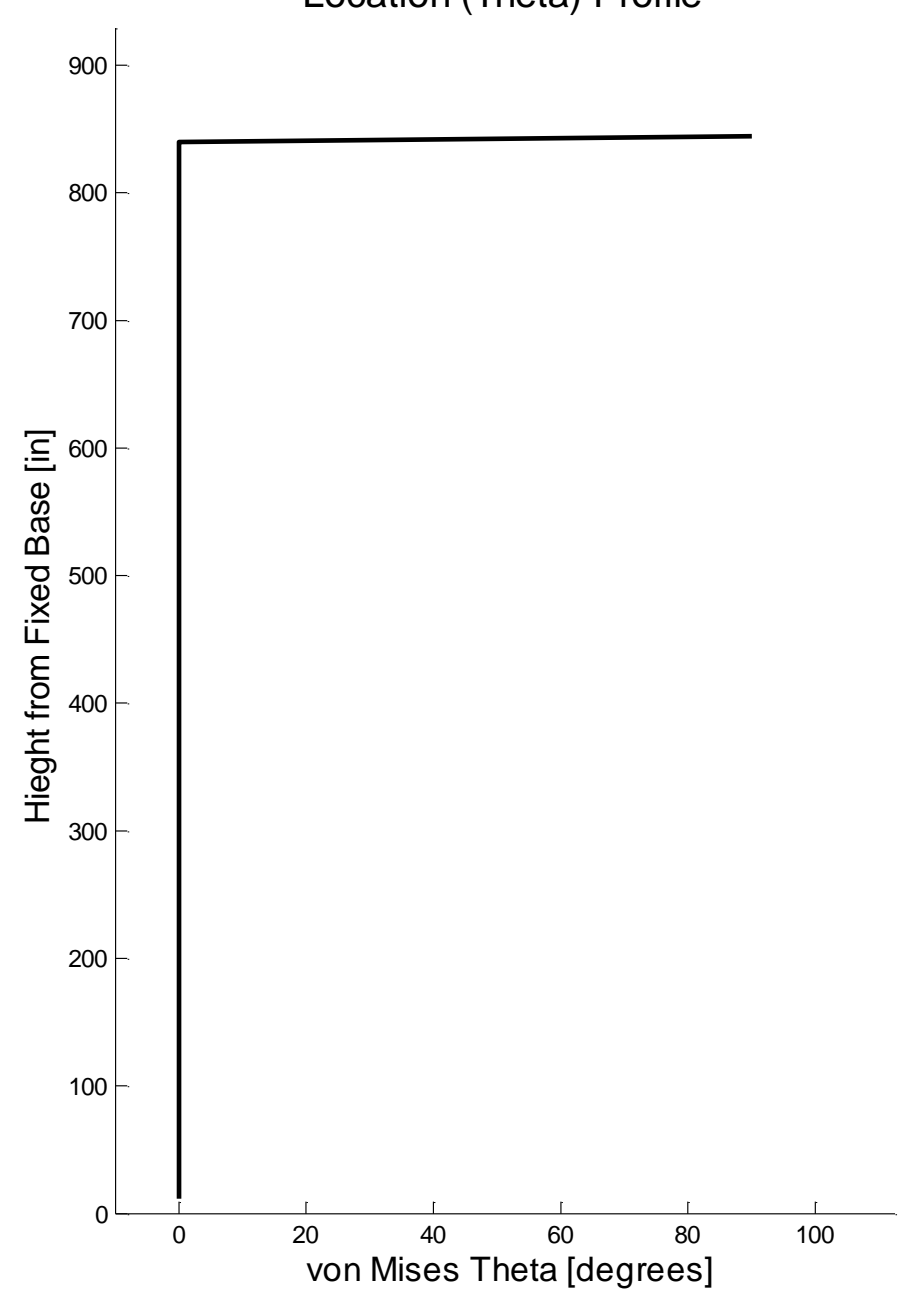




\section{Weak Axis Load Case H - Dynamic Swept Sine}




\section{Load Case H: Overview}

- Sinusoidal tower tip load applied in the X-Axis.

- Results are displayed normalized to tower tip load amplitude

- Starting Frequency: $0.10 \mathrm{~Hz}$

- Ending Frequency: $20 \mathrm{~Hz}$

- Logarithmic Sweep Rate: 0.5 Octaves / Minute

- Nhat $=30$, see Appendix A for details

- Load applied in "Weak axis" (the load is orthogonal to the plane formed by the tower, gin-pole, and strut).

- Details of the analysis parameters are summarized and defined in the attached UserInputsWeak.xIsx file; note.

- Method:

- Same method as was used for the "2B) Baseline Blades (Thrust \& Moment)" study conducted in the Strong Axis. See chart 55 for further details. 


\section{Load Case H: Swept Sine}

\section{Overlay of Methods 1A through 3B (see Chart 55 for definitions)}

Transient Small Wind Turbine Tower Structural Analysis with Coupled Rotor Dynamic Interaction

Contents Link

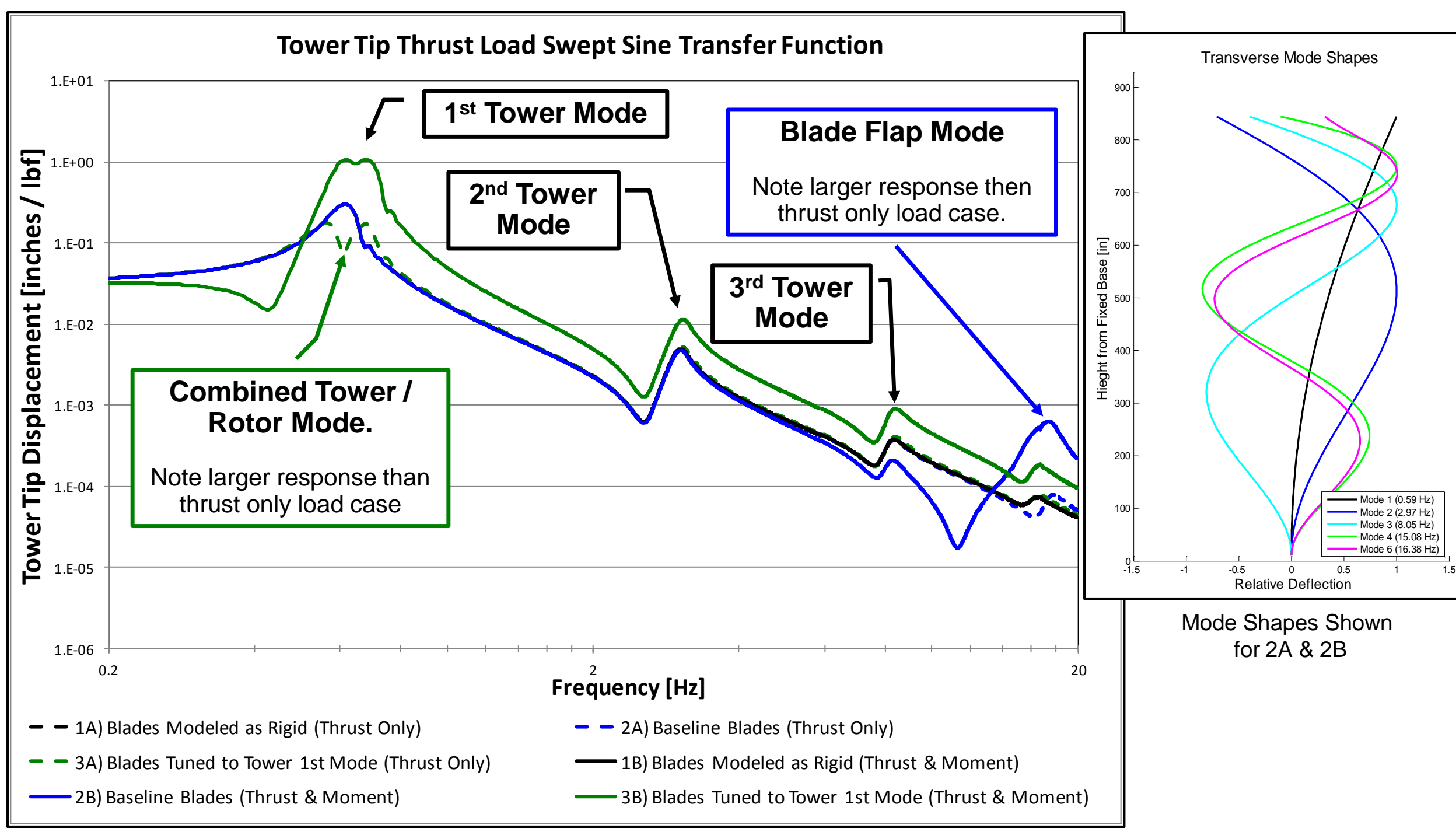

California Polytechnic State University - San Luis Obispo 


\section{Appendix C-3 Modal Truncation Convergence Study}




\section{Modal Truncation Convergence Study: Background}

Transient Small Wind Turbine Tower Structural Analysis with Coupled Rotor Dynamic Interaction

$\underline{\text { Contents Link }}$

- The transient simulations in this work use the method of ModeSuperposition.

- Mode-Superposition allows the coupled equations of motion to be uncoupled by transforming them into modal coordinates thought the use of the system eigenvectors.

- The transient simulation is performed using the uncoupled EOM, results are generated, and then transformed back into the physical coordinate system.

- When using Mode-Superposition it is common to truncate the number of modes that are included in the evaluation to reduce computational time (high order modes can require very small time steps). The number of modes included in the evaluation is represented by the parameter: $\widehat{N}$, hereafter referred to as "Nhat".

- A convergence study is required to determine what Nhat is necessary to ensure that the effects of truncation are negligible (i.e., no modes with a material impact on response are left out). 


\section{Modal Truncation Convergence Study: Method}

- The load case used for the convergence study presented in the following charts was taken from the beginning steady-state portion of the EOG load (see image below)

- Tower response was determined using static analysis

- Tower response was then determined using mode-superposition with sequentially increased Nhat.

- The results from the mode-superposition method were compared to the results from the static analysis.

- Root Mean Square (RMS) Percent Error was used as a metric to compare relative error.

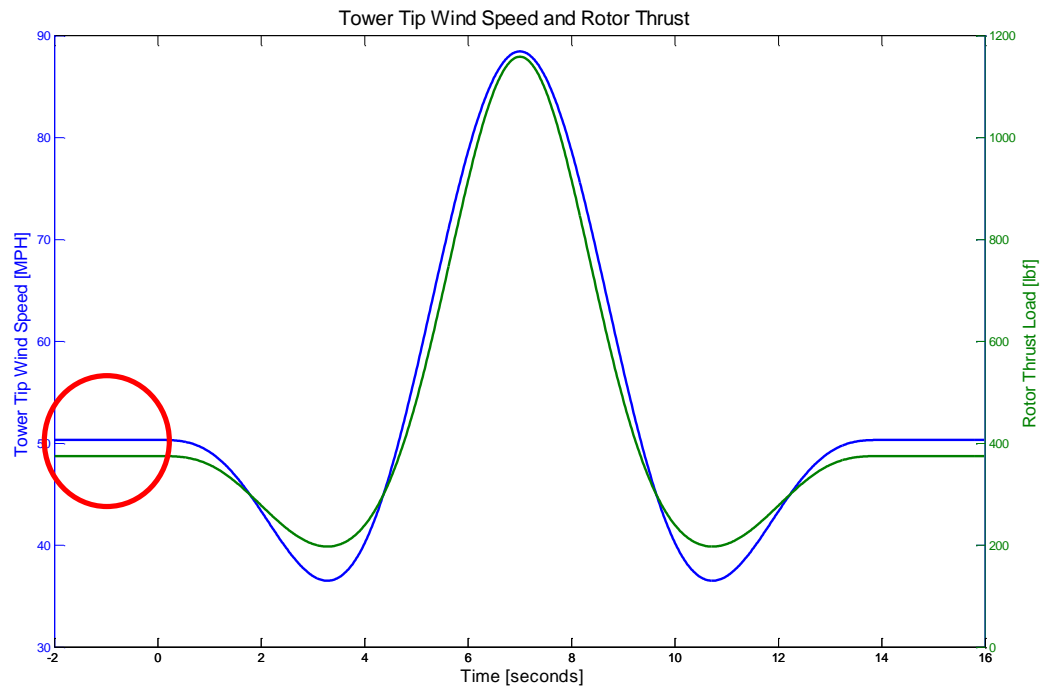




\section{Modal Truncation Convergence Study: Results Summary}

Transient Small Wind Turbine Tower Structural Analysis with Coupled Rotor Dynamic Interaction

- As shown in the table on the next chart the modesuperposition method converges to the static analysis at Nhat $=30$.

- The transient analysis results displayed in this package use Nhat $=30$.

- Of particular interest was the finding that displacement result convergence is much quicker than stress results convergence.

- Only the first 7 modes, or so, are required to produce displacement results that agree well with the static results while about 30 modes are required to produce satisfactory stress results. 


\section{Modal Truncation Convergence Study: Results Details}

Transient Small Wind Turbine Tower Structural Analysis with Coupled Rotor Dynamic Interaction

RMS Percent Error

$=$ RMS (Delta)/RMS(Static)*100, where Delta = Dynamic Result - Static Result

Number of Modes Included, "Nhat" Nhat $=1$

Nhat $=2$

Nhat $=3$

Nhat $=4$

Nhat $=5$

Nhat $=6$

Nhat $=7$

Nhat $=8$

Nhat $=9$

Nhat $=10$

Nhat $=11$

Nhat $=12$

Nhat $=13$

Nhat $=14$

Nhat $=15$

Nhat $=16$

Nhat $=17$

Nhat $=18$

Nhat $=19$

Nhat $=20$

Nhat $=25$

Nhat $=\mathbf{3 0}$

Nhat $=50$

Nhat $=75$

Nhat $=100$

Nhat $=115$ \begin{tabular}{|l|l|}
\hline Transverse (X-Axis) & Bending Moment
\end{tabular}

Bending Moment
(about Z-Axis)

Displacement

Shear Load (X-Axis)

$0.52 \%$

$0.18 \%$

$0.31 \%$

$0.43 \%$

$0.43 \%$

$0.11 \%$

$0.04 \%$

$0.04 \%$

$0.02 \%$

$0.01 \%$

$0.01 \%$

$0.01 \%$

$0.00 \%$

$0.00 \%$

$0.00 \%$

$0.00 \%$

$0.00 \%$

$0.00 \%$

$0.00 \%$

$0.00 \%$

$0.00 \%$

$\mathbf{0 . 0 0 \%}$

$0.00 \%$

$0.00 \%$

$0.00 \%$

$0.00 \%$

L.20\%

$2.52 \%$

$8.63 \%$

$8.63 \%$

$4.24 \%$

$2.35 \%$

$2.41 \%$

$1.73 \%$

$1.71 \%$

$1.70 \%$

$1.72 \%$

$1.60 \%$

$1.31 \%$

$1.01 \%$

$1.00 \%$

$0.61 \%$

$0.45 \%$

$0.18 \%$

$\mathbf{0 . 0 6 \%}$

$0.03 \%$

$0.01 \%$

$0.00 \%$

$0.00 \%$

(about Z-Axis)

von Mises Stress

\begin{tabular}{|l|l|}
\hline $2.19 \%$ & $3.75 \%$ \\
\hline $1.23 \%$ & $2.25 \%$ \\
\hline $3.14 \%$ & $4.91 \%$ \\
\hline $4.55 \%$ & $6.36 \%$ \\
\hline
\end{tabular}

\begin{tabular}{l|l}
$4.55 \%$ & $6.36 \%$ \\
\hline
\end{tabular}

\begin{tabular}{l|l}
$2.05 \%$ & $3.43 \%$ \\
\hline $0.97 \%$ & $1.92 \%$
\end{tabular}

\begin{tabular}{l|l}
$0.97 \%$ & $1.92 \%$ \\
\hline
\end{tabular}

\begin{tabular}{l|l}
$0.97 \%$ & $1.91 \%$ \\
\hline
\end{tabular}

\begin{tabular}{l|l}
$0.64 \%$ & $1.29 \%$ \\
\hline
\end{tabular}

\begin{tabular}{l|l}
$0.58 \%$ & $1.18 \%$ \\
\hline
\end{tabular}

\begin{tabular}{l|l}
$0.49 \%$ & $0.94 \%$ \\
\hline $0.49 \%$ & $0.84 \%$
\end{tabular}

\begin{tabular}{l|l}
$0.49 \%$ & $0.84 \%$ \\
\hline
\end{tabular}

\begin{tabular}{l|l}
$0.38 \%$ & $0.68 \%$ \\
\hline
\end{tabular}

\begin{tabular}{l|l}
$0.25 \%$ & $0.45 \%$ \\
\hline
\end{tabular}

\begin{tabular}{l|l}
$0.23 \%$ & $0.32 \%$ \\
\hline $0.17 \%$ & $0.28 \%$
\end{tabular}

\begin{tabular}{l|l}
$0.17 \%$ & $0.28 \%$ \\
\hline $0.17 \%$ & $0.28 \%$
\end{tabular}

\begin{tabular}{l|l}
$0.17 \%$ & $0.28 \%$ \\
\hline $0.09 \%$ & $0.16 \%$
\end{tabular}

\begin{tabular}{l|l}
$0.17 \%$ & $0.16 \%$ \\
\hline
\end{tabular}

\begin{tabular}{l|l}
$0.10 \%$ & $0.14 \%$ \\
\hline
\end{tabular}

\begin{tabular}{l|l}
$0.06 \%$ & $0.11 \%$ \\
\hline $0.02 \%$ & $0.04 \%$
\end{tabular}

\begin{tabular}{l|l}
$0.02 \%$ & $0.04 \%$ \\
\hline
\end{tabular}

\begin{tabular}{l|l}
$\mathbf{0 . 0 0 \%}$ & $\mathbf{0 . 0 1 \%}$ \\
\hline $0.00 \%$ & $0.00 \%$
\end{tabular}

\begin{tabular}{l|l}
$0.00 \%$ & $0.00 \%$ \\
\hline $0.00 \%$
\end{tabular}

\begin{tabular}{l|l}
$0.00 \%$ & $0.00 \%$ \\
\hline $0.00 \%$
\end{tabular}

\begin{tabular}{l|l}
$0.00 \%$ & $0.00 \%$ \\
\hline $0.00 \%$ & $0.00 \%$
\end{tabular}

\begin{tabular}{l|l}
$0.00 \%$ & $0.00 \%$ \\
\hline
\end{tabular}

Calitornta PoIytechnic State University - san LuIs UDIspo
Contents Link

A graphical comparison for Displacements and von Mises Stress is included in the subsequent charts 


\section{Modal Truncation Convergence Study: Displacement Overlay}

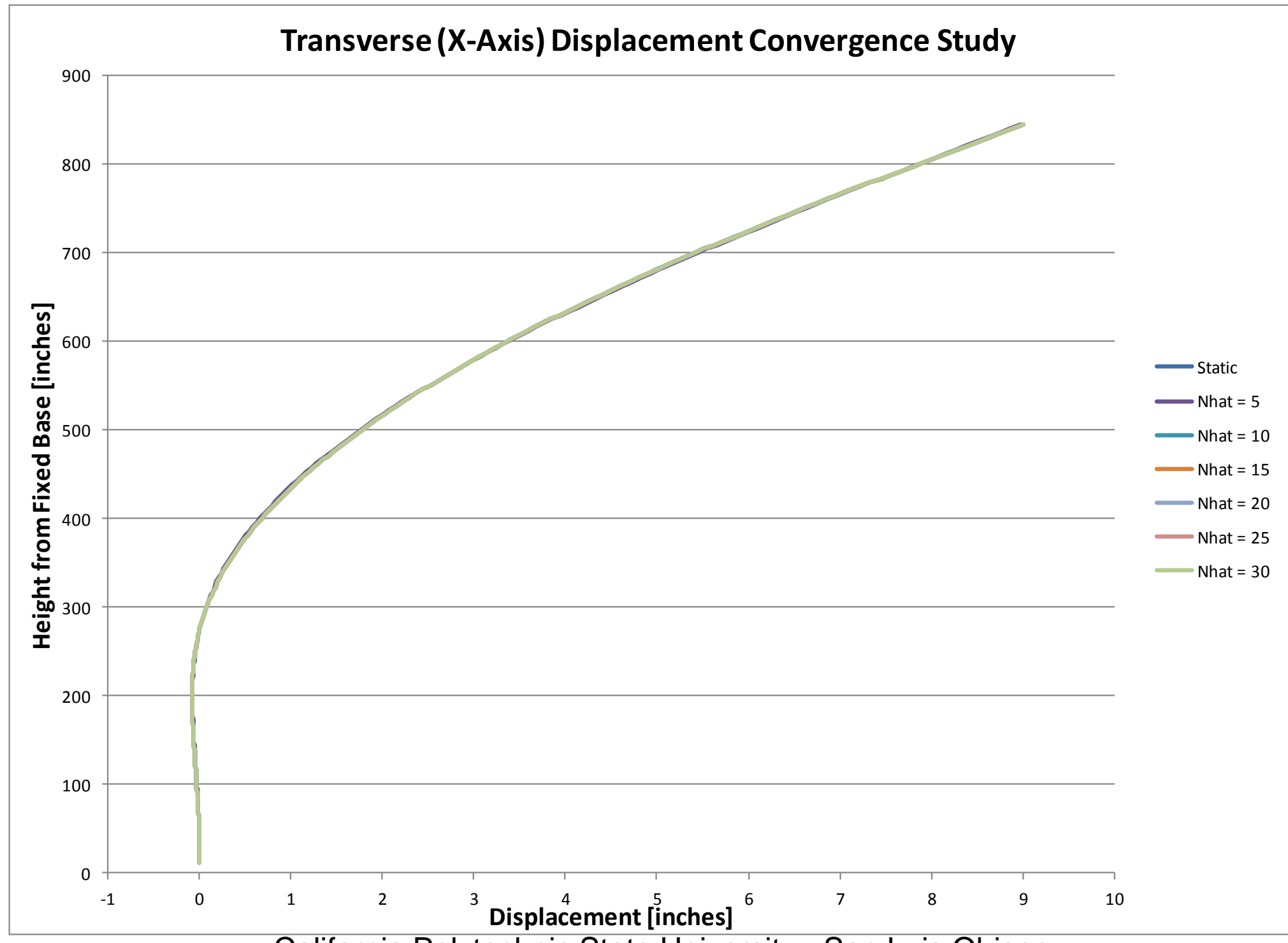

California Polytechnic State University - San Luis Obispo 


\section{Modal Truncation Convergence Study: Delta Displacement Overlay}

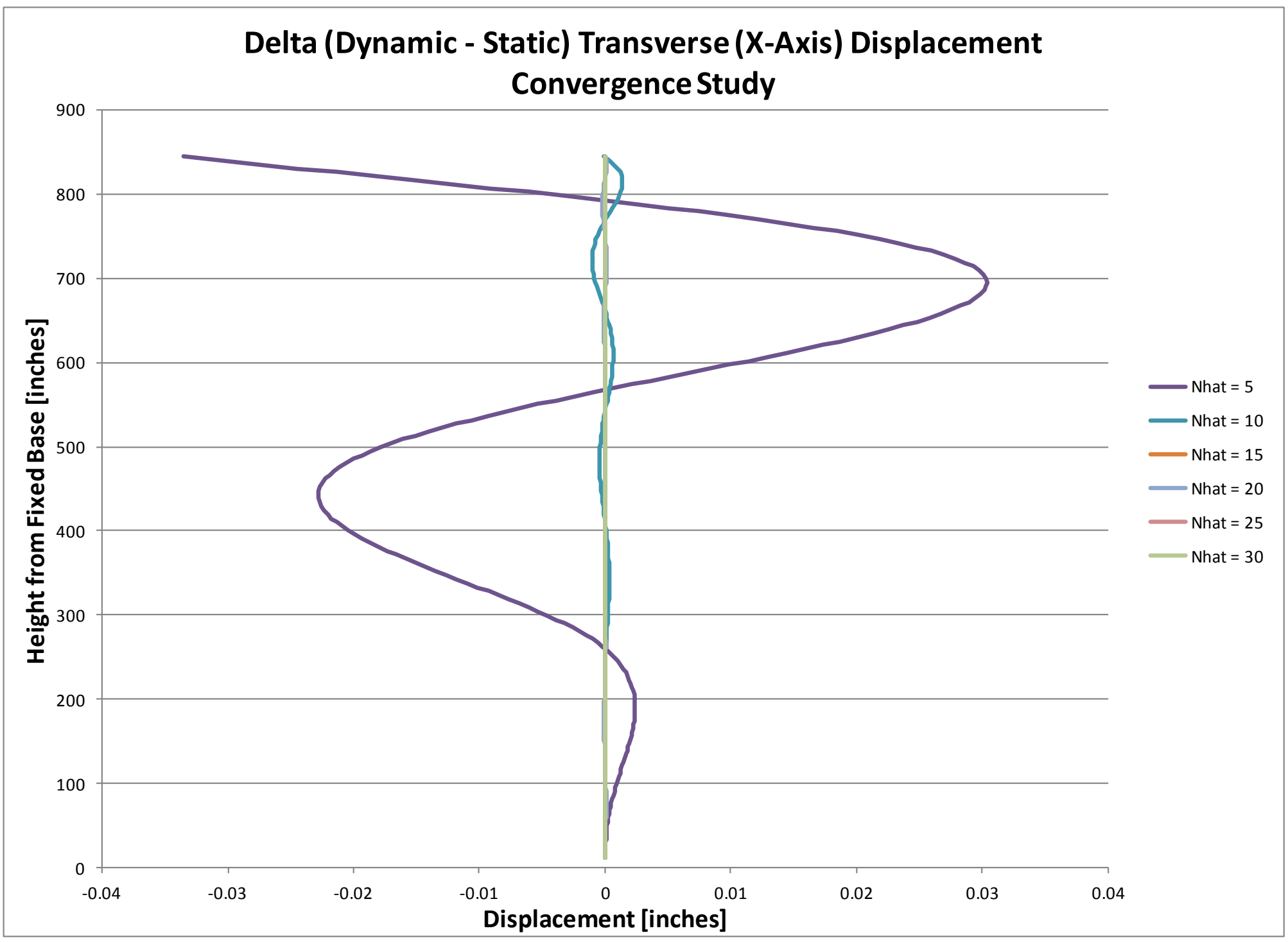

California Polytechnic State University - San Luis Obispo 


\section{Modal Truncation Convergence Study: von MOverlay}

Transient Small Wind Turbine Tower Structural Analysis with Coupled Rotor Dynamic Interaction

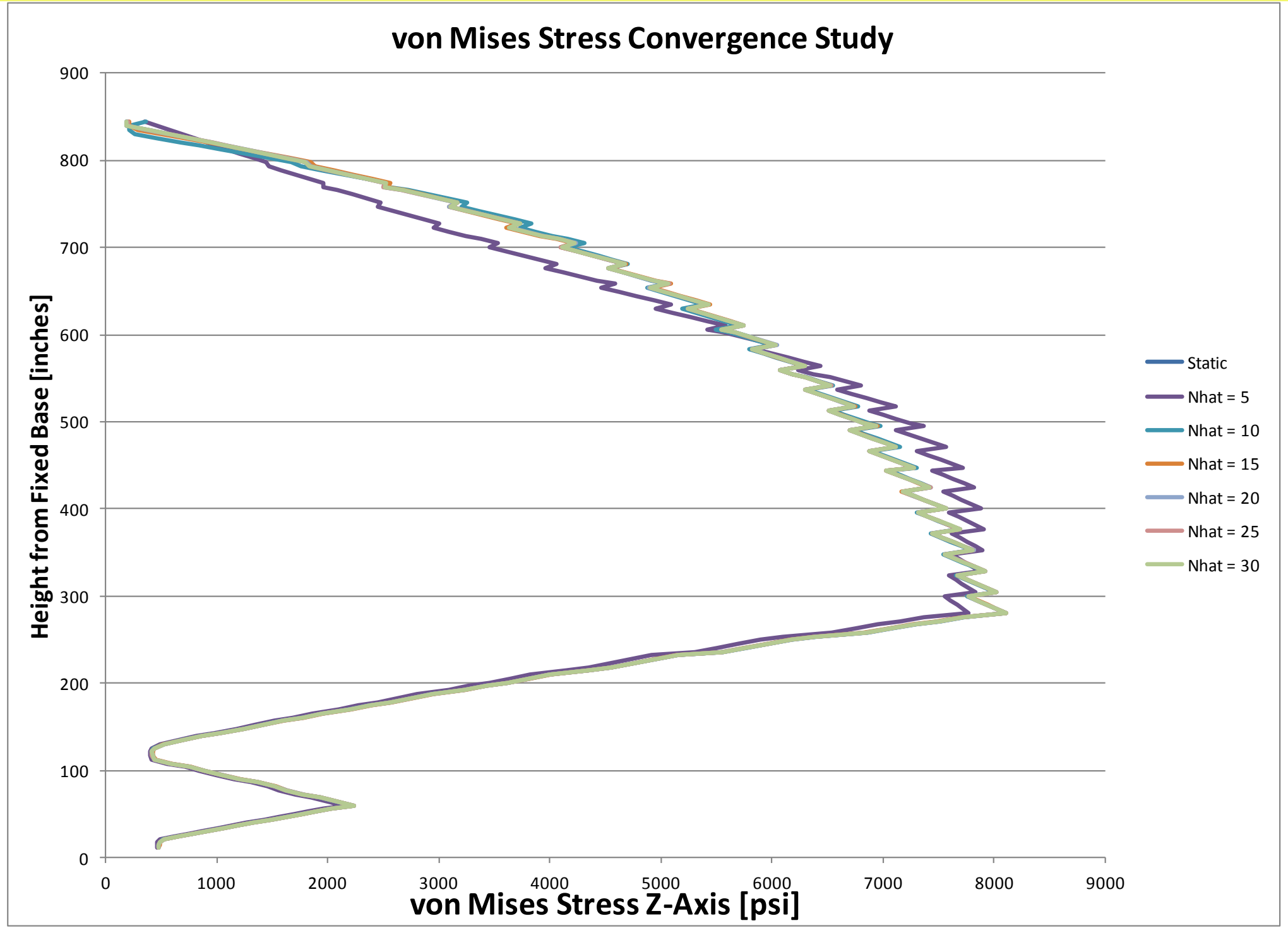

California Polytechnic State University - San Luis Obispo 


\section{Modal Truncation Convergence Study: Delta Displacement Overlay}

Transient Small Wind Turbine Tower Structural Analysis with Coupled Rotor Dynamic Interaction

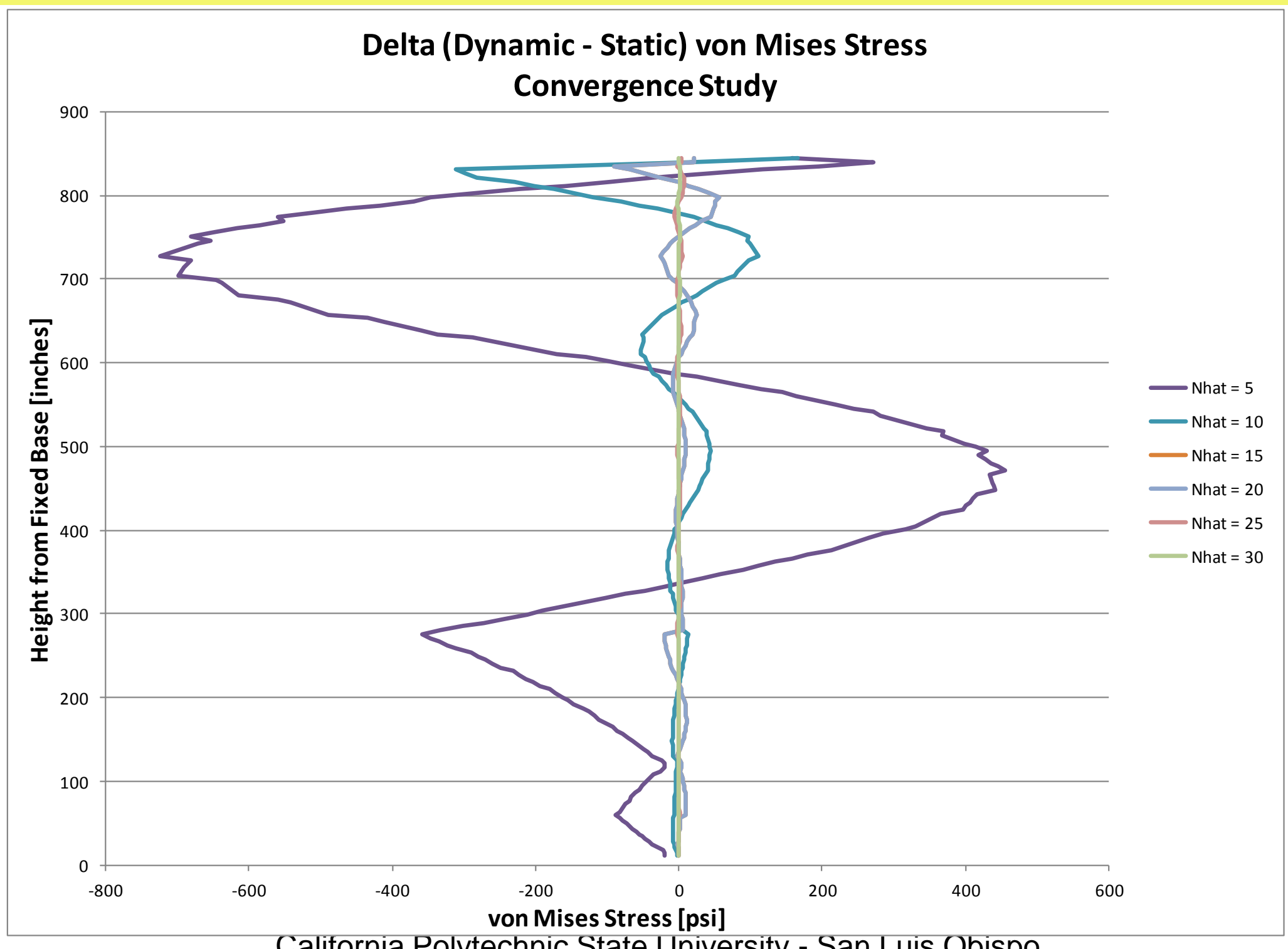

Calitornia Polytechnic State University - San Luis Obispo 


\section{Vortex Shedding - Overview}

- Wind flow past a cylindrical tower can produce cyclic excitation, orthogonal to the wind direction, due to vortex shedding.

- Vortex shedding frequency should be evaluated to ensure it does not cause detrimental resonance of the structure.

- Experimental data shows that vortex shedding frequencies can be estimated using the dimensionless Strouhal number correlation St $=f^{\star} D / V$ (Fox et al., 2004, p 442).

- Where $S t \approx 0.21$ for $\operatorname{Re}>1000$

- $f=$ frequency of vortex shedding excitation

- $D=$ tower diameter, range is below:

- 6 inches (tower tip)

- 14 inches (tower base)

- $\mathrm{V}=$ wind speed, range is below:

- $117.5 \mathrm{mph}$ (50 Year EWM, SWT Class 3)

- $5 \mathrm{mph}$ (speeds below $5 \mathrm{mph}$ are negligible)

- See next chart for results 


\section{Vortex Shedding - Frequency vs Wind Velocity}

Transient Small Wind Turbine Tower Structural Analysis with Coupled Rotor Dynamic Interaction

Contents Link

- The following plot / table display vortex shedding frequency as a function of wind velocity and tower diameter (which, for the Cal Poly Tower, varies from a base diameter of 14 in to a tip diameter of 6 in).

- Note that for a constant wind profile the vortex shedding frequency at the tower tip is higher than at the tower base

Vortex Shedding Frequency based on Strouhal Number

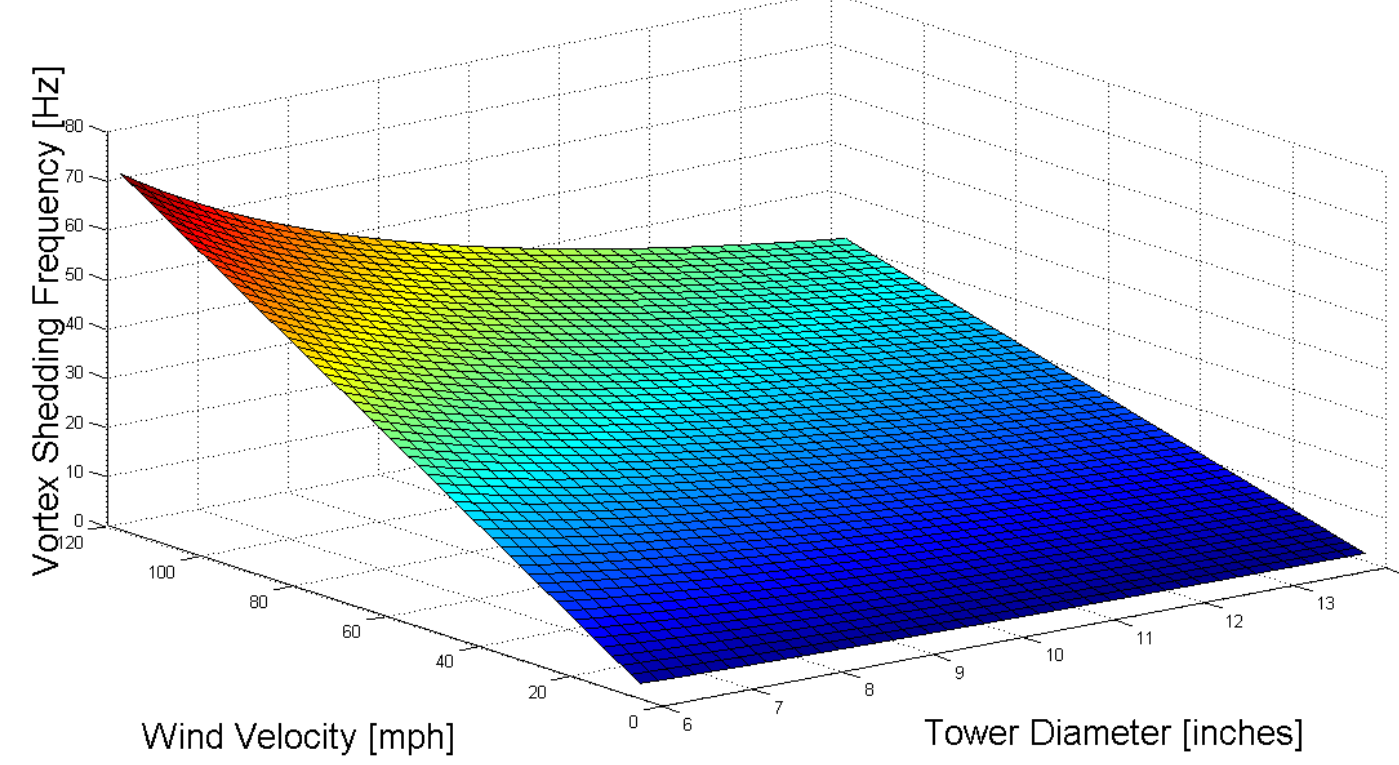

\begin{tabular}{|c|c|c|}
\hline \multirow{2}{*}{$\begin{array}{c}\text { Wind Velocity } \\
\text { (V) [mph] }\end{array}$} & \multicolumn{2}{|c|}{ Vortex Shedding Frequency [Hz] } \\
\cline { 2 - 3 } & Tower Tip (6 in Dia) & Tower Base (14 in Dia) \\
\hline 5 & 3.08 & 1.32 \\
\hline 10 & 6.16 & 2.64 \\
\hline 15 & 9.24 & 3.96 \\
\hline 20 & 12.32 & 5.28 \\
\hline 30 & 18.48 & 7.92 \\
\hline 40 & 24.64 & 10.56 \\
\hline 50 & 30.8 & 13.2 \\
\hline 70 & 43.12 & 18.48 \\
\hline 90 & 55.44 & 23.76 \\
\hline 110 & 67.76 & 29.04 \\
\hline 117.5 & 72.38 & 31.02 \\
\hline
\end{tabular}




\section{Vortex Shedding - Tower Displacement Evaluation Method}

Transient Small Wind Turbine Tower Structural Analysis with Coupled Rotor Dynamic Interaction

Contents Link

- Maximum tower displacements due to vortex shedding will occur when vortex shedding causes tower resonance (i.e., vortex shedding frequencies $=$ tower modal frequencies)

- A conservative approach has been implemented to determine maximum tower displacements due to vortex shedding using the following method:

- Step 1: Determine tower modal frequencies

- Step 2: Determine wind velocity required to produce vortex shedding frequency equal to tower modal frequencies

- A higher wind velocity will be required at the tower base than the tower tip due to

the larger diameter at the base
- Step 3: Compute vortex shedding force ( $f=\frac{1}{2} C_{L} \rho V^{2} A_{\text {Tower }}$ ) acting orthogonal to wind direction. Use the following conservative assumptions:

- Use the higher tower base wind velocity and apply it uniformly to the whole tower

- Ignore the fact that a uniform wind profile acting on a tapered tower will produce different vortex shedding frequencies along the tower's height, resulting in out of phase loading, dramatically reducing the effect of vortex shedding loads.

- Apply the entire vortex shedding force to the tower tip (instead of distributed).

- Use a Lift Coefficient (CL) of 0.5 which is on the high end of the $0.2-0.5$ range (dependent on Reynolds Number), (CAN/CSA-S6-2000).

- Use the results from the Swept Sine simulation \{Method 1A, Blades Modeled as Rigid (Thrust Only)\} and scale by the vortex shedding force to determine tower tip displacement. 


\section{Vortex Shedding - Tower Displacement Evaluation Results}

Transient Small Wind Turbine Tower Structural Analysis with Coupled Rotor Dynamic Interaction

Contents Link

\begin{tabular}{|l|c|c|c|c|c|c|}
\hline \multicolumn{2}{|c|}{} & \multicolumn{2}{c|}{$\begin{array}{c}\text { Wind Velocity [mph] } \\
\text { Required to Produce Vortex Shedding } \\
\text { Tower Modes }\end{array}$} & \multicolumn{2}{c|}{$\begin{array}{c}\text { Vortex Shedding } \\
\text { Force [Ibf] } \\
\text { Using a CL of 0.5 }\end{array}$} & $\begin{array}{c}\text { Tower } \\
\text { Deflection [in] } \\
\text { Frency that Matches Tower Mode Freq }\end{array}$ \\
Description & Freq [Hz] & $\begin{array}{c}\text { Tower Tip } \\
\text { (6 in Dia) }\end{array}$ & $\begin{array}{c}\text { Tower Base } \\
\text { (14 in Dia) }\end{array}$ & $\begin{array}{c}\text { Tower Tip } \\
\text { (6 in Dia) }\end{array}$ & $\begin{array}{c}\text { Tower Base } \\
\text { (14 in Dia) }\end{array}$ & $\begin{array}{c}\text { Using base force load } \\
\text { applied to tower tip }\end{array}$ \\
\hline Weak Axis Mode 1 & 0.59 & 0.96 & 3.63 & 0.05 & 0.78 & 0.24 \\
\hline Strong Axis Mode 1 & 0.81 & 1.31 & 4.98 & 0.10 & 1.47 & 0.28 \\
\hline Weak Axis Mode 2 & 2.97 & 4.82 & 18.26 & 1.38 & 19.83 & 0.10 \\
\hline Strong Axis Mode 2 & 4.70 & 7.63 & 28.90 & 3.46 & 49.66 & 0.09 \\
\hline Weak Axis Mode 3 & 8.05 & 13.07 & 49.50 & 10.15 & 145.67 & 0.06 \\
\hline Strong Axis Mode 3 & 12.99 & 21.09 & 79.88 & 26.44 & 379.32 & 0.05 \\
\hline Weak Axis Mode 4 & 15.08 & 24.48 & 92.73 & 35.63 & 511.20 & 0.03 \\
\hline Strong Axis Mode 4 & 16.20 & 26.30 & 99.62 & 41.12 & 589.95 & 0.04 \\
\hline Weak Axis Mode 5 & 16.38 & 26.59 & 100.72 & 42.04 & 603.14 & 0.04 \\
\hline Strong Axis Mode 5 & 16.78 & 27.24 & 103.18 & 44.11 & 632.95 & 0.04 \\
\hline
\end{tabular}

\section{Max displacement (0.28 in) is very small in comparison to other load cases.}




\section{Guy-Wire Optimization - Overview}

- For wind turbine towers that are supported with guy-wires it is important to optimize guy-wire support geometry.

- To demonstrate the ability of this tool to optimize guy-wire support geometry a hypothetical study was completed by simulating the effect of adding guy-wires to the Cal Poly Wind Turbine Tower in the Weak Axis (orthogonal to the plane produced by the Gin-Pole and Strut).

- A total of two sets of guy-wires were added (four guy-wires in total)

- Guy-Wire diameter of 0.5 inches was used

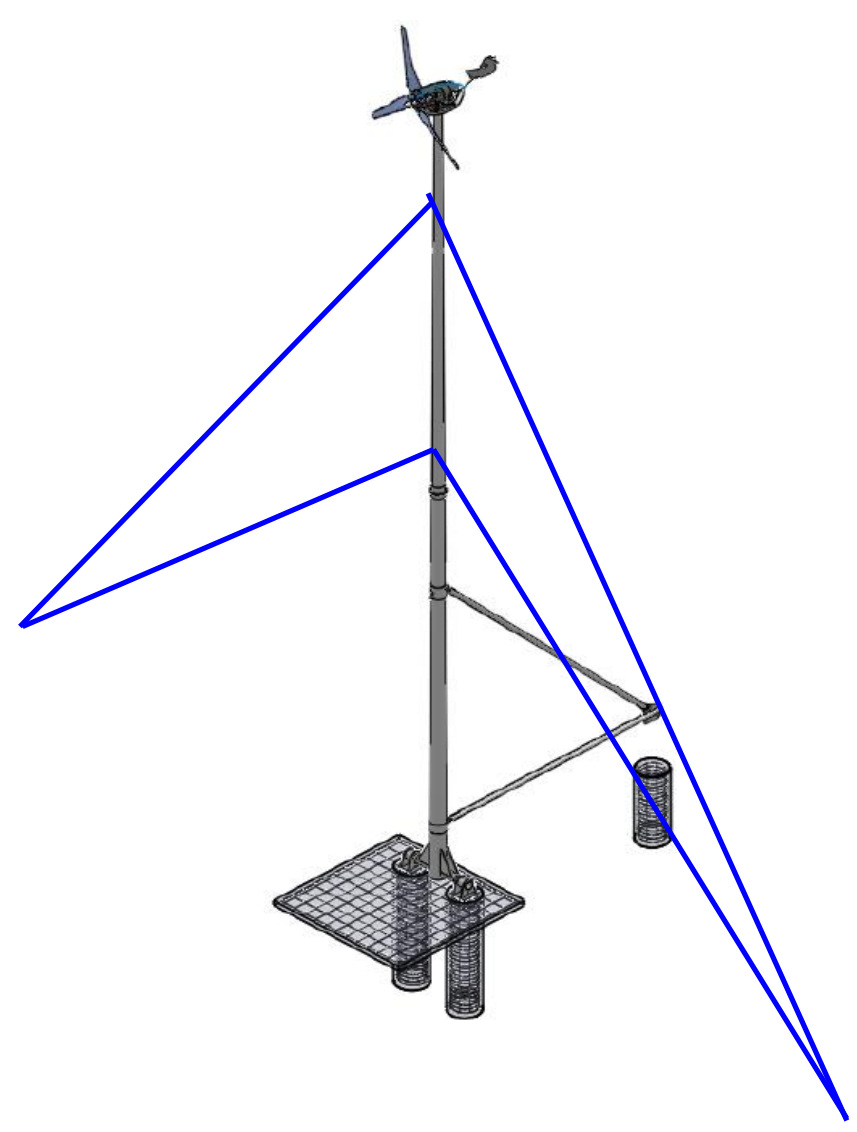




\section{Guy-Wire Optimization - Parameters}

- The guy-wire to tower interface heights were optimized for the following cases:

- Optimization A)

- Maximum Tower $1^{\text {st }}$ Mode Frequency

- Optimization B)

- Maximum Separation Between $1^{\text {st }}$ and $2^{\text {nd }}$ Tower Mode Frequencies

- Useful for modal frequency management if system design places the rotor blade flapwise natural frequencies between the tower $1^{\text {st }}$ and $2^{\text {nd }}$ modes.

- Optimization C)

- Minimum Tower von Mises Stress Due to 1 Year Extreme Wind Model

- The results are shown on the following charts 


\section{Optimization A) Maximum Tower $1^{\text {st }}$ Mode Frequency}

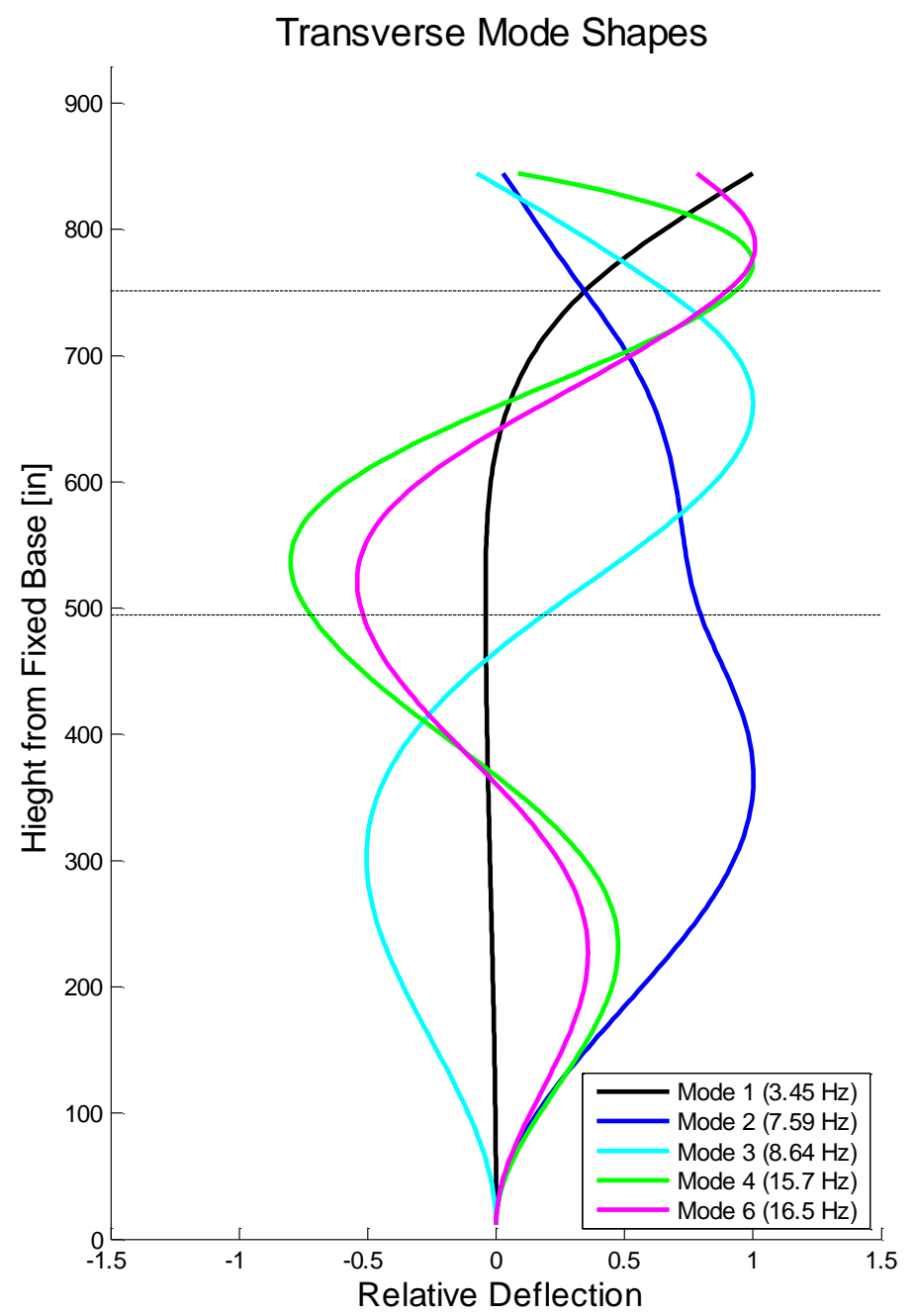

Guy-Wires to Tower Interface:

- Set 1: 495 in from ground

- Set 2: 750 in from ground

Tower First 5 Modes:

(in weak axis)

1. $3.45 \mathrm{~Hz}$

2. $7.59 \mathrm{~Hz}$

3. $8.64 \mathrm{~Hz}$

4. $15.7 \mathrm{~Hz}$

5. $16.5 \mathrm{~Hz}$ 


\section{Optimization B) Maximum Separation Between $1^{\text {st }}$ and $2^{\text {nd }}$ Modes}

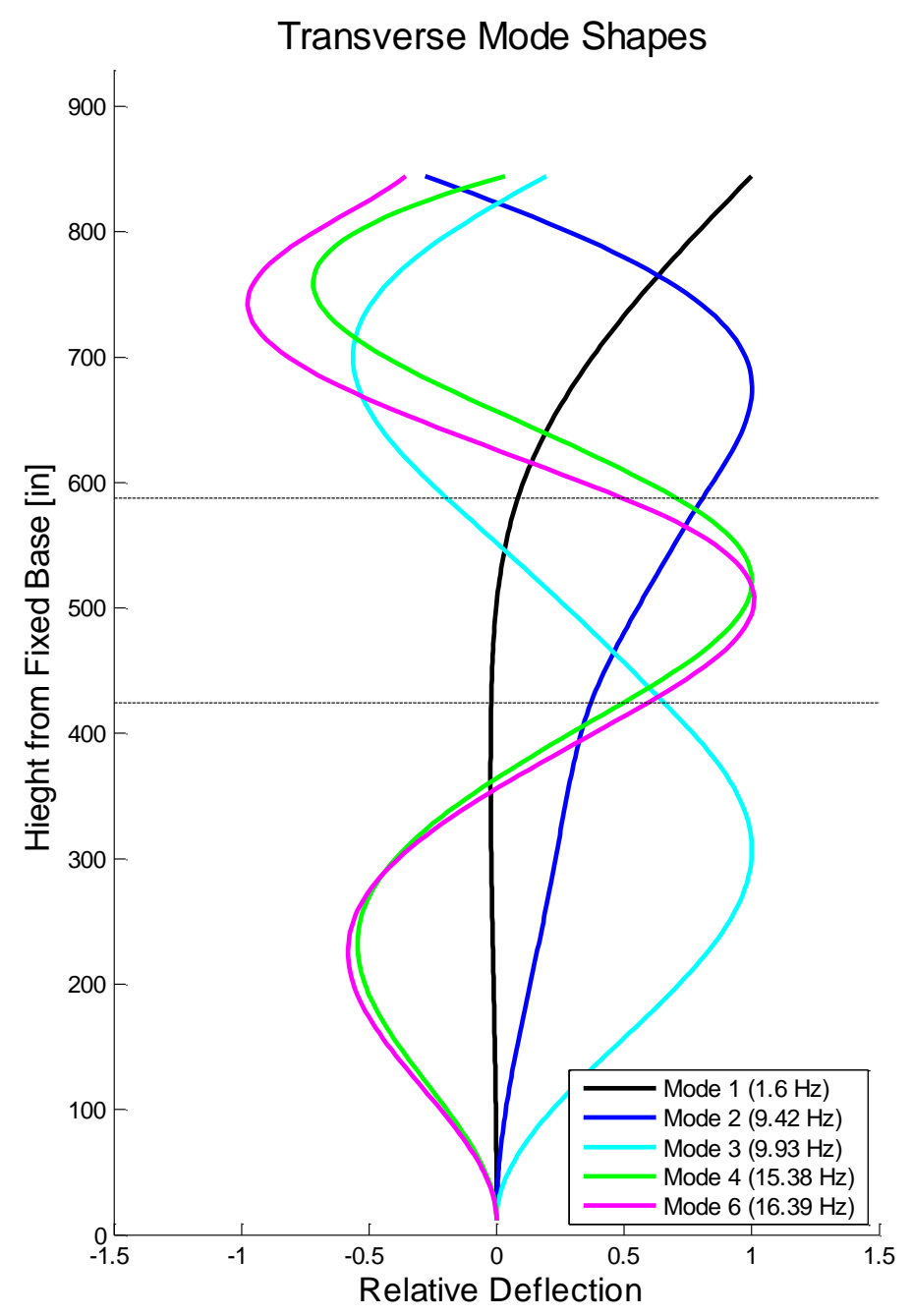

Guy-Wires to Tower Interface:

- Set 1: 425 in from ground

- Set 2: 590 in from ground

Tower First 5 Modes:

(in weak axis)

1. $1.60 \mathrm{~Hz}$

2. $9.42 \mathrm{~Hz}$

3. $9.93 \mathrm{~Hz}$

4. $15.38 \mathrm{~Hz}$

5. $16.39 \mathrm{~Hz}$ 


\section{Optimization B) Minimum Tower Stress Due to 1 Year EWM}

Max Tower von Mises Stress Profile

Max: $9767 \mathrm{lbf} / \mathrm{in}^{2}$, at hight: 751.2 in

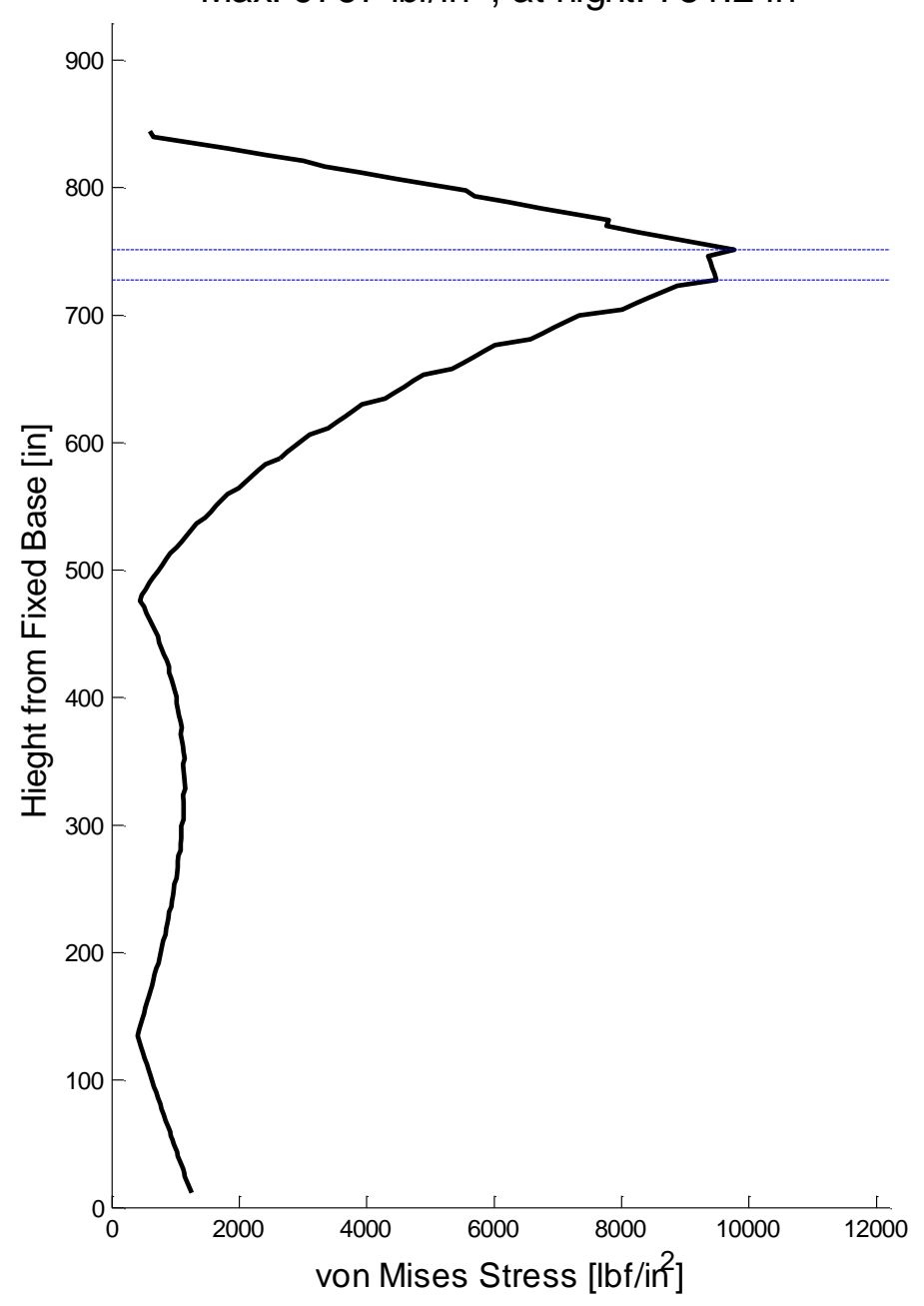

Guy-Wires to Tower Interface:

- Set 1:725 in from ground

- Set 2: 750 in from ground

Max von Mises Stress: (due to loading in weak axis)

- 9767 lbf/in² 


\section{Strut Critical Buckling Load}

Transient Small Wind Turbine Tower Structural Analysis with Coupled Rotor Dynamic Interaction

- The critical buckling load $\left(P_{\mathrm{cr}}\right)$ for the strut of the Cal Poly Wind Turbine Tower can easily be determined using the Euler column formula (Shigley et al., 2004, p 219).

- The following $\mathbf{P}_{\mathrm{cr}}$ was used to determine the strut buckling margin of safety in the results summary table.

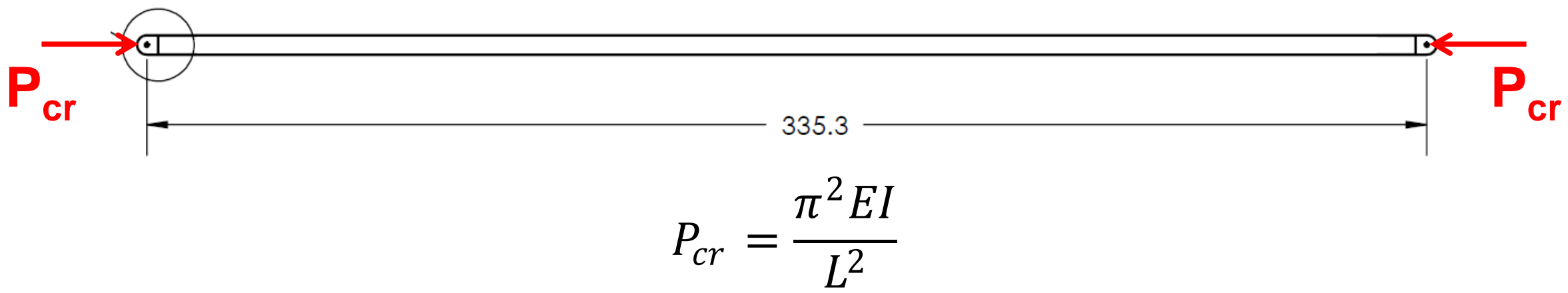

$$
P_{c r}=\frac{\pi^{2} \cdot(29.2 \mathrm{Mpsi}) \cdot\left(10.55 \mathrm{in}^{4}\right)}{(335.3 \mathrm{in})^{2}}=27,000 \mathrm{lbf}
$$




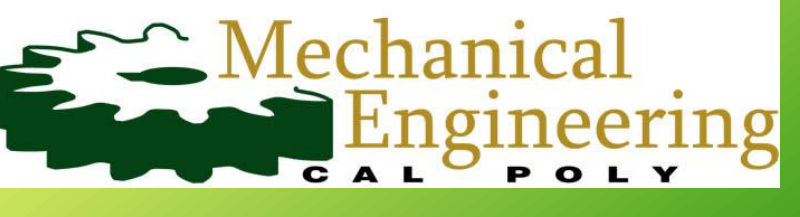

\section{Appendix C-7 Fatigue}




\section{Estimation of Fatigue Properties}

Transient Small Wind Turbine Tower Structural Analysis with Coupled Rotor Dynamic Interaction

Contents Link

\begin{tabular}{|c|c|c|c|}
\hline \multicolumn{4}{|c|}{ Fatigue S-N Curve Properties Estimates based on Shigley Method } \\
\hline Discription & Equation & Value & $\begin{array}{c}\text { Reference } \\
\text { (Shigley et al., } \\
\text { 2004, p 325) }\end{array}$ \\
\hline Endurance Limit Ratio $\left(\mathrm{f}_{\mathrm{e} 6}\right)$ & Empirical Relation & 0.504 & (Eq. 7-8) \\
\hline Low Cycle Fatigue Ratio $\left(\mathrm{f}_{\mathrm{e}}\right)$ & Empirical Relation & 0.900 & $(\operatorname{Sec} 7-8)$ \\
\hline Endurance Limit $\left(\mathrm{S}_{\mathrm{e}}\right),[\mathrm{ksi}]$ & $\mathrm{S}_{\mathrm{e}}=\mathrm{f}_{\mathrm{e} 6} \mathrm{~S}_{\mathrm{u}}$ & 32.760 & (Eq. 7-8) \\
\hline $\begin{array}{l}\text { Low Cycle (e3) Fatigue } \\
\text { Strength Ratio }\left(\mathrm{S}_{\mathrm{fe} 3}\right),[\mathrm{ksi}]\end{array}$ & $\mathrm{S}_{\mathrm{e}}=\mathrm{f}_{\mathrm{e} 3} \mathrm{~S}_{\mathrm{u}}$ & 58.500 & $(\operatorname{Sec} 7-8)$ \\
\hline Fatigue Constant (a) & $\mathrm{a}=\left(\mathrm{f}_{\mathrm{e} 3} \mathrm{~S}_{\mathrm{u}}\right)^{2} / \mathrm{S}_{\mathrm{e}}$ & 104.464 & (Eq 7-13) \\
\hline Fatigue Exponent (b) & $b=-(1 / 3) \log \left(f_{e 3} S_{u} / S_{e}\right)$ & -0.084 & (Eq 7-14) \\
\hline Fatigue Exponent (c) & $c=(\log f) / 3$ & -0.015 & (Eq 7-16) \\
\hline Fatigue Strength $\left(\mathrm{S}_{\mathrm{f}}\right)$ & - & - & - \\
\hline Low Cycles $1 \leq \mathrm{N}<10^{3}$ & $\mathrm{~S}_{\mathrm{f}}=\mathrm{S}_{\mathrm{u}} \mathrm{N}^{\mathrm{c}}$ & $S f=65 N^{\wedge}(-0.015)$ & (Eq 7-16) \\
\hline Mid Cycles $10^{3} \leq \mathrm{N}<10^{6}$ & $S_{f}=a N^{b}$ & $\mathrm{Sf}=104.46 \mathrm{~N}^{\wedge}(-0.084)$ & (Eq 7-12) \\
\hline High Cycles $10^{6} \leq \mathrm{N}$ & $\mathrm{S}_{\mathrm{f}}=\mathrm{S}_{\mathrm{e}}$ & $\mathrm{Sf}=32.76$ & (Sec 7-8) \\
\hline Cycles & \multicolumn{2}{|c|}{ Fatigue Strength $\left(\mathrm{S}_{\mathrm{f}}\right)$ [kpsi] } & - \\
\hline 1. $E+00$ & \multicolumn{2}{|c|}{65.0} & - \\
\hline 1.E+01 & \multicolumn{2}{|c|}{62.8} & - \\
\hline 1. $E+02$ & \multicolumn{2}{|c|}{60.6} & - \\
\hline 1.E+03 & \multicolumn{2}{|c|}{58.5} & - \\
\hline 1.E+03 & \multicolumn{2}{|c|}{58.5} & - \\
\hline 1.E+04 & \multicolumn{2}{|c|}{48.2} & - \\
\hline 1.E+05 & \multicolumn{2}{|c|}{39.7} & - \\
\hline 1.E+06 & \multicolumn{2}{|c|}{32.8} & - \\
\hline 1.E+07 & \multicolumn{2}{|c|}{32.8} & - \\
\hline 1. E+08 & \multicolumn{2}{|c|}{32.8} & - \\
\hline
\end{tabular}

Fatigue properties of ASTM A572 were estimated using an empirical method presented by method presented by Shigley. Results are approximate.

\begin{tabular}{|c|c|}
\hline \multicolumn{2}{|c|}{ ASTM A572, Grade 50 Tensile Strength } \\
\hline Yield $\left(\mathrm{S}_{\mathrm{y}}\right),[\mathrm{ksi}]$ & 50 \\
\hline Ultimate $\left(\mathrm{S}_{\mathrm{u}}\right),[\mathrm{ksi}]$ & 65 \\
\hline
\end{tabular}

\section{Fatigue Strength $\left(\mathrm{S}_{\mathrm{f}}\right)$}

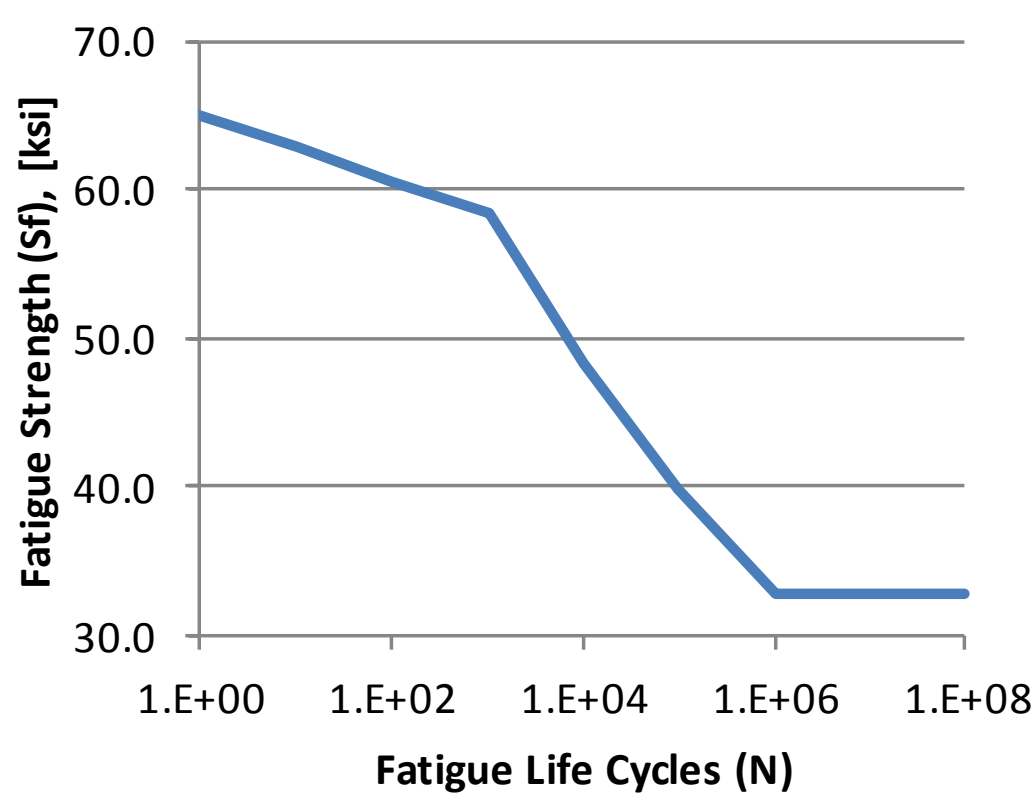




\section{Cumulative Damage Index Evaluation Method}

Transient Small Wind Turbine Tower Structural Analysis with Coupled Rotor Dynamic Interaction

- The Cumulative Damage Index (CDI) is used to determine the portion of fatigue life consumed by a time varying load profile.

- A CDI of 0 indicates full fatigue life remains

- Fatigue failure is predicted at a $\mathrm{CDI}=1$

- Per IEC 61400-2 Miner's rule can be used to determine the CDI.

- Where:

$$
C D I=\sum_{i} \frac{n_{i}}{N\left(\gamma_{f} \gamma_{m} s_{i}\right)}
$$

- $n_{i}$ : is the number of load cycles applied for a load magnitude of

- $N\left(Y_{f} Y_{m} s_{i}\right)$ is the number of load cycles to failure for a load magnitude of where and are the fatigue load and material partial factors of safety, taken as 1.0 and 1.25 respectively.

- Of the load cases considered in this work only the NTM (DLC 2.2) is used for fatigue evaluation 


\section{Cumulative Damage Index Evaluation Results}

Transient Small Wind Turbine Tower Structural Analysis with Coupled Rotor Dynamic Interaction

Contents Link

- Of the load cases considered in this work only the NTM (DLC 2.2) is used for fatigue evaluation.

- The max tower stress produced by the NTM profile is $10.05 \mathrm{Ksi}$

- This stress is relatively low because the frequency content of the NTM profile is in the rigid body range of the tower (i.e., much lower than the tower natural frequency) and thus does not induce resonance.

- The denominator of the Miner's rule equation can be simplified using the max load and evaluated to be infinity (max load is less that endurance limit). Thus the CDI is found to be zero (0).

$C D I=\sum_{i} \frac{n_{i}}{N\left(\gamma_{f} \gamma_{m} s_{\max }\right)}=\sum_{i} \frac{n_{i}}{N(1.0 \cdot 1.25 \cdot 10.05 k s i)}=\sum_{i} \frac{n_{i}}{N(12.56 k s i)}=\sum_{i} \frac{n_{i}}{\infty}=0$

- If the max load was higher than the endurance limit then a cycle counting method, such as the Rainflow Method, would need to be used to create a histogram of load magnitude vs cycle count (Manwell et al., 2002, p 161). 


\section{Myklestad Method - Background}

Transient Small Wind Turbine Tower Structural Analysis with Coupled Rotor Dynamic Interaction

- The Hinge Equivalent Blade mode requires knowledge of the blade non-rotating and rotating natural frequencies.

- These values are used to determine the blade hinge location and hinge stiffness.

- As the rotor spins centrifugal forces develop that pull the blade away from the spine axis. These centrifugal forces create a stiffening effect which causes the blade's rotating natural frequency to be higher than the blade's non-rotating natural frequency.

- The Myklestad Method (Manwell et al., 2002, p154), accounts for centrifugal stiffening, and can be used to determine the blade rotating natural frequencies.

- A simple Eigenvector / Eigenvalue analysis of a blade structure model can be used to determine the blade's non-rotating natural frequency but cannot account for centrifugal stiffening and thus cannot be used to determine the blade's rotating natural frequency. 


\section{Blades - Background}

Transient Small Wind Turbine Tower Structural Analysis with Coupled Rotor Dynamic Interaction

- The Cal Poly wind turbine blades were developed by Bryan Edwards and Dr. Patrick Lemieux in 2009 (Edwards, 2009).

- The blades feature a composite construction utilizing carbon fiber, fiberglass, and epoxy resin.

- Blade dimensional details (including airfoil cross sections) were documented by Edwards.

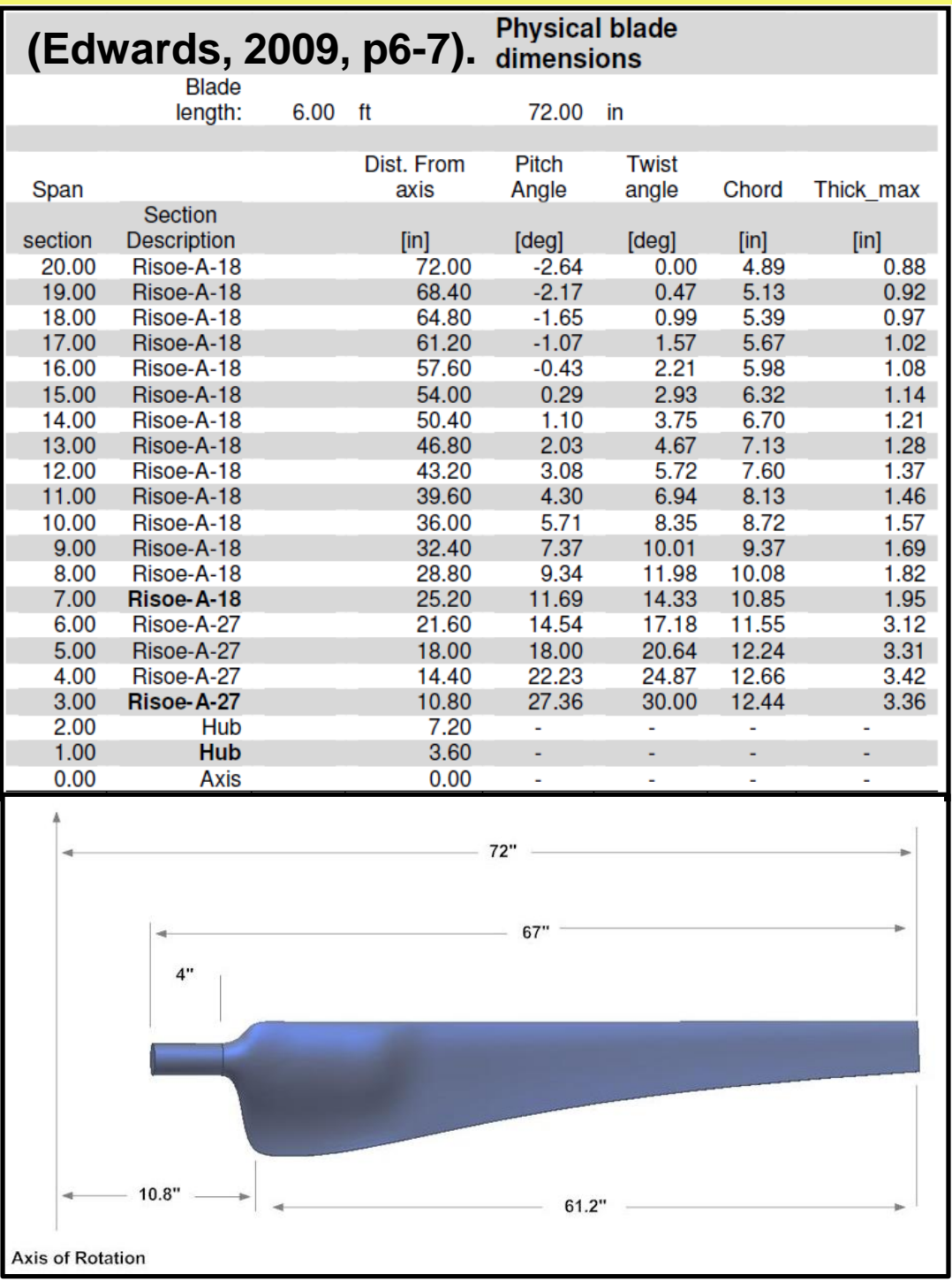


- Step 2.1: Determine the cross-sectional properties at each of the 20 span sections identified in Edwards' work by scaling the data from Step 1 based on chord length.

- Neutral Bending Axis

- Area Moment of Inertia

- Perimeter

- Material Volume per unit length (based on layup thickness documented by Edwards and perimeter)

- Step 2.2: Sum up the product of the 20 span lengths and their corresponding cross-sectional volumes. Evaluate effective layup density.

- Determine the total layup volume: 72.7 in $^{3}$

- Determine effective layup density: $0.15 \mathrm{lbs} / \mathrm{in}^{3}$

- Based on an approximate blade weight of $11 \mathrm{lbs}$

- "Effective layup density" smears the mass of the metallic root over the entire blade 


\section{Myklestad Method}

\section{Steo 3: Blade Discretization \& Stiffiness}

- Step 3.1: Create a FEM model of the blade using the 20 span sections identified in Edwards' work.

- A FEM of the blade was prepared using 2D Plane Beam elements, similar to the process used for the tower.

- Step 3.2: Determine test correlated effective isotropic Young's Modulus: 4.6 MPSI

- Per testing performed by Edwards the blade deflects 3.875 inches when acted upon by a $120 \mathrm{lbf}$ static tip load in the flapwise direction.

- The Young's Modulus was correlated to test results such that the FEM predicted deflection to the $120 \mathrm{lbf}$ tip load was the same as the test demonstrated deflection of 3.875 inches.

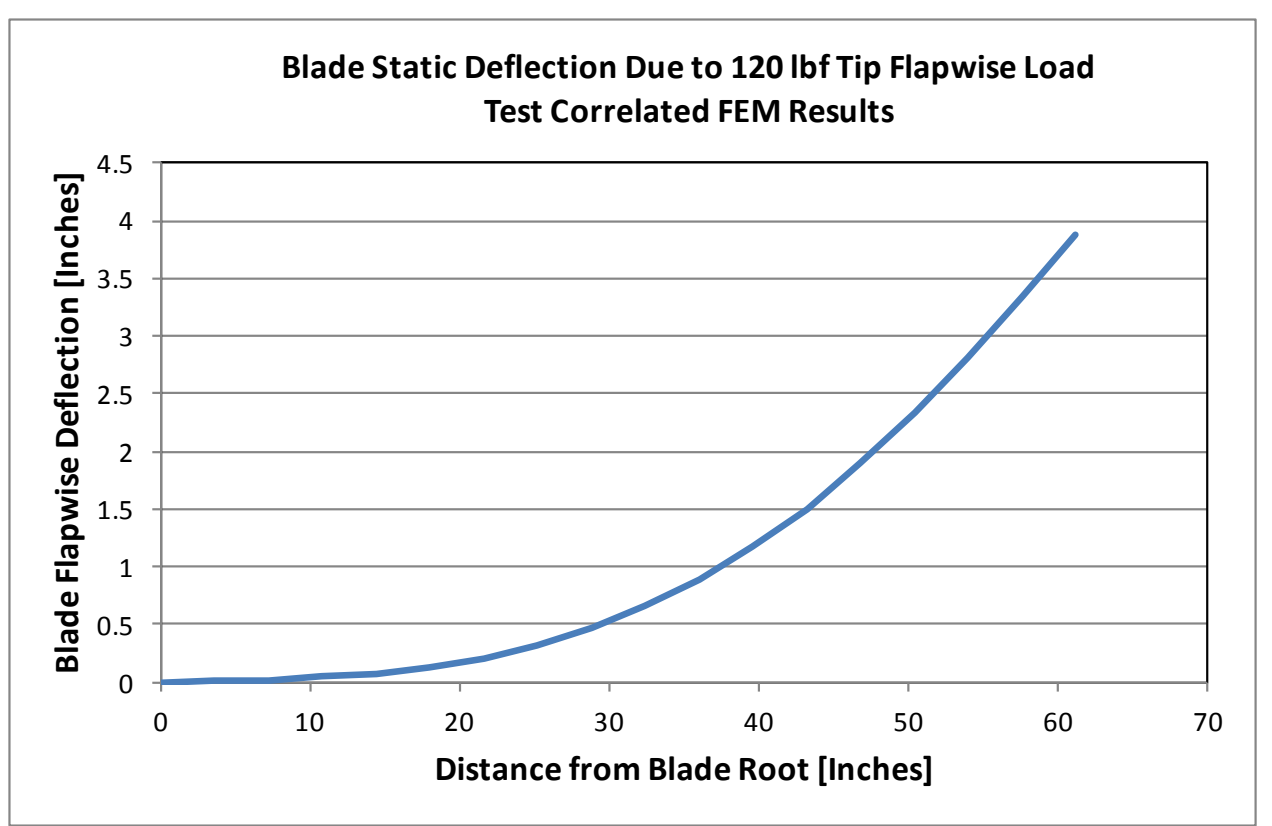


- Step 4.1: Develop the beam equations for beam flapwise deflection and rotation as detailed by (Manwell et al., 2002, p163) .

- For non-rotating case use $\Omega=0$

- For rotating case use $\Omega=230$ RPM (operational rotor speed)

- Step 4.2: Guess a blade flapwise natural frequency and then solve the deflection and rotation sequence equations.

- The equations are solved by starting at the free end of the blade and sequentially progressing to the blade root. Two sets of equations are solved:

- Equation Set 1:

- Deflection at the Free End: 1

- Rotation at the Free End: 0

- Equation Set 2:

- Deflection at the Free End: 0

- Rotation at the Free End: 1

- Evaluate the following ratios

- Ratio 1: Root Deflection from Equation Set 1 / Root Deflection from Equation Set 2

- Ratio 2: Root Rotation from Equation Set 1 / Root Rotation from Equation Set 2

- Step 4.2: Iterate until a natural frequency is found that produces a Ratio 1 = Ratio 2. 
- Application of Myklestad Method Steps $1-4$ yielded the following results for the Cal Poly Wind Turbine Blades:

- Non-Rotating Natural Frequency: $15.69 \mathrm{~Hz}$

- Rotating Natural Frequency: $19.38 \mathrm{~Hz} @$ Operational Rotor Speed of 230 RPM

- The above parameters influence the location and stiffness of the hinge in the Hinge Offset Equivalent Blade Model.

- Upon development of the Hinge Offset Equivalent Blade Model, using the above parameters, it was found that a hinge ratio of $e=0.84$ resulted (i.e., the hinge was much closer to the blade tip than the root). A high hinge ratio (e) results in reduced blade mass dynamic participation.

- To be conservative it was desired to use a lower hinge ratio, which results in increased blade mass dynamic participation. Therefore the rotating natural frequency was adjusted downward to $16.25 \mathrm{~Hz}$. This adjustment resulted in a blade hinge ratio of $\mathrm{e}=0.12$.

- This illustrates an aspect of the Hinge Offset Equivalent Blade Model that the analyst must be aware of. Adjustments to blade analytical parameters should be made such that the hinge is placed in a reasonable configuration (i.e., closer to the root than to the blade tip). 


\section{Tower Mesh (Element Count) Convergence Study}

- Overview

Transient Small Wind Turbine Tower Structural Analysis with Coupled Rotor Dynamic Interaction

- The tables on the next chart summarize the results of the convergence study used to determine an appropriate number of 2D Plane Beam Elements required to accurately model the Cal Poly Tower.

- The convergence study was performed by comparing modal frequencies and shapes for Tower modes 1-4 from models with as few as 9 elements up to a model containing 148 elements.

- It was concluded that a model featuring 37 elements offers an optimal balance of accuracy and reasonable computational time.

- A modal convergence study is the preferred approach as it accounts for both system mass and stiffness influences.

- The convergence study was performed in the Strong-Axis.

- By extension the results are more than adequate for the Weak-Axis since the Weak-Axis geometer is simpler in comparison to the Strong-Axis (no Gin-Pole / Strut). (In general simpler system geometry requires fewer elements.) 


\section{Tower Mesh (Element Count) Convergence Study}

- Results

Transient Small Wind Turbine Tower Structural Analysis with Coupled Rotor Dynamic Interaction

\section{Tower Mesh Density Modal Frequency Convergence Study}

\begin{tabular}{|c|c|c|c|c|c|}
\hline \multirow{2}{*}{ Mode } & \multicolumn{5}{|c|}{ Number of Tower Elements } \\
\cline { 2 - 6 } & $\mathbf{9 E I m}$ & $\mathbf{1 9} \mathrm{Elm}$ & $\mathbf{3 7} \mathrm{Elm}$ & $\mathbf{7 4} \mathrm{Elm}$ & $\mathbf{1 4 8} \mathrm{Elm}$ \\
\hline 1 & 0.811 & 0.813 & 0.814 & 0.814 & 0.814 \\
\hline 2 & 4.696 & 4.699 & 4.703 & 4.704 & 4.704 \\
\hline 3 & 12.980 & 12.988 & 12.994 & 12.996 & 12.996 \\
\hline 4 & 16.217 & 16.203 & 16.200 & 16.199 & 16.199 \\
\hline
\end{tabular}

Percent Error (Relative to 148 Elm Model)

\begin{tabular}{|c|c|c|c|c|c|}
\hline 1 & $-0.38 \%$ & $-0.10 \%$ & $-0.02 \%$ & $0.00 \%$ & \\
\hline 2 & $-0.16 \%$ & $-0.09 \%$ & $-0.02 \%$ & $0.00 \%$ & $\begin{array}{c}\text { Reference } \\
\text { Model }\end{array}$ \\
\hline 3 & $-0.12 \%$ & $-0.06 \%$ & $-0.01 \%$ & $0.00 \%$ & \\
\hline
\end{tabular}

\begin{tabular}{l|l|l|l|l}
\hline 4 & $0.11 \%$ & $0.02 \%$ & $<0.00 \%$ & $0.00 \%$ \\
\hline
\end{tabular}

\begin{tabular}{|c|c|c|c|c|c|}
\hline \multicolumn{6}{|c|}{$\begin{array}{l}\text { Tower Mesh Density Mode Shape Convergence Study } \\
\text { RMS Percent Error (Relative to } 148 \text { ELM Model) } \\
=R M S(\text { Delta }) / R M S(148 E L M) * 100 \text {, where Delta = Mode Shape - } \\
148 \text { ELM Mode Shape }\end{array}$} \\
\hline \multirow[b]{2}{*}{ Mode } & \multicolumn{5}{|c|}{ Number of Tower Elements } \\
\hline & $9 \mathrm{Elm}$ & $19 \mathrm{Elm}$ & $37 \mathrm{Elm}$ & $74 \mathrm{Elm}$ & 148 ELM \\
\hline 1 & $0.19 \%$ & $0.01 \%$ & $0.00 \%$ & $0.00 \%$ & \multirow{4}{*}{$\begin{array}{c}\text { Reference } \\
\text { Model }\end{array}$} \\
\hline 2 & $2.66 \%$ & $0.23 \%$ & $0.25 \%$ & $0.00 \%$ & \\
\hline 3 & $1.54 \%$ & $3.45 \%$ & $0.03 \%$ & $0.01 \%$ & \\
\hline 4 & $24.51 \%$ & $3.43 \%$ & $0.07 \%$ & $0.07 \%$ & \\
\hline
\end{tabular}

California Polytechnic State University - San Luis Obispo 
- Consider a tower supported by a four guy-wire configuration in which the spacing between guy-wires is $90^{\circ}$. A top view is shown below...

- Simplifying Assumptions:

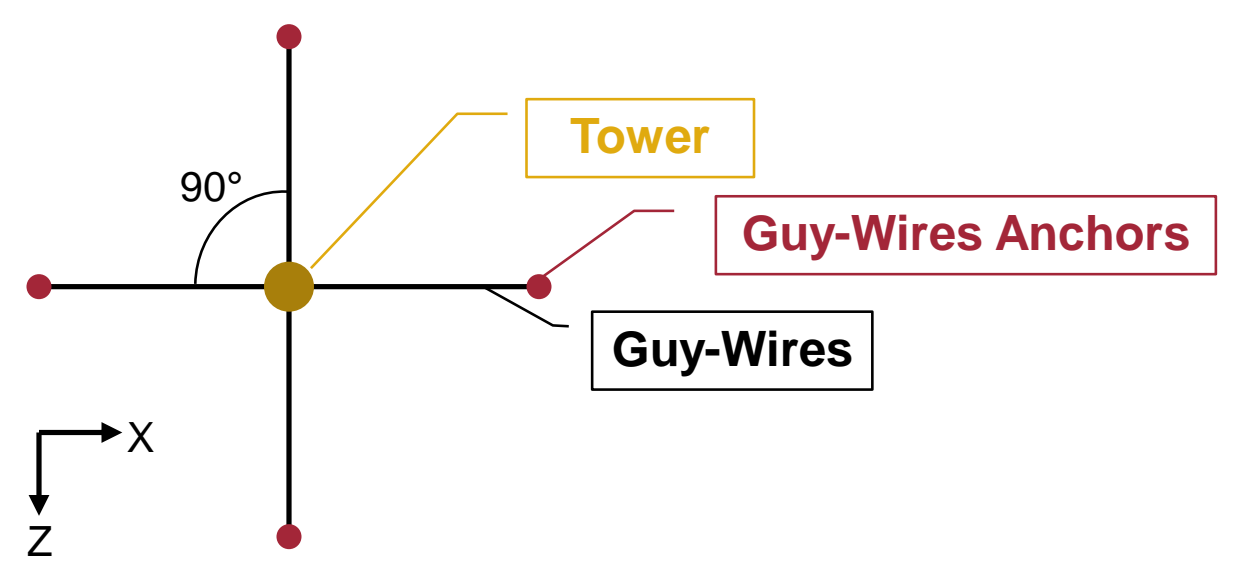

- Linear Elastic Assumption: assume that the guy-wire pretension is sufficient to prevent the guy-wires from going slack. The following derivation includes guy-wire compressive axial load (which represents a reduction in guy-wire tension below the pretension level).

- Horizontal Assumption: For simplicity, let the guy-wire anchor radius be $>>$ than the height of the guy-wire connection to the tower such that the guy-wires can be approximated by horizontal members.

- Tower Mast Stiffness Negligible: Assume that, at a tower node supported by the guywires, the stiffness contribution from the tower mast is negligible when compared to the stiffness contribution from the guy-wires.

- Let the axial stiffness of each guy-wire be K. 


\section{Four Guy-Wire Configuration, Forces and Deflections}

Transient Small Wind Turbine Tower Structural Analysis with Coupled Rotor Dynamic Interaction

- Consider an arbitrary force, F, acting at the tower node supported by the four guy-wires.

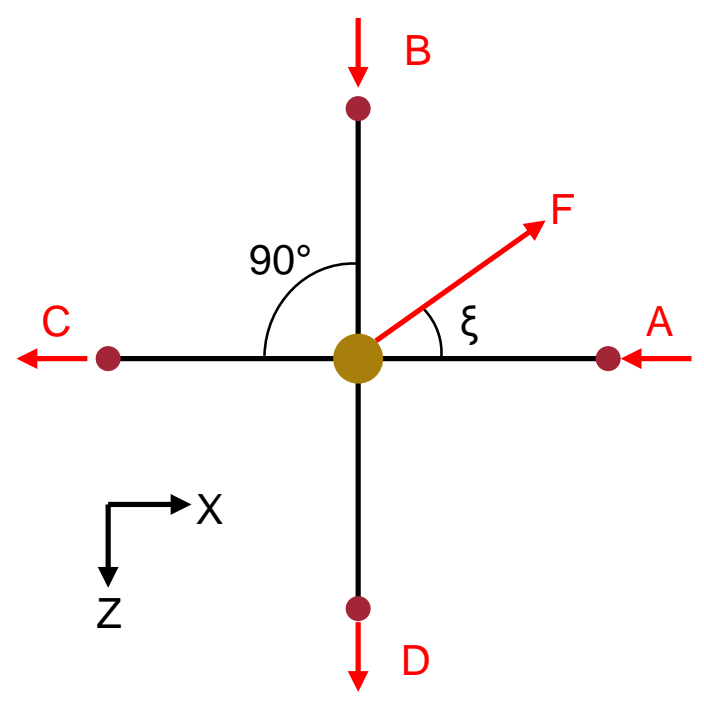

Find force acting on each member

$$
\begin{aligned}
& \sum F_{x}=0=F \cos (\xi)-A-C \\
& A l l_{-} G u y-\text { Wire_Stiffness_is_Equal } \therefore A=C \\
& A=C=\frac{F \cos (\xi)}{2} \\
& \sum F_{z}=0=F \sin (\xi)-B-D \\
& A l l_{-} \text {Guy }- \text { Wire_Stiffness_is_Equal } \therefore B=D \\
& B=D=\frac{F \sin (\xi)}{2}
\end{aligned}
$$

\section{Find Tower Deflection}

$$
\begin{aligned}
& \delta_{x}=\frac{F \cos (\xi)}{2 K} \\
& \delta_{z}=\frac{-F \sin (\xi)}{2 K} \\
& \delta_{\text {mag }}=\sqrt{\delta_{x}{ }^{2}+\delta_{z}^{2}}=\sqrt{\left(\frac{F \cos (\xi)}{2 K}\right)^{2}+\left(\frac{-F \sin (\xi)}{2 K}\right)^{2}}=\frac{F}{2 K} \sqrt{(\cos (\xi))^{2}+(-\sin (\xi))^{2}}=\frac{F}{2 K}
\end{aligned}
$$




\section{Four Guy-Wire Configuration, Conclusion}

Transient Small Wind Turbine Tower Structural Analysis with Coupled Rotor Dynamic Interaction

- As shown on the last chart, the total deflection is $F / 2 K$, where $\mathrm{K}$ is the axial stiffness of an individual guy-wire, regardless of the direction of load application.

- As such the stiffness of a four guy-wire support configuration, from the perspective of the tower mast, can be considered isotropic.

- As shown on the last chart, the maximum guy-wire load occurs when the external forces is aligned with a guy-wire and is $F / 2$. 


\section{Three Guy-Wire Configuration}

Transient Small Wind Turbine Tower Structural Analysis with Coupled Rotor Dynamic Interaction

- Consider a tower supported by a three guy-wire configuration in which the spacing between guy-wires is $90^{\circ}$. A top view is shown below...

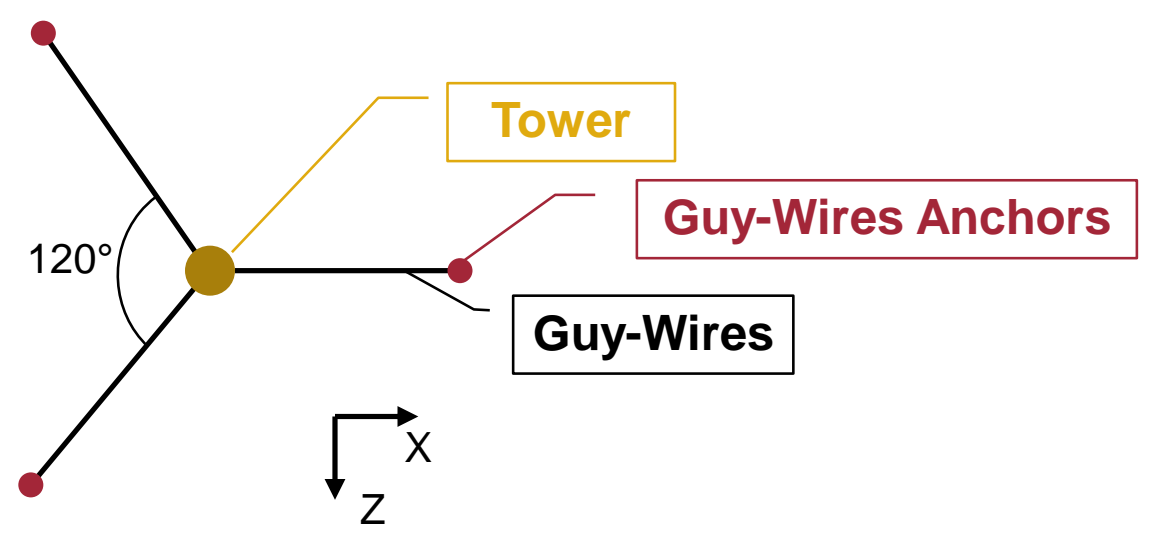

- Simplifying Assumptions:

- Linear Elastic Assumption: assume that the guy-wire pretension is sufficient to prevent the guy-wires from going slack. The following derivation includes guy-wire compressive axial load (which represents a reduction in guy-wire tension below the pretension level).

- Horizontal Assumption: For simplicity, let the guy-wire anchor radius be $>>$ than the height of the guy-wire connection to the tower such that the guy-wires can be approximated by horizontal members.

- Tower Mast Stiffness Negligible: Assume that, at a tower node supported by the guywires, the stiffness contribution from the tower mast is negligible when compared to the stiffness contribution from the guy-wires.

- Let the axial stiffness of each guy-wire be K. 


\section{Three Guy-Wire Configuration, Forces and Deflections}

Transient Small Wind Turbine Tower Structural Analysis with Coupled Rotor Dynamic Interaction

- Consider an arbitrary force, F, acting at the tower node supported by the four guy-wires.

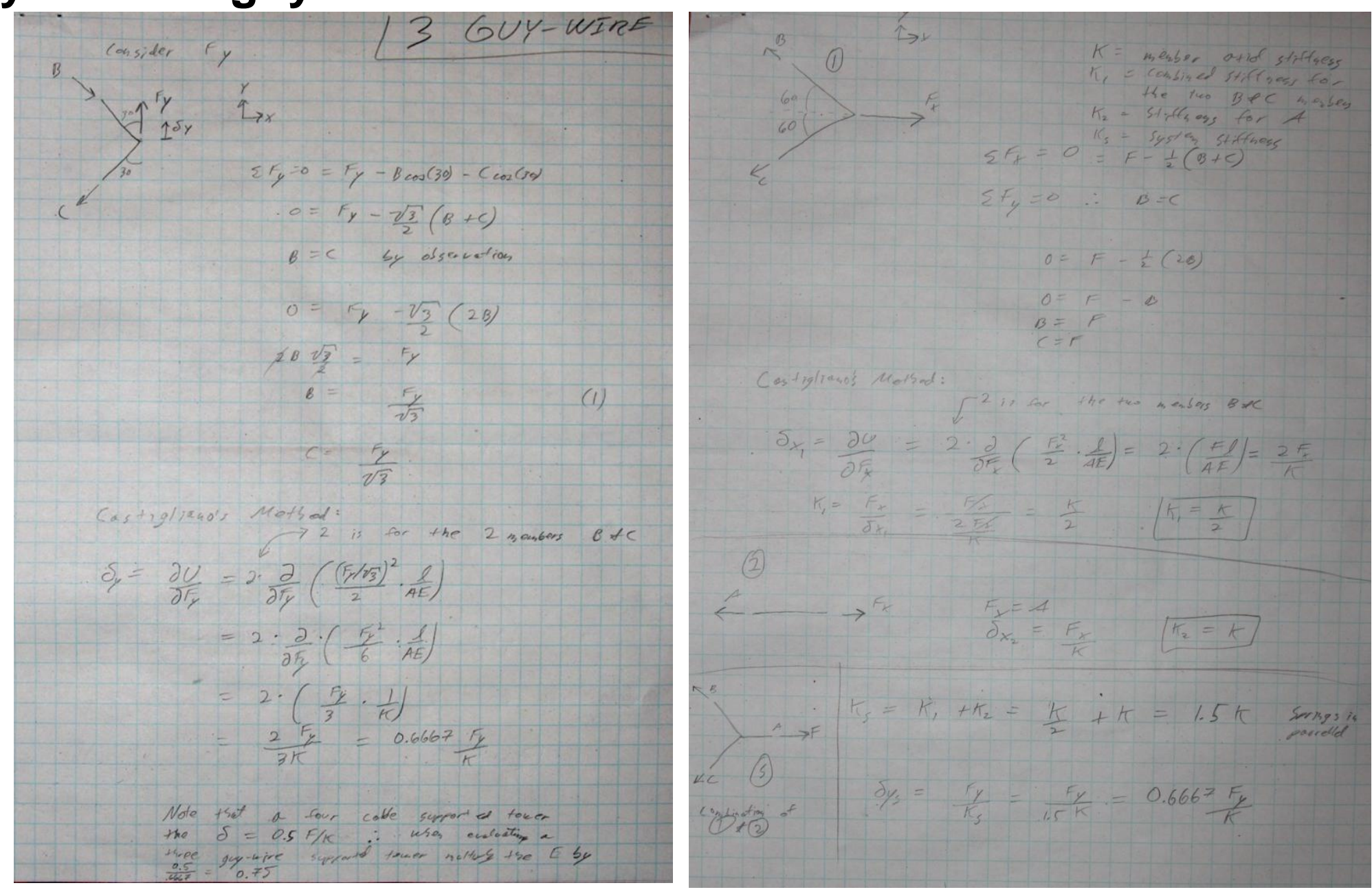




\section{Three Guy-Wire Configuration, Conclusion}

Transient Small Wind Turbine Tower Structural Analysis with Coupled Rotor Dynamic Interaction

- As shown on the last chart, the total deflection is $2 \mathrm{~F} / 3 \mathrm{~K}$, where $\mathrm{K}$ is the axial stiffness of an individual guy-wire, regardless of the direction of load application.

- As such the stiffness of a four guy-wire support configuration, from the perspective of the tower mast, can be considered isotropic.

- As shown on the last chart, the maximum guy-wire load occurs when the external forces is aligned with a guy-wire and is $2 F / 3$. 


\section{Four vs Three Guy-Wire Configuration}

Transient Small Wind Turbine Tower Structural Analysis with Coupled Rotor Dynamic Interaction

- Isotropic Nature:

- Both the four and three guy-wire configurations feature stiffness that is independent of load direction.

- Stiffness:

- For the same load, $F$, the three guy-wire configuration deflects by $(2 F) /(3 K)$ while the four guy-wire configuration deflects by $(1 \mathrm{~F}) /(2 \mathrm{~K})$. As such the three guy-wire configuration is $3 / 4$ times as stiff as the four guy-wire configuration.

- Max Tensile Load:

- For the same load, F, the three guy-wire configuration features a maximum guy-wire tensile load of $(2 / 3) F$ while the four guywire configuration features a maximum guy-wire tensile load of (1/2)F. As such the three guy-wire configuration features a maximum tensile load that is (4/3) times higher than the four guy-wire configuration. 


\section{Appendix D}

\section{Example User Input Spreadsheet}

Inputs Used for Case Study 


\begin{tabular}{|c|c|c|c|c|c|c|c|c|}
\hline \multirow{2}{*}{ 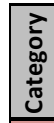 } & \multirow{2}{*}{$\begin{array}{l}\text { Parameter Name } \\
\text { (Matlab Variable } \\
\text { Name) }\end{array}$} & \multirow[b]{2}{*}{ Parameter Description } & \multirow[b]{2}{*}{ Units } & \multirow{2}{*}{$\begin{array}{l}\text { Number of required } \\
\text { User Input Values } \\
\text { (min-max) }\end{array}$} & \multicolumn{4}{|c|}{ User Input } \\
\hline & & & & & Value 1 & Value 2 & Value 3 & Value 4 \\
\hline \multicolumn{9}{|c|}{ Tower Geometry } \\
\hline & $\begin{array}{l}\text { Section Length } \\
\quad(\text { SecL) }\end{array}$ & $\begin{array}{l}\text { Enter in the length of each tower section starting with the lowest section and continuing } \\
\text { sequentially to the upper section. Note that there must be a section break anywhere that } \\
\text { there is a change in tower geometer or material and anywhere that a guy-wire is } \\
\text { connected to the tower. }\end{array}$ & Inches & $1-10$ & 48 & 220.82 & 144.18 & 420 \\
\hline & $\begin{array}{l}\text { Bottom Section } \\
\text { Height } \\
\text { (Bottom) }\end{array}$ & Enter in the height of the bottom of the lowest tower section. & Inches & 1 & 11.5 & & & \\
\hline & $\begin{array}{l}\text { Tower Outer } \\
\text { Diameter Type } \\
\text { (ODtype) }\end{array}$ & $\begin{array}{l}\text { Using the Value } 1 \text { pull down menu select one of the following: } \\
\text { Constant: } \\
\text { Tower outer diameter is constant for each of the tower sections defined in SecL. } \\
\text { Note: User must enter data for OD, data entered for ODtaper will be ignored. } \\
\text { Tapered: } \\
\text { Tower outer diameter linearly tapers as a function of distance from the tower base. } \\
\text { Note: User must enter data for ODtaper, data entered for OD will be ignored. }\end{array}$ & NA & 1 & Tapered & & & \\
\hline & $\begin{array}{c}\text { Tower Outer } \\
\text { Diameter for } \\
\text { Constant ODtype } \\
\text { (OD) }\end{array}$ & $\begin{array}{l}\text { If ODtype is Constant enter in the outer diameter of each tower section starting with the } \\
\text { lowest section and continuing sequentially to the upper section. } \\
\text { Note: if ODtype is Tapered then OD will be ignored. }\end{array}$ & Inches & $1-10$ & & & & \\
\hline & \begin{tabular}{|c|}
$\begin{array}{c}\text { Tower Outer } \\
\text { Diameter for Taper } \\
\text { ODtype } \\
\text { (ODtaper) }\end{array}$ \\
\end{tabular} & $\begin{array}{l}\text { If ODtype is Tapered enter in the outer diameter of the tower base in Value } 1 \text { and the } \\
\text { outer diameter of the tower tip in Value } 2 \text {. } \\
\text { Note: if ODtype is Constant then Odtaper will be ignored. }\end{array}$ & Inches & 2 & 13.957 & 6 & & \\
\hline & $\begin{array}{l}\text { Wall Thickness } \\
\text { (Wall) }\end{array}$ & $\begin{array}{l}\text { Enter in the tower wall thickness of each tower section starting with the lowest section } \\
\text { and continuing sequentially to the upper section. }\end{array}$ & Inches & $1-10$ & 0.375 & 0.375 & 0.375 & 0.375 \\
\hline \multicolumn{9}{|c|}{ Tower Material Properties } \\
\hline & $\begin{array}{c}\text { Tower Young's } \\
\text { Modulus of Elasticity } \\
\text { (ET) }\end{array}$ & $\begin{array}{l}\text { Enter in the Young's Modulus of Elasticity of each tower section starting with the lowest } \\
\text { section and continuing sequentially to the upper section. }\end{array}$ & |lbf/inch^2 & $1-10$ & 29200000 & 29200000 & 29200000 & 29200000 \\
\hline & $\begin{array}{l}\text { Tower material } \\
\text { weight density } \\
\text { (RhoT) }\end{array}$ & $\begin{array}{l}\text { Enter in the material weight density of each tower section starting with the lowest } \\
\text { section and continuing sequentially to the upper section. } \\
\text { Note: these values are turned into consistent mass units internally which are } \\
\text { lbf* } \sec ^{\wedge} 2 / \text { inch^}^{\wedge} 4 \text {, also know as the slinch/inch^3. }\end{array}$ & Ibf/inch^3 & $1-10$ & 0.284 & 0.284 & 0.284 & 0.284 \\
\hline
\end{tabular}




\begin{tabular}{|c|c|c|c|c|c|c|c|c|}
\hline \multirow{2}{*}{ 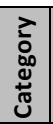 } & \multirow{2}{*}{$\begin{array}{c}\text { Parameter Name } \\
\text { (Matlab Variable } \\
\text { Name) }\end{array}$} & \multirow[b]{2}{*}{ Parameter Description } & \multirow[b]{2}{*}{ Units } & \multirow{2}{*}{$\begin{array}{c}\text { Number of required } \\
\text { User Input Values } \\
\text { (min-max) }\end{array}$} & \multicolumn{4}{|c|}{ User Input } \\
\hline & & & & & Value 1 & Value 2 & Value 3 & Value 4 \\
\hline \multicolumn{9}{|c|}{ Tower Mesh Seeding } \\
\hline & $\begin{array}{c}\begin{array}{c}\text { Elements per Section } \\
\text { (ElmPerSec) }\end{array} \\
\end{array}$ & $\begin{array}{l}\text { Enter in the number of finite elements of each tower section starting with the lowest } \\
\text { section and continuing sequentially to the upper section. It is suggested that 3-15 } \\
\text { elements be used per section. }\end{array}$ & NA & $1-10$ & 3 & 10 & 6 & 18 \\
\hline \multicolumn{9}{|c|}{ Tower Boundary Condition } \\
\hline & $\begin{array}{c}\text { Tower Base } \\
\text { Boundary Condition } \\
\text { (BC) }\end{array}$ & $\begin{array}{l}\text { Using the Value } 1 \text { pull down menu select one of the following: } \\
\text { Fixed: } \\
\text { Tower base boundary condition will be fixed (no translation or rotation of tower base } \\
\text { node allowed). } \\
\text { Pinned: } \\
\text { Tower base boundary condition will be pinned (tower base node allowed to rotate but } \\
\text { not translate). }\end{array}$ & NA & 1 & Pinned & & & \\
\hline
\end{tabular}




\begin{tabular}{|c|c|c|c|c|c|c|c|c|}
\hline \multirow{2}{*}{ 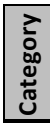 } & \multirow{2}{*}{$\begin{array}{l}\text { Parameter Name } \\
\text { (Matlab Variable } \\
\text { Name) }\end{array}$} & \multirow[b]{2}{*}{ Parameter Description } & \multirow[b]{2}{*}{ Units } & \multirow{2}{*}{$\begin{array}{l}\text { Number of required } \\
\text { User Input Values } \\
\text { (min-max) }\end{array}$} & \multicolumn{4}{|c|}{ User Input } \\
\hline & & & & & Value 1 & Value 2 & Value 3 & Value 4 \\
\hline \multicolumn{9}{|c|}{ Gin-Pole and Strut Inputs (Note, Only Gin-Pole and Strut OR Guy-Wires can be modeled - not both) } \\
\hline & $\begin{array}{l}\text { Gin-Pole and Strut } \\
\text { Usage } \\
\text { (GPS) }\end{array}$ & $\begin{array}{l}\text { Using the Value } 1 \text { pull down menu select one of the following: } \\
\text { Yes: } \\
\text { Tower is supported by a Gin-Pole and Strut configuration. } \\
\text { Note: User must enter data for all parameters included in the Gin-Pole and Strut Inputs } \\
\text { Section. } \\
\text { No: } \\
\text { Tower is not supported by a Gin-Pole and Strut configuration. } \\
\text { Note: All parameters in the Gin-Pole and Strut Inputs Section will be ignored. }\end{array}$ & NA & 1 & Yes & & & \\
\hline & $\begin{array}{l}\text { Gin-Pole and Strut } \\
\text { Connections } \\
\text { (GPSconnect) }\end{array}$ & $\begin{array}{l}\text { Enter in each tower section which attaches to a Gin-Pole or Strut at its top. The Value } 1 \\
\text { entry must be the section which attaches to the Gin-Pole at its top and the Value } 2 \text { entry } \\
\text { must be the section which attaches to the Strut at its top. }\end{array}$ & NA & 2 & 1 & 2 & & \\
\hline & $\begin{array}{l}\text { Gin-Pole and Strut } \\
\text { Ground Interface } \\
\text { (GPSground) }\end{array}$ & $\begin{array}{l}\text { Enter the distance from the base of the tower to the ground anchor for the Gin-Pole and } \\
\text { Strut. The Value } 1 \text { entry is for the Gin-Pole and the Value } 2 \text { entry is for the Strut. Positive } \\
\text { values place the ground anchor downwind from the tower and negative values place the } \\
\text { ground anchor upwind from the tower. }\end{array}$ & inches & 2 & 252.25 & 252.25 & & \\
\hline & $\begin{array}{l}\text { Gin-Pole and Strut } \\
\text { Outer Diameters } \\
\text { (GPSOD) }\end{array}$ & $\begin{array}{l}\text { Enter in the Gin-Pole and Strut outer diameter. The Value } 1 \text { entry is for the Gin-Pole and } \\
\text { the Value } 2 \text { entry is for the Strut. }\end{array}$ & inches & 2 & 5 & 5 & & \\
\hline & $\begin{array}{l}\text { Gin-Pole and Strut } \\
\text { Wall Thickness } \\
\text { (GPSWall) }\end{array}$ & $\begin{array}{l}\text { Enter in the Gin-Pole and Strut wall thickness. The Value } 1 \text { entry is for the Gin-Pole and } \\
\text { the Value } 2 \text { entry is for the Strut. }\end{array}$ & inches & 2 & 0.25 & 0.25 & & \\
\hline & $\begin{array}{l}\text { Gin-Pole and Strut } \\
\text { Young's Modulus of } \\
\text { Elasticity } \\
\text { (EGPS) }\end{array}$ & $\begin{array}{l}\text { Enter in the Young's Modulus of Elasticity of the Gin-Pole and Strut. The Value } 1 \text { entry is } \\
\text { for the Gin-Pole and the Value } 2 \text { entry is for the Strut. }\end{array}$ & Ibf/inch^2 & 2 & 29200000 & 29200000 & & \\
\hline & $\begin{array}{l}\text { Gin-Pole and Strut } \\
\text { Material Weight } \\
\text { Density } \\
\text { (RhoGPS) } \\
\end{array}$ & $\begin{array}{l}\text { Enter in the material weight density of the Gin-Pole and Strut. The Value } 1 \text { entry is for the } \\
\text { Gin-Pole and the Value } 2 \text { entry is for the Strut. } \\
\text { Note: these values are turned into consistant mass units internally which are } \\
\text { lbf* } \sec ^{\wedge} 2 / \text { inch^}^{\wedge} 4 \text {, also know as the slinch/inch^3. }\end{array}$ & Ibf/inch^3 & 2 & 0.284 & 0.284 & & \\
\hline
\end{tabular}




\begin{tabular}{|c|c|c|c|c|c|c|c|c|}
\hline \multirow{2}{*}{ 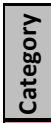 } & \multirow{2}{*}{$\begin{array}{c}\text { Parameter Name } \\
\text { (Matlab Variable } \\
\text { Name) }\end{array}$} & \multirow[b]{2}{*}{ Parameter Description } & \multirow[b]{2}{*}{ Units } & \multirow{2}{*}{$\begin{array}{c}\begin{array}{c}\text { Number of required } \\
\text { User Input Values } \\
\text { (min-max) }\end{array} \\
\end{array}$} & \multicolumn{4}{|c|}{ User Input } \\
\hline & & & & & Value 1 & Value 2 & Value 3 & Value 4 \\
\hline \multicolumn{9}{|c|}{ Guy-Wire Inputs (Note, Only Gin-Pole and Strut OR Guy-Wires can be modeled - not both) } \\
\hline & $\begin{array}{l}\text { Guy-Wire Usage } \\
\text { (GW) }\end{array}$ & $\begin{array}{l}\text { Using the Value } 1 \text { pull down menu select one of the following: } \\
\text { Yes: } \\
\text { Tower is supported by a Guy-Wire configuration. } \\
\text { Note: User must enter data for all parameters included in the Guy-Wire Section. } \\
\text { No: } \\
\text { Tower is not supported by a Guy-Wire configuration. } \\
\text { Note: All parameters in the Guy-Wire Section will be ignored. } \\
\end{array}$ & NA & 1 & No & & & \\
\hline & $\begin{array}{l}\text { Guy-Wire } \\
\text { Connections } \\
\text { (GWconnect) }\end{array}$ & $\begin{array}{l}\text { Enter in each tower section which attaches to a Guy-Wire set at its top. A set of guy-wires } \\
\text { are a group of four guy-wires that all attach to the tower at the same distance from the } \\
\text { tower base. Start with the lowest section and continue sequentially to the upper section. }\end{array}$ & NA & $1-10$ & 1 & 2 & 3 & 4 \\
\hline & $\begin{array}{l}\text { Guy-Wire Ground } \\
\text { Interface } \\
\text { (GWground) }\end{array}$ & $\begin{array}{l}\text { Enter the distance from the base of the tower to the ground anchor for each Guy-Wire set. } \\
\text { A value must be entered for each guy-wire set. Enter the same number of values as } \\
\text { GWconnect where Value } 1 \text { corresponds to the Guy-Wire set defined in GWconnect Value } \\
1 \text { and so on. The model assumes that each set of guy-wires includes four guy-wires } \\
\text { wheeled out at 90 degree angles from each other. }\end{array}$ & inches & $\begin{array}{l}\text { (must be same number } \\
\text { of values as } \\
\text { GWconnect) }\end{array}$ & 480 & 480 & 480 & 480 \\
\hline & $\begin{array}{c}\text { Guy-Wire Diameters } \\
\text { (GWdia) }\end{array}$ & $\begin{array}{l}\text { Enter in the Guy-Wire diameter for each Guy-Wire set. A value must be entered for each } \\
\text { guy-wire set. Enter the same number of values as GWconnect where Value } 1 \text { corresponds } \\
\text { to the Guy-Wire set defined in GWconnect Value } 1 \text { and so on. }\end{array}$ & inches & \begin{tabular}{|c|}
$1-10$ \\
$\begin{array}{c}\text { (must be same number } \\
\text { of values as } \\
\text { GWconnect) }\end{array}$ \\
\end{tabular} & 0.1875 & 0.1875 & 0.1875 & 0.1875 \\
\hline & $\begin{array}{c}\text { Guy-Wire Diameters } \\
\text { (EGW) }\end{array}$ & $\begin{array}{l}\text { Enter in the Guy-Wire Young's Modulus of Elasticity for each Guy-Wire set. A value must } \\
\text { be entered for each guy-wire set. Enter the same number of values as GWconnect where } \\
\text { Value } 1 \text { corresponds to the Guy-Wire set defined in GWconnect Value } 1 \text { and so on. }\end{array}$ & Ibf/inch^2 & \begin{tabular}{|c|}
$1-10$ \\
$\begin{array}{c}\text { (must be same number } \\
\text { of values as } \\
\text { GWconnect) }\end{array}$
\end{tabular} & 23000000 & 23000000 & 23000000 & 23000000 \\
\hline & $\begin{array}{l}\text { Guy-Wire Weight } \\
\text { Density } \\
\text { (RhoGW) }\end{array}$ & 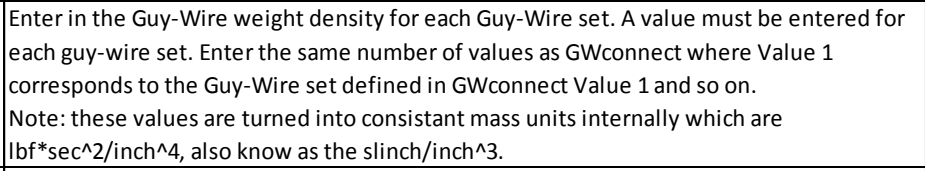 & Ibf/inch^3 & $\begin{array}{l}\text { (must be same number } \\
\text { of values as } \\
\text { GWconnect) }\end{array}$ & 0.284 & 0.284 & 0.284 & 0.284 \\
\hline & $\begin{array}{c}\text { Guy-Wire Pretention } \\
\text { (GWpretension) }\end{array}$ & $\begin{array}{l}\text { Enter in the Guy-Wire pretention for each Guy-Wire set. A value must be entered for each } \\
\text { guy-wire set. Enter the same number of values as GWconnect where Value } 1 \text { corresponds } \\
\text { to the Guy-Wire set defined in GWconnect Value } 1 \text { and so on. } \\
\text { Note: Pretention is mechanical load created by the installation process such as tightening } \\
\text { of turnbuckles. }\end{array}$ & Ibf/inch^3 & $\begin{array}{l}\text { (must be same number } \\
\text { of values as } \\
\text { GWconnect) }\end{array}$ & 1000 & 1000 & 1000 & 1000 \\
\hline
\end{tabular}




\begin{tabular}{|c|c|c|c|c|c|c|c|c|}
\hline \multirow{2}{*}{ 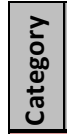 } & \multirow{2}{*}{$\begin{array}{l}\text { Parameter Name } \\
\text { (Matlab Variable } \\
\text { Name) }\end{array}$} & \multirow[b]{2}{*}{ Parameter Description } & \multirow[b]{2}{*}{ Units } & \multirow{2}{*}{$\begin{array}{c}\text { Number of required } \\
\text { User Input Values } \\
\text { (min-max) }\end{array}$} & \multicolumn{4}{|c|}{ User Input } \\
\hline & & & & & Value 1 & Value 2 & Value 3 & Value 4 \\
\hline \multicolumn{9}{|c|}{ Rotor Inputs } \\
\hline & $\begin{array}{l}\text { Rotor Usage } \\
\text { (Rotor) }\end{array}$ & $\begin{array}{l}\text { Using the Value } 1 \text { pull down menu select one of the following: } \\
\text { Yes: } \\
\text { A rotor will be included in the model. } \\
\text { Note: User must enter data for all parameters included in the Rotor Inputs Section. } \\
\text { No: } \\
\text { A rotor will not be included in the model. } \\
\text { Note: All parameters in the Rotor Inputs Section will be ignored. }\end{array}$ & NA & 1 & Yes & & & \\
\hline & $\begin{array}{c}\text { Number of Rotor } \\
\text { Blades } \\
\text { (BladeNum) }\end{array}$ & $\begin{array}{l}\text { Using the Value } 1 \text { pull down menu select one of the following: } \\
\text { 2: } \\
\text { A } 2 \text { bladed rotor will be included in the model. The blades will be } 180 \text { degrees apart. } \\
\text { 3: } \\
\text { A } 3 \text { bladed rotor will be included in the model. The blades will be } 120 \text { degrees apart. }\end{array}$ & NA & 1 & 3 & & & \\
\hline & $\begin{array}{l}\text { Rotor Blade Weight } \\
\text { (BladeM) }\end{array}$ & $\begin{array}{l}\text { Enter in the weight of each rotor blade. } \\
\text { Note: All blades are assumed to have equal weight. }\end{array}$ & Ibf & 1 & 11 & & & \\
\hline & $\begin{array}{c}\text { Rotor Blade Radius } \\
\text { (BladeM) }\end{array}$ & Enter in the radius of the rotor. & inches & 1 & 72 & & & \\
\hline & $\begin{array}{c}\text { Non-Rotating Natural } \\
\begin{array}{l}\text { Frequency } \\
\text { (BladeNF) }\end{array} \\
\end{array}$ & $\begin{array}{l}\text { Enter in the non-rotating (parked) natural frequency (first flapping mode) of the blade. } \\
\text { Note: All blades are assumed to have the same non-rotating natural frequency. }\end{array}$ & $\mathrm{Hz}$ & 1 & 15.69 & & & \\
\hline & $\begin{array}{l}\text { Rotating Natural } \\
\text { Frequency } \\
\text { (BladeRNF) }\end{array}$ & $\begin{array}{l}\text { Enter in the rotating (when rotating at the rated speed OmegaRated) natural frequency } \\
\text { (first flapping mode) of the blade. } \\
\text { Note: All blades are assumed to have the same rotating natural frequency. }\end{array}$ & $\mathrm{Hz}$ & 1 & 16.25 & & & \\
\hline & $\begin{array}{l}\text { Rated Rotor Speed } \\
\text { (OmegaRated) }\end{array}$ & Enter the rotor rated rotational speed. & RPM & 1 & 230 & & & \\
\hline & $\begin{array}{l}\text { Operational Rotor } \\
\text { Speed } \\
\text { (OmegaOp) }\end{array}$ & $\begin{array}{l}\text { Enter the rotor operational speed. } \\
\text { Note: this parameter only influences the rotor natural frequency in the simulation. The } \\
\text { rotor is still modeled as parked in the user defined azimuth orientation. }\end{array}$ & RPM & 1 & 230 & & & \\
\hline
\end{tabular}




\begin{tabular}{|c|c|c|c|c|c|c|c|c|}
\hline \multirow{2}{*}{\begin{tabular}{|l|}
3 \\
0 \\
0 \\
0 \\
0 \\
0 \\
0 \\
\end{tabular}} & \multirow{2}{*}{$\begin{array}{l}\text { Parameter Name } \\
\text { (Matlab Variable } \\
\text { Name) }\end{array}$} & \multirow[b]{2}{*}{ Parameter Description } & \multirow[b]{2}{*}{ Units } & \multirow{2}{*}{$\begin{array}{c}\text { Number of required } \\
\begin{array}{c}\text { User Input Values } \\
\text { (min-max) }\end{array}\end{array}$} & \multicolumn{4}{|c|}{ User Input } \\
\hline & & & & & Value 1 & Value 2 & Value 3 & Value 4 \\
\hline \multicolumn{9}{|c|}{ Rotor Inputs } \\
\hline & $\begin{array}{l}\text { Rotor Hub Weight } \\
\qquad \text { (HM) }\end{array}$ & $\begin{array}{l}\text { Enter the weight of the rotor hub. } \\
\text { Note: this weight must include the weight of the hub, drive train, generator, bedplate, } \\
\text { tail, and nacelle (all tower tip weight excluding the blades). } \\
\text { Note: this value is turned into consistent mass units internally which are lbf*sec^2/inch, } \\
\text { also know as the slinch. }\end{array}$ & Ibf & 1 & 427 & & & \\
\hline & $\begin{array}{l}\text { Rotor Moment of } \\
\text { Inertia about the } \\
\text { tower tip. } \\
\text { (HI) }\end{array}$ & $\begin{array}{l}\text { Enter the moment of inertia about the tower tip of the rotor hub. } \\
\text { Note: the moment of inertia must include the influence of the hub, drive train, generator, } \\
\text { bedplate, tail, and nacelle (all tower tip items excluding the blades). } \\
\text { Note: this value is turned into consistent moment of inertia units internally which are } \\
\text { lbf* } \sec ^{\wedge} 2 * \text { inch, also know as the slinch*inch^2. }\end{array}$ & $\begin{array}{c}\text { Ibf*inches } \\
\wedge 2\end{array}$ & 1 & 15372 & & & \\
\hline & $\begin{array}{l}\text { Rotor X-Axis Offset. } \\
(\mathrm{Hx})\end{array}$ & $\begin{array}{l}\text { Enter in the horizontal (X-Axis) offset between the tower tip to the center of the rotor } \\
\text { blades. } \\
\text { Note: a positive value indicates that the rotor blades are upwind from the tower while a } \\
\text { negative value indicates that the rotor blades are downwind from the tower. }\end{array}$ & inches & 1 & 16 & & & \\
\hline & $\begin{array}{l}\text { Rotor Y-Axis Offset. } \\
\text { (Hy) }\end{array}$ & $\begin{array}{l}\text { Enter in the vertical (Y-Axis) offset between the tower tip to the center of the rotor } \\
\text { blades. } \\
\text { Note: a positive value indicates that the rotor blades are above the tower tip. } \\
\end{array}$ & inches & 1 & 12 & & & \\
\hline & $\begin{array}{c}\text { Rotor Projected Area } \\
\text { (RotorArea) }\end{array}$ & $\begin{array}{l}\text { Enter in the total rotor projected area. This area must include the combined projected } \\
\text { area for all blades, the hub, nacelle, etc... }\end{array}$ & inches^2 & 1 & $\mid 1866.44$ & & & \\
\hline
\end{tabular}




\begin{tabular}{|c|c|c|c|c|c|c|c|c|}
\hline \multirow{2}{*}{ 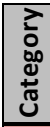 } & \multirow{2}{*}{$\begin{array}{c}\text { Parameter Name } \\
\text { (Matlab Variable } \\
\text { Name) }\end{array}$} & \multirow[b]{2}{*}{ Parameter Description } & \multirow[b]{2}{*}{ Units } & \multirow{2}{*}{$\begin{array}{c}\text { Number of required } \\
\text { User Input Values } \\
\text { (min-max) }\end{array}$} & \multicolumn{4}{|c|}{ User Input } \\
\hline & & & & & Value 1 & Value 2 & Value 3 & Value 4 \\
\hline \multicolumn{9}{|c|}{ IEC 61400-2 Small Wind Turbine Class (SWTC) } \\
\hline & SWTC & $\begin{array}{l}\text { Using the Value } 1 \text { pull down menu select an IEC } 61400-2 \text { (Table 1) SWTC. Options are 1, } 2 \text {, } \\
3 \text {, or } 4 . \\
\text { This parameter sets the reference wind speed (Vref) and the average wind speed (Vave) }\end{array}$ & NA & 1 & 3 & & & \\
\hline \multicolumn{9}{|c|}{ IEC Static Load Case - 50 Year Extreme Wind Model, IEC 61400-2 Table 4, Design Load Case 5.1. (Parked Rotor) } \\
\hline & Run_EWM50 & $\begin{array}{l}\text { Using the Value } 1 \text { pull down menu select one of the following: } \\
\text { Yes: } \\
\text { The } 50 \text { Year Extreme Wind Model Design Load Case (DLC 5.1) will be evaluated. } \\
\text { No: } \\
\text { The } 50 \text { Year Extreme Wind Model Design Load Case (DLC 5.1) will not be evaluated. }\end{array}$ & NA & 1 & Yes & & & \\
\hline \multicolumn{9}{|c|}{ IEC Static Load Case - 1 Year Extreme Wind Model, IEC 61400-2 Table 4, Design Load Case 6.1. } \\
\hline & Run_EWM1 & $\begin{array}{l}\text { Using the Value } 1 \text { pull down menu select one of the following: } \\
\text { Yes: } \\
\text { The } 1 \text { Year Extreme Wind Model Design Load Case (DLC 6.1) will be evaluated. } \\
\text { No: } \\
\text { The } 1 \text { Year Extreme Wind Model Design Load Case (DLC 6.1) will not be evaluated. }\end{array}$ & NA & 1 & Yes & & & \\
\hline \multicolumn{9}{|c|}{ Custom Static Load Case - Tower Tip Thrust Load } \\
\hline & Run_Tip_Load & $\begin{array}{l}\text { Using the Value } 1 \text { pull down menu select one of the following: } \\
\text { Yes: } \\
\text { The Simple Static Tower Tip Load Case will be evaluated. } \\
\text { No: } \\
\text { The Simple Static Tower Tip Load Case will not be evaluated. } \\
\text { Note: The Simple Static Tower Tip Load Case consists of a static tip load applied to the } \\
\text { tower tip in the X-Axis. }\end{array}$ & NA & 1 & Yes & & & \\
\hline & Tip_Load & Enter in the Tower Tip Load & $\mathrm{lbf}$ & 1 & 2100 & & & \\
\hline \multicolumn{9}{|c|}{ Custom Static Load Case - Wind Profile MPH } \\
\hline & Run_MPH & $\begin{array}{l}\text { Using the Value } 1 \text { pull down menu select one of the following: } \\
\text { Yes: } \\
\text { The Wind Profile MPH Load Case will be evaluated. } \\
\text { No: } \\
\text { The Wind Profile MPH Load Case will not be evaluated. } \\
\text { Note: The Wind Profile MPH Load Case consists of the same static wind profile that is used } \\
\text { in DLC } 6.1 \text { but is scaled to a user defined wind speed defined at the tower tip. }\end{array}$ & NA & 1 & Yes & & & \\
\hline & Tip_MPH & Enter the wind speed at the tower tip. & $\mathrm{MPH}$ & 1 & 15 & & & \\
\hline
\end{tabular}




\begin{tabular}{|c|c|c|c|c|c|c|c|c|}
\hline \multirow{2}{*}{$\begin{array}{l}7 \\
0 \\
0 \\
0 \\
\text { cँ } \\
0\end{array}$} & \multirow{2}{*}{$\begin{array}{c}\text { Parameter Name } \\
\text { (Matlab Variable } \\
\text { Name) }\end{array}$} & \multirow[b]{2}{*}{ Parameter Description } & \multirow[b]{2}{*}{ Units } & \multirow{2}{*}{$\begin{array}{c}\text { Number of required } \\
\text { User Input Values } \\
\text { (min-max) }\end{array}$} & \multicolumn{4}{|c|}{ User Input } \\
\hline & & & & & Value 1 & Value 2 & Value 3 & Value 4 \\
\hline \multicolumn{9}{|c|}{ Modal Evaluation - Modal Frequencies and Mode Shapes } \\
\hline & Run_Modal & $\begin{array}{l}\text { Using the Value } 1 \text { pull down menu select one of the following: } \\
\text { Yes: } \\
\text { The Modal Analysis will be performed. } \\
\text { No: } \\
\text { The Modal Analysis will not be performed. } \\
\text { Note: The Modal Analysis consists generates mode shapes in the transverse (X-Axis) and } \\
\text { axial (Y-Axis) directions as well as the corresponding modal frequencies. }\end{array}$ & NA & 1 & Yes & & & \\
\hline \multicolumn{9}{|c|}{ IEC Dynamic Load Cases - IEC 61400-2 Table 4, Design Load Cases 1.3, 1.5, and 2.2 } \\
\hline & Run_EOG & $\begin{array}{l}\text { Using the Value } 1 \text { pull down menu select one of the following: } \\
\text { Yes: } \\
\text { The Extreme Operating Gust Load Case (DLC 1.3) will be performed. } \\
\text { No: } \\
\text { The Extreme Operating Gust Load Case (DLC 1.3) will not be performed. }\end{array}$ & NA & 1 & Yes & & & \\
\hline & Run_ECG & $\begin{array}{l}\text { Using the Value } 1 \text { pull down menu select one of the following: } \\
\text { Yes: } \\
\text { The Extreme Coherent Gust Load Case (DLC 1.5) will be performed. } \\
\text { No: } \\
\text { The Extreme Coherent Gust Load Case (DLC 1.5) will not be performed. }\end{array}$ & NA & 1 & Yes & & & \\
\hline & Run_NTM & $\begin{array}{l}\text { Using the Value } 1 \text { pull down menu select one of the following: } \\
\text { Yes: } \\
\text { The Normal Turbulence Model Load Case (DLC 2.2) will be performed. } \\
\text { No: } \\
\text { The Normal Turbulence Model Load Case (DLC 2.2) will not be performed. }\end{array}$ & NA & 1 & Yes & & & \\
\hline \multicolumn{9}{|c|}{ Custom Dynamic Load Case - Tower Tip Thrust Swept Sine Load } \\
\hline & Run_SwptSin & $\begin{array}{l}\text { Using the Value } 1 \text { pull down menu select one of the following: } \\
\text { Yes: } \\
\text { The Tower Tip Thrust Swept Sine Load Case will be run. } \\
\text { No: } \\
\text { The Tower Tip Thrust Swept Sine Load Case will not be performed. }\end{array}$ & NA & 1 & Yes & & & \\
\hline & TipForceAmp & Enter the amplitude of the tower tip thrust load. & $\mathrm{Ibf}$ & 1 & 100 & & & \\
\hline & $\mathrm{f} 1$ & Enter the starting excitation frequency for the swept sine load case. & $\mathrm{Hz}$ & 1 & 0.1 & & & \\
\hline & $\mathrm{f2}$ & Enter the ending excitation frequency for the swept sine load case. & $\mathrm{Hz}$ & 1 & 20 & & & \\
\hline & $\mathrm{R}$ & $\begin{array}{l}\text { Enter the sweep rate for the swept sine load case. } \\
\text { The sweep rate is logarithmic and is expressed in terms of octaves per minute. } \\
\text { For example, if a swept sine sweep rate of } 1 \text { octave per minute is selected then after } 1 \\
\text { minute the frequency would have doubled and after two minutes the frequency would } \\
\text { have quadrupled. }\end{array}$ & $\begin{array}{c}\text { Octaves / } \\
\text { Minute }\end{array}$ & 1 & 0.5 & & & \\
\hline
\end{tabular}




\begin{tabular}{|c|c|c|c|c|c|c|c|c|}
\hline \multirow{2}{*}{ 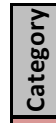 } & \multirow{2}{*}{$\begin{array}{c}\text { Parameter Name } \\
\text { (Matlab Variable } \\
\text { Name) }\end{array}$} & \multirow[b]{2}{*}{ Parameter Description } & \multirow[b]{2}{*}{ Units } & \multirow{2}{*}{$\begin{array}{c}\begin{array}{c}\text { Number of required } \\
\text { User Input Values } \\
\text { (min-max) }\end{array} \\
\end{array}$} & \multicolumn{4}{|c|}{ User Input } \\
\hline & & & & & Value 1 & Value 2 & Value 3 & Value 4 \\
\hline \multicolumn{9}{|c|}{ Dynamic Analysis Parameters } \\
\hline & Nhat & $\begin{array}{l}\text { Select the Nhat value using the pull down. Nhat is the number of modes that will be used } \\
\text { in the dynamic simulation of transient load cases which uses the Mode-Superposition } \\
\text { Method. For example, if } 5 \text { is entered then the first } 5 \text { system modes will be considered in } \\
\text { the simulation. Nhat must be } 1 \text { thru } 10 \text {. } \\
\text { Recommendation: } \\
\text { Start with Nhat }=3 \text {, run the analysis for the desired transient load cases, then } \\
\text { incrementally increase Nhat and rerun the analysis. Check for solution convergence. } \\
\text { When tower displacements and loads have converged then a sufficiently high Nhat is } \\
\text { being used. }\end{array}$ & NA & 1 & 30 & & & \\
\hline & zeta & $\begin{array}{l}\text { Enter the modal damping value for each mode that will be used in the dynamic simulation } \\
\text { of the transient load cases. The number of entered values must equal Nhat. The first } \\
\text { value corresponds to the first mode and so on. }\end{array}$ & $\begin{array}{l}\text { Unitless } \\
\text { Critical } \\
\text { Damping } \\
\text { Factor }\end{array}$ & $\begin{array}{c}1-10 \\
\text { (must be same number } \\
\text { of values as Nhat) }\end{array}$ & 0.05 & 0.05 & 0.04 & \\
\hline & $\mathrm{dt}$ & $\begin{array}{l}\text { Enter the analysis time step in the Value } 1 \text { input that will be used for the dynamic } \\
\text { simulation of transient load cases. } \\
\text { Recommendation: } \\
\text { Run the modal analysis. Then determine the modal frequency of the Nhat mode (i.e., if } \\
\text { Nhat is selected to be } 3 \text { then determine the frequency of the } 3 r d \text { mode). Then set } \mathrm{dt}= \\
1 /\left(10^{*} \text { NhatFreq), where NhatFreq is the modal frequency of the Nhat mode in } \mathrm{Hz} \text {. This }\right. \\
\text { will result in } 10 \text { simulation time steps per cycle of the highest system mode included in } \\
\text { the simulation (this will help to insure solution convergence). } \\
\text { Warning: } \\
\text { If the above recommendation is not followed then the solution may diverge. }\end{array}$ & NA & 1 & 0.01 & & & \\
\hline & DSRatio & $\begin{array}{l}\text { Enter the Down Sample Ratio. This ratio will reduce the number of time steps used in } \\
\text { output files including figures and Excel data file. } \\
\text { Example: } \\
\text { If the dynamic simulation duration is } 10 \text { seconds and dt is selected to be } 0.002 \text { seconds } \\
\text { then the wind profile and external force profile will contain } 5000 \text { time steps. If a DSRatio } \\
\text { of } 10 \text { is used then the Excel data file will contain } 500 \text { time steps thus the effective dt in } \\
\text { the Excel output file will be } 0.02 \text { seconds. Increasing this ratio reduces post processing } \\
\text { time and Excel output files size. }\end{array}$ & $\begin{array}{c}\text { NA } \\
\text { Integer } \geq 1\end{array}$ & 1 & 3 & & & \\
\hline & USRatio & $\begin{array}{l}\text { Enter the Up Sample Ratio. This ratio will increase the number of time steps used in the } \\
\text { dynamic simulation. } \\
\text { Example: } \\
\text { If the dynamic simulation duration is } 10 \text { seconds and dt is selected to be } 0.002 \text { seconds } \\
\text { then the wind profile and external force profile will contain } 5,000 \text { time steps. If a USRatio } \\
\text { of } 10 \text { is used then the dynamic simulation will contain } 50,000 \text { time steps thus the effective } \\
\text { dt used for dynamic simulation would be } 0.0002 \text { seconds. Note that the USRatio does not } \\
\text { have any effect on the number of time steps contained in the data output. Increasing this } \\
\text { parameter is allows for the simulation of long duration environments and high Nhat } \\
\text { parameters without running out of system memory. }\end{array}$ & $\begin{array}{c}\text { NA } \\
\text { Integer } \geq 1\end{array}$ & 1 & 15 & & & \\
\hline
\end{tabular}




\begin{tabular}{|c|c|c|c|c|c|c|c|c|}
\hline \multirow{2}{*}{ 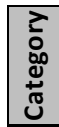 } & \multirow{2}{*}{$\begin{array}{c}\text { Parameter Name } \\
\text { (Matlab Variable } \\
\text { Name) }\end{array}$} & \multirow[b]{2}{*}{ Parameter Description } & \multirow[b]{2}{*}{ Units } & \multirow{2}{*}{$\begin{array}{c}\text { Number of required } \\
\text { User Input Values } \\
\text { (min-max) }\end{array}$} & \multicolumn{4}{|c|}{ User Input } \\
\hline & & & & & Value 1 & Value 2 & Value 3 & Value 4 \\
\hline \multicolumn{9}{|c|}{ Aerodynamic Analysis Parameters } \\
\hline & $\mathrm{Cd}$ & $\begin{array}{l}\text { Enter the tower aerodynamic drag coefficient. The tower drag coefficient is used, along } \\
\text { with the wind velocity profile, in IEC } 61400-2 \text { Eq } 45 \text { to determine tower drag load at each } \\
\text { node. } \\
\text { Recommendations: } \\
\text { Table } 3 \text { of IEC } 61400-2 \text { recommends a Cd value of } 0.7 \text { for cylinders with a characteristic } \\
\text { length of }>4 \text { inches. } \\
\text { Fox et al. (Introduction to Fluid Mechanics) gives a Cd for flow over a cylinder that varies } \\
\text { between } 0.35 \text { and } 1.0 \text { as a function of the Re number. } \\
\text { Tom Gwon (Structural Analyses of Wind Turbine Tower for } 3 \mathrm{~kW} \text { Horizontal-Axis Wind } \\
\text { Turbine) used a Cd of } 1.3 \text {. }\end{array}$ & NA & 1 & 1.3 & & & \\
\hline & $\mathrm{Ct}$ & $\begin{array}{l}\text { Enter the rotor aerodynamic thrust coefficient. The thrust coefficient is used, along with } \\
\text { the hub wind velocity, in IEC } 61400-2 \text { Eq } 32 \text { to determine the thrust load on the rotor. } \\
\text { Recommendations: } \\
\text { IEC } 61400-2 \text { recommends a Ct value of } 0.5 \text {. } \\
\text { For a ideal turbine operating at the Betz limit the Ct value is } 8 / 9 \text {. } \\
\text { The highest possible Ct value, based on one-dimensional momentum theory, is } 1.0 \text { and } \\
\text { corresponds with a downstream velocity of zero (this corresponds with an induction } \\
\text { factor of a = } 1 / 2 \text {. } \\
\text { For turbines operating in a turbulent wake state, which can occur at high tip speed ratios } \\
\text { (as is the case when a constant speed turbine operates at low wind speeds), the Glauert } \\
\text { empirical relation gives a maximum Ct value of } 2.0 \text {. }\end{array}$ & NA & 1 & 0.5 & & & \\
\hline & $\mathrm{Cf}$ & $\begin{array}{l}\text { Enter the rotor aerodynamic force (i.e., drag) coefficient for a Parked Rotor. The force } \\
\text { coefficient is used, along with the hub wind velocity, in IEC 61400-2 Eq } 45 \text { to determine } \\
\text { the thrust load on the rotor when in a parked configuration. } \\
\text { Recommendations: } \\
\text { IEC } 61400-2 \text {, table 3, recommends a Cf value of } 1.5 \text { for the rotor blades (when cord is } \\
\text { orthogonal to wind direction) and } 1.5 \text { for other blunt objects (conservative for the } \\
\text { nacelle). }\end{array}$ & NA & 1 & 1.5 & & & \\
\hline
\end{tabular}

